\title{
Cobalt-Catalyzed Asymmetric Cross-Coupling Reaction of Fluorinated Secondary Benzyl Bromides with Lithium Aryl Boronates/ZnBr2
}

Weichen Huang, Xiaolong Wan and Qilong Shen*

Key Laboratory of Organofluorine Chemistry, Center for Excellence in Molecular Synthesis, Shanghai Institute of Organic Chemistry, University of Chinese Academy of Sciences, Chinese Academy of Sciences, 345 Lingling Road, Shanghai 200032

shenql@sioc.ac.cn

\section{Table of Content}

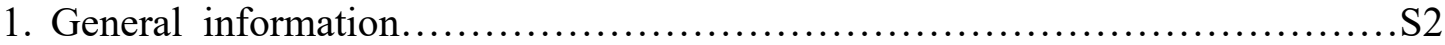

2. General procedure for the preparation of ligands........................... 3

3. General procedure for the preparation of $\alpha$-bromo- $\alpha$-fluorotoluene derivatives...S6

4. General procedure for cobalt-Catalyzed asymmetric cross-coupling $\alpha$-bromo$\alpha$-fluorotoluene derivatives.

5. Procedure for the application of the cobalt-catalyzed asymmetric coupling reaction in the preparation of druglike compounds...............................S41

6. Mechanistic study for trapping the benzylic radical by TEMPO ................S46

7. HPLC spectroscopies of compounds 3a-3ai, 5-7..........................S48

8. ${ }^{1} \mathrm{H},{ }^{19} \mathrm{~F},{ }^{13} \mathrm{C}$ NMR spectroscopies of compound L8-11, 1a-p, 3a-3ai, 5-7.......S124

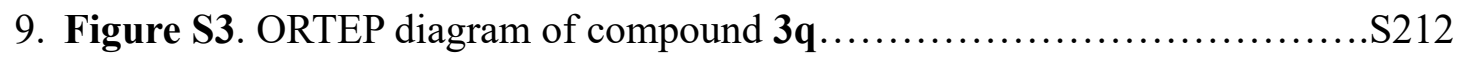




\section{General Information}

${ }^{1} \mathrm{H} \mathrm{NMR}$ and ${ }^{13} \mathrm{C}$ spectra were recorded on a $500 \mathrm{MHz}, 400 \mathrm{MHz}$ or $300 \mathrm{MHz} .{ }^{19} \mathrm{~F}$ NMR were recorded on a $376 \mathrm{MHz}$ or $282 \mathrm{MHz}$ spectrometer. ${ }^{1} \mathrm{H}$ NMR and ${ }^{13} \mathrm{C}$ NMR chemical shifts were determined relative to internal standard TMS at $\delta 0.0$ and ${ }^{19} \mathrm{~F}$ NMR chemical shifts were determined relative to $\mathrm{CFCl}_{3}$ as inter standard. Chemical shifts $(\delta)$ are reported in ppm, and coupling constants $(J)$ are in Hertz $(\mathrm{Hz})$. The following abbreviations were used to explain the multiplicities: $\mathrm{s}=$ singlet, $\mathrm{d}=$ doublet, $\mathrm{t}=$ triplet, $\mathrm{q}=$ quartet, $\mathrm{m}=$ multiplet, $\mathrm{br}=$ broad. Flash column chromatograph was carried out using 300-400 mesh silica gel at medium pressure. Oil bath was used as heating source for the reactions requiring heating. High performance liquid chromatography (HPLC) was performed on an Agilent 1260 series using Daicel Chiralcel chiral column.

All solvents were purified by standard method. Aryl pinacol boronates were purchased from BidePharm (https://www.bidepharm.com) and used without further purification. All other reagents were received from commercial sources. Solvents were freshly dried and degassed according to the purification handbook Purification of Laboratory Chemicals before using. 


\section{Procedure for the preparation of ligand L8}

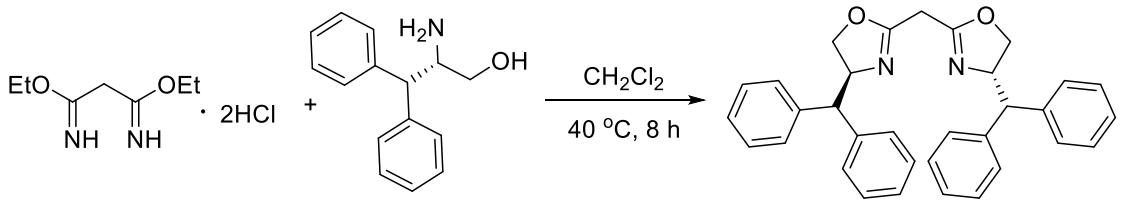

To a solution of (S)-2-amino-3,3-diphenylpropan-1-ol (4.78 g, $21.0 \mathrm{mmol}$ ) in $\mathrm{CH}_{2} \mathrm{Cl}_{2}$ $(5.0 \mathrm{~mL})$ was added diethylmalonimidate dihydrochloride $(2.31 \mathrm{~g}, 10.0 \mathrm{mmol})$. The resulting solution was stirred at $40{ }^{\circ} \mathrm{C}$ for $8 \mathrm{~h}$ (Oil bath was used as heating source). The reaction was diluted with water $(5.0 \mathrm{~mL})$ and then extracted with $\mathrm{CH}_{2} \mathrm{Cl}_{2}(5.0 \mathrm{~mL}$ $\times 3)$. The combined organic layers were washed with brine $(10.0 \mathrm{~mL})$, dried over anhydrous $\mathrm{Na}_{2} \mathrm{SO}_{4}$, filtered and concentrated. The resulting oily residue was purified by neutral alumina chromatography (Eluent: 100\% EtOAc) to give $3.6 \mathrm{~g}$ (75\% yield) of $\mathbf{L 8}$ as a white solid.

\section{Bis((S)-4-benzhydryl-4,5-dihydrooxazol-2-yl)methane L8}

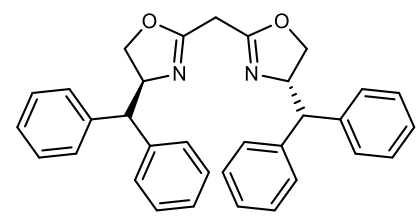

White solid (3.6 g, 75\% yield). Mp: 79-81 ${ }^{\circ} \mathrm{C} .{ }^{1} \mathrm{H}$ NMR $\left(400 \mathrm{MHz}, \mathrm{CDCl}_{3}\right) \delta 7.39-$ $7.06(\mathrm{~m}, 20 \mathrm{H}), 4.91-4.98(\mathrm{~m}, 2 \mathrm{H}), 4.27(\mathrm{~d}, J=8.0 \mathrm{~Hz}, 1 \mathrm{H}), 4.25(\mathrm{~d}, J=8.0 \mathrm{~Hz}, 1$ H), 3.91 - 3.97 (m, $4 \mathrm{H}), 3.24$ (s, $2 \mathrm{H}) ;{ }^{13} \mathrm{C} \mathrm{NMR}\left(101 \mathrm{MHz}, \mathrm{CDCl}_{3}\right) \delta$ 162.3, 142.1, $141.8,128.6,128.5,128.5,128.4,126.7,126.5,71.8,69.6,56.7,28.4$ ppm. MS (DART POS): $487\left(\mathrm{M}+\mathrm{H}^{+}\right)$; HRMS (DART POS) $\mathrm{m} / \mathrm{z}$ : $[\mathrm{M}+\mathrm{H}]^{+}$Calcd for $\mathrm{C}_{33} \mathrm{H}_{31} \mathrm{O}_{2} \mathrm{~N}_{2}$ 487.2380; Found 487.2365. IR (KBr): $v_{\max }=3073,3021,2930,2898,1686,1497$, $1467,1451,1404,1161,1002,701 \mathrm{~cm}^{-1}$.

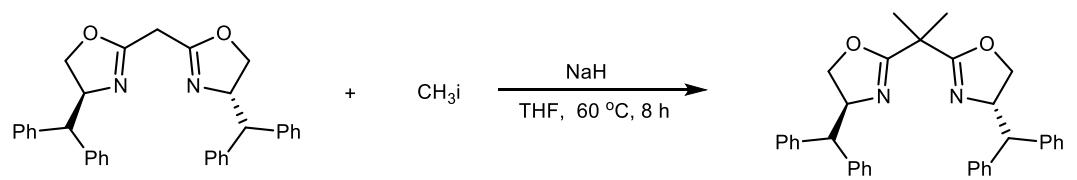

\section{General procedure for the preparation of ligands L9-11}

To a solution of bis((S)-4-benzhydryl-4,5-dihydrooxazol-2-yl)methane L8 (243 mg, $0.500 \mathrm{mmol})$ in THF $(10 \mathrm{~mL}), \mathrm{NaH}(57-63 \%$ oil dispersion) $(60 \mathrm{mg}, 1.5 \mathrm{mmol})$ was added at $0{ }^{\circ} \mathrm{C}$. After $0.5 \mathrm{~h}$ at ambient temperature, to the vigorously stirred mixture was added $\mathrm{CH}_{3} \mathrm{I}(213 \mathrm{mg}, 1.50 \mathrm{mmol})$. The resulting solution was stirred at $60{ }^{\circ} \mathrm{C}$ for 
$8 \mathrm{~h}$ (Oil bath was used as heating source). The reaction was diluted with water (5.0 $\mathrm{mL})$ and then extracted from $\mathrm{CH}_{2} \mathrm{Cl}_{2}(5.0 \mathrm{~mL} \times 3)$. The combined organic layers were washed with brine $(10.0 \mathrm{~mL})$, dried over anhydrous $\mathrm{Na}_{2} \mathrm{SO}_{4}$, filtered and concentrated. The residue was purified by column chromatography on silica gel with a gradient eluent of petroleum ether and ethyl acetate to give compound L9. Compounds L10 and L11 were prepared according to this general procedure.

(4S,4'S)-2,2'-(Propane-2,2-diyl)bis(4-benzhydryl-4,5-dihydrooxazole) L9

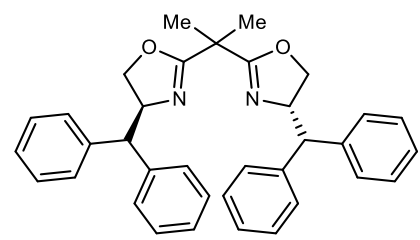

The product L9 (342 mg, 89\% yield, White solid, Mp: $111-113{ }^{\circ} \mathrm{C}$.) was purified with silica gel chromatography (petroleum ether/ethyl acetate $=4 / 1) .{ }^{1} \mathrm{H}$ NMR $(400 \mathrm{MHz}$, $\left.\mathrm{CDCl}_{3}\right) \delta 7.43-7.06(\mathrm{~m}, 20 \mathrm{H}), 4.84-4.90(\mathrm{~m}, 2 \mathrm{H}), 4.27(\mathrm{~d}, J=8.0 \mathrm{~Hz}, 1 \mathrm{H}), 4.25$ $(\mathrm{d}, J=8.0 \mathrm{~Hz}, 1 \mathrm{H}), 4.01-4.03(\mathrm{~m}, 4 \mathrm{H}), 1.27(\mathrm{~s}, 6 \mathrm{H}) ;{ }^{13} \mathrm{C} \mathrm{NMR}\left(101 \mathrm{MHz}, \mathrm{CDCl}_{3}\right)$ $\delta 169.3,141.8$ (this peak is overlapped), 129.0, 128.4, 128.1, 126.6, 126.3, 71.3, 69.1, 56.0, 38.6, $23.8 \mathrm{ppm}$ (The number of the peaks were less than expected, likely because of a few overlapping peaks that are corresponding to the carbons of the phenyl group). MS (DART POS): $515(\mathrm{M}+\mathrm{H})$; HRMS (DART POS) m/z: $[\mathrm{M}+\mathrm{H}]^{+}$ Calcd for $\mathrm{C}_{35} \mathrm{H}_{35} \mathrm{O}_{2} \mathrm{~N}_{2}$ 515.2700; Found 515.2677. IR (KBr): $v_{\max }=3414,3085,3004$, $2883,1948,1654,1496,1450,1253,1111,992,731,656 \mathrm{~cm}^{-1}$.

(4S,4'S)-2,2'-(Pentane-3,3-diyl)bis(4-benzhydryl-4,5-dihydrooxazole) L10

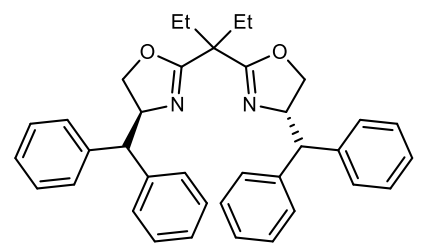

The product L10 (348 mg, 85\% yield, White solid, Mp: $40-42{ }^{\circ} \mathrm{C}$.) was purified with silica gel chromatography (petroleum ether/ethyl acetate $=4 / 1) .{ }^{1} \mathrm{H}$ NMR $(400 \mathrm{MHz}$, $\left.\mathrm{CDCl}_{3}\right) \delta 7.34-6.96(\mathrm{~m}, 20 \mathrm{H}), 4.84-4.90(\mathrm{~m}, 2 \mathrm{H}), 4.24(\mathrm{~d}, J=8.0 \mathrm{~Hz}, 1 \mathrm{H}), 4.21$ $(\mathrm{d}, J=8.0 \mathrm{~Hz}, 1 \mathrm{H}), 3.90-3.94(\mathrm{~m}, 4 \mathrm{H}), 1.93-1.73(\mathrm{~m}, 4 \mathrm{H}), 0.69(\mathrm{t}, J=7.5 \mathrm{~Hz}, 6$ 
$\mathrm{H}) ;{ }^{13} \mathrm{C} \mathrm{NMR}\left(101 \mathrm{MHz}, \mathrm{CDCl}_{3}\right) \delta 167.8,142.2,141.9,128.9,128.8,128.5,128.1$, 126.6, 126.2, 70.9, 69.6, 56.6, 46.7, 25.6, 8.4 ppm. MS (DART POS): $543\left(\mathrm{M}+\mathrm{H}^{+}\right)$; HRMS (DART POS) m/z: [M+H] $]^{+}$Calcd for $\mathrm{C}_{37} \mathrm{H}_{39} \mathrm{O}_{2} \mathrm{~N}_{2}$ 543.3006; Found 543.3000. IR $(\mathrm{KBr}): v_{\max }=3059,3026,2969,2953,2935,1652,1599,1450,1220,984$, $742,701 \mathrm{~cm}^{-1}$.

(4S,4'S)-2,2'-(Cyclopentane-1,1-diyl)bis(4-benzhydryl-4,5-dihydrooxazole) L11

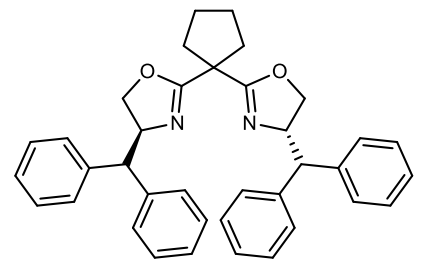

The product L11 (300 mg, 75\% yield, White solid, Mp: 43-45 ${ }^{\circ} \mathrm{C}$. ) was purified with silica gel chromatography (petroleum ether/ethyl acetate $=4 / 1) .{ }^{1} \mathrm{H}$ NMR $(400 \mathrm{MHz}$, $\left.\mathrm{CDCl}_{3}\right) \delta 7.36-6.98(\mathrm{~m}, 20 \mathrm{H}), 4.85-4.88(\mathrm{~m}, 2 \mathrm{H}), 4.27(\mathrm{~d}, J=8.0 \mathrm{~Hz}, 1 \mathrm{H}), 4.25$ $(\mathrm{d}, J=8.0 \mathrm{~Hz}, 1 \mathrm{H}), 3.99-4.03(\mathrm{~m}, 4 \mathrm{H}), 2.27-2.08(\mathrm{~m}, 2 \mathrm{H}), 1.85-1.66(\mathrm{~m}, 2 \mathrm{H})$, $1.61-1.48(\mathrm{~m}, 4 \mathrm{H}) ;{ }^{13} \mathrm{C}$ NMR $\left(101 \mathrm{MHz}, \mathrm{CDCl}_{3}\right) \delta 168.8,141.9,141.8,129.0,129.0$, 128.4, 128.1, 126.6, 126.3, 71.4, 69.1, 56.1, 48.9, 35.0, 25.3 ppm. MS (DART POS): $541\left(\mathrm{M}+\mathrm{H}^{+}\right)$; HRMS (DART POS) $\mathrm{m} / \mathrm{z}$ : $[\mathrm{M}+\mathrm{H}]^{+}$Calcd for $\mathrm{C}_{37} \mathrm{H}_{37} \mathrm{O}_{2} \mathrm{~N}_{2}$ 541.2850; Found 541.2836. IR (KBr): $v_{\max }=3312,3059,3026,2872,1949,1889,1753,1655$, $1494,1471,1450,1355,1265,1157,999,739,702,632 \mathrm{~cm}^{-1}$. 
General procedure for the preparation of $\alpha$-bromo- $\alpha$-fluorotoluene derivatives<smiles>COC(=O)c1ccc(C(F)(F)Br)cc1</smiles>

Into a $100 \mathrm{~mL}$ round bottom flask was added methyl 4-(hydroxymethyl)benzoate (5.0 $\mathrm{g}, 30 \mathrm{mmol})$, and $\mathrm{CH}_{2} \mathrm{CI}_{2}(50.0 \mathrm{~mL})$. The resulting solution was cooled in an ice bath and DAST (6.0 mL, $45 \mathrm{mmol})$ was added dropwise. After stirring for several minutes at $0{ }^{\circ} \mathrm{C}$, the ice bath was removed and the reaction mixture was allowed to warm to room temperature. After $12 \mathrm{~h}$ at room temperature, the reaction mixture was cooled in an ice bath and carefully quenched by the addition of saturated aqueous solution of $\mathrm{NaHCO}_{3}$ with vigorous stirring. After warming to $\mathrm{rt}$ and sitting for $1 \mathrm{~h}$, the organic layer was separated and the aqueous layer was further extracted with $\mathrm{CH}_{2} \mathrm{Cl}_{2}(10.0$ $\mathrm{mL} \times 3$ ). The combined organic extracts were dried over anhydrous $\mathrm{Na}_{2} \mathrm{SO}_{4}$, filtered and concentrated in vacuo to afford a dark brown oil. The residue was purified by short chromatography to give methyl 4-(fluoromethyl)benzoate which was used without further purification.

Methyl 4-(fluoromethyl)benzoate obtained from the previous step was dissolved in carbon tetrachloride $(35.0 \mathrm{~mL})$ and NBS $(5.3 \mathrm{~g}, 30 \mathrm{mmol})$ were added under an argon atmosphere. The mixture was refluxed for $8 \mathrm{~h}$ under irradiation of a $200 \mathrm{~W}$ incandescent lamp (Oil bath was used as heating source). The reaction mixture was then cooled to room temperature, and filtered through a short plug of silica gel. The solvent was evaporated in vacuo. The residue was purified by column chromatography on silica gel with a gradient eluent of petroleum ether and ethyl acetate to give compound $1 \mathbf{a}$ as a yellow liquid (4.0 g, 55\%).

Methyl 4-(bromofluoromethyl)benzoate 1a 


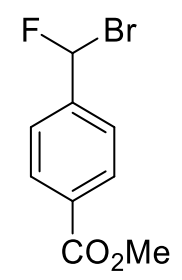

The product 1a (4.0 g, 55\% yield, yellow liquid) was purified with silica gel chromatography (petroleum ether/ethyl acetate $=20 / 1) .{ }^{1} \mathrm{H}$ NMR $\left(400 \mathrm{MHz}, \mathrm{CDCl}_{3}\right)$ $\delta 8.09$ (d, $J=8.2 \mathrm{~Hz}, 2 \mathrm{H}), 7.57$ (d, $J=8.2 \mathrm{~Hz}, 2 \mathrm{H}), 7.44$ (d, $J=49.4 \mathrm{~Hz}, 1 \mathrm{H}), 3.94$ $(\mathrm{s}, 3 \mathrm{H}) ;{ }^{19} \mathrm{~F}$ NMR $\left(376 \mathrm{MHz}, \mathrm{CDCl}_{3}\right) \delta-134.17(\mathrm{~d}, J=49.4 \mathrm{~Hz}, 1 \mathrm{~F}) ;{ }^{13} \mathrm{C}$ NMR $(101$ $\left.\mathrm{MHz}, \mathrm{CDCl}_{3}\right) \delta 166.1,142.9(\mathrm{~d}, J=20.0 \mathrm{~Hz}), 131.6,130.0,125.0(\mathrm{~d}, J=6.2 \mathrm{~Hz})$, 90.8 (d, $J=253.8 \mathrm{~Hz}), 52.3$ ppm. MS (EI): 167 (100), $246\left(\mathrm{M}^{+}\right)$; HRMS (EI) m/z: $\left[\mathrm{M}^{+}\right]$Calcd for $\mathrm{C}_{9} \mathrm{H}_{8} \mathrm{O}_{2} \mathrm{FBr}$ 245.9700; Found 245.9701. IR (KBr): $v_{\max }=3001,2953$, $2844,1724,1613,1311,1281,1112,705 \mathrm{~cm}^{-1}$.

\section{Methyl 3-(bromofluoromethyl)benzoate 1b}<smiles>COC(=O)c1cccc(C(F)Br)c1</smiles>

The product $1 \mathrm{~b}$ (1.1 g, 76\% yield, yellow liquid) was purified with silica gel chromatography (petroleum ether/ethyl acetate $=20 / 1) .{ }^{1} \mathrm{H}$ NMR $\left(400 \mathrm{MHz}, \mathrm{CDCl}_{3}\right)$ $\delta 8.16(\mathrm{~s}, 1 \mathrm{H}), 8.08(\mathrm{~d}, J=7.8 \mathrm{~Hz}, 1 \mathrm{H}), 7.71(\mathrm{~d}, J=7.7 \mathrm{~Hz}, 1 \mathrm{H}), 7.52(\mathrm{dd}, J=7.8$, $7.7 \mathrm{~Hz}, 1 \mathrm{H}), 7.44(\mathrm{~d}, J=49.3 \mathrm{~Hz}, 1 \mathrm{H}), 3.95(\mathrm{~s}, 3 \mathrm{H}) ;{ }^{19} \mathrm{~F} \mathrm{NMR}\left(376 \mathrm{MHz}, \mathrm{CDCl}_{3}\right) \delta$ $-131.94(\mathrm{~d}, J=49.2 \mathrm{~Hz}, 1 \mathrm{~F}) ;{ }^{13} \mathrm{C}$ NMR $\left(101 \mathrm{MHz}, \mathrm{CDCl}_{3}\right) \delta 166.1,139.2(\mathrm{~d}, J=20.2$ $\mathrm{Hz}), 131.2,130.8,129.3(\mathrm{~d}, J=5.8 \mathrm{~Hz}), 129.0,126.2(\mathrm{~d}, J=6.4 \mathrm{~Hz}), 91.0(\mathrm{~d}, J=$ 253.5 Hz), 52.4 ppm. MS (EI): 167 (100), $254\left(\mathrm{M}^{+}\right)$; HRMS (EI) m/z: [M+] Calcd for $\mathrm{C}_{9} \mathrm{H}_{8} \mathrm{O}_{2} \mathrm{FBr}$ 245.9692; Found 245.9702. IR (KBr): $v_{\max }=3001,2952,2903,2844$, $1724,1609,1507,1449,1434,1292,1215,1110,890 \mathrm{~cm}^{-1}$.

\section{Ethyl 4-(bromofluoromethyl)benzoate 1c}


<smiles>CCOC(=O)c1ccc(C(F)Br)cc1</smiles>

The product 1c (970 mg, 68\% yield, yellow liquid) was purified with silica gel chromatography (petroleum ether/ethyl acetate $=20 / 1) .{ }^{1} \mathrm{H} \mathrm{NMR}\left(400 \mathrm{MHz}, \mathrm{CDCl}_{3}\right)$ $\delta 8.08(\mathrm{~d}, J=8.1 \mathrm{~Hz}, 2 \mathrm{H}), 7.54(\mathrm{~d}, J=8.3 \mathrm{~Hz}, 2 \mathrm{H}), 7.42(\mathrm{~d}, J=49.4 \mathrm{~Hz}, 1 \mathrm{H}), 4.38$ $(\mathrm{q}, J=7.1 \mathrm{~Hz}, 2 \mathrm{H}), 1.38(\mathrm{t}, J=7.1 \mathrm{~Hz}, 3 \mathrm{H}) ;{ }^{19} \mathrm{~F}$ NMR $\left(376 \mathrm{MHz}, \mathrm{CDCl}_{3}\right) \delta-134.73$ $(\mathrm{d}, J=49.2 \mathrm{~Hz}, 1 \mathrm{~F}) ;{ }^{13} \mathrm{C} \mathrm{NMR}\left(101 \mathrm{MHz}, \mathrm{CDCl}_{3}\right) \delta 165.6,142.8(\mathrm{~d}, J=20.0 \mathrm{~Hz})$, 132.0, 129.9, 124.9 (d, $J=6.3 \mathrm{~Hz}), 90.8$ (d, $J=253.8 \mathrm{~Hz}), 61.3,14.3$ ppm. MS (EI): 181 (100), 214 [M-OC $\left.{ }_{2} \mathrm{H}_{5}\right]^{+}$; HRMS (EI) m/z: [M+ $]$Calcd for $\mathrm{C}_{8} \mathrm{H}_{5} \mathrm{OFBr} 214.9508$; Found 214.9511. IR (KBr): $v_{\max }=2982,1720,1614,1581,1416,1310,1277,1222$, $1106,1019,705 \mathrm{~cm}^{-1}$.

\section{3-(Bromofluoromethyl)benzonitrile 1d}<smiles>N#Cc1cccc(C(F)Br)c1</smiles>

The product 1d (980 mg, 61\% yield, white solid, Mp: $56-58{ }^{\circ} \mathrm{C}$.) was purified with silica gel chromatography (petroleum ether/ethyl acetate $=20 / 1) .{ }^{1} \mathrm{H}$ NMR $(400 \mathrm{MHz}$, $\left.\mathrm{CDCl}_{3}\right) \delta 7.78(\mathrm{~s}, 1 \mathrm{H}), 7.72(\mathrm{~d}, J=7.9 \mathrm{~Hz}, 1 \mathrm{H}), 7.68(\mathrm{~d}, J=7.8 \mathrm{~Hz}, 1 \mathrm{H}), 7.55(\mathrm{t}, J=$ $7.8 \mathrm{~Hz}, 1 \mathrm{H}), 7.39$ (d, $J=49.2 \mathrm{~Hz}, 1 \mathrm{H}) ;{ }^{19} \mathrm{~F} \mathrm{NMR}\left(376 \mathrm{MHz}, \mathrm{CDCl}_{3}\right) \delta-134.73$ (d, $J$ $=49.2 \mathrm{~Hz}, 1 \mathrm{~F}) ;{ }^{13} \mathrm{C} \mathrm{NMR}\left(101 \mathrm{MHz}, \mathrm{CDCl}_{3}\right) \delta 140.17(\mathrm{~d}, J=21.1 \mathrm{~Hz}), 133.5,129.8$, $129.3(\mathrm{~d}, J=5.8 \mathrm{~Hz}), 128.6(\mathrm{~d}, J=6.6 \mathrm{~Hz}), 117.8,113.1,89.7$ (d, $J=254.7 \mathrm{~Hz}) \mathrm{ppm}$. MS (EI): 134 (100), $213\left(\mathrm{M}^{+}\right)$; HRMS (EI) m/z: [M+] Calcd for $\mathrm{C}_{8} \mathrm{H}_{5} \mathrm{NFBr} 212.9589$; Found 212.9585. IR (KBr): $v_{\max }=3073,2234,1606,1483,1346,1244,1041,889$, $805,740 \mathrm{~cm}^{-1}$.

\section{4-(Bromofluoromethyl)benzonitrile 1e}




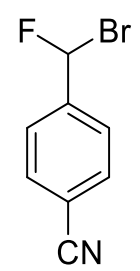

The product 1e $\left(840 \mathrm{mg}, 76 \%\right.$ yield, white solid, Mp: $49-51^{\circ} \mathrm{C}$.) was purified with silica gel chromatography (petroleum ether/ethyl acetate $=20 / 1) .{ }^{1} \mathrm{H}$ NMR $(400 \mathrm{MHz}$, $\left.\mathrm{CDCl}_{3}\right) \delta 7.74(\mathrm{~d}, J=8.2 \mathrm{~Hz}, 2 \mathrm{H}), 7.61(\mathrm{~d}, J=8.4 \mathrm{~Hz}, 2 \mathrm{H}), 7.43(\mathrm{~d}, J=49.3 \mathrm{~Hz}, 1$ $\mathrm{H}) ;{ }^{19} \mathrm{~F}$ NMR $\left(376 \mathrm{MHz}, \mathrm{CDCl}_{3}\right) \delta-136.29(\mathrm{~d}, J=49.3 \mathrm{~Hz}, 1 \mathrm{~F}) ;{ }^{13} \mathrm{C}$ NMR $(101 \mathrm{MHz}$, $\left.\mathrm{CDCl}_{3}\right) \delta 143.1(\mathrm{~d}, J=20.5 \mathrm{~Hz}), 132.6,125.7(\mathrm{~d}, J=6.4 \mathrm{~Hz}), 117.9,113.9,89.8(\mathrm{~d}, J$

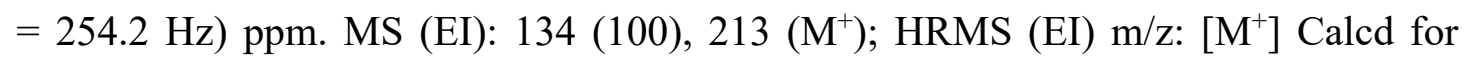
$\mathrm{C}_{8} \mathrm{H}_{5} \mathrm{NFBr}$ 212.9589; Found 212.9600. IR (KBr): $v_{\max }=3480.3096,3045,3006$, 2227, 1937, 1611, 1506, 1417, 1361, 1226, 1040, 1019, $849 \mathrm{~cm}^{-1}$.

\section{4-(Bromofluoromethyl)-2-fluorobenzonitrile 1f}<smiles>N#Cc1ccc(C(F)Br)cc1F</smiles>

The product 1 f $(530 \mathrm{mg}, 23 \%$ yield, yellow liquid) was purified with silica gel chromatography (petroleum ether/ethyl acetate $=20 / 1) .{ }^{1} \mathrm{H}$ NMR $\left(400 \mathrm{MHz}, \mathrm{CDCl}_{3}\right)$ $\delta 7.75-7.63(\mathrm{~m}, 1 \mathrm{H}), 7.39(\mathrm{~d}, J=49.2 \mathrm{~Hz}, 1 \mathrm{H}), 7.36(\mathrm{~d}, J=8.5 \mathrm{~Hz}, 2 \mathrm{H}) ;{ }^{19} \mathrm{~F}$ NMR $\left(376 \mathrm{MHz}, \mathrm{CDCl}_{3}\right) \delta-101.39--108.07(\mathrm{~m}, 1 \mathrm{~F}),-137.67(\mathrm{~d}, J=49.2 \mathrm{~Hz}, 1 \mathrm{~F}) ;{ }^{13} \mathrm{C}$ NMR (101 MHz, $\left.\mathrm{CDCl}_{3}\right) \delta 162.9(\mathrm{~d}, J=260.9 \mathrm{~Hz}), 145.8(\mathrm{dd}, J=21.3,7.6 \mathrm{~Hz})$, 134.1, 121.2 (dd, $J=6.3,4.0 \mathrm{~Hz}), 113.3$ (dd, $J=21.9,7.1 \mathrm{~Hz}), 113.1,103.0$ (d, $J=$ $15.7 \mathrm{~Hz}), 88.6(\mathrm{~d}, J=254.9 \mathrm{~Hz}) \mathrm{ppm}$. MS (EI): $132(100), 230\left(\mathrm{M}^{+}\right)$; HRMS (EI) m/z: $\left[\mathrm{M}^{+}\right]$Calcd for $\mathrm{C}_{8} \mathrm{H}_{4} \mathrm{NF}_{2} \mathrm{Br} 230.9495$; Found 230.9506. IR (KBr): $v_{\max }=3556,3095$, $3009,2241,1624,1575,1504,1434,1351,1116,1051,945,881,768,725,689 \mathrm{~cm}^{-1}$

\section{4-(Bromofluoromethyl)-3-fluorobenzonitrile $1 \mathrm{~g}$}<smiles>N#Cc1ccc(C(F)Br)c(F)c1</smiles> 
The product $1 \mathrm{~g}$ (1.38 g, $60 \%$ yield, yellow liquid) was purified with silica gel chromatography (petroleum ether/ethyl acetate $=20 / 1) .{ }^{1} \mathrm{H} \mathrm{NMR}\left(400 \mathrm{MHz}, \mathrm{CDCl}_{3}\right)$ $\delta 7.86-7.76(\mathrm{~m}, 1 \mathrm{H}), 7.60(\mathrm{~d}, J=8.1 \mathrm{~Hz}, 1 \mathrm{H}), 7.60(\mathrm{~d}, J=48.6 \mathrm{~Hz}, 1 \mathrm{H}), 7.44(\mathrm{dt}, J$ =9.4, $1.2 \mathrm{~Hz}, 1 \mathrm{H}) ;{ }^{19} \mathrm{~F}$ NMR $\left(376 \mathrm{MHz}, \mathrm{CDCl}_{3}\right) \delta-113.74(\mathrm{ddd}, J=9.5,7.1,2.6 \mathrm{~Hz}$, $1 \mathrm{~F}),-142.62(\mathrm{dd}, J=48.5,2.1 \mathrm{~Hz}, 1 \mathrm{~F}) ;{ }^{13} \mathrm{C} \mathrm{NMR}\left(101 \mathrm{MHz}, \mathrm{CDCl}_{3}\right) \delta 157.1$ (dd, $J$ = 255.0, $5.6 \mathrm{~Hz}), 131.6(\mathrm{dd}, J=22.4,12.1 \mathrm{~Hz}), 128.7(\mathrm{~d}, J=4.1 \mathrm{~Hz}), 127.6(\mathrm{dd}, J=$ 7.2, $2.7 \mathrm{~Hz}), 119.7$ (d, $J=24.1 \mathrm{~Hz}), 116.7$ (d, $J=2.8 \mathrm{~Hz}), 115.4$ (d, $J=9.5 \mathrm{~Hz}), 83.3$ (dd, $J=252.6,4.9 \mathrm{~Hz})$ ppm. MS (EI): $152(100), 231\left(\mathrm{M}^{+}\right) ; \operatorname{HRMS}(\mathrm{EI}) \mathrm{m} / \mathrm{z}:\left[\mathrm{M}^{+}\right]$ Calcd for $\mathrm{C}_{8} \mathrm{H}_{4} \mathrm{NF}_{2} \mathrm{Br} 230.9495$; Found 230.9501. IR (KBr): $v_{\max }=3675,3097,3029$, 2237, 1621, 1577, 1499, 1418, 1260, 1221, 1109, 1049, 949, 767, $659 \mathrm{~cm}^{-1}$.

\section{1-(Bromofluoromethyl)-4-(trifluoromethyl)benzene $1 \mathrm{~h}$}<smiles>FC(Br)c1ccc(C(F)(F)F)cc1</smiles>

The product $1 \mathrm{~h}$ (1.2 g, 85\% yield, yellow liquid) was purified with silica gel chromatography (petroleum ether/ethyl acetate $=80 / 1) .{ }^{1} \mathrm{H} \mathrm{NMR}\left(400 \mathrm{MHz}, \mathrm{CDCl}_{3}\right)$ $\delta 7.68(\mathrm{~d}, J=8.3 \mathrm{~Hz}, 2 \mathrm{H}), 7.61(\mathrm{~d}, J=8.2 \mathrm{~Hz}, 2 \mathrm{H}), 7.43(\mathrm{~d}, J=49.3 \mathrm{~Hz}, 1 \mathrm{H}) ;{ }^{19} \mathrm{~F}$ NMR (376 MHz, $\left.\mathrm{CDCl}_{3}\right) \delta-62.98(\mathrm{~s}, 3 \mathrm{~F}),-134.46(\mathrm{~d}, J=49.3 \mathrm{~Hz}, 1 \mathrm{~F}) ;{ }^{13} \mathrm{C} \mathrm{NMR}$ $\left(101 \mathrm{MHz}, \mathrm{CDCl}_{3}\right) \delta 142.2(\mathrm{~d}, J=20.3 \mathrm{~Hz}), 132.2(\mathrm{q}, J=33.1 \mathrm{~Hz}), 125.8(\mathrm{q}, J=3.7$ $\mathrm{Hz}), 125.4(\mathrm{~d}, J=6.3 \mathrm{~Hz}), 123.5$ (q, $J=272.4 \mathrm{~Hz}), 90.3$ (d, $J=253.8 \mathrm{~Hz}) \mathrm{ppm} . \mathrm{MS}$ (EI): 177 (100), $256\left(\mathrm{M}^{+}\right)$; HRMS (EI) m/z: [M+ Calcd for $\mathrm{C}_{8} \mathrm{H}_{5} \mathrm{~F}_{4} \mathrm{Br} 255.9511$; Found 255.9504. IR (KBr): $v_{\max }=3069,2924,1593,1450,1327,1268,1127,797$, $701 \mathrm{~cm}^{-1}$.

\section{1-(Bromofluoromethyl)-3-(trifluoromethyl)benzene 1i}<smiles>FC(Br)c1cccc(C(F)(F)F)c1</smiles>

The product 1i (1.2 g, 82\% yield, yellow liquid) was purified with silica gel chromatography (petroleum ether/ethyl acetate $=80 / 1) .{ }^{1} \mathrm{H} \mathrm{NMR}\left(400 \mathrm{MHz}, \mathrm{CDCl}_{3}\right)$ 
$\delta 7.74(\mathrm{~s}, 1 \mathrm{H}), 7.67(\mathrm{t}, J=8.4 \mathrm{~Hz}, 2 \mathrm{H}), 7.55(\mathrm{t}, J=7.8 \mathrm{~Hz}, 1 \mathrm{H}), 7.43(\mathrm{~d}, J=49.2 \mathrm{~Hz}$, $1 \mathrm{H}) ;{ }^{19} \mathrm{~F} \mathrm{NMR}\left(376 \mathrm{MHz}, \mathrm{CDCl}_{3}\right) \delta-62.89(\mathrm{~s}, 3 \mathrm{~F}),-133.27(\mathrm{~d}, J=49.2 \mathrm{~Hz}, 1 \mathrm{~F}) ;{ }^{13} \mathrm{C}$ NMR (101 MHz, $\left.\mathrm{CDCl}_{3}\right) \delta 139.6(\mathrm{~d}, J=20.5 \mathrm{~Hz}), 131.3(\mathrm{q}, J=33.1 \mathrm{~Hz}), 129.4$, $128.4(\mathrm{~d}, J=5.0 \mathrm{~Hz}), 126.9(\mathrm{~d}, J=2.8 \mathrm{~Hz}), 123.5(\mathrm{q}, J=272.5 \mathrm{~Hz}), 122.0(\mathrm{dq}, J=$ 7.8, $3.9 \mathrm{~Hz}), 90.4$ (d, $J=253.5 \mathrm{~Hz})$ ppm. MS (EI): $177(100), 256\left(\mathrm{M}^{+}\right)$; HRMS (EI) $\mathrm{m} / \mathrm{z}:\left[\mathrm{M}^{+}\right]$Calcd for $\mathrm{C}_{8} \mathrm{H}_{5} \mathrm{~F}_{4} \mathrm{Br}$ 255.9511; Found 255.9512. IR (KBr): $v_{\max }=30335$, $2922,1621,1496,1415,1326,1167,1127,1067,1018,920,810,750 \mathrm{~cm}^{-1}$.

\section{1-Bromo-4-(bromofluoromethyl)benzene $1 \mathbf{j}$}

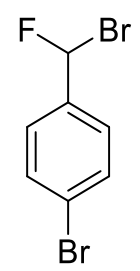

The product 1j (1.05 g, 40\% yield, yellow liquid) was purified with silica gel chromatography (petroleum ether/ethyl acetate = 100/1). ${ }^{1} \mathrm{H}$ NMR (400 $\left.\mathrm{MHz}, \mathrm{CDCl}_{3}\right)$ $\delta 7.54(\mathrm{~d}, J=8.3 \mathrm{~Hz}, 2 \mathrm{H}), 7.36(\mathrm{~d}, J=8.4 \mathrm{~Hz}, 2 \mathrm{H}), 7.34(\mathrm{~d}, J=49.2 \mathrm{~Hz}, 1 \mathrm{H}) ;{ }^{19} \mathrm{~F}$ $\operatorname{NMR}\left(376 \mathrm{MHz}, \mathrm{CDCl}_{3}\right) \delta-131.42(\mathrm{~d}, J=49.2 \mathrm{~Hz}, 1 \mathrm{~F}) ;{ }^{13} \mathrm{C} \mathrm{NMR}\left(101 \mathrm{MHz}, \mathrm{CDCl}_{3}\right)$ $\delta 137.7(\mathrm{~d}, J=20.2 \mathrm{~Hz}), 131.9,126.7(\mathrm{~d}, J=6.1 \mathrm{~Hz}), 124.4,91.0(\mathrm{~d}, J=253.2 \mathrm{~Hz})$ ppm. MS (EI): 187 (100), $265\left(\mathrm{M}^{+}\right)$; HRMS (EI) m/z: [M+] Calcd for $\mathrm{C}_{7} \mathrm{H}_{5} \mathrm{FBr}_{2}$ 265.8742; Found 265.8746. IR (KBr): $v_{\max }=3089,2924,2852,1903,1699,1594$, $1488,1405,1382,1219,1071,1044,827,719 \mathrm{~cm}^{-1}$.

\section{1-Bromo-3-(bromofluoromethyl)-5-fluorobenzene $1 \mathrm{k}$}<smiles>Fc1cc(Br)cc(C(F)Br)c1</smiles>

The product 1k (1.39 g, 51\% yield, yellow liquid) was purified with silica gel chromatography (petroleum ether/ethyl acetate =100/1). ${ }^{1} \mathrm{H}$ NMR $\left(400 \mathrm{MHz}, \mathrm{CDCl}_{3}\right)$ $\delta 7.41(\mathrm{~s}, 1 \mathrm{H}), 7.31(\mathrm{~d}, J=49.3 \mathrm{~Hz}, 1 \mathrm{H}), 7.29-7.25(\mathrm{~m}, 1 \mathrm{H}), 7.15(\mathrm{~d}, J=8.6 \mathrm{~Hz}, 1$ $\mathrm{H}) ;{ }^{19} \mathrm{~F}$ NMR $\left(376 \mathrm{MHz}, \mathrm{CDCl}_{3}\right) \delta-108.71(\mathrm{t}, J=8.3 \mathrm{~Hz}, 1 \mathrm{~F}),-134.21(\mathrm{~d}, J=49.2$ $\mathrm{Hz}, 1 \mathrm{~F}) ;{ }^{13} \mathrm{C} \mathrm{NMR}\left(101 \mathrm{MHz}, \mathrm{CDCl}_{3}\right) \delta 162.3(\mathrm{~d}, J=253.3 \mathrm{~Hz}), 142.1$ (dd, $J=21.1$, $8.2 \mathrm{~Hz}), 124.0(\mathrm{dd}, J=6.6,3.4 \mathrm{~Hz}), 122.9$ (d, $J=9.5 \mathrm{~Hz}), 120.8(\mathrm{~d}, J=24.5 \mathrm{~Hz})$, 
$111.4(\mathrm{dd}, J=23.6,6.6 \mathrm{~Hz}), 89.2(\mathrm{dd}, J=254.5,2.4 \mathrm{~Hz}) \mathrm{ppm}$. MS (EI): 205 (100), $283\left(\mathrm{M}^{+}\right)$; HRMS (EI) m/z: [M+] Calcd for $\mathrm{C}_{7} \mathrm{H}_{4} \mathrm{~F}_{2} \mathrm{Br}_{2}$ 283.8648; Found 283.8653. IR $(\mathrm{KBr}): v_{\max }=2917,2848,1958,1261,1180,1075,1029,966,801,668 \mathrm{~cm}^{-1}$.

\section{2-Bromo-4-(bromofluoromethyl)-1-chlorobenzene 11}<smiles>FC(Br)c1ccc(Cl)c(Br)c1</smiles>

The product 11 (1.2 g, 41\% yield, yellow liquid) was purified with silica gel chromatography (petroleum ether/ethyl acetate $=100 / 1) .{ }^{1} \mathrm{H}$ NMR $\left(400 \mathrm{MHz}, \mathrm{CDCl}_{3}\right)$ $\delta 7.79(\mathrm{~d}, J=1.9 \mathrm{~Hz}, 1 \mathrm{H}), 7.53(\mathrm{~d}, J=8.2 \mathrm{~Hz}, 1 \mathrm{H}), 7.40$ (d, $J=2.0 \mathrm{~Hz}, 1 \mathrm{H}), 7.36$ (d, $J=49.1 \mathrm{~Hz}, 1 \mathrm{H}) ;{ }^{19} \mathrm{~F} \mathrm{NMR}\left(376 \mathrm{MHz}, \mathrm{CDCl}_{3}\right) \delta-132.63(\mathrm{~d}, J=49.1 \mathrm{~Hz}, 1 \mathrm{~F}) ;{ }^{13} \mathrm{C}$ NMR (101 MHz, $\left.\mathrm{CDCl}_{3}\right) \delta 138.6(\mathrm{~d}, J=20.7 \mathrm{~Hz}), 136.4,130.6,130.3(\mathrm{~d}, J=6.5 \mathrm{~Hz})$, $125.0(\mathrm{~d}, J=5.9 \mathrm{~Hz}), 122.8,89.6$ (d, $J=253.9 \mathrm{~Hz})$ ppm. MS (EI): 223 (100), 299 $\left(\mathrm{M}^{+}\right)$; HRMS (EI) $\mathrm{m} / \mathrm{z}$ : $\left[\mathrm{M}^{+}\right]$Calcd for $\mathrm{C}_{7} \mathrm{H}_{4} \mathrm{ClFBr}_{2}$ 299.8352; Found 299.8353. IR $(\mathrm{KBr}): v_{\max }=3089,1592,1468,1396,1332,1273,1217,1048,8692,823,712 \mathrm{~cm}^{-1}$.

\section{1-(Bromofluoromethyl)-4-chlorobenzene 1m}<smiles>FC(Br)c1ccc(Cl)cc1</smiles>

The product $1 \mathrm{~m}$ (910 mg, 41\% yield, yellow liquid) was purified with silica gel chromatography (petroleum ether/ethyl acetate =100/1). ${ }^{1} \mathrm{H}$ NMR $\left(400 \mathrm{MHz}, \mathrm{CDCl}_{3}\right)$ $\delta 7.43(\mathrm{~d}, J=8.3 \mathrm{~Hz}, 2 \mathrm{H}), 7.38(\mathrm{~d}, J=8.6 \mathrm{~Hz}, 2 \mathrm{H}), 7.27(\mathrm{~d}, J=21.8 \mathrm{~Hz}, 1 \mathrm{H}) ;{ }^{19} \mathrm{~F}$ NMR (376 MHz, $\left.\mathrm{CDCl}_{3}\right) \delta-130.92(\mathrm{~d}, J=49.2 \mathrm{~Hz}, 1 \mathrm{~F}) ;{ }^{13} \mathrm{C} \mathrm{NMR}\left(101 \mathrm{MHz}, \mathrm{CDCl}_{3}\right)$ $\delta 137.2(\mathrm{~d}, J=20.2 \mathrm{~Hz}), 136.1,129.0,126.4(\mathrm{~d}, J=6.1 \mathrm{~Hz}), 91.0(\mathrm{~d}, J=253.1 \mathrm{~Hz})$ ppm. MS (EI): 155 (100), $221\left(\mathrm{M}^{+}\right)$; HRMS (EI) m/z: [M+] Calcd for Calcd for $\mathrm{C}_{7} \mathrm{H}_{5} \mathrm{ClFBr}: 221.9247$; Found 221.9250. IR (KBr): $v_{\max }=2925,2849,1904,1701$, $1600,1493,1410,1221,1093,1044,1014,832,723,651 \mathrm{~cm}^{-1}$.

\section{4-(Bromofluoromethyl)-1,2-dichlorobenzene 1n}




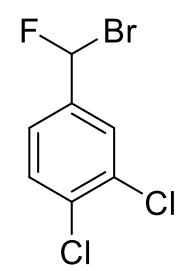

The product 1n (850 mg, 35\% yield, yellow liquid) was purified with silica gel chromatography (petroleum ether/ethyl acetate $=100 / 1) .{ }^{1} \mathrm{H}$ NMR $\left(400 \mathrm{MHz}, \mathrm{CDCl}_{3}\right)$ $\delta 7.58(\mathrm{~d}, J=2.0 \mathrm{~Hz}, 1 \mathrm{H}), 7.49(\mathrm{~d}, J=8.4 \mathrm{~Hz}, 1 \mathrm{H}), 7.32(\mathrm{~d}, J=49.1 \mathrm{~Hz}, 1 \mathrm{H}), 7.31$ $(\mathrm{dd}, J=8.4,1.8 \mathrm{~Hz}, 1 \mathrm{H}) ;{ }^{19} \mathrm{~F}$ NMR $\left(376 \mathrm{MHz}, \mathrm{CDCl}_{3}\right) \delta-132.80(\mathrm{~d}, J=49.1 \mathrm{~Hz}, 1$ F); $\left.{ }^{13} \mathrm{C} \mathrm{NMR} \mathrm{(101} \mathrm{MHz,} \mathrm{CDCl}_{3}\right) \delta 138.6(\mathrm{~d}, J=20.7 \mathrm{~Hz}), 134.4,133.1,130.8,127.1$ (d, $J=6.6 \mathrm{~Hz}), 124.3$ (d, $J=6.0 \mathrm{~Hz}), 89.7$ (d, $J=253.8 \mathrm{~Hz})$ ppm. MS (EI): 177 (100), $256\left(\mathrm{M}^{+}\right)$; HRMS (EI) m/z: $\left[\mathrm{M}^{+}\right]$Calcd for $\mathrm{C}_{7} \mathrm{H}_{4} \mathrm{FCl}_{2} \mathrm{Br}$ 255.8857; Found 255.8862. IR (KBr): $v_{\max }=3094,1593,1472,1400,1332,1217,1034,909,881,824,712,665$ $\mathrm{cm}^{-1}$.

\section{1-(Bromofluoromethyl)-3,5-dichlorobenzene 10}<smiles>FC(Br)c1cc(Cl)cc(Cl)c1</smiles>

The product 10 (740 mg, 51\% yield, yellow liquid) was purified with silica gel chromatography (petroleum ether/ethyl acetate =100/1). ${ }^{1} \mathrm{H}$ NMR $\left(400 \mathrm{MHz}, \mathrm{CDCl}_{3}\right)$ $\delta 7.41-7.38(\mathrm{~m}, 3 \mathrm{H}), 7.31(\mathrm{~d}, J=49.3 \mathrm{~Hz}, 1 \mathrm{H}) ;{ }^{19} \mathrm{~F} \mathrm{NMR}\left(376 \mathrm{MHz}, \mathrm{CDCl}_{3}\right) \delta$ $-134.39(\mathrm{~d}, J=49.1 \mathrm{~Hz}, 1 \mathrm{~F}) ;{ }^{13} \mathrm{C} \mathrm{NMR}\left(101 \mathrm{MHz}, \mathrm{CDCl}_{3}\right) \delta 141.58(\mathrm{~d}, J=20.9 \mathrm{~Hz})$, 135.5, 130.2, 123.6 (d, $J=6.5 \mathrm{~Hz}), 89.3$ (d, $J=254.5 \mathrm{~Hz})$ ppm. MS (EI): 177 (100), $256\left(\mathrm{M}^{+}\right)$; HRMS (EI) m/z: $\left[\mathrm{M}^{+}\right]$Calcd for $\mathrm{C}_{7} \mathrm{H}_{4} \mathrm{Cl}_{2} \mathrm{FBr} 255.8857$; Found 255.8855 . IR $(\mathrm{KBr}): v_{\max }=3081,1593,1573,1433,1333,1220,1119,1017,1053,863,833$ $700 \mathrm{~cm}^{-1}$.

\section{4-(Bromofluoromethyl)-1-chloro-2-fluorobenzene 1p}<smiles>Fc1cc(C(F)Br)ccc1Cl</smiles> 
The product 1p (1.1 g, 41\% yield, yellow liquid) was purified with silica gel chromatography (petroleum ether/ethyl acetate $=100 / 1) .{ }^{1} \mathrm{H}$ NMR $\left(400 \mathrm{MHz}, \mathrm{CDCl}_{3}\right)$ $\delta 7.46(\mathrm{t}, J=7.8 \mathrm{~Hz}, 1 \mathrm{H}), 7.36(\mathrm{~d}, J=49.1 \mathrm{~Hz}, 1 \mathrm{H}), 7.32(\mathrm{dd}, J=9.3,1.9 \mathrm{~Hz}, 1 \mathrm{H})$, $7.22(\mathrm{~d}, J=8.0 \mathrm{~Hz}, 1 \mathrm{H}) ;{ }^{19} \mathrm{~F}$ NMR $\left(376 \mathrm{MHz}, \mathrm{CDCl}_{3}\right) \delta-109.13--116.31(\mathrm{~m}, 1 \mathrm{~F})$, $-132.66(\mathrm{~d}, J=49.1 \mathrm{~Hz}, 1 \mathrm{~F}) ;{ }^{13} \mathrm{C} \mathrm{NMR}\left(101 \mathrm{MHz}, \mathrm{CDCl}_{3}\right) \delta 157.9(\mathrm{~d}, J=251.1 \mathrm{~Hz})$, $139.2(\mathrm{dd}, J=20.9,6.6 \mathrm{~Hz}), 131.1,123.0(\mathrm{~d}, J=17.8 \mathrm{~Hz}), 121.3(\mathrm{dd}, J=6.3,4.0 \mathrm{~Hz})$, $113.6(\mathrm{dd}, J=23.2,6.7 \mathrm{~Hz}), 89.8$ (d, $J=255.4 \mathrm{~Hz})$ ppm. MS (EI): 161 (100), 239 $\left(\mathrm{M}^{+}\right)$; HRMS (EI) m/z: [M+] Calcd for $\mathrm{C}_{7} \mathrm{H}_{4} \mathrm{ClF}_{2} \mathrm{Br}$ 239.9153; Found 239.9145. IR $(\mathrm{KBr}): v_{\max }=3074,1609,1585,1491,12145,1199,1068,1047,940,767,711,678$ $\mathrm{cm}^{-1}$. 
General procedure for cobalt-Catalyzed asymmetric cross-coupling $\alpha$-bromo$\alpha$-fluorotoluene derivatives

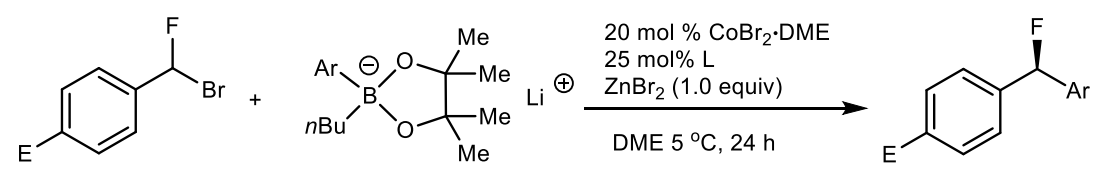

$1 \mathbf{a}\left(\mathrm{E}=\mathrm{CO}_{2} \mathrm{Me}\right)$

In a glove box, phenyl pinacol boronate ester (5.1 g, $25 \mathrm{mmol})$ was weighted into a $100 \mathrm{~mL}$ Schlenk tube, and $40 \mathrm{~mL}$ of dry THF was added. The mixture was taken out from the glove box and cooled at $-20{ }^{\circ} \mathrm{C}$. $n$-BuLi (10 mL, 2.5 M in Hexanes, $\left.25 \mathrm{mmol}\right)$ was added. The mixture was stirred at $-20{ }^{\circ} \mathrm{C}$ for $2 \mathrm{~h}$. Then the Schlenk tube was taken into the glove box, the solvents were removed by oil pump to give lithium phenyl $n$-butyl pinacol boronate.

In an argon-filled glove box, lithium organoboronate (371 mg, $0.900 \mathrm{mmol}, 3.00$ equiv.), ligand $\mathbf{L 8}$ (36.5 mg, $0.0750 \mathrm{mmol}, 0.250$ equiv.), $\mathrm{ZnBr}_{2}(67.5 \mathrm{mg}, 0.300$ mmol, 1.00 equiv.) and $\mathrm{CoBr}_{2} \cdot \mathrm{DME}(18.5 \mathrm{mg}, 0.0600 \mathrm{mmol}, 0.200$ equiv.) were placed into a $25 \mathrm{~mL}$ Schlenk tube. To this vial was added $3.0 \mathrm{~mL}$ of anhydrous DME. The Schlenk tube was taken out from the glove box and cooled at $5{ }^{\circ} \mathrm{C}$. Methyl 4-(bromofluoromethyl)benzoate $1 \mathrm{a}(73.8 \mathrm{mg}, 0.300 \mathrm{mmol})$ was added and the mixture was stirred at $5{ }^{\circ} \mathrm{C}$ for $24 \mathrm{~h}$. The mixture was quenched by addition of water $\left(5.0 \mathrm{~mL}\right.$ and extracted with $\mathrm{Et}_{2} \mathrm{O}(10.0 \mathrm{~mL} \times 3)$. The organic layer was combined, dried over anhydrous $\mathrm{Na}_{2} \mathrm{SO}_{4}$, filtered and concentrated under vacuum. The crude product was purified by column chromatography on silica gel with pentane/ethyl acetate as the eluent to give Methyl (S)-4-(fluoro(phenyl)methyl)benzoate 3a as a yellow liquid.

\section{Methyl (S)-4-(fluoro(phenyl)methyl)benzoate 3a}<smiles>COC(=O)c1ccc(C(F)c2ccccc2)cc1</smiles> 
The product 3a (55 mg, 75\% yield, yellow liquid) was purified with silica gel chromatography (petroleum ether/ethyl acetate $=20 / 1) .{ }^{1} \mathrm{H}$ NMR $\left(400 \mathrm{MHz}, \mathrm{CDCl}_{3}\right)$ $\delta 8.05(\mathrm{~d}, J=8.0 \mathrm{~Hz}, 2 \mathrm{H}), 7.43(\mathrm{~d}, J=8.5 \mathrm{~Hz}, 2 \mathrm{H}), 7.39-7.31(\mathrm{~m}, 5 \mathrm{H}), 6.51(\mathrm{~d}, J=$ $47.2 \mathrm{~Hz}, 1 \mathrm{H}), 3.92(\mathrm{~s}, 3 \mathrm{H}) ;{ }^{19} \mathrm{~F}$ NMR $\left(376 \mathrm{MHz}, \mathrm{CDCl}_{3}\right) \delta-168.60(\mathrm{~d}, J=47.5 \mathrm{~Hz}, 1$ F); ${ }^{13} \mathrm{C}$ NMR $\left(101 \mathrm{MHz}, \mathrm{CDCl}_{3}\right) \delta 166.6,144.7(\mathrm{~d}, J=22.2 \mathrm{~Hz}), 139.0(\mathrm{~d}, J=21.2$ Hz), 130.0, 129.8, 128.8 (d, $J=2.3 \mathrm{~Hz}), 128.6,126.8$ (d, $J=6.0 \mathrm{~Hz}), 126.1$ (d, $J=6.8$ Hz), 93.9 (d, $J=174.4$ Hz), 52.1 ppm. MS (EI): 185 (100), $244\left(\mathrm{M}^{+}\right)$; HRMS (EI) m/z: $\left[\mathrm{M}^{+}\right]$Calcd for $\mathrm{C}_{15} \mathrm{H}_{13} \mathrm{O}_{2} \mathrm{~F}$ 244.0900; Found 244.0911. IR (KBr): $v_{\max }=3429,3064$, $2843,1941,1723,1653,1455,1435,1280,1111,1020,755 \mathrm{~cm}^{-1}$.

HPLC (IG, $0.46 \times 25 \mathrm{~cm}, 5 \mu \mathrm{m}$, hexane/isopropanol = 97/3 (v/v \%), flow $0.7 \mathrm{~mL} / \mathrm{min}$, $\mathrm{UV}$ detection at $214 \mathrm{~nm}$ ), retention time $=15.84 \mathrm{~min}$ (minor) and $16.46 \mathrm{~min}$ (major). $[\alpha]_{\mathrm{D}}^{25}=+23.91\left(\mathrm{c}=0.230, \mathrm{CHCl}_{3}, 95: 5\right.$ e.r $)$.

Gram-scale preparation of compound 3a. In an argon-filled glove box, lithium organoboronate (6.2 g, $15.0 \mathrm{mmol}, 3.00$ equiv.), ligand $\mathbf{L 8}$ (608 mg, $1.25 \mathrm{mmol}, 0.250$ equiv.), $\mathrm{ZnBr}_{2}$ (1.12 g, $5.00 \mathrm{mmol}, 1.00$ equiv.) and $\mathrm{CoBr}_{2} \cdot \mathrm{DME}$ (308 mg, $1.00 \mathrm{mmol}$, 0.200 equiv.) were placed into a $25 \mathrm{~mL}$ Schlenk tube. To this vial was added $3.0 \mathrm{~mL}$ of anhydrous DME. The Schlenk tube was taken out from the glove box and cooled at $5{ }^{\circ} \mathrm{C}$. Methyl 4-(bromofluoromethyl)benzoate 1a (1.23 g, $\left.5.00 \mathrm{mmol}\right)$ was added and the mixture was stirred at $5{ }^{\circ} \mathrm{C}$ for $24 \mathrm{~h}$. The mixture was quenched by addition of water $\left(5.0 \mathrm{~mL}\right.$ and extracted with $\mathrm{Et}_{2} \mathrm{O}(10.0 \mathrm{~mL} \times 3)$. The organic layer was combined, dried over anhydrous $\mathrm{Na}_{2} \mathrm{SO}_{4}$, filtered and concentrated under vacuum. The crude product was purified by column chromatography on silica gel with pentane/ethyl acetate $(\mathrm{v} / \mathrm{v}=20: 1)$ as the eluent to give methyl (S)-4-(fluoro(phenyl)methyl)benzoate 3a as a yellow liquid (68\% yield, $829 \mathrm{mg}$ ) and dimethyl 4,4'-(1,2-difluoroethane-1,2-diyl)dibenzoate ( $\mathrm{dr}=1: 1) \mathbf{3 a}$ ' (125 mg, 15\% yield) as a yellow liquid.

Dimethyl 4,4'-(1,2-difluoroethane-1,2-diyl)dibenzoate $(\mathrm{dr}=1: 1) 3 \mathrm{a}^{\prime}$ 


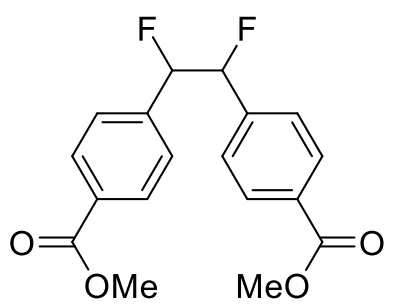

The product 3a' (125 mg, 15\% yield, yellow liquid) was purified with silica gel chromatography (petroleum ether/ethyl acetate $=10 / 1) .{ }^{1} \mathrm{H}$ NMR $\left(400 \mathrm{MHz}, \mathrm{CDCl}_{3}\right)$ $\delta 7.97(\mathrm{~d}, J=8.2 \mathrm{~Hz}, 2 \mathrm{H}), 7.93(\mathrm{~d}, J=8.2 \mathrm{~Hz}, 2 \mathrm{H}), 7.18(\mathrm{~d}, J=8.1 \mathrm{~Hz}, 2 \mathrm{H}), 7.15(\mathrm{~d}$, $J=8.1 \mathrm{~Hz}, 2 \mathrm{H}), 5.62-5.80(\mathrm{~m}, 1 \mathrm{H}), 5.62-5.80(\mathrm{~m}, 1 \mathrm{H}), 3.90(\mathrm{~s}, 3 \mathrm{H}), 3.89(\mathrm{~s}, 3 \mathrm{H})$; ${ }^{19} \mathrm{~F}$ NMR $\left(376 \mathrm{MHz}, \mathrm{CDCl}_{3}\right) \delta-186.77--187.50(\mathrm{~m}),-189.44--190.04(\mathrm{~m}) ;{ }^{13} \mathrm{C}$ NMR (101 MHz, $\left.\mathrm{CDCl}_{3}\right) \delta 166.5,166.4,139.4$ - 139.0 (m), 138.9 - 138.5 (m), 130.8, 130.8, 129.5, 129.4, 126.6, 126.5, 94.3 (t, $J=26.9 \mathrm{~Hz}), 92.83(\mathrm{t}, J=26.9 \mathrm{~Hz}), 52.26$ ( this peak is overlapped). MS (EI): 167 (100), $334\left(\mathrm{M}^{+}\right)$; HRMS (EI): Calcd for $\mathrm{C}_{18} \mathrm{H}_{16} \mathrm{O}_{4} \mathrm{~F}_{2}$ : 334.1017; Found 334.1021.

\section{Methyl (R)-4-(fluoro(m-tolyl)methyl)benzoate 3b}

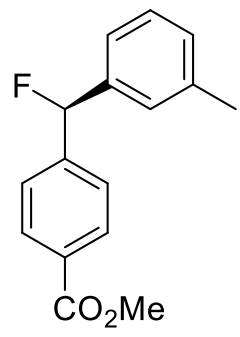

The product 3b (59 mg, 76\% yield, yellow liquid) was purified with silica gel chromatography (petroleum ether/ethyl acetate $=20 / 1) .{ }^{1} \mathrm{H}$ NMR $\left(400 \mathrm{MHz}, \mathrm{CDCl}_{3}\right)$ $\delta 8.03(\mathrm{~d}, J=8.2 \mathrm{~Hz}, 2 \mathrm{H}), 7.41(\mathrm{~d}, J=8.2 \mathrm{~Hz}, 2 \mathrm{H}), 7.25(\mathrm{dd}, J=9.7,5.2 \mathrm{~Hz}, 1 \mathrm{H})$, $7.10-7.16$ (m, $3 \mathrm{H}), 6.45$ (d, J=47.3 Hz, $1 \mathrm{H}), 3.90$ (s, $3 \mathrm{H}), 2.33$ (s, $3 \mathrm{H}) ;{ }^{19} \mathrm{~F}$ NMR $\left(376 \mathrm{MHz}, \mathrm{CDCl}_{3}\right) \delta-168.56(\mathrm{~d}, J=47.2 \mathrm{~Hz}, 1 \mathrm{~F}) ;{ }^{13} \mathrm{C} \mathrm{NMR}\left(101 \mathrm{MHz}, \mathrm{CDCl}_{3}\right) \delta$ 166.7, $144.8(\mathrm{~d}, J=22.3 \mathrm{~Hz}), 139.0(\mathrm{~d}, J=20.8 \mathrm{~Hz}), 138.4,130.0,129.7,129.6$ (d, $J$ $=2.3 \mathrm{~Hz}), 128.5,127.4(\mathrm{~d}, J=5.8 \mathrm{~Hz}), 126.1(\mathrm{~d}, J=6.8 \mathrm{~Hz}), 123.9(\mathrm{~d}, J=6.0 \mathrm{~Hz})$, $94.0(\mathrm{~d}, J=174.3 \mathrm{~Hz}), 52.1,21.4$ ppm. MS (EI): 199 (100), $258\left(\mathrm{M}^{+}\right)$; HRMS (EI) m/z: $\left[\mathrm{M}^{+}\right]$Calcd for $\mathrm{C}_{16} \mathrm{H}_{15} \mathrm{O}_{2} \mathrm{~F}$ 258.1056; Found 258.1061. IR $(\mathrm{KBr}): v_{\max }=2951$, $2922,1724,1435,1301,1280,1109,910,748,708 \mathrm{~cm}^{-1}$. 
HPLC (IE3, $0.46 \times 25 \mathrm{~cm}, 5 \mu \mathrm{m}$, hexane/isopropanol = 98/2 (v/v \%), flow 0.7 $\mathrm{mL} / \mathrm{min}$, UV detection at $214 \mathrm{~nm}$ ), retention time $=13.62 \mathrm{~min}$ (minor) and $16.54 \mathrm{~min}$ (major). $[\alpha]_{\mathrm{D}}{ }^{25}=-27.83\left(\mathrm{c}=0.230, \mathrm{CHCl}_{3}, 96: 4\right.$ e.r).

Methyl (R)-4-(fluoro(3-morpholinophenyl)methyl)benzoate 3c<smiles>CC(=O)c1ccc(C(F)c2cccc(N3CCOCC3)c2)cc1</smiles>

The product 3c (40 mg, 41\% yield, yellow liquid) was purified with silica gel chromatography (petroleum ether/ethyl acetate $=10 / 1) .{ }^{1} \mathrm{H}$ NMR $\left(400 \mathrm{MHz}, \mathrm{CDCl}_{3}\right)$ $\delta 8.02(\mathrm{~d}, J=8.0 \mathrm{~Hz}, 2 \mathrm{H}), 7.41(\mathrm{~d}, J=8.3 \mathrm{~Hz}, 2 \mathrm{H}), 7.24(\mathrm{t}, J=4.0 \mathrm{~Hz}, 1 \mathrm{H}), 6.87$ (d, $J=6.4 \mathrm{~Hz}, 2 \mathrm{H}), 6.78(\mathrm{~d}, J=7.3 \mathrm{~Hz}, 1 \mathrm{H}), 6.43$ (d, $J=47.3 \mathrm{~Hz}, 1 \mathrm{H}), 3.90$ (s, $3 \mathrm{H})$, $3.85-3.78(\mathrm{~m}, 4 \mathrm{H}), 3.20-2.99(\mathrm{~m}, 4 \mathrm{H}) ;{ }^{19} \mathrm{~F}$ NMR $\left(376 \mathrm{MHz}, \mathrm{CDCl}_{3}\right) \delta-168.77(\mathrm{~d}$, $J=47.3 \mathrm{~Hz}, 1 \mathrm{~F}) ;{ }^{13} \mathrm{C} \mathrm{NMR}\left(101 \mathrm{MHz}, \mathrm{CDCl}_{3}\right) \delta 166.7,151.5,144.7$ (d, $\left.J=22.0 \mathrm{~Hz}\right)$, $140.0(\mathrm{~d}, J=20.8 \mathrm{~Hz}), 130.0,129.7,129.4,126.2$ (d, $J=6.7 \mathrm{~Hz}), 118.3$ (d, $J=5.9$ $\mathrm{Hz}), 115.8(\mathrm{~d}, J=2.1 \mathrm{~Hz}), 113.7(\mathrm{~d}, J=6.5 \mathrm{~Hz}), 94.1$ (d, $J=174.6 \mathrm{~Hz}), 66.8,52.2$, 49.0 ppm. MS (EI): 329 (100), $329\left(\mathrm{M}^{+}\right)$; HRMS (EI) m/z: $\left[\mathrm{M}^{+}\right]$Calcd for $\mathrm{C}_{19} \mathrm{H}_{20} \mathrm{NO}_{3} \mathrm{~F}$ 329.1427; Found 329.1439. IR (KBr): $v_{\max }=2954,2854,1721,1602$, $1447,1280,1241,1120,1056,994,781 \mathrm{~cm}^{-1}$.

HPLC (AD-H, $0.46 \times 25 \mathrm{~cm}, 5 \mu \mathrm{m}$, hexane/isopropanol = 95/5 (v/v \%), flow 0.7 $\mathrm{mL} / \mathrm{min}$, UV detection at $214 \mathrm{~nm}$ ), retention time $=29.92 \mathrm{~min}$ (minor) and $34.85 \mathrm{~min}$ (major). $[\alpha]_{\mathrm{D}}^{25}=+4.00\left(\mathrm{c}=0.150, \mathrm{CHCl}_{3}, 92: 8\right.$ e.r).

Methyl (R)-4-(fluoro(3-methoxyphenyl)methyl)benzoate 3d<smiles>COc1cccc(C(F)c2ccc(C(C)=O)cc2)c1</smiles> 
The product 3d (54 mg, 66\% yield, yellow liquid) was purified with silica gel chromatography (petroleum ether/ethyl acetate $=10 / 1) .{ }^{1} \mathrm{H}$ NMR $\left(400 \mathrm{MHz}, \mathrm{CDCl}_{3}\right)$ $\delta 8.04(\mathrm{~d}, J=8.0 \mathrm{~Hz}, 2 \mathrm{H}), 7.43(\mathrm{~d}, J=8.5 \mathrm{~Hz}, 2 \mathrm{H}), 7.32-7.26(\mathrm{~m}, 1 \mathrm{H}), 6.98-6.73$ (m, $3 \mathrm{H}), 6.47$ (d, J=47.2 Hz, $1 \mathrm{H}), 3.92(\mathrm{~s}, 3 \mathrm{H}), 3.79$ (s, $3 \mathrm{H}) ;{ }^{19} \mathrm{~F}$ NMR $(376 \mathrm{MHz}$, $\left.\mathrm{CDCl}_{3}\right) \delta-169.14(\mathrm{~d}, J=47.2 \mathrm{~Hz}, 1 \mathrm{~F}) ;{ }^{13} \mathrm{C} \mathrm{NMR}\left(101 \mathrm{MHz}, \mathrm{CDCl}_{3}\right) \delta 166.6,159.8$, $144.5(\mathrm{~d}, J=22.0 \mathrm{~Hz}), 140.6(\mathrm{~d}, J=21.2 \mathrm{~Hz}), 130.1,129.8,129.7,126.1$ (d, $J=6.7$ Hz), 119.0 (d, $J=6.2 \mathrm{~Hz}), 114.2(\mathrm{~d}, J=2.1 \mathrm{~Hz}), 112.2$ (d, $J=6.6 \mathrm{~Hz}), 93.8$ (d, $J=$ $174.9 \mathrm{~Hz})$, 55.2, 52.1 ppm. MS (EI): 274 (100), $274\left(\mathrm{M}^{+}\right)$; HRMS (EI) m/z: [M+] Calcd for $\mathrm{C}_{16} \mathrm{H}_{15} \mathrm{O}_{3} \mathrm{~F}: 274.1005$; Found 274.1016. IR (KBr): $v_{\max }=3649,3002,2837$, $1936,1720,1602,1456,1311,1278,1191,1019,861,748 \mathrm{~cm}^{-1}$.

HPLC (AD-H, $0.46 \times 25 \mathrm{~cm}, 5 \mu \mathrm{m}$, hexane/isopropanol = 98/2 (v/v \%), flow 0.7 $\mathrm{mL} / \mathrm{min}$, UV detection at $214 \mathrm{~nm}$ ), retention time $=23.14 \mathrm{~min}$ (minor) and $26.51 \mathrm{~min}$ (major). $[\alpha]_{\mathrm{D}}^{25}=-5.76\left(\mathrm{c}=0.250, \mathrm{CHCl}_{3}, 94: 6\right.$ e.r).

\section{Methyl (R)-4-(fluoro(3-fluorophenyl)methyl)benzoate 3e}

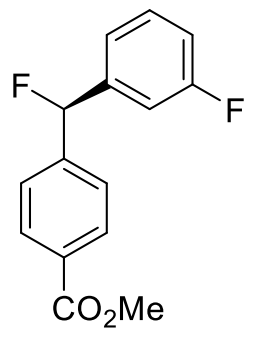

The product 3e (56 mg, 72\% yield, yellow liquid) was purified with silica gel chromatography (petroleum ether/ethyl acetate $=20 / 1) .{ }^{1} \mathrm{H}$ NMR $\left(400 \mathrm{MHz}, \mathrm{CDCl}_{3}\right)$ $\delta 8.06(\mathrm{~d}, J=8.1 \mathrm{~Hz}, 2 \mathrm{H}), 7.42(\mathrm{~d}, J=8.1 \mathrm{~Hz}, 2 \mathrm{H}), 7.38-7.30(\mathrm{~m}, 1 \mathrm{H}), 7.13-7.08$ (m, $1 \mathrm{H}), 7.13-7.04(\mathrm{~m}, 2 \mathrm{H}), 6.48(\mathrm{~d}, J=47.0 \mathrm{~Hz}, 1 \mathrm{H}), 3.92(\mathrm{~s}, 3 \mathrm{H}) ;{ }^{19} \mathrm{~F}$ NMR $\left(376 \mathrm{MHz}, \mathrm{CDCl}_{3}\right) \delta-110.13--115.03(\mathrm{~m}, 1 \mathrm{~F}),-169.61(\mathrm{~d}, J=47.0 \mathrm{~Hz}, 1 \mathrm{~F}) ;{ }^{13} \mathrm{C}$ NMR (101 MHz, $\left.\mathrm{CDCl}_{3}\right) \delta 166.5,162.8(\mathrm{~d}, J=247.2 \mathrm{~Hz}), 143.9(\mathrm{~d}, J=21.7 \mathrm{~Hz})$, $141.5(\mathrm{dd}, J=21.9,6.9 \mathrm{~Hz}), 130.4(\mathrm{~d}, J=1.7 \mathrm{~Hz}), 130.3(\mathrm{~d}, J=8.2 \mathrm{~Hz}), 129.9,126.2$ $(\mathrm{d}, J=6.6 \mathrm{~Hz}), 122.2(\mathrm{dd}, J=6.3,3.0 \mathrm{~Hz}), 115.7(\mathrm{dd}, J=21.1,2.0 \mathrm{~Hz}), 113.6(\mathrm{dd}, J$ = 22.7, $6.8 \mathrm{~Hz}), 93.1(\mathrm{~d}, J=175.8 \mathrm{~Hz}), 52.2$ ppm. MS (EI): 203 (100), $262\left(\mathrm{M}^{+}\right)$; HRMS (EI) m/z: $\left[\mathrm{M}^{+}\right]$Calcd for $\mathrm{C}_{15} \mathrm{H}_{12} \mathrm{O}_{2} \mathrm{~F}_{2}$ 262.0805; Found 262.0796. IR (KBr): 
$v_{\max }=3066,2953,2845,1941, \mathrm{M} 1723,1612,1449,1280,1192,1018,743$ 707, 694 $\mathrm{cm}^{-1}$.

HPLC (OJ-H, $0.46 \times 25 \mathrm{~cm}, 5 \mu \mathrm{m}$, hexane/isopropanol = 98/2 (v/v \%), flow 0.7 $\mathrm{mL} / \mathrm{min}$, UV detection at $214 \mathrm{~nm}$ ), retention time $=39.57 \mathrm{~min}$ (minor) and $45.85 \mathrm{~min}$ (major). $[\alpha]_{\mathrm{D}}{ }^{25}=-4.44\left(\mathrm{c}=0.090, \mathrm{CHCl}_{3}, 95: 5\right.$ e.r).

Methyl (R)-4-(fluoro(4-fluorophenyl)methyl)benzoate 3f<smiles>COC(=O)c1ccc(C(F)c2ccc(F)cc2)cc1</smiles>

The product 3f (51 mg, 65\% yield, yellow liquid) was purified with silica gel chromatography (petroleum ether/ethyl acetate $=20 / 1) .{ }^{1} \mathrm{H}$ NMR $\left(400 \mathrm{MHz}, \mathrm{CDCl}_{3}\right)$ $\delta 8.08(\mathrm{~d}, J=8.0 \mathrm{~Hz}, 2 \mathrm{H}), 7.43(\mathrm{~d}, J=8.5 \mathrm{~Hz}, 2 \mathrm{H}), 7.33(\mathrm{ddd}, J=6.5,4.4,1.3 \mathrm{~Hz}, 2$ H), $7.09(\mathrm{t}, J=8.4 \mathrm{~Hz}, 2 \mathrm{H}), 6.51(\mathrm{~d}, J=47.2 \mathrm{~Hz}, 1 \mathrm{H}), 3.94(\mathrm{~s}, 3 \mathrm{H}) ;{ }^{19} \mathrm{~F}$ NMR $(376$ $\left.\mathrm{MHz}, \mathrm{CDCl}_{3}\right) \delta-112.58(\mathrm{td}, J=8.6,4.0 \mathrm{~Hz}, 1 \mathrm{~F}),-166.80(\mathrm{~d}, J=47.2 \mathrm{~Hz}, 1 \mathrm{~F}) ;{ }^{13} \mathrm{C}$ NMR (101 MHz, $\left.\mathrm{CDCl}_{3}\right) \delta 166.6,162.9(\mathrm{~d}, J=248.1 \mathrm{~Hz}), 144.4(\mathrm{~d}, J=22.4 \mathrm{~Hz})$, $134.9(\mathrm{~d}, J=21.6 \mathrm{~Hz}), 130.2,129.8,128.8(\mathrm{dd}, J=8.4,5.8 \mathrm{~Hz}), 126.0(\mathrm{~d}, J=6.8 \mathrm{~Hz})$, 115.6 (d, $J=21.7 \mathrm{~Hz}), 93.3$ (d, $J=174.6 \mathrm{~Hz}$ ), 52.2 ppm. MS (EI): 203 (100), 262 $\left(\mathrm{M}^{+}\right)$; HRMS (EI) m/z: $\left[\mathrm{M}^{+}\right]$Calcd for $\mathrm{C}_{15} \mathrm{H}_{12} \mathrm{O}_{2} \mathrm{~F}_{2}$ 262.0805; Found 262.0808. IR $(\mathrm{KBr}): v_{\max }=2953,1936,1724,1606,1510,1311,1281,1111,1017,838,792 \mathrm{~cm}^{-1}$. HPLC (AD-H, $0.46 \times 25 \mathrm{~cm}, 5 \mu \mathrm{m}$, hexane/isopropanol = 98/2 (v/v \%), flow 0.7 $\mathrm{mL} / \mathrm{min}$, UV detection at $214 \mathrm{~nm}$ ), retention time $=18.16 \mathrm{~min}$ (minor) and $19.67 \mathrm{~min}$ (major). $[\alpha]_{\mathrm{D}}^{25}=-58.73\left(\mathrm{c}=0.110, \mathrm{CHCl}_{3}, 93: 7\right.$ e.r).

\section{Methyl (R)-4-(fluoro(3-fluoro-4-methylphenyl)methyl)benzoate 3g}<smiles>COC(=O)c1ccc(C(F)c2ccc(C)c(F)c2)cc1</smiles> 
The product $\mathbf{3 g}$ (51 mg, 62\% yield, yellow liquid) was purified with silica gel chromatography (petroleum ether/ethyl acetate $=20 / 1) .{ }^{1} \mathrm{H}$ NMR $\left(400 \mathrm{MHz}, \mathrm{CDCl}_{3}\right)$ $\delta 8.05(\mathrm{~d}, J=8.2 \mathrm{~Hz}, 2 \mathrm{H}), 7.41(\mathrm{~d}, J=8.1 \mathrm{~Hz}, 2 \mathrm{H}), 7.18(\mathrm{t}, J=7.8 \mathrm{~Hz}, 1 \mathrm{H}), 6.96-$ $7.00(\mathrm{~m}, 2 \mathrm{H}), 6.45(\mathrm{~d}, J=47.1 \mathrm{~Hz}, 1 \mathrm{H}), 3.92(\mathrm{~s}, 3 \mathrm{H}), 2.27(\mathrm{~s}, 3 \mathrm{H}) ;{ }^{19} \mathrm{~F}$ NMR $(376$ $\left.\mathrm{MHz} \mathrm{CDCl}_{3}\right) \delta-116.36(\mathrm{ddd}, J=10.0,8.1,1.8 \mathrm{~Hz}, 1 \mathrm{~F}),-168.43(\mathrm{~d}, J=47.1 \mathrm{~Hz}, 1 \mathrm{~F})$; ${ }^{13} \mathrm{C}$ NMR $\left(101 \mathrm{MHz}, \mathrm{CDCl}_{3}\right) \delta 166.6,161.2(\mathrm{~d}, J=245.9 \mathrm{~Hz}), 144.2(\mathrm{~d}, J=22.1 \mathrm{~Hz})$, $138.7(\mathrm{dd}, J=21.7,7.1 \mathrm{~Hz}), 131.6(\mathrm{~d}, J=5.4 \mathrm{~Hz}), 130.2,129.8,126.1$ (d, $J=6.7 \mathrm{~Hz})$, $125.5(\mathrm{~d}, J=17.3 \mathrm{~Hz}), 122.1(\mathrm{dd}, J=6.1,3.4 \mathrm{~Hz}), 113.4(\mathrm{dd}, J=23.6,6.4 \mathrm{~Hz}), 93.1$ (d, $J=175.1 \mathrm{~Hz}), 52.2,14.3(\mathrm{~d}, J=3.5 \mathrm{~Hz})$ ppm. MS (EI): $217(100), 276\left(\mathrm{M}^{+}\right)$; HRMS (EI) m/z: [M+] Calcd for $\mathrm{C}_{16} \mathrm{H}_{14} \mathrm{O}_{2} \mathrm{~F}_{2}$ 276.0962; Found 276.0958. IR (KBr): $V_{\max }=2999,2953,1934,1724,1614,1510,1435,1310,1279,1147,1111,847 \mathrm{~cm}^{-1}$. HPLC (AD-H, $0.46 \times 25 \mathrm{~cm}, 5 \mu \mathrm{m}$, hexane/isopropanol = 98/2 (v/v \%), flow 0.7 $\mathrm{mL} / \mathrm{min}$, UV detection at $214 \mathrm{~nm}$ ), retention time $=15.48 \mathrm{~min}$ (minor) and $17.22 \mathrm{~min}$ (major). $[\alpha]_{\mathrm{D}}{ }^{25}=-12.94\left(\mathrm{c}=0.170, \mathrm{CHCl}_{3}, 94: 6\right.$ e.r).

\section{Methyl (S)-3-(fluoro(phenyl)methyl)benzoate $3 \mathrm{~h}$}

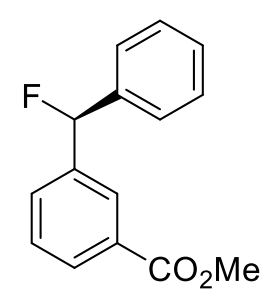

The product $3 \mathrm{~h}$ (42 mg, 58\% yield, yellow liquid) was purified with silica gel chromatography (petroleum ether/ethyl acetate $=20 / 1) .{ }^{1} \mathrm{H}$ NMR $\left(400 \mathrm{MHz}, \mathrm{CDCl}_{3}\right)$ $\delta 8.05(\mathrm{~d}, J=0.7 \mathrm{~Hz}, 1 \mathrm{H}), 8.02(\mathrm{~d}, J=8.0 \mathrm{~Hz}, 1 \mathrm{H}), 7.55(\mathrm{~d}, J=7.7 \mathrm{~Hz}, 1 \mathrm{H}), 7.46$ (t, $J=7.7 \mathrm{~Hz}, 1 \mathrm{H}), 7.43-7.30(\mathrm{~m}, 5 \mathrm{H}), 6.51(\mathrm{~d}, J=47.1 \mathrm{~Hz}, 1 \mathrm{H}), 3.91(\mathrm{~s}, 3 \mathrm{H}) ;{ }^{19} \mathrm{~F}$ NMR $\left(376 \mathrm{MHz}, \mathrm{CDCl}_{3}\right) \delta-167.68(\mathrm{~d}, J=47.1 \mathrm{~Hz}, 1 \mathrm{~F}) ;{ }^{13} \mathrm{C} \mathrm{NMR}\left(101 \mathrm{MHz}, \mathrm{CDCl}_{3}\right)$ $\delta 166.7,140.3(\mathrm{~d}, J=22.4 \mathrm{~Hz}), 139.3$ (d, $J=21.5 \mathrm{~Hz}), 130.7$ (d, $J=6.3 \mathrm{~Hz}), 130.5$, $129.5(\mathrm{~d}, J=1.7 \mathrm{~Hz}), 128.7,128.6,128.6,127.5$ (d, $J=6.8 \mathrm{~Hz}), 126.6$ (d, $J=6.2 \mathrm{~Hz})$, $94.0(\mathrm{~d}, J=174.0 \mathrm{~Hz}), 52.2$ ppm. MS (EI): 185 (100), $244\left(\mathrm{M}^{+}\right)$; HRMS (EI) m/z: $\left[\mathrm{M}^{+}\right]$Calcd for $\mathrm{C}_{15} \mathrm{H}_{13} \mathrm{O}_{2} \mathrm{~F}$ 244.0900; Found 244.0908. IR (KBr): $v_{\max }=3065,3032$, $1724,1609,1448,1290,1204,1181,749 \mathrm{~cm}^{-1}$. 
HPLC (OD-H, $0.46 \times 25 \mathrm{~cm}, 5 \mu \mathrm{m}$, hexane/isopropanol = 98/2 (v/v \%), flow 0.7 $\mathrm{mL} / \mathrm{min}$, UV detection at $214 \mathrm{~nm}$ ), retention time $=12.61 \mathrm{~min}$ (minor) and $15.28 \mathrm{~min}$ (major). $[\alpha]_{\mathrm{D}^{25}}=-0.95\left(\mathrm{c}=0.210, \mathrm{CHCl}_{3}, 93: 7\right.$ e.r).

\section{Methyl (R)-3-(fluoro(3-fluorophenyl)methyl)benzoate 3i}<smiles>CC(=O)c1cccc(C(F)c2cccc(F)c2)c1</smiles>

The product 3i (51 mg, 66\% yield, yellow liquid) was purified with silica gel chromatography (petroleum ether/ethyl acetate $=20 / 1) .{ }^{1} \mathrm{H}$ NMR $\left(400 \mathrm{MHz}, \mathrm{CDCl}_{3}\right)$ $\delta 8.03(\mathrm{~d}, J=6.5 \mathrm{~Hz}, 2 \mathrm{H}), 7.56-7.50(\mathrm{~m}, 1 \mathrm{H}), 7.47(\mathrm{t}, J=8.0 \mathrm{~Hz}, 1 \mathrm{H}), 7.35(\mathrm{dd}, J$ $=13.7,7.8 \mathrm{~Hz}, 1 \mathrm{H}), 7.11(\mathrm{~d}, J=7.7 \mathrm{~Hz}, 1 \mathrm{H}), 7.09-6.99(\mathrm{~m}, 2 \mathrm{H}), 6.49(\mathrm{~d}, J=47.0$ $\mathrm{Hz}, 1 \mathrm{H}), 3.92(\mathrm{~s}, 3 \mathrm{H}) ;{ }^{19} \mathrm{~F} \mathrm{NMR}\left(376 \mathrm{MHz}, \mathrm{CDCl}_{3}\right) \delta-112.11(\mathrm{td}, J=9.0,5.8 \mathrm{~Hz}, 1$ F), $-168.48(\mathrm{~d}, J=46.9 \mathrm{~Hz}, 1 \mathrm{~F}) ;{ }^{13} \mathrm{C} \mathrm{NMR}\left(101 \mathrm{MHz}, \mathrm{CDCl}_{3}\right) \delta 166.5,162.8(\mathrm{~d}, J=$ $247.1 \mathrm{~Hz}), 141.8$ (dd, $J=22.3,7.1 \mathrm{~Hz}), 139.7$ (d, $J=22.0 \mathrm{~Hz}), 130.8$ (d, $J=6.2 \mathrm{~Hz})$, 130.6, 130.2 (d, $J=8.2 \mathrm{~Hz}), 129.8$ (d, $J=1.8 \mathrm{~Hz}), 128.8,127.66$ (d, $J=6.6 \mathrm{~Hz})$, $122.0(\mathrm{dd}, J=6.5,3.0 \mathrm{~Hz}), 115.5(\mathrm{dd}, J=21.1,1.8 \mathrm{~Hz}), 113.4(\mathrm{dd}, J=22.7,7.0 \mathrm{~Hz})$, $93.2(\mathrm{dd}, J=175.4,1.9 \mathrm{~Hz}), 52.2$ ppm. MS (EI): 262 (100), $262\left(\mathrm{M}^{+}\right)$; HRMS (EI) $\mathrm{m} / \mathrm{z}:\left[\mathrm{M}^{+}\right]$Calcd for $\mathrm{C}_{15} \mathrm{H}_{12} \mathrm{O}_{2} \mathrm{~F}_{2}$ 262.0805; Found 262.0798. IR $(\mathrm{KBr}): v_{\max }=3433$, $3070,2953,2847,1724,1614,1592,1488,1290,1200,1109,1086,982,771 \mathrm{~cm}^{-1}$. HPLC (OD-H, $0.46 \times 25 \mathrm{~cm}, 5 \mu \mathrm{m}$, hexane/isopropanol = 95/5 (v/v \%), flow 0.7 $\mathrm{mL} / \mathrm{min}$, UV detection at $214 \mathrm{~nm}$ ), retention time $=9.57 \mathrm{~min}$ (minor) and $11.35 \mathrm{~min}$ (major). $[\alpha]_{\mathrm{D}}{ }^{25}=+4.22\left(\mathrm{c}=0.270, \mathrm{CHCl}_{3}, 96: 4\right.$ e.r).

\section{Ethyl (S)-4-(fluoro(phenyl)methyl)benzoate 3j}<smiles>CCOC(=O)c1ccc(C(F)c2ccccc2)cc1</smiles> 
The product 3j (53 mg, 68\% yield, yellow liquid) was purified with silica gel chromatography (petroleum ether/ethyl acetate $=20 / 1) .{ }^{1} \mathrm{H}$ NMR $\left(400 \mathrm{MHz}, \mathrm{CDCl}_{3}\right)$ $\delta 8.06(\mathrm{~d}, J=8.0 \mathrm{~Hz}, 2 \mathrm{H}), 7.48-7.29(\mathrm{~m}, 7 \mathrm{H}), 6.51(\mathrm{~d}, J=47.2 \mathrm{~Hz}, 1 \mathrm{H}), 4.38(\mathrm{q}, J$ $=7.1 \mathrm{~Hz}, 2 \mathrm{H}), 1.39(\mathrm{t}, J=7.1 \mathrm{~Hz}, 3 \mathrm{H}) ;{ }^{19} \mathrm{~F} \mathrm{NMR}\left(376 \mathrm{MHz}, \mathrm{CDCl}_{3}\right) \delta-168.63(\mathrm{~d}, J$ $=47.2 \mathrm{~Hz}, 1 \mathrm{~F}) ;{ }^{13} \mathrm{C} \mathrm{NMR}\left(101 \mathrm{MHz}, \mathrm{CDCl}_{3}\right) \delta 166.2,144.5(\mathrm{~d}, J=22.2 \mathrm{~Hz}), 139.1$ $(\mathrm{d}, J=21.1 \mathrm{~Hz}), 130.4,129.7,128.8(\mathrm{~d}, J=2.4 \mathrm{~Hz}), 128.6,126.8(\mathrm{~d}, J=6.1 \mathrm{~Hz})$, 126.1 (d, $J=6.8 \mathrm{~Hz}), 93.9$ (d, $J=174.3 \mathrm{~Hz}), 61.0,14.3$ ppm. MS (EI): 213 (100), 258 $\left(\mathrm{M}^{+}\right)$; HRMS (EI) $\mathrm{m} / \mathrm{z}$ : $\left[\mathrm{M}^{+}\right]$Calcd for Calcd for $\mathrm{C}_{16} \mathrm{H}_{15} \mathrm{O}_{2} \mathrm{~F}$ : 258.1056; Found 258.1050. IR (KBr): $v_{\max }=3064,1716,1614,1455,1391,1277,1105,1021,699$ $\mathrm{cm}^{-1}$.

HPLC (AD-H, $0.46 \times 25 \mathrm{~cm}, 5 \mu \mathrm{m}$, hexane/isopropanol = 98/2 (v/v \%), flow 0.7 $\mathrm{mL} / \mathrm{min}$, UV detection at $214 \mathrm{~nm}$ ), retention time $=14.96 \mathrm{~min}$ (minor) and $16.08 \mathrm{~min}$ (major). $[\alpha]_{\mathrm{D}}{ }^{25}=-13.83\left(\mathrm{c}=0.120, \mathrm{CHCl}_{3}, 92: 8\right.$ e.r).

(S)-3-(Fluoro(phenyl)methyl)benzonitrile 3k<smiles>N#Cc1cccc(C(F)c2ccccc2)c1</smiles>

The product 3k (41 mg, 65\% yield, yellow liquid) was purified with silica gel chromatography (petroleum ether/ethyl acetate $=20 / 1) .{ }^{1} \mathrm{H}$ NMR $\left(400 \mathrm{MHz}, \mathrm{CDCl}_{3}\right)$ $\delta 7.59(\mathrm{dd}, J=13.6,7.9 \mathrm{~Hz}, 3 \mathrm{H}), 7.48$ (t, $J=7.7 \mathrm{~Hz}, 1 \mathrm{H}), 7.42-7.35$ (m, $3 \mathrm{H}), 7.32$ $(\mathrm{t}, J=7.9 \mathrm{~Hz}, 2 \mathrm{H}), 6.46(\mathrm{~d}, J=47.0 \mathrm{~Hz}, 1 \mathrm{H}) ;{ }^{19} \mathrm{~F}$ NMR $\left(376 \mathrm{MHz}, \mathrm{CDCl}_{3}\right) \delta$ $-168.63(\mathrm{~d}, J=47.0 \mathrm{~Hz}, 1 \mathrm{~F}) ;{ }^{13} \mathrm{C} \mathrm{NMR}\left(101 \mathrm{MHz}, \mathrm{CDCl}_{3}\right) \delta 141.5(\mathrm{~d}, J=23.1 \mathrm{~Hz})$, $138.4(\mathrm{~d}, J=21.0 \mathrm{~Hz}), 131.9$ (d, $J=1.6 \mathrm{~Hz}), 130.6$ (d, $J=6.4 \mathrm{~Hz}), 129.8$ (d, $J=7.1$ $\mathrm{Hz}), 129.4,129.1$ (d, $J=2.3 \mathrm{~Hz}), 128.8,126.7$ (d, $J=6.0 \mathrm{~Hz}), 118.4,112.7,93.3$ (d, $J$ $=175.3 \mathrm{~Hz})$ ppm. MS (EI): $211(100), 211\left(\mathrm{M}^{+}\right)$; HRMS (EI) m/z: [M+] Calcd for $\mathrm{C}_{14} \mathrm{H}_{10} \mathrm{NF} 211.0797$; Found 211.0800. IR (KBr): $v_{\max }=3065,3034,2921,2231,1604$, $1494,1455,1304,1198,1174,1002,943,919 \mathrm{~cm}^{-1}$. 
HPLC (OD-H, $0.46 \times 25 \mathrm{~cm}, 5 \mu \mathrm{m}$, hexane/isopropanol = 97/3 (v/v \%), flow 0.7 $\mathrm{mL} / \mathrm{min}$, UV detection at $214 \mathrm{~nm}$ ), retention time $=14.61 \mathrm{~min}$ (minor) and $16.39 \mathrm{~min}$ (major). $[\alpha]_{\mathrm{D}}{ }^{25}=-32.00\left(\mathrm{c}=0.200, \mathrm{CHCl}_{3}, 93: 7\right.$ e.r).

(R)-3-(Fluoro(3-fluorophenyl)methyl)benzonitrile 31<smiles>N#Cc1cccc(C(F)c2cccc(F)c2)c1</smiles>

The product 31 (49 $\mathrm{mg}, 71 \%$ yield, yellow liquid) was purified with silica gel chromatography (petroleum ether/ethyl acetate $=20 / 1) .{ }^{1} \mathrm{H}$ NMR $\left(400 \mathrm{MHz}, \mathrm{CDCl}_{3}\right)$ $\delta 7.68-7.61(\mathrm{~m}, 2 \mathrm{H}), 7.58(\mathrm{~d}, J=7.9 \mathrm{~Hz}, 1 \mathrm{H}), 7.51(\mathrm{t}, J=7.7 \mathrm{~Hz}, 1 \mathrm{H}), 7.38(\mathrm{dd}, J$ $=13.7,7.9 \mathrm{~Hz}, 1 \mathrm{H}), 7.13-6.98(\mathrm{~m}, 3 \mathrm{H}), 6.46(\mathrm{~d}, J=46.8 \mathrm{~Hz}, 1 \mathrm{H}) ;{ }^{19} \mathrm{~F}$ NMR $(376$ $\left.\mathrm{MHz} \mathrm{CDCl}_{3}\right) \delta-111.58(\mathrm{td}, J=8.9,5.8 \mathrm{~Hz}, 1 \mathrm{~F}),-169.47(\mathrm{~d}, J=46.8 \mathrm{~Hz}, 1 \mathrm{~F}) ;{ }^{13} \mathrm{C}$ NMR (101 MHz, $\left.\mathrm{CDCl}_{3}\right) \delta 162.9(\mathrm{~d}, J=247.6 \mathrm{~Hz}), 140.9(\mathrm{~d}, J=21.7 \mathrm{~Hz}), 140.8(\mathrm{~d}, J$ $=22.7 \mathrm{~Hz}), 132.2(\mathrm{~d}, J=1.7 \mathrm{~Hz}), 130.7(\mathrm{~d}, J=6.3 \mathrm{~Hz}), 130.5(\mathrm{~d}, J=8.2 \mathrm{~Hz}), 129.9$ (d, $J=7.0 \mathrm{~Hz}), 129.5,122.1(\mathrm{dd}, J=6.3,3.0 \mathrm{~Hz}), 118.3,116.0(\mathrm{dd}, J=21.2,1.9 \mathrm{~Hz})$, 113.5 (dd, $J=22.7,6.8 \mathrm{~Hz}), 112.9,92.5$ (d, $J=174.9 \mathrm{~Hz})$ ppm. MS (EI): 229 (100), $229\left(\mathrm{M}^{+}\right)$; HRMS (EI) m/z: $\left[\mathrm{M}^{+}\right]$Calcd for $\mathrm{C}_{14} \mathrm{H}_{9} \mathrm{NF}_{2}$ 229.0703; Found 229.0701. IR $(\mathrm{KBr}): v_{\max }=3070,2923,2232,1958,1615,1487,1449,1268,1139,875 \mathrm{~cm}^{-1}$.

HPLC (AD-H, $0.46 \times 25 \mathrm{~cm}, 5 \mu \mathrm{m}$, hexane/isopropanol = 99/1 (v/v \%), flow 0.7 $\mathrm{mL} / \mathrm{min}$, UV detection at $214 \mathrm{~nm}$ ), retention time $=23.99 \mathrm{~min}$ (minor) and $25.56 \mathrm{~min}$ (major). $[\alpha]_{\mathrm{D}}^{25}=-8.00\left(\mathrm{c}=0.090, \mathrm{CHCl}_{3}, 96: 4\right.$ e.r).

\section{(S)-3-(Fluoro(4-fluorophenyl)methyl)benzonitrile 3m}

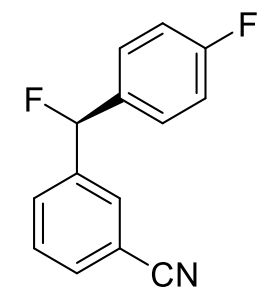

The product 3m (46 mg, 67\% yield, yellow liquid) was purified with silica gel chromatography (petroleum ether/ethyl acetate $=30 / 1) .{ }^{1} \mathrm{H}$ NMR $\left(400 \mathrm{MHz}, \mathrm{CDCl}_{3}\right)$ $\delta 7.61(\mathrm{dd}, J=5.9,4.9 \mathrm{~Hz}, 2 \mathrm{H}), 7.57-7.43(\mathrm{~m}, 2 \mathrm{H}), 7.34-7.25(\mathrm{~m}, 2 \mathrm{H}), 7.08$ (t, $J$ 
$=8.4 \mathrm{~Hz}, 2 \mathrm{H}), 6.44(\mathrm{~d}, J=47.0 \mathrm{~Hz}, 1 \mathrm{H}) ;{ }^{19} \mathrm{~F} \mathrm{NMR}\left(376 \mathrm{MHz}, \mathrm{CDCl}_{3}\right) \delta-112.03$ $(\mathrm{ddd}, J=13.7,8.8,4.5 \mathrm{~Hz}, 1 \mathrm{~F}),-166.85(\mathrm{~d}, J=47.0 \mathrm{~Hz}, 1 \mathrm{~F}) ;{ }^{13} \mathrm{C}$ NMR $(101 \mathrm{MHz}$, $\left.\mathrm{CDCl}_{3}\right) \delta 163.0(\mathrm{~d}, J=246.1 \mathrm{~Hz}), 141.2(\mathrm{~d}, J=23.2 \mathrm{~Hz}), 134.3(\mathrm{~d}, J=24.7 \mathrm{~Hz})$, $132.0(\mathrm{~d}, J=1.6 \mathrm{~Hz}), 130.5$ (d, $J=6.4 \mathrm{~Hz}), 129.7$ (d, $J=7.2 \mathrm{~Hz}), 129.4,128.8$ (dd, $J$ $=8.5,5.9 \mathrm{~Hz}), 118.3,115.9(\mathrm{~d}, J=21.8 \mathrm{~Hz}), 112.8,92.6(\mathrm{~d}, J=175.7 \mathrm{~Hz}) \mathrm{ppm} . \mathrm{MS}$ (EI): 229 (100), $229\left(\mathrm{M}^{+}\right)$; HRMS (EI) m/z: [M+] Calcd for $\mathrm{C}_{14} \mathrm{H}_{9} \mathrm{NF}_{2} 229.0703$; Found 229.0710. IR (KBr): $v_{\max }=3075,2920,2232,1605,1511,1226,1159,1012$, $839,689 \mathrm{~cm}^{-1}$.

HPLC (OD-H, $0.46 \times 25 \mathrm{~cm}, 5 \mu \mathrm{m}$, hexane/isopropanol = 98/2 (v/v \%), flow 0.7 $\mathrm{mL} / \mathrm{min}$, UV detection at $214 \mathrm{~nm}$ ), retention time $=18.83 \mathrm{~min}$ (minor) and $21.63 \mathrm{~min}$ (major). $[\alpha]_{\mathrm{D}}^{25}=-4.80\left(\mathrm{c}=0.100, \mathrm{CHCl}_{3}, 94: 6\right.$ e.r).

(S)-3-(Fluoro(m-tolyl)methyl)benzonitrile 3n

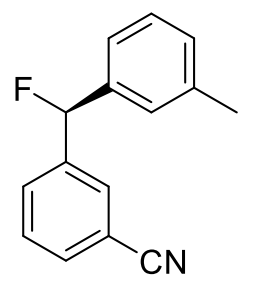

The product 3n (47 mg, 70\% yield, yellow liquid) was purified with silica gel chromatography (petroleum ether/ethyl acetate $=20 / 1) .{ }^{1} \mathrm{H} \mathrm{NMR}\left(400 \mathrm{MHz}, \mathrm{CDCl}_{3}\right)$ $\delta 7.65-7.54(\mathrm{~m}, 3 \mathrm{H}), 7.47(\mathrm{t}, J=7.6 \mathrm{~Hz}, 1 \mathrm{H}), 7.28(\mathrm{t}, J=8.0,1 \mathrm{H}), 7.18(\mathrm{~d}, J=7.6$ $\mathrm{Hz}, 1 \mathrm{H}), 7.10$ (d, $J=9.3 \mathrm{~Hz}, 2 \mathrm{H}), 6.42$ (d, $J=47.1 \mathrm{~Hz}, 1 \mathrm{H}), 2.35$ (s, $3 \mathrm{H}) ;{ }^{19} \mathrm{~F} \mathrm{NMR}$ $\left(376 \mathrm{MHz}, \mathrm{CDCl}_{3}\right) \delta-168.65(\mathrm{~d}, J=47.0 \mathrm{~Hz}, 1 \mathrm{~F}) ;{ }^{13} \mathrm{C} \mathrm{NMR}\left(101 \mathrm{MHz}, \mathrm{CDCl}_{3}\right) \delta$ $141.6(\mathrm{~d}, J=23.2 \mathrm{~Hz}), 138.7,138.3(\mathrm{~d}, J=20.9 \mathrm{~Hz}), 131.8(\mathrm{~d}, J=1.6 \mathrm{~Hz}), 130.6(\mathrm{~d}, J$ $=6.5 \mathrm{~Hz}), 129.9(\mathrm{~d}, J=2.6 \mathrm{~Hz}), 129.8,129.3,128.7,127.3(\mathrm{~d}, J=6.0 \mathrm{~Hz}), 123.8(\mathrm{~d}, J$ $=6.1 \mathrm{~Hz}), 118.5,112.7,93.3(\mathrm{~d}, J=175.2 \mathrm{~Hz}), 21.4$ ppm. MS (EI): 210 (100), 225 $\left(\mathrm{M}^{+}\right)$; HRMS (EI) $\mathrm{m} / \mathrm{z}$ : $\left[\mathrm{M}^{+}\right]$Calcd for $\mathrm{C}_{15} \mathrm{H}_{12} \mathrm{NF}$ 225.0954; Found 225.0960. IR $(\mathrm{KBr}): v_{\max }=3031,2867,2231,1958,1608,1483,1436,1283,1014,702 \mathrm{~cm}^{-1}$.

HPLC (AD-H, $0.46 \times 25 \mathrm{~cm}, 5 \mu \mathrm{m}$, hexane/isopropanol = 98/2 (v/v \%), flow 0.7 $\mathrm{mL} / \mathrm{min}$, UV detection at $214 \mathrm{~nm}$ ), retention time $=11.55 \mathrm{~min}$ (minor) and $12.82 \mathrm{~min}$ (major). $[\alpha]_{\mathrm{D}}{ }^{25}=-41.60\left(\mathrm{c}=0.250, \mathrm{CHCl}_{3}, 94: 6\right.$ e.r).

(R)-3-(Fluoro(3-methoxyphenyl)methyl)benzonitrile 30 


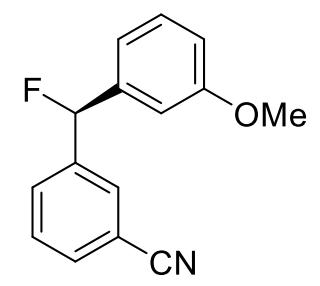

The product 3o (59 mg, 82\% yield, yellow liquid) was purified with silica gel chromatography (petroleum ether/ethyl acetate $=20 / 1) .{ }^{1} \mathrm{H} \mathrm{NMR}\left(400 \mathrm{MHz}, \mathrm{CDCl}_{3}\right)$ $\delta 7.70-7.55$ (m, $3 \mathrm{H}), 7.49$ (t, $J=7.6 \mathrm{~Hz}, 1 \mathrm{H}), 7.32$ (t, $J=7.9 \mathrm{~Hz}, 1 \mathrm{H}), 6.99-6.81$ $(\mathrm{m}, 3 \mathrm{H}), 6.44(\mathrm{~d}, J=47.0 \mathrm{~Hz}, 1 \mathrm{H}), 3.81(\mathrm{~s}, 3 \mathrm{H}) ;{ }^{19} \mathrm{~F}$ NMR $\left(376 \mathrm{MHz}, \mathrm{CDCl}_{3}\right) \delta$ $-169.15(\mathrm{~d}, J=47.0 \mathrm{~Hz}, 1 \mathrm{~F}) ;{ }^{13} \mathrm{C} \mathrm{NMR}\left(101 \mathrm{MHz}, \mathrm{CDCl}_{3}\right) \delta 159.9,141.4(\mathrm{~d}, J=22.9$ Hz), 139.9 (d, $J=21.2 \mathrm{~Hz}), 132.0$ (d, $J=1.6 \mathrm{~Hz}), 130.6$ (d, $J=6.4 \mathrm{~Hz}), 129.9$ (d, $J=$ $2.4 \mathrm{~Hz}), 129.8,129.3,118.8(\mathrm{~d}, J=6.2 \mathrm{~Hz}), 118.4,114.3(\mathrm{~d}, J=2.0 \mathrm{~Hz}), 112.7,112.3$ (d, $J=6.6 \mathrm{~Hz}), 93.1$ (d, $J=175.9 \mathrm{~Hz}), 55.3$ ppm. MS (EI): $241(100), 241\left(\mathrm{M}^{+}\right)$; HRMS (EI) m/z: [M+] Calcd for $\mathrm{C}_{15} \mathrm{H}_{12} \mathrm{NOF}$ 241.0903; Found 241.0898. IR (KBr): $v_{\max }=3064,2938,2231,1601,1489,1456,1288,1262,1190,1050,808,744 \mathrm{~cm}^{-1}$.

HPLC (AD-H, $0.46 \times 25 \mathrm{~cm}, 5 \mu \mathrm{m}$, hexane/isopropanol = 98/2 (v/v \%), flow 0.7 $\mathrm{mL} / \mathrm{min}$, UV detection at $214 \mathrm{~nm}$ ), retention time $=21.76 \mathrm{~min}$ (minor) and $23.41 \mathrm{~min}$ (major). $[\alpha]_{\mathrm{D}}{ }^{25}=-12.97\left(\mathrm{c}=0.370, \mathrm{CHCl}_{3}, 93: 7\right.$ e.r).

\section{(R)-3-(Fluoro(3-fluoro-4-methylphenyl)methyl)benzonitrile 3p}<smiles>Cc1ccc(C(F)c2cccc(C#N)c2)cc1F</smiles>

The product 3p (52 mg, 72\% yield, yellow liquid) was purified with silica gel chromatography (petroleum ether/ethyl acetate $=20 / 1) .{ }^{1} \mathrm{H} \mathrm{NMR}\left(400 \mathrm{MHz}, \mathrm{CDCl}_{3}\right)$ $\delta 7.66-7.59(\mathrm{~m}, 2 \mathrm{H}), 7.58(\mathrm{~d}, J=7.8 \mathrm{~Hz}, 1 \mathrm{H}), 7.50(\mathrm{t}, J=7.7 \mathrm{~Hz}, 1 \mathrm{H}), 7.22(\mathrm{t}, J=$ $7.8 \mathrm{~Hz}, 1 \mathrm{H}), 6.98$ (t, $J=8.0 \mathrm{~Hz}, 1 \mathrm{H}), 6.96(\mathrm{t}, J=8.0 \mathrm{~Hz}, 1 \mathrm{H}), 6.42(\mathrm{~d}, J=46.9 \mathrm{~Hz}$, $1 \mathrm{H}), 2.28(\mathrm{~s}, 3 \mathrm{H}) ;{ }^{19} \mathrm{~F}$ NMR $\left(376 \mathrm{MHz}, \mathrm{CDCl}_{3}\right) \delta-111.41--120.65(\mathrm{~m}, 1 \mathrm{~F})$, $-168.37(\mathrm{~d}, J=46.9 \mathrm{~Hz}, 1 \mathrm{~F}) ;{ }^{13} \mathrm{C} \mathrm{NMR}\left(101 \mathrm{MHz}, \mathrm{CDCl}_{3}\right) \delta 161.3(\mathrm{~d}, J=246.3 \mathrm{~Hz})$, $141.1(\mathrm{~d}, J=22.9 \mathrm{~Hz}), 138.0(\mathrm{dd}, J=21.7,7.1 \mathrm{~Hz}), 132.1(\mathrm{~d}, J=1.6 \mathrm{~Hz}), 131.9(\mathrm{~d}, J$ 
$=5.4 \mathrm{~Hz}), 130.6(\mathrm{~d}, J=6.4 \mathrm{~Hz}), 129.8(\mathrm{~d}, J=7.0 \mathrm{~Hz}), 129.4,126.0(\mathrm{~d}, J=15.2 \mathrm{~Hz})$, $122.0(\mathrm{dd}, J=6.1,3.5 \mathrm{~Hz}), 118.3,113.3(\mathrm{dd}, J=23.7,6.4 \mathrm{~Hz}), 112.8,92.5(\mathrm{~d}, J=$ $177.6 \mathrm{~Hz}), 14.3$ (d, $J=3.5 \mathrm{~Hz})$ ppm. MS (EI): 228 (100), $243\left(\mathrm{M}^{+}\right)$; HRMS (EI) m/z: $\left[\mathrm{M}^{+}\right]$Calcd for $\mathrm{C}_{15} \mathrm{H}_{11} \mathrm{NF}_{2}$ 243.0860; Found 243.0863. IR (KBr): $v_{\max }=3067,3929$, $2231,1585,1509,1421,1267,1115,1015,875,774 \mathrm{~cm}^{-1}$.

HPLC (AD-H, $0.46 \times 25 \mathrm{~cm}, 5 \mu \mathrm{m}$, hexane/isopropanol = 98/2 (v/v \%), flow 0.7 $\mathrm{mL} / \mathrm{min}$, UV detection at $214 \mathrm{~nm}$ ), retention time $=14.21 \mathrm{~min}$ (minor) and $15.00 \mathrm{~min}$ (major). $[\alpha]_{\mathrm{D}}{ }^{25}=-18.00\left(\mathrm{c}=0.250, \mathrm{CHCl}_{3}, 95: 5\right.$ e.r).

(S)-4-(Fluoro(phenyl)methyl)benzonitrile 3q<smiles>N#Cc1ccc(C(F)c2ccccc2)cc1</smiles>

The product 3q (51 mg, 81\% yield, White solid, Mp: $56-58{ }^{\circ} \mathrm{C}$ ) was purified with silica gel chromatography (petroleum ether/ethyl acetate $=20 / 1) .{ }^{1} \mathrm{H}$ NMR $(400 \mathrm{MHz}$, $\left.\mathrm{CDCl}_{3}\right) \delta 7.67(\mathrm{~d}, J=8.0 \mathrm{~Hz}, 2 \mathrm{H}), 7.47(\mathrm{~d}, J=8.0 \mathrm{~Hz}, 2 \mathrm{H}), 7.42-7.37$ (m, $\left.3 \mathrm{H}\right)$, $7.32(\mathrm{dd}, J=6.3,1.6 \mathrm{~Hz}, 2 \mathrm{H}), 6.49$ (d, $J=47.1 \mathrm{~Hz}, 1 \mathrm{H}) ;{ }^{19} \mathrm{~F}$ NMR $(376 \mathrm{MHz}$, $\left.\mathrm{CDCl}_{3}\right) \delta-169.37(\mathrm{~d}, J=47.1 \mathrm{~Hz}, 1 \mathrm{~F}) ;{ }^{13} \mathrm{C} \mathrm{NMR}\left(101 \mathrm{MHz}, \mathrm{CDCl}_{3}\right) \delta 144.9(\mathrm{~d}, J=$ $22.7 \mathrm{~Hz}), 138.4(\mathrm{~d}, J=20.9 \mathrm{~Hz}), 132.3,129.1$ (d, $J=2.4 \mathrm{~Hz}), 128.8,126.8$ (d, $J=6.0$ Hz), 126.7 (d, $J=7.1 \mathrm{~Hz}), 118.5,112.2,93.5$ (d, $J=175.6 \mathrm{~Hz})$ ppm. MS (EI): 211 (100), $211\left(\mathrm{M}^{+}\right)$; HRMS (EI) m/z: [M+] Calcd for $\mathrm{C}_{14} \mathrm{H}_{10} \mathrm{NF}$ 211.0797; Found 211.0803. IR (KBr): $v_{\max }=3396,2923,2228,1980,1660,1567,1384,1180,1029$, $862,700 \mathrm{~cm}^{-1}$.

HPLC (OD-H, $0.46 \times 25 \mathrm{~cm}, 5 \mu \mathrm{m}$, hexane/isopropanol = 97/3 (v/v \%), flow 0.7 $\mathrm{mL} / \mathrm{min}$, UV detection at $214 \mathrm{~nm}$ ), retention time $=18.63 \mathrm{~min}$ (minor) and $20.24 \mathrm{~min}$ (major). $[\alpha]_{\mathrm{D}}^{25}=-1.11\left(\mathrm{c}=0.090, \mathrm{CHCl}_{3}, 95: 5\right.$ e.r).

Method for the single crystal growth. $5.0 \mathrm{Mg}$ of compound $\mathbf{3 q}$ was dissolved 1.0 $\mathrm{mL}$ of acetone in a vial at room temperature. The lid of the vial was not completely 
tightened, which allowed the slow evaporation of acetone. The crystals that are suitable for X-ray diffusion study were formed two weeks later.

Method for X-ray diffraction study. A crystal with size of $0.08 \times 0.12 \times 0.16 \mathrm{~mm}$ was obtained by solvent-evaporation method using acetone. The data were collected on a Bruker SMART CCD area-detector diffractometer at room temperature using $\mathrm{CuK} \alpha$ radiation by $\omega / \varphi$ scan mode. 5230 reflections were collected, of which 2016 reflections were unique $(\operatorname{Rint}=0.0615)$.

(R)-4-(Fluoro(m-tolyl)methyl)benzonitrile 3r<smiles>Cc1cccc(C(F)c2ccc(C#N)cc2)c1</smiles>

The product $3 \mathrm{r}$ (37 mg, 55\% yield, yellow liquid) was purified with silica gel chromatography (petroleum ether/ethyl acetate $=20 / 1) .{ }^{1} \mathrm{H}$ NMR $\left(400 \mathrm{MHz}, \mathrm{CDCl}_{3}\right)$ $\delta 7.65(\mathrm{~d}, J=8.0 \mathrm{~Hz}, 2 \mathrm{H}), 7.45(\mathrm{~d}, J=8.0 \mathrm{~Hz}, 2 \mathrm{H}), 7.26(\mathrm{dd}, J=12.4,4.9 \mathrm{~Hz}, 1 \mathrm{H})$, 7.17 (d, $J=7.6 \mathrm{~Hz}, 1 \mathrm{H}), 7.09$ (d, $J=8.5 \mathrm{~Hz}, 2 \mathrm{H}), 6.43$ (d, $J=47.1 \mathrm{~Hz}, 1 \mathrm{H}), 2.34$ (s, $3 \mathrm{H}) ;{ }^{19} \mathrm{~F}$ NMR $\left(376 \mathrm{MHz}, \mathrm{CDCl}_{3}\right) \delta-169.40(\mathrm{~d}, J=47.1 \mathrm{~Hz}, 1 \mathrm{~F}) ;{ }^{13} \mathrm{C}$ NMR $(101$ $\left.\mathrm{MHz}, \mathrm{CDCl}_{3}\right) \delta 145.1(\mathrm{~d}, J=22.8 \mathrm{~Hz}), 138.6,138.3(\mathrm{~d}, J=20.7 \mathrm{~Hz}), 132.3,129.9(\mathrm{~d}$, $J=2.4 \mathrm{~Hz}), 128.7,127.4(\mathrm{~d}, J=5.8 \mathrm{~Hz}), 126.7(\mathrm{~d}, J=7.1 \mathrm{~Hz}), 123.9(\mathrm{~d}, J=5.9 \mathrm{~Hz})$, 118.5, 112.1, 93.6 (d, $J=175.5 \mathrm{~Hz}), 21.4$ ppm. MS (EI): $210(100), 225\left(\mathrm{M}^{+}\right)$; HRMS (EI) m/z: [M+] Calcd for $\mathrm{C}_{15} \mathrm{H}_{12} \mathrm{NF} 225.0954$; Found 225.0959. IR (KBr): $v_{\max }=3675$, $3028,2867,2229,1928,1608,1506,1409,1303,1273,1001,885,700 \mathrm{~cm}^{-1}$.

HPLC $(\mathrm{PC} 3,0.46 \times 25 \mathrm{~cm}, 5 \mu \mathrm{m}$, acetonitrile/water $=80 / 20(\mathrm{v} / \mathrm{v} \%)$,flow $0.7 \mathrm{~mL} / \mathrm{min}$, $\mathrm{UV}$ detection at $214 \mathrm{~nm}$ ), retention time $=6.30 \mathrm{~min}$ (minor) and $6.54 \mathrm{~min}$ (major). $[\alpha]_{\mathrm{D}}^{25}=-22.17\left(\mathrm{c}=0.230, \mathrm{CHCl}_{3}, 94: 6\right.$ e.r $)$.

\section{(R)-3-(Fluoro(3-fluorophenyl)methyl)benzonitrile 3s}




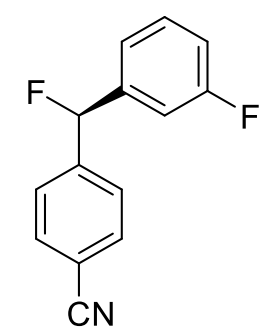

The product 3s (38 mg, 55\% yield, yellow liquid) was purified with silica gel chromatography (petroleum ether/ethyl acetate $=20 / 1) .{ }^{1} \mathrm{H} \mathrm{NMR}\left(400 \mathrm{MHz}, \mathrm{CDCl}_{3}\right)$ $\delta 7.69(\mathrm{~d}, J=8.1 \mathrm{~Hz}, 2 \mathrm{H}), 7.46(\mathrm{~d}, J=8.1 \mathrm{~Hz}, 2 \mathrm{H}), 7.37$ (dd, $J=13.8,7.8 \mathrm{~Hz}, 1 \mathrm{H})$, $7.21-6.98(\mathrm{~m}, 3 \mathrm{H}), 6.47(\mathrm{~d}, J=46.8 \mathrm{~Hz}, 1 \mathrm{H}) ;{ }^{19} \mathrm{~F}$ NMR $\left(376 \mathrm{MHz}, \mathrm{CDCl}_{3}\right) \delta$ $-111.60(\mathrm{td}, J=8.9,5.8 \mathrm{~Hz}, 1 \mathrm{~F}),-170.43(\mathrm{~d}, J=46.8 \mathrm{~Hz}, 1 \mathrm{~F}) ;{ }^{13} \mathrm{C} \mathrm{NMR}(101 \mathrm{MHz}$, $\left.\mathrm{CDCl}_{3}\right) \delta 162.9(\mathrm{~d}, J=247.8 \mathrm{~Hz}), 144.2(\mathrm{~d}, J=22.3 \mathrm{~Hz}), 140.8(\mathrm{dd}, J=21.7,7.1 \mathrm{~Hz})$, 132.4, 130.5 (d, $J=8.2 \mathrm{~Hz}), 126.8$ (d, $J=6.9 \mathrm{~Hz}), 122.2(\mathrm{dd}, J=6.2,3.1 \mathrm{~Hz}), 118.3$, $116.1(\mathrm{dd}, J=21.1,2.0 \mathrm{~Hz}), 113.6(\mathrm{dd}, J=22.7,6.6 \mathrm{~Hz}), 112.5,92.7(\mathrm{~d}, J=178.9 \mathrm{~Hz})$ ppm. MS (EI): $229(100), 229\left(\mathrm{M}^{+}\right)$; HRMS (EI) m/z: [M+ Calcd for $\mathrm{C}_{14} \mathrm{H}_{9} \mathrm{NF}_{2}$ 229.0703; Found 229.0710. IR (KBr): $v_{\max }=3068,2927,2230,1611,1489,1269$, $1178,1012,930,777,559 \mathrm{~cm}^{-1}$.

HPLC (AD-H, $0.46 \times 25 \mathrm{~cm}, 5 \mu \mathrm{m}$, hexane/isopropanol = 99/1 (v/v \%), flow 0.7 $\mathrm{mL} / \mathrm{min}$, UV detection at $214 \mathrm{~nm}$ ), retention time $=23.99 \mathrm{~min}$ (minor) and $25.55 \mathrm{~min}$ (major). $[\alpha]_{\mathrm{D}}{ }^{25}=-26.84\left(\mathrm{c}=0.190, \mathrm{CHCl}_{3}, 96: 4\right.$ e.r).

(S)-2-Fluoro-4-(fluoro(3-fluorophenyl)methyl)benzonitrile 3t

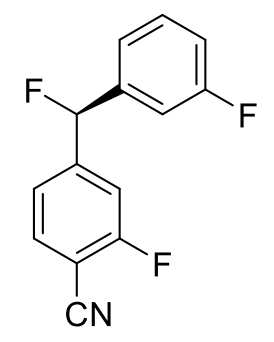

The product 3t (53 mg, 71\% yield, yellow liquid) was purified with silica gel chromatography (petroleum ether/ethyl acetate $=20 / 1) .{ }^{1} \mathrm{H} \mathrm{NMR}\left(400 \mathrm{MHz}, \mathrm{CDCl}_{3}\right)$ $\delta 7.65(\mathrm{t}, J=7.1 \mathrm{~Hz}, 1 \mathrm{H}), 7.39(\mathrm{dd}, J=13.8,7.8 \mathrm{~Hz}, 1 \mathrm{H}), 7.23(\mathrm{~d}, J=8.6 \mathrm{~Hz}, 2 \mathrm{H})$, $7.09(\mathrm{t}, J=8.7 \mathrm{~Hz}, 2 \mathrm{H}), 7.03(\mathrm{~d}, J=9.1 \mathrm{~Hz}, 1 \mathrm{H}), 6.45(\mathrm{~d}, J=46.7 \mathrm{~Hz}, 1 \mathrm{H}) ;{ }^{19} \mathrm{~F}$ NMR $\left(376 \mathrm{MHz}, \mathrm{CDCl}_{3}\right) \delta-105.03(\mathrm{dd}, J=9.0,6.8 \mathrm{~Hz}, 1 \mathrm{~F}),-111.25(\mathrm{td}, J=8.8,5.9$ 
$\mathrm{Hz}, 1 \mathrm{~F}),-170.64(\mathrm{~d}, J=46.7 \mathrm{~Hz}, 1 \mathrm{~F}) ;{ }^{13} \mathrm{C} \mathrm{NMR}\left(101 \mathrm{MHz}, \mathrm{CDCl}_{3}\right) \delta 163.2(\mathrm{~d}, J=$ $260.4 \mathrm{~Hz}), 162.9$ (d, $J=248.1 \mathrm{~Hz}), 147.3$ (dd, $J=23.2,7.4 \mathrm{~Hz}), 140.1$ (dd, $J=21.3$, $7.1 \mathrm{~Hz}), 133.8,130.7(\mathrm{~d}, J=8.2 \mathrm{~Hz}), 122.3(\mathrm{dd}, J=6.4,3.1 \mathrm{~Hz}), 122.2,116.4(\mathrm{dd}, J=$ 21.1, 2.1 Hz), $114.1(\mathrm{dd}, J=21.1,7.9 \mathrm{~Hz}), 113.7(\mathrm{dd}, J=22.7,6.5 \mathrm{~Hz}), 113.5,101.5$ (d, $J=17.0 \mathrm{~Hz}), 92.1$ (d, $J=178.6 \mathrm{~Hz})$ ppm. MS (EI): 247 (100), $247\left(\mathrm{M}^{+}\right)$; HRMS (EI) m/z: [M+] Calcd for $\mathrm{C}_{14} \mathrm{H}_{8} \mathrm{NF}_{3}$ 247.0609; Found 247.0608. IR (KBr): $v_{\max }=3092$, $2240,1616,1501,1269,1155,1023,797,693 \mathrm{~cm}^{-1}$.

HPLC (OD-H, $0.46 \times 25 \mathrm{~cm}, 5 \mu \mathrm{m}$, hexane/isopropanol = 95/5 (v/v \%), flow 0.7 $\mathrm{mL} / \mathrm{min}$, UV detection at $214 \mathrm{~nm}$ ), retention time $=16.12 \mathrm{~min}$ (minor) and $17.82 \mathrm{~min}$ (major). $[\alpha]_{\mathrm{D}}^{25}=+16.88\left(\mathrm{c}=0.160, \mathrm{CHCl}_{3}, 96: 4\right.$ e.r).

\section{(S)-3-Fluoro-4-(fluoro(3-fluorophenyl)methyl)benzonitrile 3u}

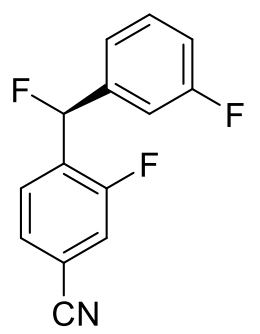

The product 3u (30 mg, 40\% yield, yellow liquid) was purified with silica gel chromatography (petroleum ether/ethyl acetate $=20 / 1) .{ }^{1} \mathrm{H}$ NMR $\left(400 \mathrm{MHz}, \mathrm{CDCl}_{3}\right)$ $\delta 7.68-7.60(\mathrm{~m}, 1 \mathrm{H}), 7.54(\mathrm{dd}, J=8.0,1.2 \mathrm{~Hz}, 1 \mathrm{H}), 7.44-7.32(\mathrm{~m}, 2 \mathrm{H}), 7.16-$ $7.08(\mathrm{~m}, 1 \mathrm{H}), 7.10-7.02(\mathrm{~m}, 2 \mathrm{H}), 6.73(\mathrm{~d}, J=45.9 \mathrm{~Hz}, 1 \mathrm{H}) ;{ }^{19} \mathrm{~F}$ NMR $(376 \mathrm{MHz}$, $\left.\mathrm{CDCl}_{3}\right) \delta-109.97--112.53(\mathrm{~m}, 1 \mathrm{~F}),-113.31--116.15(\mathrm{~m}, 1 \mathrm{~F}),-176.28(\mathrm{~d}, J=45.9$ $\mathrm{Hz}, 1 \mathrm{~F}) ;{ }^{13} \mathrm{C}$ NMR $\left(101 \mathrm{MHz}, \mathrm{CDCl}_{3}\right) \delta 162.8(\mathrm{~d}, J=247.7 \mathrm{~Hz}), 158.7$ (dd, $J=251.8$, $5.6 \mathrm{~Hz}), 139.8(\mathrm{dd}, J=22.3,7.1 \mathrm{~Hz}), 132.4(\mathrm{dd}, J=24.0,13.2 \mathrm{~Hz}), 130.5(\mathrm{~d}, J=8.2$ $\mathrm{Hz}), 128.6(\mathrm{~d}, J=3.9 \mathrm{~Hz}), 128.3$ (dd, $J=8.4,4.0 \mathrm{~Hz}), 121.8$ (d, $J=6.3 \mathrm{~Hz}), 119.4$ (d, $J=24.6 \mathrm{~Hz}), 117.0,116.3(\mathrm{~d}, J=2.0 \mathrm{~Hz}), 114.0(\mathrm{~d}, J=7.9 \mathrm{~Hz}), 113.3(\mathrm{dd}, J=23.1$, $6.5 \mathrm{~Hz}), 87.4$ (d, $J=177.2 \mathrm{~Hz})$ ppm. MS (EI): 247 (100), $247\left(\mathrm{M}^{+}\right)$; HRMS (EI) m/z: $\left[\mathrm{M}^{+}\right]$Calcd for $\mathrm{C}_{14} \mathrm{H}_{8} \mathrm{NF}_{3}$ 247.0609; Found 247.0610. IR (KBr): $v_{\max }=3079,2925$, $2236,1593,1491,1416,1287,1269,1162,1018,878,814 \mathrm{~cm}^{-1}$. 
HPLC (IE3, $0.46 \times 25 \mathrm{~cm}, 5 \mu \mathrm{m}$, hexane/isopropanol = 98/2 (v/v \%), flow 0.7 $\mathrm{mL} / \mathrm{min}$, UV detection at $214 \mathrm{~nm}$ ), retention time $=12.00 \mathrm{~min}$ (minor) and $12.68 \mathrm{~min}$ (major). $[\alpha]_{\mathrm{D}}{ }^{25}=+0.71\left(\mathrm{c}=0.280, \mathrm{CHCl}_{3}, 91: 9\right.$ e.r).

(S)-1-(Fluoro(phenyl)methyl)-4-(trifluoromethyl)benzene 3v<smiles>FC(c1ccccc1)c1ccc(C(F)(F)F)cc1</smiles>

The product 3v (52 mg, 68\% yield, yellow liquid) was purified with silica gel chromatography (petroleum ether/ethyl acetate $=80 / 1) .{ }^{1} \mathrm{H}$ NMR $\left(400 \mathrm{MHz}, \mathrm{CDCl}_{3}\right)$ $\delta 7.64(\mathrm{~d}, J=8.2 \mathrm{~Hz}, 2 \mathrm{H}), 7.48(\mathrm{~d}, J=8.0 \mathrm{~Hz}, 2 \mathrm{H}), 7.44-7.29(\mathrm{~m}, 5 \mathrm{H}), 6.51(\mathrm{~d}, J=$ $47.2 \mathrm{~Hz}, 1 \mathrm{H}) ;{ }^{19} \mathrm{~F}$ NMR $\left(376 \mathrm{MHz}, \mathrm{CDCl}_{3}\right) \delta-62.68(\mathrm{~s}, 3 \mathrm{~F}),-168.70(\mathrm{~d}, J=47.2 \mathrm{~Hz}$, $1 \mathrm{~F}) ;{ }^{13} \mathrm{C} \mathrm{NMR}\left(101 \mathrm{MHz}, \mathrm{CDCl}_{3}\right) \delta 143.7$ (d, $\left.J=22.4 \mathrm{~Hz}\right), 138.9(\mathrm{~d}, J=21.1 \mathrm{~Hz})$, 130.5 (q, $J=32.5 \mathrm{~Hz}), 128.9$ (d, J = 2.3 Hz), 128.7, 126.7 (d, $J=6.1 \mathrm{~Hz}), 126.5$ (d, $J$ $=6.8 \mathrm{~Hz}), 125.4$ (q, $J=3.8 \mathrm{~Hz}), 123.9$ (q, $J=272.2 \mathrm{~Hz}), 93.7$ (d, $J=174.6 \mathrm{~Hz}) \mathrm{ppm}$. MS (EI): 185 (100), $254\left(\mathrm{M}^{+}\right)$; HRMS (EI) m/z: [M+] Calcd for $\mathrm{C}_{14} \mathrm{H}_{10} \mathrm{~F}_{4}$ 254.0719; Found 254.0725. IR (KBr): $v_{\max }=3066,2922,1621,1455,1326,1166,1067,1018$, $864 \mathrm{~cm}^{-1}$.

HPLC (OD-H, $0.46 \times 25 \mathrm{~cm}, 5 \mu \mathrm{m}$, hexane/isopropanol = 98/2 (v/v \%), flow 0.7 $\mathrm{mL} / \mathrm{min}$, UV detection at $214 \mathrm{~nm}$ ), retention time $=7.25 \mathrm{~min}$ (minor) and $8.50 \mathrm{~min}$ (major). $[\alpha]_{\mathrm{D}}{ }^{25}=-24.00\left(\mathrm{c}=0.080, \mathrm{CHCl}_{3}, 93: 7\right.$ e.r).

(R)-1-Fluoro-3-(fluoro(4-(trifluoromethyl)phenyl)methyl)benzene 3w<smiles>Fc1cccc(C(F)c2ccc(C(F)(F)F)cc2)c1</smiles>

The product 3w (61 mg, 75\% yield, yellow liquid) was purified with silica gel chromatography (petroleum ether/ethyl acetate $=80 / 1) .{ }^{1} \mathrm{H} \mathrm{NMR}\left(400 \mathrm{MHz}, \mathrm{CDCl}_{3}\right)$ 
$\delta 7.66(\mathrm{~d}, J=8.1 \mathrm{~Hz}, 2 \mathrm{H}), 7.47(\mathrm{~d}, J=8.1 \mathrm{~Hz}, 2 \mathrm{H}), 7.37(\mathrm{dd}, J=14.2,8.3 \mathrm{~Hz}, 1 \mathrm{H})$, $7.11(\mathrm{~d}, J=7.6 \mathrm{~Hz}, 1 \mathrm{H}), 7.05(\mathrm{~d}, J=8.8 \mathrm{~Hz}, 2 \mathrm{H}), 6.49(\mathrm{~d}, J=47.0 \mathrm{~Hz}, 1 \mathrm{H}) ;{ }^{19} \mathrm{~F}$ NMR $\left(376 \mathrm{MHz}, \mathrm{CDCl}_{3}\right) \delta-62.75(\mathrm{~s}, 1 \mathrm{~F}),-111.90(\mathrm{td}, J=8.9,5.8 \mathrm{~Hz}, 1 \mathrm{~F}),-169.60$ $(\mathrm{d}, J=46.9 \mathrm{~Hz}, 1 \mathrm{~F}) ;{ }^{13} \mathrm{C} \mathrm{NMR}\left(101 \mathrm{MHz}, \mathrm{CDCl}_{3}\right) \delta 162.8(\mathrm{~d}, J=247.4 \mathrm{~Hz}), 143.0(\mathrm{~d}$, $J=21.9 \mathrm{~Hz}), 141.3(\mathrm{dd}, J=21.9,7.1 \mathrm{~Hz}), 130.8(\mathrm{q}, J=31.3 \mathrm{~Hz}), 130.3(\mathrm{~d}, J=8.2$ $\mathrm{Hz}), 126.6(\mathrm{~d}, J=6.7 \mathrm{~Hz}), 125.6(\mathrm{q}, J=3.8 \mathrm{~Hz}), 123.8$ (q, $J=272.3 \mathrm{~Hz}), 122.2(\mathrm{dd}, J$ $=6.4,3.1 \mathrm{~Hz}), 115.8(\mathrm{dd}, J=21.1,1.9 \mathrm{~Hz}), 113.6(\mathrm{dd}, J=22.6,6.8 \mathrm{~Hz}), 92.9(\mathrm{~d}, J=$ $176.0 \mathrm{~Hz})$ ppm. MS (EI): 203 (100), $272\left(\mathrm{M}^{+}\right)$; HRMS (EI) m/z: [M+ Calcd for $\mathrm{C}_{14} \mathrm{H}_{9} \mathrm{~F}_{5}$ 272.0624; Found 272.0629. IR (KBr): $v_{\max }=3072,1926,1615,1450,1269$, $1128,850,694 \mathrm{~cm}^{-1}$.

HPLC (OD-H, $0.46 \times 25 \mathrm{~cm}, 5 \mu \mathrm{m}$, hexane/isopropanol $=100 / 0(\mathrm{v} / \mathrm{v} \%)$, flow 0.7 $\mathrm{mL} / \mathrm{min}$, UV detection at $214 \mathrm{~nm}$ ), retention time $=13.77 \mathrm{~min}$ (minor) and $14.46 \mathrm{~min}$ (major). $[\alpha]_{\mathrm{D}}^{25}=+0.91\left(\mathrm{c}=0.110, \mathrm{CHCl}_{3}, 95: 5\right.$ e.r).

\section{(S)-1-(Fluoro(phenyl)methyl)-3-(trifluoromethyl)benzene 3x}

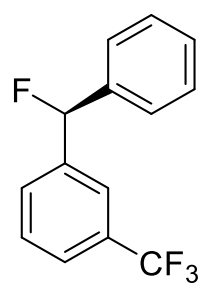

The product 3x (47 mg, 62\% yield, yellow liquid) was purified with silica gel chromatography (petroleum ether/ethyl acetate $=80 / 1) .{ }^{1} \mathrm{H} \mathrm{NMR}\left(400 \mathrm{MHz}, \mathrm{CDCl}_{3}\right)$ $\delta 7.63(\mathrm{~s}, 1 \mathrm{H}), 7.59$ (d, J=6.8 Hz, $1 \mathrm{H}), 7.52-7.44$ (m, $2 \mathrm{H}), 7.43-7.29$ (m, $5 \mathrm{H})$, $6.50(\mathrm{~d}, J=47.1 \mathrm{~Hz}, 1 \mathrm{H}) ;{ }^{19} \mathrm{~F}$ NMR $\left(376 \mathrm{MHz}, \mathrm{CDCl}_{3}\right) \delta-62.69(\mathrm{~s}, 3 \mathrm{~F}),-167.78(\mathrm{~d}$, $J=47.1 \mathrm{~Hz}, 1 \mathrm{~F}) ;{ }^{13} \mathrm{C} \mathrm{NMR}\left(101 \mathrm{MHz}, \mathrm{CDCl}_{3}\right) \delta 140.9(\mathrm{~d}, J=22.7 \mathrm{~Hz}), 138.9(\mathrm{~d}, J=$ $21.2 \mathrm{~Hz}), 130.9$ (q, $J=32.4 \mathrm{~Hz}), 129.7$ (d, $J=6.3 \mathrm{~Hz}), 129.0,128.9$ (d, $J=2.3 \mathrm{~Hz}$ ), 128.7, 126.7 (d, $J=6.1 \mathrm{~Hz}), 125.2(\mathrm{~d}, J=3.7 \mathrm{~Hz}), 123.9$ (q, $J=272.4 \mathrm{~Hz}), 123.1$ (dq, $J=7.7,3.8 \mathrm{~Hz}), 93.7$ (d, $J=174.4 \mathrm{~Hz})$ ppm. MS (EI): 185 (100), $254\left(\mathrm{M}^{+}\right)$; HRMS (EI) $\mathrm{m} / \mathrm{z}:\left[\mathrm{M}^{+}\right]$Calcd for $\mathrm{C}_{14} \mathrm{H}_{10} \mathrm{~F}_{4}$ 254.0719; Found 254.0730. IR (KBr): $v_{\max }=2924$, $1717,1653,1507,1328,1131,1075,1021,798,701 \mathrm{~cm}^{-1}$. 
HPLC (OD-H, $0.46 \times 25 \mathrm{~cm}, 5 \mu \mathrm{m}$, hexane/isopropanol = 98/2 (v/v \%), flow 0.7 $\mathrm{mL} / \mathrm{min}$, UV detection at $214 \mathrm{~nm}$ ), retention time $=6.95 \mathrm{~min}$ (minor) and $7.57 \mathrm{~min}$ (major). $[\alpha]_{\mathrm{D}}{ }^{25}=-18.75\left(\mathrm{c}=0.160, \mathrm{CHCl}_{3}, 93: 7\right.$ e.r).

(R)-1-((4-Bromophenyl)fluoromethyl)-3-fluorobenzene 3y<smiles>Fc1cccc(C(F)c2ccc(Br)cc2)c1</smiles>

The product 3w (35 mg, 42\% yield, yellow liquid) was purified with silica gel chromatography (petroleum ether/ethyl acetate $=80 / 1) .{ }^{1} \mathrm{H}$ NMR $\left(400 \mathrm{MHz}, \mathrm{CDCl}_{3}\right)$ $\delta 7.52(\mathrm{~d}, J=7.9 \mathrm{~Hz}, 2 \mathrm{H}), 7.39-7.31(\mathrm{~m}, 1 \mathrm{H}), 7.21(\mathrm{~d}, J=8.1 \mathrm{~Hz}, 2 \mathrm{H}), 7.12-6.98$ $(\mathrm{m}, 3 \mathrm{H}), 6.40(\mathrm{~d}, J=47.1 \mathrm{~Hz}, 1 \mathrm{H}) ;{ }^{19} \mathrm{~F}$ NMR $\left(376 \mathrm{MHz}, \mathrm{CDCl}_{3}\right) \delta-112.06(\mathrm{td}, J=$ 9.0, $5.8 \mathrm{~Hz}, 1 \mathrm{~F}),-167.75(\mathrm{~d}, J=47.1 \mathrm{~Hz}, 1 \mathrm{~F}) ;{ }^{13} \mathrm{C} \mathrm{NMR}\left(101 \mathrm{MHz}, \mathrm{CDCl}_{3}\right) \delta 162.8$ $(\mathrm{d}, J=247.0 \mathrm{~Hz}), 141.7(\mathrm{dd}, J=22.3,7.1 \mathrm{~Hz}), 138.2(\mathrm{~d}, J=21.8 \mathrm{~Hz}), 131.8,130.2(\mathrm{~d}$, $J=8.1 \mathrm{~Hz}), 128.2(\mathrm{~d}, J=6.2 \mathrm{~Hz}), 122.8(\mathrm{~d}, J=2.7 \mathrm{~Hz}), 122.0(\mathrm{dd}, J=6.5,3.0 \mathrm{~Hz})$, $115.5(\mathrm{dd}, J=21.1,1.8 \mathrm{~Hz}), 113.4(\mathrm{dd}, J=22.7,7.0 \mathrm{~Hz}), 93.0(\mathrm{~d}, J=175.2 \mathrm{~Hz}) \mathrm{ppm}$. MS (EI): 203 (100), $282\left(\mathrm{M}^{+}\right)$; HRMS (EI) m/z: [M+] Calcd for $\mathrm{C}_{13} \mathrm{H}_{9} \mathrm{~F}_{2} \mathrm{Br} 281.9856$; Found 281.9860. IR (KBr): $v_{\max }=3069,2924,1903,1614,1592,1488,1294,1270$, $1180,1105,771 \mathrm{~cm}^{-1}$.

HPLC (AD-H, $0.46 \times 25 \mathrm{~cm}, 5 \mu \mathrm{m}$, hexane/isopropanol = 98/2 (v/v \%), flow 0.7 $\mathrm{mL} / \mathrm{min}$, UV detection at $214 \mathrm{~nm}$ ), retention time $=7.54 \mathrm{~min}$ (minor) and $7.86 \mathrm{~min}$ (major). $[\alpha]_{\mathrm{D}}{ }^{25}=-13.64\left(\mathrm{c}=0.110, \mathrm{CHCl}_{3}, 94: 6\right.$ e.r).

(S)-1-Bromo-3-fluoro-5-(fluoro(3-fluorophenyl)methyl)benzene 3z<smiles>Fc1cccc(C(F)c2cc(F)cc(Br)c2)c1</smiles>

The product 3z (43 mg, 48\% yield, yellow liquid) was purified with silica gel chromatography (petroleum ether/ethyl acetate $=80 / 1) .{ }^{1} \mathrm{H} \mathrm{NMR}\left(400 \mathrm{MHz}, \mathrm{CDCl}_{3}\right)$ 
$\delta 7.38(\mathrm{dd}, J=13.7,7.9 \mathrm{~Hz}, 1 \mathrm{H}), 7.28$ (s, $1 \mathrm{H}), 7.23$ (dd, $J=8.0,2.0 \mathrm{~Hz}, 1 \mathrm{H}), 7.13-$ $6.96(\mathrm{~m}, 4 \mathrm{H}), 6.37$ (d, $J=46.8 \mathrm{~Hz}, 1 \mathrm{H}) ;{ }^{19} \mathrm{~F} \mathrm{NMR}\left(376 \mathrm{MHz}, \mathrm{CDCl}_{3}\right) \delta-109.46(\mathrm{t}, J$ $=8.5 \mathrm{~Hz}, 1 \mathrm{~F}),-111.62(\mathrm{td}, J=8.9,5.7 \mathrm{~Hz}, 1 \mathrm{~F}),-168.99(\mathrm{~d}, J=46.8 \mathrm{~Hz}, 1 \mathrm{~F}) ;{ }^{13} \mathrm{C}$ NMR (101 MHz, $\left.\mathrm{CDCl}_{3}\right) \delta 162.9(\mathrm{~d}, J=247.5 \mathrm{~Hz}), 162.6(\mathrm{~d}, J=252.0 \mathrm{~Hz}), 143.1$ $(\mathrm{dd}, J=22.8,7.6 \mathrm{~Hz}), 140.8(\mathrm{dd}, J=21.7,7.1 \mathrm{~Hz}), 130.4$ (d, $J=8.2 \mathrm{~Hz}), 125.2(\mathrm{dd}, J$ $=7.0,3.3 \mathrm{~Hz}), 122.9(\mathrm{~d}, J=9.6 \mathrm{~Hz}), 122.1(\mathrm{dd}, J=6.3,3.1 \mathrm{~Hz}), 119.3(\mathrm{~d}, J=24.4$ $\mathrm{Hz}), 116.0(\mathrm{dd}, J=21.1,1.9 \mathrm{~Hz}), 113.6(\mathrm{dd}, J=22.7,6.8 \mathrm{~Hz}), 112.4(\mathrm{dd}, J=22.7,7.1$

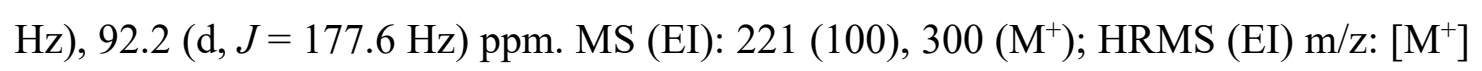

Calcd for $\mathrm{C}_{13} \mathrm{H}_{8} \mathrm{~F}_{3} \mathrm{Br}$ 299.9761; Found 299.9749. IR (KBr): $v_{\max }=3086,2926,1733$, $1606,1585,1451,1271,1253,1163,1019,859,762,709 \mathrm{~cm}^{-1}$.

HPLC (AD-H, $0.46 \times 25 \mathrm{~cm}, 5 \mu \mathrm{m}$, hexane/isopropanol = 98/2 (v/v \%), flow 0.7 $\mathrm{mL} / \mathrm{min}$, UV detection at $214 \mathrm{~nm}$ ), retention time $=12.28 \mathrm{~min}$ (minor) and $13.14 \mathrm{~min}$ (major). $[\alpha]_{\mathrm{D}}^{25}=-8.67$ (c $=0.090, \mathrm{CHCl}_{3}, 95: 5$ e.r).

(S)-2-Bromo-1-chloro-4-(fluoro(phenyl)methyl)benzene 3aa<smiles>FC(c1ccccc1)c1ccc(Cl)c(Br)c1</smiles>

The product 3aa (50 mg, 56\% yield, yellow liquid) was purified with silica gel chromatography (petroleum ether/ethyl acetate $=80 / 1) .{ }^{1} \mathrm{H} \mathrm{NMR}\left(400 \mathrm{MHz}, \mathrm{CDCl}_{3}\right)$ $\delta 7.65-7.57(\mathrm{~m}, 1 \mathrm{H}), 7.47-7.29(\mathrm{~m}, 6 \mathrm{H}), 7.24-7.18(\mathrm{~m}, 1 \mathrm{H}), 6.40(\mathrm{~d}, J=47.1$ $\mathrm{Hz}, 1 \mathrm{H}) ;{ }^{19} \mathrm{~F}$ NMR $\left(376 \mathrm{MHz}, \mathrm{CDCl}_{3}\right) \delta-167.37(\mathrm{~d}, J=47.1 \mathrm{~Hz}, 1 \mathrm{~F}) ;{ }^{13} \mathrm{C}$ NMR $(101$ $\left.\mathrm{MHz} \mathrm{CDCl}_{3}\right) \delta 140.1(\mathrm{~d}, J=22.8 \mathrm{~Hz}), 138.6(\mathrm{~d}, J=21.3 \mathrm{~Hz}), 134.4,131.6(\mathrm{~d}, J=7.1$ $\mathrm{Hz}), 130.3,128.9$ (d, $J=2.2 \mathrm{~Hz}), 128.7,126.6(\mathrm{~d}, J=6.1 \mathrm{~Hz}), 126.4(\mathrm{~d}, J=6.4 \mathrm{~Hz})$, 122.6, 93.0 (d, $J=175.1 \mathrm{~Hz})$ ppm. MS (EI): 183(100), $316\left(\mathrm{M}^{+}\right)$; HRMS (EI) m/z: $\left[\mathrm{M}^{+}\right]$Calcd for $\mathrm{C}_{13} \mathrm{H}_{9} \mathrm{FCLBr} 297.9560$; Found 297.9564. IR (KBr): $v_{\max }=3587,3064$, $1591,1466,1204,1025,842,630 \mathrm{~cm}^{-1}$. 
HPLC (OD-H, $0.46 \times 25 \mathrm{~cm}, 5 \mu \mathrm{m}$, hexane/isopropanol = 95/5 (v/v \%), flow 0.7 $\mathrm{mL} / \mathrm{min}$, UV detection at $214 \mathrm{~nm}$ ), retention time $=7.47 \mathrm{~min}$ (minor) and $8.57 \mathrm{~min}$ (major). $[\alpha]_{\mathrm{D}}{ }^{25}=-37.89\left(\mathrm{c}=0.180, \mathrm{CHCl}_{3}, 92: 8\right.$ e.r).

(R)-1-((4-Chlorophenyl)fluoromethyl)-3-fluorobenzene 3ab<smiles>Fc1cccc(C(F)c2ccc(Cl)cc2)c1</smiles>

The product 3ab (45 mg, 63\% yield, yellow liquid) was purified with silica gel chromatography (petroleum ether/ethyl acetate $=80 / 1) .{ }^{1} \mathrm{H}$ NMR $\left(400 \mathrm{MHz}, \mathrm{CDCl}_{3}\right)$ $\delta 7.39-7.28(\mathrm{~m}, 3 \mathrm{H}), 7.25(\mathrm{~d}, J=8.3 \mathrm{~Hz}, 2 \mathrm{H}), 7.07$ (d, $J=7.8 \mathrm{~Hz}, 1 \mathrm{H}), 7.03(\mathrm{~d}, J=$ $8.8 \mathrm{~Hz}, 2 \mathrm{H}), 6.40(\mathrm{~d}, J=47.1 \mathrm{~Hz}, 1 \mathrm{H}) ;{ }^{19} \mathrm{~F} \mathrm{NMR}\left(376 \mathrm{MHz}, \mathrm{CDCl}_{3}\right) \delta-112.13(\mathrm{td}, J$ $=9.0,5.8 \mathrm{~Hz}, 1 \mathrm{~F}),-167.44(\mathrm{~d}, J=47.1 \mathrm{~Hz}, 1 \mathrm{~F}) ;{ }^{13} \mathrm{C} \mathrm{NMR}\left(101 \mathrm{MHz}, \mathrm{CDCl}_{3}\right) \delta$ $162.8(\mathrm{~d}, J=247.0 \mathrm{~Hz}), 141.8(\mathrm{dd}, J=22.4,7.1 \mathrm{~Hz}), 137.6(\mathrm{~d}, J=21.8 \mathrm{~Hz}), 134.7$, $130.2(\mathrm{~d}, J=8.2 \mathrm{~Hz}), 128.8,128.0(\mathrm{~d}, J=6.2 \mathrm{~Hz}), 122.0(\mathrm{dd}, J=6.4,3.0 \mathrm{~Hz}), 115.5$ $(\mathrm{dd}, J=21.2,1.8 \mathrm{~Hz}), 113.4(\mathrm{dd}, J=22.7,7.0 \mathrm{~Hz}), 93.0(\mathrm{~d}, J=176.9 \mathrm{~Hz}) \mathrm{ppm} . \mathrm{MS}$ (EI): 203 (100), $238\left(\mathrm{M}^{+}\right)$; HRMS (EI) m/z: [M+] Calcd for $\mathrm{C}_{13} \mathrm{H}_{9} \mathrm{~F}_{2} \mathrm{Cl}$ 238.0361; Found 238.0365. IR (KBr): $v_{\max }=3070,2924,1615,1491,1449,1294,1179,1091$, $1015,961,833,789,760 \mathrm{~cm}^{-1}$.

HPLC (OD-H, $0.46 \times 25 \mathrm{~cm}, 5 \mu \mathrm{m}$, hexane/isopropanol = 98/2 (v/v \%), flow 0.7 $\mathrm{mL} / \mathrm{min}$, UV detection at $214 \mathrm{~nm}$ ), retention time $=8.66 \mathrm{~min}$ (minor) and $9.09 \mathrm{~min}$ (major). $[\alpha]_{\mathrm{D}}{ }^{25}=+9.43\left(\mathrm{c}=0.280, \mathrm{CHCl}_{3}, 94: 6\right.$ e.r).

(S)-1,2-Dichloro-4-(fluoro(3-fluorophenyl)methyl)benzene 3ac<smiles>Fc1cccc(C(F)c2ccc(Cl)c(Cl)c2)c1</smiles> 
The product 3ac (66 mg, 81\% yield, yellow liquid) was purified with silica gel chromatography (petroleum ether/ethyl acetate $=80 / 1) .{ }^{1} \mathrm{H} \mathrm{NMR}\left(400 \mathrm{MHz}, \mathrm{CDCl}_{3}\right)$ $\delta 7.48-7.41(\mathrm{~m}, 2 \mathrm{H}), 7.40-7.32(\mathrm{~m}, 1 \mathrm{H}), 7.19-7.14(\mathrm{~m}, 1 \mathrm{H}), 7.11-6.99(\mathrm{~m}, 3$ $\mathrm{H}), 6.38(\mathrm{~d}, J=46.9 \mathrm{~Hz}, 1 \mathrm{H}) ;{ }^{19} \mathrm{~F}$ NMR $\left(376 \mathrm{MHz}, \mathrm{CDCl}_{3}\right) \delta-111.71(\mathrm{td}, J=9.0,5.8$ $\mathrm{Hz}, 1 \mathrm{~F}),-168.27$ (d, $J=46.9 \mathrm{~Hz}, 1 \mathrm{~F}) ;{ }^{13} \mathrm{C} \mathrm{NMR}\left(101 \mathrm{MHz}, \mathrm{CDCl}_{3}\right) \delta 162.8(\mathrm{~d}, J=$ $247.5 \mathrm{~Hz}), 141.1$ (dd, $J=22.0,7.1 \mathrm{~Hz}), 139.3(\mathrm{~d}, J=22.4 \mathrm{~Hz}), 132.9,132.9,130.7$, $130.4(\mathrm{~d}, J=8.2 \mathrm{~Hz}), 128.5(\mathrm{~d}, J=6.9 \mathrm{~Hz}), 125.7$ (d, $J=6.3 \mathrm{~Hz}), 122.0(\mathrm{dd}, J=6.4$, $3.0 \mathrm{~Hz}), 115.9(\mathrm{dd}, J=21.2,1.8 \mathrm{~Hz}), 113.5(\mathrm{dd}, J=22.7,6.9 \mathrm{~Hz}), 92.3(\mathrm{dd}, J=176.5$, $1.9 \mathrm{~Hz})$ ppm. MS (EI): 237 (100), $272\left(\mathrm{M}^{+}\right)$; HRMS (EI) m/z: [M+] Calcd for $\mathrm{C}_{13} \mathrm{H}_{8} \mathrm{~F}_{2} \mathrm{Cl}_{2}$ 271.9971; Found 271.9976. IR (KBr): $v_{\max }=3071,2925,1615,1593$, $1489,1471,1449,1398,1271,1135,1032,915,792,760,739,687 \mathrm{~cm}^{-1}$.

HPLC (ADRH, $0.46 \times 15 \mathrm{~cm}, 5 \mu \mathrm{m}$, acetonitrile/water $=60 / 40(\mathrm{v} / \mathrm{v} \%)$, flow 0.7 $\mathrm{mL} / \mathrm{min}$, UV detection at $214 \mathrm{~nm}$ ), retention time $=7.54 \mathrm{~min}$ (minor) and $7.86 \mathrm{~min}$ (major). $[\alpha]_{\mathrm{D}}^{25}=-1.88\left(\mathrm{c}=0.160, \mathrm{CHCl}_{3}, 93: 7\right.$ e.r).

(S)-1,3-Dichloro-5-(fluoro(3-fluorophenyl)methyl)benzene 3ad

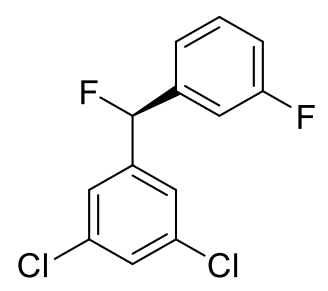

The product 3ad (62 mg, 76\% yield, yellow liquid) was purified with silica gel chromatography (petroleum ether/ethyl acetate $=80 / 1) .{ }^{1} \mathrm{H}$ NMR $\left(400 \mathrm{MHz}, \mathrm{CDCl}_{3}\right)$ $\delta 7.41-7.36(\mathrm{~m}, 1 \mathrm{H}), 7.34(\mathrm{~s}, 1 \mathrm{H}), 7.22(\mathrm{~s}, 2 \mathrm{H}), 7.13-6.99(\mathrm{~m}, 3 \mathrm{H}), 6.36(\mathrm{~d}, J=$ $46.8 \mathrm{~Hz}, 1 \mathrm{H}) ;{ }^{19} \mathrm{~F}$ NMR $\left(376 \mathrm{MHz}, \mathrm{CDCl}_{3}\right) \delta-111.62(\mathrm{td}, J=8.9,5.9 \mathrm{~Hz}, 1 \mathrm{~F})$, $-169.32(\mathrm{~d}, J=46.8 \mathrm{~Hz}, 1 \mathrm{~F}) ;{ }^{13} \mathrm{C} \mathrm{NMR}\left(101 \mathrm{MHz}, \mathrm{CDCl}_{3}\right) \delta 162.9(\mathrm{~d}, J=247.7 \mathrm{~Hz})$, $142.4(\mathrm{~d}, J=22.7 \mathrm{~Hz}), 140.7(\mathrm{dd}, J=21.8,7.2 \mathrm{~Hz}), 135.4,130.5(\mathrm{~d}, J=8.2 \mathrm{~Hz})$, $128.8(\mathrm{~d}, J=1.4 \mathrm{~Hz}), 124.8(\mathrm{~d}, J=7.0 \mathrm{~Hz}), 122.1(\mathrm{dd}, J=6.4,3.1 \mathrm{~Hz}), 116.0(\mathrm{~d}, J=$ $23.0 \mathrm{~Hz}), 113.6(\mathrm{dd}, J=22.7,6.8 \mathrm{~Hz}), 92.2$ (d, $J=177.7 \mathrm{~Hz}) \mathrm{ppm} . \mathrm{MS}(\mathrm{EI}): 237$ (100), $272\left(\mathrm{M}^{+}\right)$; HRMS (EI) m/z: [M $]$Calcd for $\mathrm{C}_{13} \mathrm{H}_{8} \mathrm{~F}_{2} \mathrm{Cl}_{2}$ 271.9971; Found 271.9961. IR (KBr): $v_{\max }=3082,2926,1940,1747,1615,1571,1450,1434,1269$, $1252,1140,1103,1018,862,761 \mathrm{~cm}^{-1}$. 
HPLC (OD-H, $0.46 \times 25 \mathrm{~cm}, 5 \mu \mathrm{m}$, hexane/isopropanol = 98/2 (v/v \%), flow 0.7 $\mathrm{mL} / \mathrm{min}$, UV detection at $214 \mathrm{~nm}$ ), retention time $=8.48 \mathrm{~min}$ (minor) and $9.04 \mathrm{~min}$ (major). $[\alpha]_{\mathrm{D}}{ }^{25}=-45.25\left(\mathrm{c}=0.160, \mathrm{CHCl}_{3}, 95: 5\right.$ e.r).

(S)-1,3-Dichloro-5-(fluoro(phenyl)methyl)benzene 3ae<smiles>FC(c1ccccc1)c1cc(Cl)cc(Cl)c1</smiles>

The product 3ae (62 mg, 81\% yield, yellow liquid) was purified with silica gel chromatography (petroleum ether/ethyl acetate $=80 / 1) .{ }^{1} \mathrm{H}$ NMR $\left(400 \mathrm{MHz}, \mathrm{CDCl}_{3}\right)$ $\delta 7.47-7.35(\mathrm{~m}, 3 \mathrm{H}), 7.35-7.30(\mathrm{~m}, 3 \mathrm{H}), 7.26-7.20(\mathrm{~m}, 2 \mathrm{H}), 6.38(\mathrm{~d}, J=47.0$ $\mathrm{Hz}, 1 \mathrm{H}) ;{ }^{19} \mathrm{~F} \mathrm{NMR}\left(376 \mathrm{MHz}, \mathrm{CDCl}_{3}\right) \delta-168.39(\mathrm{~d}, J=47.0 \mathrm{~Hz}, 1 \mathrm{~F}) ;{ }^{13} \mathrm{C} \mathrm{NMR}(101$ $\left.\mathrm{MHz}, \mathrm{CDCl}_{3}\right) \delta 143.2(\mathrm{~d}, J=23.2 \mathrm{~Hz}), 138.3(\mathrm{~d}, J=21.0 \mathrm{~Hz}), 135.2,129.1(\mathrm{~d}, J=2.3$ $\mathrm{Hz}), 128.8,128.4,126.7$ (d, $J=6.1 \mathrm{~Hz}), 124.7$ (d, $J=7.1 \mathrm{~Hz}), 93.1$ (d, $J=176.2 \mathrm{~Hz})$ ppm. MS (EI): 219 (100), $254\left(\mathrm{M}^{+}\right)$; HRMS (EI) m/z: [M+ Calcd for $\mathrm{C}_{13} \mathrm{H}_{9} \mathrm{FCl}_{2}$ 254.0065; Found 254.0057. IR (KBr): $v_{\max }=3081,2923,1958,1590,1430,1301$, $1206,1101,700 \mathrm{~cm}^{-1}$.

HPLC (OD-H, $0.46 \times 25 \mathrm{~cm}, 5 \mu \mathrm{m}$, hexane/isopropanol = 98/2 (v/v \%), flow 0.7 $\mathrm{mL} / \mathrm{min}$, UV detection at $214 \mathrm{~nm}$ ), retention time $=7.30 \mathrm{~min}$ (minor) and $8.19 \mathrm{~min}$ (major). $[\alpha]_{\mathrm{D}}{ }^{25}=-33.18\left(\mathrm{c}=0.220, \mathrm{CHCl}_{3}, 94: 6\right.$ e.r).

(S)-1,3-Dichloro-5-((4-chlorophenyl)fluoromethyl)benzene 3af<smiles>FC(c1ccc(Cl)cc1)c1cc(Cl)cc(Cl)c1</smiles>

The product 3af (68 mg, 78\% yield, yellow liquid) was purified with silica gel chromatography (petroleum ether/ethyl acetate $=80 / 1) .{ }^{1} \mathrm{H}$ NMR $\left(400 \mathrm{MHz}, \mathrm{CDCl}_{3}\right)$ $\delta 7.37$ (d, $J=8.3 \mathrm{~Hz}, 2 \mathrm{H}), 7.32$ (s, $1 \mathrm{H}), 7.24$ (d, $J=8.2 \mathrm{~Hz}, 2 \mathrm{H}), 7.19$ (s, $2 \mathrm{H}), 6.34$ $(\mathrm{d}, J=46.9 \mathrm{~Hz}, 1 \mathrm{H}) ;{ }^{19} \mathrm{~F} \mathrm{NMR}\left(376 \mathrm{MHz}, \mathrm{CDCl}_{3}\right) \delta-168.34(\mathrm{~d}, J=46.9 \mathrm{~Hz}, 1 \mathrm{~F})$; ${ }^{13} \mathrm{C}$ NMR $\left(101 \mathrm{MHz}, \mathrm{CDCl}_{3}\right) \delta 142.6(\mathrm{~d}, J=22.9 \mathrm{~Hz}), 136.8(\mathrm{~d}, J=21.5 \mathrm{~Hz}), 135.3$, 
135.1, 129.0, $128.7,128.0(\mathrm{~d}, J=6.1 \mathrm{~Hz}), 124.7(\mathrm{~d}, J=7.0 \mathrm{~Hz}), 92.4(\mathrm{~d}, J=177.1$ Hz) ppm. MS (EI): 253 (100), $288\left(\mathrm{M}^{+}\right)$; HRMS (EI) m/z: [M+ $\mathrm{M}^{+}$Calcd for $\mathrm{C}_{13} \mathrm{H}_{8} \mathrm{FCl}_{3}$ : 287.9676 Found 287.9684. IR (KBr): $v_{\max }=3082,2924,1906,1590,1570,1434$, $1310,1205,1092,797 \mathrm{~cm}^{-1}$.

HPLC (AD-H, $0.46 \times 25 \mathrm{~cm}, 5 \mu \mathrm{m}$, hexane/isopropanol = 100/0 (v/v \%), flow 0.7 $\mathrm{mL} / \mathrm{min}$, UV detection at $214 \mathrm{~nm}$ ), retention time $=15.98 \mathrm{~min}$ (minor) and $19.32 \mathrm{~min}$ (major). $[\alpha]_{\mathrm{D}}{ }^{25}=-13.40\left(\mathrm{c}=0.100, \mathrm{CHCl}_{3}, 94: 6\right.$ e.r).

\section{(S)-1,3-Dichloro-5-((3-chlorophenyl)fluoromethyl)benzene 3ag}<smiles>FC(c1cccc(Cl)c1)c1cc(Cl)cc(Cl)c1</smiles>

The product 3ag (44 mg, 51\% yield, yellow liquid) was purified with silica gel chromatography (petroleum ether/ethyl acetate $=80 / 1) .{ }^{1} \mathrm{H}$ NMR $\left(400 \mathrm{MHz}, \mathrm{CDCl}_{3}\right)$ $\delta 7.40-7.29(\mathrm{~m}, 4 \mathrm{H}), 7.25-7.16(\mathrm{~m}, 3 \mathrm{H}), 6.34(\mathrm{~d}, J=46.8 \mathrm{~Hz}, 1 \mathrm{H}) ;{ }^{19} \mathrm{~F}$ NMR $\left(376 \mathrm{MHz}, \mathrm{CDCl}_{3}\right) \delta-169.48(\mathrm{~d}, J=47.0 \mathrm{~Hz}, 1 \mathrm{~F}) ;{ }^{13} \mathrm{C} \mathrm{NMR}\left(101 \mathrm{MHz}, \mathrm{CDCl}_{3}\right) \delta$ $142.4(\mathrm{~d}, J=22.8 \mathrm{~Hz}), 140.3(\mathrm{~d}, J=21.8 \mathrm{~Hz}), 135.4,134.8,130.1,129.2(\mathrm{~d}, J=2.0$ Hz), 128.8, 126.6 (d, $J=6.7 \mathrm{~Hz}), 124.8$ (d, $J=7.0 \mathrm{~Hz}), 124.6(\mathrm{~d}, J=6.2 \mathrm{~Hz}), 92.3$ (d,

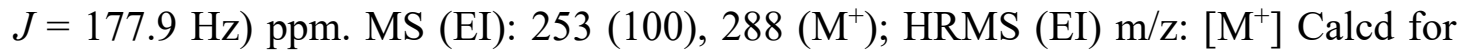
$\mathrm{C}_{13} \mathrm{H}_{8} \mathrm{FCl}_{3}$ : 287.9676; Found 287.9669. IR (KBr): $v_{\max }=3081,2925,1733,1589$, $1571,1434,1304,1195,1103,1016,800 \mathrm{~cm}^{-1}$.

HPLC (OD-H, $0.46 \times 25 \mathrm{~cm}, 5 \mu \mathrm{m}$, hexane/isopropanol = 97/3 (v/v \%), flow 0.7 $\mathrm{mL} / \mathrm{min}$, UV detection at $214 \mathrm{~nm}$ ), retention time $=6.62 \mathrm{~min}$ (minor) and $6.96 \mathrm{~min}$ (major). $[\alpha]_{\mathrm{D}}^{25}=-9.38\left(\mathrm{c}=0.160, \mathrm{CHCl}_{3}, 93: 7\right.$ e.r).

(S)-1-Chloro-2-fluoro-4-(fluoro(phenyl)methyl)benzene 3ah<smiles>Fc1cc(C(F)c2ccccc2)ccc1Cl</smiles> 
The product 3ah (38 mg, 54\% yield, yellow liquid) was purified with silica gel chromatography (petroleum ether/ethyl acetate $=80 / 1) .{ }^{1} \mathrm{H}$ NMR $\left(400 \mathrm{MHz}, \mathrm{CDCl}_{3}\right)$ $\delta 7.45-7.29(\mathrm{~m}, 6 \mathrm{H}), 7.18-7.10(\mathrm{~m}, 1 \mathrm{H}), 7.07$ (dd, $J=8.3,0.9 \mathrm{~Hz}, 1 \mathrm{H}), 6.42(\mathrm{~d}, J$ $=47.1 \mathrm{~Hz}, 1 \mathrm{H}) ;{ }^{19} \mathrm{~F}$ NMR $\left(376 \mathrm{MHz}, \mathrm{CDCl}_{3}\right) \delta-114.48(\mathrm{dd}, J=9.5,7.6 \mathrm{~Hz}, 1 \mathrm{~F})$, -167.27 (d, $J=47.1 \mathrm{~Hz}, 1 \mathrm{~F}) ;{ }^{13} \mathrm{C} \mathrm{NMR}\left(101 \mathrm{MHz}, \mathrm{CDCl}_{3}\right) \delta 158.0(\mathrm{~d}, J=249.7 \mathrm{~Hz})$, $140.8(\mathrm{dd}, J=23.0,6.2 \mathrm{~Hz}), 138.7$ (d, $J=21.1 \mathrm{~Hz}), 130.7,128.9$ (d, $J=2.2 \mathrm{~Hz})$, 128.7, $126.6(\mathrm{~d}, J=6.1 \mathrm{~Hz}), 122.7$ (dd, $J=6.6,3.8 \mathrm{~Hz}), 120.9$ (d, $J=17.7 \mathrm{~Hz}), 114.7$ (dd, $J=22.3,7.2 \mathrm{~Hz}), 93.2(\mathrm{~d}, J=173.5 \mathrm{~Hz}) \mathrm{ppm}$. MS (EI): $117(100), 238\left(\mathrm{M}^{+}\right)$; HRMS (EI) m/z: [M+] Calcd for $\mathrm{C}_{13} \mathrm{H}_{9} \mathrm{~F}_{2} \mathrm{Cl}$ 238.0361; Found 238.0370. IR (KBr): $v_{\max }=3063,3030,1889,1602,1581,1488,1421,1242,1083,815,701 \mathrm{~cm}^{-1}$.

HPLC (OJ-H, $0.46 \times 25 \mathrm{~cm}, 5 \mu \mathrm{m}$, hexane/isopropanol = 99/1 (v/v \%), flow 0.7 $\mathrm{mL} / \mathrm{min}$, UV detection at $214 \mathrm{~nm}$ ), retention time $=14.42 \mathrm{~min}$ (minor) and $15.75 \mathrm{~min}$ (major). $[\alpha]_{\mathrm{D}}^{25}=+0.67$ (c=0.150, $\mathrm{CHCl}_{3}, 93: 7$ e.r).

(S)-1-Chloro-2-fluoro-4-(fluoro(3-fluorophenyl)methyl)benzene 3ai

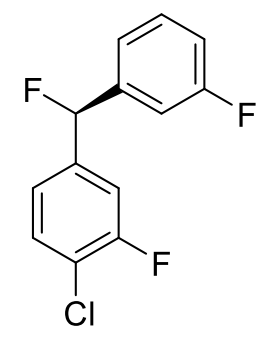

The product 3ai (49 mg, 64\% yield, yellow liquid) was purified with silica gel chromatography (petroleum ether/ethyl acetate $=80 / 1) .{ }^{1} \mathrm{H} \mathrm{NMR}\left(400 \mathrm{MHz}, \mathrm{CDCl}_{3}\right)$ $\delta 7.51-7.29(\mathrm{~m}, 2 \mathrm{H}), 7.20-6.98(\mathrm{~m}, 5 \mathrm{H}), 6.40(\mathrm{~d}, J=46.9 \mathrm{~Hz}, 1 \mathrm{H}) ;{ }^{19} \mathrm{~F} \mathrm{NMR}$ $\left(376 \mathrm{MHz}, \mathrm{CDCl}_{3}\right) \delta-111.81(\mathrm{~d}, J=5.7 \mathrm{~Hz}, 1 \mathrm{~F}),-112.97--116.81(\mathrm{~m}, 1 \mathrm{~F}),-168.12$ $(\mathrm{d}, J=46.9 \mathrm{~Hz}, 1 \mathrm{~F}) ;{ }^{13} \mathrm{C} \mathrm{NMR}\left(101 \mathrm{MHz}, \mathrm{CDCl}_{3}\right) \delta 162.8(\mathrm{~d}, J=247.5 \mathrm{~Hz}), 158.1$ (d, $J=250.1 \mathrm{~Hz}), 141.1(\mathrm{dd}, J=22.0,7.1 \mathrm{~Hz}), 140.0(\mathrm{dd}, J=22.5,6.3 \mathrm{~Hz}), 130.8,130.4$ $(\mathrm{d}, J=8.2 \mathrm{~Hz}), 122.8(\mathrm{dd}, J=6.5,3.8 \mathrm{~Hz}), 122.0(\mathrm{dd}, J=6.3,3.0 \mathrm{~Hz}), 121.4(\mathrm{~d}, J=$ $17.7 \mathrm{~Hz}), 115.8(\mathrm{dd}, J=21.2,1.8 \mathrm{~Hz}), 114.8(\mathrm{dd}, J=22.4,7.0 \mathrm{~Hz}), 113.5(\mathrm{dd}, J=$ 22.7, $6.9 \mathrm{~Hz}), 92.4$ (d, $J=176.4 \mathrm{~Hz})$ ppm. MS (EI): 221 (100), $256\left(\mathrm{M}^{+}\right)$; HRMS (EI) $\mathrm{m} / \mathrm{z}$ : $\left[\mathrm{M}^{+}\right]$Calcd for $\mathrm{C}_{13} \mathrm{H}_{8} \mathrm{~F}_{3} \mathrm{Cl}$ 256.0267; Found 256.0277. IR (KBr): $v_{\max }=3072$, $2924,1751,1593,1488,1422,1246,1063,1016,876,795,694 \mathrm{~cm}^{-1}$. 
HPLC (OD-H, $0.46 \times 25 \mathrm{~cm}, 5 \mu \mathrm{m}$, hexane/isopropanol = 100/0 (v/v \%), flow 0.7 $\mathrm{mL} / \mathrm{min}$, UV detection at $214 \mathrm{~nm}$ ), retention time $=16.71 \mathrm{~min}$ (minor) and $17.67 \mathrm{~min}$ (major). $[\alpha]_{\mathrm{D}}{ }^{25}=-6.30\left(\mathrm{c}=0.270, \mathrm{CHCl}_{3}, 96: 4\right.$ e.r). 
Procedure for the application of the cobalt-catalyzed asymmetric coupling reaction in the preparation of druglike compounds

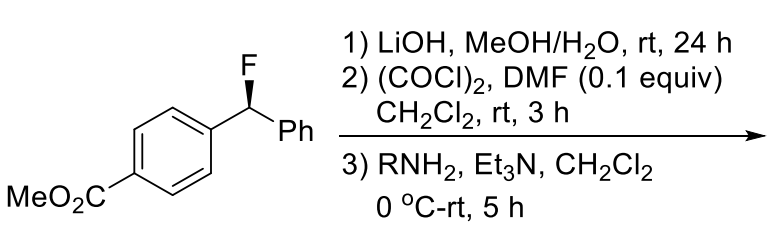

3a, $75 \%$ yield, $95: 5$ e.r<smiles>Cc1cc([N+](=O)[O-])c(CNC(=O)c2ccc(C(F)c3ccccc3)cc2)c(O)n1</smiles>

5, 71\% yield, 95:5 e.r

In a $25 \mathrm{~mL}$ flask, compound 3a (122 mg, $0.500 \mathrm{mmol})$ was dissolved in $\mathrm{MeOH}(5.0$ $\mathrm{mL})$. A solution of $\mathrm{LiOH}(18.0 \mathrm{mg}, 0.75 \mathrm{mmol})$ in $\mathrm{H}_{2} \mathrm{O}(5.0 \mathrm{~mL})$ was added. The mixture was stirred at room temperature for $24 \mathrm{~h}$ and quenched by an aqueous solution of $\mathrm{HCl}(2.0 \mathrm{~mL}, 3.0 \mathrm{~N})$. The mixture was extracted with $\mathrm{CH}_{2} \mathrm{Cl}_{2}(20 \mathrm{~mL} \times 3)$ and the organic layer was combined, dried over anhydrous $\mathrm{Na}_{2} \mathrm{SO}_{4}$, filtered and concentrated under vacuum. The crude product was used in the next step without further purification.

To a stirred solution of benzoic acid derivative obtained from previous step and DMF (5.5 $\mu \mathrm{L}, 0.10$ equiv.) in $\mathrm{CH}_{2} \mathrm{Cl}_{2}(10.0 \mathrm{~mL})$ was added oxalyl chloride (140 $\mu \mathrm{L}$, 3.00 equiv.) at $0{ }^{\circ} \mathrm{C}$. The mixture was stirred at ambient temperature for $3 \mathrm{~h}$ and extra oxalyl chloride and the solvent were removed by evaporation under vacuum. The residue was dissolved in $\mathrm{CH}_{2} \mathrm{Cl}_{2} \quad(5.0 \mathrm{~mL})$ and a solution of 3-(aminomethyl)-4,6-dimethylpyridin-2-ol (92.0 mg, 1.20 equiv.) and $\mathrm{Et}_{3} \mathrm{~N}$ (300 $\mu \mathrm{L}$, 4.00 equiv.) in $\mathrm{CH}_{2} \mathrm{Cl}_{2}(5.0 \mathrm{~mL})$ was added dropwise at $0{ }^{\circ} \mathrm{C}$. The mixture was further stirred at room temperature for $5 \mathrm{~h}$ before it was quenched by addition of water (1.0 $\mathrm{mL})$. The mixture was extracted with $\mathrm{CH}_{2} \mathrm{Cl}_{2}(20.0 \mathrm{~mL} \times 3)$ and the organic layer was dried over anhydrous $\mathrm{Na}_{2} \mathrm{SO}_{4}$, filtered and concentrated under vacuum. The residue was purified by column chromatography on silica gel with a gradient eluent of petroleum ether and ethyl acetate to give (S)-4-(fluoro(phenyl)methyl)- $N$ ((2-hydroxy-4,6-dimethylpyridin-3-yl)methyl)benzamide 5 (129 mg, 71\% yield).

(S)-4-(Fluoro(phenyl)methyl)- $N$-((2-hydroxy-4,6-dimethylpyridin-3-yl)methyl)be

\section{nzamide 5}




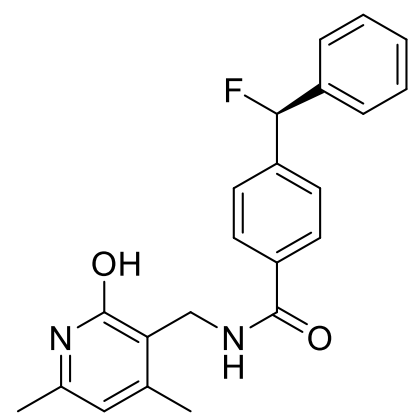

The product 5 (129 mg, 71\% yield, white solid, Mp: $162-163{ }^{\circ} \mathrm{C}$ ) was purified with silica gel chromatography (petroleum ether/ethyl acetate $=1 / 1) .{ }^{1} \mathrm{H}$ NMR $(400 \mathrm{MHz}$, $\left.\mathrm{CDCl}_{3}\right) \delta 7.94(\mathrm{~s}, 1 \mathrm{H}), 7.81(\mathrm{~s}, 2 \mathrm{H}), 7.50-7.05(\mathrm{~m}, 7 \mathrm{H}), 6.48(\mathrm{~d}, J=47.3 \mathrm{~Hz}, 1 \mathrm{H})$, $5.99(\mathrm{~s}, 1 \mathrm{H}), 4.59(\mathrm{~s}, 2 \mathrm{H}), 2.42(\mathrm{~s}, 3 \mathrm{H}), 2.28(\mathrm{~s}, 3 \mathrm{H}) ;{ }^{19} \mathrm{~F} \mathrm{NMR}\left(376 \mathrm{MHz}, \mathrm{CDCl}_{3}\right) \delta$ $-168.07(\mathrm{~d}, J=50.3 \mathrm{~Hz}, 1 \mathrm{~F}) ;{ }^{13} \mathrm{C} \mathrm{NMR}\left(101 \mathrm{MHz}, \mathrm{CDCl}_{3}\right) \delta 166.5,165.1,151.0$, $143.0(\mathrm{~d}, J=22.1 \mathrm{~Hz}), 139.2(\mathrm{~d}, J=21.2 \mathrm{~Hz}), 134.6,130.1,128.6,128.5,127.2$, $126.6(\mathrm{~d}, J=6.1 \mathrm{~Hz}), 126.3(\mathrm{~d}, J=6.6 \mathrm{~Hz}), 121.8,110.5,93.9$ (d, $J=174.1 \mathrm{~Hz}), 36.5$, 19.6, 18.6 ppm. MS (ESI): 365 (100), $365(\mathrm{M}+\mathrm{H})^{+}$; HRMS (EI) m/z: [M+] Calcd for $\mathrm{C}_{22} \mathrm{H}_{22} \mathrm{O}_{2} \mathrm{~N}_{2} \mathrm{~F}$ 365.1660; Found 365.1656. IR (KBr): $v_{\max }=3363,3254,2914,1640$, $1540,1502,1380,1208,1002,848,693,620,572 \mathrm{~cm}^{-1}$.

SFC $\left(\mathrm{OJ}-\mathrm{H}, 0.46 \times 25 \mathrm{~cm}, 5 \mu \mathrm{m}, \mathrm{CO}_{2} / \mathrm{MeOH}=92 / 8\right.$, flow $2.0 \mathrm{~mL} / \mathrm{min}$, column Temperature: $40{ }^{\circ} \mathrm{C}$, background press: $2000 \mathrm{psi}$, UV detection at $214 \mathrm{~nm}$ ), retention time $=30.03 \min$ (major) and $31.68 \min ($ minor $) .[\alpha]_{\mathrm{D}}{ }^{25}=-13.33\left(\mathrm{c}=0.120, \mathrm{CHCl}_{3}\right.$, 94:6 e.r).<smiles>COC(=O)c1ccc(C(F)c2ccccc2)cc1</smiles>

3a, 95:5 e.r.
1) $\mathrm{LiOH}, \mathrm{MeOH} / \mathrm{H}_{2} \mathrm{O}$, rt, $24 \mathrm{~h}$

2) $(\mathrm{COCl})_{2}$, DMF (0.1 equiv.) $\mathrm{CH}_{2} \mathrm{Cl}_{2}, \mathrm{rt}, 3 \mathrm{~h}$

3) $\mathrm{R}_{2} \mathrm{NH}, \mathrm{Et}_{3} \mathrm{~N}, \mathrm{CH}_{2} \mathrm{Cl}_{2}$ $0^{\circ} \mathrm{C}-\mathrm{rt}, 5 \mathrm{~h}$

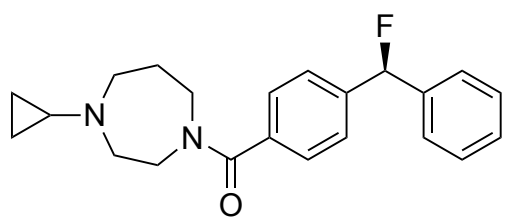

Fluorinated mimic of histamine $\mathrm{H} 3$ receptor 6 $84 \%$ yield, $95: 5$ e.r.

In a $25 \mathrm{~mL}$ flask, compound 3a (122 mg $0.500 \mathrm{mmol})$ was dissolved in $\mathrm{MeOH}(5.0$ $\mathrm{mL})$. A solution of $\mathrm{LiOH}(18.0 \mathrm{mg}, 0.75 \mathrm{mmol})$ in $\mathrm{H}_{2} \mathrm{O}(5.0 \mathrm{~mL})$ was added. The mixture was stirred at room temperature for $24 \mathrm{~h}$ and quenched by an aqueous solution of $\mathrm{HCl}(2.0 \mathrm{~mL}, 3.0 \mathrm{~N})$. The mixture was extracted with $\mathrm{CH}_{2} \mathrm{Cl}_{2}(20.0 \mathrm{~mL} \times$ 3) and the organic layer was combined, dried over anhydrous $\mathrm{Na}_{2} \mathrm{SO}_{4}$ and 
concentrated under vacuum. The crude product was used in the next step without further purification.

To a stirred solution of benzoic acid derivative obtained from previous step and DMF (5.5 $\mu \mathrm{L}, 0.10$ equiv.) in $\mathrm{CH}_{2} \mathrm{Cl}_{2}(10.0 \mathrm{~mL})$ was added oxalyl chloride (140 $\mu \mathrm{L}$, 3.00 equiv.) at $0{ }^{\circ} \mathrm{C}$. The mixture was stirred at ambient temperature for $3 \mathrm{~h}$ and extra oxalyl chloride and the solvent were removed by evaporation under vacuum. The residue was dissolved in $\mathrm{CH}_{2} \mathrm{Cl}_{2} \quad\left(\begin{array}{lll}5.0 & \mathrm{~mL}\end{array}\right)$ and a solution of 1-cyclopropyl-1,4-diazepane (128.0 mg, 1.20 equiv.) and $\mathrm{Et}_{3} \mathrm{~N}$ (300 $\mu \mathrm{L}, 4.00$ equiv.) in $\mathrm{CH}_{2} \mathrm{Cl}_{2}(5.0 \mathrm{~mL})$ was added dropwise at $0{ }^{\circ} \mathrm{C}$. The mixture was further stirred at room temperature for $5 \mathrm{~h}$ before it was quenched by adding water $(1.0 \mathrm{~mL})$. The mixture was extracted with $\mathrm{CH}_{2} \mathrm{Cl}_{2}(20 \mathrm{~mL} \times 3)$ and the organic layer was dried over anhydrous $\mathrm{Na}_{2} \mathrm{SO}_{4}$, filtered and concentrated under vacuum. The residue was purified by column chromatography on silica gel with a gradient eluent of petroleum ether and ethyl acetate to give (S)-(4-Cyclopropyl-1,4-diazepan-1-yl)(4-(fluoro(phenyl)methyl) phenyl)methanone 6.

\section{(S)-(4-Cyclopropyl-1,4-diazepan-1-yl)(4-(fluoro(phenyl)methyl)phenyl)methanon} e 6

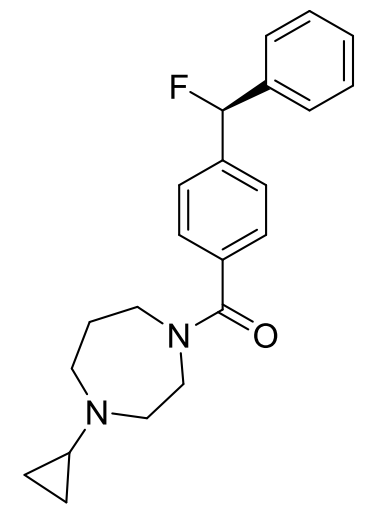

The product 6 (147 $\mathrm{mg}, 84 \%$ yield, yellow liquid) was purified with silica gel chromatography (petroleum ether/ethyl acetate $=80 / 1) .{ }^{1} \mathrm{H}$ NMR $\left(400 \mathrm{MHz}, \mathrm{CDCl}_{3}\right)$ $\delta 7.76-7.26(\mathrm{~m}, 9 \mathrm{H}), 6.48(\mathrm{~d}, J=47.3 \mathrm{~Hz}, 1 \mathrm{H}), 3.94-3.54(\mathrm{~m}, 2 \mathrm{H}), 3.46(\mathrm{~d}, J=$ $5.9 \mathrm{~Hz}, 2 \mathrm{H}), 3.06$ (s, $1 \mathrm{H}), 2.89$ (s, $2 \mathrm{H}), 2.79$ (s, $1 \mathrm{H}), 2.07$ - 1.72 (m, $3 \mathrm{H}), 0.65$ (s, 1 $\mathrm{H}), 0.68-0.30(\mathrm{~m}, 3 \mathrm{H}) ;{ }^{19} \mathrm{~F}$ NMR $\left(376 \mathrm{MHz}, \mathrm{CDCl}_{3}\right) \delta-167.56(\mathrm{~d}, J=48.1 \mathrm{~Hz}, 1 \mathrm{~F})$; ${ }^{13} \mathrm{C} \mathrm{NMR}\left(101 \mathrm{MHz}, \mathrm{CDCl}_{3}\right) \delta 171.1,141.1(\mathrm{~d}, J=27.4 \mathrm{~Hz}), 139.2(\mathrm{~d}, J=20.4 \mathrm{~Hz})$, 
128.6, 128.5, 126.9, 126.8, 126.6, 126.6, $94.0(\mathrm{~d}, J=171.6 \mathrm{~Hz}), 56.7,55.8,55.1,54.5$, 48.4, 45.4, 7.1 ppm. MS (EI): 149 (100), $352\left(\mathrm{M}^{+}\right)$; HRMS (EI) m/z: [M+] Calcd for $\mathrm{C}_{22} \mathrm{H}_{25} \mathrm{~N}_{2} \mathrm{OF}$ 352.1951; Found 352.1942. IR (KBr): $v_{\max }=3447,3032,2816,1719$, $1628,1495,1424,1191,1017,996,873 \mathrm{~cm}^{-1}$.

HPLC (AD-H, $0.46 \times 25 \mathrm{~cm}, 5 \mu \mathrm{m}$, hexane/isopropanol = 90/10 (v/v \%), flow 0.7 $\mathrm{mL} / \mathrm{min}$, UV detection at $214 \mathrm{~nm}$ ), retention time $=32.17 \mathrm{~min}$ (minor) and $33.68 \mathrm{~min}$ (major). $[\alpha]_{\mathrm{D}}^{25}=-6.78\left(\mathrm{c}=0.180, \mathrm{CHCl}_{3}, 95: 5\right.$ e.r).

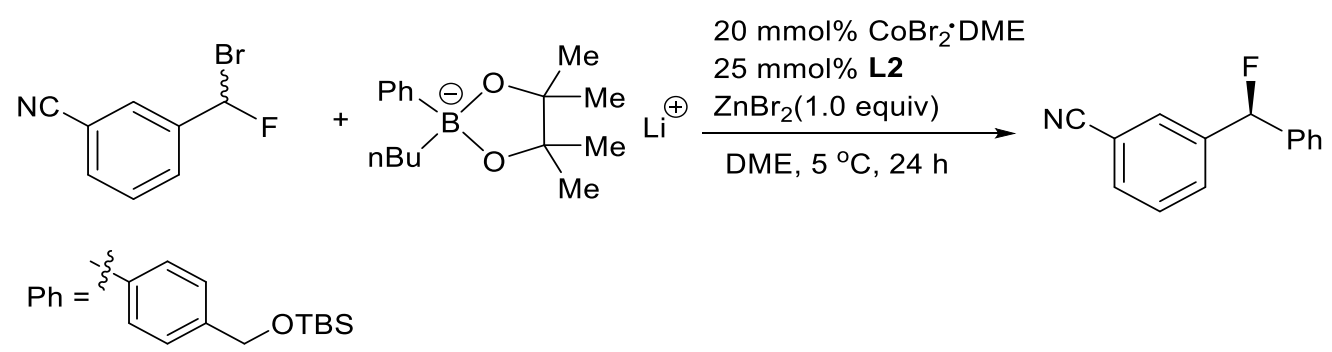

In a glove box, tert-butyldimethyl((4-(4,4,5,5-tetramethyl-1,3,2-dioxaborolan-2-yl) benzyl)oxy)silane (1.74 g, $5.00 \mathrm{mmol})$ was weighted into a $100 \mathrm{~mL}$ Schlenk tube, and $20.0 \mathrm{~mL}$ of dry THF was added. The mixture was taken out from the glove box and cooled at $-20{ }^{\circ} \mathrm{C} . n$-BuLi $(2.0 \mathrm{~mL}, 2.5 \mathrm{M}$ in Hexanes, $5.0 \mathrm{mmol})$ was added. The mixture was stirred at $-20{ }^{\circ} \mathrm{C}$ for $2 \mathrm{~h}$. Then the Schlenk tube was taken into the glove box, the solvents were removed by oil pump to give lithium phenyl $n$-butyl pinacol boronate.

In an argon-filled glove box, lithium organoboronate $(500 \mathrm{mg}, 0.900 \mathrm{mmol}, 3.00$ equiv.), ligand $\mathbf{L 8}$ (36.5 mg, $0.0750 \mathrm{mmol}, 0.250$ equiv.), $\mathrm{ZnBr}_{2}(67.5 \mathrm{mg}, 0.300 \mathrm{mmol}$, 1.00 equiv.) and $\mathrm{CoBr}_{2} \cdot \mathrm{DME}(18.5 \mathrm{mg}, 0.0600 \mathrm{mmol}, 0.200$ equiv.) were placed into a 25 $\mathrm{mL}$ Schlenk tube. To this vial was added $3.0 \mathrm{~mL}$ of anhydrous DME. The Schlenk tube was taken out from the glove box and cooled at $5{ }^{\circ} \mathrm{C}$. 3-(Bromofluoromethyl)benzonitrile 1d $(63.9 \mathrm{mg}, 0.300 \mathrm{mmol})$ was added and the mixture was stirred at $5{ }^{\circ} \mathrm{C}$ for $24 \mathrm{~h}$. The mixture was quenched by addition of water $(5.0$ $\mathrm{mL})$ and extracted with $\mathrm{Et}_{2} \mathrm{O}(10.0 \mathrm{~mL} \times 3)$. The organic layer was combined, dried over anhydrous $\mathrm{Na}_{2} \mathrm{SO}_{4}$, filtered and concentrated under vacuum. The crude product was purified by column chromatography on silica gel with pentane/ethyl acetate as the eluent 
to give (S)-3-((4-(((tert-butyldimethylsilyl)oxy)methyl)phenyl)fluoromethyl) benzonitrile 7 .

\section{(S)-3-((4-(((tert-Butyldimethylsilyl)oxy)methyl)phenyl)fluoromethyl)benzonitrile} 7<smiles>N#Cc1cccc(C(F)c2ccc(CO[Sb])cc2)c1</smiles>

The product 7 (57 mg, 54\% yield, yellow liquid) was purified with silica gel chromatography (petroleum ether/ethyl acetate $=80 / 1) .{ }^{1} \mathrm{H}$ NMR $\left(400 \mathrm{MHz}, \mathrm{CDCl}_{3}\right)$ $\delta 7.65-7.54(\mathrm{~m}, 3 \mathrm{H}), 7.47$ (t, $J=7.6 \mathrm{~Hz}, 1 \mathrm{H}), 7.36(\mathrm{~d}, J=7.7 \mathrm{~Hz}, 2 \mathrm{H}), 7.27$ (d, $J=$ $7.7 \mathrm{~Hz}, 2 \mathrm{H}), 6.46(\mathrm{~d}, J=47.1 \mathrm{~Hz}, 1 \mathrm{H}), 4.75(\mathrm{~s}, 2 \mathrm{H}), 0.94(\mathrm{~s}, 9 \mathrm{H}), 0.10(\mathrm{~s}, 6 \mathrm{H}) ;{ }^{19} \mathrm{~F}$ NMR $\left(376 \mathrm{MHz} \mathrm{CDCl}_{3}\right) \delta-167.89(\mathrm{~d}, J=47.0 \mathrm{~Hz}, 1 \mathrm{~F}) ;{ }^{13} \mathrm{C} \mathrm{NMR}\left(101 \mathrm{MHz}, \mathrm{CDCl}_{3}\right)$ $\delta 137.9,136.8(\mathrm{~d}, J=23.4 \mathrm{~Hz}), 132.1(\mathrm{~d}, J=21.1 \mathrm{~Hz}), 127.1,125.8(\mathrm{~d}, J=6.4 \mathrm{~Hz})$, $125.0(\mathrm{~d}, J=7.1 \mathrm{~Hz}), 124.5,121.9(\mathrm{~d}, J=5.9 \mathrm{~Hz}), 121.5,113.7,107.9,88.4$ (d, $J=$ 175.1 Hz), 59.7, 21.1, 13.6, -10.0 ppm. MS (EI): 191 (100), $355\left(\mathrm{M}^{+}\right)$; HRMS (EI) $\mathrm{m} / \mathrm{z}$ : $\left[\mathrm{M}^{+}\right]$Calcd for $\mathrm{C}_{21} \mathrm{H}_{26} \mathrm{NOFSi} 355.1768$; Found 355.1758. IR $(\mathrm{KBr}): v_{\max }=3455$, $2960,2231,1615,1416,1361,1260,1092,1022,800,689 \mathrm{~cm}^{-1}$.

HPLC (IG, $0.46 \times 25 \mathrm{~cm}, 5 \mu \mathrm{m}$, hexane/isopropanol = 95/5 (v/v \%), flow $0.7 \mathrm{~mL} / \mathrm{min}$, $\mathrm{UV}$ detection at $214 \mathrm{~nm}$ ), retention time $=7.52 \mathrm{~min}$ (minor) and $7.94 \mathrm{~min}$ (major). $[\alpha]_{\mathrm{D}}^{25}=-33.44\left(\mathrm{c}=0.180, \mathrm{CHCl}_{3}, 92: 8\right.$ e.r $)$. 
Mechanistic study for trapping the benzylic radical by TEMPO<smiles>CCCC[B-]1([O-])OC(C)(C)C(C)(C)O1</smiles>

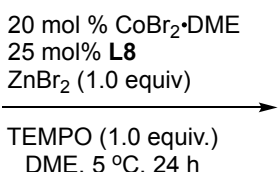<smiles>COC(=O)c1ccc([C@@H](F)ON2C3(C)CCCC2(C)CC3)cc1</smiles>

${ }^{19}$ F NMR: $-104.8 \mathrm{ppm}$ MS (ESI): 324.2

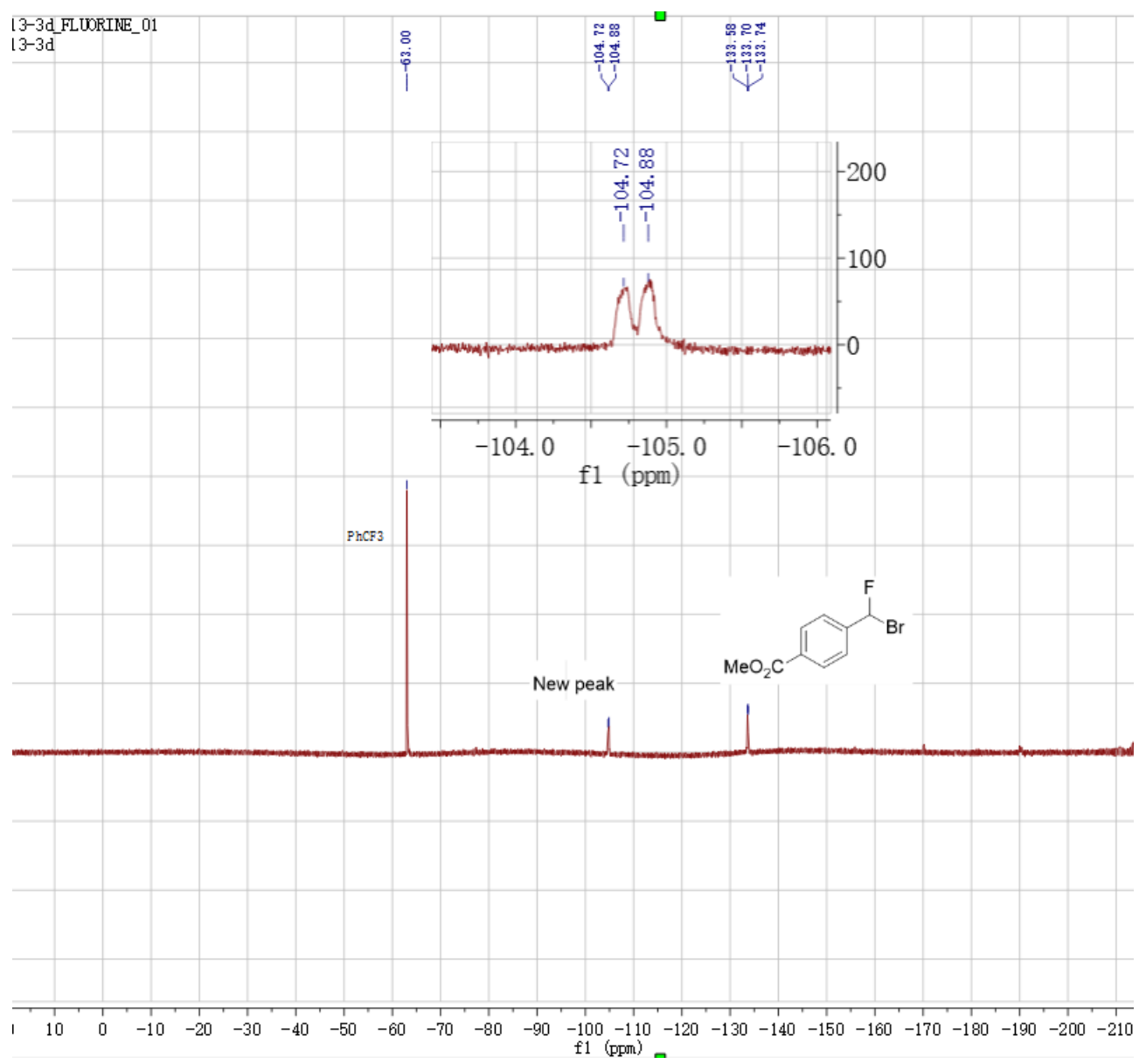

Figure S1. ${ }^{19} \mathrm{~F}$ NMR spectroscopy of cobalt-catalyzed asymmetric coupling of 4-(bromofluoromethyl)benzoate 1a with lithium phenyl $n$-butyl pinacol boronate in the presence of 1.0 equivalent of TEMPO. 


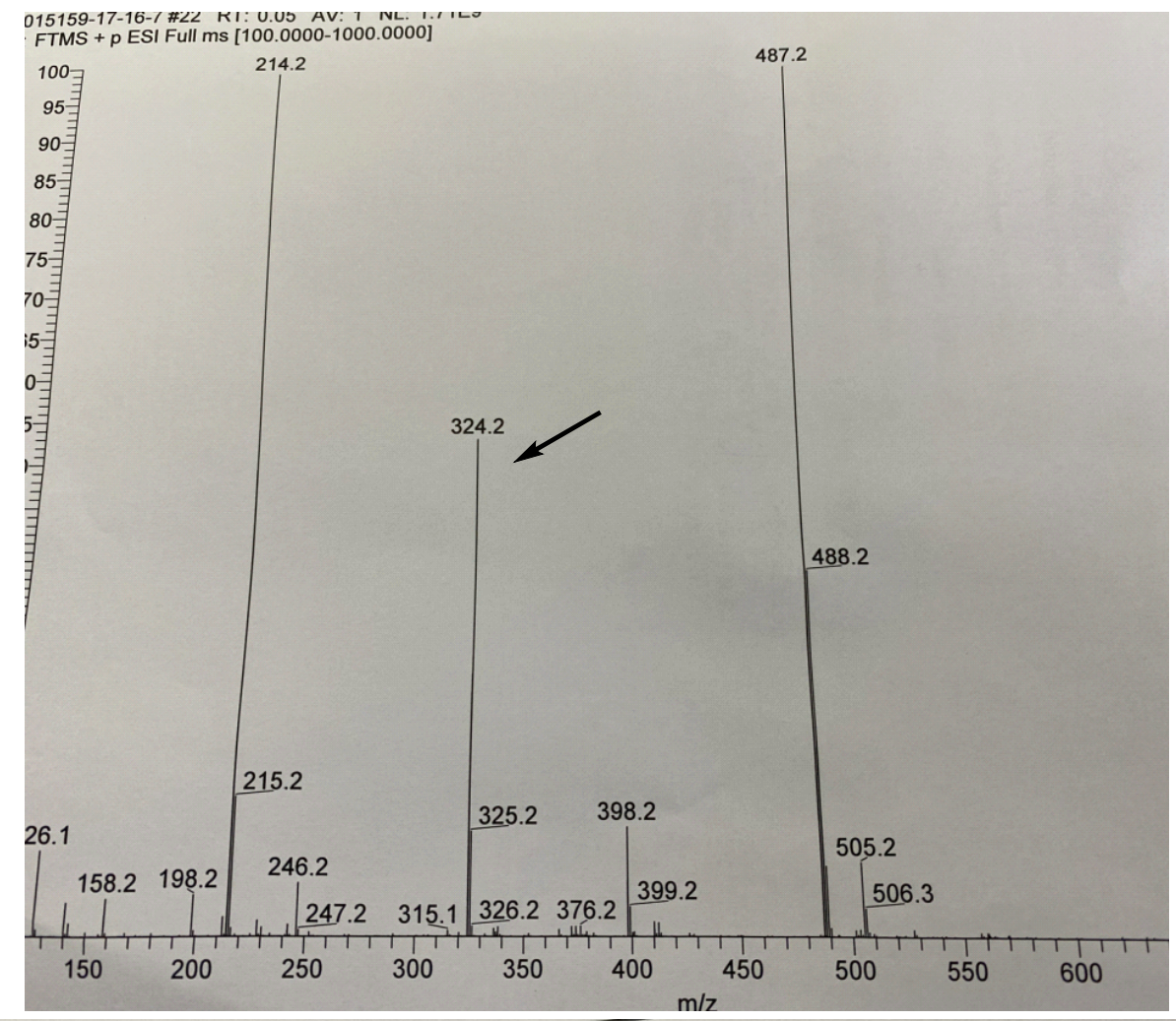

Instrument: Thermo Scientific Q Exactive HF Orbitrap-FTMS

Card Serial Number: H190836

Sample Serial Number: 2015159-17-16-7

Operator: QHL

Operation Mode: ESI Positive Ion Mode

Elemental composition search on mass 324.20

\begin{tabular}{|c|c|c|c|c|}
\hline $\begin{array}{c}m / z=319 . \\
m / z\end{array}$ & $\begin{array}{c}20-329.20 \\
\text { Theo. }\end{array}$ & $\begin{array}{l}\text { Delta } \\
\text { (ppm) }\end{array}$ & $\begin{array}{l}\text { RDB } \\
\text { equiv. }\end{array}$ & Compositior \\
\hline 324. & 324.1969 & -1.66 & 5.5 & $\mathrm{C}_{18} \mathrm{H}_{27} \mathrm{O}_{3} \mathrm{~N} \mathrm{~F}$ \\
\hline & 324.1958 & 1.86 & 9.5 & $\mathrm{C}_{21} \mathrm{H}_{26} \mathrm{O}_{2} \mathrm{~N}$ \\
\hline
\end{tabular}

Figure S2. MS (ESI) (top) and HRMS (ESI) (down) spectroscopies of TEMPO-R (R =1-fluoro-(4-methoxycarbonylbenzyl)). 


\section{HPLC analysis of Compounds 3a-3ai}<smiles>CCOC(=O)c1ccc(C(F)c2ccccc2)cc1</smiles>

Compound 3a. HPLC (IG, $0.46 \times 25 \mathrm{~cm}, 5 \mu \mathrm{m}$, hexane/isopropanol $=97 / 3(\mathrm{v} / \mathrm{v} \%)$, flow $0.7 \mathrm{~mL} / \mathrm{min}$, UV detection at $214 \mathrm{~nm}$ ), retention time $=15.84 \min$ (minor) and $16.46 \min$ (major). $[\alpha]_{\mathrm{D}}{ }^{25}=+23.91\left(\mathrm{c}=0.230, \mathrm{CHCl}_{3}, 95: 5\right.$ e.r $)$.

\section{HWC-15-24-3 IG 9732140.7}

\begin{tabular}{|llll|}
\hline Sample Name: & HWC-15-24-3 IG 973 214 0.7 & Injection Volume: & $\mathbf{2 . 0}$ \\
Vial Number: & RC7 & Channel: & UV_VIS_2 \\
Sample Type: & unknown & Wavelength: & $\mathbf{2 1 4 . 0}$ \\
Control Program: & test-dad6 & Bandwidth: & $\mathbf{4}$ \\
Quantif. Method: & $\mathbf{2 0 1 7 0 6 0 8}$ & Dilution Factor: & $\mathbf{1 . 0 0 0 0}$ \\
Recording Time: & $\mathbf{2 0 1 9 - 5 - 3 0 1 2 : 4 1}$ & Sample Weight: & $\mathbf{1 . 0 0 0 0}$ \\
Run Time (min): & $\mathbf{3 0 . 0 0}$ & Sample Amount: & $\mathbf{1 . 0 0 0 0}$ \\
\hline
\end{tabular}

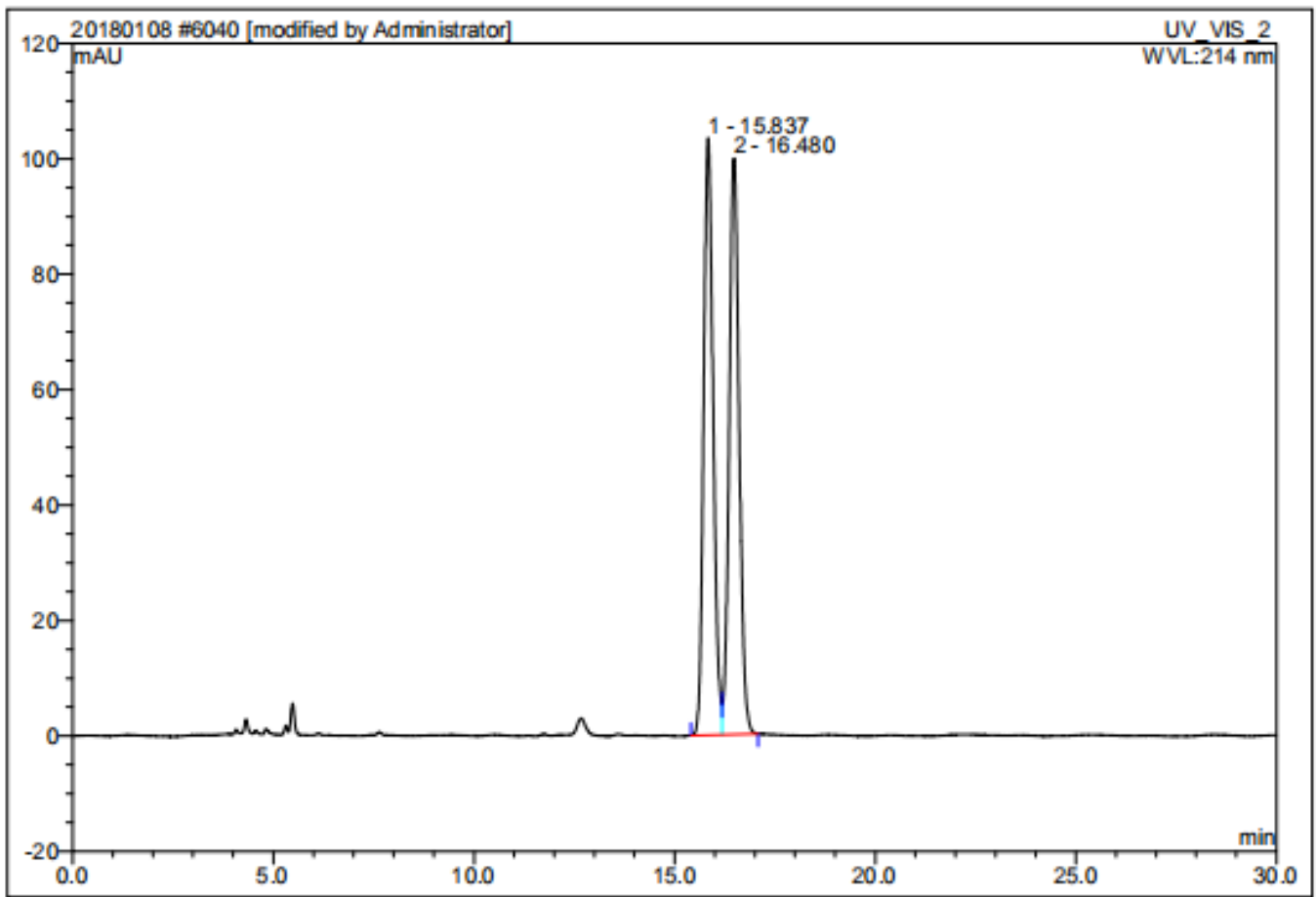

\begin{tabular}{|r|ccrrrrr|}
\hline No. & $\begin{array}{c}\text { Ret.Time } \\
\text { min }\end{array}$ & Peak Name & $\begin{array}{c}\text { Height } \\
\text { mAU }\end{array}$ & $\begin{array}{c}\text { Area } \\
\text { mAU*min }\end{array}$ & $\begin{array}{c}\text { Rel.Area } \\
\%\end{array}$ & Amount & Type \\
\hline 1 & 15.84 & n.a. & 103.529 & 28.547 & 49.85 & n.a. & BM \\
2 & 16.48 & n.a. & 99.846 & 28.724 & 50.15 & n.a. & MB \\
\hline Total: & & & 203.375 & 57.271 & 100.00 & 0.000 & \\
\hline
\end{tabular}


6041 HWC-15-25-3A IG 9732140.7

Sample Name: $\quad$ HWC-15-25-3A IG 9732140.7

Vial Number: $\quad$ RC8

Sample Type:

Contral Program:

Quantif. Method.

Recording Time:

Run Time (min): unknown

test-dad6

20170608

2019-5-30 13: 16

30.00 injection Volume:

Channel:

Wavelength:

Bandwidth:

Dĩution Factor:

Sample Weight:

Sample Amount:
2.0 UV_VIS_2 214.0 4 1.0000 1.0000 1.0000

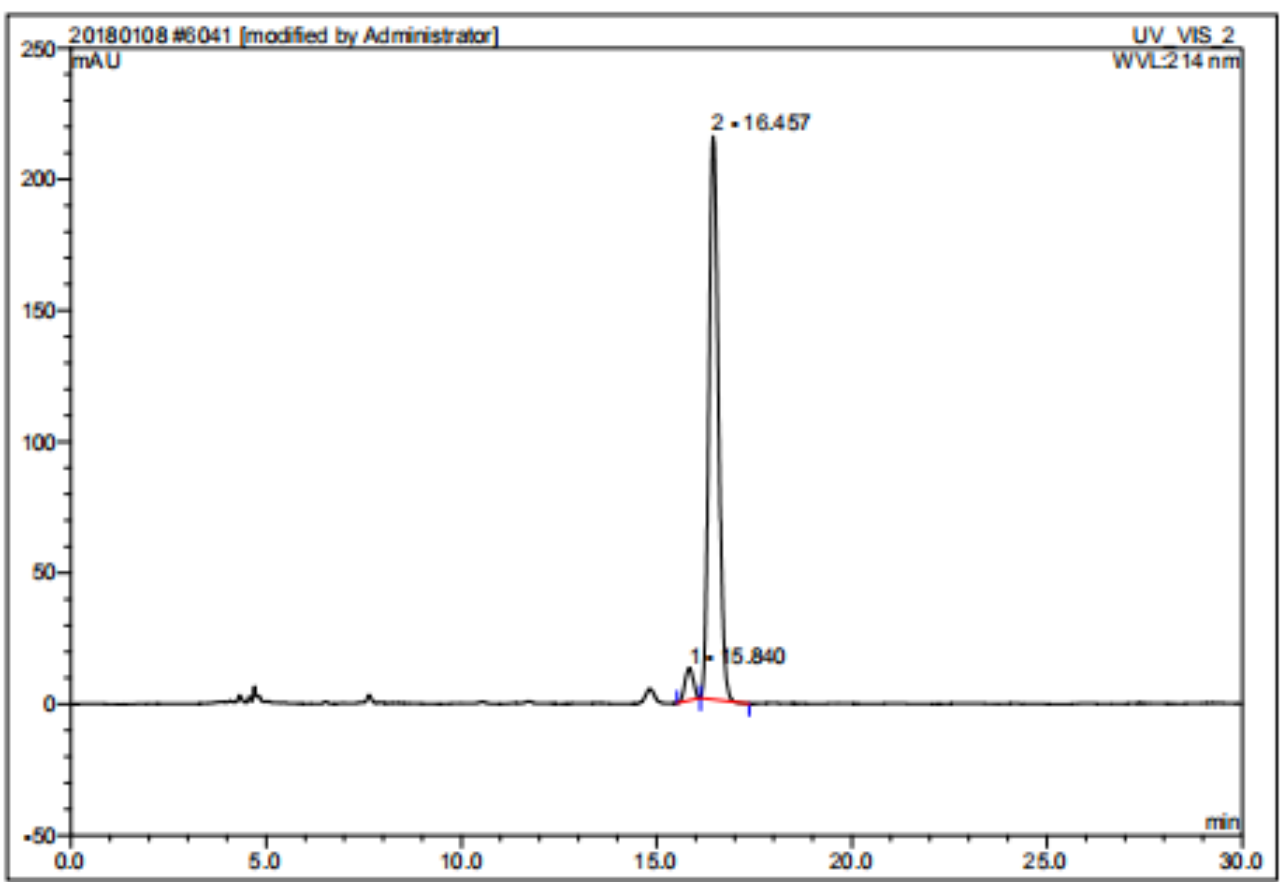

\begin{tabular}{|c|c|c|c|c|c|c|c|}
\hline No. & $\begin{array}{c}\text { Ret. Time } \\
\text { min }\end{array}$ & Peak Name & $\begin{array}{c}\text { Height } \\
\text { mAU }\end{array}$ & $\begin{array}{c}\text { Area } \\
\text { mAU*min }\end{array}$ & $\begin{array}{c}\text { Rel.Area } \\
\%\end{array}$ & Amount & Type \\
\hline 1 & 15.84 & n.a. & 12.561 & 3.108 & 4.86 & na. & BMb* \\
\hline 2 & 16.46 & n.a. & 214.789 & 60.887 & 95.14 & n.a. & $\mathrm{bMB}^{*}$ \\
\hline Total: & & & 227.350 & 63.995 & 100.00 & 0.000 & \\
\hline
\end{tabular}




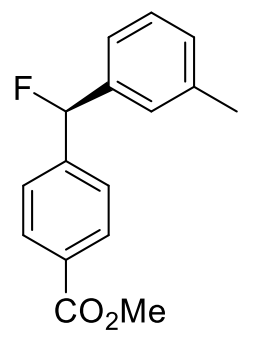

Compound 3b. HPLC (IE3, $0.46 \times 25 \mathrm{~cm}, 5 \mu \mathrm{m}$, hexane/isopropanol = 98/2 (v/v \%), flow $0.7 \mathrm{~mL} / \mathrm{min}$, UV detection at $214 \mathrm{~nm}$ ), retention time $=13.62 \mathrm{~min}$ (minor) and $16.54 \min$ (major). $[\alpha]_{\mathrm{D}}^{25}=-27.83\left(\mathrm{c}=0.230, \mathrm{CHCl}_{3}, 96: 4\right.$ e.r).

\begin{tabular}{|llll|}
\hline 5762 HWC-14-73-3A+- IE3 9822140.7 & & \\
& & & \\
\hline Sample Name: & HWC-14-73-3A+- IE3 982 214 0.7 & njection Volume: & 3.0 \\
Vial Number: & RA6 & Channel: & UV_VIS_2 \\
Sample Type: & unknown & Wavelength: & 254.0 \\
Cantral Program: & test-dad4 & Bandwidth: & 4 \\
Quantif. Method: & 20170608 & Dilutian Factor: & 1.0000 \\
Recording Time: & $2019-5-511: 12$ & Sample Weight: & 1.0000 \\
Run Time (min): & 25.00 & Sample Amount: & 1.0000 \\
\hline
\end{tabular}

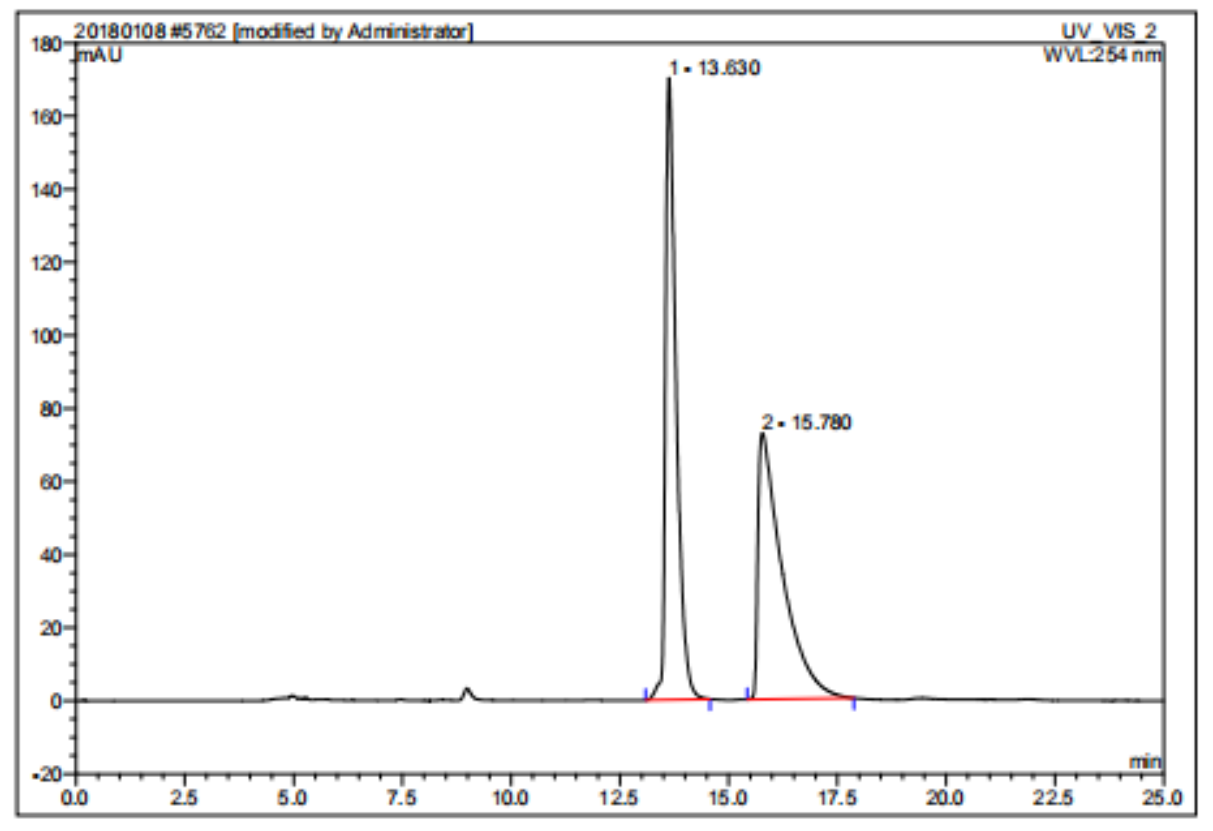

\begin{tabular}{|r|ccrrrrr|}
\hline No. & $\begin{array}{c}\text { Ret. Time } \\
\text { min }\end{array}$ & Peak Name & $\begin{array}{c}\text { Height } \\
\text { mAU }\end{array}$ & $\begin{array}{c}\text { Area } \\
\text { mAU*min }\end{array}$ & $\begin{array}{r}\text { Rel.Area } \\
\%\end{array}$ & Amount & Type \\
\hline 1 & 13.63 & n.a. & 170.212 & 48.524 & 50.61 & n.a. & BMB \\
2 & 15.78 & n.a. & 72.802 & 47.350 & 49.39 & n.a. & BMB \\
\hline Total: & & & 243.013 & 95.874 & 100.00 & 0.000 & \\
\hline
\end{tabular}




\begin{tabular}{|llll|}
\hline 5761 HWC-14-72-3A IE3 9822140.7 & & \\
& & & \\
\hline Sample Name: & HWC-14-72-3A IE3 9822140.7 & hjection Volume: & 3.0 \\
Vial Number: & RB6 & Channel: & UV_VIS_2 \\
Sample Type: & unknown & Wavelength: & 254.0 \\
Cantrol Program: & test-dad4 & Bandwidth: & 4 \\
Quantif. Method: & 20170608 & Dilutian Factor: & 1.0000 \\
Recording Time: & $2019-5-510: 45$ & Sample Weight: & 1.0000 \\
Run Time (min): & 25.00 & Sample Amount: & 1.0000 \\
\hline
\end{tabular}

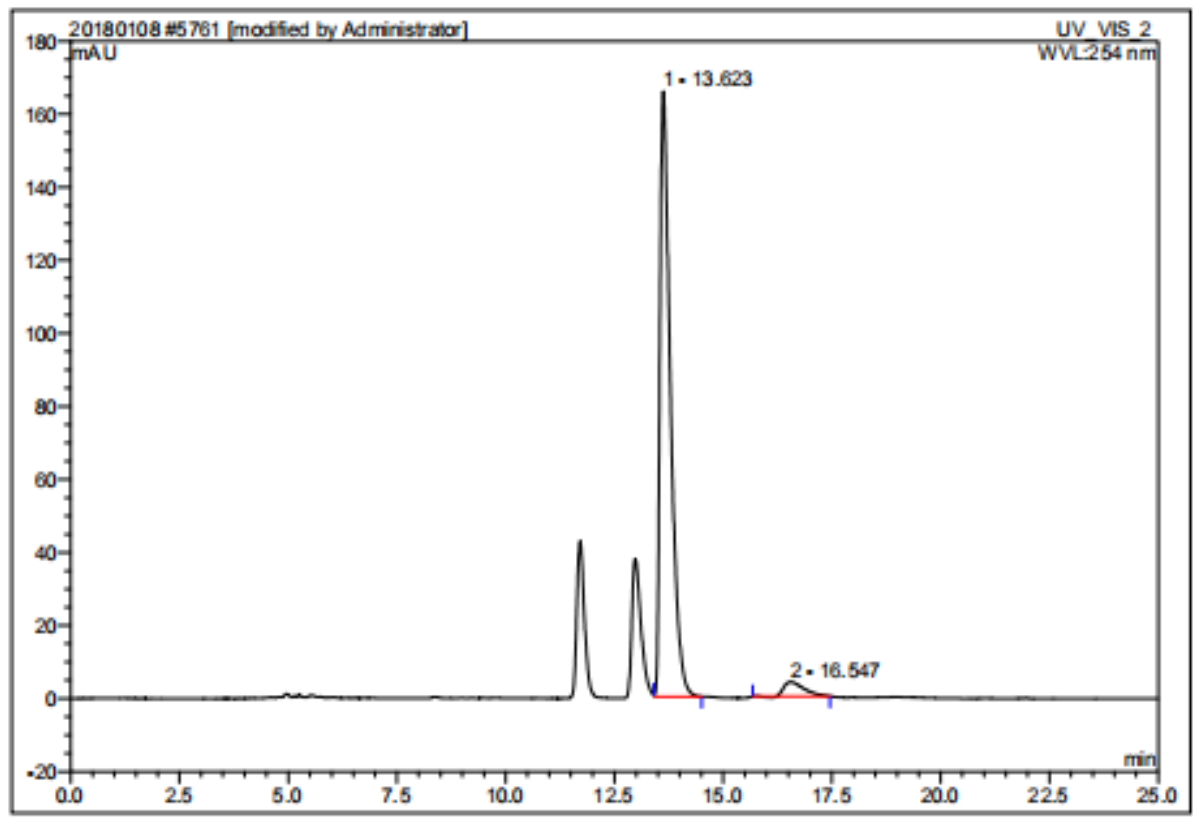

\begin{tabular}{|r|ccrrrrr|}
\hline No. & $\begin{array}{c}\text { Ret. Time } \\
\text { min }\end{array}$ & Peak Name & $\begin{array}{c}\text { Height } \\
\text { mAU }\end{array}$ & $\begin{array}{c}\text { Area } \\
\text { mAU*min }\end{array}$ & $\begin{array}{r}\text { Rel.Area } \\
\%\end{array}$ & Amount & Type \\
\hline 1 & 13.62 & n.a. & 165.828 & 46.297 & 95.68 & n.a. & MB $^{*}$ \\
2 & 16.55 & n.a. & 3.951 & 2.091 & 4.32 & n.a. & BMB* $^{*}$ \\
\hline Total: & & & 169.779 & 48.388 & 100.00 & 0.000 & \\
\hline
\end{tabular}




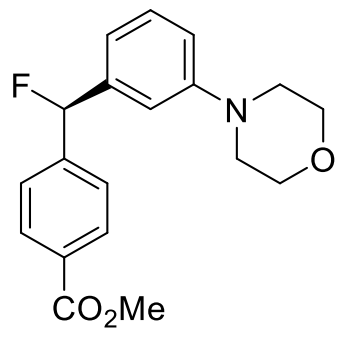

Compound 3c. HPLC (AD-H, $0.46 \times 25 \mathrm{~cm}, 5 \mu \mathrm{m}$, hexane/isopropanol = 95/5 (v/v \%), flow $0.7 \mathrm{~mL} / \mathrm{min}$, UV detection at $214 \mathrm{~nm}$ ), retention time $=29.92 \mathrm{~min}$ (minor) and $34.85 \min$ (major). $[\alpha]_{\mathrm{D}}{ }^{25}=+4.00\left(\mathrm{c}=0.150, \mathrm{CHCl}_{3}, 92: 8\right.$ e.r).

\begin{tabular}{|llll|}
\hline 5879 HWC-14-94-3B+- ADH 9552140.7 & & \\
& & & \\
\hline Sample Name: & HWC-14-94-3B+- ADH 9552140.7 & mjection Volume: & 3.0 \\
Vial Number: & RE7 & Channel: & UV_VIS_2 \\
Sample Type: & unknown & Wavelength: & 214.0 \\
Control Program: & test-dad6 & Bandwidth: & 4 \\
Quantif. Method: & 20170608 & Dïution Factor: & 1.0000 \\
Recording Time: & $2019-5-139: 45$ & Sample Weight: & 1.0000 \\
Run Time (min): & 46.04 & Sample Amount: & 1.0000 \\
\hline
\end{tabular}

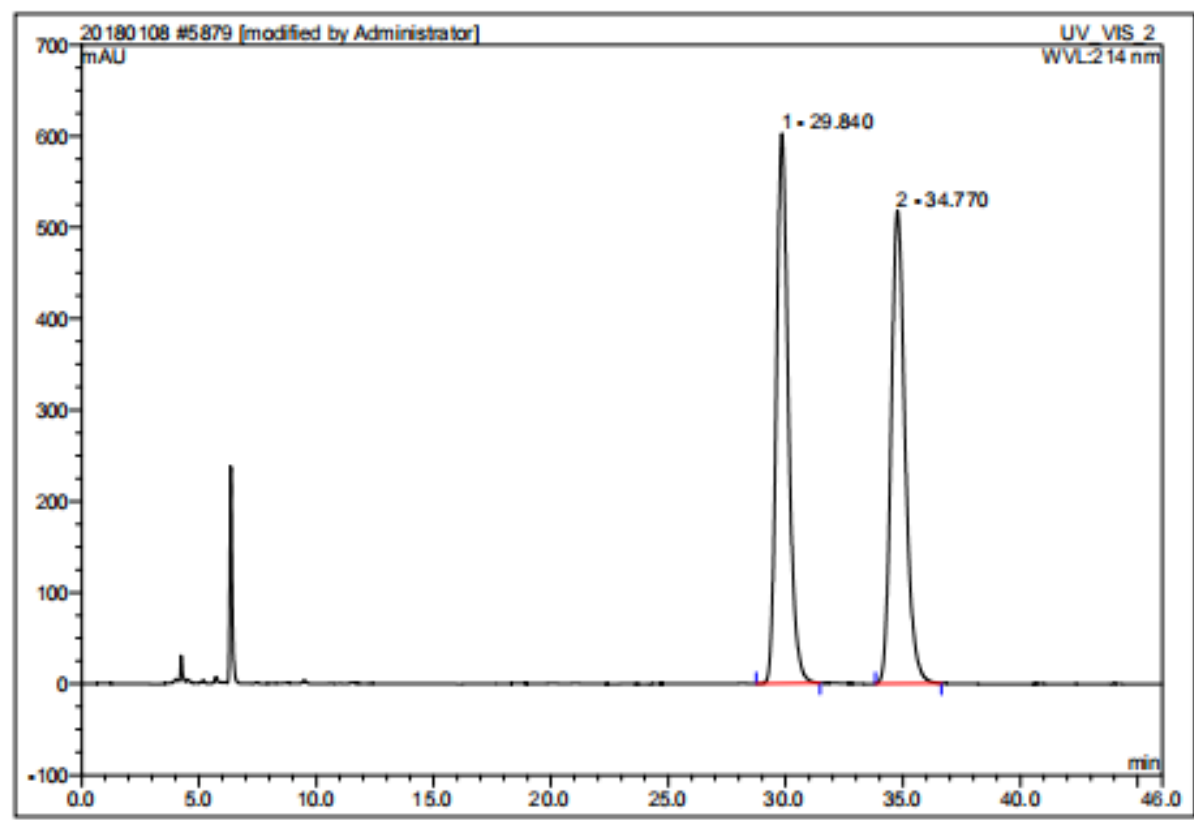

\begin{tabular}{|r|ccccccc|}
\hline No. & $\begin{array}{c}\text { Ret.Time } \\
\text { min }\end{array}$ & Peak Name & $\begin{array}{c}\text { Height } \\
\text { mAU }\end{array}$ & $\begin{array}{c}\text { Area } \\
\text { mAU*min }\end{array}$ & $\begin{array}{c}\text { Rel.Area } \\
\%\end{array}$ & Amount & Type \\
\hline 1 & 29.84 & n.a. & 601.800 & 357.172 & 49.90 & n.a. & BMB $^{*}$ \\
2 & 34.77 & n.a. & 518.411 & 358.621 & 50.10 & n.a. & BMB \\
\hline Total: & & & 1120.210 & 715.794 & 100.00 & 0.000 & \\
\hline
\end{tabular}


5880 HWC-14-94-3A ADH 9552140.7

\begin{tabular}{llll}
\hline Sample Name: & HWC-14-94-3A ADH 9552140.7 & hjection Volume: & 3.0 \\
Vial Number: & RE4 & Channel: & UV_VIS_2 \\
Sample Type: & unknown & Wavelength: & 214.0 \\
Control Program: & test-dad6 & Bandwidth: & 4 \\
Quantif. Method: & 20170608 & Dilution Factor: & 1.0000 \\
Recording Time: & $2019-5-1312: 09$ & Sample Weight: & 1.0000 \\
Run Time (min): & 62.07 & Sample Amount: & 1.0000 \\
\hline
\end{tabular}

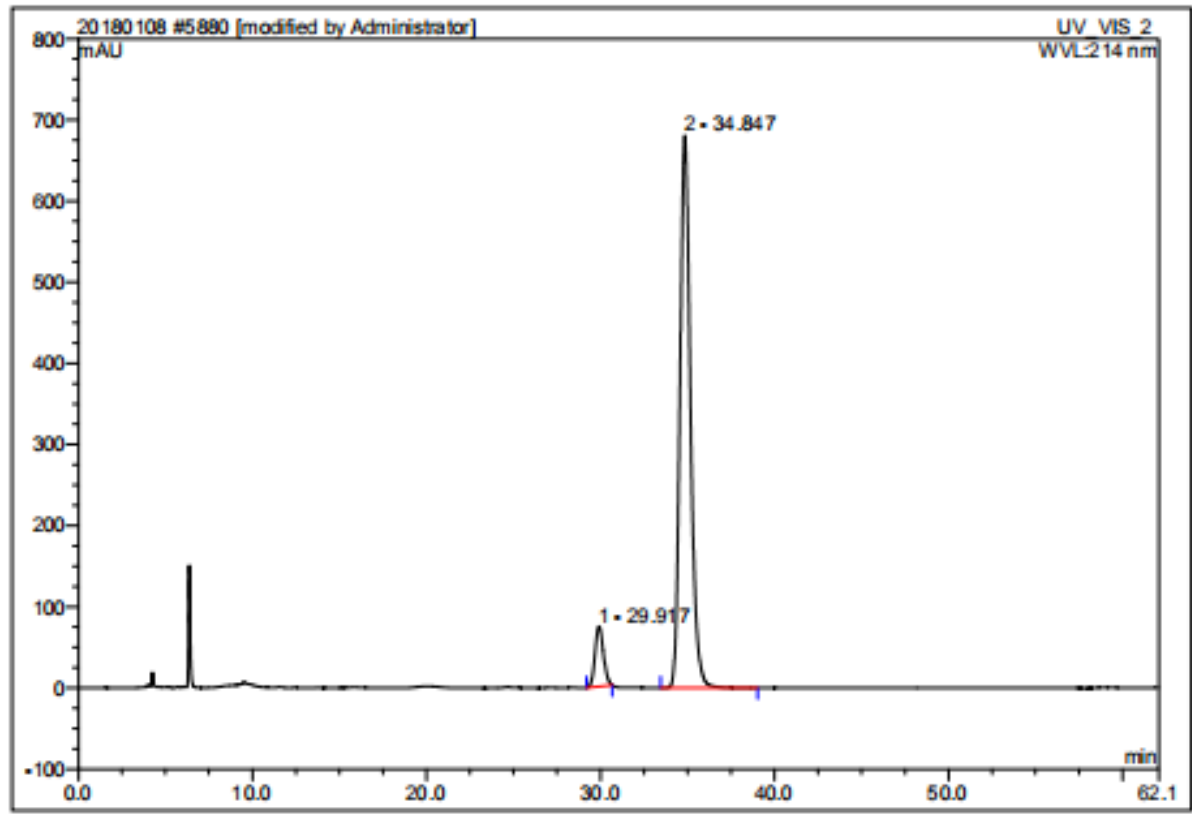

\begin{tabular}{|c|c|c|c|c|c|c|c|}
\hline No. & $\begin{array}{c}\text { Ret.Time } \\
\text { min }\end{array}$ & Peak Name & $\begin{array}{c}\text { Height } \\
\text { mAU }\end{array}$ & $\begin{array}{c}\text { Area } \\
\text { mAU*min }\end{array}$ & $\begin{array}{c}\text { Rel.Area } \\
\%\end{array}$ & Amount & Type \\
\hline 1 & 29.92 & n.a. & 73.844 & 41.319 & 8.01 & na. & BMB* \\
\hline 2 & 34.85 & n.a. & 680.919 & 474.690 & 91.99 & n.a. & $\mathrm{BMB}^{*}$ \\
\hline Total: & & & 754.762 & 516.009 & 100.00 & 0.000 & \\
\hline
\end{tabular}




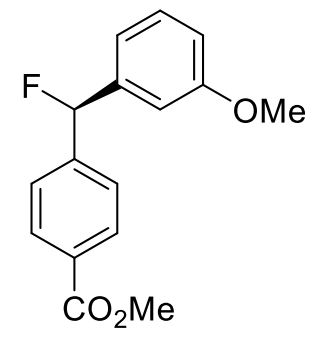

Compound 3d. HPLC (AD-H, $0.46 \times 25 \mathrm{~cm}, 5 \mu \mathrm{m}$, hexane/isopropanol $=98 / 2$ $(\mathrm{v} / \mathrm{v} \%)$, flow $0.7 \mathrm{~mL} / \mathrm{min}$, UV detection at $214 \mathrm{~nm})$, retention time $=23.14 \mathrm{~min}$ (minor) and $26.51 \mathrm{~min}$ (major). $[\alpha]_{\mathrm{D}}{ }^{25}=-5.76\left(\mathrm{c}=0.250, \mathrm{CHCl}_{3}, 94: 6\right.$ e.r).

\begin{tabular}{|llll|}
\hline 5819 HWC-14-80-3A+- ADH 9822140.7 & & \\
& & & \\
\hline Sample Name: & HWC-14-80-3A+-ADH 9822140.7 & hjection Volume: & 2.0 \\
Vial Number. & RB7 & Channel: & UV_VIS_2 \\
Sample Type: & unknown & Wavelength: & 214.0 \\
Cantral Program: & test-dad6 & Bandwidth: & 4 \\
Quantif. Method: & 20170608 & Dïufion Factor: & 1.0000 \\
Recording Time: & $2019-5-810: 22$ & Sample Weight: & 1.0000 \\
Run Time (min): & 31.08 & Sample Amount: & 1.0000 \\
\hline
\end{tabular}

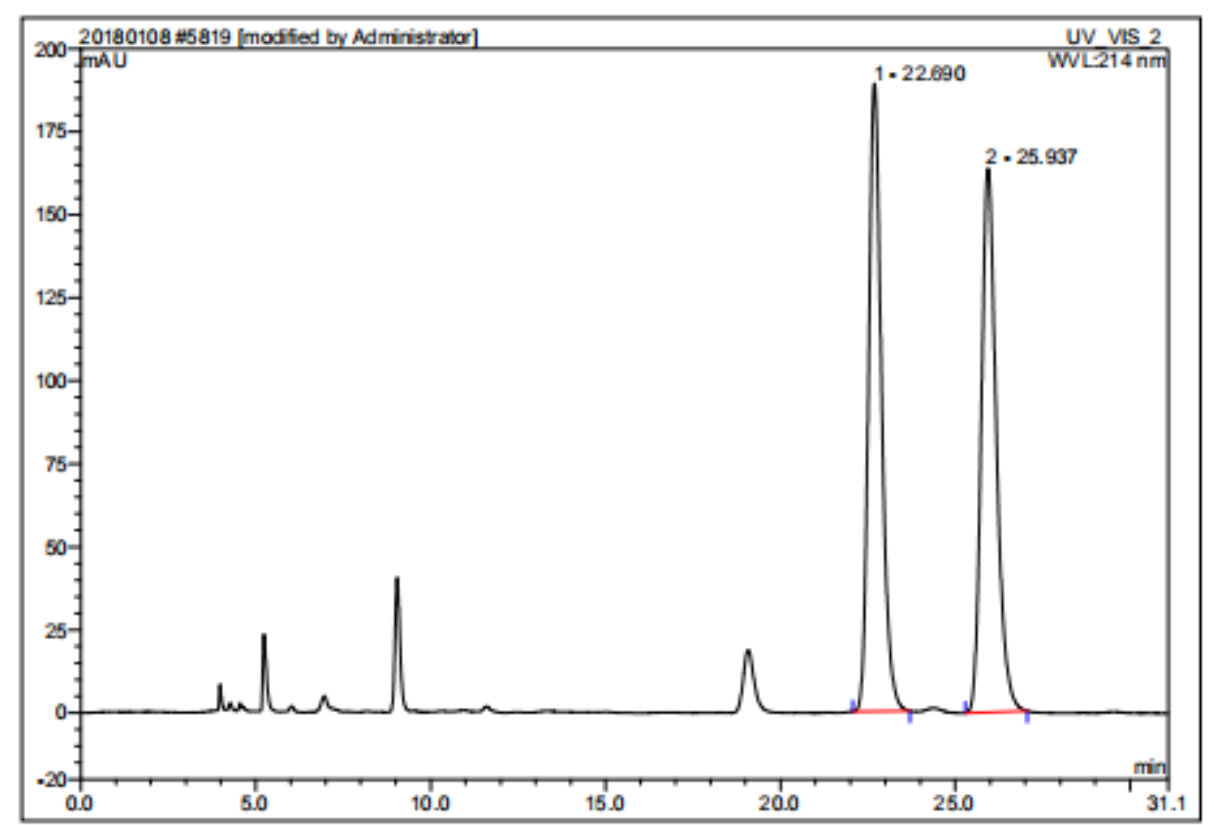

\begin{tabular}{|r|ccrrrrr|}
\hline No. & $\begin{array}{c}\text { Ret.Time } \\
\text { min }\end{array}$ & Peak Name & $\begin{array}{c}\text { Height } \\
\text { mAU }\end{array}$ & $\begin{array}{c}\text { Area } \\
\text { mAU*min }\end{array}$ & $\begin{array}{c}\text { Rel.Area } \\
\%\end{array}$ & Amount & Type \\
\hline 1 & 22.69 & n.a. & 189.039 & 81.115 & 49.96 & n.a. & BMB \\
2 & 25.94 & n.a. & 163.859 & 81.255 & 50.04 & n.a. & BMB \\
\hline Total: & & & 352.898 & 162.370 & 100.00 & 0.000 & \\
\hline
\end{tabular}




\begin{tabular}{|llll|}
\hline 5820 HWC-14-79-3A ADH 9822140.7 & & \\
& & & \\
\hline Sample Name: & HWC-14-79-3A ADH 9822140.7 & njection Volume: & 2.0 \\
Vial Number: & RD7 & Channel: & UV_VIS_2 \\
Sample Type: & unknown & Wavelength: & 214.0 \\
Control Program:: & test-dad6 & Bandwidth: & 4 \\
Quantif. Method: & 20170608 & Dilution Factor: & 1.0000 \\
Recording Time: & $2019-5-89: 04$ & Sample Weight: & 1.0000 \\
Run Time (min): & 40.00 & Sample Amount: & 1.0000 \\
\hline
\end{tabular}
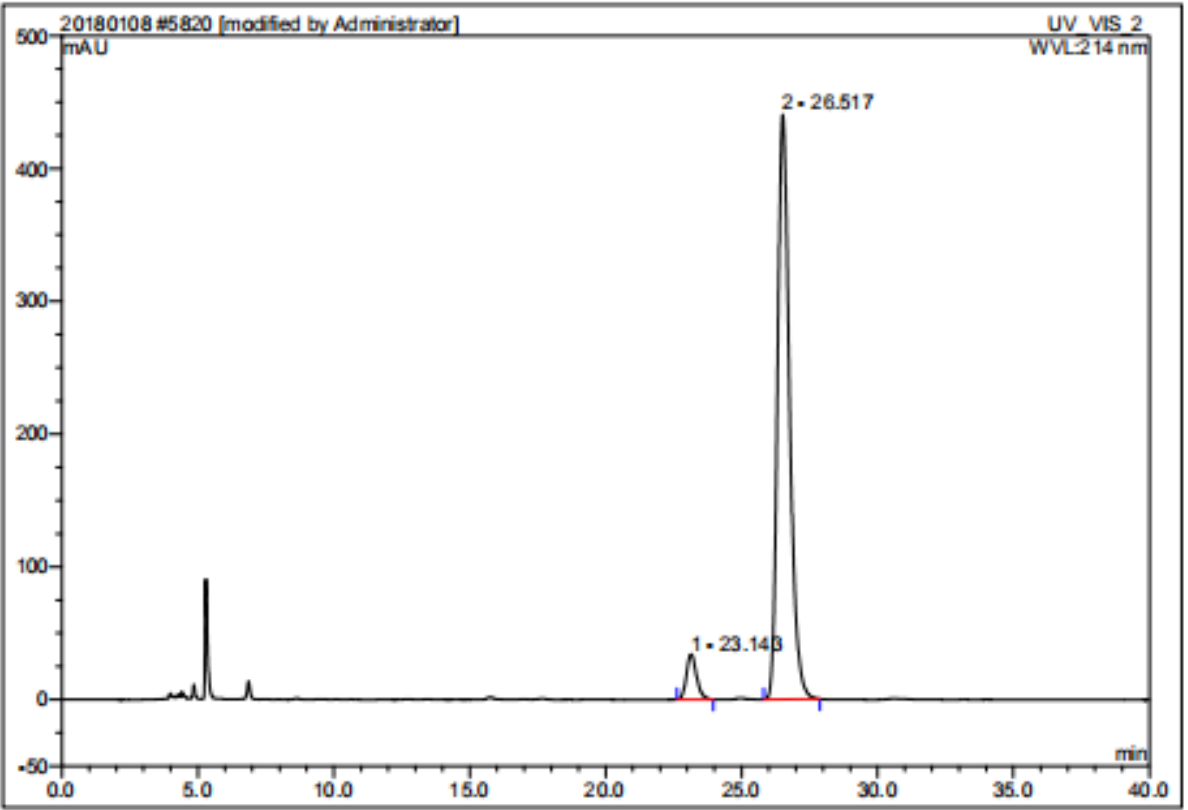

\begin{tabular}{|r|ccrrrrr|}
\hline No. & $\begin{array}{c}\text { Ret. Time } \\
\text { min }\end{array}$ & Peak Name & $\begin{array}{c}\text { Height } \\
\text { mAU }\end{array}$ & $\begin{array}{c}\text { Area } \\
\text { mAU*min }\end{array}$ & $\begin{array}{r}\text { Rel.Area } \\
\%\end{array}$ & Amount & Type \\
\hline 1 & 23.14 & n.a. & 34.033 & 14.656 & 6.11 & n.a. & BMB \\
2 & 26.52 & n.a. & 440.479 & 225.309 & 93.89 & n.a. & BMB \\
\hline Total: & & & 474.512 & 239.966 & 100.00 & 0.000 & \\
\hline
\end{tabular}




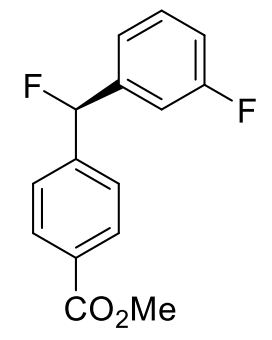

Compound 3e. HPLC (OJ-H, $0.46 \times 25 \mathrm{~cm}, 5 \mu \mathrm{m}$, hexane/isopropanol = 98/2 (v/v \%), flow $0.7 \mathrm{~mL} / \mathrm{min}$, UV detection at $214 \mathrm{~nm}$ ), retention time $=39.57 \mathrm{~min}$ (minor) and $45.85 \min$ (major). $[\alpha]_{\mathrm{D}}{ }^{25}=-4.44\left(\mathrm{c}=0.090, \mathrm{CHCl}_{3}, 95: 5\right.$ e.r).

\section{Empower " 3}

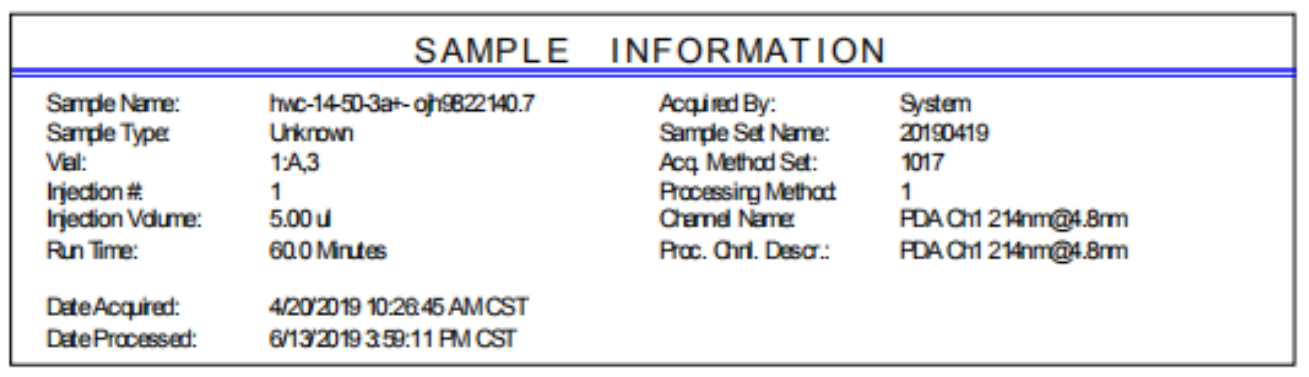

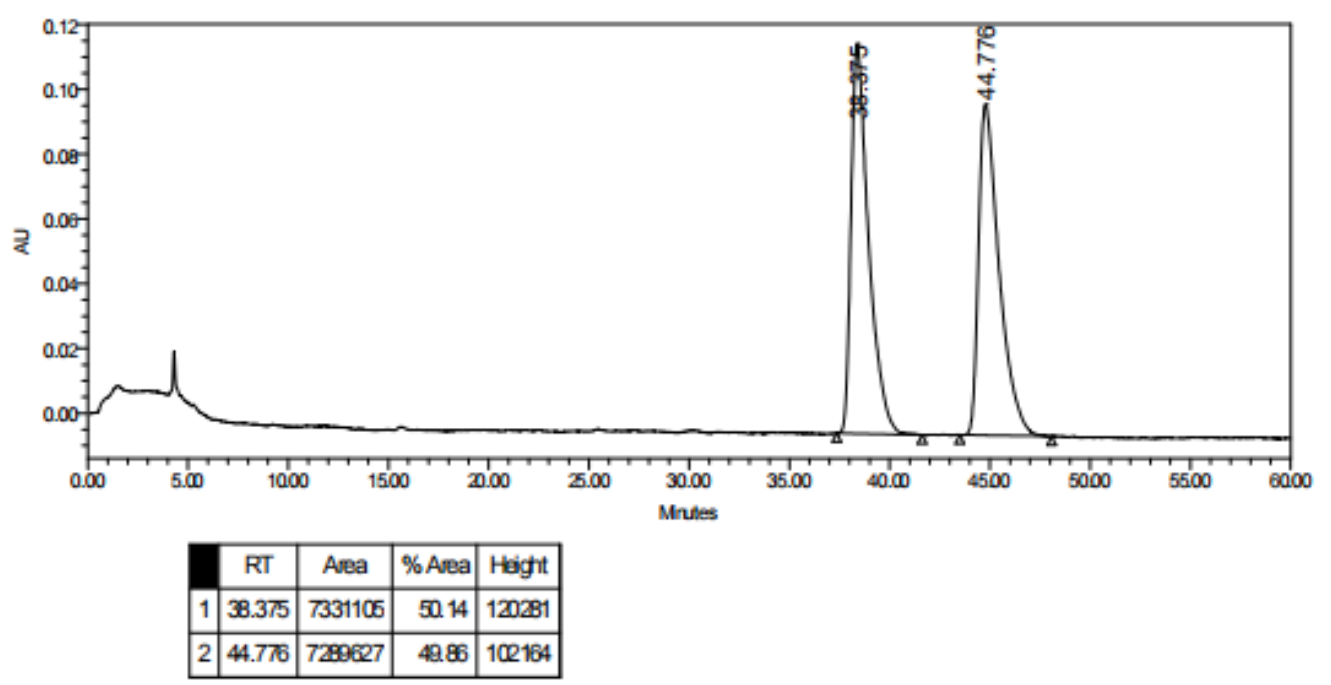




\begin{tabular}{|c|c|c|c|}
\hline & SAMPLE & INFORMATION & \\
\hline Sample Name: & hwc-1449-3agihg622140.7 & Acquired By: & System \\
\hline Sample Type: & Utknomn & Sample Set Name & 20190419 \\
\hline Vial: & $1 \cdot \mathrm{A}, 8$ & Aor Method Set: & 1017 \\
\hline Irjection\#. & 1 & Processing Method & 1 \\
\hline Irjection Vdume: & $5.00 \mathrm{u}$ & Chanel Name: & PDA Ch1 214mm@4.8m \\
\hline Run Time: & 60.0 Minutes & Proc. Onnl. Desa.: & PDA Ch1 214mm@4.8m \\
\hline Date Acquired & 4/19/20199.44:23PMCST & & \\
\hline Date Processed. & 6/13/20194:00:17PMCST & & \\
\hline
\end{tabular}

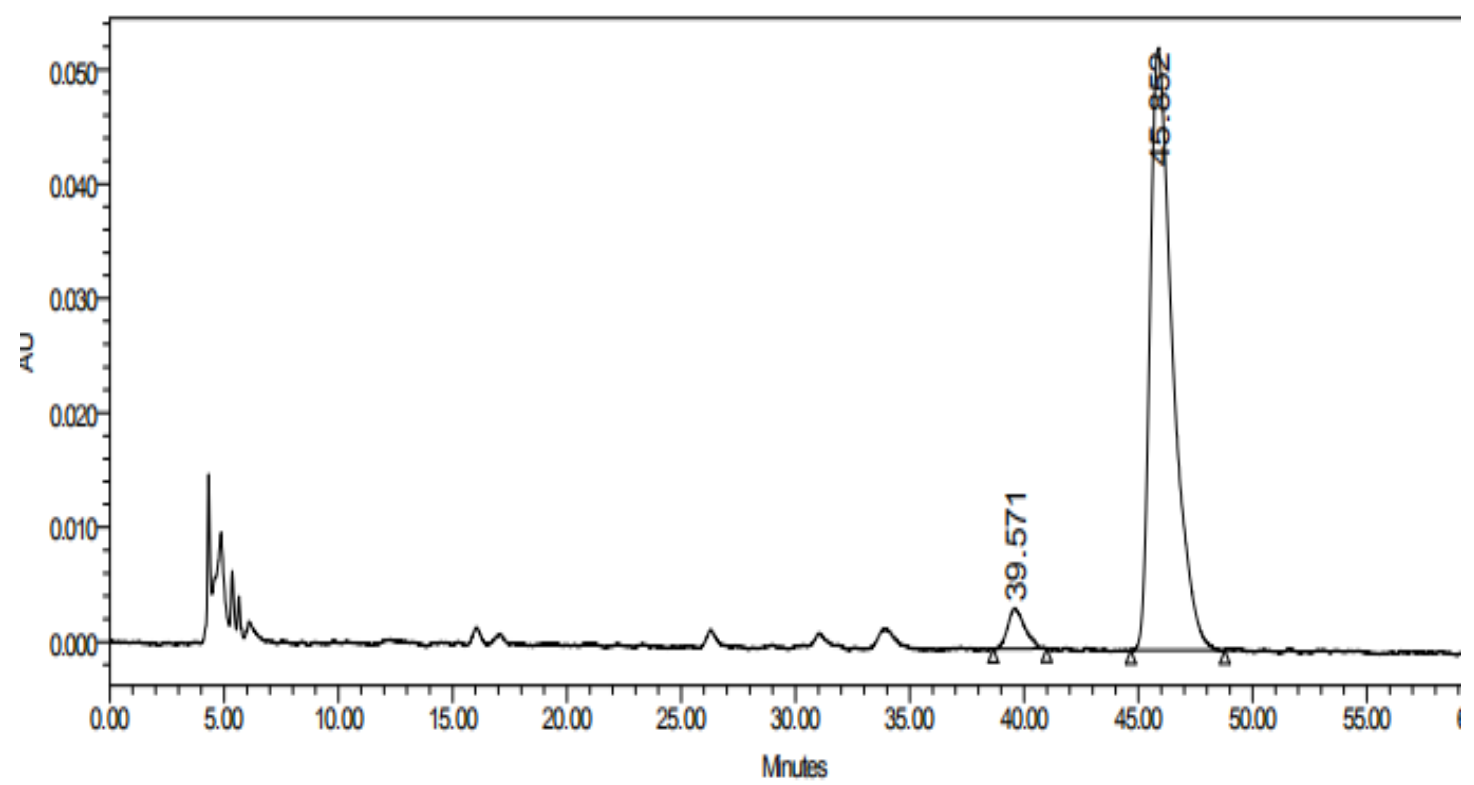

\begin{tabular}{|c|c|c|r|r|}
\hline & RT & Arø & \% Arø & Height \\
\hline 1 & 39.571 & 181708 & 4.80 & 3492 \\
\hline 2 & 45.852 & 3600884 & 95.20 & 52542 \\
\hline
\end{tabular}




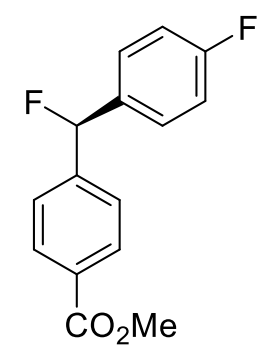

Compound 3f.HPLC (AD-H, $0.46 \times 25 \mathrm{~cm}, 5 \mu \mathrm{m}$, hexane/isopropanol = 98/2 (v/v \%), flow $0.7 \mathrm{~mL} / \mathrm{min}$, UV detection at $214 \mathrm{~nm}$ ), retention time $=18.16 \mathrm{~min}$ (minor) and $19.67 \min$ (major). $[\alpha]_{\mathrm{D}}^{25}=-58.73\left(\mathrm{c}=0.110, \mathrm{CHCl}_{3}, 93: 7\right.$ e.r).

5493 HWC-14-41-3A+- ADH 9822140.7

\begin{tabular}{llll|}
\hline Sample Name: & HWC-14-41-3A+- ADH 9822140.7 & Injection Volume: & 2.0 \\
VialNumber: & RB4 & Channel: & UV_VIS_2 \\
Sample Type: & unknown & Wavelength: & 214.0 \\
Control Program: & test-dad3 & Bandwidth: & 4 \\
Quantif. Method: & 20170608 & Dilution Factor: & 1.0000 \\
Recording Time: & $2019-4-1614: 16$ & Sample Weight: & 1.0000 \\
Run Time (min): & 25.53 & Sample Amount: & 1.0000
\end{tabular}

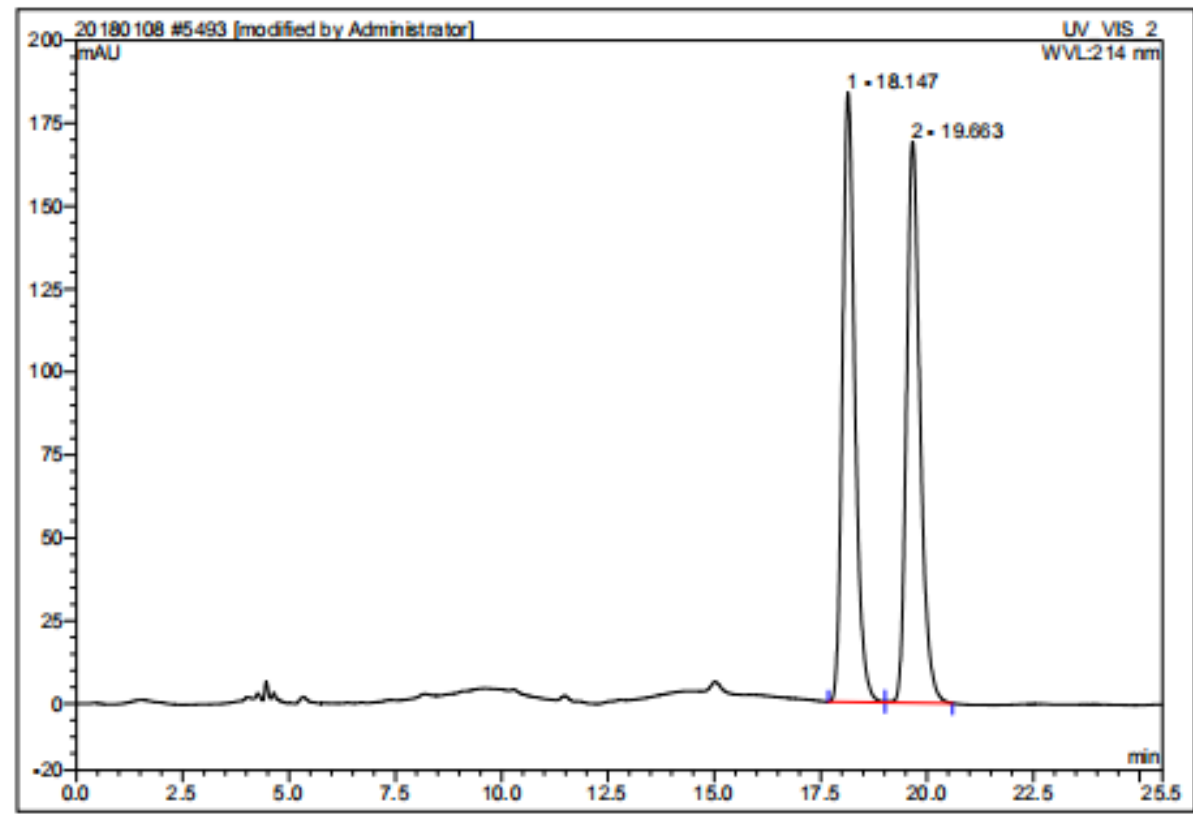

\begin{tabular}{|r|ccrrrrr|}
\hline No. & $\begin{array}{c}\text { Ret.Time } \\
\text { min }\end{array}$ & Peak Name & $\begin{array}{c}\text { Height } \\
\text { mAU }\end{array}$ & $\begin{array}{c}\text { Area } \\
\text { mAU*min }\end{array}$ & $\begin{array}{c}\text { Rel.Area } \\
\%\end{array}$ & Amount & Type \\
\hline 1 & 18.15 & n.a. & 183.822 & 62.291 & 49.89 & n.a. & BM \\
2 & 19.66 & n.a. & 169.134 & 62.576 & 50.11 & n.a. & MB \\
\hline Total: & & & 352.956 & 124.867 & 100.00 & 0.000 & \\
\hline
\end{tabular}




\section{HWC-14-40-3A ADH 9822140.7}

\begin{tabular}{|llll|}
\hline Sample Name: & HWC-14-40-3A ADH 982 214 0.7 & injection Volume: & 2.0 \\
Vial Number: & RA4 & Channet & UV_uS_2 \\
Sample Type: & unknown & Wavelength: & 214.0 \\
Control Program: & test-dad3 & Bandwidth: & 4 \\
Quantf. Method: & 20170608 & Dllubion Factor: & 1.0000 \\
Recording Time: & $2019-4-1614: 43$ & Sample Weight: & 1.0000 \\
Run Time (min): & 26.14 & Sample Amount: & 1.0000 \\
\hline
\end{tabular}

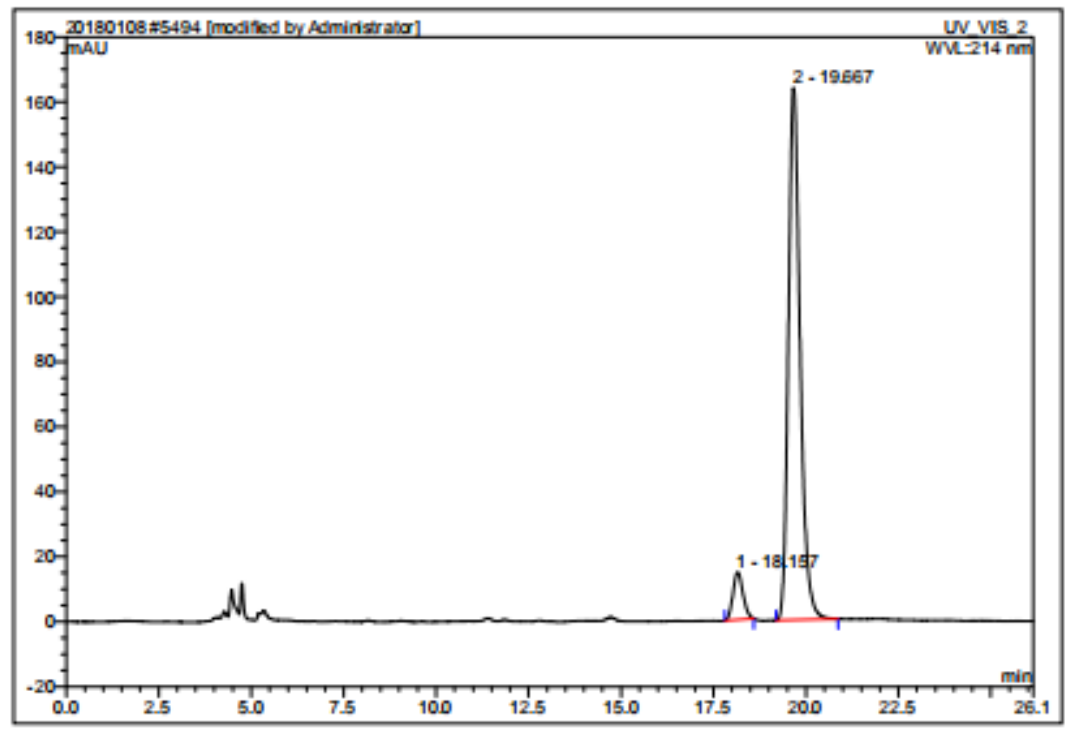

\begin{tabular}{|r|ccrrrrr|}
\hline No. & $\begin{array}{c}\text { Ret.Time } \\
\text { min }\end{array}$ & Peak Name & $\begin{array}{c}\text { Height } \\
\text { mAU }\end{array}$ & $\begin{array}{c}\text { Area } \\
\text { mAU*min }\end{array}$ & $\begin{array}{r}\text { RelArea } \\
\%\end{array}$ & Amount & Type \\
\hline 1 & 18.16 & n.a. & 14.596 & 4.603 & 7.08 & n.a. & BMB $^{*}$ \\
2 & 19.67 & n.a. & 163.872 & 60.386 & 92.92 & n.a. & BMB $^{*}$ \\
\hline Total: & & & 178.468 & 64.988 & 100.00 & 0.000 & \\
\hline
\end{tabular}




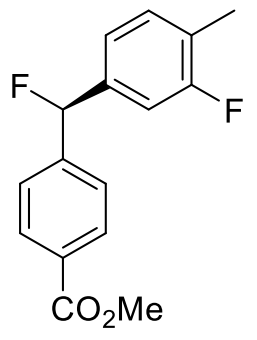

Compound 3g. HPLC (AD-H, $0.46 \times 25 \mathrm{~cm}, 5 \mu \mathrm{m}$, hexane/isopropanol $=98 / 2$ $(\mathrm{v} / \mathrm{v} \%)$, flow $0.7 \mathrm{~mL} / \mathrm{min}$, UV detection at $214 \mathrm{~nm})$, retention time $=15.48 \mathrm{~min}$ (minor) and $17.22 \mathrm{~min}$ (major). $[\alpha]_{\mathrm{D}}{ }^{25}=-12.94$ (c $=0.170, \mathrm{CHCl}_{3}, 94: 6$ e.r).

\begin{tabular}{|llll|}
\hline 5837 HWC-14-91-3B+- ADH 9822140.7 & & \\
& & & \\
\hline Sample Name: & HWC-14-91-3B+- ADH 9822140.7 & hjection Volume: & 2.0 \\
Vial Number: & RB7 & Channel: & UV_VIS_2 \\
Sample Type: & unknown & Wavelength: & 214.0 \\
Control Program: & test-dad6 & Bandwidth: & 4 \\
Quantif. Method: & 20170608 & Dîutian Factor: & 1.0000 \\
Recording Time: & $2019-5-916: 13$ & Sample Weight: & 1.0000 \\
Run Time (min): & 32.53 & Sample Amount: & 1.0000 \\
\hline
\end{tabular}

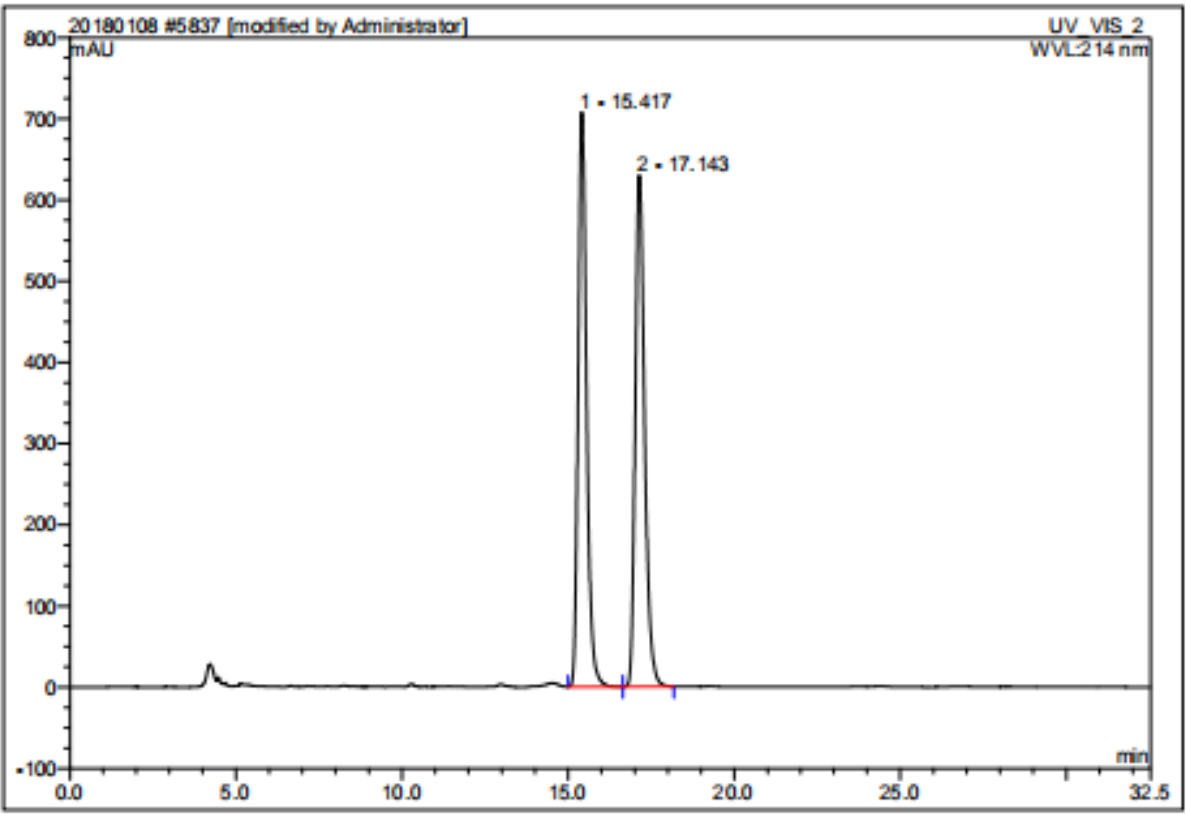

\begin{tabular}{|r|ccccccc|}
\hline No. & $\begin{array}{c}\text { Ret.Time } \\
\text { min }\end{array}$ & Peak Name & $\begin{array}{c}\text { Height } \\
\text { mAU }\end{array}$ & $\begin{array}{c}\text { Area } \\
\text { mAU*min }\end{array}$ & $\begin{array}{c}\text { Rel.Area } \\
\%\end{array}$ & Amount & Type \\
\hline 1 & 15.42 & n.a. & 707.774 & 207.433 & 50.13 & n.a. & BM \\
2 & 17.14 & n.a. & 630.421 & 206.359 & 49.87 & n.a. & MB \\
\hline Total: & & & 1338.195 & 413.792 & 100.00 & 0.000 & \\
\hline
\end{tabular}




\begin{tabular}{|llll|}
\hline 5838 HWC-14-91-3A ADH 9822140.7 & & \\
& & & \\
\hline Sample Name: & HWC-14-91-3A ADH 9822140.7 & njection Volume: & 2.0 \\
Vial Number: & RB8 & Channel: & UV_VIS_2 \\
Sample Type: & unknown & Wavelength: & 214.0 \\
Contral Program: & test-dad6 & Bandwidth: & 4 \\
Quantif. Method:: & 20170608 & Dilution Factor: & 1.0000 \\
Recording Time: & $2019-5-916: 48$ & Sample Weight: & 1.0000 \\
Run Time (min): & 25.00 & Sample Amount: & 1.0000 \\
\hline
\end{tabular}

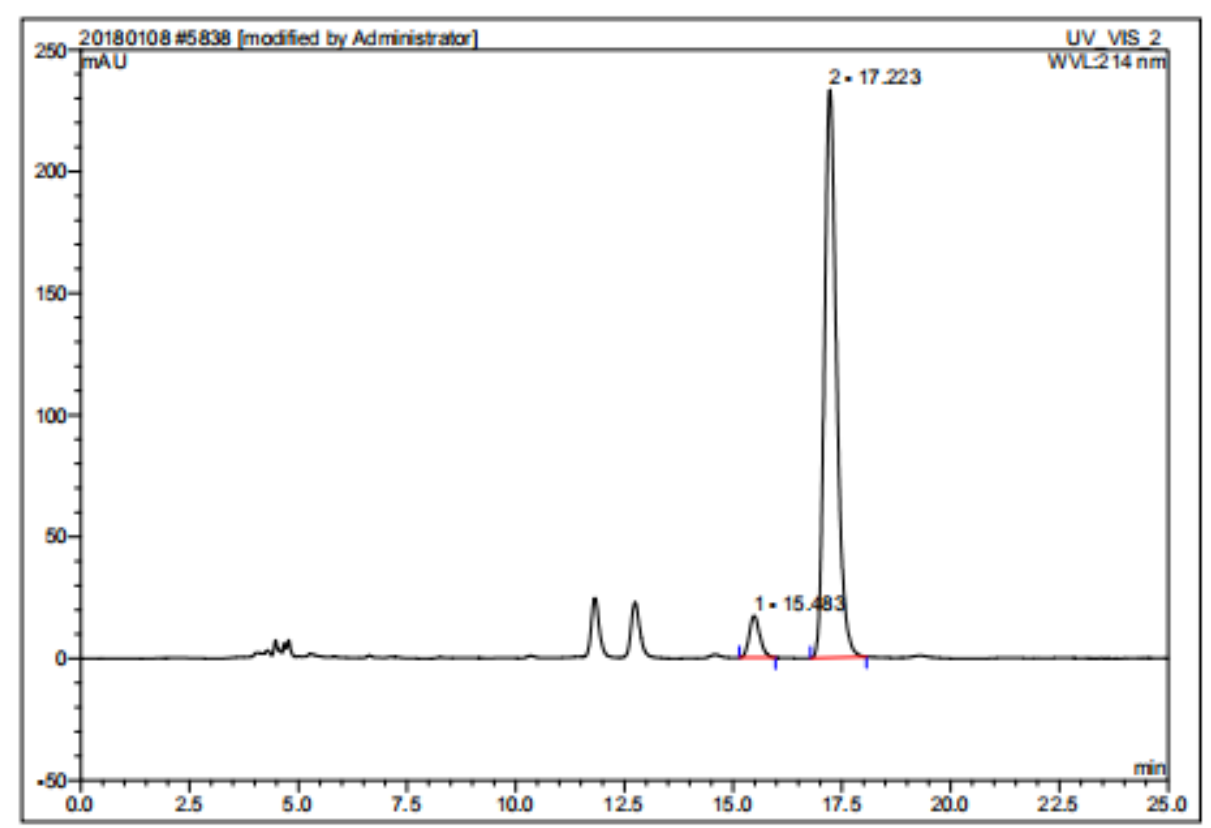

\begin{tabular}{|r|ccrrrrr|}
\hline No. & $\begin{array}{c}\text { Ret. Time } \\
\text { min }\end{array}$ & Peak Name & $\begin{array}{c}\text { Height } \\
\text { mAU }\end{array}$ & $\begin{array}{r}\text { Area } \\
\text { mAU*min }\end{array}$ & $\begin{array}{r}\text { Rel.Area } \\
\text { \% }\end{array}$ & Amount & Type \\
\hline 1 & 15.48 & n.a. & 17.242 & 4.886 & 6.07 & n.a. & BMB \\
2 & 17.22 & n.a. & 233.367 & 75.670 & 93.93 & n.a. & BMB \\
\hline Total: & & & 250.609 & 80.557 & 100.00 & 0.000 & \\
\hline
\end{tabular}




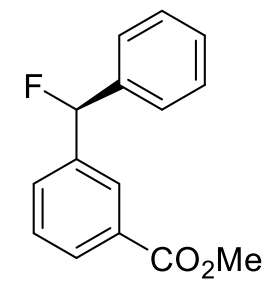

Compound 3h. HPLC (OD-H, $0.46 \times 25 \mathrm{~cm}, 5 \mu \mathrm{m}$, hexane/isopropanol $=98 / 2$ (v/v \%), flow $0.7 \mathrm{~mL} / \mathrm{min}$, UV detection at $214 \mathrm{~nm}$ ), retention time $=12.61 \mathrm{~min}$ (minor) and $15.28 \mathrm{~min}$ (major). $[\alpha]_{\mathrm{D}}{ }^{25}=-0.95\left(\mathrm{c}=0.210, \mathrm{CHCl}_{3}, 93: 7\right.$ e.r).

\begin{tabular}{|llll|}
\hline 5438 HWC-14-36-3C+- ODH 9822140.7 & & \\
& & & \\
\hline Sample Name: & HWC-14-36-3C+- ODH 9822140.7 & njection Volume: & 2.0 \\
Vial Number: & RD6 & Channel: & UV_VIS_2 \\
Sample Type: & unknown & Wavelength: & 214.0 \\
Contral Program: & test-dad4 & Bandwidth: & 4 \\
Quantif. Method:: & 20170608 & Dïution Factor: & 1.0000 \\
Recording Time: & $2019-4-1116: 03$ & Sample Weight: & 1.0000 \\
Run Time (min): & 18.46 & Sample Amount: & 1.0000 \\
\hline
\end{tabular}

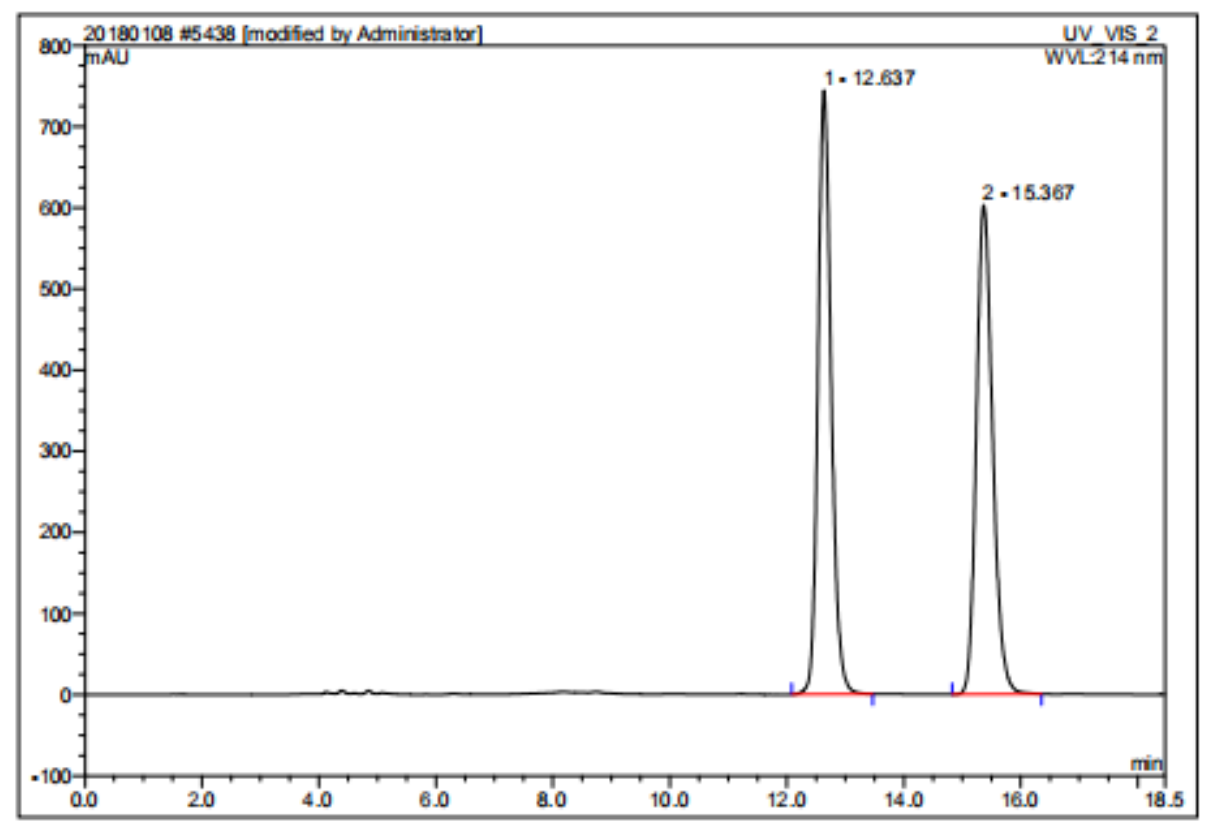

\begin{tabular}{|r|ccccccc|}
\hline No. & $\begin{array}{c}\text { Ret. Time } \\
\text { min }\end{array}$ & Peak Name & $\begin{array}{c}\text { Height } \\
\text { mAU }\end{array}$ & $\begin{array}{c}\text { Area } \\
\text { mAU*min }\end{array}$ & $\begin{array}{c}\text { Rel.Area } \\
\%\end{array}$ & Amount & Type \\
\hline 1 & 12.64 & n.a. & 744.414 & 195.883 & 50.18 & n.a. & BMB \\
2 & 15.37 & n.a. & 603.006 & 194.459 & 49.82 & n.a. & BMB \\
\hline Total: & & & 1347.420 & 390.341 & 100.00 & 0.000 & \\
\hline
\end{tabular}


i465 HWC-13-38-3A ODH 9822140.7

\begin{tabular}{llll}
\hline iample Name: & HWC-13-38-3A ODH 982 214 0.7 & hjection Volume: & 3.0 \\
lial Number: & RE6 & Channel: & UV_VIS_2 \\
iample Type: & unknown & Wavelength: & $\mathbf{2 1 4 . 0}$ \\
iontrol Program: & test-dad4 & Bandwidth: & 4 \\
Zuantif. Method: & 20170608 & Dilution Factor: & 1.0000 \\
Recording Time: & $2019-4-1218: 05$ & Sample Weight: & 1.0000 \\
Run Time (min): & 20.00 & Sample Amount: & 1.0000 \\
\hline
\end{tabular}

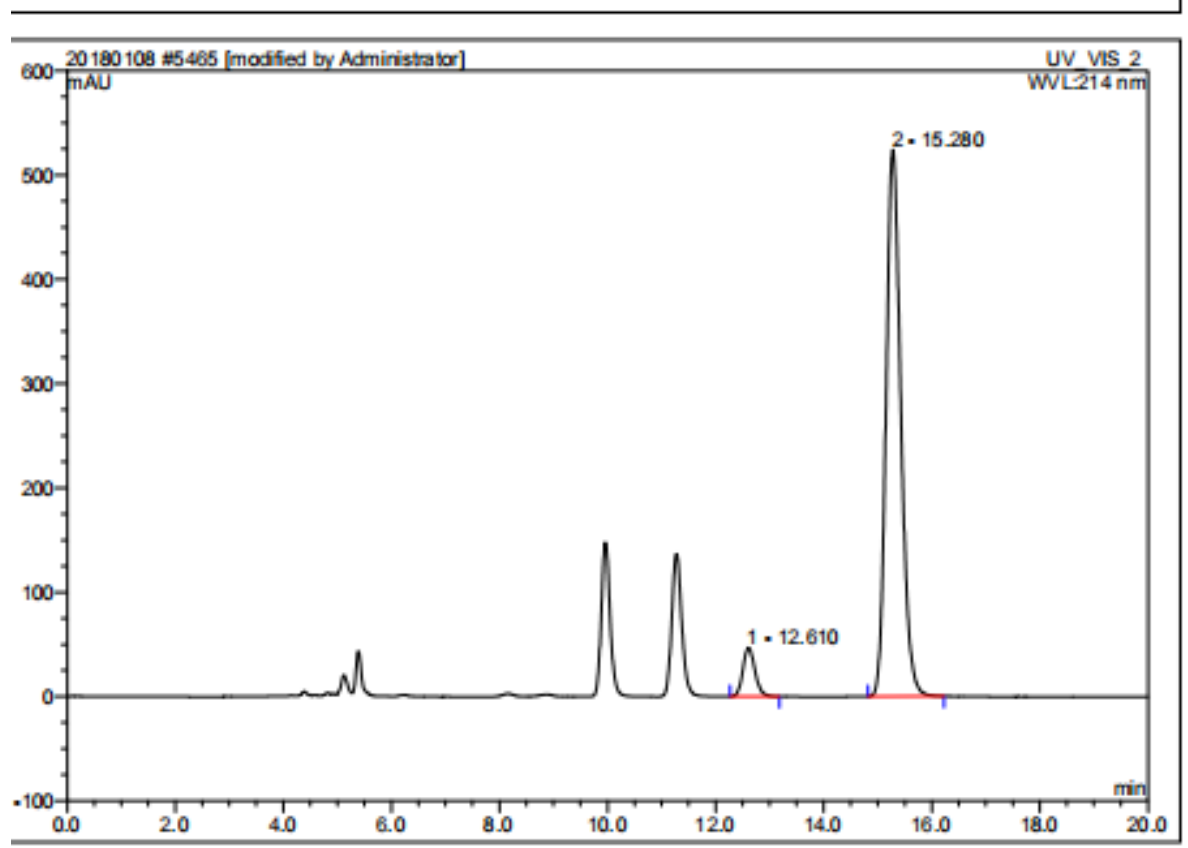

\begin{tabular}{r|ccrrrrr|}
\hline No. & $\begin{array}{c}\text { Ret.Time } \\
\text { min }\end{array}$ & Peak Name & $\begin{array}{c}\text { Height } \\
\text { mAU }\end{array}$ & $\begin{array}{c}\text { Area } \\
\text { mAU*min }\end{array}$ & $\begin{array}{c}\text { Rel.Area } \\
\%\end{array}$ & Amount & Type \\
\hline 1 & 12.61 & n.a. & 47.092 & 12.142 & 6.83 & n.a. & BMB \\
2 & 15.28 & n.a. & 524.156 & 165.569 & 93.17 & n.a. & BMB \\
\hline otal: & & & 571.248 & 177.711 & 100.00 & 0.000 & \\
\hline
\end{tabular}




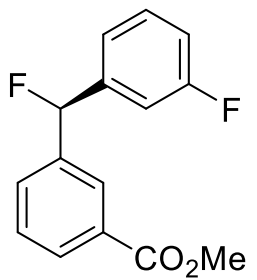

Compound 3i. HPLC (OD-H, $0.46 \times 25 \mathrm{~cm}, 5 \mu \mathrm{m}$, hexane/isopropanol = 95/5 (v/v \%), flow $0.7 \mathrm{~mL} / \mathrm{min}$, UV detection at $214 \mathrm{~nm}$ ), retention time $=9.57 \mathrm{~min}$ (minor) and $11.35 \min$ (major). $[\alpha]_{\mathrm{D}}^{25}=+4.22\left(\mathrm{c}=0.270, \mathrm{CHCl}_{3}, 96: 4\right.$ e.r).

\begin{tabular}{|c|c|c|c|}
\hline & SAMPLE & INFORMATION & \\
\hline Sample Name: & HMC-1456-3A+-CDHE652 140.7 & Acquired By. & System \\
\hline Sample Type: & Urkrown & SampleSet Name & 20190422 \\
\hline Val: & 1:E,1 & Acq. Method Set: & 1017 \\
\hline Irjection\# \# & 1 & Processing Method: & 1 \\
\hline Irjection Volume & $6.00 \mathrm{ul}$ & Crame Name: & PDACh1 214rm@4.8rm \\
\hline Ren Time: & 40.0Mintes & Proc. Onl. Descr.: & PDACh1 214m@4.8rm \\
\hline Date Acquired: & 4/24/20197.@241 PMCST & & \\
\hline Date Processed: & 6/1320194.47:23 PMCST & & \\
\hline
\end{tabular}

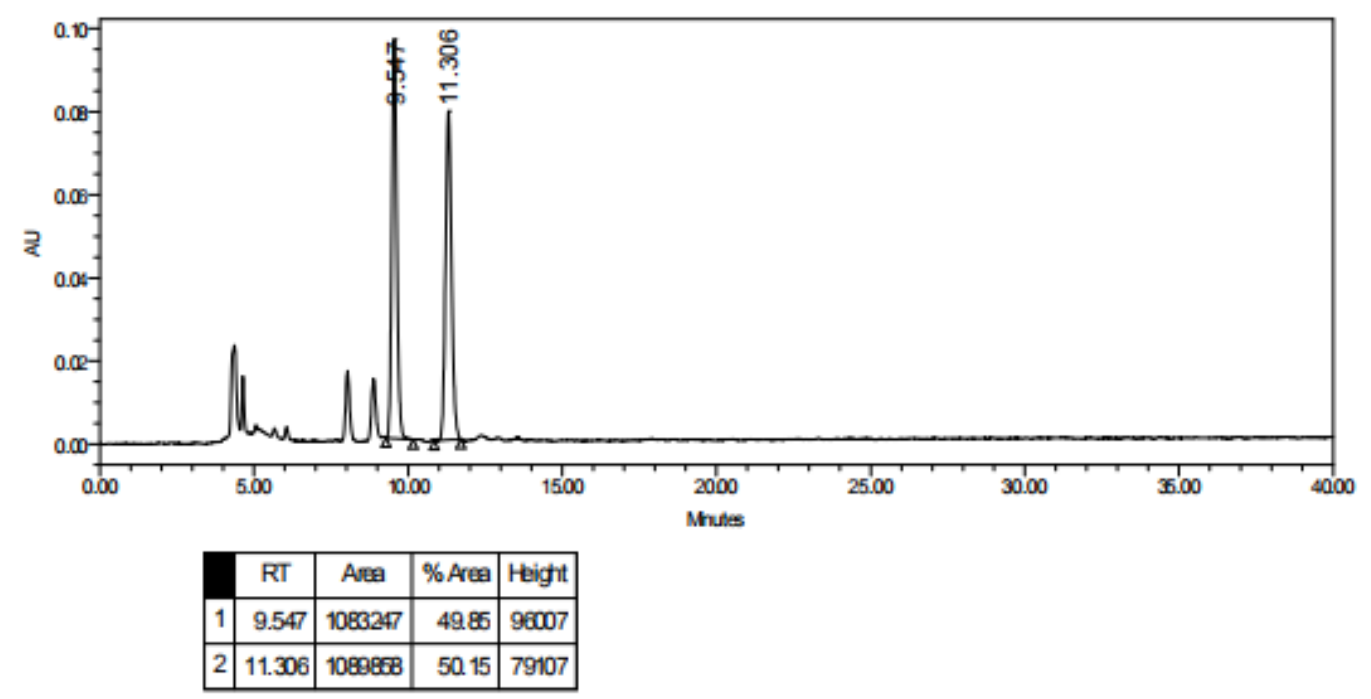




\begin{tabular}{|c|c|c|c|}
\hline & SAMPLE & INFORMATION & \\
\hline Sample Name: & 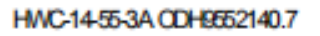 & Acqined By: & System \\
\hline Sample Type & Urkrown & Sample Set Name: & 20190422 \\
\hline Val: & $1: E, 2$ & Acq Method Set: & 1017 \\
\hline Irjection \#\# & 1 & Pocessing Methat & \\
\hline Irjection Vaume: & $6.00 \mathrm{u}$ & Creme Name & PDAOM1 214nm@4.8m \\
\hline Pen Time: & 40.0 Mintes & Proc. Orri. Descr.: & PDAOM1 214nm@4.8m \\
\hline DateAcquired: & 4/24/2019 7.43.26 PM CST & & \\
\hline DateProcessed: & 6/132019 4.46:31 RM CST & & \\
\hline
\end{tabular}

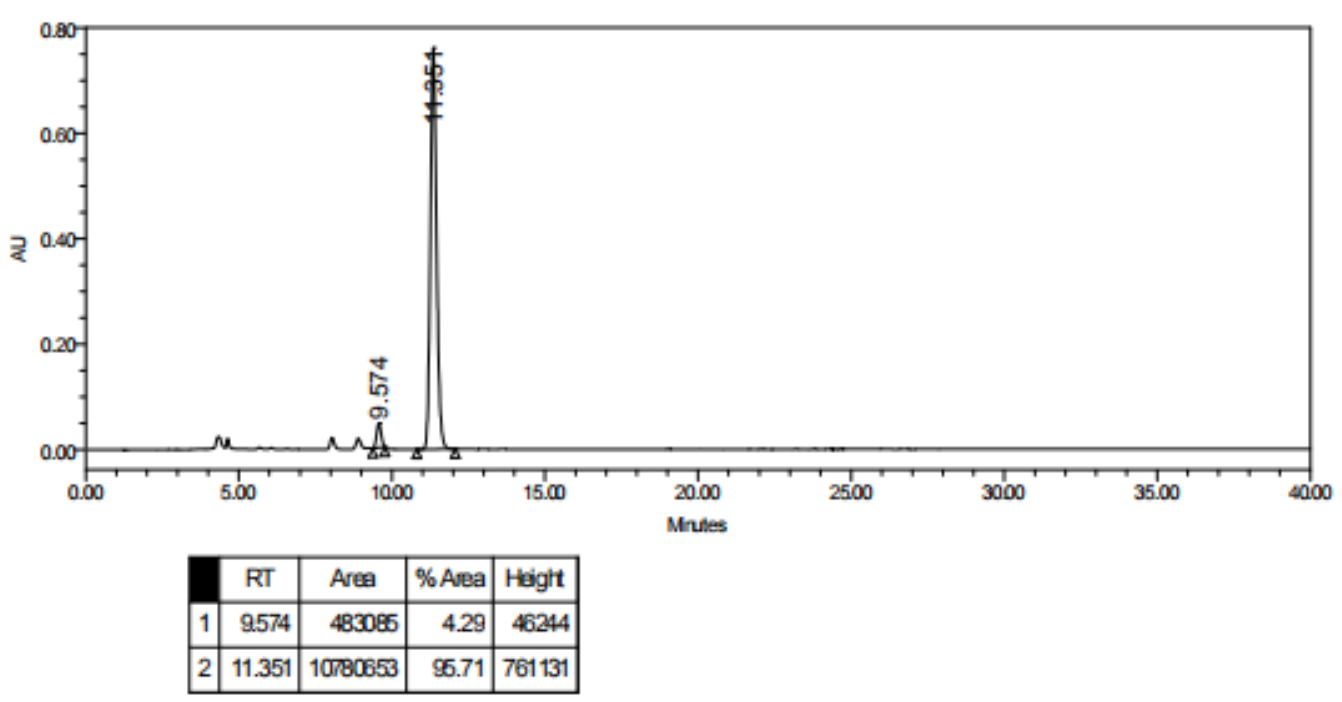


<smiles>CCOC(=O)c1ccc(C(F)c2ccccc2)cc1</smiles>

Compound 3j.HPLC (AD-H, $0.46 \times 25 \mathrm{~cm}, 5 \mu \mathrm{m}$, hexane/isopropanol = 98/2 (v/v \%), flow $0.7 \mathrm{~mL} / \mathrm{min}$, UV detection at $214 \mathrm{~nm}$ ), retention time $=14.96 \mathrm{~min}$ (minor) and $16.08 \min$ (major). $[\alpha]_{\mathrm{D}^{25}}=-13.83\left(\mathrm{c}=0.120, \mathrm{CHCl}_{3}, 92: 8\right.$ e.r).

5518 HWC-14-44-3C+- ADH 9822140.7

\begin{tabular}{|llll|}
\hline Sample Name: & HWC-14-44-3C +- ADH 9822140.7 & injection Volume: & 2.0 \\
Vial Number: & RC4 & Channel: & UV_VIS_2 \\
Sample Type: & unknown & Wavelength: & 214.0 \\
Control Program: & test-dad3 & Bandwidth: & 4 \\
Quantif. Method: & 20170608 & Dilution Factor: & 1.0000 \\
Recording Time: & $2019-4-1716: 46$ & Sample Weight: & 1.0000 \\
Run Time (min): & 25.98 & Sample Amount: & 1.0000 \\
\hline
\end{tabular}

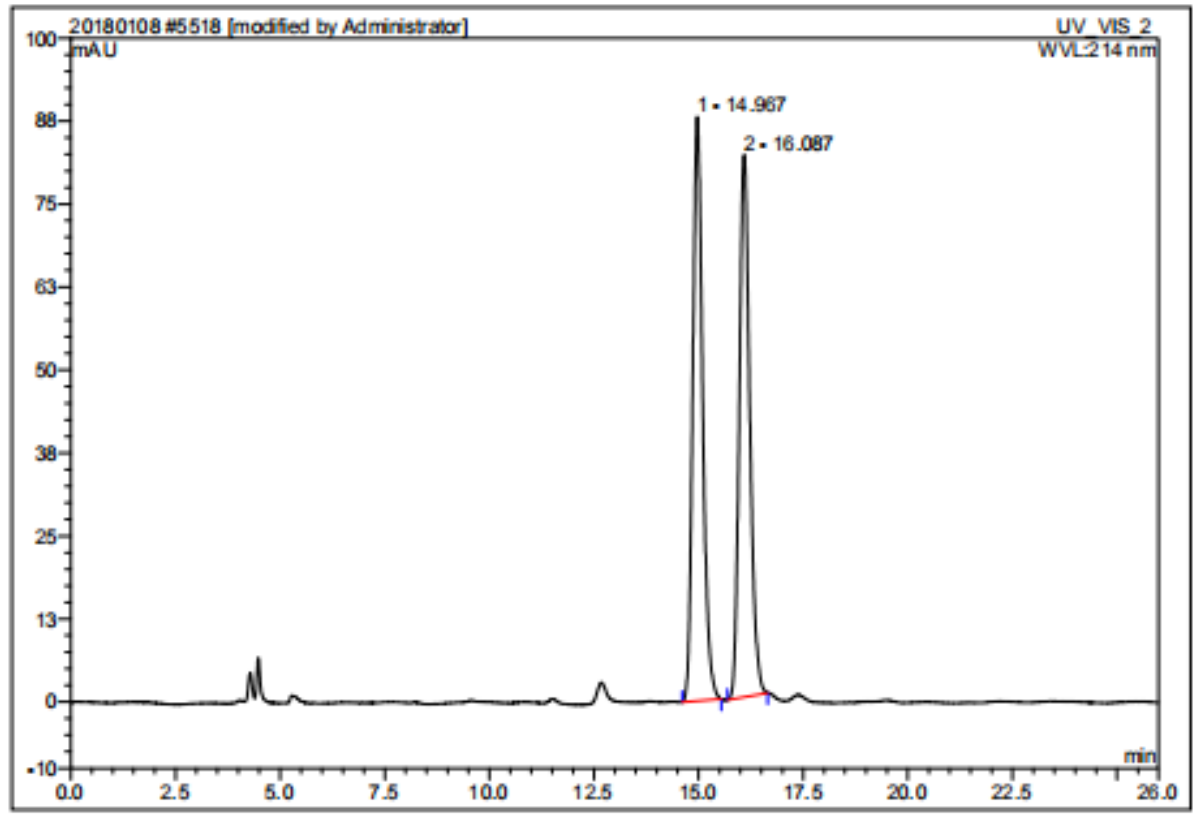

\begin{tabular}{|c|c|c|c|c|c|c|c|}
\hline No. & $\begin{array}{c}\text { Ret.Time } \\
\text { min }\end{array}$ & Peak Name & $\begin{array}{l}\text { Height } \\
\text { mAU }\end{array}$ & $\begin{array}{c}\text { Area } \\
\text { mAU*min }\end{array}$ & $\begin{array}{c}\text { Rel.Area } \\
\%\end{array}$ & Amount & Type \\
\hline 1 & 14.97 & n.a. & 88.057 & 24.204 & 50.43 & na. & BMB \\
\hline 2 & 16.09 & n.a. & 81.695 & 23.788 & 49.57 & na. & $\mathrm{BMB}$ \\
\hline Total: & & & 169.752 & 47.992 & 100.00 & 0.000 & \\
\hline
\end{tabular}




\begin{tabular}{|llll|}
\hline 5519 HWC-14-43-3C ADH 9822140.7 & & \\
& & & \\
\hline Sample Name: & HWC-14-43-3C ADH 9822140.7 & Injection Volume: & 2.0 \\
Vial Number: & RD4 & Channel: & UV_VIS_2 \\
Sample Type: & unknown & Wavelength: & 214.0 \\
Control Program: & test-dad3 & Bandwidth: & 4 \\
Quantif. Method: & 20170608 & Dilution Factor: & 1.0000 \\
Recording Time: & $2019-4-17$ 17:14 & Sample Weight: & 1.0000 \\
Run Time (min): & 22.34 & Sample Amount: & 1.0000 \\
\hline
\end{tabular}

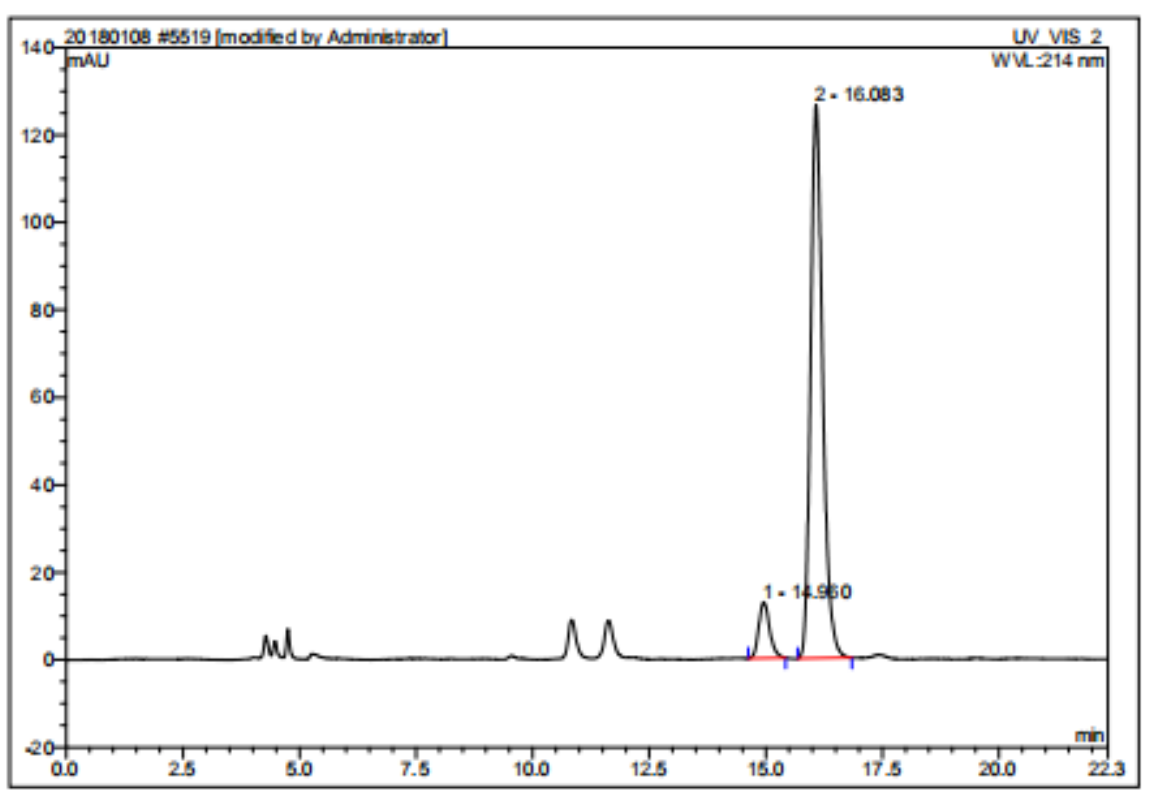

\begin{tabular}{|r|ccrrrrr|}
\hline No. & $\begin{array}{c}\text { Ret.Time } \\
\text { min }\end{array}$ & Peak Name & $\begin{array}{c}\text { Height } \\
\text { mAU }\end{array}$ & $\begin{array}{c}\text { Area } \\
\text { mAU*min }\end{array}$ & $\begin{array}{c}\text { Rel.Area } \\
\%\end{array}$ & Amount & Type \\
\hline 1 & 14.96 & n.a. & 12.874 & 3.478 & 8.41 & n.a. & BMB \\
2 & 16.08 & n.a. & 126.531 & 37.904 & 91.59 & n.a. & BMB \\
\hline Total: & & & 139.405 & 41.382 & 100.00 & 0.000 & \\
\hline
\end{tabular}


<smiles>N#Cc1cccc(C(F)c2ccccc2)c1</smiles>

Compound 3k. HPLC (OD-H, $0.46 \times 25 \mathrm{~cm}, 5 \mu \mathrm{m}$, hexane/isopropanol $=97 / 3$ (v/v \%), flow $0.7 \mathrm{~mL} / \mathrm{min}$, UV detection at $214 \mathrm{~nm}$ ), retention time $=14.61 \mathrm{~min}$ (minor) and $16.39 \min$ (major). $[\alpha]_{\mathrm{D}}{ }^{25}=-32.00\left(\mathrm{c}=0.200, \mathrm{CHCl}_{3}, 93: 7\right.$ e.r).

2019/6/13 $3: 4$

9041 HWC-13-102-3B ODH 9732140.7

\begin{tabular}{llll}
\hline Sample Name: & HWC-13-102-3B ODH 973 214 0.7 & injection Volume: & 3.0 \\
Vial Number: & GC2 & Channel: & UV_VIS_1 \\
Sample Type: & unknown & Wavelength: & 214 \\
Control Program: & $201701-6$ & Bandwidth: & n.a. \\
Quantif. Method: & 201701 & Dilution Factor: & 1.0000 \\
Recording Time: & $2019 / 3 / 1815: 39$ & Sample Weight: & 1.0000 \\
Run Time (min): & 60.01 & Sample Amount: & 1.0000 \\
\hline
\end{tabular}

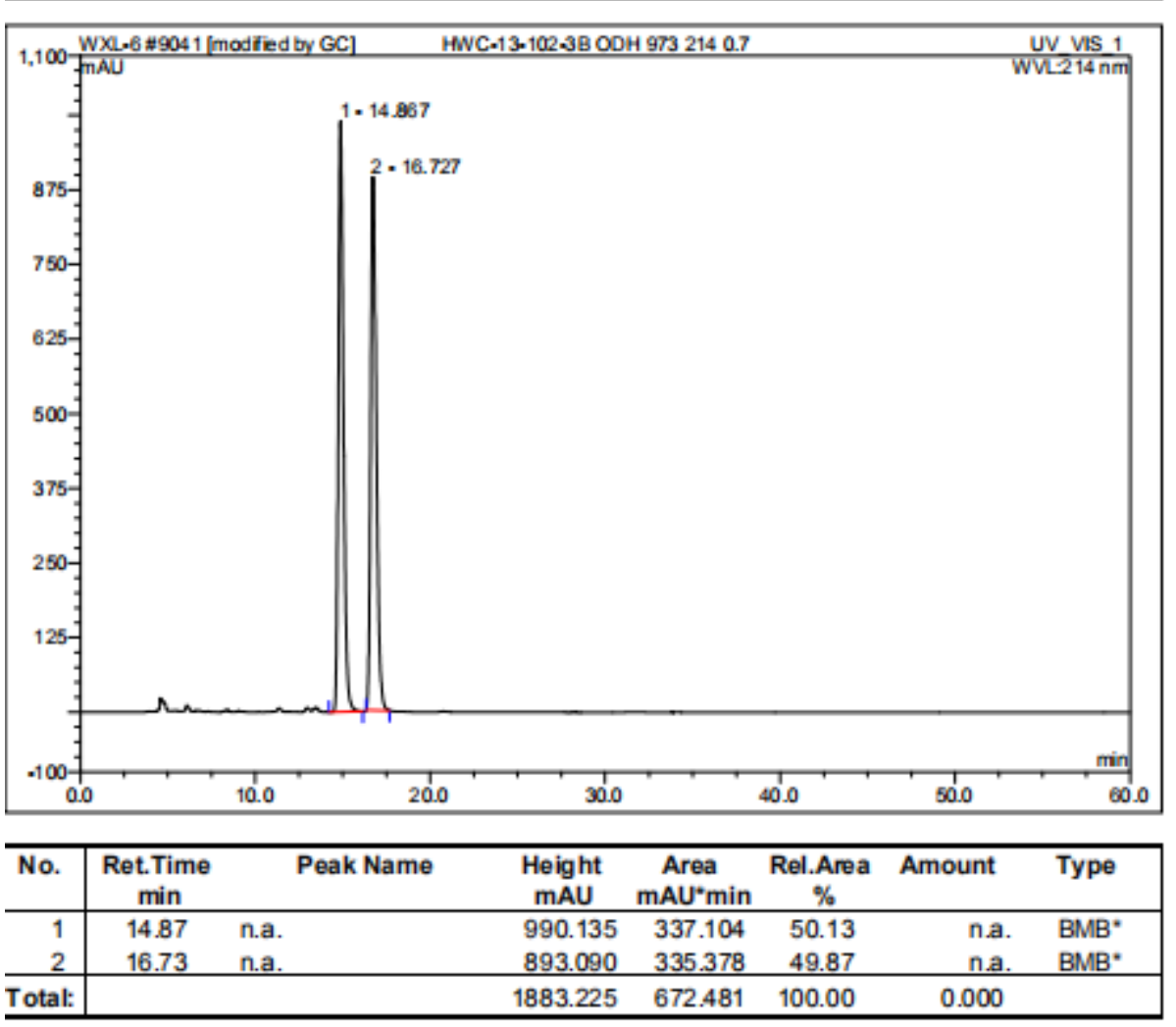


9042 HWC-13-103-3B ODH 9732140.7

\begin{tabular}{|llll|}
\hline Sample Name: & HWC-13-103-3B ODH973214 0.7 & Injection Volume: & 3.0 \\
Vial Number: & GB1 & Channel: & UV_VIS_1 \\
Sample Type: & unknown & Wavelength: & 214 \\
Control Program: & $201701-6$ & Bandwidth: & n.a. \\
Quantif. Method: & 201701 & Dïution Factor: & 1.0000 \\
Recording Time: & $2019 / 3 / 1817: 11$ & Sample Weight: & 1.0000 \\
Run Time (min): & 21.68 & Sample Amount: & 1.0000 \\
\hline
\end{tabular}

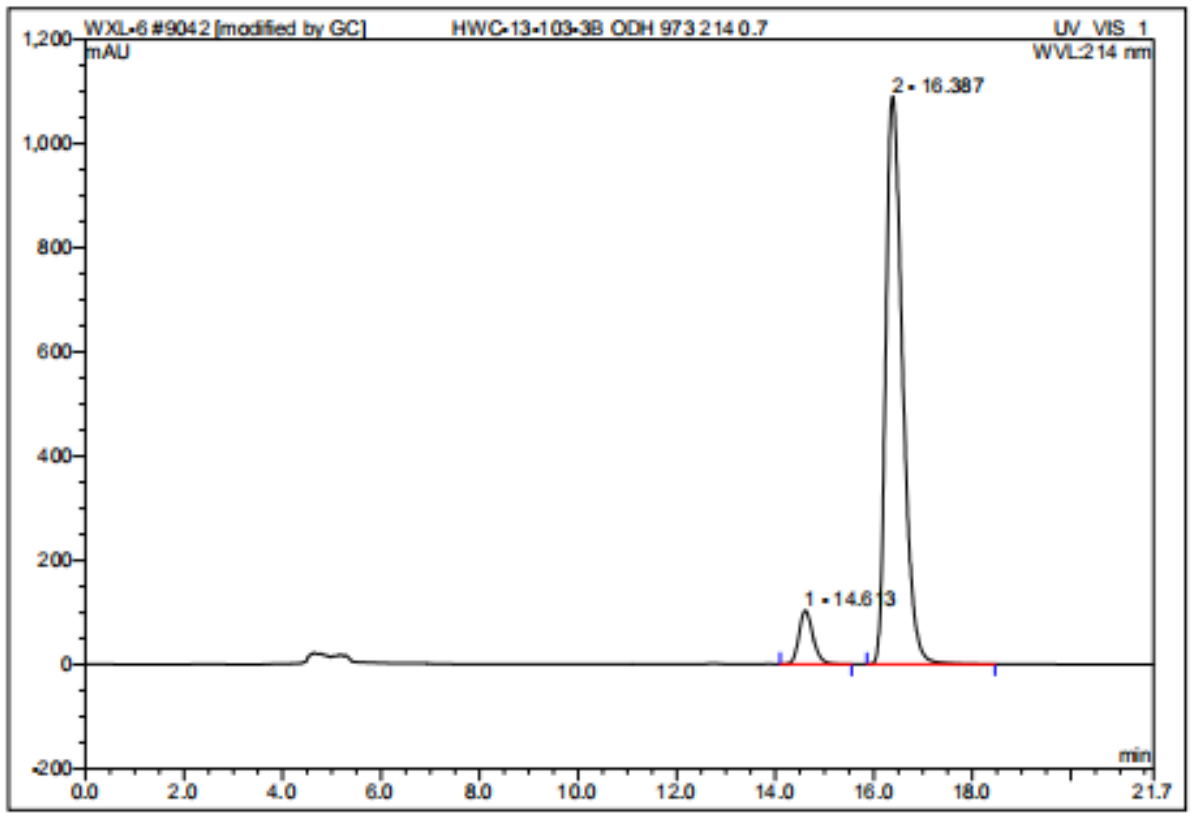

\begin{tabular}{|r|ccrrrrr|}
\hline No. & $\begin{array}{c}\text { Ret.Time } \\
\text { min }\end{array}$ & Peak Name & $\begin{array}{c}\text { Height } \\
\text { mAU }\end{array}$ & $\begin{array}{c}\text { Area } \\
\text { mAU*min }\end{array}$ & $\begin{array}{r}\text { Rel.Area } \\
\%\end{array}$ & Amount & Type \\
\hline 1 & 14.61 & n.a. & 103.799 & 34.026 & 7.32 & n.a. & BMB \\
2 & 16.39 & n.a. & 1089.853 & 430.844 & 92.68 & n.a. & BMB \\
\hline Total: & & & 1193.652 & 464.869 & 100.00 & 0.000 & \\
\hline
\end{tabular}


<smiles>N#Cc1cccc(C(F)c2cccc(F)c2)c1</smiles>

Compound 31. HPLC (AD-H, $0.46 \times 25 \mathrm{~cm}, 5 \mu \mathrm{m}$, hexane/isopropanol = 99/1 (v/v \%), flow $0.7 \mathrm{~mL} / \mathrm{min}$, UV detection at $214 \mathrm{~nm}$ ), retention time $=23.99 \mathrm{~min}$ (minor) and $25.56 \min$ (major). $[\alpha]_{\mathrm{D}}{ }^{25}=-8.00\left(\mathrm{c}=0.090, \mathrm{CHCl}_{3}, 96: 4\right.$ e.r).

\begin{tabular}{|llll|}
\hline 5661 HWC-14-50-3C+- ADH 9912140.7 & & \\
& & & \\
\hline Sample Name: & HWC-14-50-3C+- ADH 9912140.7 & Injection Volume: & 3.0 \\
Vial Number: & RC6 & Channel: & UV_VIS_2 \\
Sample Type: & unknown & Wavelength: & 214.0 \\
Contral Program: & test-dad6 & Bandwidth: & 4 \\
Quantif. Method: & 20170608 & Dilution Factor: & 1.0000 \\
Recording Time: & $2019-4-2617: 02$ & Sample Weight: & 1.0000 \\
Run Time (min): & 29.95 & Sample Amount: & 1.0000 \\
\hline
\end{tabular}

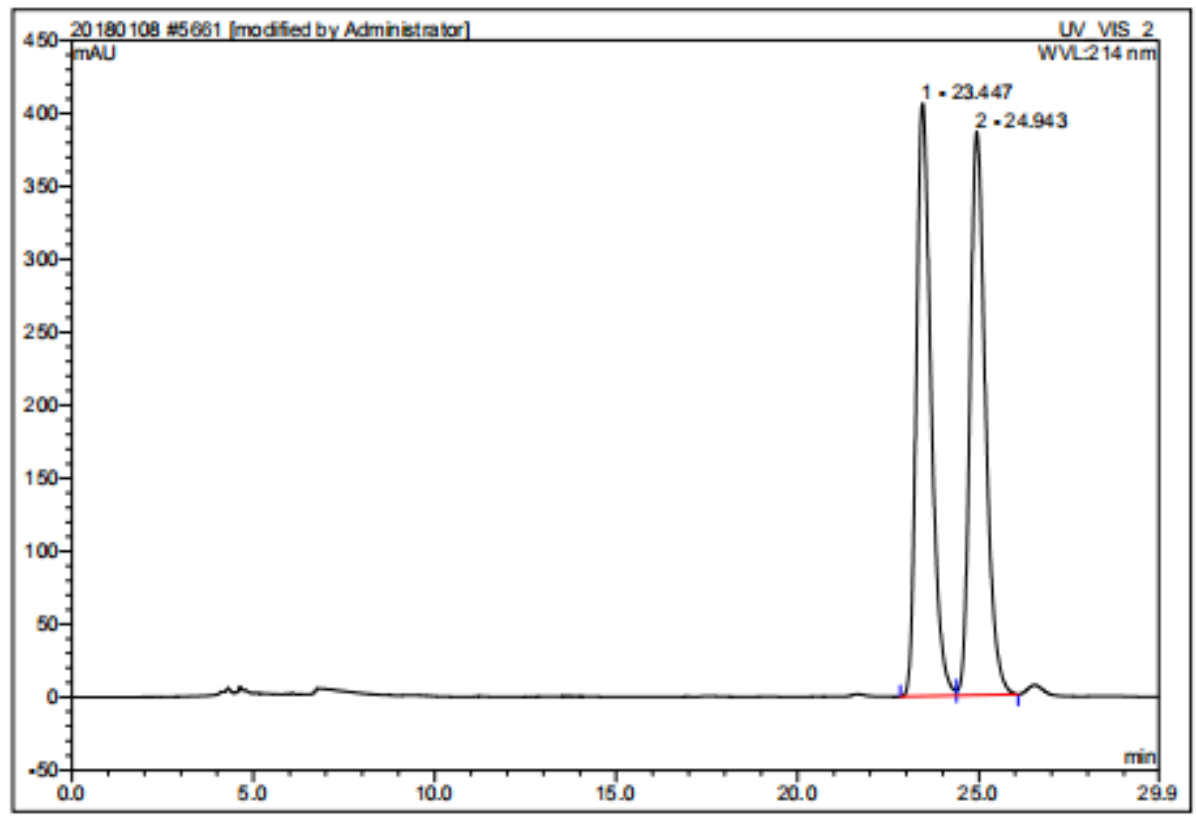

\begin{tabular}{|c|c|c|c|c|c|c|c|}
\hline No. & $\begin{array}{c}\text { Ret.Time } \\
\text { min }\end{array}$ & Peak Name & $\begin{array}{c}\text { Height } \\
\text { mAU }\end{array}$ & $\begin{array}{c}\text { Area } \\
\text { mAU*min }\end{array}$ & $\begin{array}{c}\text { Rel.Area } \\
\%\end{array}$ & Amount & Type \\
\hline 1 & 23.45 & n.a. & 406.343 & 191.008 & 50.02 & n.a. & $\mathrm{BM}^{*}$ \\
\hline 2 & 24.94 & n.a. & 386.227 & 190.828 & 49.98 & n.a. & $\mathrm{MB}^{*}$ \\
\hline Total: & & & 792.570 & 381.836 & 100.00 & 0.000 & \\
\hline
\end{tabular}




\begin{tabular}{|llll|}
\hline 5662 HWC-14-49-3C ADH 9912140.7 & & \\
& & & \\
\hline Sample Name: & HWC-14-49-3C ADH 9912140.7 & Injection Volume: & 3.0 \\
Vial Number: & RC4 & Channel: & UV_VIS_2 \\
Sample Type: & unknown & Wavelength: & 214.0 \\
Contral Program: & test-dad6 & Bandwidth: & 4 \\
Quantif. Method: & 20170608 & Dilution Factor: & 1.0000 \\
Recording Time: & $2019-4-2616: 28$ & Sample Weight & 1.0000 \\
Run Time (min): & 32.37 & Sample Amount: & 1.0000 \\
\hline
\end{tabular}

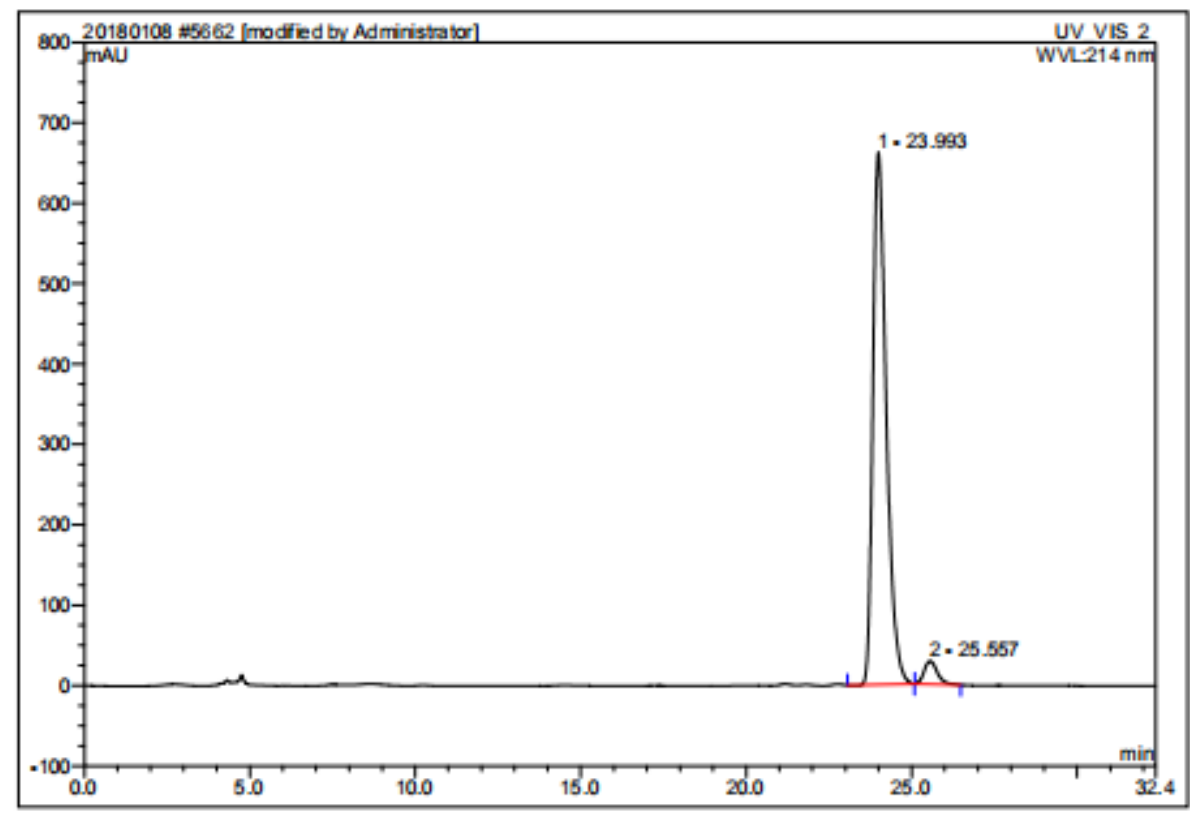

\begin{tabular}{|c|ccrrrrr|}
\hline No. & $\begin{array}{c}\text { Ret.Time } \\
\text { min }\end{array}$ & Peak Name & $\begin{array}{c}\text { Height } \\
\text { mAU }\end{array}$ & $\begin{array}{c}\text { Area } \\
\text { mAU*min }\end{array}$ & $\begin{array}{r}\text { Rel_Area } \\
\%\end{array}$ & Amount & Type \\
\hline 1 & 23.99 & n.a. & 662.065 & 309.534 & 95.88 & n.a. & BMB* $^{*}$ \\
2 & 25.56 & n.a. & 28.996 & 13.285 & 4.12 & n.a. & BMB* $^{*}$ \\
\hline Total: & & & 691.061 & 322.819 & 100.00 & 0.000 & \\
\hline
\end{tabular}




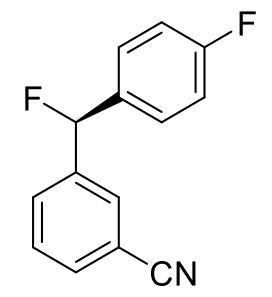

Compound 3m. HPLC (OD-H, $0.46 \times 25 \mathrm{~cm}, 5 \mu \mathrm{m}$, hexane/isopropanol $=98 / 2$ (v/v \%), flow $0.7 \mathrm{~mL} / \mathrm{min}$, UV detection at $214 \mathrm{~nm})$, retention time $=18.83 \mathrm{~min}$ (minor) and $21.63 \min$ (major). $[\alpha]_{\mathrm{D}}{ }^{25}=-4.80\left(\mathrm{c}=0.100, \mathrm{CHCl}_{3}, 94: 6\right.$ e.r).

\begin{tabular}{|c|c|c|c|}
\hline & SAMPLE & INFORMATION & \\
\hline Sample Name: & HMC-1444-3At-ODH 9822140.7 & Acqined By: & System \\
\hline Sample Type & Urkrown & Sample Set Name: & 20190409 \\
\hline Val: & $1: C, 1$ & Acq Method Set: & 1017 \\
\hline Irjection \#\#. & 1 & Processing Methat & 1 \\
\hline Irjection Vdume: & $2.00 \mathrm{u}$ & Orame Name & PDAOM1 214nm@4.8m \\
\hline Run Time: & 30.0 Mintes & Proc. Onri. Descr.: & FDAOM1 214nm@4.8m \\
\hline DateAcquired: & 4/17/2019320:30RMCST & & \\
\hline DateProcessed: & 6/1320194: 10:35 PMCST & & \\
\hline
\end{tabular}

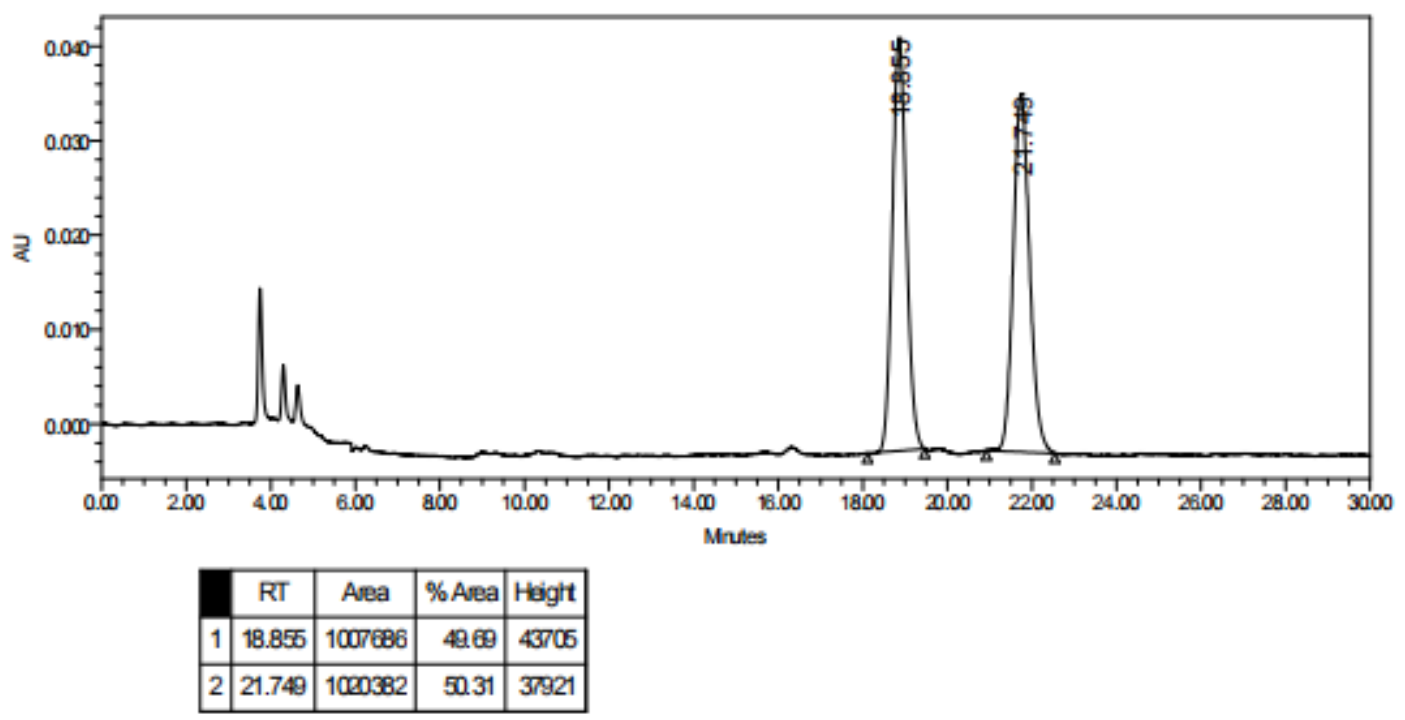




\section{Empower' 3}

\begin{tabular}{|c|c|c|c|}
\hline & SAMPLE & INFORMATION & \\
\hline Sample Name: & HMC-14-43-3A COH982 2140.7 & Acqined By: & System \\
\hline Sample Type & Urkrown & Sample Set Name: & 20190409 \\
\hline Val: & $1: \mathrm{C}, 2$ & Acq Methad Set: & 1017 \\
\hline Irjection\# & 1 & Processirg Methat & 1 \\
\hline Irjection Vaume: & $2.00 \mathrm{u}$ & Creme Name & PDAOM1 214nm@4.8m \\
\hline Run Time: & 30.0 Mintes & Proc. Onri. Descr.: & PDAOM1 214nm@4.8m \\
\hline DateAcquired: & 4/17/201935:08 RM CST & & \\
\hline DateProcessed: & 6/1320194: 10:05 RM CST & & \\
\hline
\end{tabular}

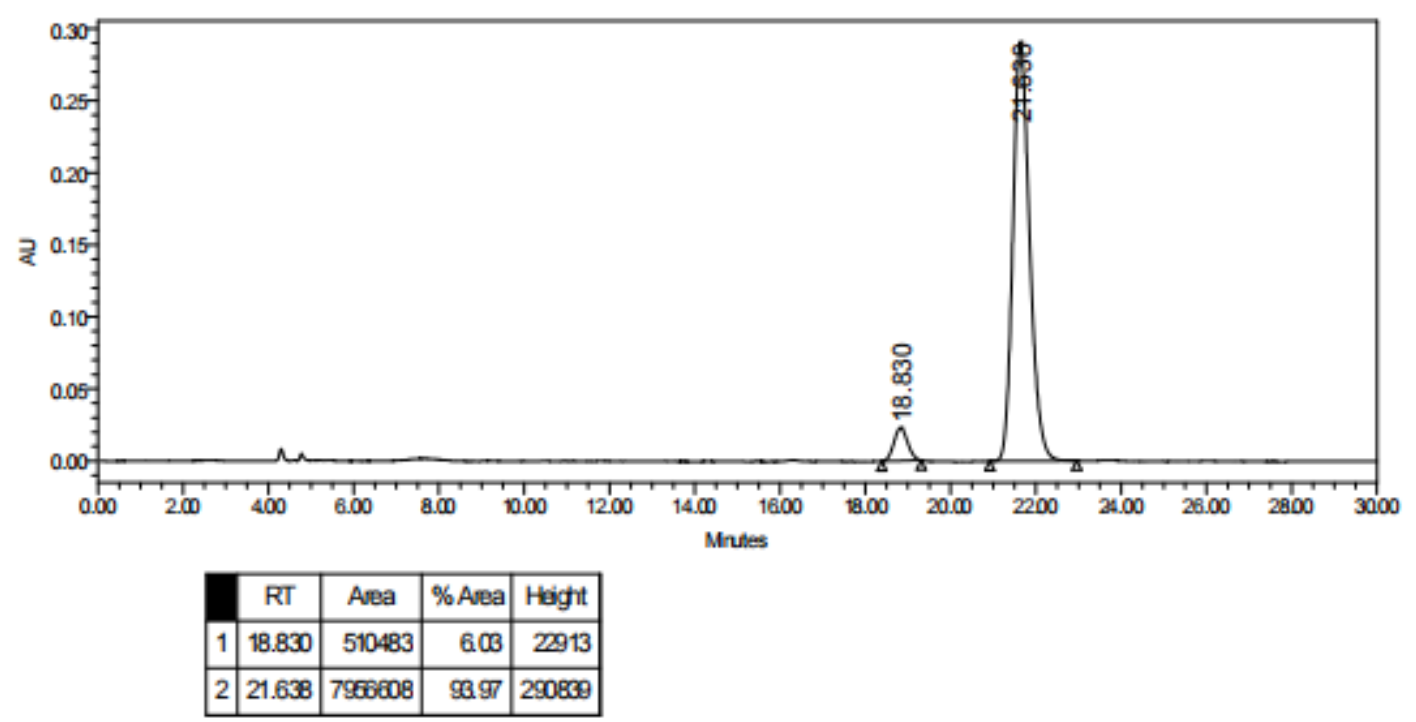


<smiles>Cc1cccc(C(F)c2cccc(C#N)c2)c1</smiles>

Compound 3n. HPLC (AD-H, $0.46 \times 25 \mathrm{~cm}, 5 \mu \mathrm{m}$, hexane/isopropanol $=98 / 2$ (v/v \%), flow $0.7 \mathrm{~mL} / \mathrm{min}$, UV detection at $214 \mathrm{~nm}$ ), retention time $=11.55 \mathrm{~min}$ (minor) and $12.82 \mathrm{~min}$ (major). $[\alpha]_{\mathrm{D}}{ }^{25}=-41.60\left(\mathrm{c}=0.250, \mathrm{CHCl}_{3}, 94: 6\right.$ e.r).

5984 HWC-15-11-3+- ADH 9822140.7

\begin{tabular}{|llll|}
\hline Sample Name: & HWC-15-11-3+-ADH 9822140.7 & Injection Volume: & 3.0 \\
VialNumber: & RA2 & Channel: & UV_VIS_2 \\
Sample Type: & unknown & Wavelength: & 214.0 \\
Control Program: & test-dad6 & Bandwidth: & 4 \\
Quantif. Method: & 20170608 & Dilution Factor: & 1.0000 \\
Recording Time: & $2019-5-249: 02$ & Sample Weight: & 1.0000 \\
Run Time (min): & 20.00 & Sample Amount: & 1.0000 \\
\hline
\end{tabular}

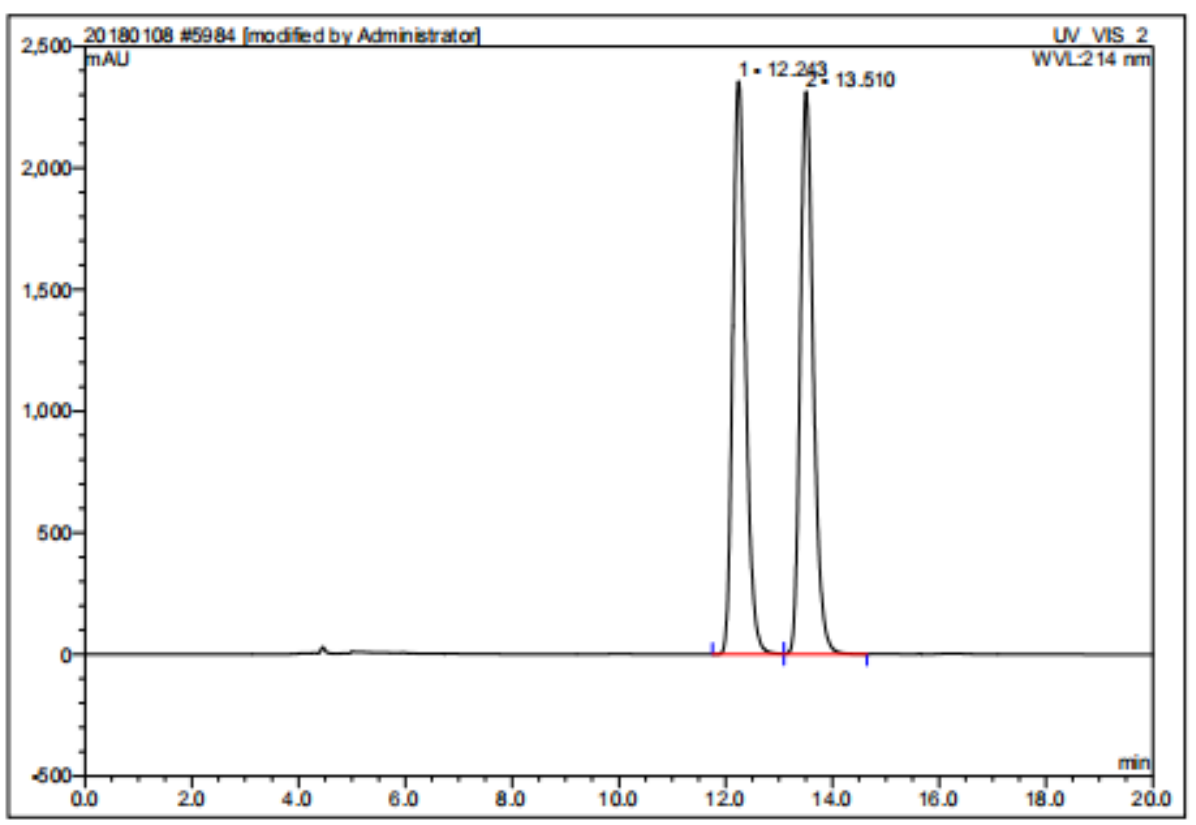

\begin{tabular}{|r|cccrrrr|}
\hline No. & $\begin{array}{c}\text { Ret.Time } \\
\text { min }\end{array}$ & Peak Name & $\begin{array}{c}\text { Height } \\
\text { mAU }\end{array}$ & $\begin{array}{c}\text { Area } \\
\text { mAU*min }\end{array}$ & $\begin{array}{c}\text { Rel.Area } \\
\%\end{array}$ & Amount & Type \\
\hline 1 & 12.24 & n.a. & 2357.352 & 655.002 & 49.84 & n.a. & BM \\
2 & 13.51 & n.a. & 2314.116 & 659.093 & 50.16 & n.a. & MB \\
\hline Total: & & & 4671.467 & 1314.095 & 100.00 & 0.000 & \\
\hline
\end{tabular}




\begin{tabular}{|llll|}
\hline 5985 HWC-14-105-3D ADH 982 & 2140.7 & & \\
& & & \\
\hline Sample Name: & HWC-14-105-3D ADH 9822140.7 & Injection Volume: & 3.0 \\
Vial Number: & RB2 & Channel: & UV_VIS_2 \\
Sample Type: & unknown & Wavelength: & 214.0 \\
Control Program: & test-dad6 & Bandwidth: & 4 \\
Quantif. Method: & 20170608 & Dilution Factor: & 1.0000 \\
Recording Time: & $2019-5-249: 23$ & Sample Weight: & 1.0000 \\
Run Time (min): & 20.00 & Sample Amount: & 1.0000 \\
\hline
\end{tabular}

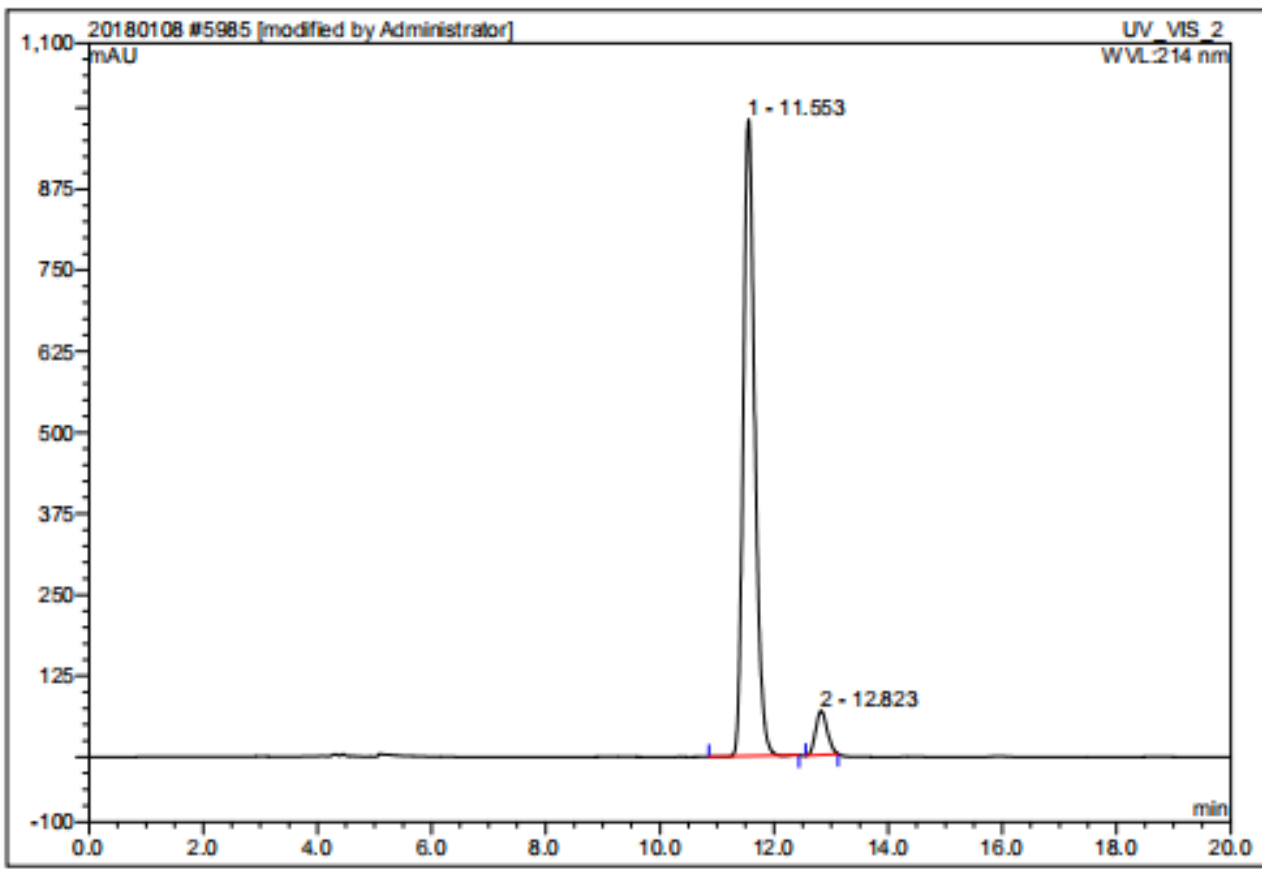

\begin{tabular}{|r|ccrrrrr|}
\hline No. & $\begin{array}{c}\text { Ret. Time } \\
\text { min }\end{array}$ & Peak Name & $\begin{array}{c}\text { Height } \\
\text { mAU }\end{array}$ & $\begin{array}{c}\text { Area } \\
\text { mAU*min }\end{array}$ & $\begin{array}{r}\text { Rel.Area } \\
\%\end{array}$ & Amount & Type \\
\hline 1 & 11.55 & n.a. & 981.155 & 229.671 & 93.50 & n.a. & BMB $^{*}$ \\
2 & 12.82 & n.a. & 67.718 & 15.960 & 6.50 & n.a. & BMB $^{*}$ \\
\hline Total: & & & 1048.874 & 245.631 & 100.00 & 0.000 & \\
\hline
\end{tabular}


<smiles>COc1cccc(C(F)c2cccc(C#N)c2)c1</smiles>

Compound 3o. HPLC $($ AD-H, $0.46 \times 25 \mathrm{~cm}, 5 \mu \mathrm{m}$, hexane/isopropanol $=98 / 2$ (v/v \%), flow $0.7 \mathrm{~mL} / \mathrm{min}$, UV detection at $214 \mathrm{~nm}$ ), retention time $=21.76 \mathrm{~min}$ (minor) and $23.41 \mathrm{~min}$ (major). $[\alpha]_{\mathrm{D}}{ }^{25}=-12.97$ (c $=0.370, \mathrm{CHCl}_{3}, 93: 7$ e.r).

Operator:Administrator Timebase:HPLC Sequence 20180108 $2019-6-13 \quad 2: 54$

5814 HWC-14-80-3B+- ADH 9822140.7

\begin{tabular}{|llll|}
\hline Sample Name: & HWC-14-80-3B+-ADH 982 214 0.7 & injection Volume: & 2.0 \\
Vial Number: & RB8 & Channet & UV_uS_2 \\
Sample Type: & unknown & Wavelength: & 214.0 \\
Control Program: & test-dad6 & Bandwibth: & 4 \\
Quanti. Method: & 20170608 & Dilution Factor: & 1.0000 \\
Recording Time: & $\mathbf{2 0 1 9 - 5 - 7 1 6 : 3 1}$ & Sample Weight: & 1.0000 \\
Run Time (min): & $\mathbf{4 0 . 0 0}$ & Sample Amount: & 1.0000 \\
\hline
\end{tabular}

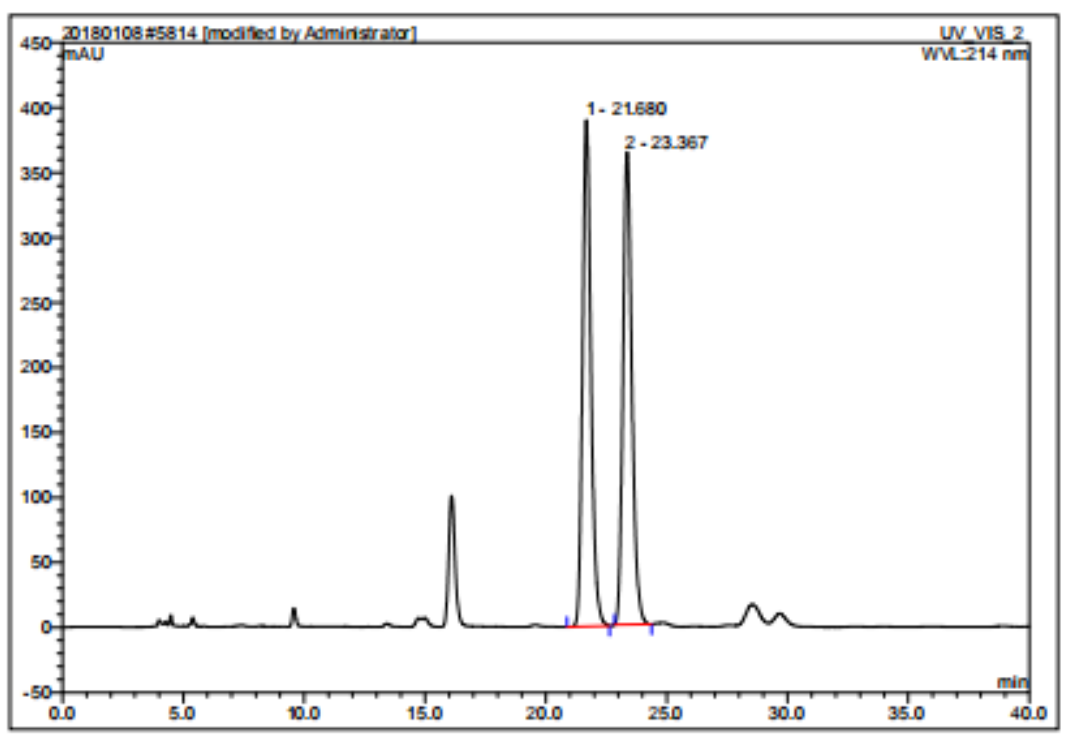

\begin{tabular}{|r|cccrrrr|}
\hline No. & $\begin{array}{c}\text { Ret.Time } \\
\text { min }\end{array}$ & Peak Name & $\begin{array}{c}\text { Height } \\
\text { mAU }\end{array}$ & $\begin{array}{r}\text { Area } \\
\text { mAU'min }\end{array}$ & $\begin{array}{r}\text { RelArea } \\
\%\end{array}$ & Amount & Type \\
\hline 1 & 21.68 & n.a. & 389.923 & 157.739 & 50.30 & n.a. & BMB $^{*}$ \\
2 & 23.37 & n.a. & 363.554 & 155.829 & 49.70 & n.a. & BMB $^{*}$ \\
\hline Total: & & & 753.478 & 313.568 & 100.00 & 0.000 & \\
\hline
\end{tabular}




\section{HWC-14-79-3B ADH 9822140.7}

\begin{tabular}{llll|}
\hline Sample Name: & HWC-14-79-3B ADH 9822140.7 & injection Volume: & 2.0 \\
Vial Number: & RC7 & Channet & UV_uS_2 \\
Sample Type: & unknown & Wavelength & 214.0 \\
Control Program: & test-dad6 & Bandwidth: & 4 \\
Quanbif. Method: & 20170608 & Dilution Factor: & 1.0000 \\
Recording Time: & $2019-5-717: 13$ & Sample Weight: & 1.0000 \\
Run Time (min): & 40.00 & Sample Amount: & 1.0000 \\
\hline
\end{tabular}

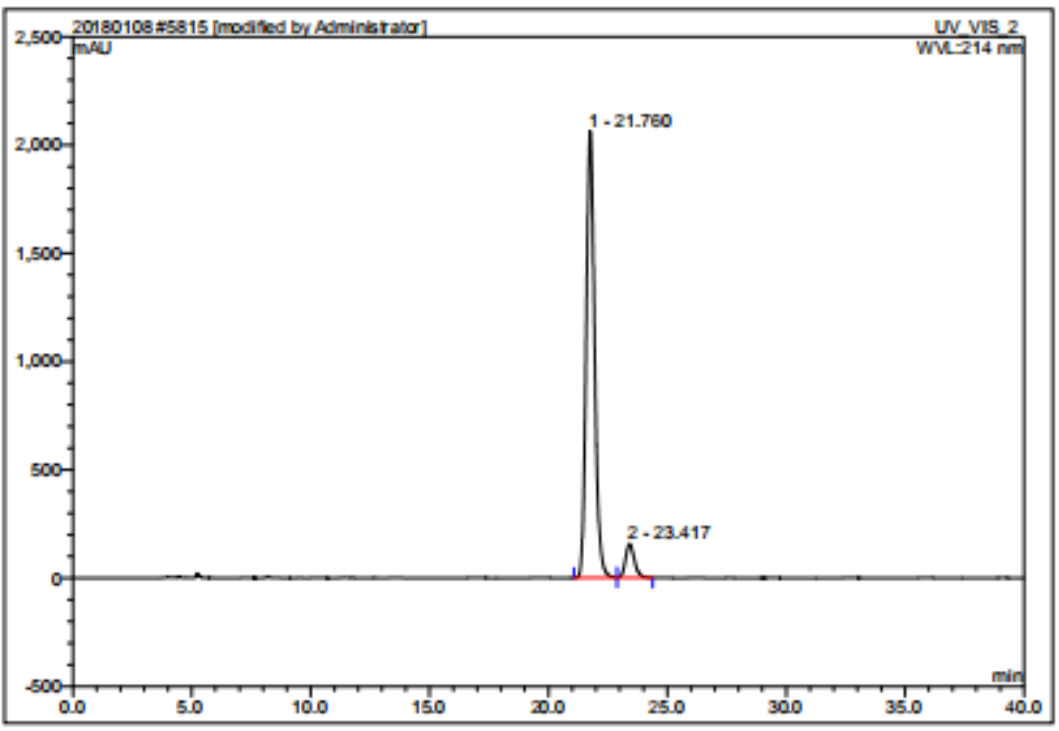

\begin{tabular}{|r|ccrrrrr|}
\hline No. & $\begin{array}{c}\text { Ret.Time } \\
\text { min }\end{array}$ & Peak Name & $\begin{array}{c}\text { Height } \\
\text { mAU }\end{array}$ & $\begin{array}{c}\text { Area } \\
\text { mAU'min }\end{array}$ & $\begin{array}{r}\text { RelArea } \\
\text { \% }\end{array}$ & Amount & Type \\
\hline 1 & 21.76 & n.a. & 2064.362 & 843.953 & 92.51 & n.a. & BM \\
2 & 23.42 & na. & 158.113 & 68.332 & 7.49 & n.a. & MB \\
\hline Total: & & & 2222.475 & 912.284 & 100.00 & 0.000 & \\
\hline
\end{tabular}


<smiles>Cc1ccc(C(F)c2cccc(C#N)c2)cc1F</smiles>

Compound 3p.HPLC (AD-H, $0.46 \times 25 \mathrm{~cm}, 5 \mu \mathrm{m}$, hexane/isopropanol = 98/2 (v/v \%), flow $0.7 \mathrm{~mL} / \mathrm{min}$, UV detection at $214 \mathrm{~nm}$ ), retention time $=14.21 \mathrm{~min}$ (minor) and $15.00 \min$ (major). $[\alpha]_{\mathrm{D}}^{25}=-18.00\left(\mathrm{c}=0.250, \mathrm{CHCl}_{3}, 95: 5\right.$ e.r $)$.

5839 HWC-14-92-3B+- ADH 9822140.7

\begin{tabular}{llll}
\hline Sample Name: & HWC-14-92-3B+- ADH 982 214 0.7 & hjection Volume: & 2.0 \\
Vial Number: & RC7 & Channel: & UV_VIS_2 \\
Sample Type: & unknown & Wavelength: & 214.0 \\
Control Program: & test-dad6 & Bandwidth: & 4 \\
Quantif. Method: & 20170608 & Dïution Factor: & 1.0000 \\
Recording Time: & $2019-5-917: 14$ & Sample Weight: & 1.0000 \\
Run Time (min): & 35.00 & Sample Amount: & 1.0000 \\
\hline
\end{tabular}

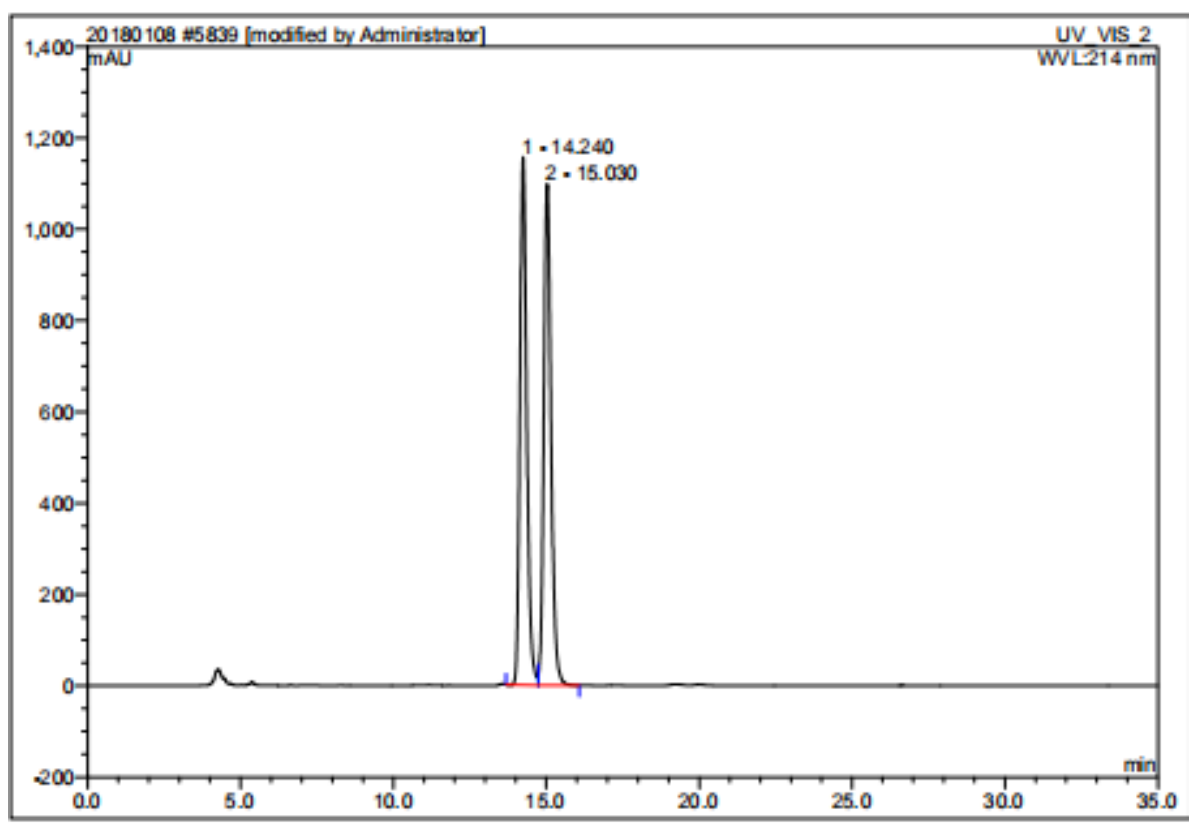

\begin{tabular}{r|ccccccc}
\hline No. & $\begin{array}{c}\text { Ret.Time } \\
\text { min }\end{array}$ & Peak Name & $\begin{array}{c}\text { Height } \\
\text { mAU }\end{array}$ & $\begin{array}{c}\text { Area } \\
\text { mAU*min }\end{array}$ & $\begin{array}{c}\text { Rel.Area } \\
\%\end{array}$ & Amount & Type \\
\hline 1 & 14.24 & n.a. & 1156.658 & 301.766 & 49.95 & n.a. & BM $^{*}$ \\
2 & 15.03 & n.a. & 1096.623 & 302.367 & 50.05 & n.a. & MB $^{*}$ \\
\hline Total: & & & 2253.281 & 604.133 & 100.00 & 0.000 & \\
\hline
\end{tabular}




\section{HWC-14-92-3A ADH 9822140.7}

\begin{tabular}{|llll|}
\hline Sample Name: & HWC-14-92-3A ADH 982 214 0.7 & Injection Volume: & 2.0 \\
Vial Number: & RC8 & Channel: & UV_VIS_2 \\
Sample Type: & unknown & Wavelength: & 214.0 \\
Cantrol Program: & test-dad6 & Bandwidth: & 4 \\
Quantif. Method: & 20170608 & Dilution Factor: & 1.0000 \\
Recording Time: & $2019-5-917: 50$ & Sample Weight: & 1.0000 \\
Run Time (min): & 20.00 & Sample Amount: & 1.0000 \\
\hline
\end{tabular}

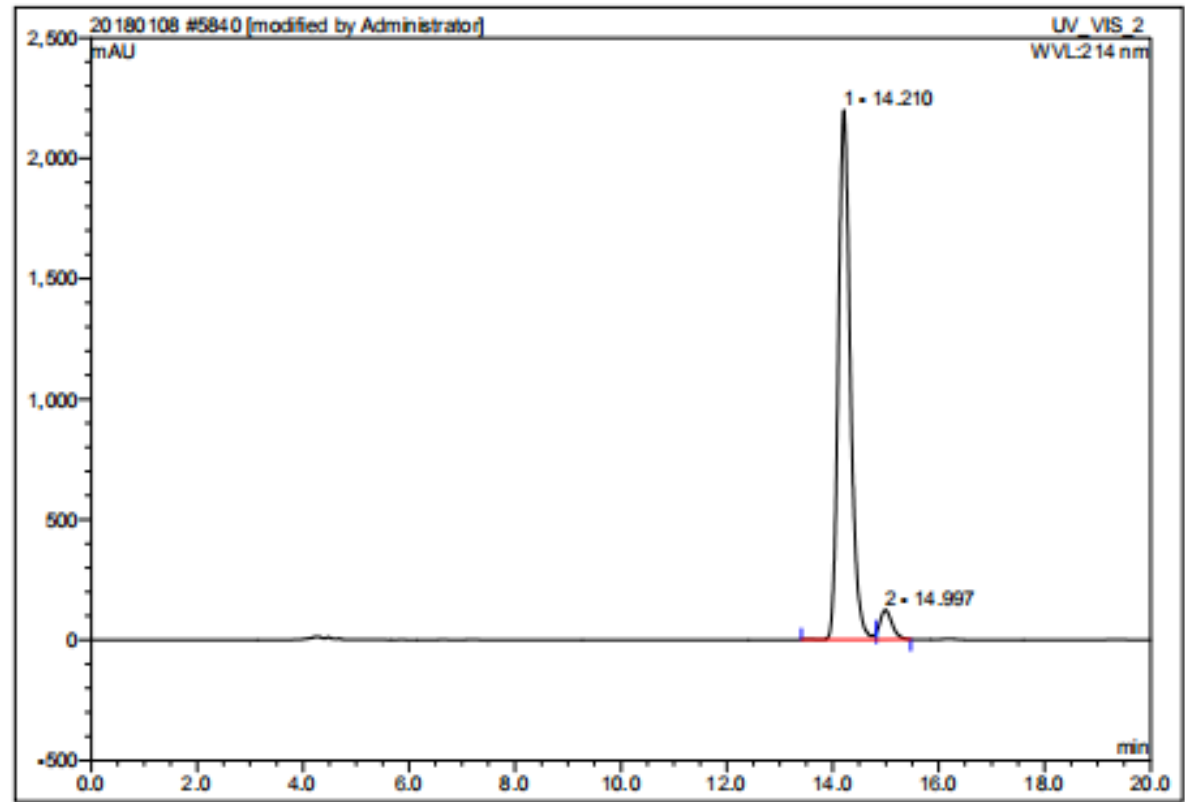

\begin{tabular}{|c|ccrrrrr|}
\hline No. & $\begin{array}{c}\text { Ret. Time } \\
\text { min }\end{array}$ & Peak Name & $\begin{array}{c}\text { Height } \\
\text { mAU }\end{array}$ & $\begin{array}{c}\text { Area } \\
\text { mAU } \text { min }\end{array}$ & $\begin{array}{r}\text { Rel.Area } \\
\%\end{array}$ & Amount & Type \\
\hline 1 & 14.21 & n.a. & 2199.243 & 577.460 & 94.65 & n.a. & BM $^{*}$ \\
2 & 15.00 & n.a. & 123.813 & 32.625 & 5.35 & n.a. & MB $^{*}$ \\
\hline Total: & & & 2323.056 & 610.085 & 100.00 & 0.000 & \\
\hline
\end{tabular}


<smiles>N#Cc1ccc(C(F)c2ccccc2)cc1</smiles>

Compound 3q. HPLC (OD-H, $0.46 \times 25 \mathrm{~cm}, 5 \mu \mathrm{m}$, hexane/isopropanol $=97 / 3$ $(\mathrm{v} / \mathrm{v} \%)$, flow $0.7 \mathrm{~mL} / \mathrm{min}$, $\mathrm{UV}$ detection at $214 \mathrm{~nm})$, retention time $=18.63 \mathrm{~min}$ (minor) and $20.24 \mathrm{~min}$ (major). $[\alpha]_{\mathrm{D}}{ }^{25}=-1.11\left(\mathrm{c}=0.090, \mathrm{CHCl}_{3}, 95: 5\right.$ e.r).

\begin{tabular}{|llll|}
\hline 4958 HWC-13-78-3A+- ODH 9732140.7 & & \\
& & & \\
\hline Sample Name: & HWC-13-78-3A+-ODH 9732140.7 & hjection Volume: & 2.0 \\
Vial Number: & RC7 & Channel: & UV_VIS_2 \\
Sample Type: & unknown & Wavelength: & 214.0 \\
Contral Program: & test-dad4 & Bandwidth: & 4 \\
Quantif. Method: & 20170608 & Dilution Factor: & 1.0000 \\
Recording Time: & $2019-3-516: 11$ & Sample Weight: & 1.0000 \\
Run Time (min): & 35.56 & Sample Amount: & 1.0000 \\
\hline
\end{tabular}

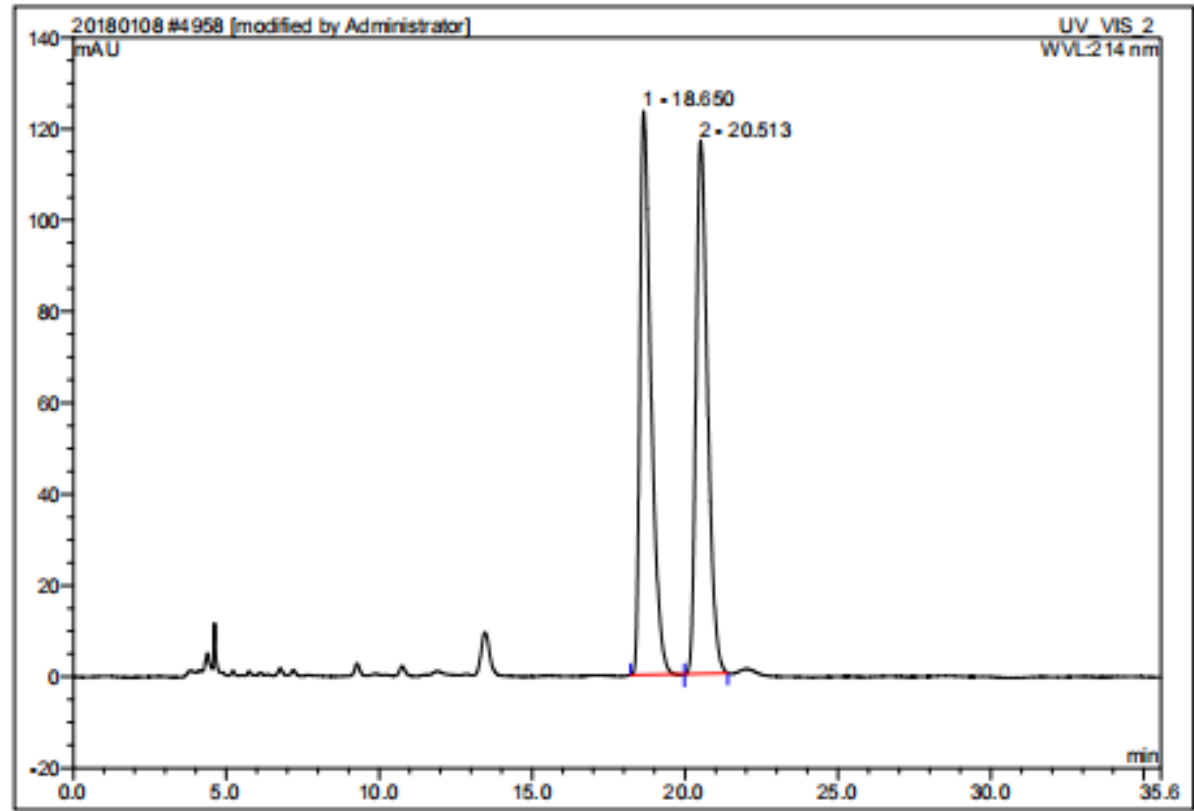

\begin{tabular}{|r|ccrrrrr|}
\hline No. & $\begin{array}{c}\text { Ret. Time } \\
\text { min }\end{array}$ & Peak Name & $\begin{array}{c}\text { Height } \\
\text { mAU }\end{array}$ & $\begin{array}{c}\text { Area } \\
\text { mAU*min }\end{array}$ & $\begin{array}{r}\text { Rel.Area } \\
\%\end{array}$ & Amount & Type \\
\hline 1 & 18.65 & n.a. & 123.555 & 51.938 & 50.29 & n.a. & BM \\
2 & 20.51 & n.a. & 116.882 & 51.342 & 49.71 & n.a. & MB \\
\hline Total: & & & 240.437 & 103.280 & 100.00 & 0.000 & \\
\hline
\end{tabular}


4959 HWC-13-75-3A ODH 9732140.7

\begin{tabular}{llll|}
\hline Sample Name: & HWC-13-75-3A ODH 973 214 0.7 & hjection Volume: & 2.0 \\
Vial Number: & RC8 & Channel: & UV_VIS_2 \\
Sample Type: & unknown & Wavelength: & 214.0 \\
Control Program: & test-dad4 & Bandwidth: & 4 \\
Quantif. Method: & 20170608 & Dilution Factor: & 1.0000 \\
Recording Time: & $2019-3-515: 25$ & Sample Weight: & 1.0000 \\
Run Time (min): & 31.87 & Sample Amount: & 1.0000 \\
\hline
\end{tabular}

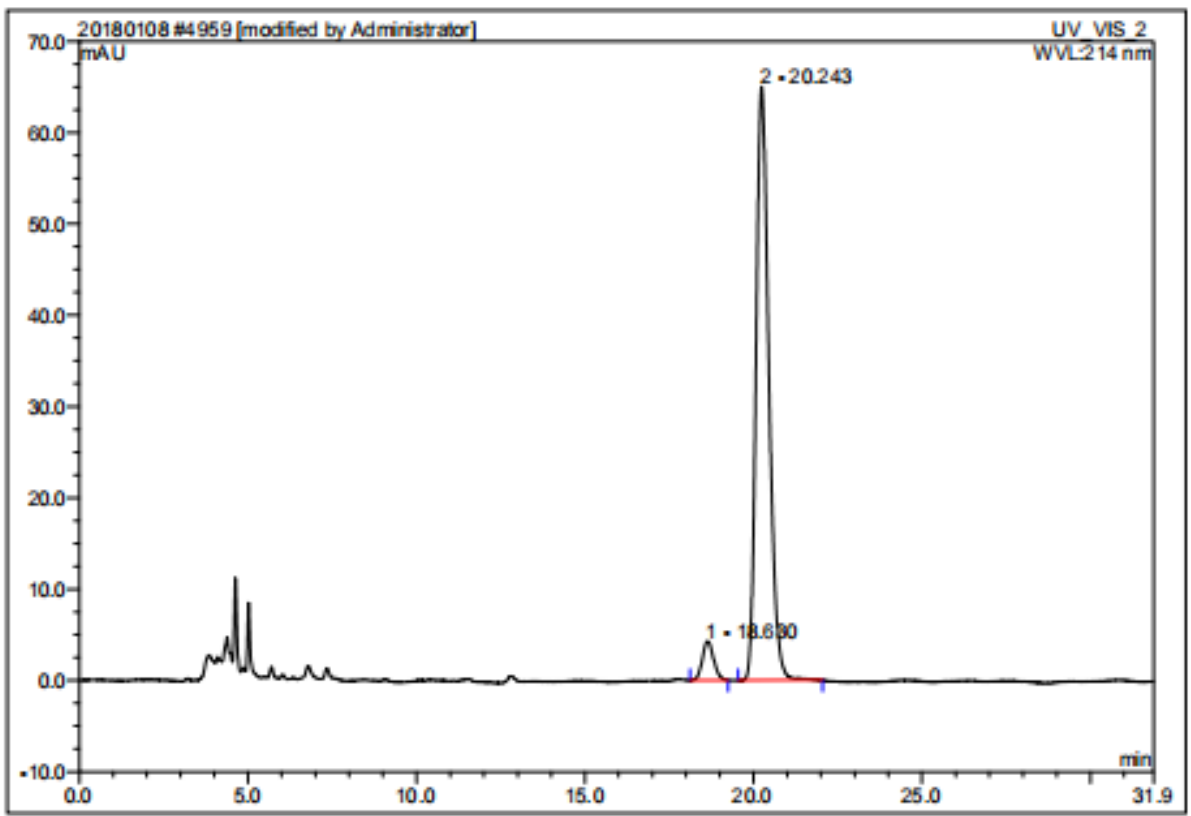

\begin{tabular}{|r|ccrrrrr|}
\hline No. & $\begin{array}{c}\text { Ret. Time } \\
\text { min }\end{array}$ & Peak Name & $\begin{array}{c}\text { Height } \\
\text { mAU }\end{array}$ & $\begin{array}{c}\text { Area } \\
\text { mAU*min }\end{array}$ & $\begin{array}{r}\text { Rel.Area } \\
\%\end{array}$ & Amount & Type \\
\hline 1 & 18.63 & n.a. & 4.258 & 1.654 & 5.48 & n.a. & BMB* $^{*}$ \\
2 & 20.24 & n.a. & 65.022 & 28.518 & 94.52 & n.a. & BMB $^{*}$ \\
\hline Total: & & & 69.280 & 30.173 & 100.00 & 0.000 & \\
\hline
\end{tabular}




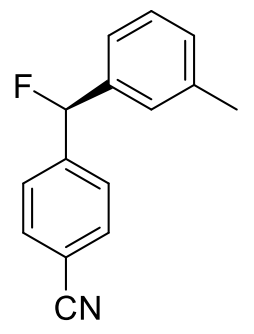

Compound 3r. HPLC $(\mathrm{PC} 3,0.46 \times 25 \mathrm{~cm}, 5 \mu \mathrm{m}$, acetonitrile/water $=80 / 20$ $(\mathrm{v} / \mathrm{v} \%$ ), flow $0.7 \mathrm{~mL} / \mathrm{min}, \mathrm{UV}$ detection at $214 \mathrm{~nm}$ ), retention time $=6.30 \mathrm{~min}$ (minor) and 6.54 min (major). $[\alpha]_{\mathrm{D}^{25}}=-22.17\left(\mathrm{c}=0.230, \mathrm{CHCl}_{3}, 94: 6\right.$ e.r).

\begin{tabular}{|c|c|c|c|}
\hline & SAMPLE & INFORMATION & \\
\hline SampleNate: & hwc-14733ct- pc3ABNR21407 & AcqiredBy. & System \\
\hline Sample Type & Unkrown & SampeSet Nane: & 20190411 \\
\hline Via: & 1:E3 & Aoq Method Set: & 1008 \\
\hline Irjection\#\# & 1 & Proossing Methoot & 1 \\
\hline Inection Vdume & $200 u$ & Chand Nane: & PDAG1214m@4.am \\
\hline RunTime & 160.0 Mrutes & Poc Orl. Desa: & PDAG1214m@4.amm \\
\hline DeteAcquired & 551201922139PMCST & & \\
\hline DateProcessed & 562019 104852AMCST & & \\
\hline
\end{tabular}

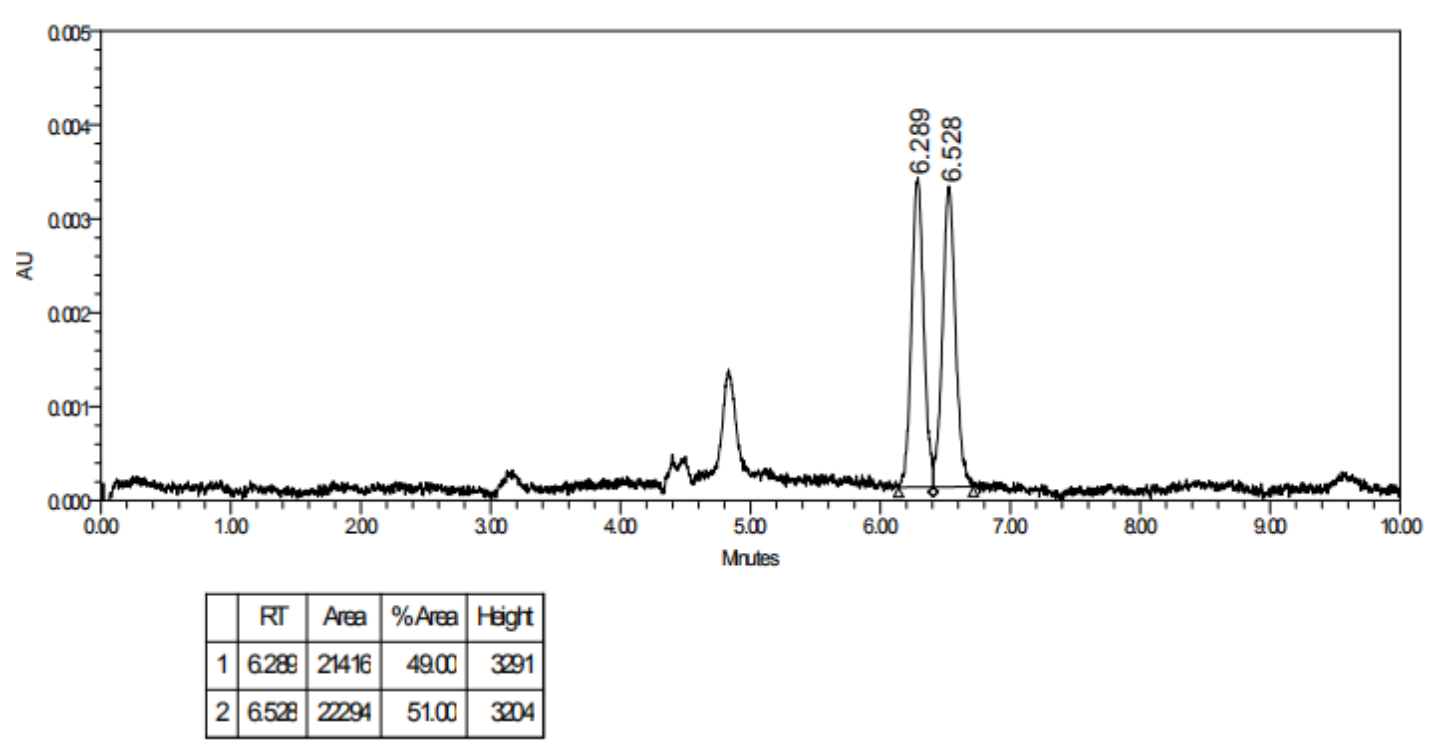




\begin{tabular}{|llll|}
\hline & \multicolumn{2}{c|}{ S A M P L E I N F O R M A T IO N } \\
\hline \hline Sample Name: & hwc-14-72-3c pc3 A8W2 214 0.7 & Acquired By: & System \\
Sample Type: & Unknow n & Sample Set Name: & 20190411 \\
Vial: & $1:$ E.4 & Acq. Method Set: & 1008 \\
njection \#: & 1 & Processing Method: & 1 \\
hjection Volume: & 3.00 ul & Channel Name: & PDA Ch1 214nm@4.8nm \\
Run Time: & 16.0 Mnutes & Proc. Chnl. Descr.: & PDA Ch1 214nm@4.8nm \\
& & & \\
Date Acquired: & $5 / 5 / 2019$ 5:22:27 PM CST & & \\
Date Processed: & $6 / 13 / 2019$ 4:29:55 PM CST & & \\
\hline
\end{tabular}

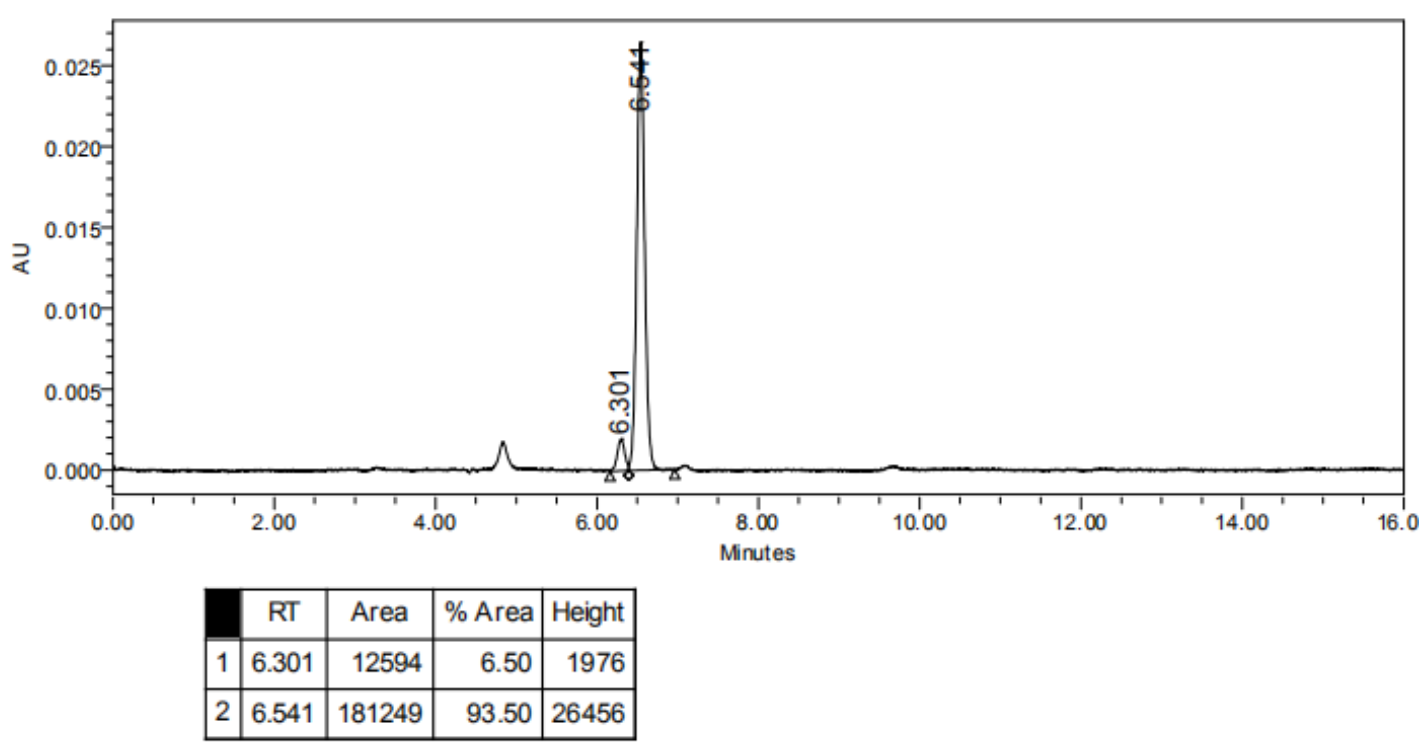


<smiles>N#Cc1ccc(C(F)c2cccc(F)c2)cc1</smiles>

Compound 3s. HPLC (AD-H, $0.46 \times 25 \mathrm{~cm}, 5 \mu \mathrm{m}$, hexane/isopropanol = 99/1 (v/v \%), flow $0.7 \mathrm{~mL} / \mathrm{min}$, UV detection at $214 \mathrm{~nm}$ ), retention time $=23.99 \mathrm{~min}$ (minor) and $25.55 \min$ (major). $[\alpha]_{\mathrm{D}}^{25}=-26.84\left(\mathrm{c}=0.190, \mathrm{CHCl}_{3}, 96: 4\right.$ e.r).

5661 HWC-14-50-3C+- ADH 9912140.7

Sample Name: Vial Number: Sample Type: Control Program: Quantif. Method: Recording Time: Run Time (min):

\begin{tabular}{lll|} 
HWC-14-50-3C+- ADH 9912140.7 & Injection Volume: & 3.0 \\
RC6 & Channel: & UV_VIS_2 \\
unknown & Wavelength: & 214.0 \\
test-dad6 & Bandwidth: & 4 \\
20170608 & Dilution Factor: & 1.0000 \\
$2019-4-2617: 02$ & Sample Weight: & 1.0000 \\
29.95 & Sample Amount: & 1.0000
\end{tabular}

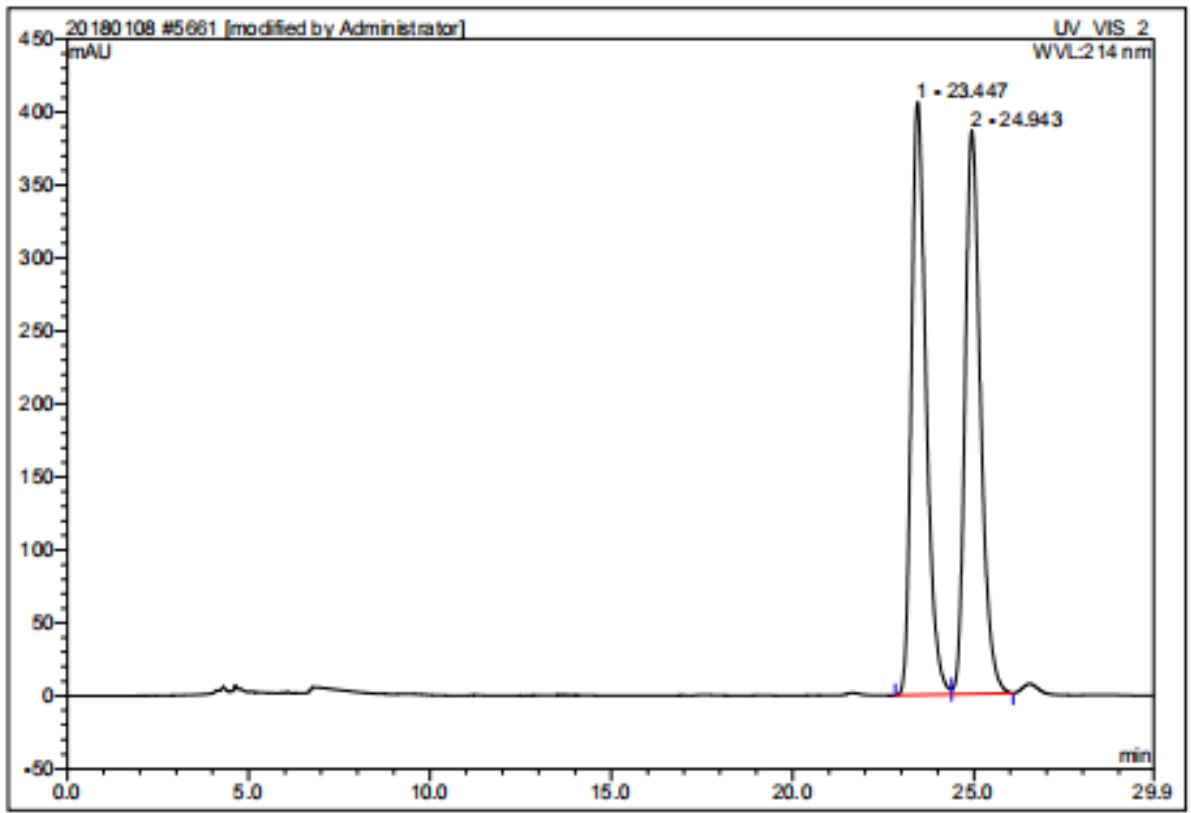

\begin{tabular}{|c|c|c|c|c|c|c|c|}
\hline No. & $\begin{array}{c}\text { Ret.Time } \\
\text { min }\end{array}$ & Peak Name & $\begin{array}{c}\text { Height } \\
\text { mAU }\end{array}$ & $\begin{array}{c}\text { Area } \\
\text { mAU*min }\end{array}$ & $\begin{array}{c}\text { Rel.Area } \\
\%\end{array}$ & Amount & Type \\
\hline 1 & 23.45 & n.a. & 406.343 & 191.008 & 50.02 & n.a. & $\mathrm{BM}^{*}$ \\
\hline 2 & 24.94 & n.a. & 386.227 & 190.828 & 49.98 & n.a. & $\mathrm{MB}^{*}$ \\
\hline Total: & & & 792.570 & 381.836 & 100.00 & 0.000 & \\
\hline
\end{tabular}




\begin{tabular}{|llll|}
\hline 5662 HWC-14-49-3C ADH 9912140.7 & & \\
& & & \\
\hline Sample Name: & HWC-14-49-3C ADH 9912140.7 & Injection Volume: & 3.0 \\
Vial Number: & RC4 & Channel: & UV_VIS_2 \\
Sample Type: & unknown & Wavelength: & 214.0 \\
Control Program: & test-dad6 & Bandwidth: & 4 \\
Quantif. Method: & 20170608 & Dilution Factor: & 1.0000 \\
Recording Time: & $2019-4-2616: 28$ & Sample Weight: & 1.0000 \\
Run Time (min): & 32.37 & Sample Amount: & 1.0000 \\
\hline
\end{tabular}

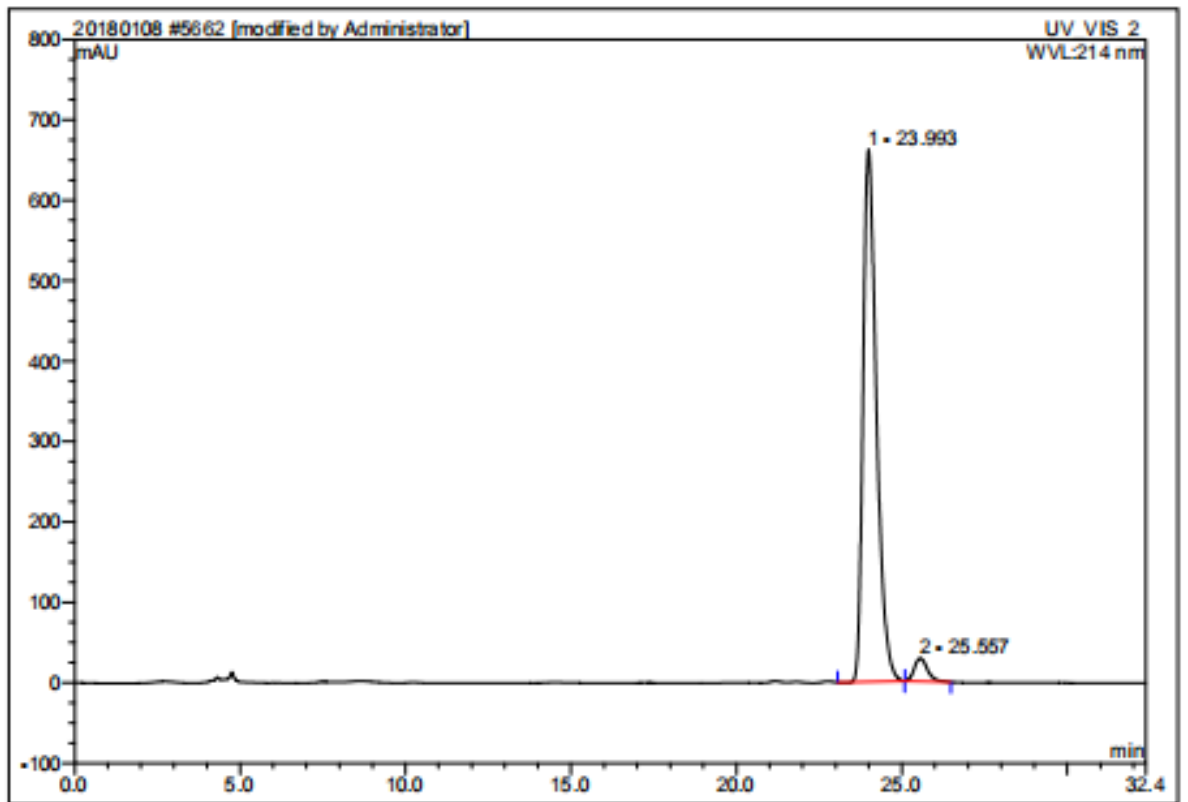

\begin{tabular}{|c|c|c|c|c|c|c|c|}
\hline No. & $\begin{array}{c}\begin{array}{c}\text { Ret. Time } \\
\text { min }\end{array} \\
\end{array}$ & Peak Name & $\begin{array}{c}\text { Height } \\
\text { mAU }\end{array}$ & $\begin{array}{c}\text { Area } \\
\text { mAU*min }\end{array}$ & $\begin{array}{c}\text { Rel_Area } \\
\%\end{array}$ & Amount & Type \\
\hline 1 & 23.99 & n.a. & 662.065 & 309.534 & 95.88 & n.a. & BMB* $^{*}$ \\
\hline 2 & 25.56 & n.a. & 28.996 & 13.285 & 4.12 & n.a. & BMB* \\
\hline Total: & & & 691.061 & 322.819 & 100.00 & 0.000 & \\
\hline
\end{tabular}


<smiles>N#Cc1ccc(C(F)c2cccc(F)c2)cc1F</smiles>

Compound 3t.HPLC (OD-H, $0.46 \times 25 \mathrm{~cm}, 5 \mu \mathrm{m}$, hexane/isopropanol = 95/5 (v/v \%), flow $0.7 \mathrm{~mL} / \mathrm{min}$, UV detection at $214 \mathrm{~nm}$ ), retention time $=16.12 \mathrm{~min}$ (minor) and $17.82 \min$ (major). $[\alpha]_{\mathrm{D}}{ }^{25}=+16.88\left(\mathrm{c}=0.160, \mathrm{CHCl}_{3}, 96: 4\right.$ e.r).

\section{Empower"3}

\begin{tabular}{|c|c|c|c|}
\hline & SAMPLE & INFORMATION & \\
\hline Sample Name & HMC-1450-30+- CDHE652140.7 & Acquired By. & System \\
\hline Sample Type & Urknown & Sample Set Name & 20190422 \\
\hline Va: & 1:E,4 & Acq Mathod Set: & 1017 \\
\hline hjection\#: & 1 & Processing Method & 1 \\
\hline hjection Vodume & $3.00 \mathrm{u}$ & Oreme Name & PDACh1214nm@4.8nm \\
\hline Run Time & 40.0 Mnutes & Proc. Onri. Descr.: & PDACh1214nm@4.8nm \\
\hline Date Acquired & 4/25/201984323 AMCST & & \\
\hline Date Processect & 6/132019538 14 PMCST & & \\
\hline
\end{tabular}

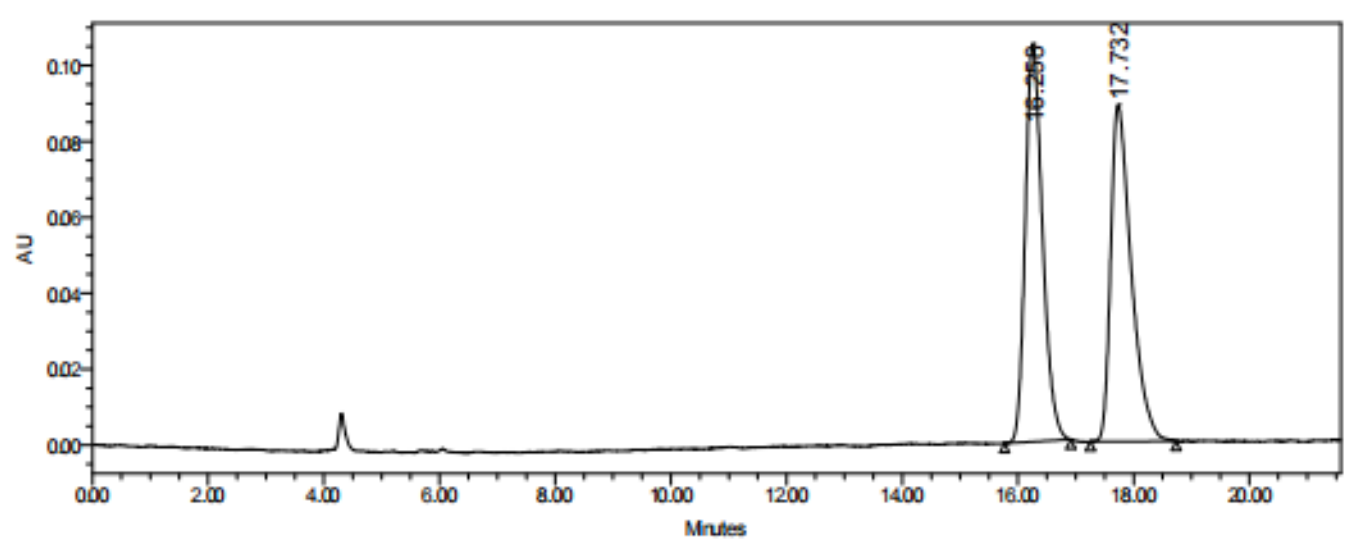

\begin{tabular}{|c|c|c|c|c|}
\hline & RT & Avea & \% Ares & Heigit \\
\hline 1 & 16258 & 2170781 & 49.81 & 104838 \\
\hline 2 & 17.732 & 2187317 & 50.19 & 88627 \\
\hline
\end{tabular}




\begin{tabular}{|c|c|c|c|}
\hline & SAMPLE & INFORMATION & \\
\hline $\begin{array}{l}\text { Sample Name: } \\
\text { Sample Type: }\end{array}$ & HMG-14-493D CDHEES2140.7 & Acquired By: & System \\
\hline $\begin{array}{l}\text { Sample Type: } \\
\text { Ma: }\end{array}$ & $\begin{array}{l}\text { Urknown } \\
\text { 1:E.5. }\end{array}$ & $\begin{array}{l}\text { Sample Set Name: } \\
\text { Aca Mathod Set: }\end{array}$ & $\begin{array}{l}20190422 \\
1017\end{array}$ \\
\hline Injection & 1 & Prowessing Mathod. & 1 \\
\hline Injection Vdume: & $3.00 u$ & Channel Name: & PDA On1 214mgat.8m \\
\hline Rin Time: & 30.0Mnutes & Prac. Ont. Descr:: & PDA Ch1 214mgat.8m \\
\hline Date Acqured. & 42572019906605 AM CST & & \\
\hline Date Processed. & 6/13/2019 5:37:50 PM CST & & \\
\hline
\end{tabular}

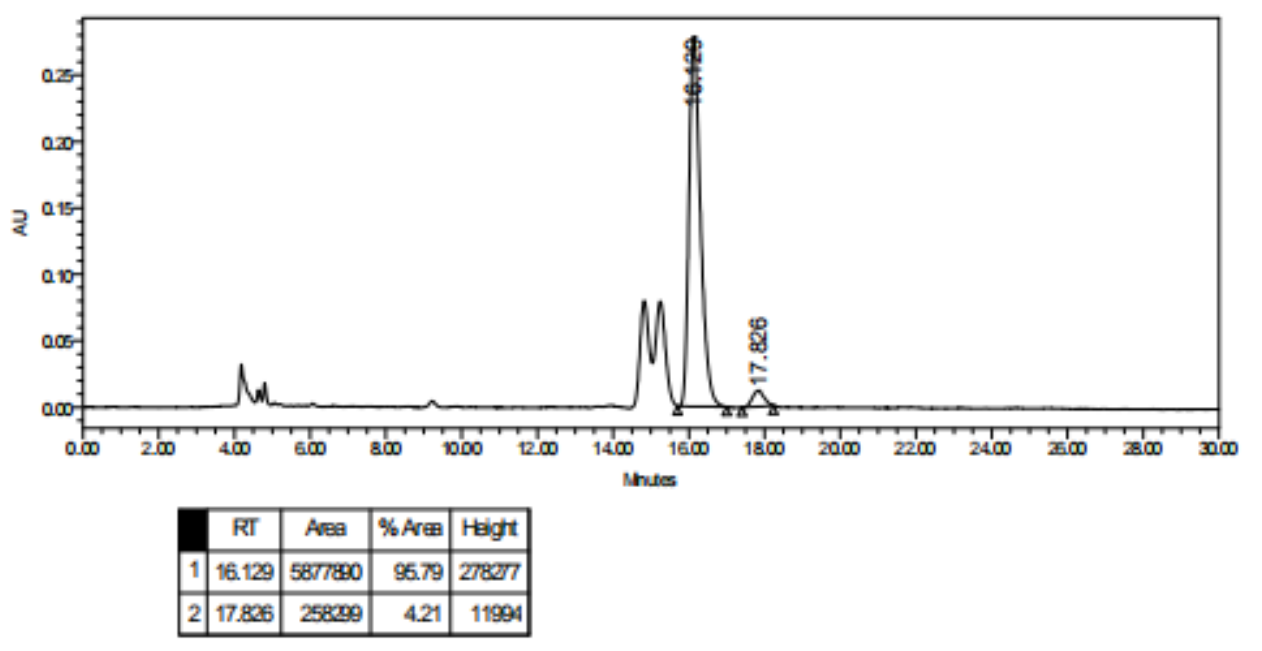

Reported by Uber. System

Project Name: Defalts

Report Mathod. 1

Report Method ID. 67:6734

Date Pinted.

Dane. 1 of?

अ-27 DA DP 
<smiles>N#Cc1ccc(C(F)c2cccc(F)c2)c(F)c1</smiles>

Compound 3u. HPLC (IE3, $0.46 \times 25 \mathrm{~cm}, 5 \mu \mathrm{m}$, hexane/isopropanol = 98/2 (v/v \%), flow $0.7 \mathrm{~mL} / \mathrm{min}$, UV detection at $214 \mathrm{~nm}$ ), retention time $=12.00 \mathrm{~min}$ (minor) and $12.68 \min$ (major). $[\alpha]_{\mathrm{D}}{ }^{25}=+0.71\left(\mathrm{c}=0.280, \mathrm{CHCl}_{3}, 91: 9\right.$ e.r).

\begin{tabular}{|llll|}
\hline 5756 HWC-14-68-3A+- IE3 9822140.7 & & \\
& & & \\
\hline Sample Name: & HWC-14-68-3A+- IE3 9822140.7 & njection Volume: & 3.0 \\
Vial Number: & RE5 & Channel: & UV_VIS_2 \\
Sample Type: & unknown & Wavelength: & 254.0 \\
Cantrol Program: & test-dad4 & Bandwidth: & 4 \\
Quantif. Method: & 20170608 & Dilution Factor: & 1.0000 \\
Recording Time: & $2019-4-3018: 29$ & Sample Weight: & 1.0000 \\
Run Time (min): & 20.00 & Sample Amount: & 1.0000 \\
\hline
\end{tabular}

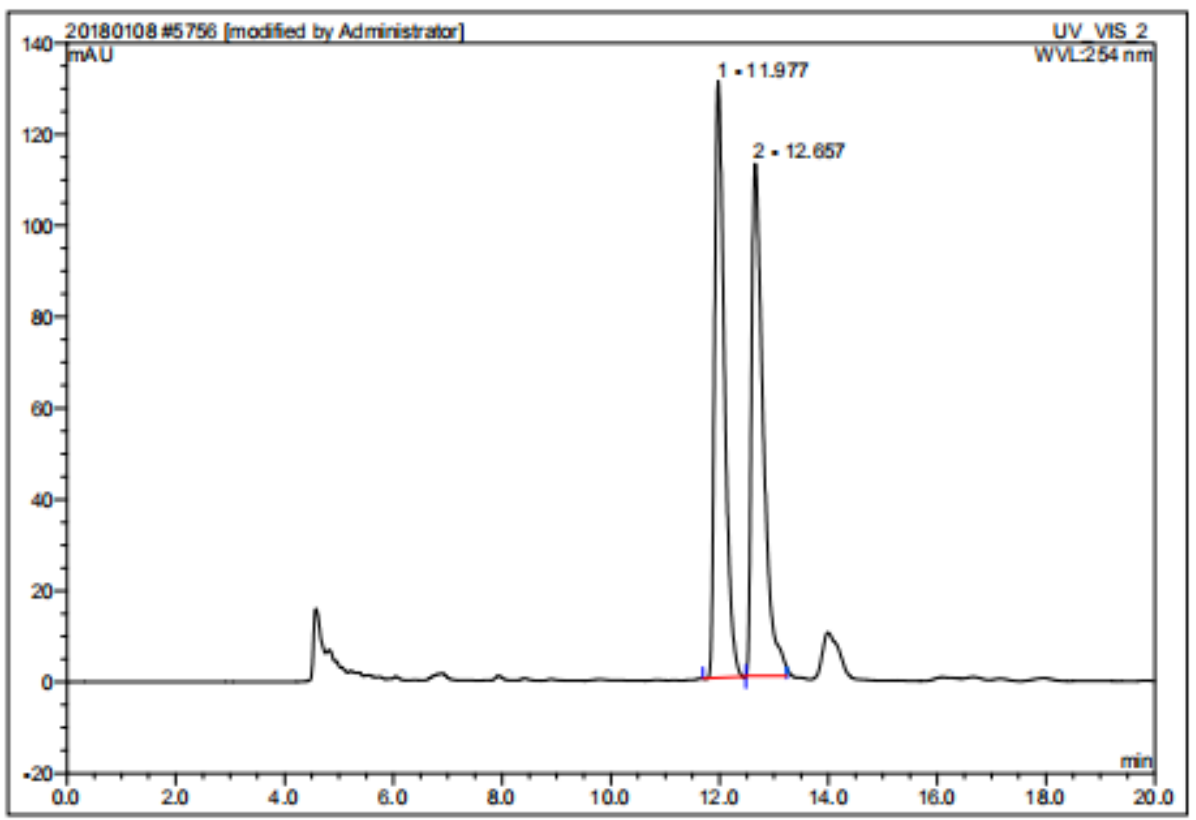

\begin{tabular}{|r|cccrrrr|}
\hline No. & $\begin{array}{c}\text { Ret. Time } \\
\text { min }\end{array}$ & Peak Name & $\begin{array}{c}\text { Height } \\
\text { mAU }\end{array}$ & $\begin{array}{c}\text { Area } \\
\text { mAU*min }\end{array}$ & $\begin{array}{c}\text { Rel.Area } \\
\%\end{array}$ & Amount & Type \\
\hline 1 & 11.98 & n.a. & 130.804 & 27.345 & 49.47 & n.a. & BMB* $^{*}$ \\
2 & 12.66 & n.a. & 112.274 & 27.931 & 50.53 & n.a. & BM $^{*}$ \\
\hline Total: & & & 243.079 & 55.276 & 100.00 & 0.000 & \\
\hline
\end{tabular}




\begin{tabular}{|llll|}
\hline 5757 HWC-14-67-3A IE3 9822140.7 & & \\
& & & \\
\hline Sample Name: & HWC-14-67-3A IE3 9822140.7 & njection Volume: & 3.0 \\
Vial Number: & RE4 & Channel: & UV_VIS_2 \\
Sample Type: & unknown & Wavelength: & 254.0 \\
Control Program: & test-dad4 & Bandwidth: & 4 \\
Quantif. Method:: & 20170608 & Dïution Factor: & 1.0000 \\
Recording Time: & $2019-4-3018: 51$ & Sample Weight: & 1.0000 \\
Run Time (min): & 20.00 & Sample Amount & 1.0000 \\
\hline
\end{tabular}

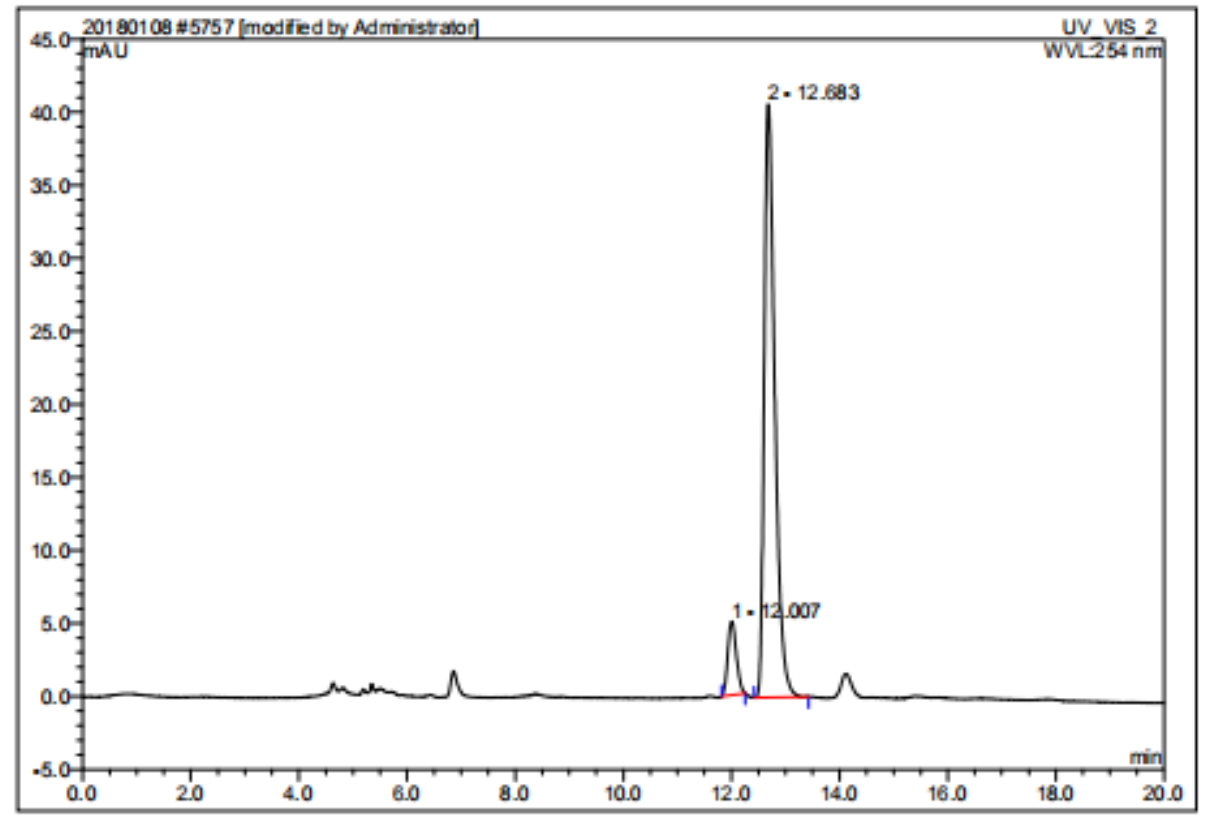

\begin{tabular}{|c|c|c|c|c|c|c|c|}
\hline No. & $\begin{array}{c}\text { Ret.Time } \\
\text { min }\end{array}$ & Peak Name & $\begin{array}{c}\text { Height } \\
\text { mAU }\end{array}$ & $\begin{array}{c}\text { Area } \\
\text { mAU*min }\end{array}$ & $\begin{array}{c}\text { Rel.Area } \\
\%\end{array}$ & Amount & Type \\
\hline 1 & 12.01 & n.a. & 5.057 & 0.929 & 9.14 & n.a. & BMB $^{*}$ \\
\hline 2 & 12.68 & n.a. & 40.630 & 9.228 & 90.86 & n.a. & BMB $^{*}$ \\
\hline Total: & & & 45.687 & 10.156 & 100.00 & 0.000 & \\
\hline
\end{tabular}


<smiles>FC(c1ccccc1)c1ccc(C(F)(F)F)cc1</smiles>

Compound 3v.HPLC (OD-H, $0.46 \times 25 \mathrm{~cm}, 5 \mu \mathrm{m}$, hexane/isopropanol = 98/2 (v/v \%), flow $0.7 \mathrm{~mL} / \mathrm{min}$, $\mathrm{UV}$ detection at $214 \mathrm{~nm}$ ), retention time $=7.25 \mathrm{~min}$ (minor) and $8.50 \min$ (major). $[\alpha]_{\mathrm{D}}{ }^{25}=-24.00\left(\mathrm{c}=0.080, \mathrm{CHCl}_{3}, 93: 7\right.$ e.r).

\begin{tabular}{|lllll|}
\hline 3496 HWC-12-21-3+- ODH 982 & 2140.7 & & \\
& & & \\
\hline Sample Name: & HWC-12-21-3+-ODH 9822140.7 & Injection Volume: & 1.0 \\
Vial Number: & GB6 & Channel: & UV_VIS_2 \\
Sample Type: & unknown & Wavelength: & 214.0 \\
Control Program: & test-dad3 & Bandwidth: & 4 \\
Quantif. Method: & 20170608 & Dilution Factor: & 1.0000 \\
Recording Time: & $2018-10-3014: 47$ & Sample Weight: & 1.0000 \\
Run Time (min): & 10.41 & Sample Amount: & 1.0000 \\
\hline
\end{tabular}

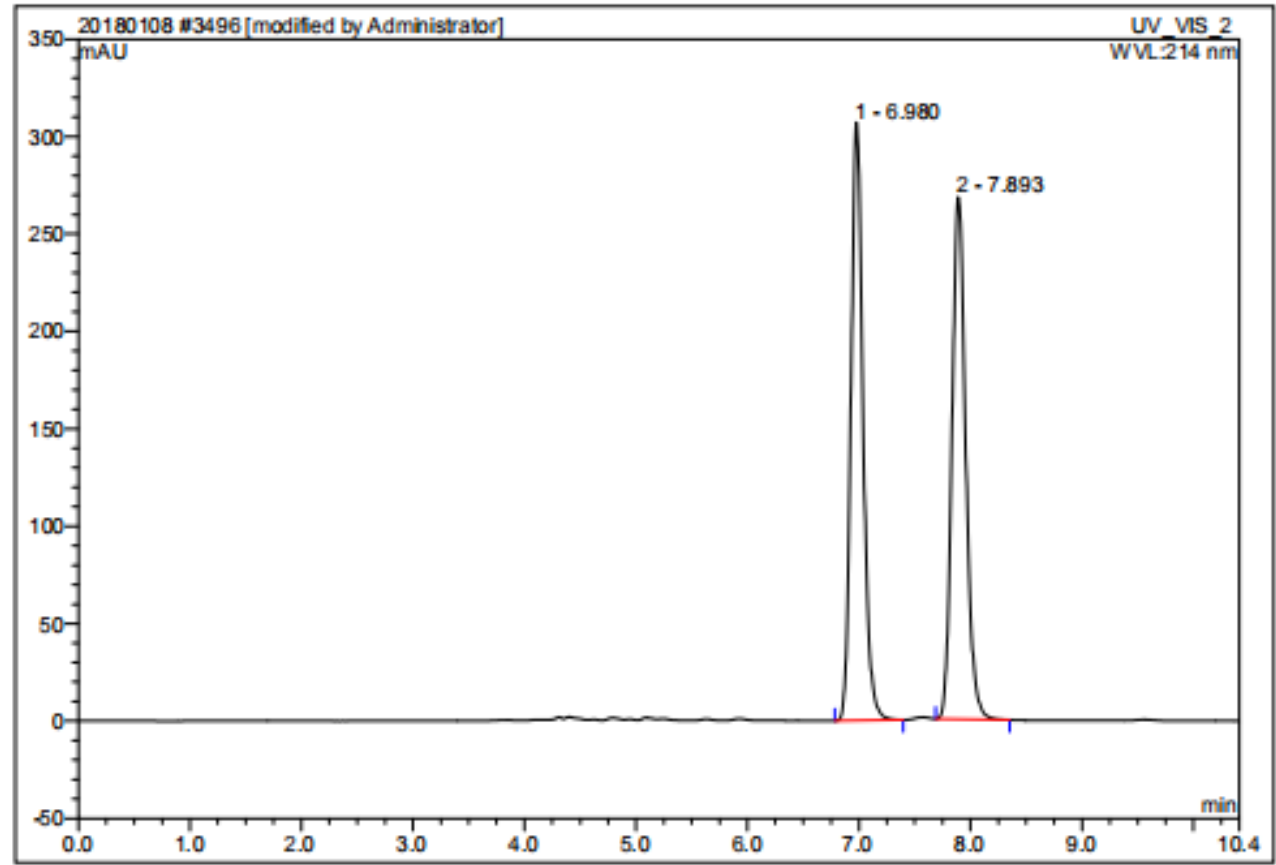

\begin{tabular}{|r|cccrrrr|}
\hline No. & $\begin{array}{c}\text { Ret.Time } \\
\text { min }\end{array}$ & Peak Name & $\begin{array}{c}\text { Height } \\
\text { mAU }\end{array}$ & $\begin{array}{c}\text { Area } \\
\text { mAU*min }\end{array}$ & $\begin{array}{c}\text { Rel.Area } \\
\%\end{array}$ & Amount & Type \\
\hline 1 & 6.98 & n.a. & 307.044 & 38.587 & 50.16 & n.a. & BMB* $^{*}$ \\
2 & 7.89 & n.a. & 268.194 & 38.340 & 49.84 & n.a. & BMB $^{*}$ \\
\hline Total: & & & 575.238 & 76.927 & 100.00 & 0.000 & \\
\hline
\end{tabular}




\begin{tabular}{|llll|}
\hline 4976 HWC-13-82-3B ODH 9822140.7 & & \\
& & & \\
& & & \\
\hline Sample Name: & HWC-13-82-3B ODH 9822140.7 & hjection Volume: & 3.0 \\
Vial Number: & RD1 & Channel: & UV_VIS_2 \\
Sample Type: & unknown & Wavelength: & 214.0 \\
Control Program: & test-dad6 & Bandwidth: & 4 \\
Quantif. Method: & 20170608 & Dilution Factor: & 1.0000 \\
Recording Time: & $2019-3-619: 03$ & Sample Weight: & 1.0000 \\
Run Time (min): & 12.66 & Sample Amount: & 1.0000 \\
\hline
\end{tabular}

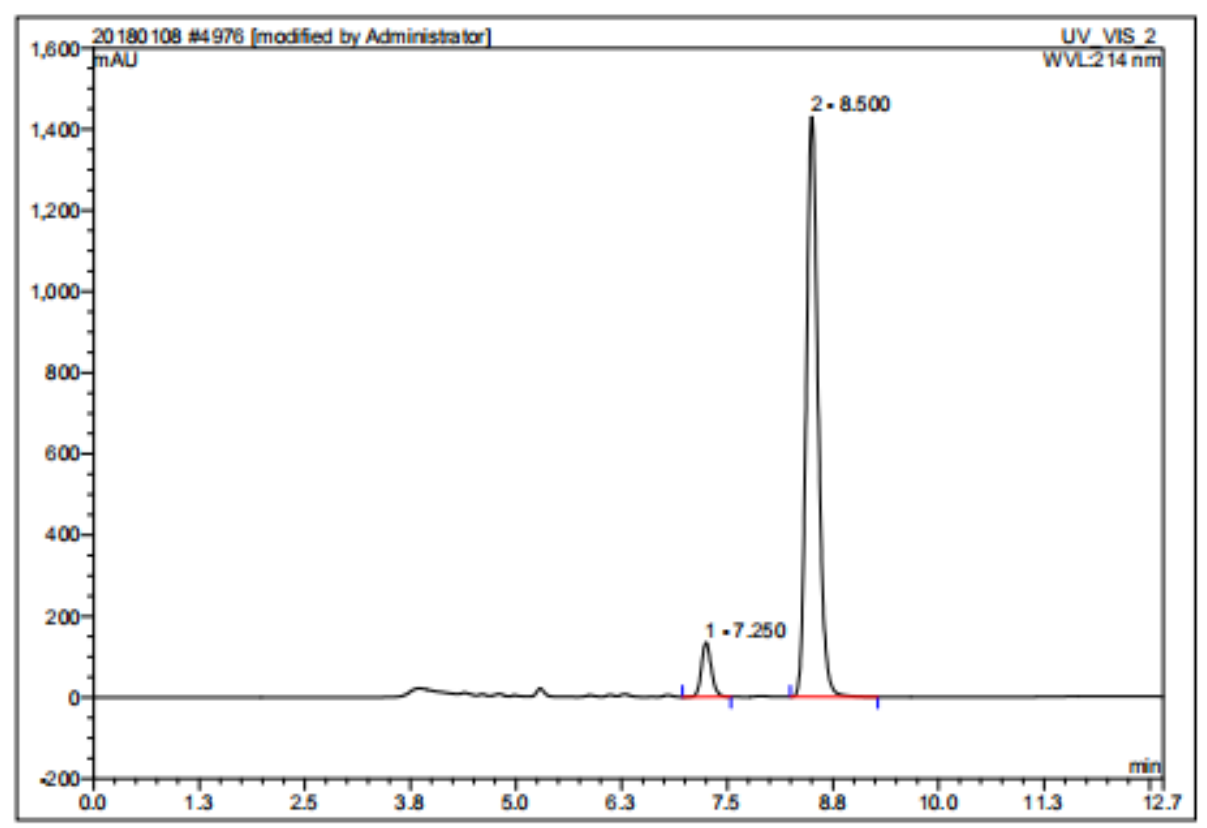

\begin{tabular}{|r|rrrrrrr|}
\hline No. & $\begin{array}{c}\text { Ret. Time } \\
\text { min }\end{array}$ & Peak Name & $\begin{array}{c}\text { Height } \\
\text { mAU }\end{array}$ & $\begin{array}{c}\text { Area } \\
\text { mAU*min }\end{array}$ & $\begin{array}{c}\text { Rel.Area } \\
\%\end{array}$ & Amount & Type \\
\hline 1 & 7.25 & n.a. & 134.363 & 18.173 & 7.30 & n.a. & BMB \\
2 & 8.50 & n.a. & 1430.043 & 230.653 & 92.70 & n.a. & BMB \\
\hline Total: & & & 1564.406 & 248.826 & 100.00 & 0.000 & \\
\hline
\end{tabular}




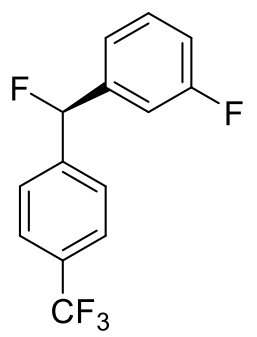

Compound 3w.HPLC (OD-H, $0.46 \times 25 \mathrm{~cm}, 5 \mu \mathrm{m}$, hexane/isopropanol $=100 / 0$ (v/v \%), flow $0.7 \mathrm{~mL} / \mathrm{min}, \mathrm{UV}$ detection at $214 \mathrm{~nm}$ ), retention time $=13.77 \mathrm{~min}$ (minor) and $14.46 \mathrm{~min}$ (major). $[\alpha]_{\mathrm{D}}{ }^{25}=+0.91\left(\mathrm{c}=0.110, \mathrm{CHCl}_{3}, 95: 5\right.$ e.r).

\section{Empower'3}

\begin{tabular}{|c|c|c|c|}
\hline & SAMPLE & INFORMATION & \\
\hline Sampe Name: & HMC-1453-3A+-ODH12140.7 & Acquired By: & System \\
\hline Sample Type & Urkrown & Sample Set Name: & 20190422 \\
\hline Val: & $1: D, 3$ & Acq Methad Set: & 1017 \\
\hline Irjection \# & 1 & Processirg Methat & 1 \\
\hline Irijection Vdume: & $3.00 \mathrm{u}$ & Crand Name & PDAOM1 214nm@4.8rm \\
\hline Run Time: & 200 Mrutes & Prc. Onri. Descr.: & PDAOM1 214nm@4.8rm \\
\hline DateAcquired: & 4/232019 1:48:00 PMCST & & \\
\hline DateProcessed: & 6/132019 4:33:47 RM CST & & \\
\hline
\end{tabular}

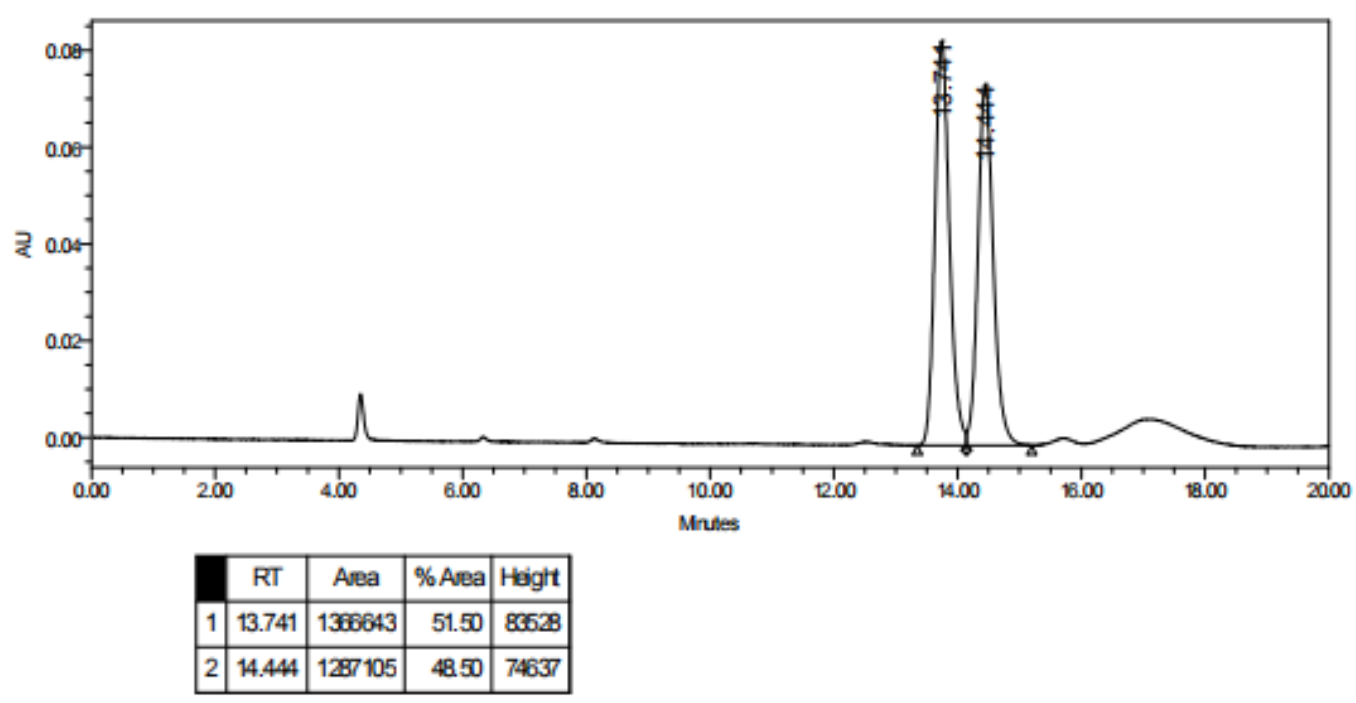




\begin{tabular}{|c|c|c|c|}
\hline & SAMPLE & INFORMATION & \\
\hline Sampie Name: & HMC-1452-3A CDH12140.7 & Acquired By: & System \\
\hline Sanpe Type & Urkrown & Sample Set Name: & 20190422 \\
\hline Val: & 1:D,4 & Acq Method Set: & 1017 \\
\hline Iriection\# & 1 & Rocessing Methot & 1 \\
\hline Irjection Vdume: & $3.00 \mathrm{u}$ & Orame Name & PDAOM1 214nm@4.8rm \\
\hline Ren Time: & 100.0 Minutes & Roc. Onri. Descr.: & PDAOM1 214nm@4.8rm \\
\hline DateAcquired: & 4/23:192 13:13 PM CST & & \\
\hline DateProcessed: & 6/13:2019 4:34:42 PM CST & & \\
\hline
\end{tabular}

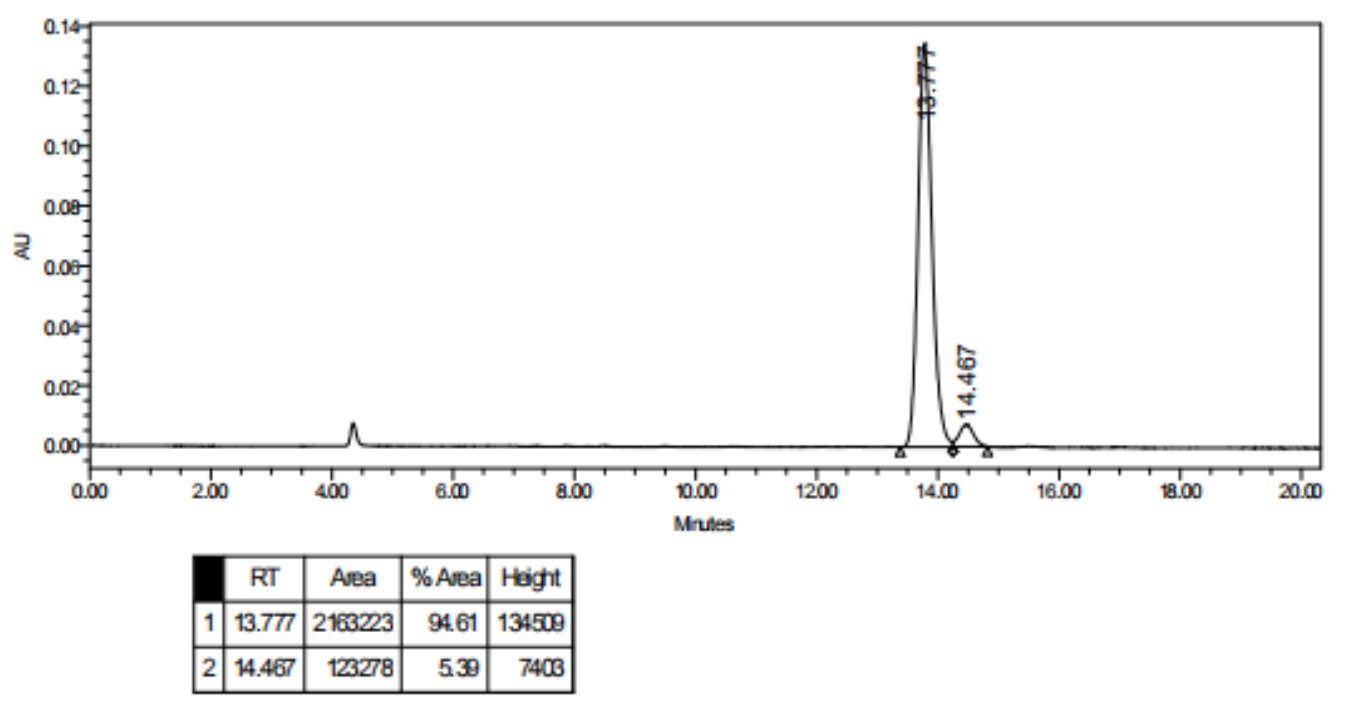


<smiles>FC(c1ccccc1)c1cccc(C(F)(F)F)c1</smiles>

Compound 3x.HPLC (OD-H, $0.46 \times 25 \mathrm{~cm}, 5 \mu \mathrm{m}$, hexane/isopropanol = 98/2 (v/v \%), flow $0.7 \mathrm{~mL} / \mathrm{min}$, UV detection at $214 \mathrm{~nm}$ ), retention time $=6.95 \mathrm{~min}$ (minor) and 7.57 min (major). $[\alpha]_{\mathrm{D}}{ }^{25}=-18.75\left(\mathrm{c}=0.160, \mathrm{CHCl}_{3}, 93: 7\right.$ e.r).

\begin{tabular}{|llll|}
\hline 5436 HWC-14-36-3D+- ODH 9822140.7 & & \\
& & & \\
\hline Sample Name: & HWC-14-36-3D+- ODH 9822140.7 & Mjection Volume: & 2.0 \\
Vial Number: & RE6 & Channel: & UV_VIS_2 \\
Sample Type: & unknown & Wavelength: & 214.0 \\
Cantrol Program: & test-dad4 & Bandwidth: & 4 \\
Quantif. Method: & 20170608 & Dïutian Factor: & 1.0000 \\
Recording Time: & $2019-4-1115: 18$ & Sample Weight: & 1.0000 \\
Run Time (min): & 18.12 & Sample Amount: & 1.0000 \\
\hline
\end{tabular}

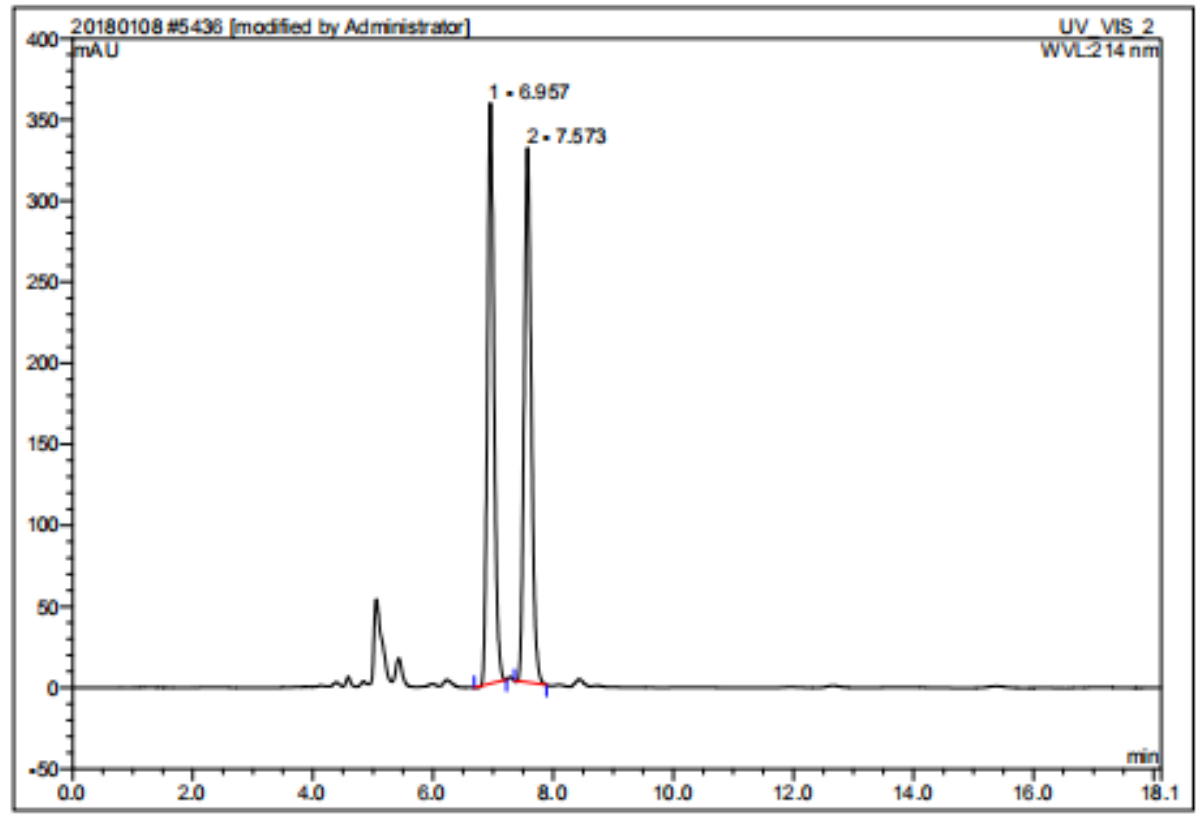

\begin{tabular}{|r|cccrrrr|}
\hline No. & $\begin{array}{c}\text { Ret. Time } \\
\text { min }\end{array}$ & Peak Name & $\begin{array}{c}\text { Height } \\
\text { mAU }\end{array}$ & $\begin{array}{c}\text { Area } \\
\text { mAU*min }\end{array}$ & $\begin{array}{c}\text { Rel.Area } \\
\%\end{array}$ & Amount & Type \\
\hline 1 & 6.96 & n.a. & 357.898 & 45.464 & 49.90 & n.a. & BMB* $^{*}$ \\
2 & 7.57 & n.a. & 329.548 & 45.652 & 50.10 & n.a. & BMB* $^{*}$ \\
\hline Total: & & & 687.446 & 91.115 & 100.00 & 0.000 & \\
\hline
\end{tabular}




\begin{tabular}{|llll|}
\hline 5437 HWC-14-35-3D ODH 982 & 2140.7 & & \\
& & & \\
\hline Sample Name: & HWC-14-35-3D ODH 9822140.7 & injection Volume: & 2.0 \\
Vial Number: & RE5 & Channet & UV_vS_2 \\
Sample Type: & unknown & Wavelength & 214.0 \\
Control Program: & test-dad4 & Bandwidth: & 4 \\
Quandf. Method: & 20170608 & Dilution Factor: & 1.0000 \\
Recording Time: & $2019-4-11$ 15:38 & Sample Weight: & 1.0000 \\
Run Time (min): & 22.72 & Sample Amount: & 1.0000 \\
\hline
\end{tabular}

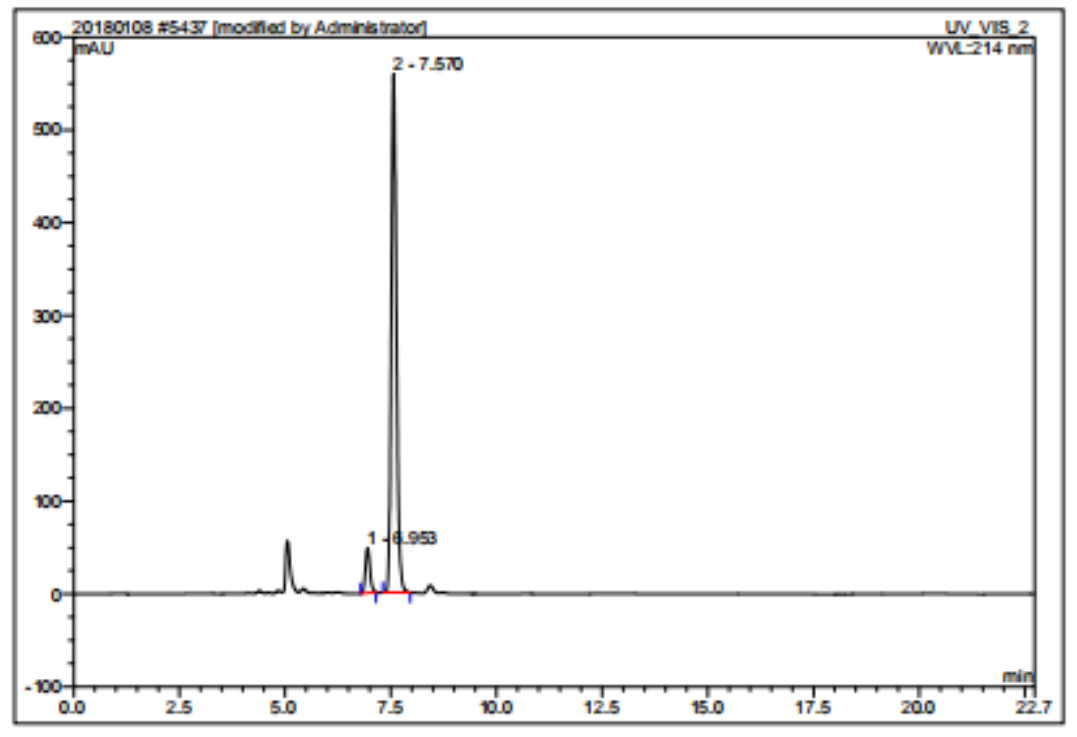

\begin{tabular}{|r|ccrrrrr|}
\hline No. & $\begin{array}{c}\text { Ret.Time } \\
\text { min }\end{array}$ & Peak Name & $\begin{array}{c}\text { Height } \\
\text { mAU }\end{array}$ & $\begin{array}{r}\text { Area } \\
\text { mAU'min }\end{array}$ & $\begin{array}{r}\text { RelArea } \\
\%\end{array}$ & Amount & Type \\
\hline 1 & 6.95 & n.a. & 48.340 & 6.077 & 7.18 & n.a. & BMB* $^{*}$ \\
2 & 7.57 & na. & 558.835 & 78.592 & 92.82 & n.a. & BMB $^{*}$ \\
\hline Total: & & & 607.175 & 84.670 & 100.00 & 0.000 & \\
\hline
\end{tabular}




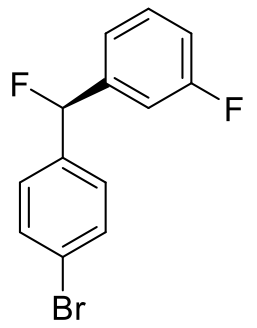

Compound 3y. HPLC (AD-H, $0.46 \times 25 \mathrm{~cm}, 5 \mu \mathrm{m}$, hexane/isopropanol $=98 / 2$ $(\mathrm{v} / \mathrm{v} \%$ ), flow $0.7 \mathrm{~mL} / \mathrm{min}$, UV detection at $214 \mathrm{~nm})$, retention time $=7.54 \mathrm{~min}$ (minor) and $7.86 \min$ (major). $[\alpha]_{\mathrm{D}}{ }^{25}=-13.64\left(\mathrm{c}=0.110, \mathrm{CHCl}_{3}, 94: 6\right.$ e.r).

\begin{tabular}{|llll|}
\hline 5665 HWC-14-63-3At- ADH 9822140.7 & & \\
& & & \\
\hline Sample Name: & HWC-14-63-3A+-ADH 9822140.7 & injection Volume: & 3.0 \\
Vial Number: & RB7 & Channet & UV_uS_2 \\
Sample Type: & unknown & Wavelength: & 214.0 \\
Cortrol Program: & test-dad6 & Bandwibth: & 4 \\
Quandf. Method: & 20170608 & Dilution Factor: & 1.0000 \\
Recording Time: & $2019-4-2610: 22$ & Sample Weight: & 1.0000 \\
Run Time (min): & 18.22 & Sample Amount: & 1.0000 \\
\hline
\end{tabular}

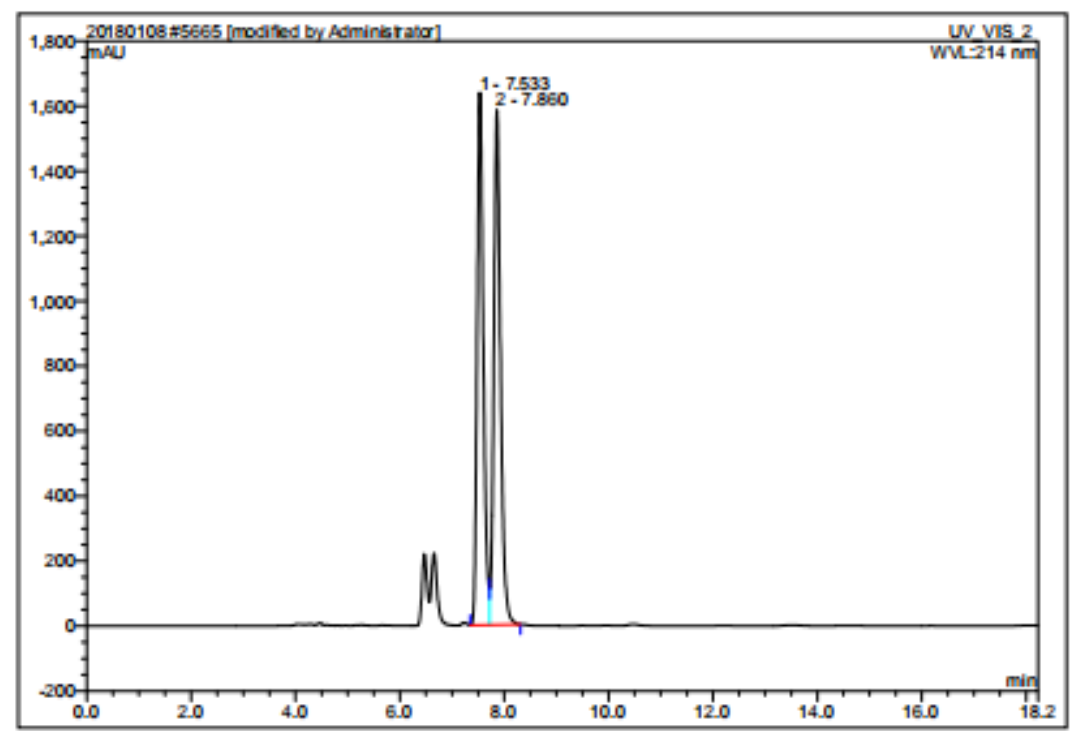

\begin{tabular}{|c|c|c|c|c|c|c|c|}
\hline No. & $\begin{array}{c}\text { Ret.Time } \\
\text { min }\end{array}$ & Peak Name & $\begin{array}{c}\text { Height } \\
\text { mAU }\end{array}$ & $\begin{array}{c}\text { Area } \\
\text { mAU'min }\end{array}$ & $\begin{array}{c}\text { RelArea } \\
\%\end{array}$ & Amount & Type \\
\hline 1 & 7.53 & na. & 1639.088 & 226.344 & 49.06 & n.a & $\mathrm{BM}^{*}$ \\
\hline 2 & 7.86 & na. & 1584.081 & 234.997 & 50.94 & n.a & $\mathrm{MB}^{*}$ \\
\hline Total: & & & 3223.169 & 461.342 & 100.00 & 0.000 & \\
\hline
\end{tabular}




\begin{tabular}{|llll|}
\hline 5666 HWC-14-63-3B ADH 9822140.7 & & \\
& & & \\
\hline Sample Name: & HWC-14-63-3B ADH 9822140.7 & Injection Volume: & 3.0 \\
Vial Number: & RB6 & Channel: & UV_VIS_2 \\
Sample Type: & unknown & Wavelength: & 214.0 \\
Cantral Program: & test-dad6 & Bandwidth: & 4 \\
Quantif. Method: & 20170608 & Dilution Factor: & 1.0000 \\
Recording Time: & $2019-4-26 ~ 10: 42$ & Sample Weight: & 1.0000 \\
Run Time (min): & 24.27 & Sample Amount: & 1.0000 \\
\hline
\end{tabular}

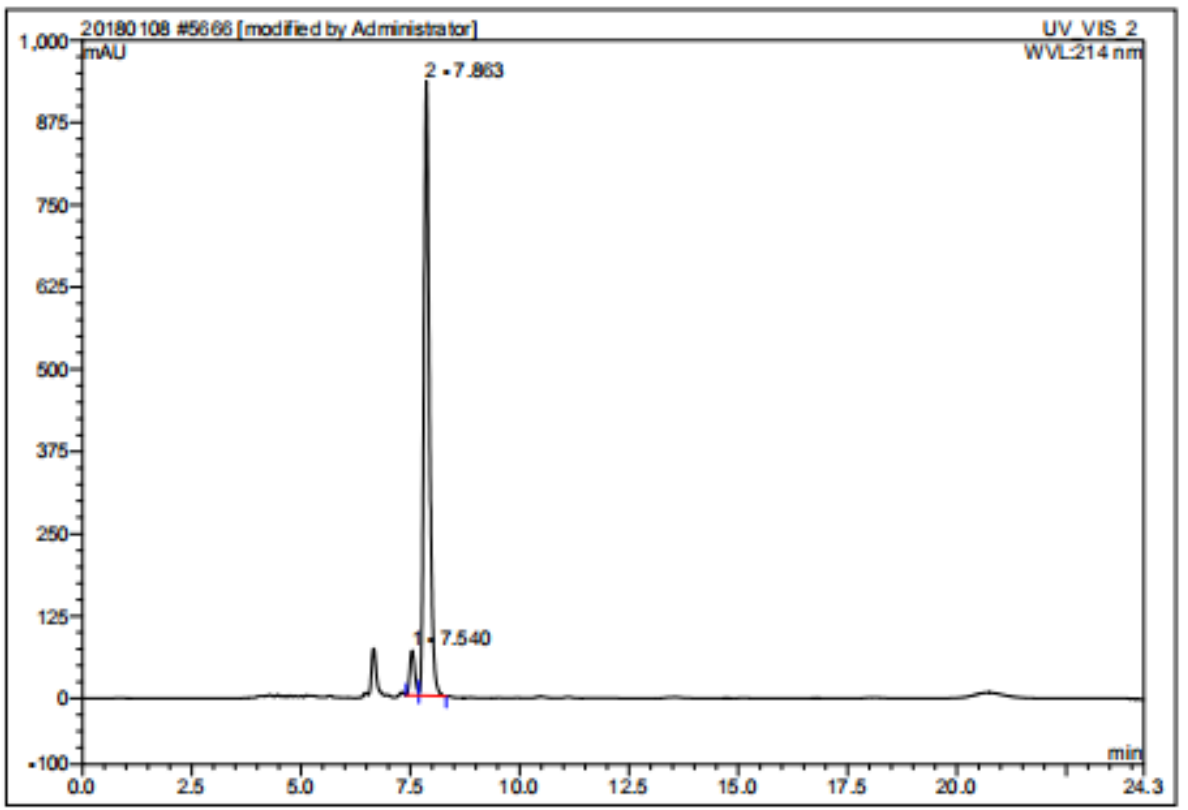

\begin{tabular}{|r|ccrrrrr|}
\hline No. & $\begin{array}{c}\text { Ret. Time } \\
\text { min }\end{array}$ & Peak Name & $\begin{array}{c}\text { Height } \\
\text { mAU }\end{array}$ & $\begin{array}{c}\text { Area } \\
\text { mAU*min }\end{array}$ & $\begin{array}{r}\text { Rel.Area } \\
\%\end{array}$ & Amount & Type \\
\hline 1 & 7.54 & n.a. & 67.868 & 8.851 & 6.13 & n.a. & BM $^{*}$ \\
2 & 7.86 & n.a. & 933.887 & 135.591 & 93.87 & n.a. & MB $^{*}$ \\
\hline Total: & & & 1001.756 & 144.442 & 100.00 & 0.000 & \\
\hline
\end{tabular}


<smiles>Fc1cccc(C(F)c2cc(F)cc(Br)c2)c1</smiles>

Compound 3z. HPLC (AD-H, $0.46 \times 25 \mathrm{~cm}, 5 \mu \mathrm{m}$, hexane/isopropanol = 98/2 (v/v \%), flow $0.7 \mathrm{~mL} / \mathrm{min}$, UV detection at $214 \mathrm{~nm}$ ), retention time $=12.28 \mathrm{~min}$ (minor) and $13.14 \min$ (major). $[\alpha]_{\mathrm{D}}{ }^{25}=-8.67$ (c $=0.090, \mathrm{CHCl}_{3}, 95: 5$ e.r).

\section{Empower' 3}

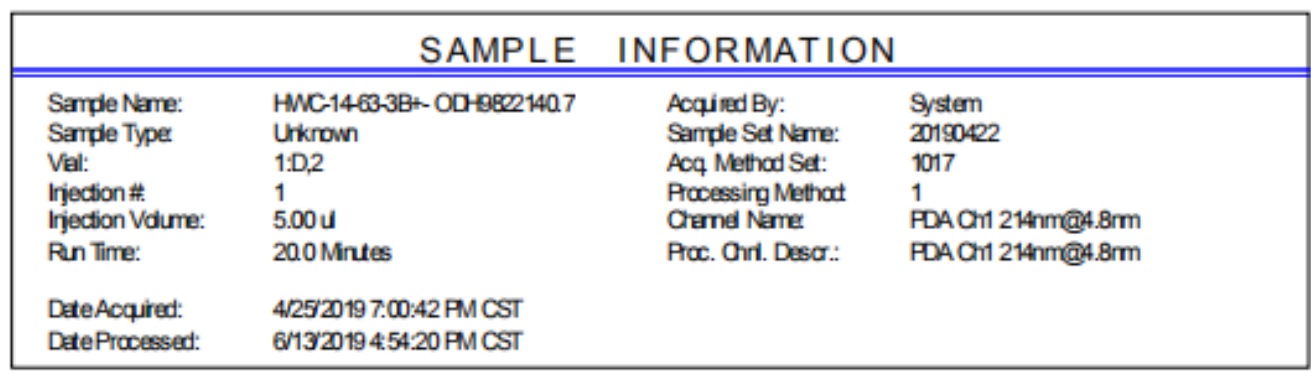

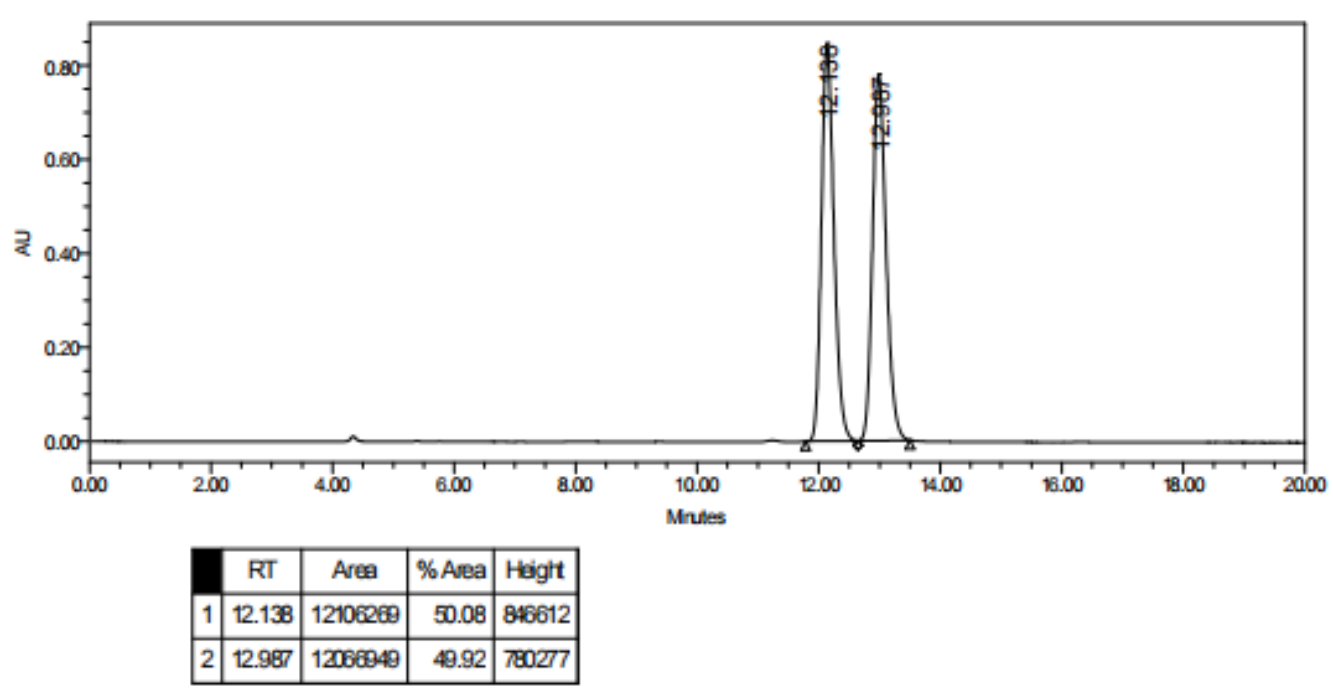


SAMPLE INFORMATION

\begin{tabular}{|c|c|c|c|}
\hline Sample Name & HMC-1462-3B CDHCB22140.7 & Acquired By: & System \\
\hline Sample Type & Urkramn & Sample Set Name: & 20190422 \\
\hline Via: & $1: D, 3$ & Acq. Method Set: & 1017 \\
\hline Injectian\# & 1 & Processing Methat & 1 \\
\hline Injection Vdume: & 5.00 ul & Crarrel Name: & PDAG1 214m@4.8m \\
\hline Run Time & 200 Minutes & Proc. Ori. Desar:: & PDA G1 $214 \mathrm{~mm} 94.8 \mathrm{rm}$ \\
\hline Date Acquired & 4/25/2019 7:21:24 PMCST & & \\
\hline Date Processed: & 6/13/2019 4:535B PMCST & & \\
\hline
\end{tabular}

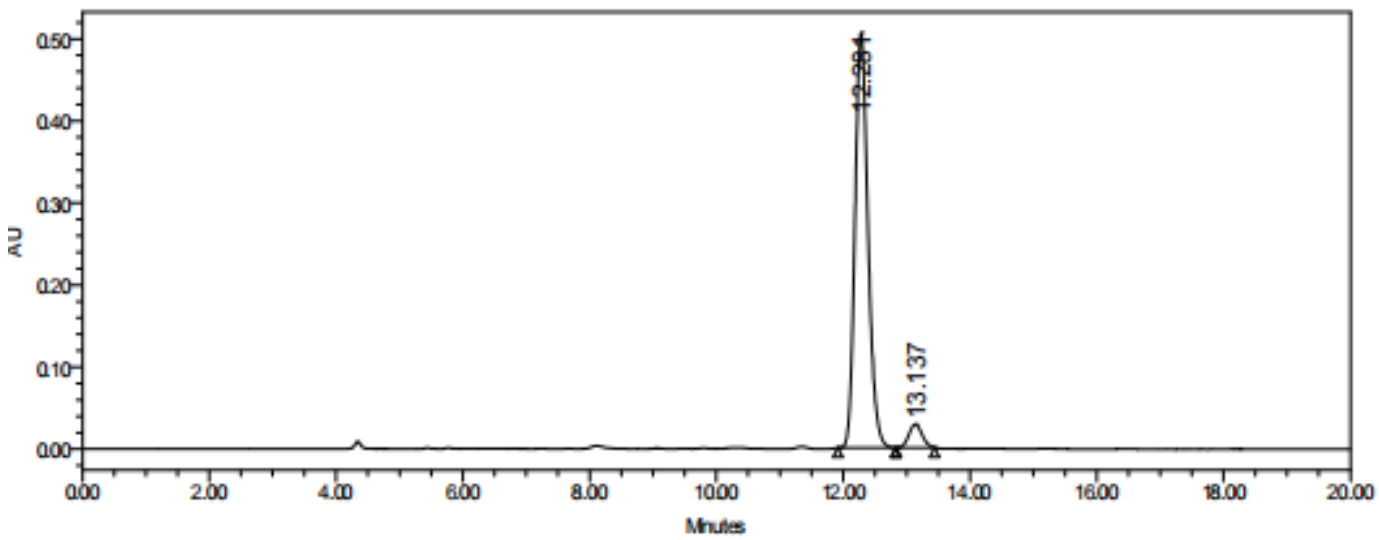

\begin{tabular}{|c|c|r|r|r|}
\hline & RT & \multicolumn{1}{c|}{ Aver } & \% Are日 & Height \\
\hline 1 & 12.281 & 7200096 & 94.60 & 506500 \\
\hline 2 & 13.137 & 411127 & 5.40 & 28440 \\
\hline
\end{tabular}


<smiles>FC(c1ccccc1)c1ccc(Cl)c(Br)c1</smiles>

Compound 3aa. HPLC (OD-H, $0.46 \times 25 \mathrm{~cm}, 5 \mu \mathrm{m}$, hexane/isopropanol $=95 / 5$ $(\mathrm{v} / \mathrm{v} \%$ ), flow $0.7 \mathrm{~mL} / \mathrm{min}$, UV detection at $214 \mathrm{~nm})$, retention time $=7.47 \mathrm{~min}$ (minor) and $8.57 \min$ (major). $[\alpha]_{\mathrm{D}}{ }^{25}=-37.89\left(\mathrm{c}=0.180, \mathrm{CHCl}_{3}, 92: 8\right.$ e.r).

\section{HWC-14-3-3A ODH 9552140.7}

\section{Sample Name:} Vial Number.

Sample Type:

Control Program:

Quantif. Method:

Recording Time:

Run Time (min):

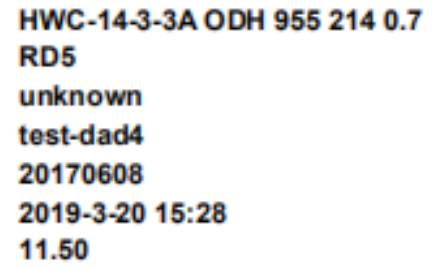

3.0 UV_VIS_2 214.0 4 1.0000 1.0000 1.0000

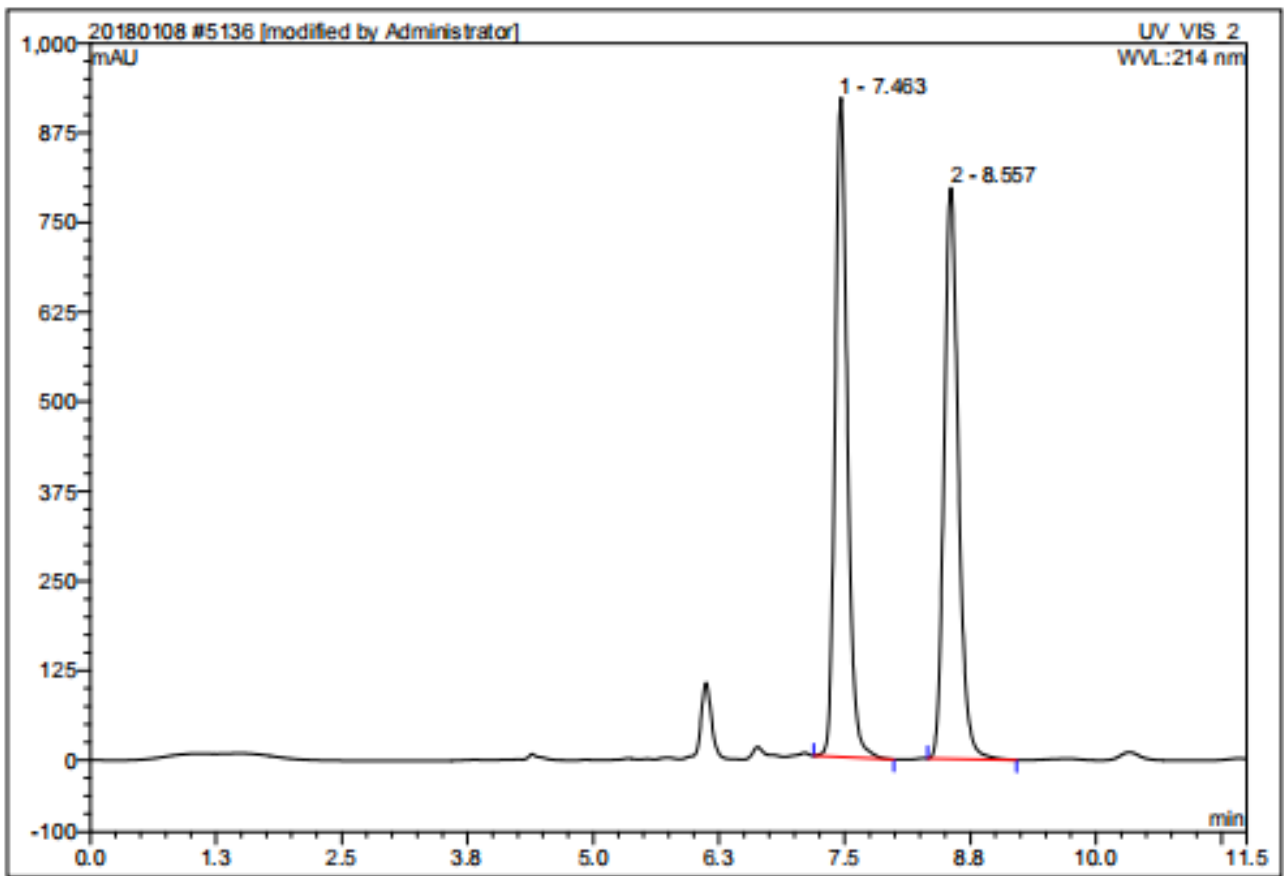

\begin{tabular}{|r|ccrrrrr|}
\hline No. & $\begin{array}{c}\text { Ret.Time } \\
\text { min }\end{array}$ & Peak Name & $\begin{array}{c}\text { Height } \\
\text { mAU }\end{array}$ & $\begin{array}{c}\text { Area } \\
\text { mAU*min }\end{array}$ & $\begin{array}{c}\text { Rel.Area } \\
\%\end{array}$ & Amount & Type \\
\hline 1 & 7.46 & n.a. & 919.549 & 130.586 & 49.93 & n.a. & BMB $^{*}$ \\
2 & 8.56 & n.a. & 796.750 & 130.928 & 50.07 & n.a. & BMB \\
\hline Total: & & & 1716.298 & 261.514 & 100.00 & 0.000 & \\
\hline
\end{tabular}




\begin{tabular}{|llll|}
\hline 5137 HWC-14-3-3B ODH 9552140.7 & & \\
& & & \\
\hline Sample Name: & HWC-14-3-3B ODH 9552140.7 & injection Volume: & 3.0 \\
Vial Number: & RE4 & Channel: & UV_VIS_2 \\
Sample Type: & unknown & Wavelength: & 214.0 \\
Contral Program: & test-dad4 & Bandwidth: & 4 \\
Quantif. Method: & 20170608 & Dilution Factor: & 1.0000 \\
Recording Time: & $2019-3-2015: 41$ & Sample Weight: & 1.0000 \\
Run Time (min): & 10.00 & Sample Amount: & 1.0000 \\
\hline
\end{tabular}

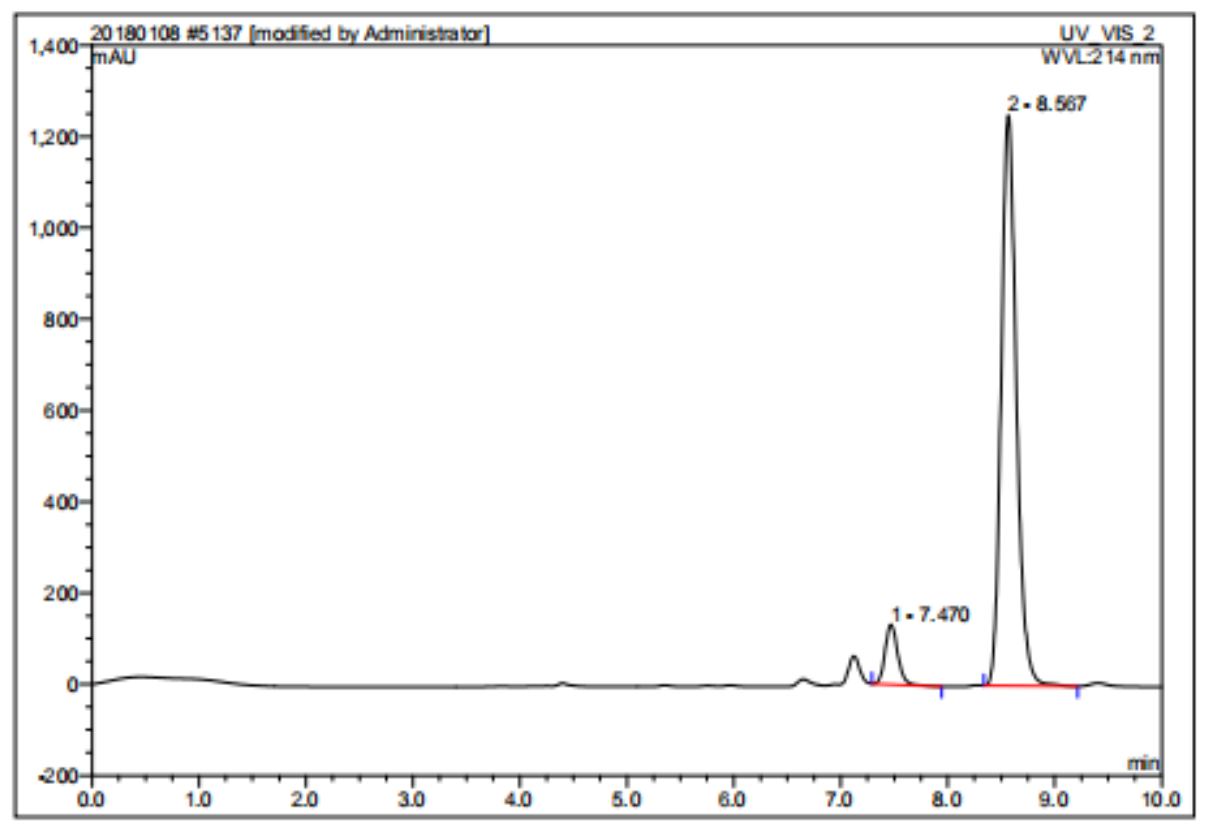

\begin{tabular}{|r|ccrrrrr|}
\hline No. & $\begin{array}{c}\text { Ret.Time } \\
\text { min }\end{array}$ & Peak Name & $\begin{array}{c}\text { Height } \\
\text { mAU }\end{array}$ & $\begin{array}{r}\text { Area } \\
\text { mAU*min }\end{array}$ & $\begin{array}{r}\text { Rel.Area } \\
\%\end{array}$ & Amount & Type \\
\hline 1 & 7.47 & n.a. & 131.668 & 17.896 & 8.01 & n.a. & BMB* $^{*}$ \\
2 & 8.57 & n.a. & 1249.856 & 205.634 & 91.99 & n.a. & BMB $^{*}$ \\
\hline Total: & & & 1381.524 & 223.530 & 100.00 & 0.000 & \\
\hline
\end{tabular}




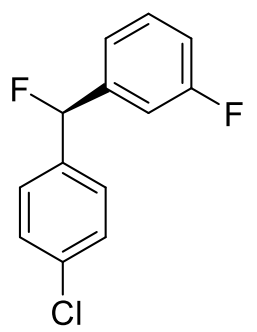

Compound 3ab. HPLC (OD-H, $0.46 \times 25 \mathrm{~cm}, 5 \mu \mathrm{m}$, hexane/isopropanol = 98/2 $(\mathrm{v} / \mathrm{v} \%)$, flow $0.7 \mathrm{~mL} / \mathrm{min}, \mathrm{UV}$ detection at $214 \mathrm{~nm})$, retention time $=8.66 \mathrm{~min}$ (minor) and $9.09 \min$ (major). $[\alpha]_{\mathrm{D}}{ }^{25}=+9.43\left(\mathrm{c}=0.280, \mathrm{CHCl}_{3}, 94: 6\right.$ e.r).

\section{Empower'3}

\begin{tabular}{|c|c|c|c|}
\hline & SAMPLE & INFORMATION & \\
\hline Sample Name: & HMC-1456-3B+-OD-9822 140.7 & Acqied By: & System \\
\hline Sampe Type & Urkrown & Sample Set Name: & 20190422 \\
\hline Val: & $1: C, 3$ & Acq Method Set: & 1017 \\
\hline Irịection\# \# & 1 & Rocessirg Methat & 1 \\
\hline Irjection Vodume: & $10 \infty u l$ & Creme Name & PDAOM1 214nm@4.8rm \\
\hline Run Time: & 40.0 Mintes & Prc. Onri. Descr.: & PDAOM1 214nm@4.8m \\
\hline DateAcquired: & 4/222019@:51:58 RM CST & & \\
\hline DateProcessed: & 6/1320194: 19:03 PM CST & & \\
\hline
\end{tabular}

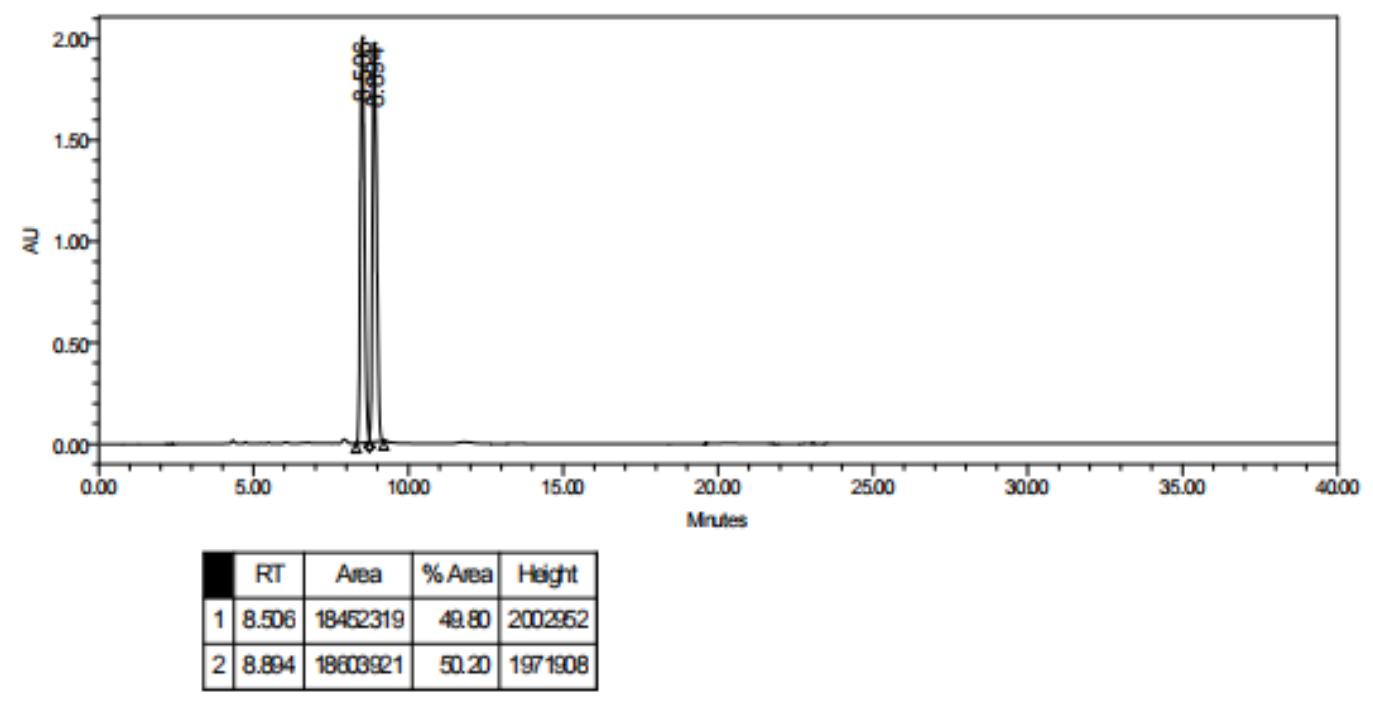




\begin{tabular}{|c|c|c|c|}
\hline & SAMPLE & INFORMATION & \\
\hline Sample Name: & HMC-145-3B CDHBE22140.7 & Acqied By: & System \\
\hline Sample Type & Urkrown & Sample Set Name: & 20190422 \\
\hline Val: & $1: D, 1$ & Acq Method Set: & 1017 \\
\hline Iriection \#\# & 1 & Pocessirg Methat & 1 \\
\hline Irjection Vdume: & $2.00 \mathrm{u}$ & Creme Name & PDA On1 214nm@4.8m \\
\hline Pen Time: & 20.0 Mintes & Proc. Onri. Descr.: & PDAOM1 214nm@4.8m \\
\hline DateAcquired: & 4/232019845.21 AMCST & & \\
\hline DateProcessed: & 6/13:20194:17:44 PM CST & & \\
\hline
\end{tabular}

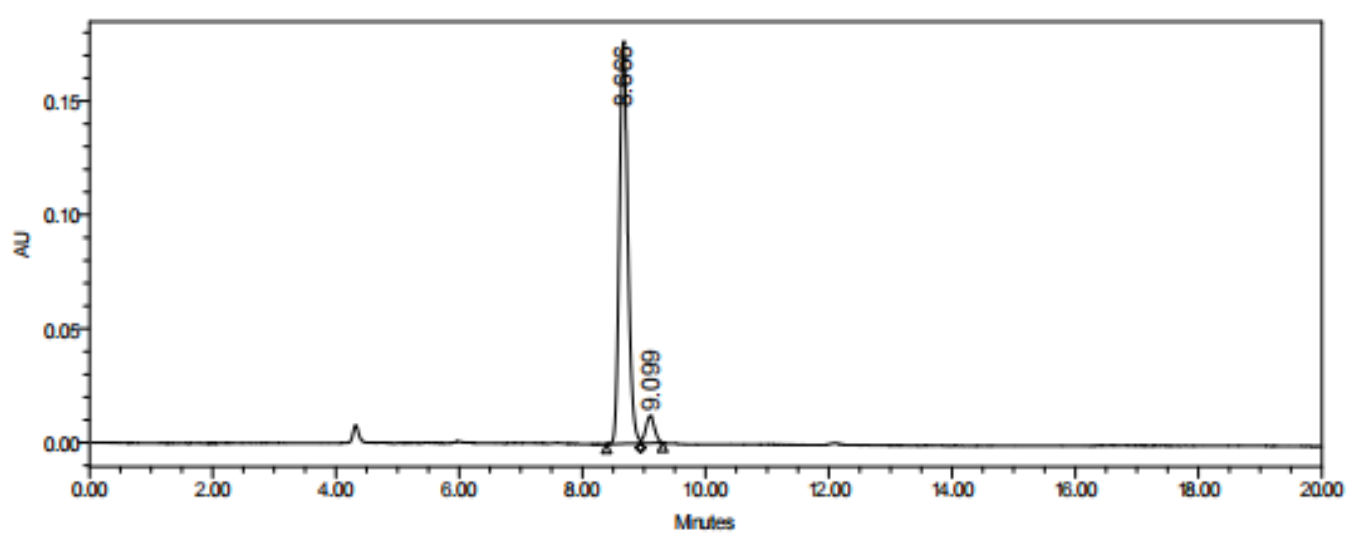

\begin{tabular}{|r|c|r|r|r|}
\hline & RT & \multicolumn{1}{|c|}{ Are日 } & \% Are日 & Heigtt \\
\hline 1 & 8.606 & 1674825 & 9356 & 176283 \\
\hline 2 & 9.099 & 115303 & 644 & 11775 \\
\hline
\end{tabular}




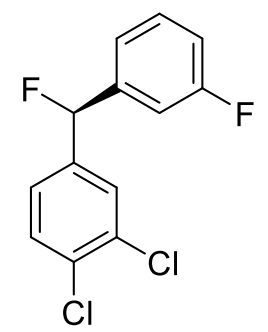

Compound 3ac. HPLC (ADRH, $0.46 \times 15 \mathrm{~cm}, 5 \mu \mathrm{m}$, acetonitrile/water $=60 / 40$ $(\mathrm{v} / \mathrm{v} \%)$, flow $0.7 \mathrm{~mL} / \mathrm{min}, \mathrm{UV}$ detection at $214 \mathrm{~nm})$, retention time $=12.87 \mathrm{~min}$ (minor) and $13.43 \mathrm{~min}$ (major). $[\alpha]_{\mathrm{D}}{ }^{25}=-1.88\left(\mathrm{c}=0.160, \mathrm{CHCl}_{3}, 93: 7\right.$ e.r).

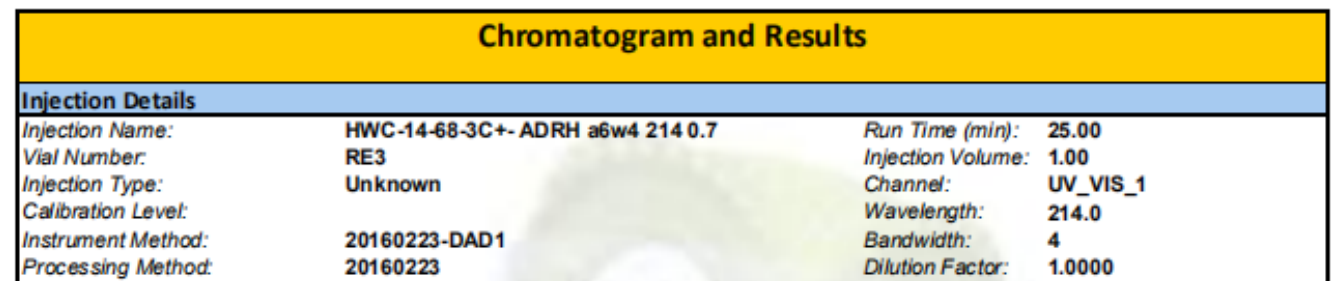

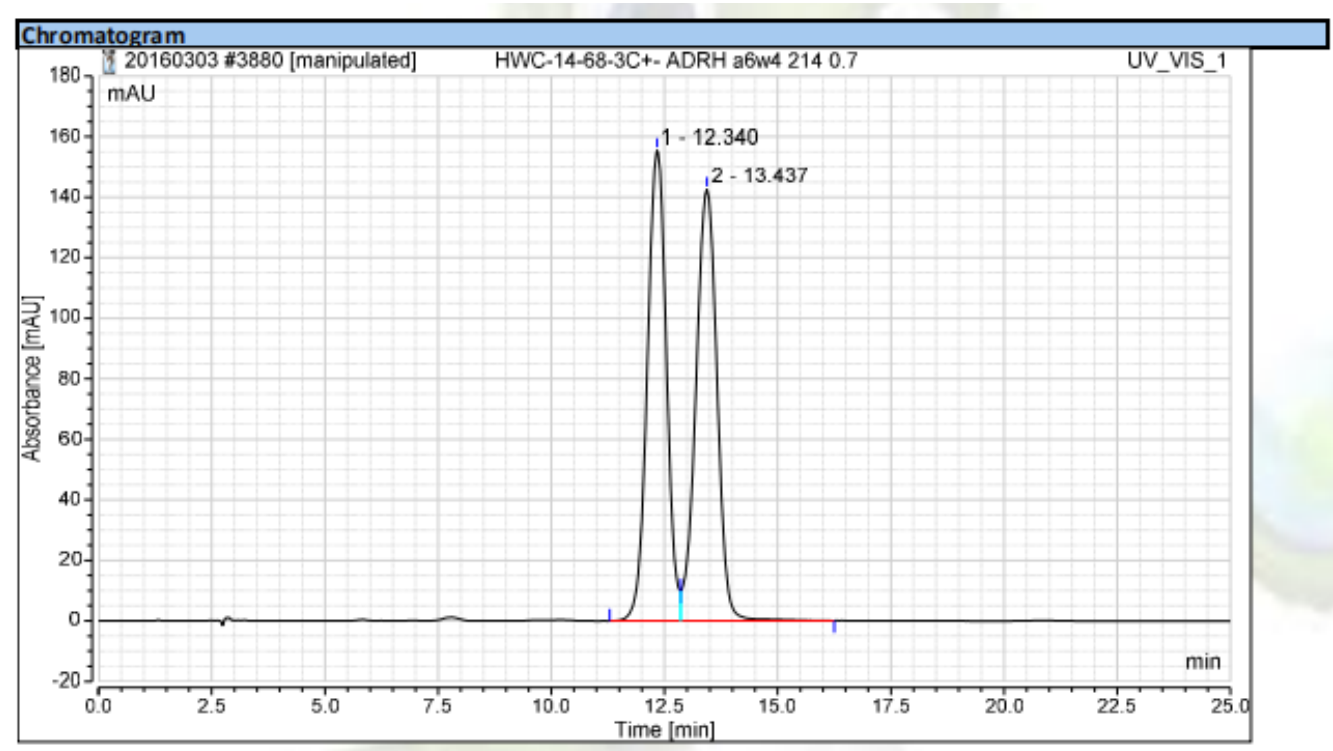

\begin{tabular}{|l|c|c|c|c|}
\hline \multicolumn{3}{|l|}{ Integration Results } \\
\hline No. & $\begin{array}{c}\text { Retention Time } \\
\text { min }\end{array}$ & $\begin{array}{c}\text { Area } \\
\text { mAU*min }\end{array}$ & $\begin{array}{c}\text { Height } \\
\text { mAU }\end{array}$ & $\begin{array}{c}\text { Relative Area } \\
\%\end{array}$ \\
\hline 1 & 12.340 & 75.9987 & 155.4936 & 49.584 \\
2 & 13.437 & 77.2742 & 142.6348 & 50.416 \\
\hline Total: & 153.273 & 1401.998 & 100.000 \\
\hline
\end{tabular}




\begin{tabular}{|c|c|c|c|}
\hline \multicolumn{4}{|c|}{ Chromatogram and Results } \\
\hline \multicolumn{4}{|l|}{ Injection Details } \\
\hline Injection Name: & HWC-14.67.3C ADRH a6w4 2140.7 & Run Time (min) & 18.85 \\
\hline Vial Number: & RE4 & Injection Volume: & 2.00 \\
\hline Injection Type: & Unknown & Channet & UV_vis_1 \\
\hline Calbration Level: & & Wavelength: & 214.0 \\
\hline Instrument Methodt & $20160223 \cdot D A D 1$ & Bandwiath: & 4 \\
\hline Processing Methodt & 20160223 & Dikition Factor: & 1.0000 \\
\hline
\end{tabular}

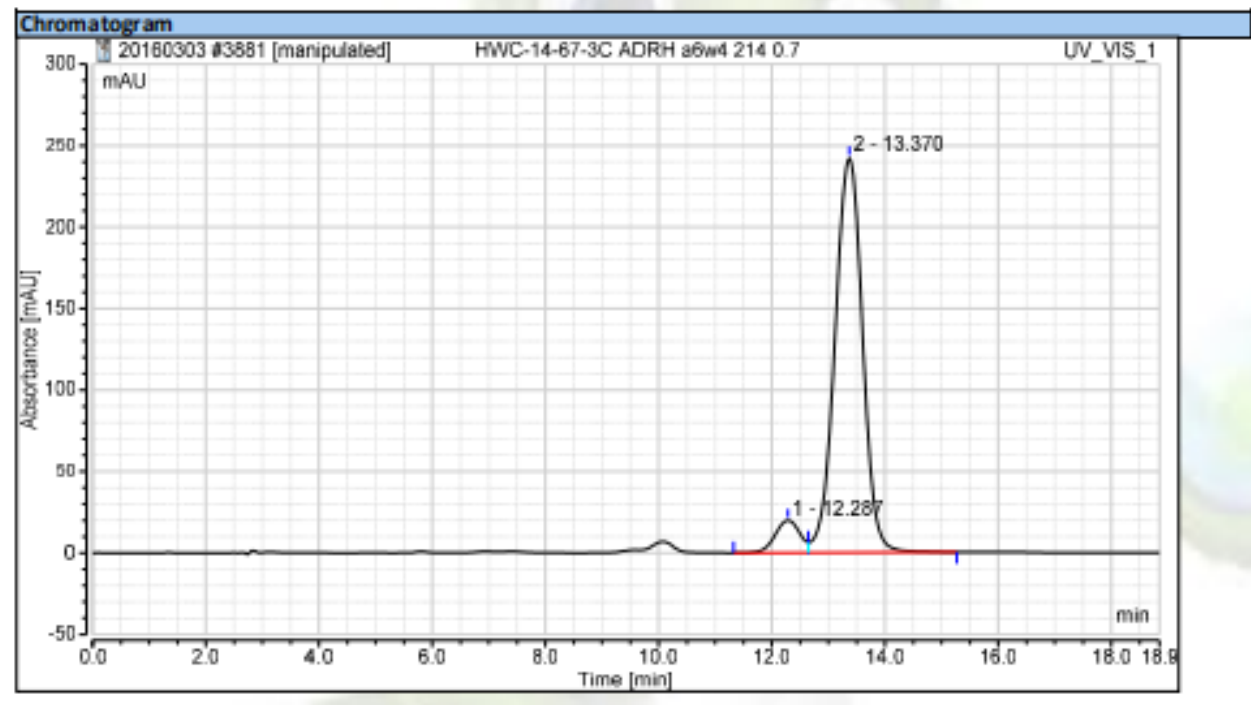

\begin{tabular}{|c|c|c|c|c|}
\hline \multicolumn{5}{|c|}{ Integration Results } \\
\hline No. & $\begin{array}{c}\text { Retenfion Time } \\
\text { min }\end{array}$ & $\begin{array}{c}\text { Area } \\
\text { mAu'min }\end{array}$ & $\begin{array}{l}\text { Height } \\
\text { mAU }\end{array}$ & $\begin{array}{c}\text { Relafive Area } \\
\%\end{array}$ \\
\hline 1 & 12.287 & 10.0047 & 19.8049 & 6.810 \\
\hline 2 & 13.370 & 136.9044 & 242.1106 & 93.190 \\
\hline \multicolumn{2}{|c|}{ Total: } & 146.909 & 1401.998 & 100.000 \\
\hline
\end{tabular}


<smiles>Fc1cccc(C(F)c2cc(Cl)cc(Cl)c2)c1</smiles>

Compound 3ad. HPLC (OD-H, $0.46 \times 25 \mathrm{~cm}, 5 \mu \mathrm{m}$, hexane/isopropanol $=98 / 2$ (v/v \%), flow $0.7 \mathrm{~mL} / \mathrm{min}$, UV detection at $214 \mathrm{~nm}$ ), retention time $=8.48 \mathrm{~min}$ (minor) and $9.04 \min$ (major). $[\alpha]_{\mathrm{D}}{ }^{25}=-45.25\left(\mathrm{c}=0.160, \mathrm{CHCl}_{3}, 95: 5\right.$ e.r).

\begin{tabular}{|c|c|c|c|}
\hline \multicolumn{2}{|r|}{ SAMPLE } & \multicolumn{2}{|c|}{ INFORMATION } \\
\hline $\begin{array}{l}\text { Sample Name } \\
\text { Sample Type } \\
\text { Via: } \\
\text { lijedion \#t } \\
\text { lijection Vdume } \\
\text { Ran Time }\end{array}$ & 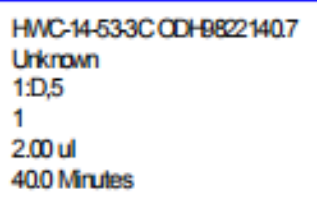 & $\begin{array}{l}\text { Acquired By. } \\
\text { Sample Sel Name } \\
\text { Acq. Method Set. } \\
\text { Processing Method } \\
\text { Crarrel Name } \\
\text { Proc. Ori. Descr.: }\end{array}$ & 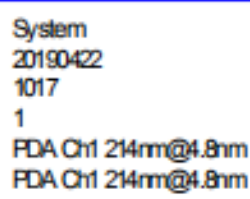 \\
\hline $\begin{array}{l}\text { DateAcquired } \\
\text { DateProcessedt }\end{array}$ & $\begin{array}{l}\text { 4/2322019 11:4806 AM CST } \\
\text { 6/13220195:C2:51 PMCST }\end{array}$ & & \\
\hline
\end{tabular}

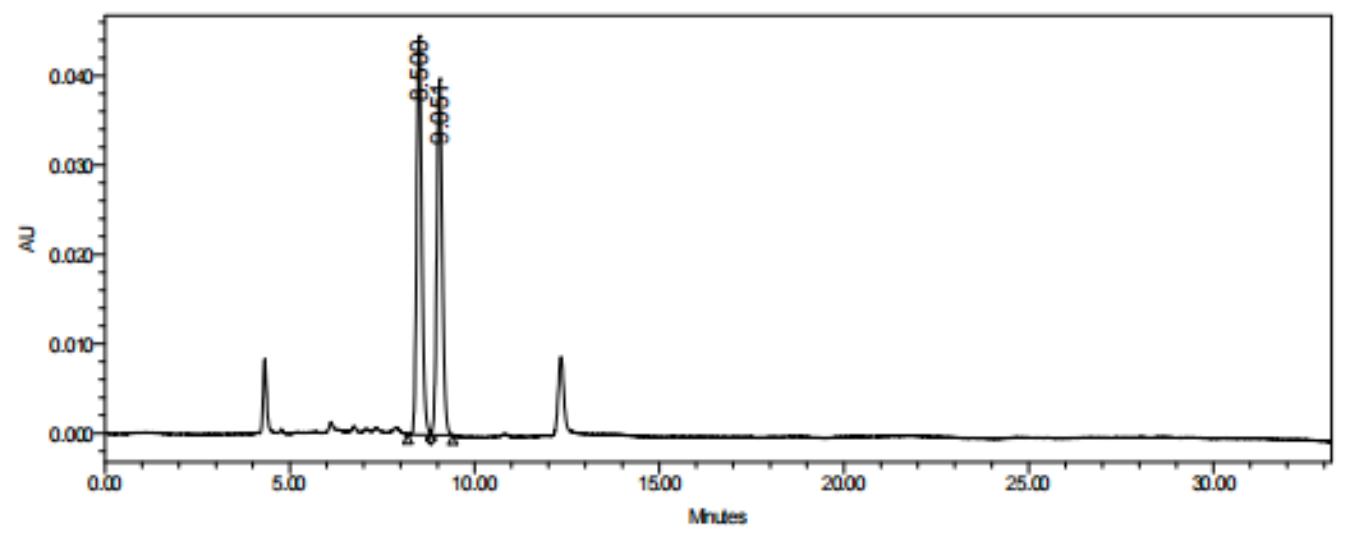

\begin{tabular}{|c|c|c|r|c|}
\hline & RT & Area & $\%$ Area & Heignt \\
\hline 1 & 8.500 & 428519 & 51.33 & 44609 \\
\hline 2 & 9.051 & 406291 & 48.67 & 39850 \\
\hline
\end{tabular}




\begin{tabular}{|c|c|c|c|}
\hline & SAMPLE & INFORMATION & \\
\hline Sample Name: & HMC-1452-3CODHEER2140.7 & Acqined By: & System \\
\hline Sample Type & Urkrown & Sample Set Name: & 20190422 \\
\hline Val: & $1: D, 6$ & Acq Method Set: & 1017 \\
\hline Irjection \# & 1 & Processirg Methout & 1 \\
\hline Irjection Vdume: & $2.00 \mathrm{u}$ & Cremel Name & PDAOM1 214nm@4.8m \\
\hline Run Time: & 40.0 Mintes & Prc. Onri. Descr.: & PDAOM1 214nm@4.8rm \\
\hline DateAcquired: & 4/232019 12:29 16 PMCST & & \\
\hline DateProcessed: & 6/1320195@::17PMCST & & \\
\hline
\end{tabular}

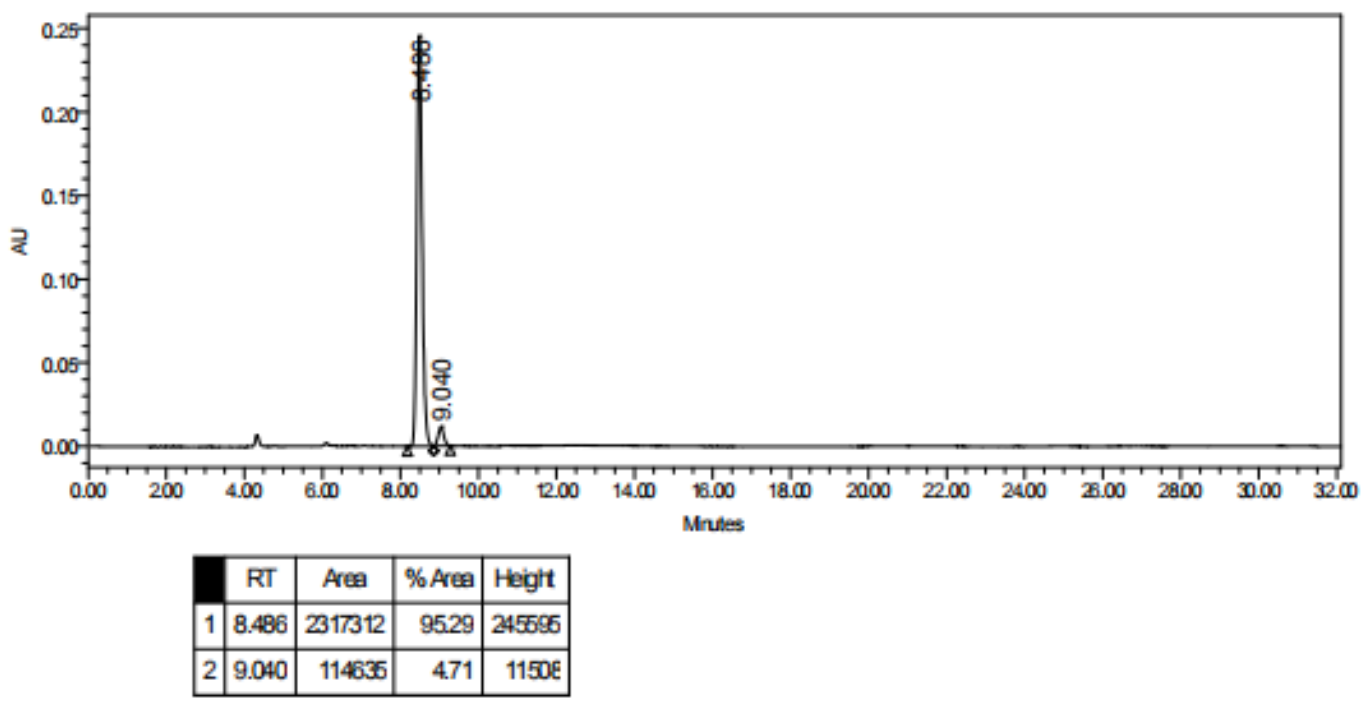


<smiles>FC(c1ccccc1)c1cc(Cl)cc(Cl)c1</smiles>

Compound 3ae. HPLC (OD-H, $0.46 \times 25 \mathrm{~cm}, 5 \mu \mathrm{m}$, hexane/isopropanol $=98 / 2$ (v/v \%), flow $0.7 \mathrm{~mL} / \mathrm{min}$, UV detection at $214 \mathrm{~nm}$ ), retention time $=7.30 \mathrm{~min}$ (minor) and $8.19 \min$ (major). $[\alpha]_{\mathrm{D}}{ }^{25}=-33.18\left(\mathrm{c}=0.220, \mathrm{CHCl}_{3}, 94: 6\right.$ e.r).

5947 HWC-14-36-3B ODH 9822140.7

\begin{tabular}{llll|}
\hline Sample Name: & HWC-14-36-3B ODH 9822140.7 & Injection Volume: & 2.0 \\
Vial Number: & RC6 & Channel: & UV_VIS_2 \\
Sample Type: & unknown & Wavelength: & 214.0 \\
Control Program: & test-dad6 & Bandwidth: & 4 \\
Quantif. Method: & 20170608 & Dilution Factor: & 1.0000 \\
Recording Time: & $2019-5-2012: 37$ & Sample Weight: & 1.0000 \\
Run Time (min): & 14.78 & Sample Amount: & 1.0000 \\
\hline
\end{tabular}

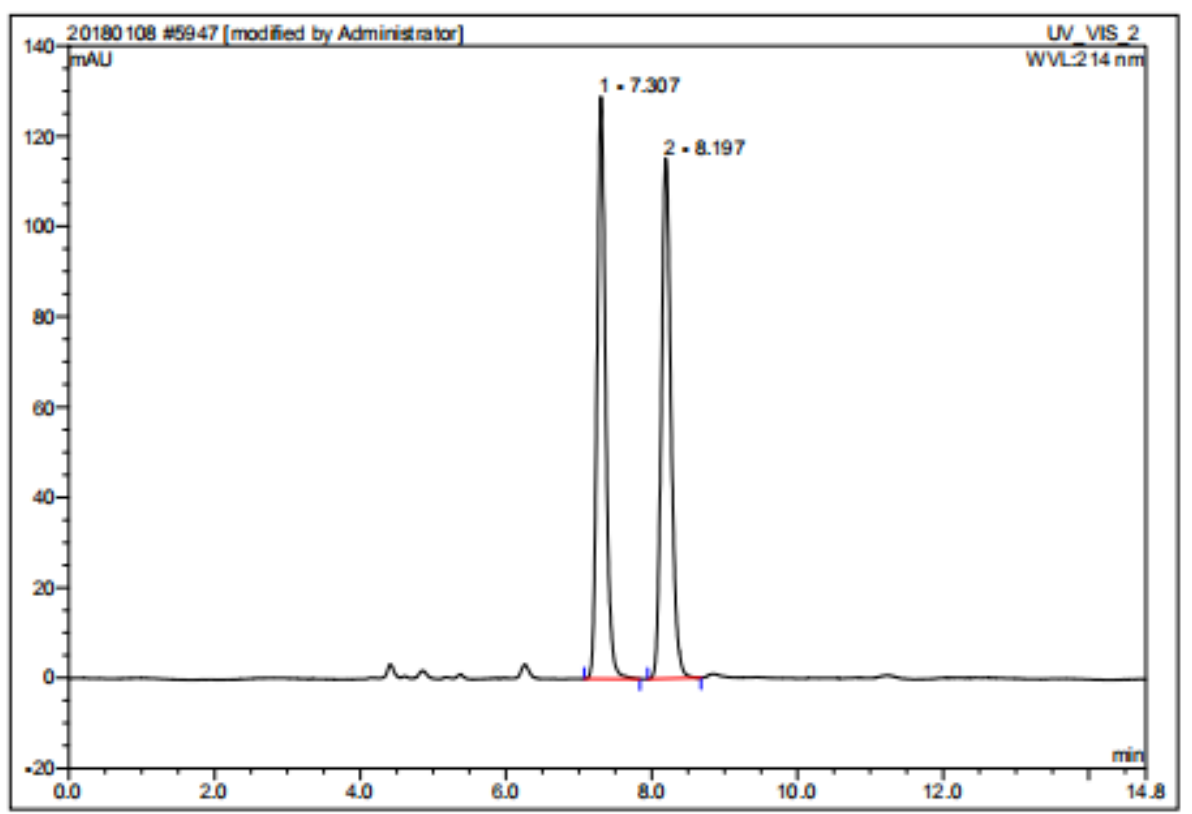

\begin{tabular}{|r|cccrrrr|}
\hline No. & $\begin{array}{c}\text { Ret. Time } \\
\text { min }\end{array}$ & Peak Name & $\begin{array}{c}\text { Height } \\
\text { mAU }\end{array}$ & $\begin{array}{c}\text { Area } \\
\text { mAU*min }\end{array}$ & $\begin{array}{c}\text { Rel.Area } \\
\%\end{array}$ & Amount & Type \\
\hline 1 & 7.31 & n.a. & 128.966 & 17.486 & 49.51 & n.a. & BMB \\
2 & 8.20 & n.a. & 115.281 & 17.833 & 50.49 & n.a. & BMB $^{*}$ \\
\hline Total: & & & 244.247 & 35.319 & 100.00 & 0.000 & \\
\hline
\end{tabular}


5948 HWC-14-35-3B ODH 9822140.7

\begin{tabular}{llll|}
\hline Sample Name: & HWC-14-35-3B ODH 982 214 0.7 & injection Volume: & 2.0 \\
Vial Number: & RC7 & Channet & UV_vS_2 \\
Sample Type: & unknown & Wavelength & 214.0 \\
Control Program: & test-dad6 & Bandwidth: & 4 \\
Quantf. Method: & 20170608 & Dilution Factor: & 1.0000 \\
Recording Time: & $2019-5-2012: 54$ & Sample Weight: & 1.0000 \\
Run Time (min): & 40.00 & Sample Amount: & 1.0000 \\
\hline
\end{tabular}

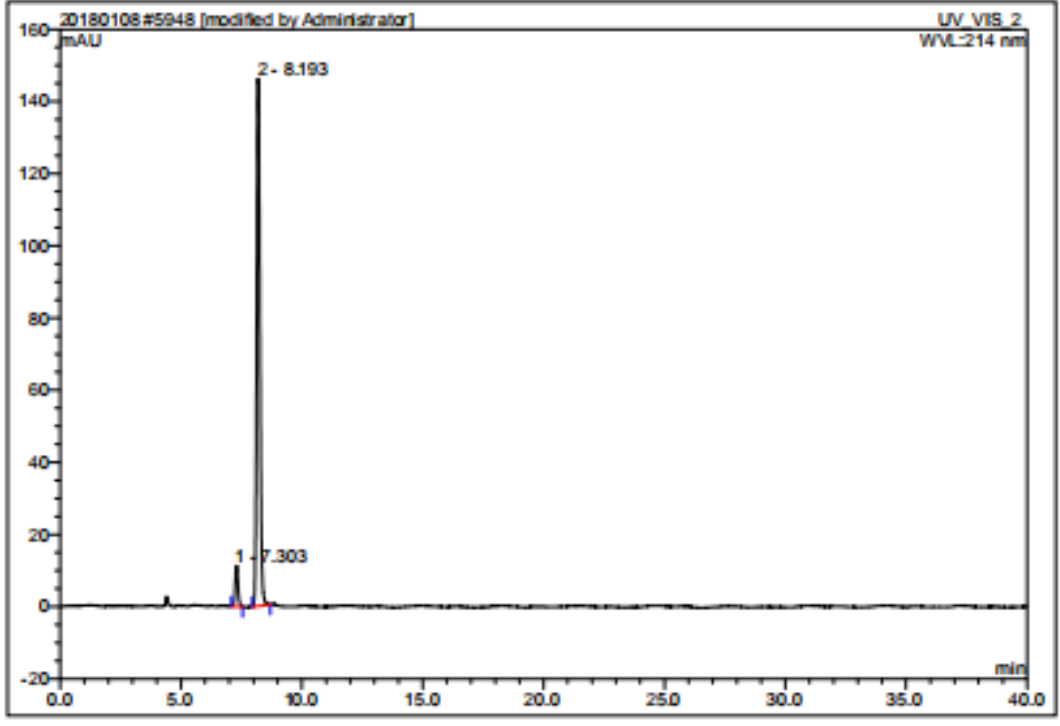

\begin{tabular}{|r|ccrrrrr|}
\hline No. & $\begin{array}{c}\text { Ret.Time } \\
\text { min }\end{array}$ & Peak Name & $\begin{array}{c}\text { Height } \\
\text { mAU }\end{array}$ & $\begin{array}{c}\text { Area } \\
\text { mAU*min }\end{array}$ & $\begin{array}{r}\text { RelArea } \\
\%\end{array}$ & Amount & Type \\
\hline 1 & 7.30 & n.a. & 11.353 & 1.554 & 6.46 & n.a. & BMB \\
2 & 8.19 & n.a. & 146.203 & 22.508 & 93.54 & n.a. & BMB \\
\hline Total: & & & 157.556 & 24.062 & 100.00 & 0.000 & \\
\hline
\end{tabular}


<smiles>FC(c1ccc(Cl)cc1)c1cc(Cl)cc(Cl)c1</smiles>

Compound 3af. HPLC (AD-H, $0.46 \times 25 \mathrm{~cm}, 5 \mu \mathrm{m}$, hexane/isopropanol $=100 / 0$ (v/v \%), flow $0.7 \mathrm{~mL} / \mathrm{min}$, UV detection at $214 \mathrm{~nm}$ ), retention time $=15.98 \mathrm{~min}$ (minor) and $19.32 \mathrm{~min}$ (major). $[\alpha]_{\mathrm{D}}^{25}=-13.40\left(\mathrm{c}=0.100, \mathrm{CHCl}_{3}, 94: 6\right.$ e.r).

$2019-6-13 \quad 3: 31$

\begin{tabular}{|llll|}
\hline 5614 HWC-14-58-3F+- ADH 12140.7 & & \\
& & & \\
\hline Sample Name: & HWC-14-58-3F+-ADH 1 214 0.7 & Injection Volume: & $\mathbf{5 . 0}$ \\
Vial Number: & RE3 & Channel: & UV_VIS_2 \\
Sample Type: & unknown & Wavelength: & 214.0 \\
Control Program: & test-dad4 & Bandwidth: & 4 \\
Quantif. Method: & 20170608 & Dilution Factor: & 1.0000 \\
Recording Time: & $2019-4-2418: 06$ & Sample Weight: & 1.0000 \\
Run Time (min): & 25.00 & Sample Amount: & 1.0000 \\
\hline
\end{tabular}

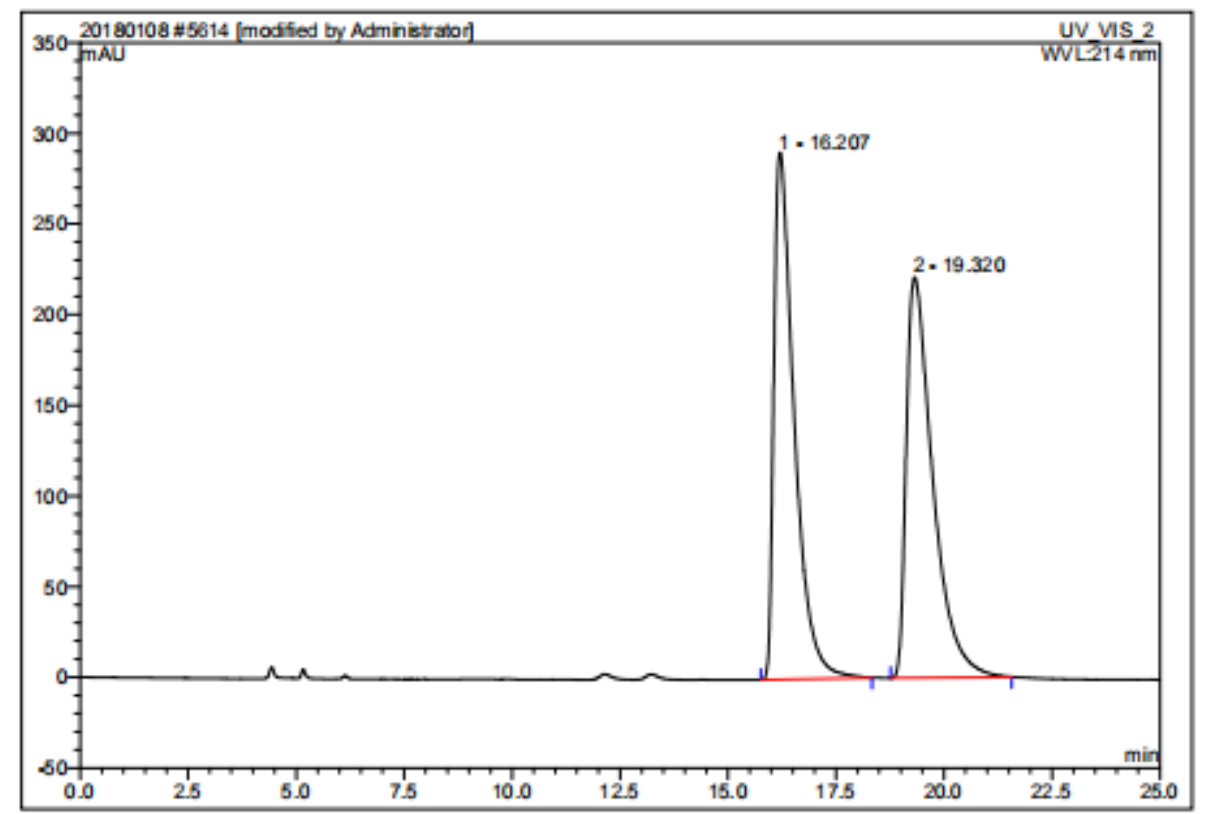

\begin{tabular}{|r|cccrrrr|}
\hline No. & $\begin{array}{c}\text { Ret. Time } \\
\text { min }\end{array}$ & Peak Name & $\begin{array}{c}\text { Height } \\
\text { mAU }\end{array}$ & $\begin{array}{c}\text { Area } \\
\text { mAU*min }\end{array}$ & $\begin{array}{c}\text { Rel.Area } \\
\%\end{array}$ & Amount & Type \\
\hline 1 & 16.21 & n.a. & 290.751 & 158.828 & 50.25 & n.a. & BMB \\
2 & 19.32 & n.a. & 221.027 & 157.259 & 49.75 & n.a. & BMB \\
\hline Total: & & & 511.779 & 316.086 & 100.00 & 0.000 & \\
\hline
\end{tabular}




\begin{tabular}{|llll|}
\hline 5615 HWC-14-59-3F ADH 1214 & 0.7 & & \\
& & & \\
\hline Sample Name: & HWC-14-50-3F ADH 1214 0.7 & injection Volume: & $\mathbf{5 . 0}$ \\
Vial Number: & RE4 & Channet & UV_VS_2 \\
Sample Type: & unknown & Wavelength: & 214.0 \\
Control Program: & test-dad4 & Bandwioth: & 4 \\
Quandf. Method: & 20170608 & Dllution Factor: & 1.0000 \\
Recording Time: & $2019-4-2418: 33$ & Sample Weight: & 1.0000 \\
Run Time (min): & 25.00 & Sample Amount: & 1.0000 \\
\hline
\end{tabular}

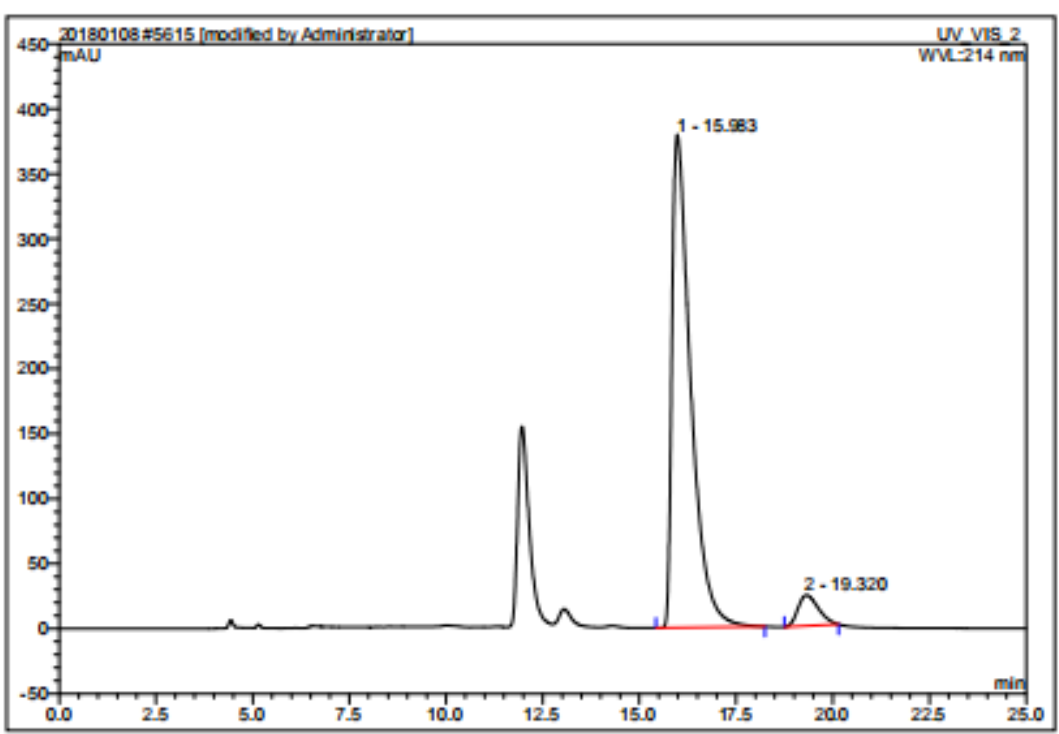

\begin{tabular}{|r|rlrrrrr|}
\hline No. & $\begin{array}{c}\text { Ret.Time } \\
\text { min }\end{array}$ & Peak Name & $\begin{array}{c}\text { Height } \\
\text { mAU }\end{array}$ & $\begin{array}{c}\text { Area } \\
\text { mAU*min }\end{array}$ & $\begin{array}{r}\text { RelArea } \\
\%\end{array}$ & Amount & Type \\
\hline 1 & 15.98 & na. & 379.556 & 212.848 & 93.63 & n.a. & BMB $^{*}$ \\
2 & 19.32 & n.a. & 23.651 & 14.489 & 6.37 & n.a. & BMB $^{*}$ \\
\hline Total: & & & 403.207 & 227.337 & 100.00 & 0.000 & \\
\hline
\end{tabular}


<smiles>FC(c1cccc(Cl)c1)c1cc(Cl)cc(Cl)c1</smiles>

Compound 3ag. HPLC (OD-H, $0.46 \times 25 \mathrm{~cm}, 5 \mu \mathrm{m}$, hexane/isopropanol $=97 / 3$ (v/v \%), flow $0.7 \mathrm{~mL} / \mathrm{min}, \mathrm{UV}$ detection at $214 \mathrm{~nm}$ ), retention time $=6.62 \mathrm{~min}$ (minor) and $6.96 \min$ (major). $[\alpha]_{\mathrm{D}}{ }^{25}=-9.38\left(\mathrm{c}=0.160, \mathrm{CHCl}_{3}, 93: 7\right.$ e.r).

\section{Empower' 3}

\begin{tabular}{|c|c|c|c|}
\hline & SAMPLE & INFORMATION & \\
\hline Sample Name: & HMC-14ळ-3B+-OD 9732140.7 & Acqined By: & System \\
\hline Sample Type & Urkrown & Sample Set Name: & 20190428 \\
\hline Val: & $1 \mathrm{~A}, 3$ & Acq Method Set: & $4 B$ \\
\hline Irjection\# & 1 & Processing Methat & 1 \\
\hline Irjedion Vaume: & $1.00 \mathrm{u}$ & Crand Name. & PDAOM1 214nm@4.8m \\
\hline Ren Time: & 140.0 Minutes & Prc. Orri. Desa.: & PDAOM1 214nm@4.8rm \\
\hline DateAcquired: & 4/29:2019344:06 PM CST & & \\
\hline DateProcessed: & 6/13/2019:4:57:50RM CST & & \\
\hline
\end{tabular}

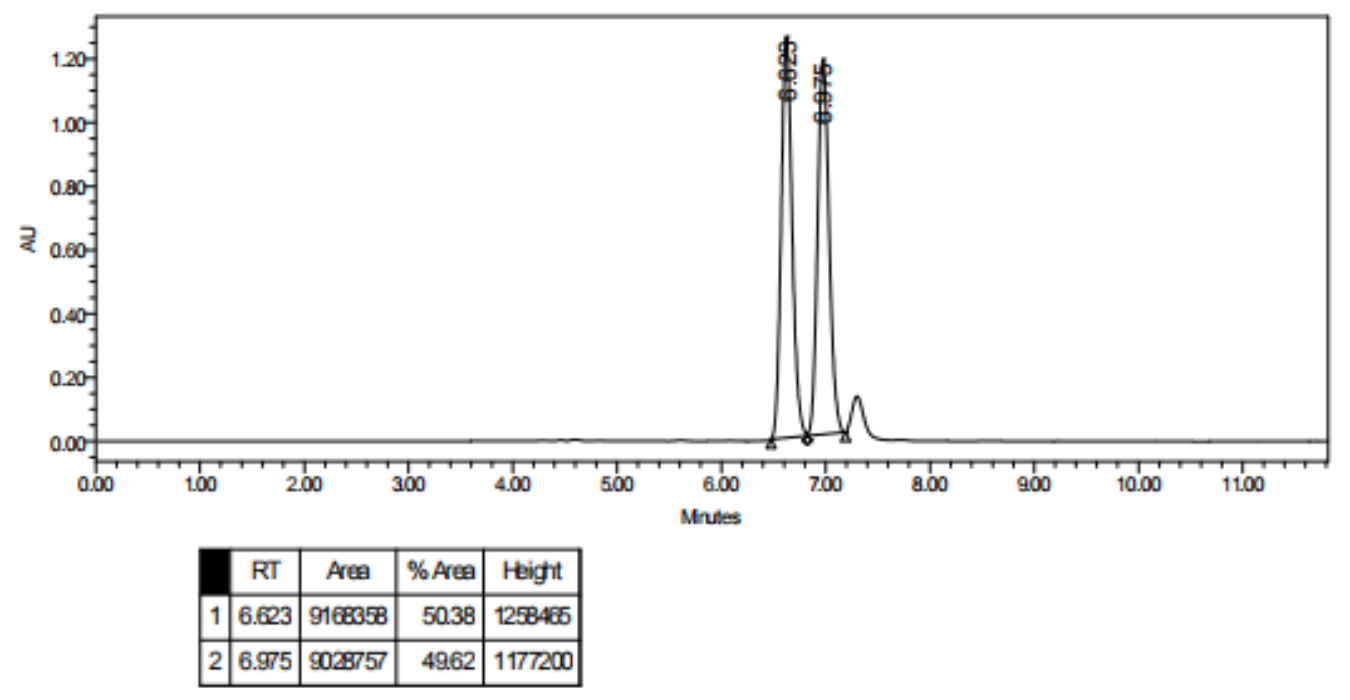




\section{SAMPLE INFORMATION}

\begin{tabular}{|c|c|c|c|}
\hline $\begin{array}{l}\text { Sample Name } \\
\text { Sample Type }\end{array}$ & HNC-14643B CDH-97321407 & Acquired By. & System \\
\hline & $\begin{array}{l}\text { Urkrown } \\
1 \text {;A,4 }\end{array}$ & $\begin{array}{l}\text { Sample Set Name } \\
\text { Acq Method Set: }\end{array}$ & $\begin{array}{l}20190428 \\
428\end{array}$ \\
\hline hjection\#: & 1 & Rocessirg Method & 1 \\
\hline hjection Vdume & $1.00 \mathrm{u}$ & Creme Name & PDACh1214nm@4.8nm \\
\hline Run Time & 140.0 Minutes & Prc. Onri. Descr.: & PDACh1214nm@4.8nm \\
\hline Date Acquiredt & 4/29/201935052 PMCST & & \\
\hline Date Piocessect & 6/132019 4:57.21 PMCST & & \\
\hline
\end{tabular}

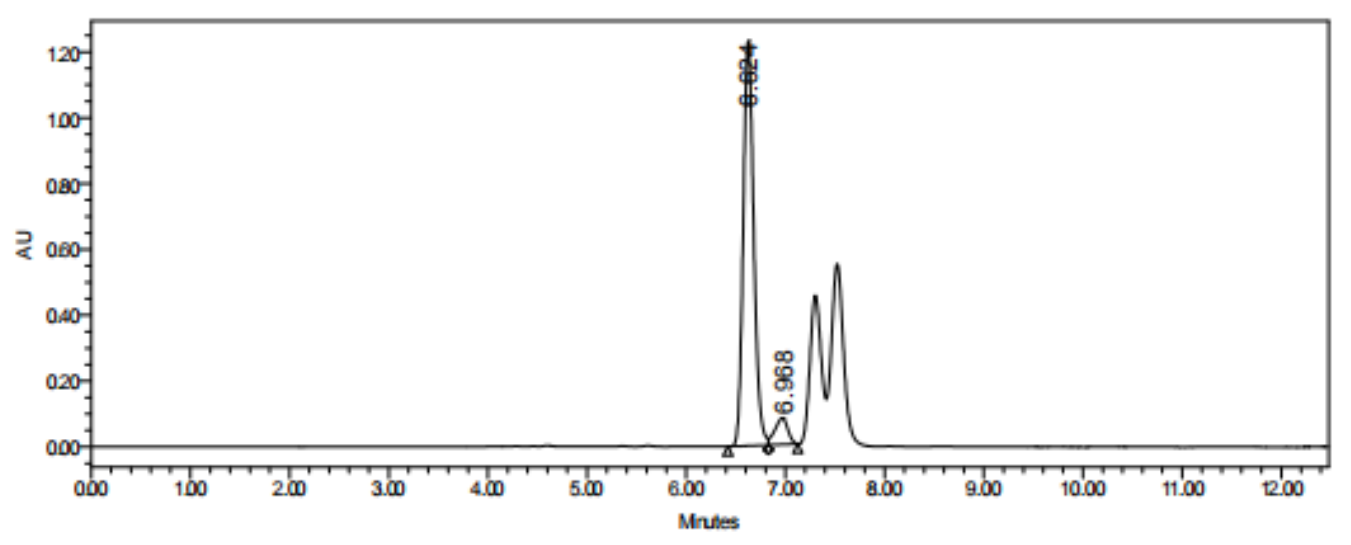

\begin{tabular}{|r|c|c|r|r|}
\hline & RT & \multicolumn{1}{c|}{ Area } & \% Avea & Height \\
\hline 1 & 6.624 & 9041960 & 92.50 & 1229022 \\
\hline 2 & 6.968 & 730801 & 7.50 & 80621 \\
\hline
\end{tabular}


<smiles>Fc1cc(C(F)c2ccccc2)ccc1Cl</smiles>

Compound 3ah. HPLC (OJ-H, $0.46 \times 25 \mathrm{~cm}, 5 \mu \mathrm{m}$, hexane/isopropanol = 99/1 $(\mathrm{v} / \mathrm{v} \%)$, flow $0.7 \mathrm{~mL} / \mathrm{min}$, UV detection at $214 \mathrm{~nm})$, retention time $=14.42 \mathrm{~min}$ (minor) and $15.75 \mathrm{~min}$ (major). $[\alpha]_{\mathrm{D}}^{25}=+0.67\left(\mathrm{c}=0.150, \mathrm{CHCl}_{3}, 93: 7\right.$ e.r).

\begin{tabular}{|llll|}
\hline 5400 HWC-14-27-3B+- OJH 9912140.7 & & \\
& & & \\
\hline Sample Name: & HWC-14-27-3B+-OJH 9912140.7 & hjection Volume: & 6.0 \\
Vial Number: & RC5 & Channel: & UV_VIS_2 \\
Sample Type: & unknown & Wavelength: & 214.0 \\
Control Program: & test-dad4 & Bandwidth: & 4 \\
Quantif. Method: & 20170608 & Dilution Factor: & 1.0000 \\
Recording Time: & $2019-4-49: 32$ & Sample Weight: & 1.0000 \\
Run Time (min): & 22.16 & Sample Amount: & 1.0000 \\
\hline
\end{tabular}

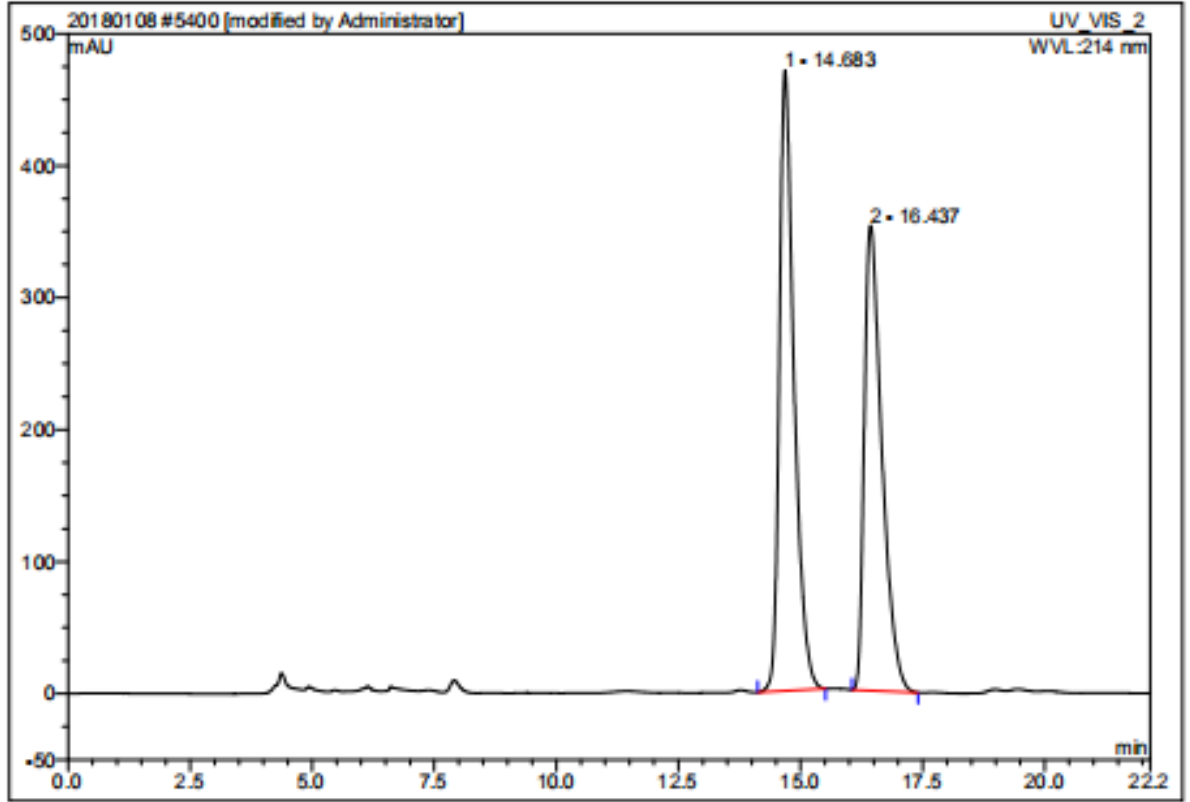

\begin{tabular}{|c|ccccccc|}
\hline No. & $\begin{array}{c}\text { Ret. Time } \\
\text { min }\end{array}$ & Peak Name & $\begin{array}{c}\text { Height } \\
\text { mAU }\end{array}$ & $\begin{array}{c}\text { Area } \\
\text { mAU*min }\end{array}$ & $\begin{array}{c}\text { Rel.Area } \\
\%\end{array}$ & Amount & Type \\
\hline 1 & 14.68 & n.a. & 470.040 & 163.630 & 52.74 & n.a. & BMB \\
2 & 16.44 & n.a. & 352.210 & 146.611 & 47.26 & n.a. & BMB \\
\hline Total: & & & 822.249 & 310.241 & 100.00 & 0.000 & \\
\hline
\end{tabular}


5401 HWC-14-27-3A OJH 9912140.7

\begin{tabular}{|llll|}
\hline Sample Name: & HWC-14-27-3A OJH 9912140.7 & Injection Volume: & 6.0 \\
Vial Number: & RC6 & Channel: & UV_VIS_2 \\
Sample Type: & unknown & Wavelength: & 214.0 \\
Control Program: & test-dad4 & Bandwidth: & 4 \\
Quantif. Method: & 20170608 & Dilution Factor: & 1.0000 \\
Recording Time: & $2019-4-49: 58$ & Sample Weight: & 1.0000 \\
Run Time (min): & 25.00 & Sample Amount: & 1.0000 \\
\hline
\end{tabular}

\begin{tabular}{|c|c|}
\hline 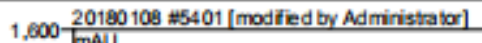 & UV VIS 2 \\
\hline
\end{tabular}

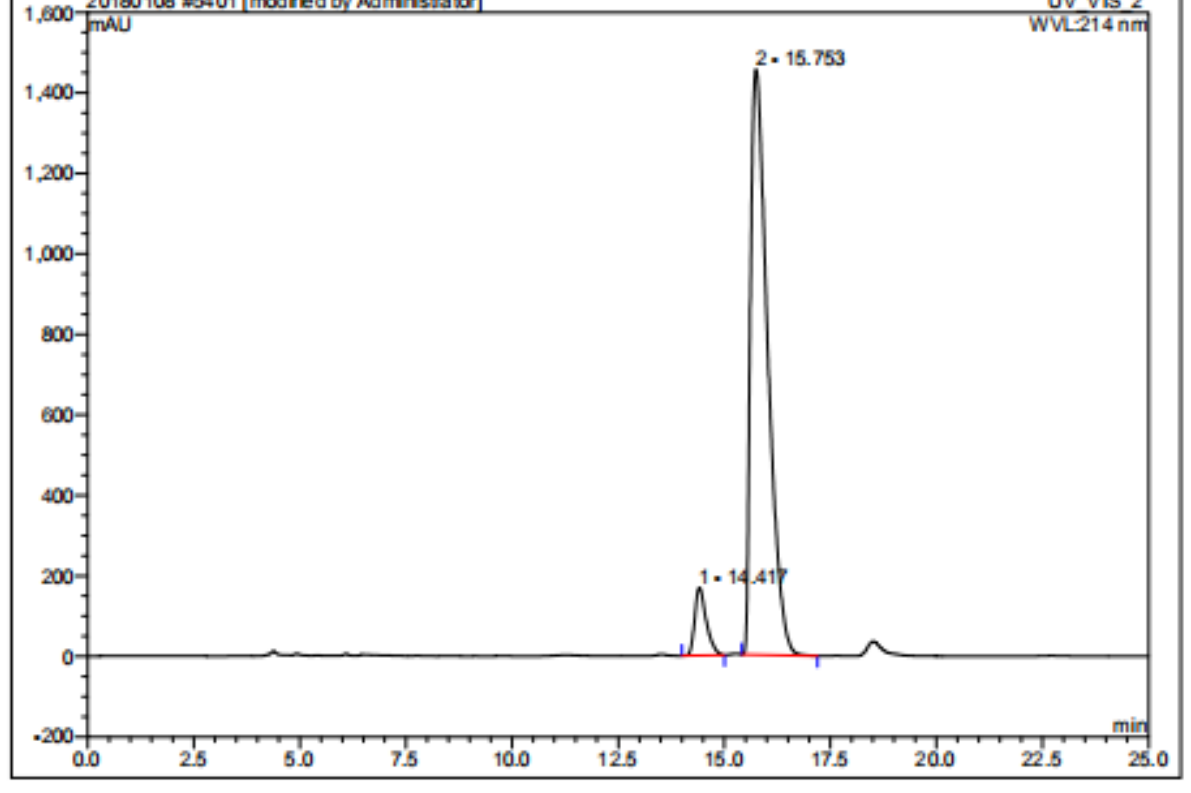

\begin{tabular}{|r|rrrrrrr|}
\hline No. & $\begin{array}{c}\text { Ret.Time } \\
\text { min }\end{array}$ & Peak Name & $\begin{array}{c}\text { Height } \\
\text { mAU }\end{array}$ & $\begin{array}{c}\text { Area } \\
\text { mAU*min }\end{array}$ & $\begin{array}{r}\text { Rel.Area } \\
\%\end{array}$ & Amount & Type \\
\hline 1 & 14.42 & n.a. & 168.843 & 52.996 & 7.22 & n.a. & BMB $^{*}$ \\
2 & 15.75 & n.a. & 1453.913 & 681.329 & 92.78 & n.a. & BMB $^{*}$ \\
\hline Total: & & & 1622.756 & 734.325 & 100.00 & 0.000 & \\
\hline
\end{tabular}




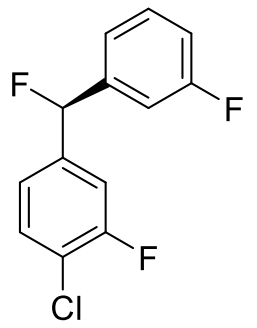

Compound 3ai. HPLC (OD-H, $0.46 \times 25 \mathrm{~cm}, 5 \mu \mathrm{m}$, hexane/isopropanol $=100 / 0$ $(\mathrm{v} / \mathrm{v} \%$ ), flow $0.7 \mathrm{~mL} / \mathrm{min}, \mathrm{UV}$ detection at $214 \mathrm{~nm})$, retention time $=16.71 \mathrm{~min}$ (minor) and $17.67 \mathrm{~min}$ (major). $[\alpha]_{\mathrm{D}}{ }^{25}=-6.30\left(\mathrm{c}=0.270, \mathrm{CHCl}_{3}, 96: 4\right.$ e.r).

\section{Empower"3}

\begin{tabular}{|c|c|c|c|}
\hline & SAMPLE & INFORMATION & \\
\hline SampleName: & HMG $14533 \mathrm{D}-\mathrm{ODH} 12140.7$ & Acquired By. & System \\
\hline Sample Type & Urknown & Sample Set Name & 20190422 \\
\hline Via: & 1:D1 & Acq MetrodSet: & 1017 \\
\hline Injection\# \# & 1 & Processing Method: & 1 \\
\hline Injection Voume & $300 u$ & Crand Narre: & PDAOM1 214rm@4.8rm \\
\hline Run Time: & 100.0Minutes & Proc. Onri. Descr.: & PDAOM1 214rm@4.8rm \\
\hline Date Acquired: & 4/23/2019239.22 RM CST & & \\
\hline Date Rocessed: & ब 13/2019 4:37:06 PM CST & & \\
\hline
\end{tabular}

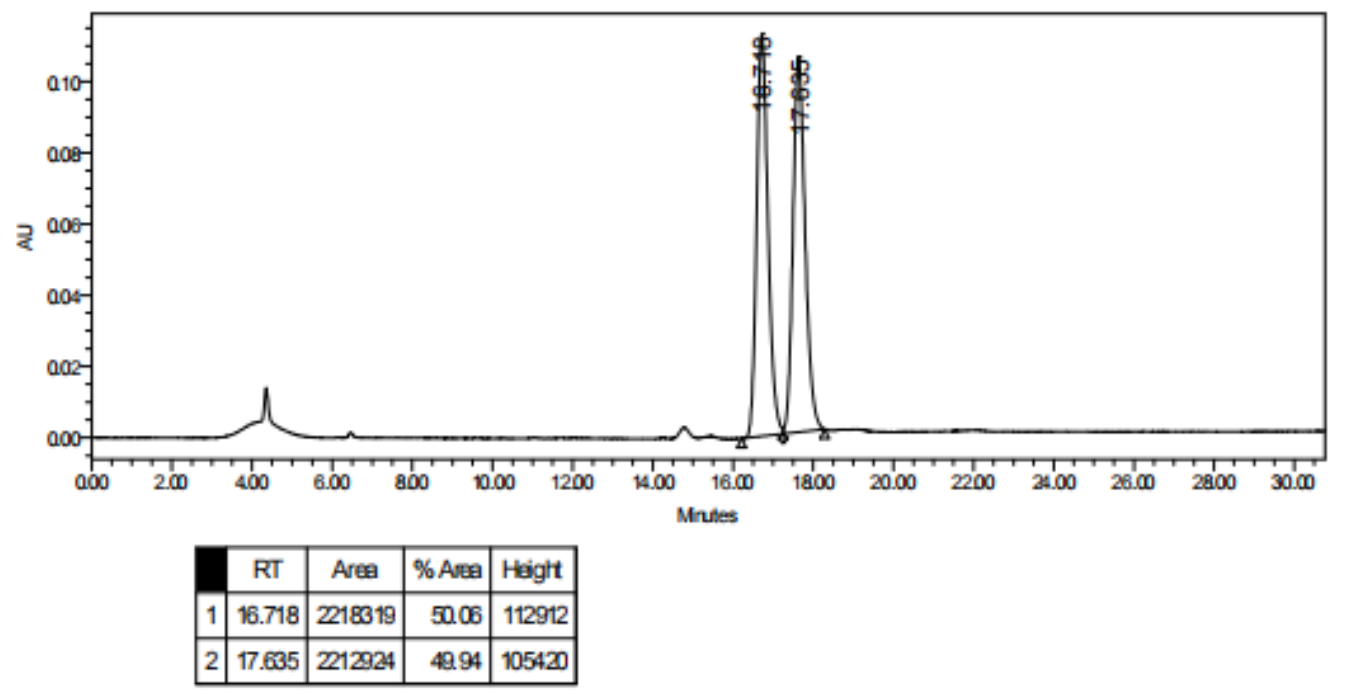




\begin{tabular}{|c|c|c|c|}
\hline & SAMPLE & INFORMATION & \\
\hline $\begin{array}{l}\text { Sample Name: } \\
\text { Sample Type: }\end{array}$ & $\begin{array}{l}\text { HMG-1452-30 CDH12140.7 } \\
\text { Unknown }\end{array}$ & $\begin{array}{l}\text { Acquied By: } \\
\text { Sample Set Name: }\end{array}$ & $\begin{array}{l}\text { System } \\
\text { 20190422 }\end{array}$ \\
\hline Ma: & $\mathrm{t}: \mathrm{D}, 2$ & Acq Mathod Set: & 1017 \\
\hline Injation & 1 & Prowessing Mathod: & 1 \\
\hline Injection Vdume: & $3.00 \mathrm{u}$ & Chand Name. & PDA On1 214mgas. $8 \mathrm{~mm}$ \\
\hline Run Time: & 100.0 Mntes & Pra. Ond. Descr: & PDA Ch1 214m gas.8m \\
\hline Date Acquired. & 4/23/20193:13:22 PM CST & & \\
\hline Date Processed. & G/132019 4:36:42PMM CST & & \\
\hline
\end{tabular}

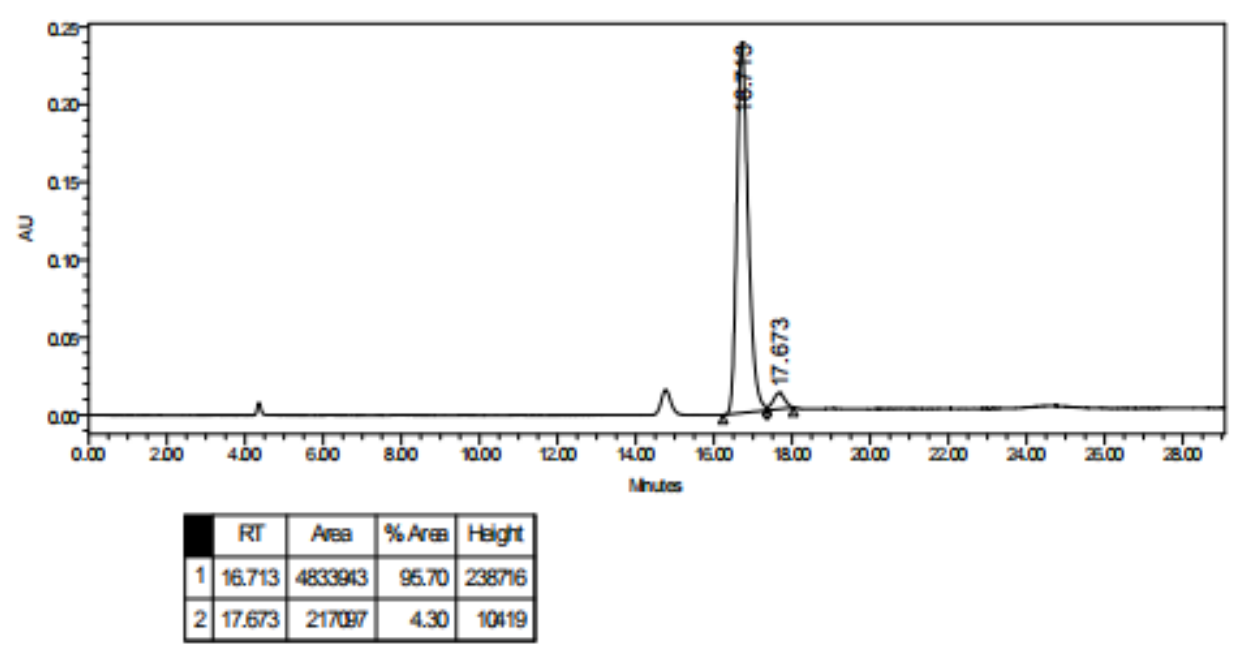


<smiles>Cc1cc(C)c(CNC(=O)c2ccc(C(F)c3ccccc3)cc2)c(O)n1</smiles>

Compound 5. SFC (OJ-H, $0.46 \times 25 \mathrm{~cm}, 5 \mu \mathrm{m}, \mathrm{CO}_{2} / \mathrm{MeOH}=92 / 8$, flow $2.0 \mathrm{~mL} / \mathrm{min}$, column Temperature: $40{ }^{\circ} \mathrm{C}$, background press: 2000 psi, UV detection at $214 \mathrm{~nm}$ ), retention time $=30.03 \min \left(\right.$ major) and $31.68 \min \left(\right.$ minor) $.[\alpha]_{\mathrm{D}}^{25}=-13.33(\mathrm{c}=0.120$, $\mathrm{CHCl}_{3}, 94: 6$ e.r).

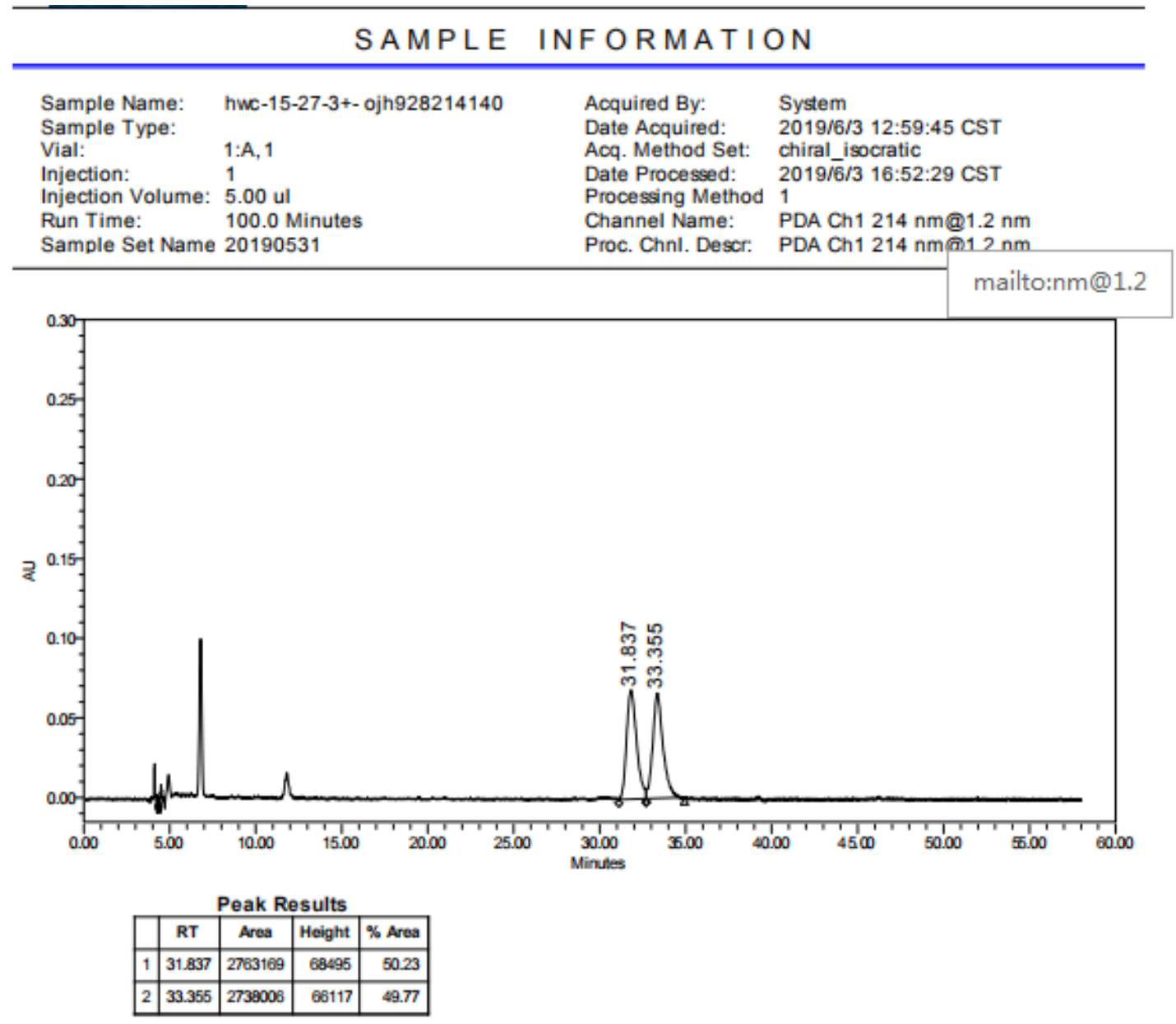




\section{Empower" 3}

SAMPLE INFORMATION

$\begin{array}{llll}\text { Sample Name: } & \text { hwc-15-33-3a ojh928214140 } & \text { Acquired By: } & \text { System } \\ \text { Sample Type: } & & \text { Date Acquired: } & \text { 2019/6/3 13:59:02 CST } \\ \text { Vial: } & \text { 1:A,2 } & \text { Acq. Method Set: } & \text { chiral_isocratic } \\ \text { Injection: } & 1 & \text { Date Processed: } & 2019 / 6 / 316: 50: 31 \text { CST } \\ \text { Injection Volume: } & 5.00 \text { ul } & \text { Processing Method } & 1 \\ \text { Run Time: } & 100.0 \text { Minutes } & \text { Channel Name: } & \text { PDA Ch1 214 nm@1.2 nm } \\ \text { Sample Set Name } & 20190531 & \text { Proc. Chnl. Descr: } & \text { PDA Ch1 214 nm@1.2 nm }\end{array}$

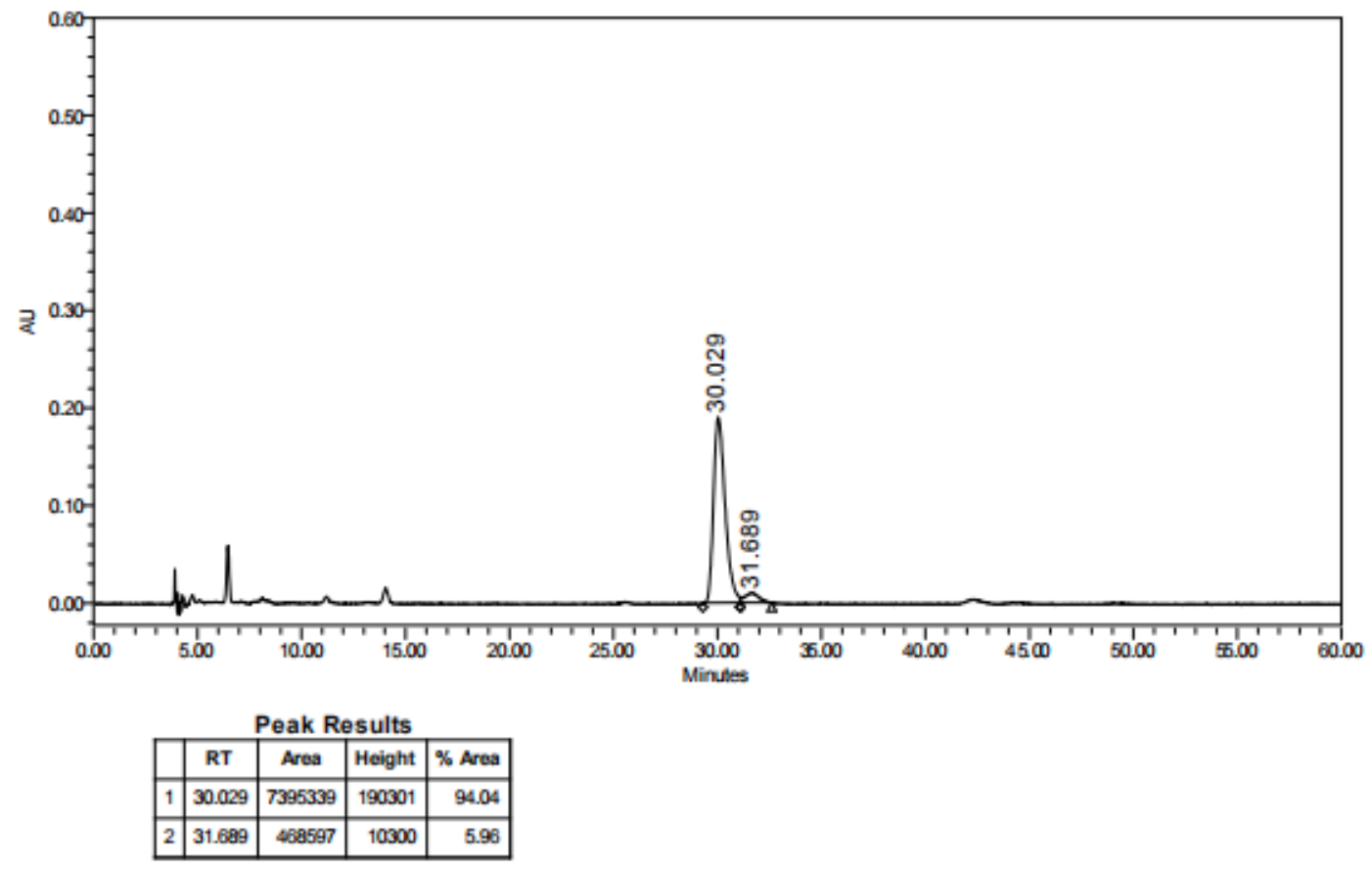




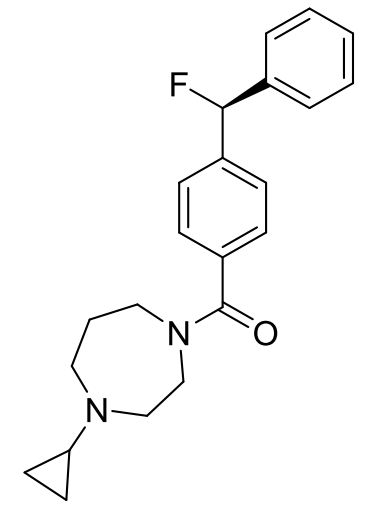

Compound 6. HPLC (AD-H, $0.46 \times 25 \mathrm{~cm}, 5 \mu \mathrm{m}$, hexane/isopropanol $=90 / 10$ (v/v \%), flow $0.7 \mathrm{~mL} / \mathrm{min}$, UV detection at $214 \mathrm{~nm}$ ), retention time $=32.17 \mathrm{~min}$ (minor) and $33.68 \mathrm{~min}$ (major). $[\alpha]_{\mathrm{D}}{ }^{25}=-6.78\left(\mathrm{c}=0.180, \mathrm{CHCl}_{3}, 95: 5\right.$ e.r).

\begin{tabular}{|llll|}
\hline 6145 HWC-15-49-3B+- ADH 912140.7 & & \\
& & & \\
\hline Sample Name: & HWC-15-49-3B+-ADH 912140.7 & injection Volume: & 3.0 \\
Vial Number: & RA5 & Channet & UV_VS_2 \\
Sample Type: & unknown & Wavelength: & 214.0 \\
Control Program: & test-dad6 & Bandwibth: & 4 \\
Quantf. Method: & 20170608 & Dllution Factor: & 1.0000 \\
Recording Time: & $2019-6-128: 36$ & Sample Weight: & 1.0000 \\
Run Time (min): & 55.48 & Sample Amount: & 1.0000 \\
\hline
\end{tabular}

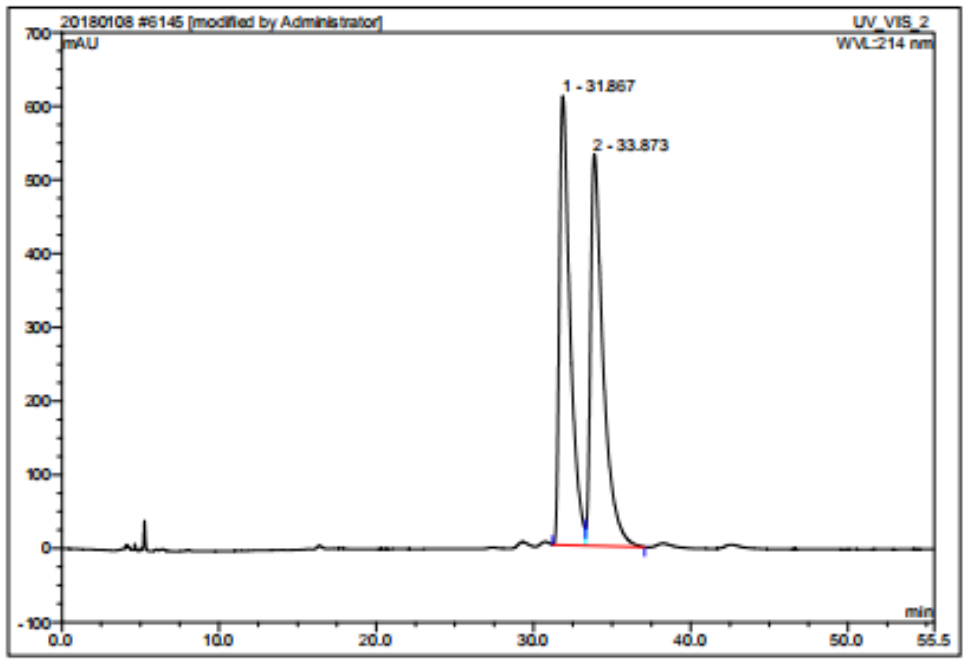

\begin{tabular}{|r|ccrrrrr|}
\hline No. & $\begin{array}{c}\text { Ret.Time } \\
\text { min }\end{array}$ & Peak Name & $\begin{array}{c}\text { Height } \\
\text { mAU }\end{array}$ & $\begin{array}{c}\text { Area } \\
\text { mAU'min }\end{array}$ & $\begin{array}{c}\text { Rel.Area } \\
\%\end{array}$ & Amount & Type \\
\hline 1 & 31.87 & n.a. & 609.554 & 481.940 & 49.41 & n.a. & BM \\
2 & 33.87 & na. & 531.191 & 493.368 & 50.59 & n.a. & MB \\
\hline Total: & & & 1140.745 & 975.308 & 100.00 & 0.000 & \\
\hline
\end{tabular}




\begin{tabular}{|llll|}
\hline 6146 HWC-15-33-3B ADH 912140.7 & & \\
& & & \\
\hline Sample Name: & HWC-15-33-3B ADH 912140.7 & injection Volume: & 2.0 \\
Vial Number: & RA4 & Channel: & UV_VIS_2 \\
Sample Type: & unknown & Wavelength: & 214.0 \\
Control Program: & test-dad6 & Bandwidth: & 4 \\
Quantif. Method: & 20170608 & Dilution Factor: & 1.0000 \\
Recording Time: & $2019-6-1117: 41$ & Sample Weight: & 1.0000 \\
Run Time (min): & 60.00 & Sample Amount: & 1.0000 \\
\hline
\end{tabular}

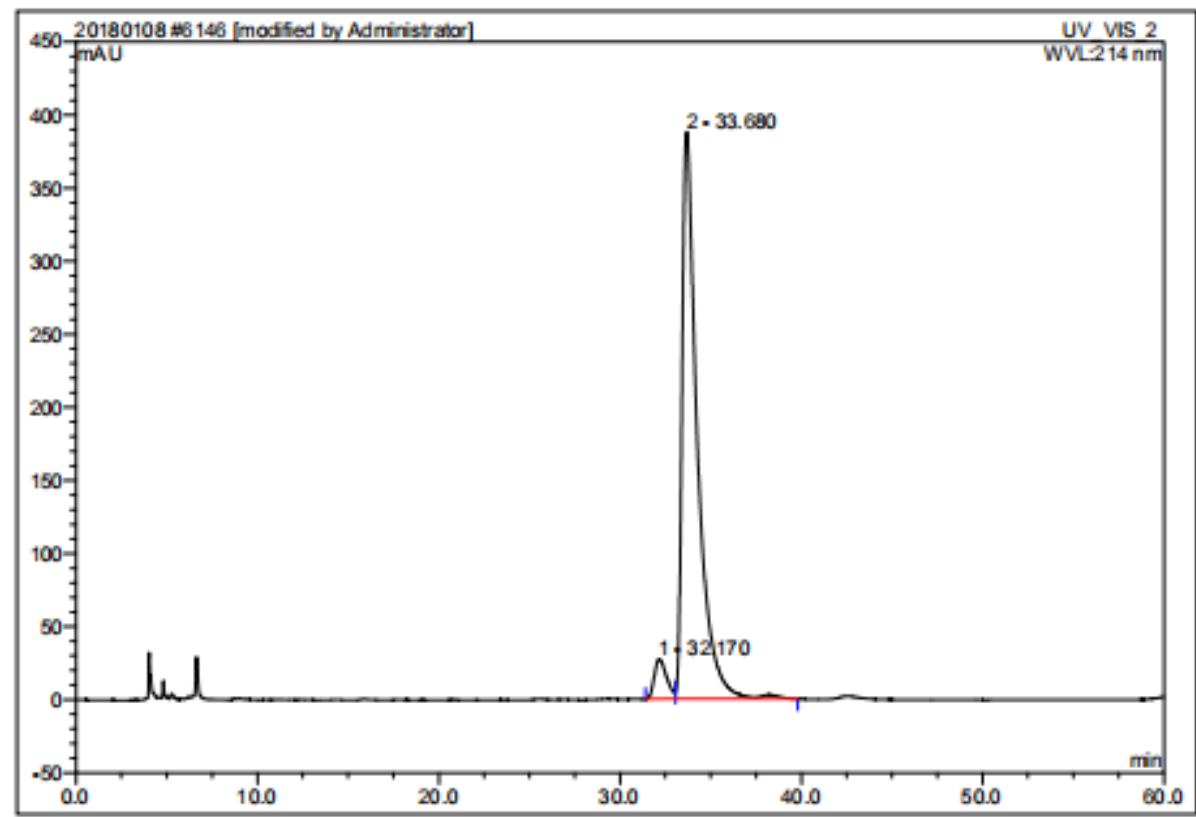

\begin{tabular}{|c|c|c|c|c|c|c|c|}
\hline No. & $\begin{array}{c}\text { Ret.Time } \\
\text { min }\end{array}$ & Peak Name & $\begin{array}{c}\text { Height } \\
\text { mAU }\end{array}$ & $\begin{array}{c}\text { Area } \\
\text { mAU*min }\end{array}$ & $\begin{array}{c}\text { Rel.Area } \\
\%\end{array}$ & Amount & Type \\
\hline 1 & 32.17 & n.a. & 27.277 & 21.527 & 5.38 & na. & $\mathrm{BM}^{*}$ \\
\hline 2 & 33.68 & n.a. & 388.042 & 378.769 & 94.62 & n.a. & $\mathrm{MB}^{*}$ \\
\hline Total: & & & 415.319 & 400.296 & 100.00 & 0.000 & \\
\hline
\end{tabular}


<smiles>N#Cc1cccc(C(F)c2ccc(CO[Sb])cc2)c1</smiles>

Compound 7. HPLC (IG, $0.46 \times 25 \mathrm{~cm}, 5 \mu \mathrm{m}$, hexane/isopropanol $=95 / 5(\mathrm{v} / \mathrm{v} \%)$, flow $0.7 \mathrm{~mL} / \mathrm{min}$, UV detection at $214 \mathrm{~nm}$ ), retention time $=7.52 \mathrm{~min}$ (minor) and $7.94 \min$ (major). $[\alpha]_{\mathrm{D}}{ }^{25}=-33.44\left(\mathrm{c}=0.180, \mathrm{CHCl}_{3}, 92: 8\right.$ e.r).

6065 HWC-15-26-3A+- IG 9552140.7

\begin{tabular}{|llll|}
\hline Sample Name: & HWC-15-26-3A+- IG 955 214 0.7 & hjection Volume: & 1.0 \\
Vial Number: & RE6 & Channel: & UV_VIS_2 \\
Sample Type: & unknown & Wavelength: & 214.0 \\
Contral Program: & test-dad6 & Bandwidth: & 4 \\
Quantif. Method: & 20170608 & Dilution Factor: & 1.0000 \\
Recording Time: & $2019-6-38: 48$ & Sample Weight: & 1.0000 \\
Run Time (min): & 16.47 & Sample Amount: & 1.0000 \\
\hline
\end{tabular}

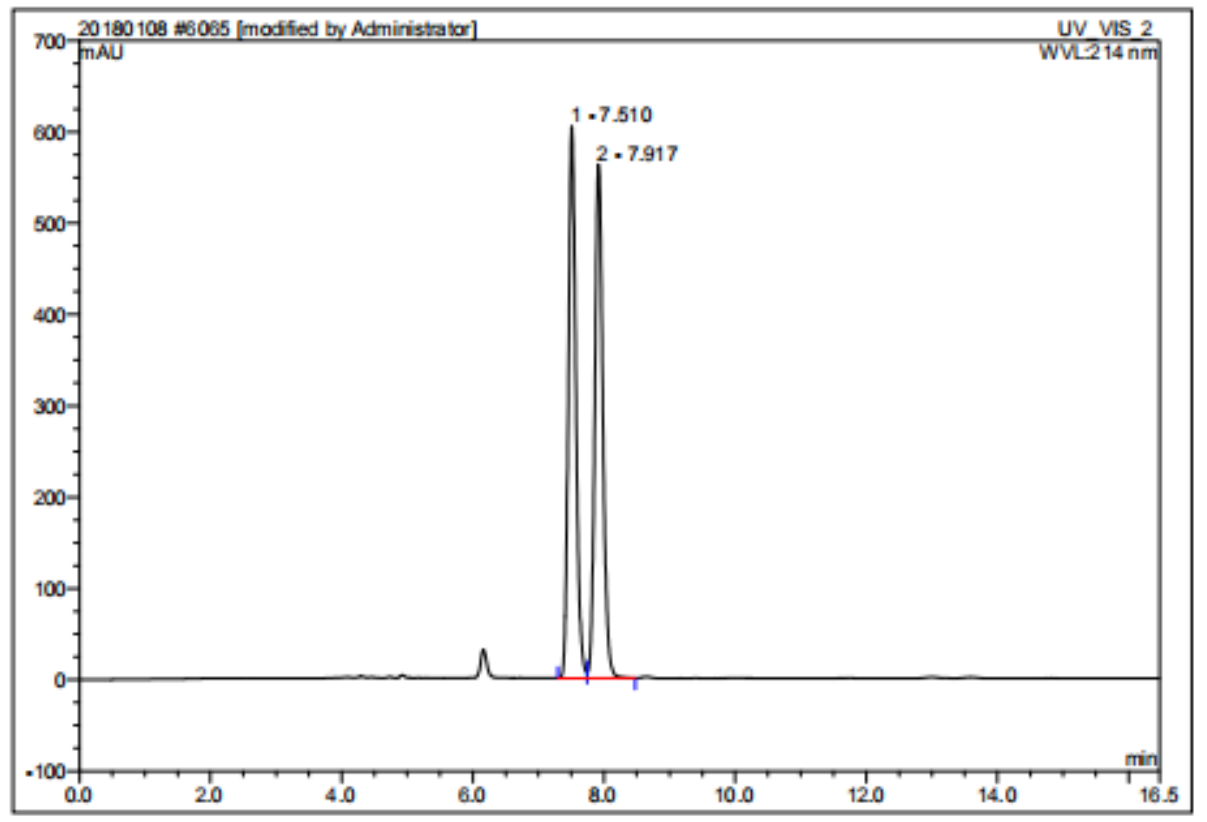

\begin{tabular}{|r|ccrrrrr|}
\hline No. & $\begin{array}{c}\text { Ret. Time } \\
\text { min }\end{array}$ & Peak Name & $\begin{array}{c}\text { Height } \\
\text { mAU }\end{array}$ & $\begin{array}{c}\text { Area } \\
\text { mAU*min }\end{array}$ & $\begin{array}{c}\text { Rel.Area } \\
\%\end{array}$ & Amount & Type \\
\hline 1 & 7.51 & n.a. & 604.443 & 80.011 & 50.01 & n.a. & BM $^{*}$ \\
2 & 7.92 & n.a. & 562.538 & 79.979 & 49.99 & n.a. & MB $^{*}$ \\
\hline Total: & & & 1166.981 & 159.990 & 100.00 & 0.000 & \\
\hline
\end{tabular}




\begin{tabular}{|lllll|}
\hline 6066 HWC-15-31-3A IG 9552140.7 & & \\
& & & \\
\hline Sample Name: & HWC-15-31-3A IG 9552140.7 & injection Volume: & 1.0 \\
Vial Number: & RE5 & Channet & UV_vS_2 \\
Sample Type: & unknown & Wavelength: & 214.0 \\
Cortrol Program: & test-dad6 & Bandwidth: & 4 \\
Quandf. Method: & 20170608 & Dilution Factor: & 1.0000 \\
Recording Time: & $2019-6-39: 07$ & Sample Weight: & 1.0000 \\
Run Time (min): & 20.00 & Sample Amount: & 1.0000 \\
\hline
\end{tabular}

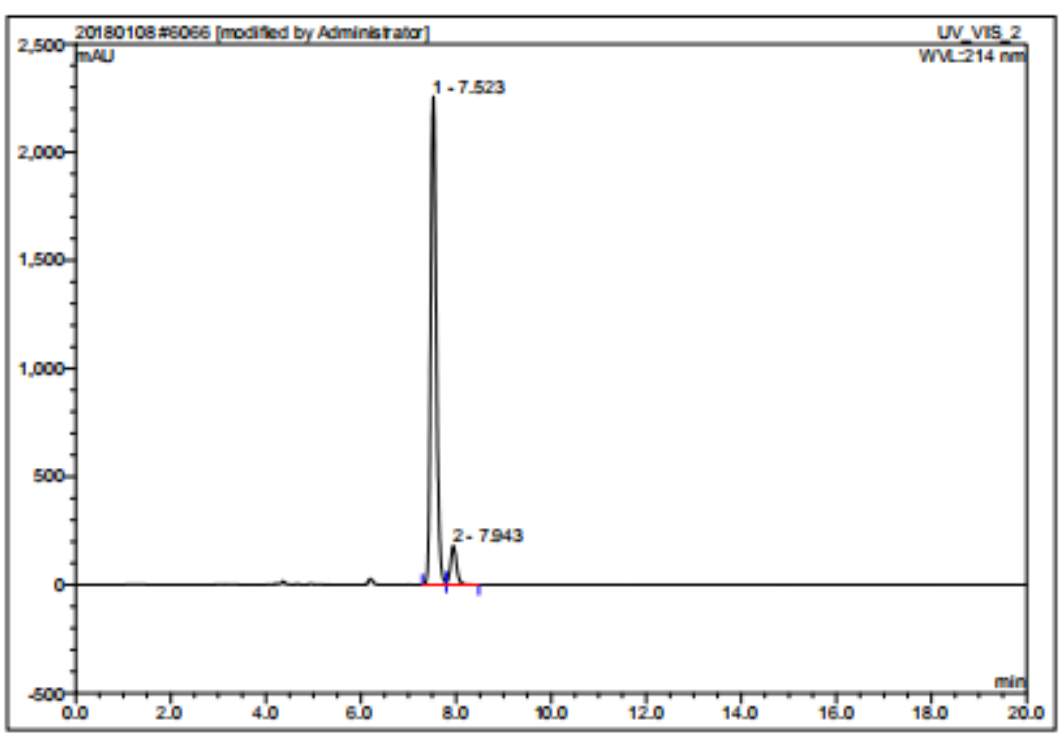

\begin{tabular}{|r|rlrrrrr|}
\hline No. & $\begin{array}{c}\text { Ret.Time } \\
\text { min }\end{array}$ & Peak Name & $\begin{array}{c}\text { Height } \\
\text { mAU }\end{array}$ & $\begin{array}{c}\text { Area } \\
\text { mAU'min }\end{array}$ & $\begin{array}{r}\text { RelArea } \\
\%\end{array}$ & Amount & Type \\
\hline 1 & 7.52 & n.a. & 2258.202 & 303.060 & 92.01 & n.a. & BM \\
2 & 7.94 & n.a. & 180.970 & 26.321 & 7.99 & n.a. & MB \\
\hline Total: & & & 2439.172 & 329.381 & 100.00 & 0.000 & \\
\hline
\end{tabular}


${ }^{1} \mathrm{H}$ NMR (400 MHz, $\left.\mathrm{CDCl}_{3}\right)$ spectrum of bis((S)-4-benzhydryl-4,5-dihydrooxazol-2-yl)methane L8

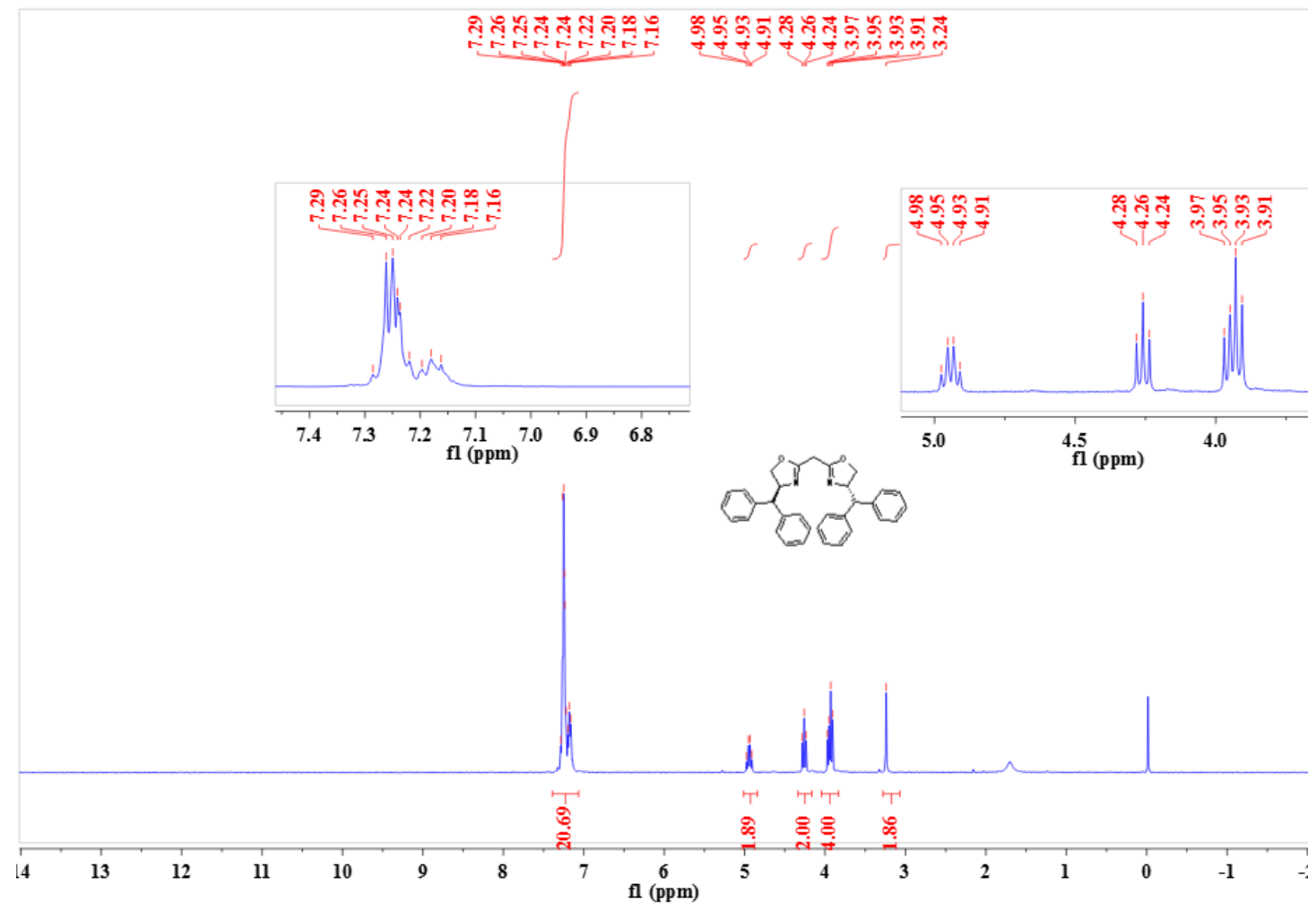

${ }^{13} \mathrm{C}$ NMR (101 MHz, $\left.\mathrm{CDCl}_{3}\right)$ spectrum of bis((S)-4-benzhydryl-4,5-dihydrooxazol-2-yl)methane $\mathbf{L 8}$

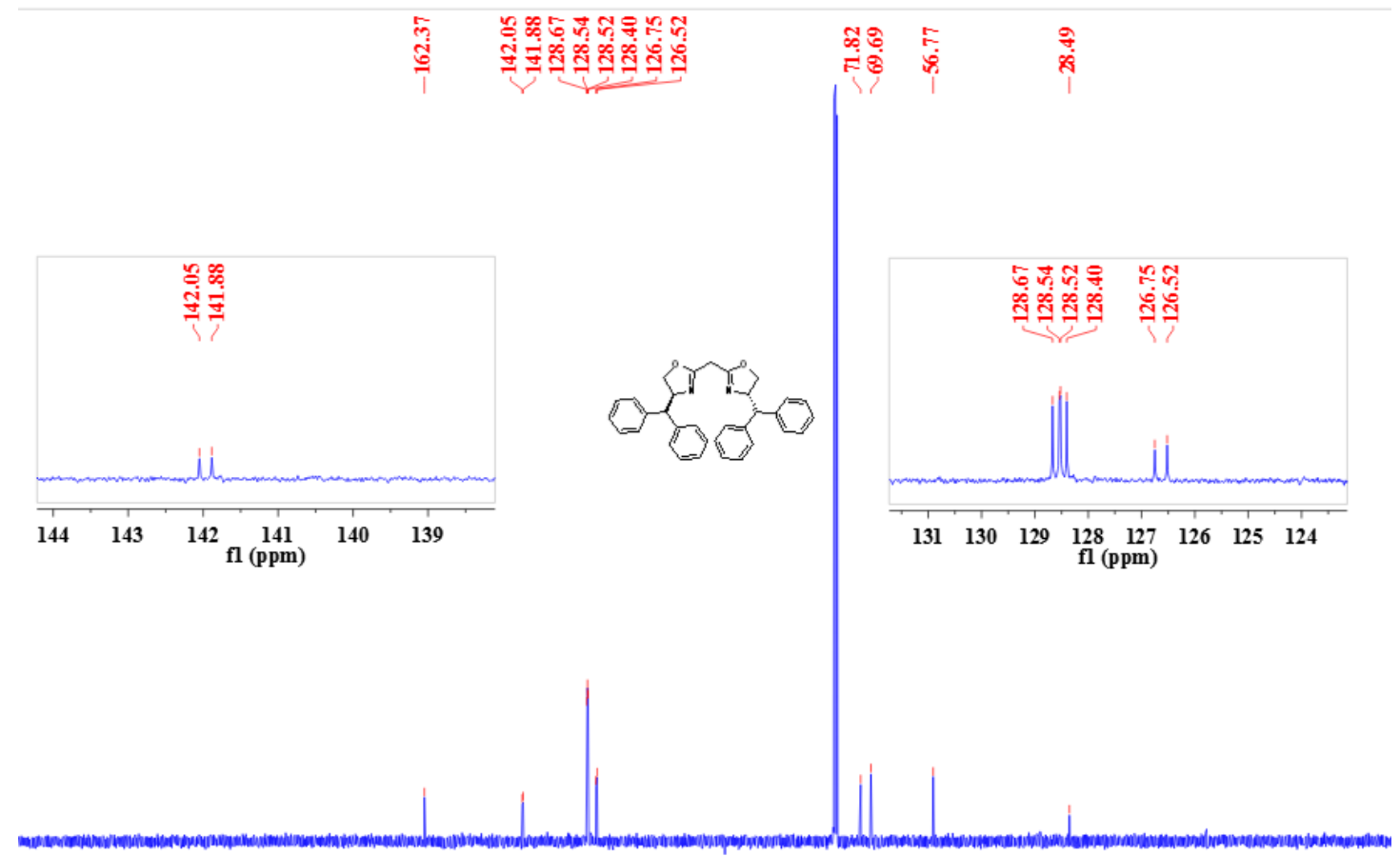

$\begin{array}{llllllllllllllllllllllllllllllllllllll}240 & 230 & 220 & 210 & 200 & 190 & 180 & 170 & 160 & 150 & 140 & 130 & 120 & 110 & 100 & 90 & 80 & 70 & 60 & 50 & 40 & 30 & 20 & 10 & 0 & -10 & -20 & -30 & -\end{array}$ 
${ }^{1} \mathrm{H}$ NMR (400 MHz, $\left.\mathrm{CDCl}_{3}\right)$ spectrum of (4S,4'S)-2,2'-(propane-2,2-diyl)bis(4-benzhydryl-4,5-dihydrooxazole) L9

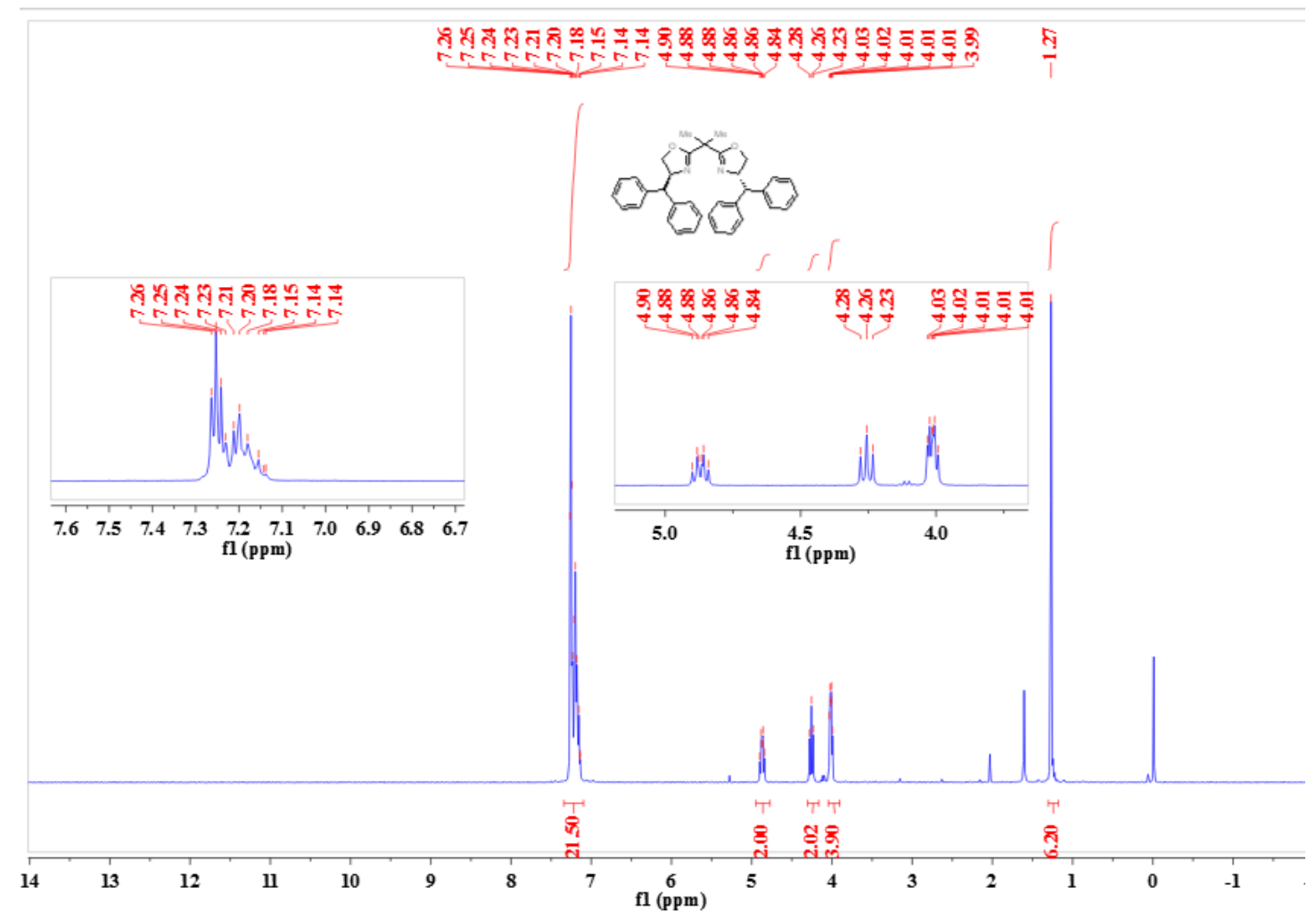

${ }^{13} \mathrm{C}$ NMR (101 MHz, $\left.\mathrm{CDCl}_{3}\right)$ spectrum of (4S,4'S)-2,2'-(propane-2,2-diyl)bis(4-benzhydryl-4,5-dihydrooxazole) L9

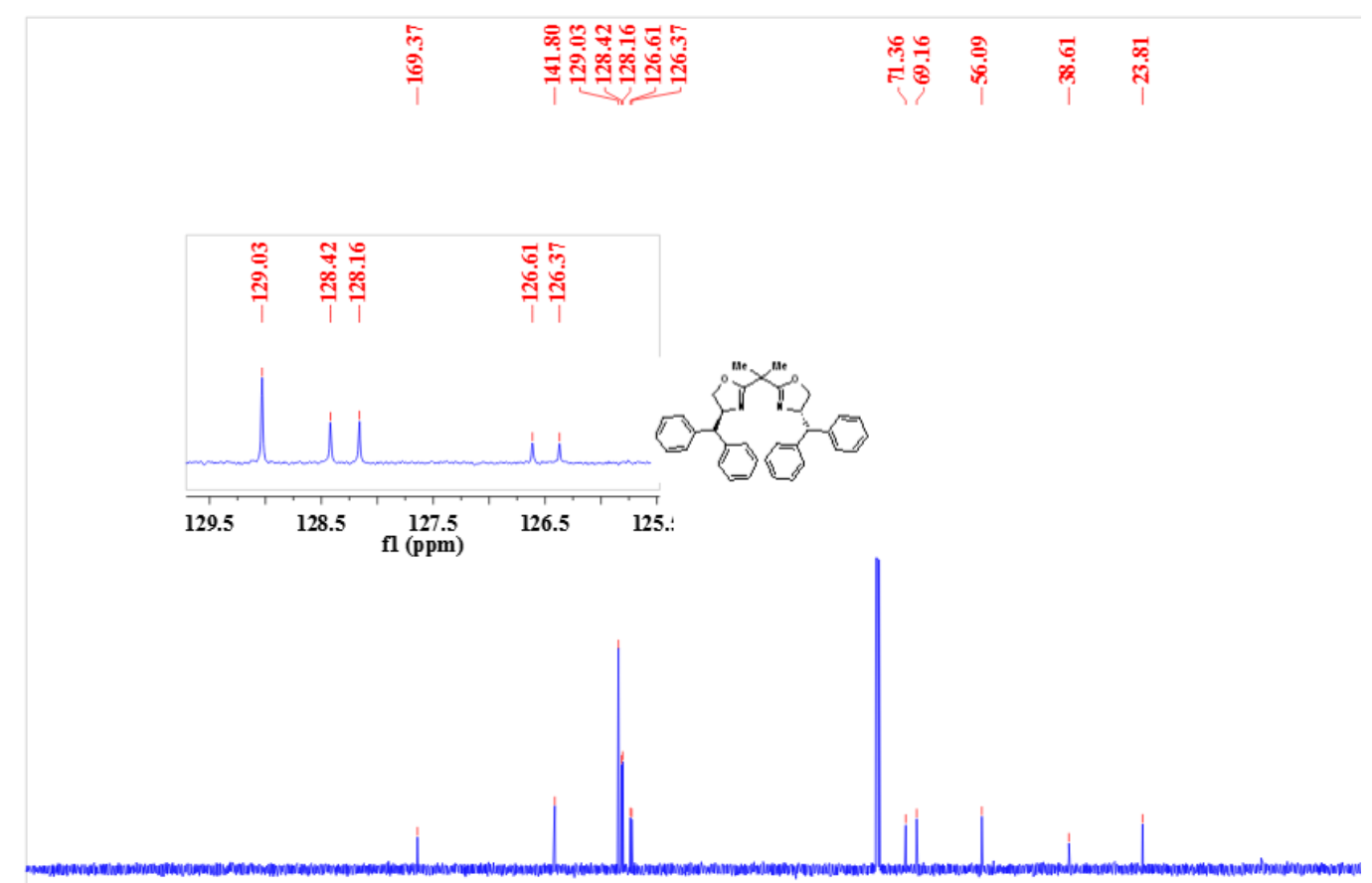

$\begin{array}{llllllllllllllllllllllllllllllllllll}240 & 230 & 220 & 210 & 200 & 190 & 180 & 170 & 160 & 150 & 140 & 130 & 120 & 110 & 100 & 90 & 80 & 70 & 60 & 50 & 40 & 30 & 20 & 10 & 0 & -10 & -2\end{array}$ 
${ }^{1} \mathrm{H}$ NMR (400 MHz, $\left.\mathrm{CDCl}_{3}\right)$ spectrum of (4S,4'S)-2,2'-(pentane-3,3-diyl)bis(4-benzhydryl-4,5-dihydrooxazole) L10

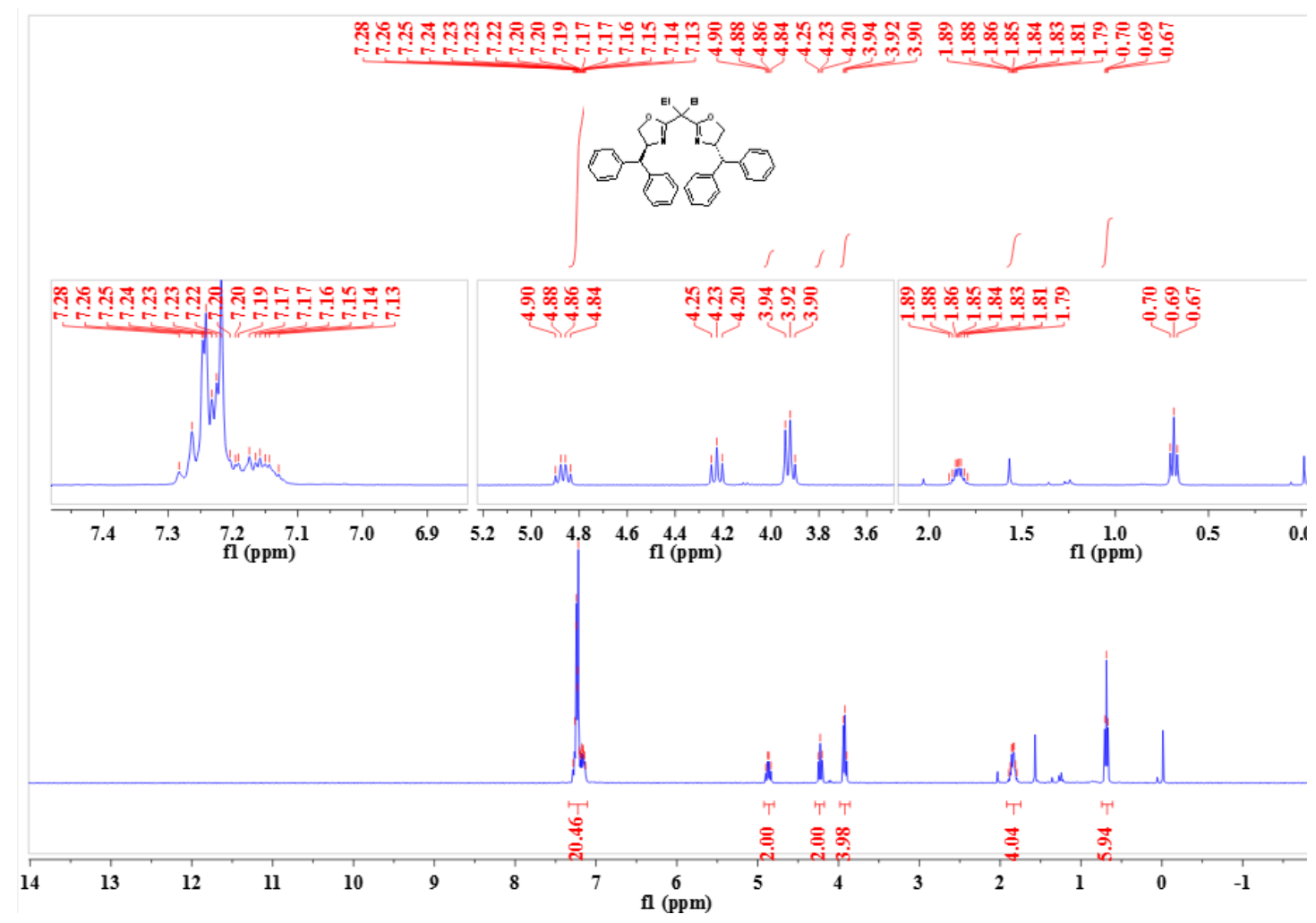

${ }^{13} \mathrm{C}$ NMR (101 MHz, $\left.\mathrm{CDCl}_{3}\right)$ spectrum of (4S,4'S)-2,2'-(pentane-3,3-diyl)bis(4-benzhydryl-4,5-dihydrooxazole) L10

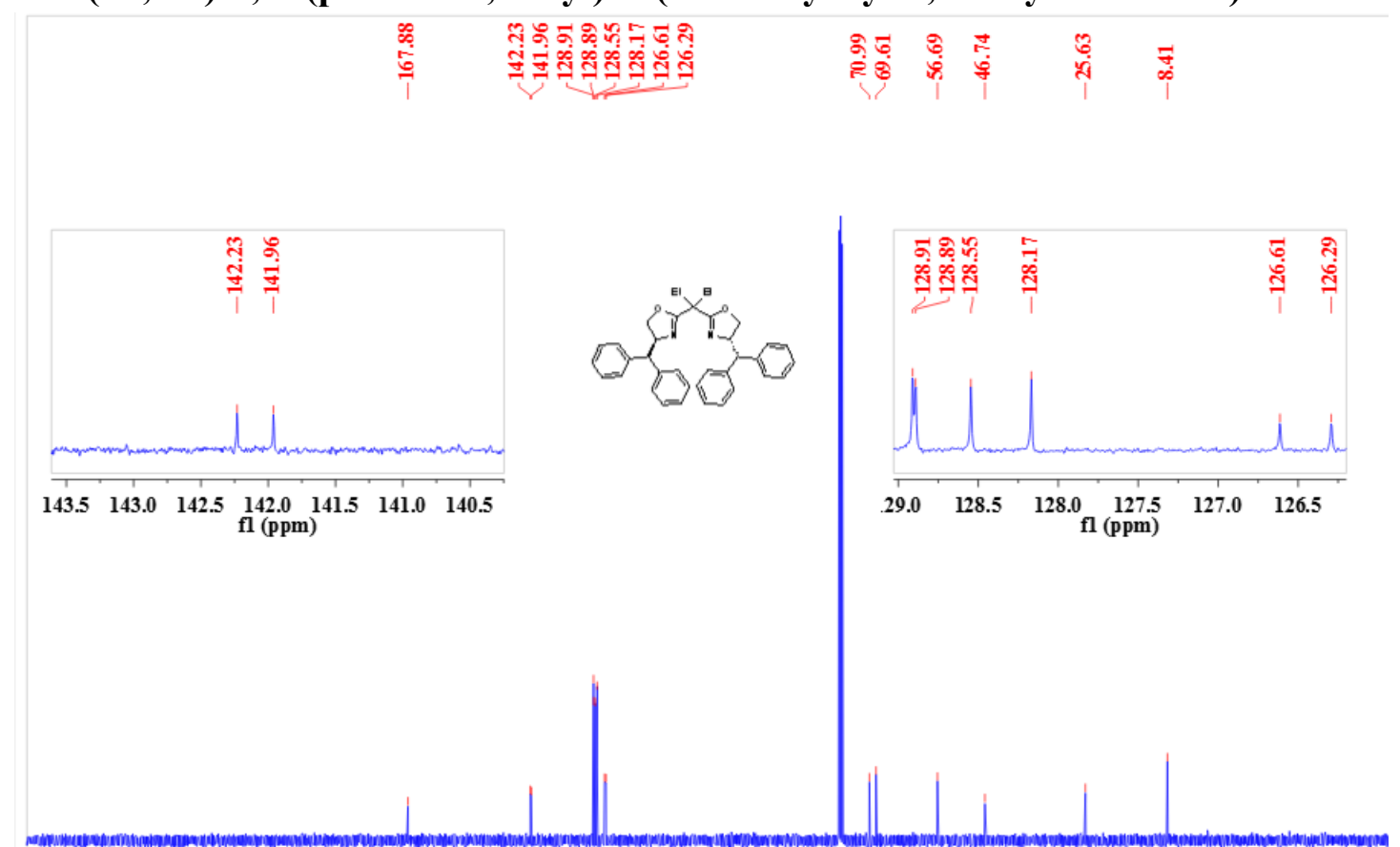

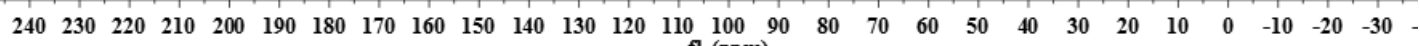


${ }^{1} \mathrm{H}$ NMR (400 MHz, $\left.\mathrm{CDCl}_{3}\right)$ spectrum of (4S,4'S)-2,2'-(cyclopentane-1,1-diyl)bis(4-benzhydryl-4,5-dihydrooxazole) L11

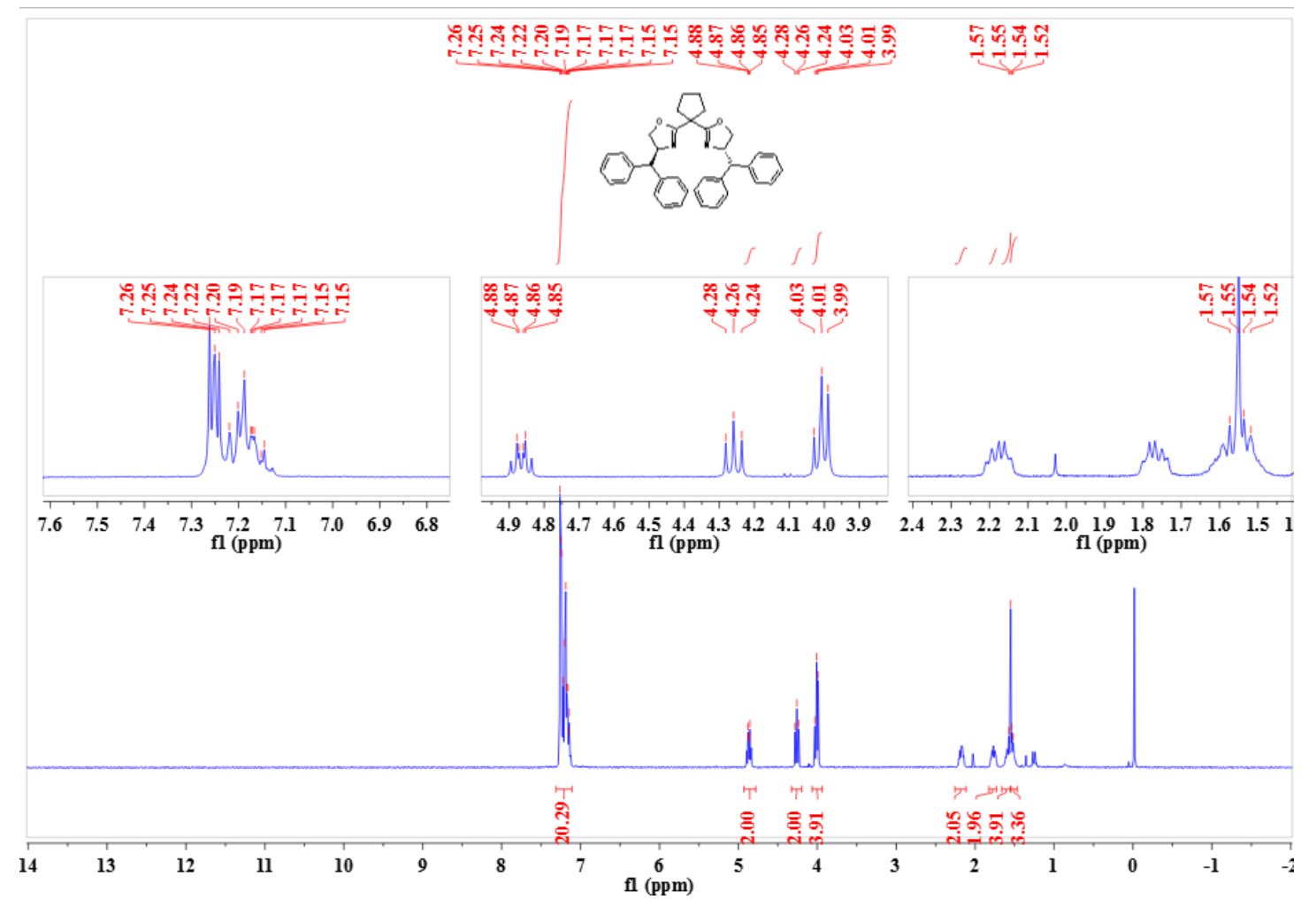

${ }^{13} \mathrm{C}$ NMR (101 MHz, $\left.\mathrm{CDCl}_{3}\right)$ spectrum of (4S,4'S)-2,2'-(cyclopentane-1,1-diyl)bis(4-benzhydryl-4,5-dihydrooxazole) L11

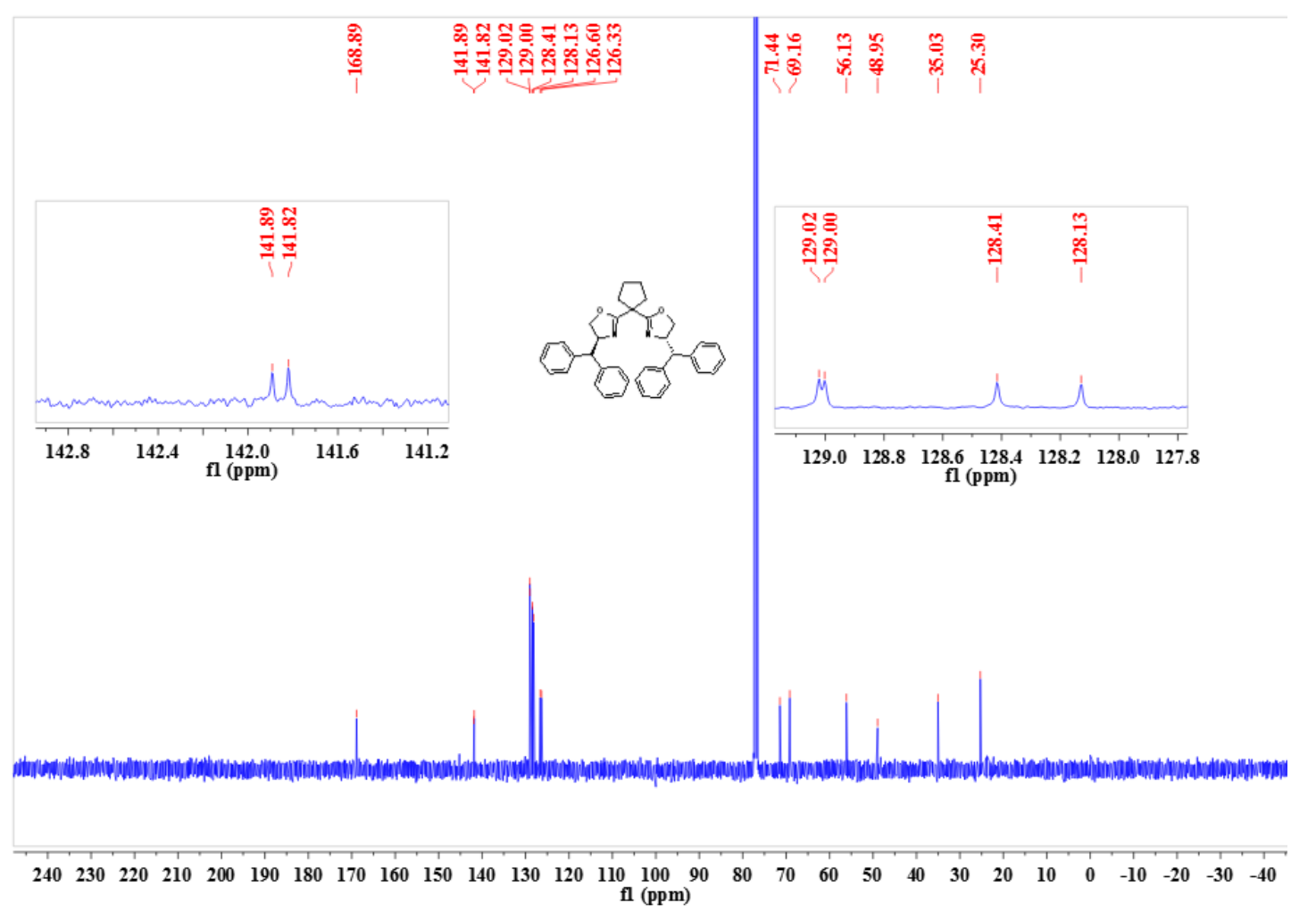


${ }^{1} \mathrm{H}$ NMR (400 MHz, $\mathrm{CDCl}_{3}$ ) spectrum of methyl 4-(bromofluoromethyl)benzoate 1a

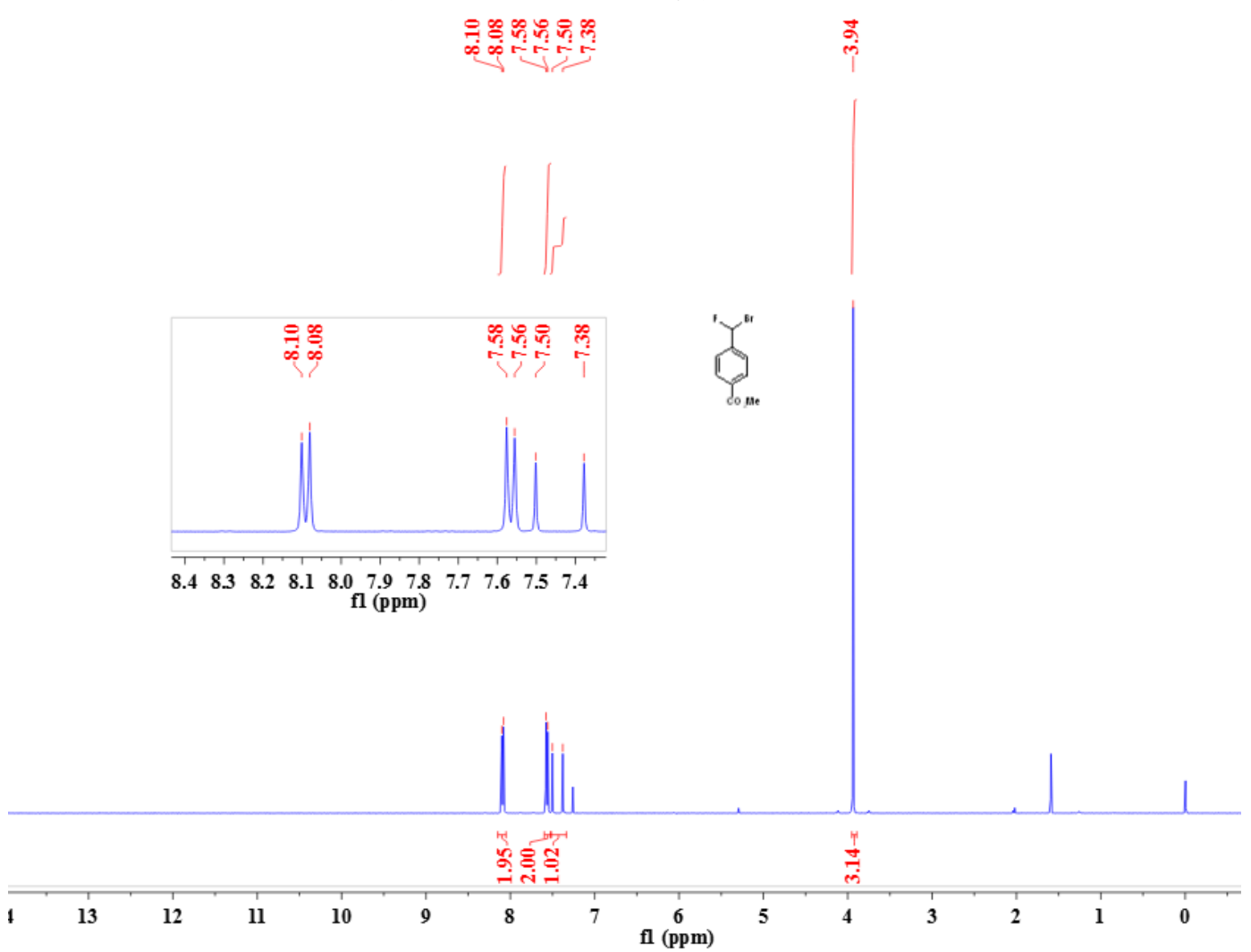

${ }^{19}$ F NMR (376 MHz, CDCl 3 ) spectrum of methyl 4-(bromofluoromethyl)benzoate 1a
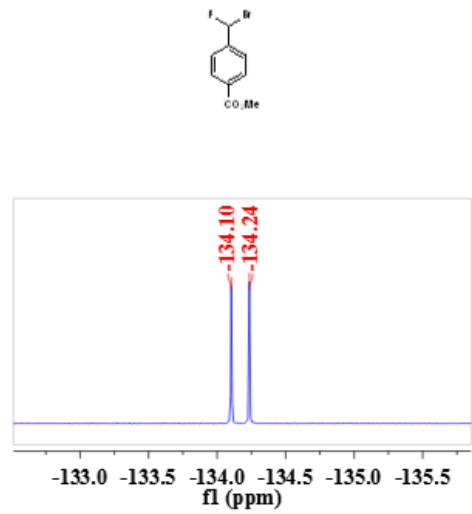
${ }^{13} \mathrm{C}$ NMR (101 MHz, $\mathrm{CDCl}_{3}$ ) spectrum of methyl 4-(bromofluoromethyl)benzoate 1a

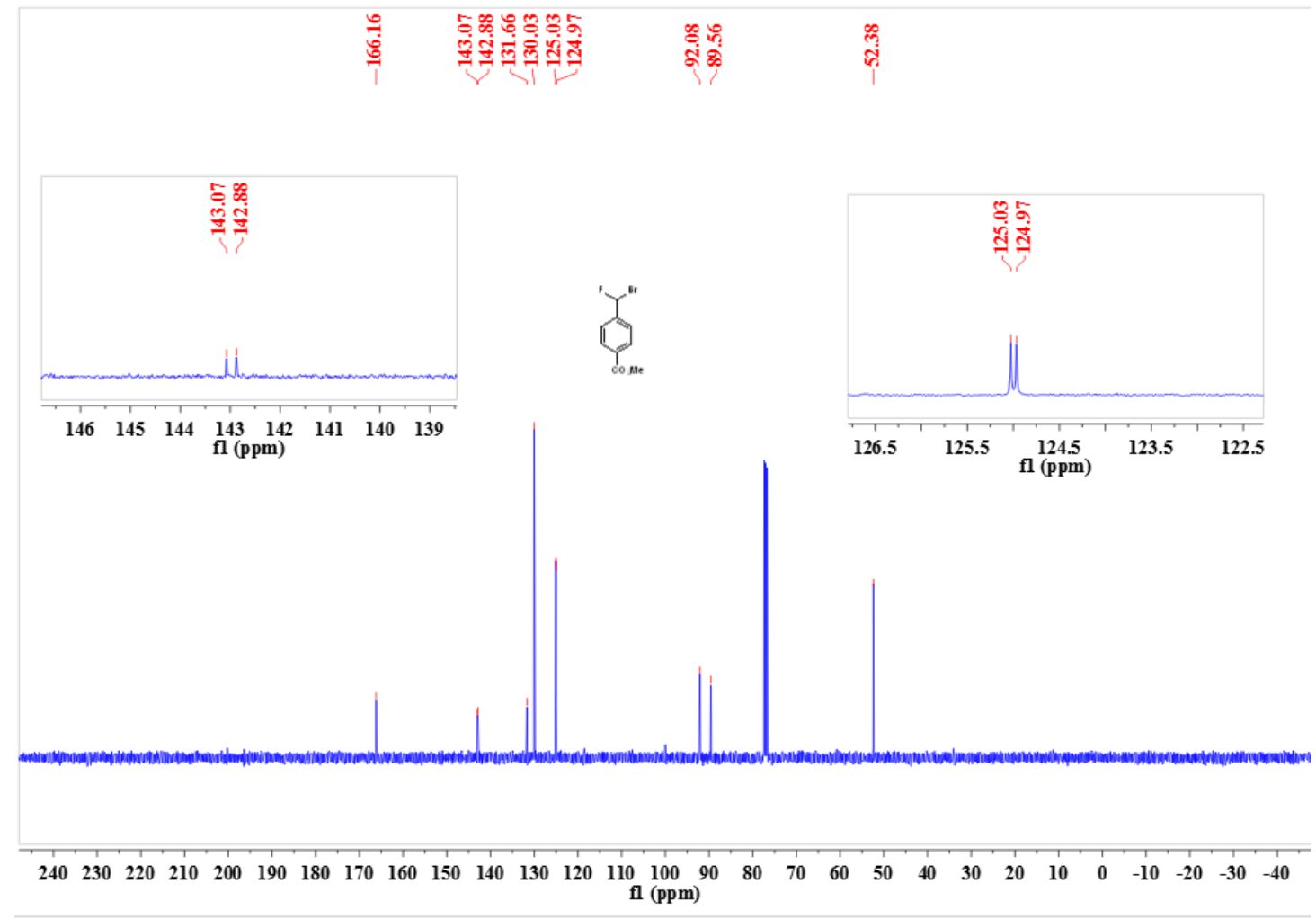

${ }^{1} \mathrm{H}$ NMR (400 $\mathrm{MHz}, \mathrm{CDCl}_{3}$ ) spectrum of methyl 3-(bromofluoromethyl)benzoate 1b

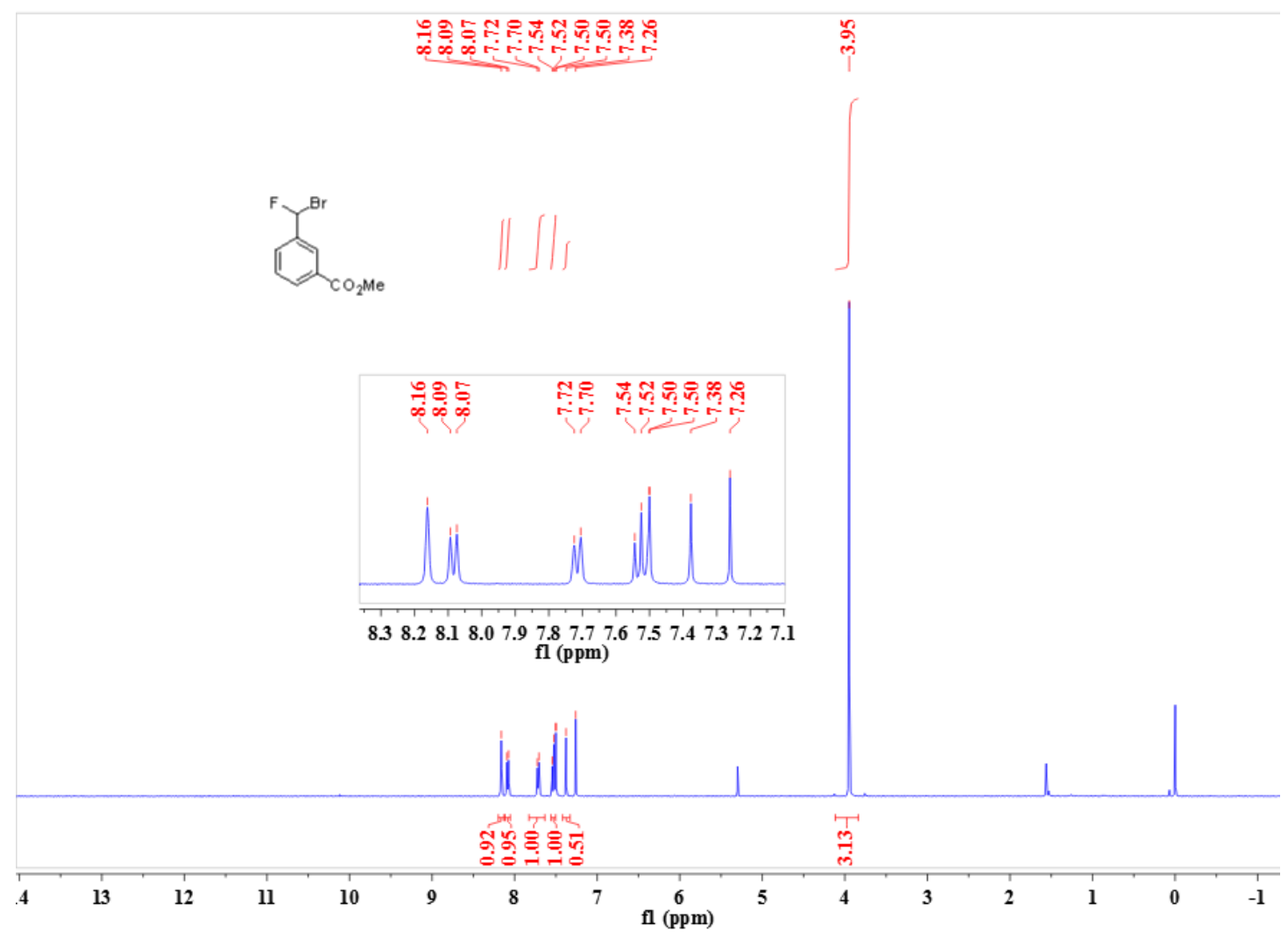


${ }^{19} \mathrm{~F}$ NMR (376 $\mathrm{MHz}, \mathrm{CDCl}_{3}$ ) spectrum of methyl 3-(bromofluoromethyl)benzoate $1 \mathrm{~b}$

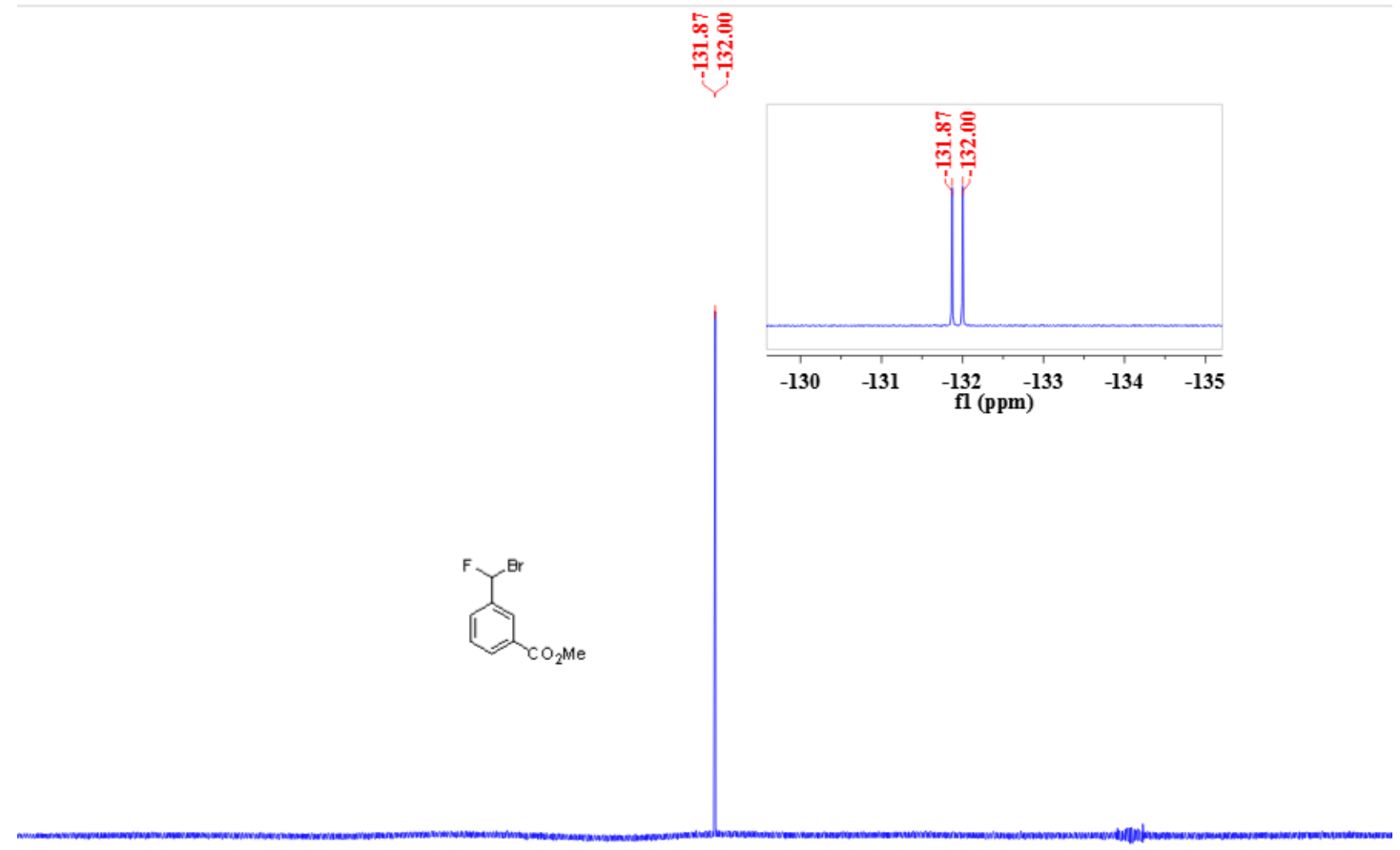

${ }^{13} \mathrm{C}$ NMR (101 MHz, $\left.\mathrm{CDCl}_{3}\right)$ spectrum of methyl 3-(bromofluoromethyl)benzoate 1b

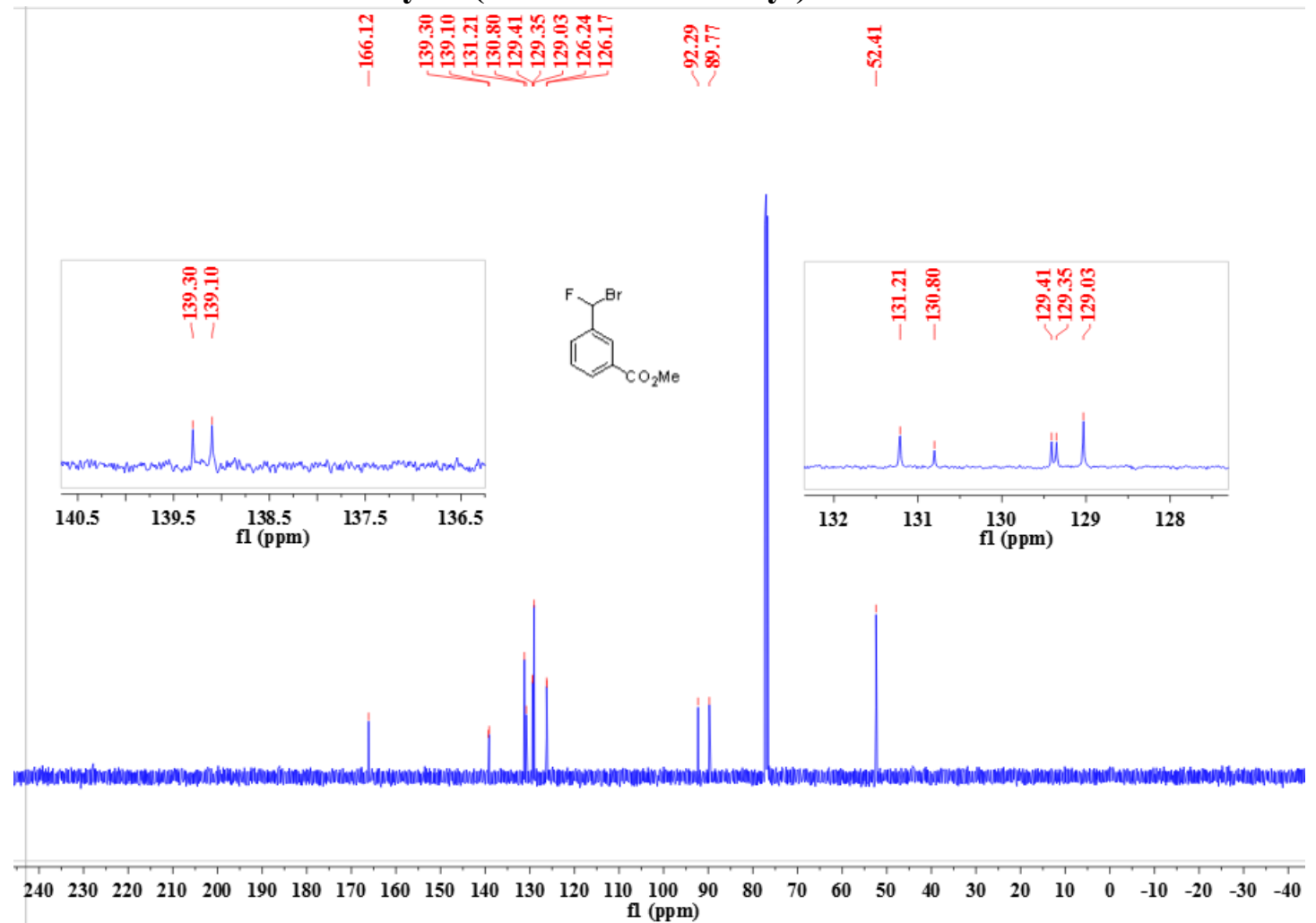


${ }^{1} \mathrm{H}$ NMR (400 $\mathrm{MHz}, \mathrm{CDCl}_{3}$ ) spectrum of ethyl 4-(bromofluoromethyl)benzoate 1c

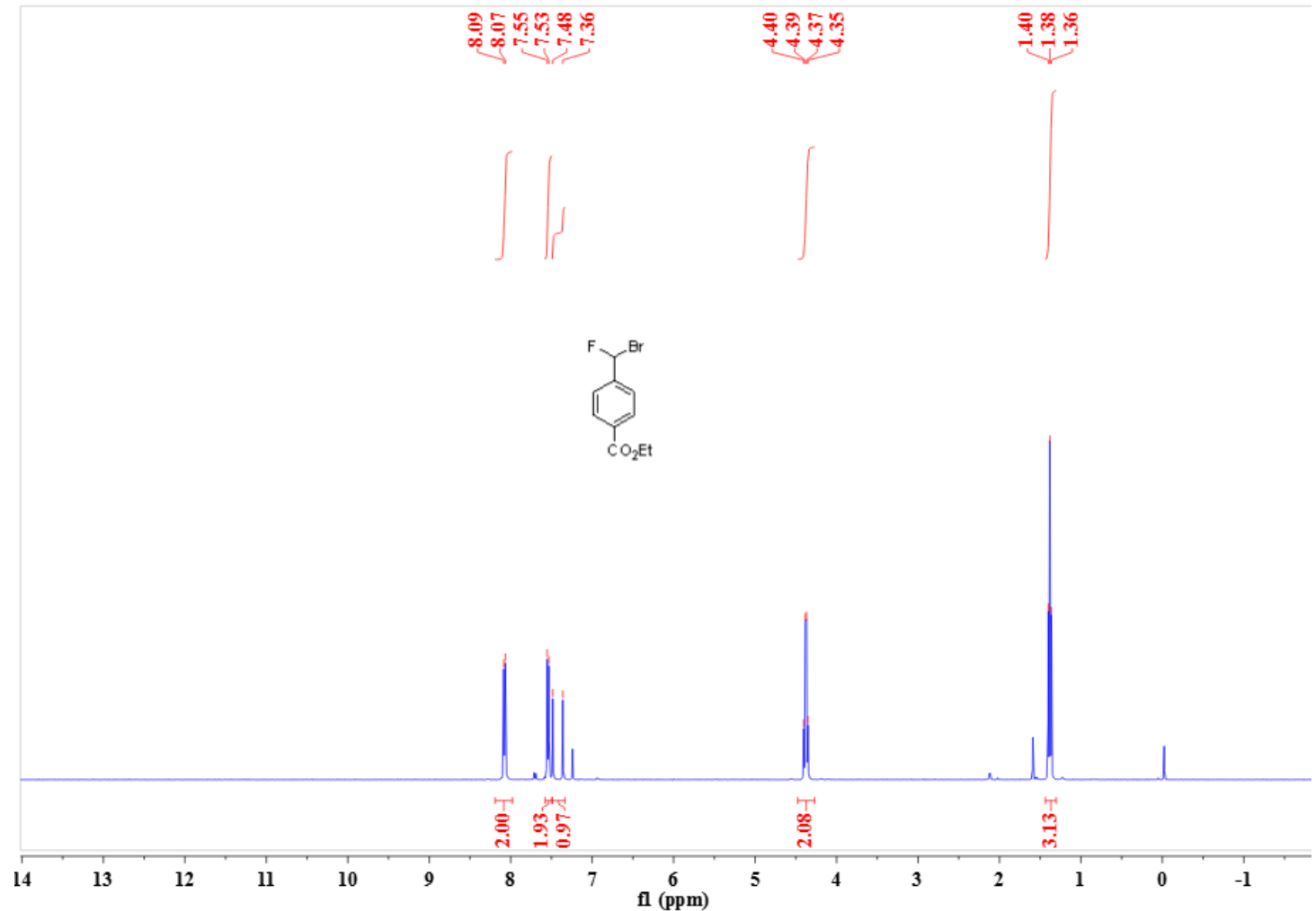

${ }^{19}$ F NMR (376 MHz, $\left.\mathrm{CDCl}_{3}\right)$ spectrum of ethyl 4-(bromofluoromethyl)benzoate 1c

고ำ
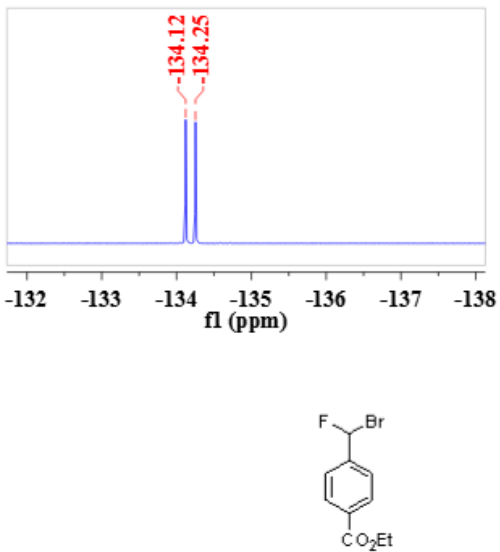

$\begin{array}{llllllllllllllllllllllllll}30 & 20 & 10 & 0 & -10 & -20 & -30 & -40 & -50 & -60 & -70 & -80 & -90 & -100 & -110 & -120 & -130 & -140 & -150 & -160 & -170 & -180 & -190 & -\end{array}$ 
${ }^{13} \mathrm{C}$ NMR (101 MHz, $\left.\mathrm{CDCl}_{3}\right)$ spectrum of ethyl 4-(bromofluoromethyl)benzoate 1c

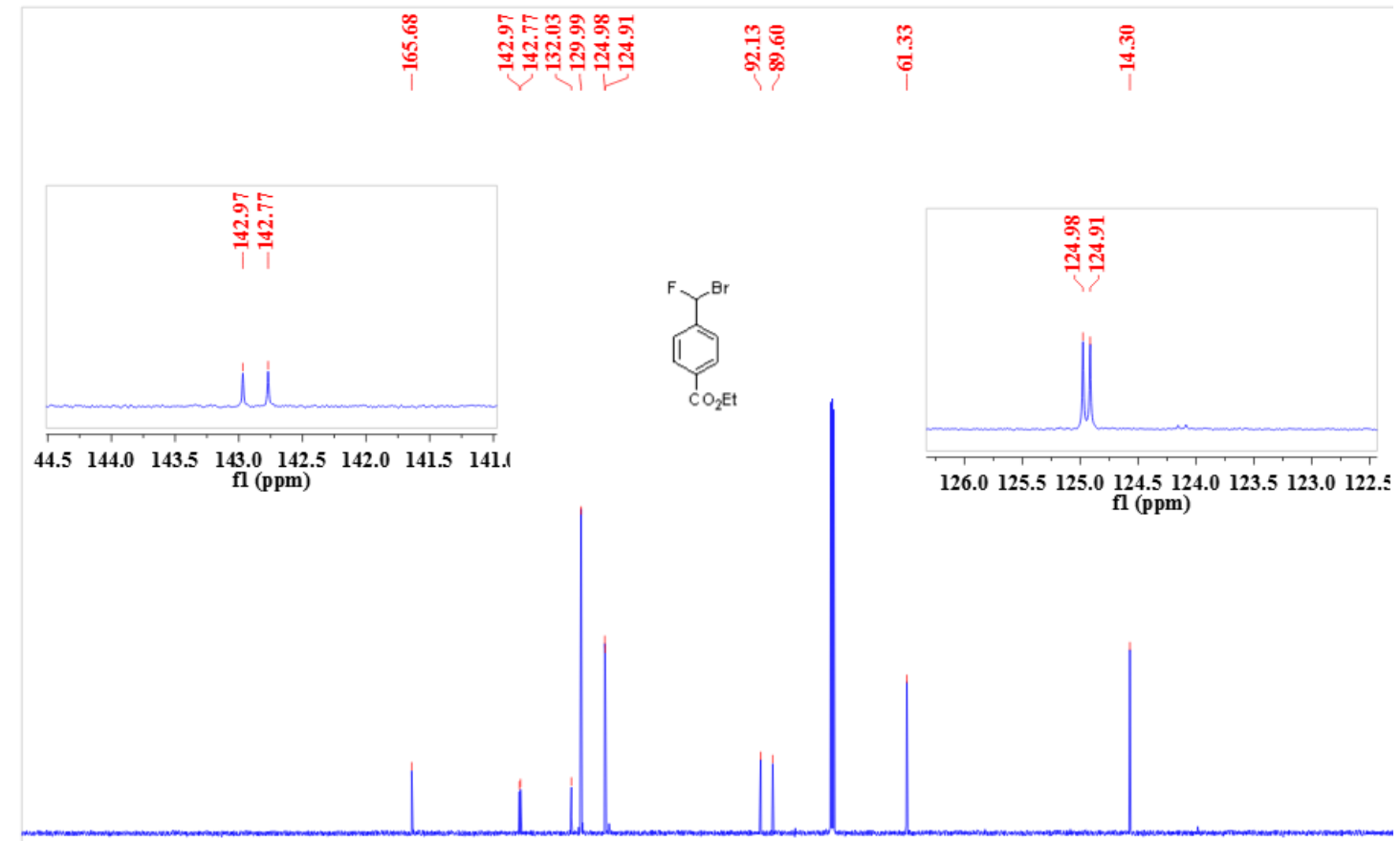

$\begin{array}{lllllllllllllllllllllllllllll}240 & 230 & 220 & 210 & 200 & 190 & 180 & 170 & 160 & 150 & 140 & 130 & 120 & 110 & 100 & 90 & 80 & 70 & 60 & 50 & 40 & 30 & 20 & 10 & 0 & -10 & -20 & -30 & -4\end{array}$ fl (ppm)

${ }^{1} \mathrm{H}$ NMR (400 $\mathrm{MHz}, \mathrm{CDCl}_{3}$ ) spectrum of

3-(bromofluoromethyl)benzonitrile 1d

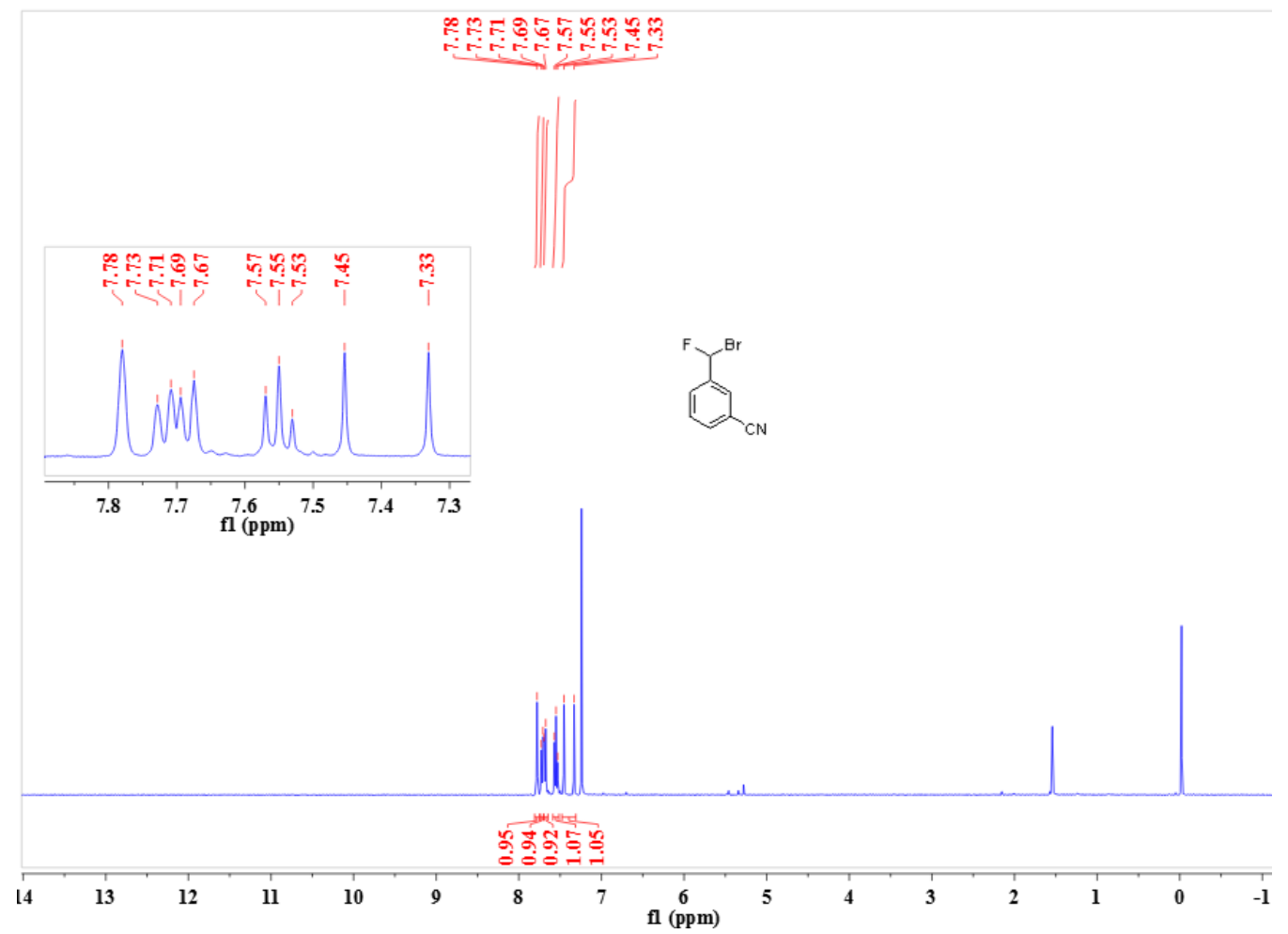


${ }^{19}$ F NMR (376 $\mathrm{MHz}, \mathrm{CDCl}_{3}$ ) spectrum of 3-(bromofluoromethyl)benzonitrile 1d

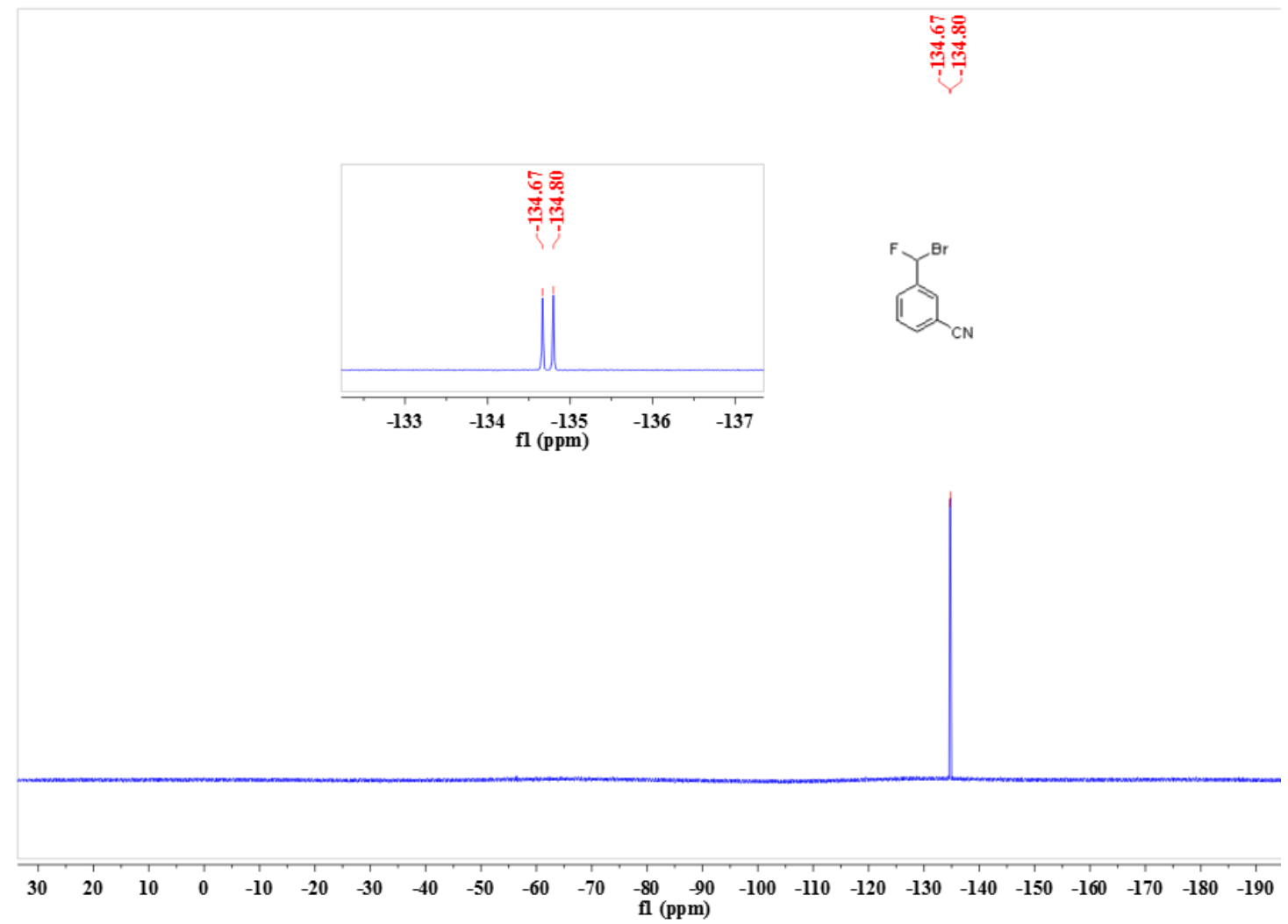

${ }^{13} \mathrm{C}$ NMR (101 MHz, $\left.\mathrm{CDCl}_{3}\right)$ spectrum of

3-(bromofluoromethyl)benzonitrile 1d

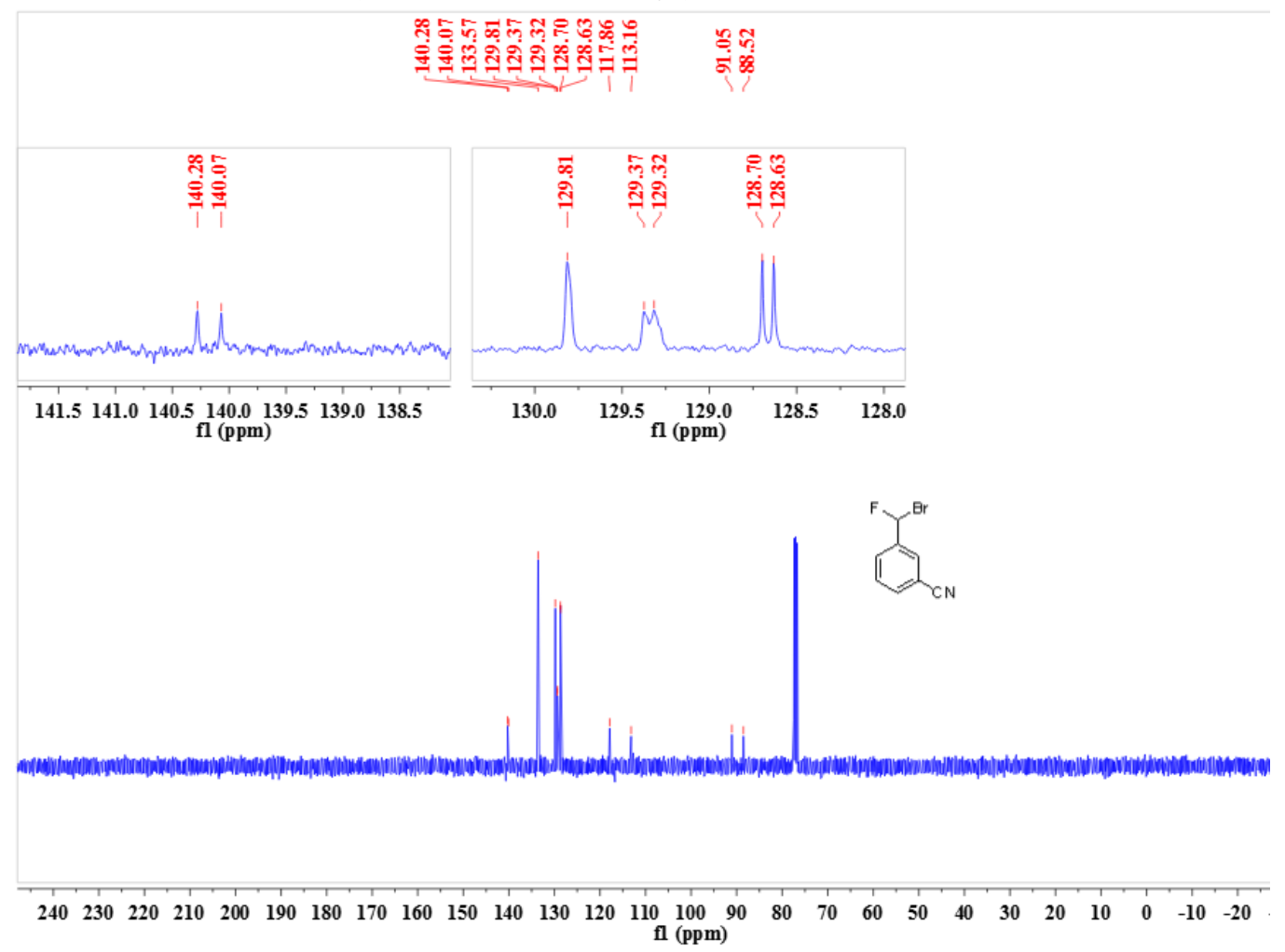




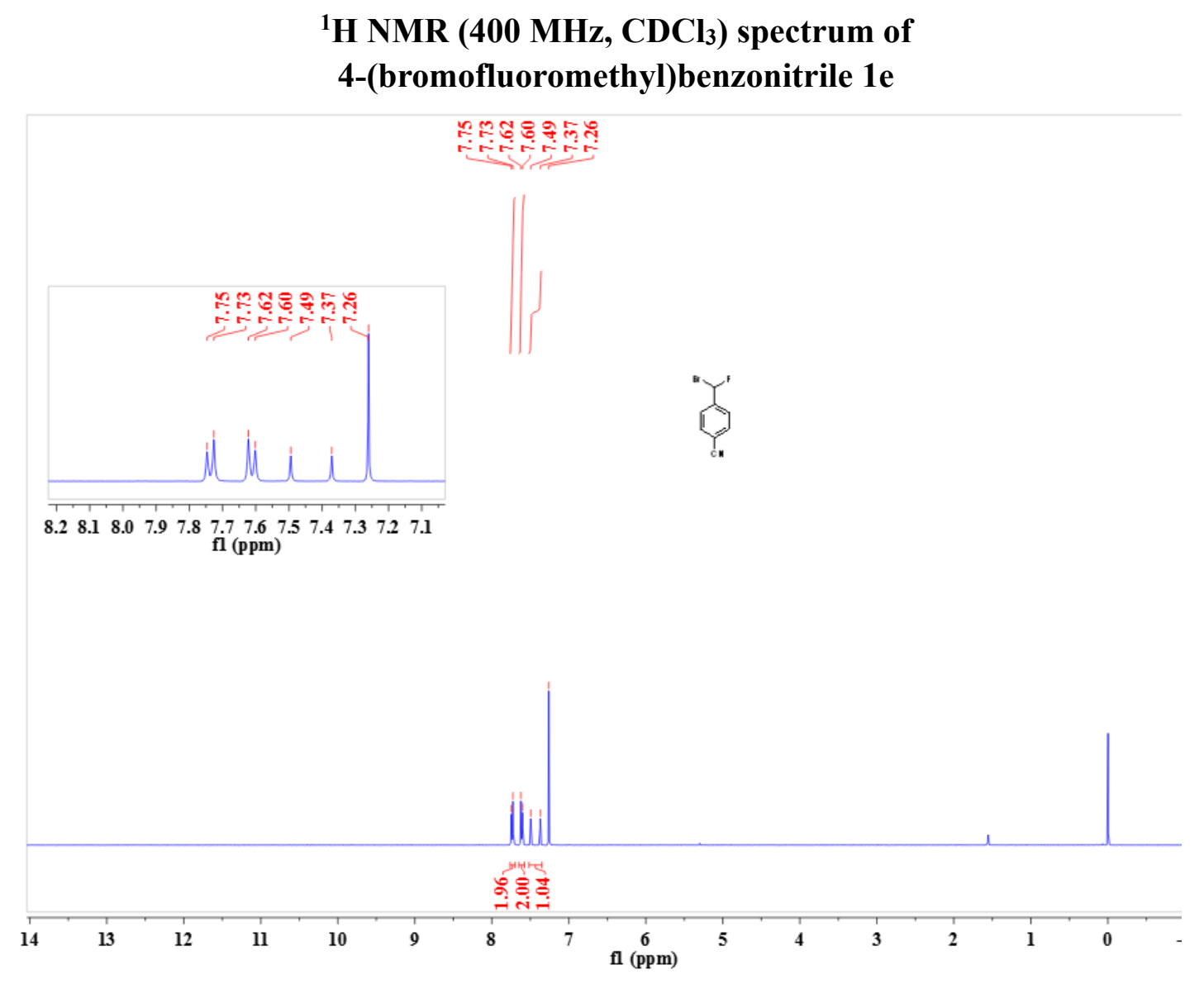

${ }^{19}$ F NMR (376 MHz, $\mathrm{CDCl}_{3}$ ) spectrum of 4-(bromofluoromethyl)benzonitrile $1 \mathrm{e}$

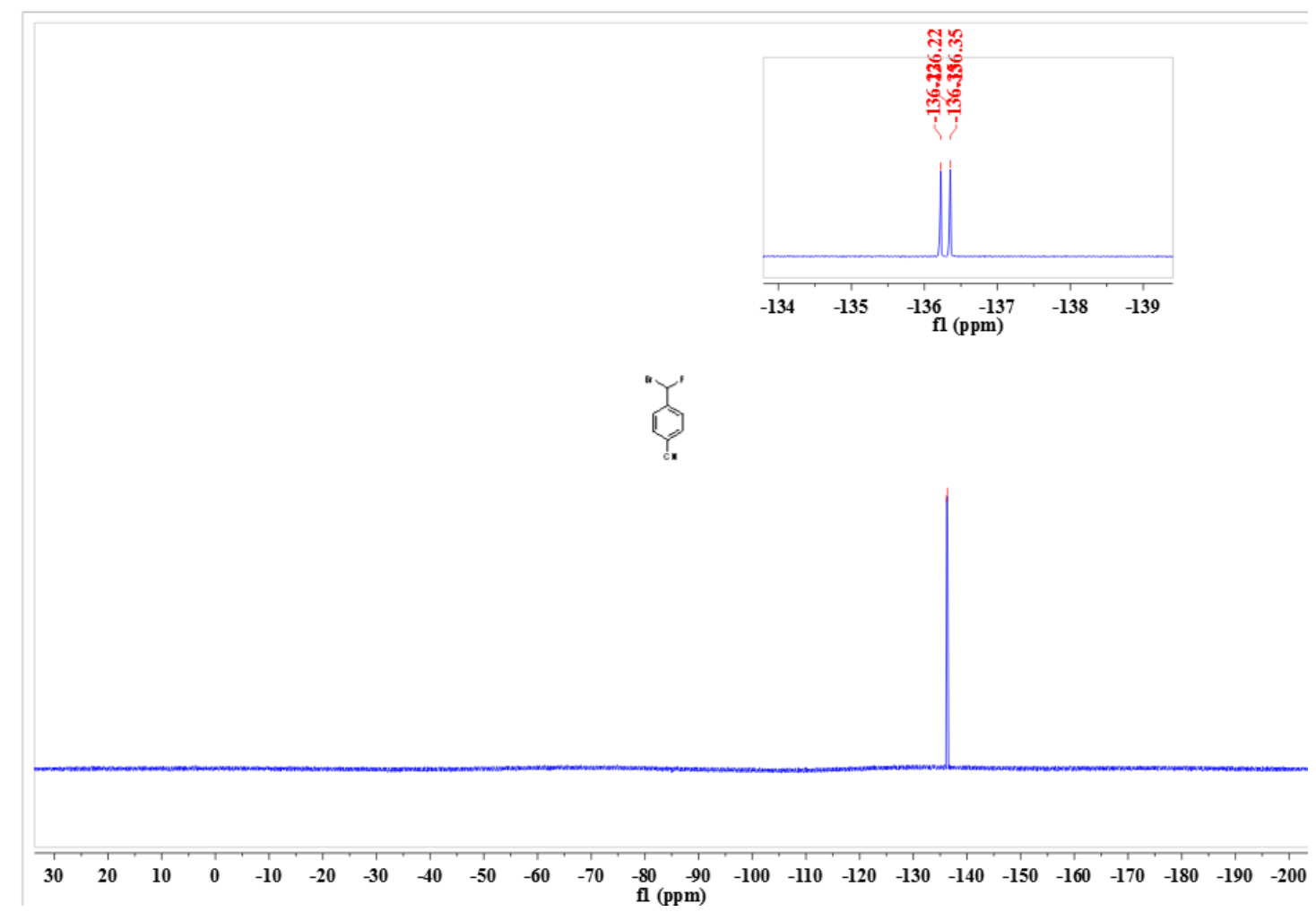


${ }^{13} \mathrm{C}$ NMR (101 MHz, $\left.\mathrm{CDCl}_{3}\right)$ spectrum of 4-(bromofluoromethyl)benzonitrile $1 \mathrm{e}$

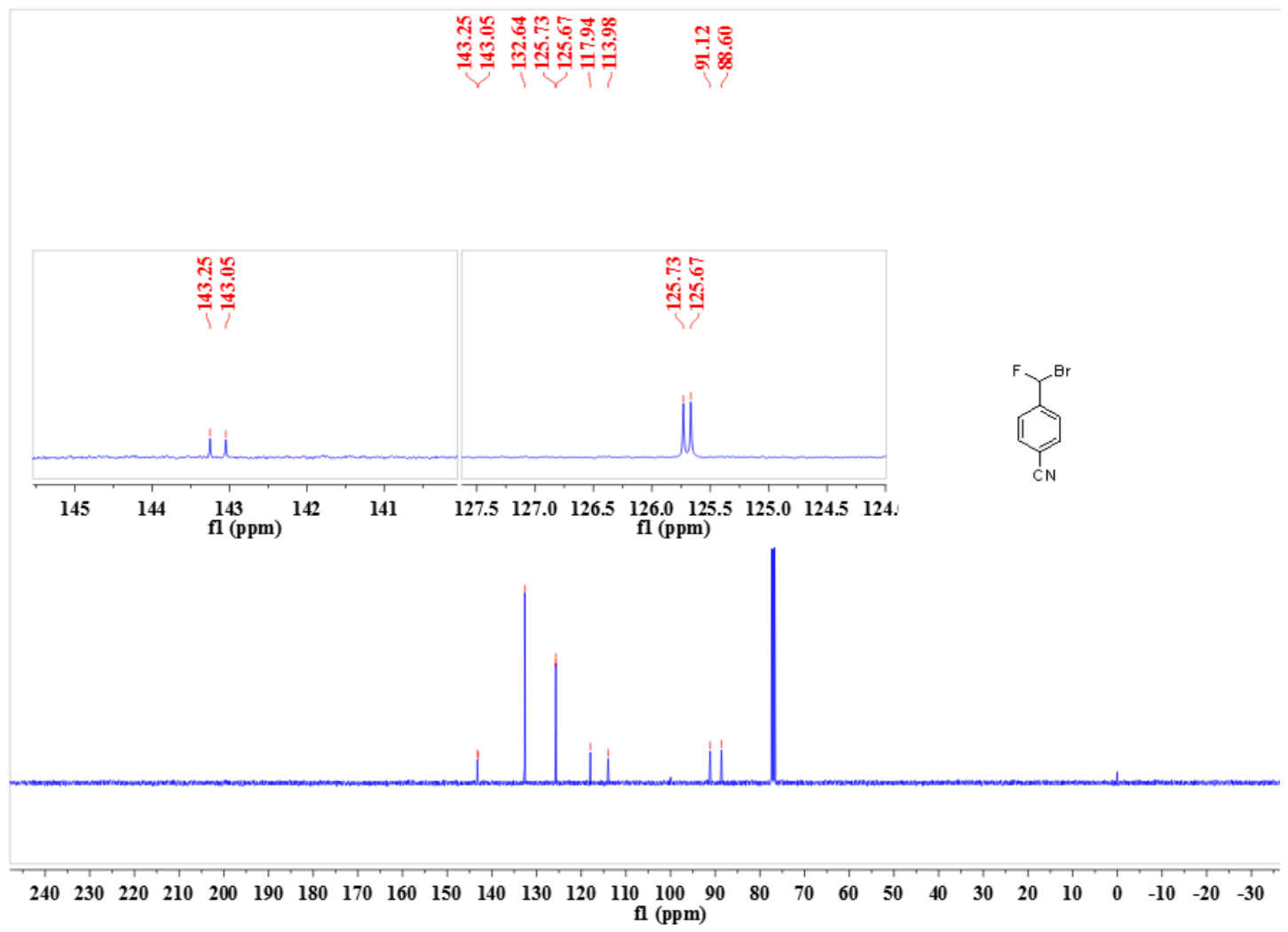

${ }^{1} \mathrm{H}$ NMR (400 MHz, $\left.\mathrm{CDCl}_{3}\right)$ spectrum of 4-(bromofluoromethyl)-2-fluorobenzonitrile $1 f$

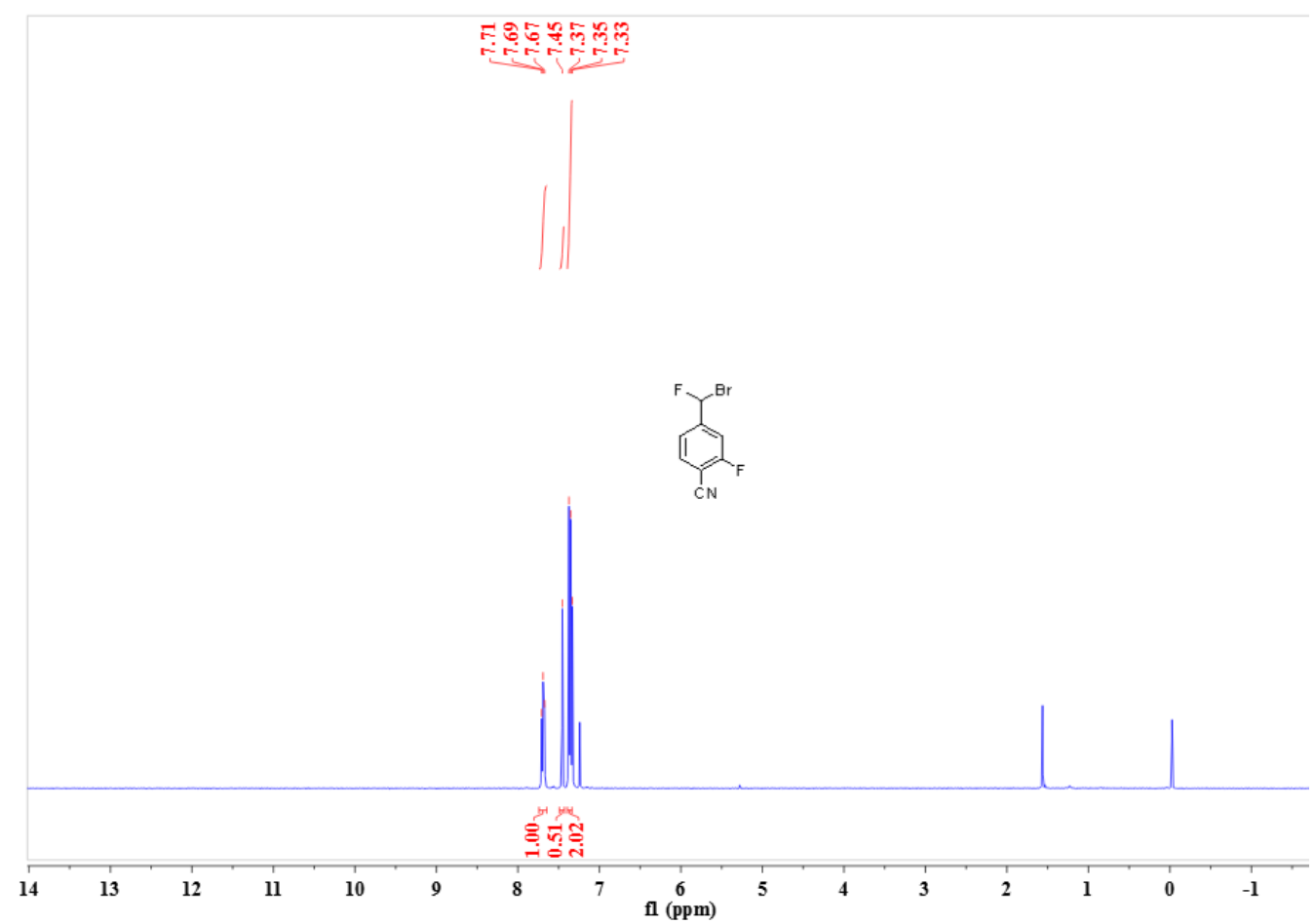


${ }^{19}$ F NMR (376 $\mathrm{MHz}, \mathrm{CDCl}_{3}$ ) spectrum of 4-(bromofluoromethyl)-2-fluorobenzonitrile $1 f$

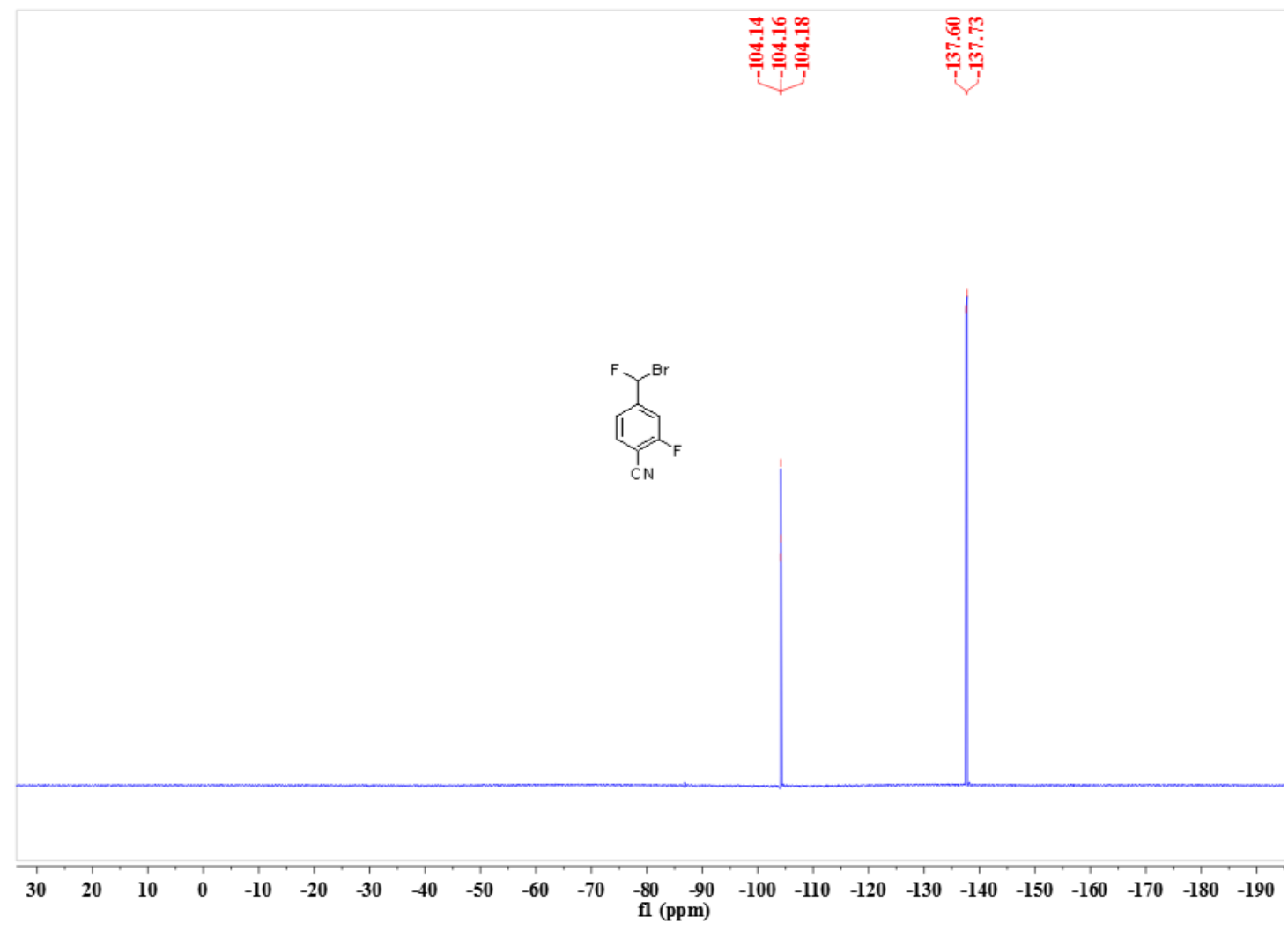

${ }^{13} \mathrm{C}$ NMR (101 MHz, $\left.\mathrm{CDCl}_{3}\right)$ spectrum of 4-(bromofluoromethyl)-2-fluorobenzonitrile $1 f$

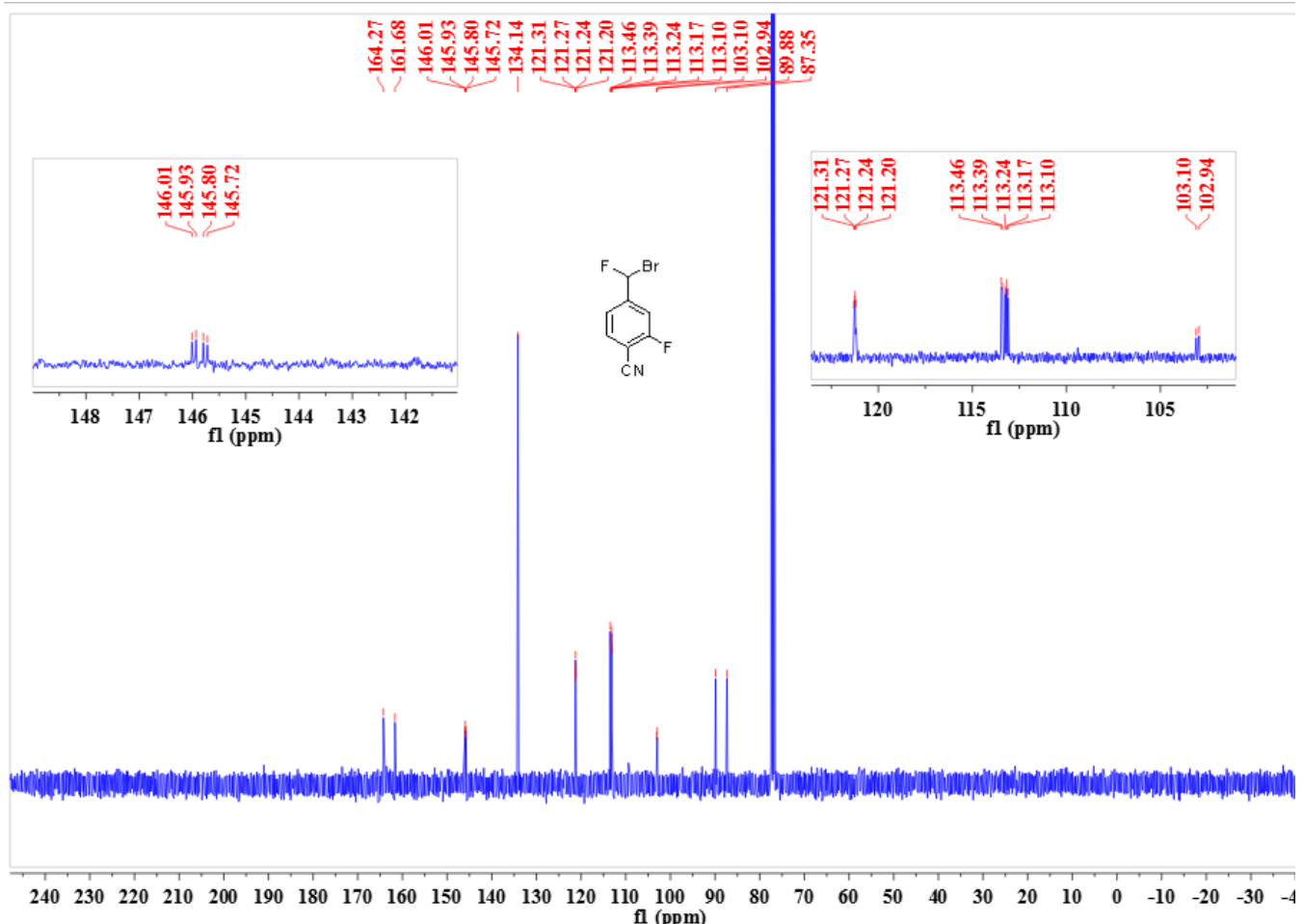

fl (ppm) 
${ }^{1} \mathrm{H}$ NMR (400 MHz, $\left.\mathrm{CDCl}_{3}\right)$ spectrum of 4-(bromofluoromethyl)-3-fluorobenzonitrile $1 \mathrm{~g}$
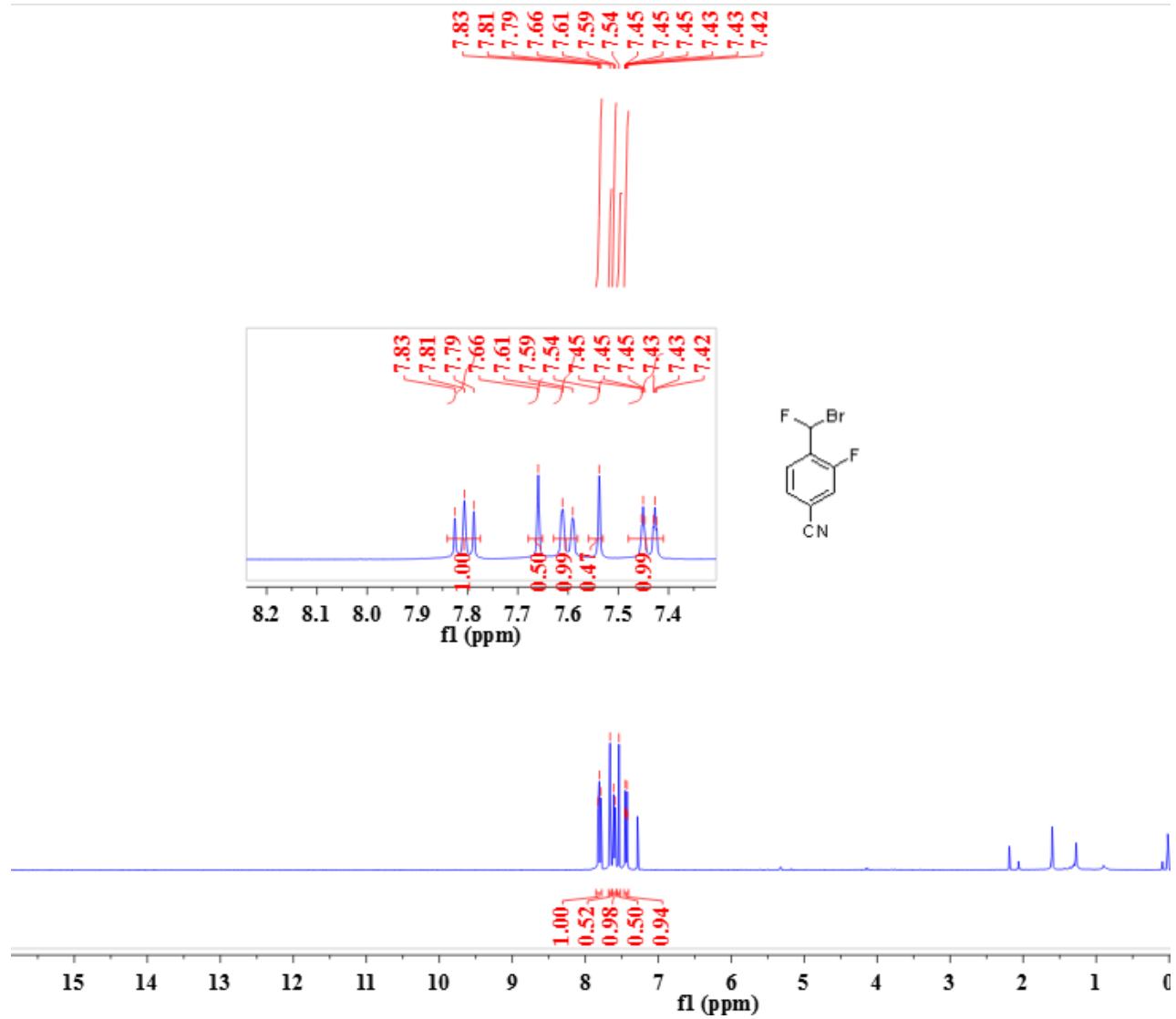

${ }^{19} \mathrm{~F}$ NMR (376 $\left.\mathrm{MHz}, \mathrm{CDCl}_{3}\right)$ spectrum of 4-(bromofluoromethyl)-3-fluorobenzonitrile $1 \mathrm{~g}$

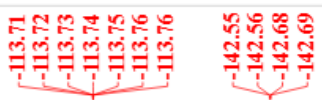
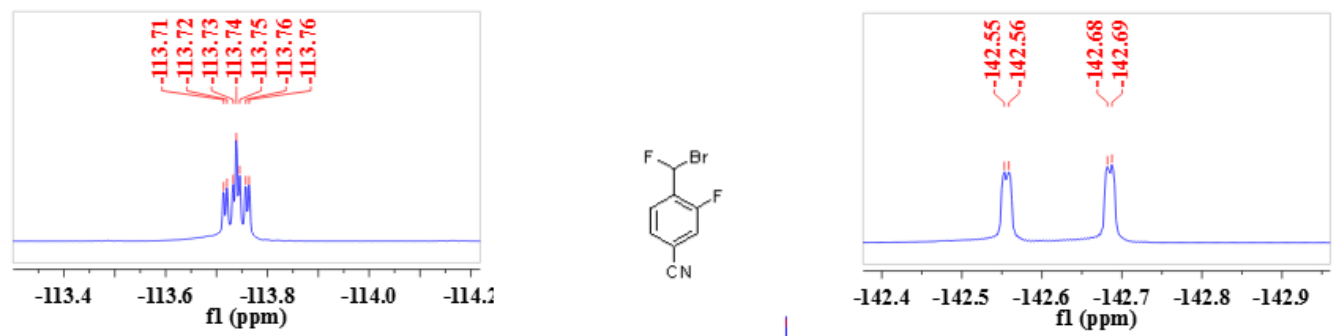

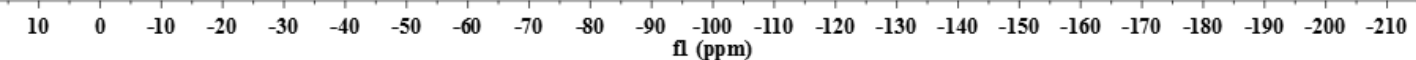


${ }^{13} \mathrm{C}$ NMR (101 $\left.\mathrm{MHz}, \mathrm{CDCl}_{3}\right)$ spectrum of 4-(bromofluoromethyl)-3-fluorobenzonitrile $1 \mathrm{~g}$

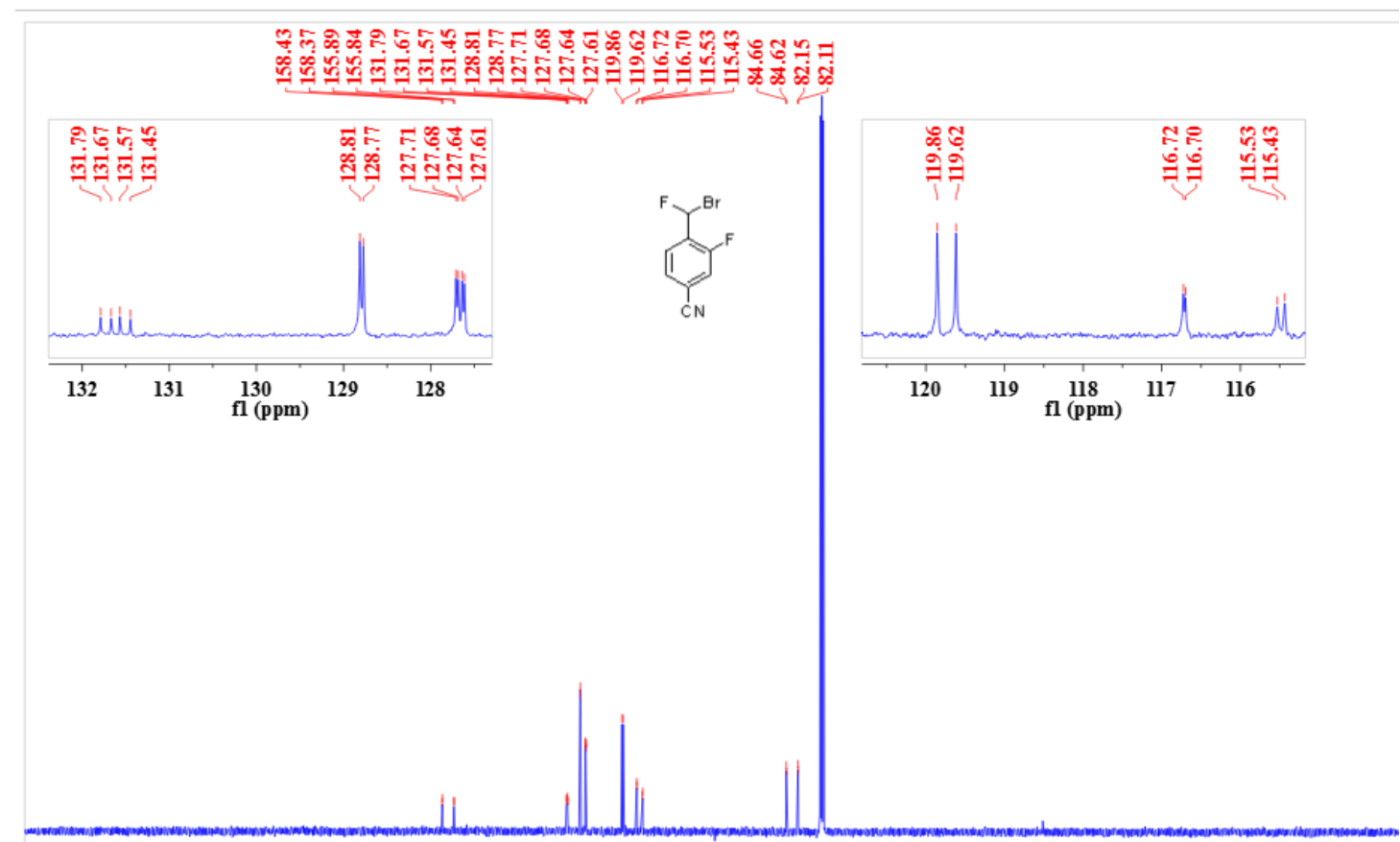

$\begin{array}{lllllllllllllllllllllllllllll}240 & 230 & 220 & 210 & 200 & 190 & 180 & 170 & 160 & 150 & 140 & 130 & 120 & 110 & 100 & 90 & 80 & 70 & 60 & 50 & 40 & 30 & 20 & 10 & 0 & -10 & -20 & -30 & -40\end{array}$ fl (ppm)

${ }^{1} \mathrm{H}$ NMR (400 MHz, CDCl 3$)$ spectrum of 1-(bromofluoromethyl)-4-(trifluoromethyl)benzene $1 \mathrm{~h}$

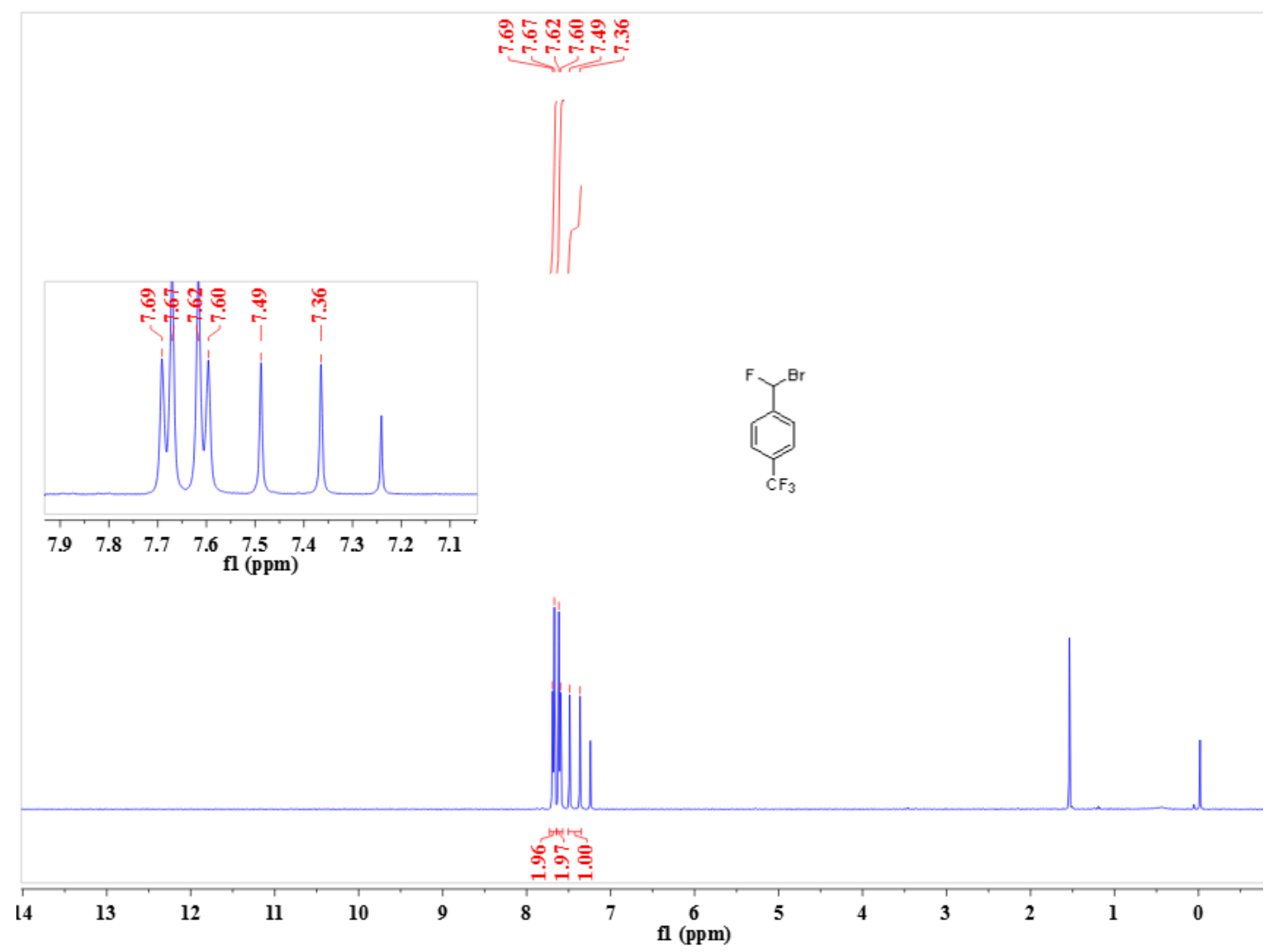


${ }^{19}$ F NMR (376 MHz, $\mathrm{CDCl}_{3}$ ) spectrum of 1-(bromofluoromethyl)-4-(trifluoromethyl)benzene $1 \mathrm{~h}$

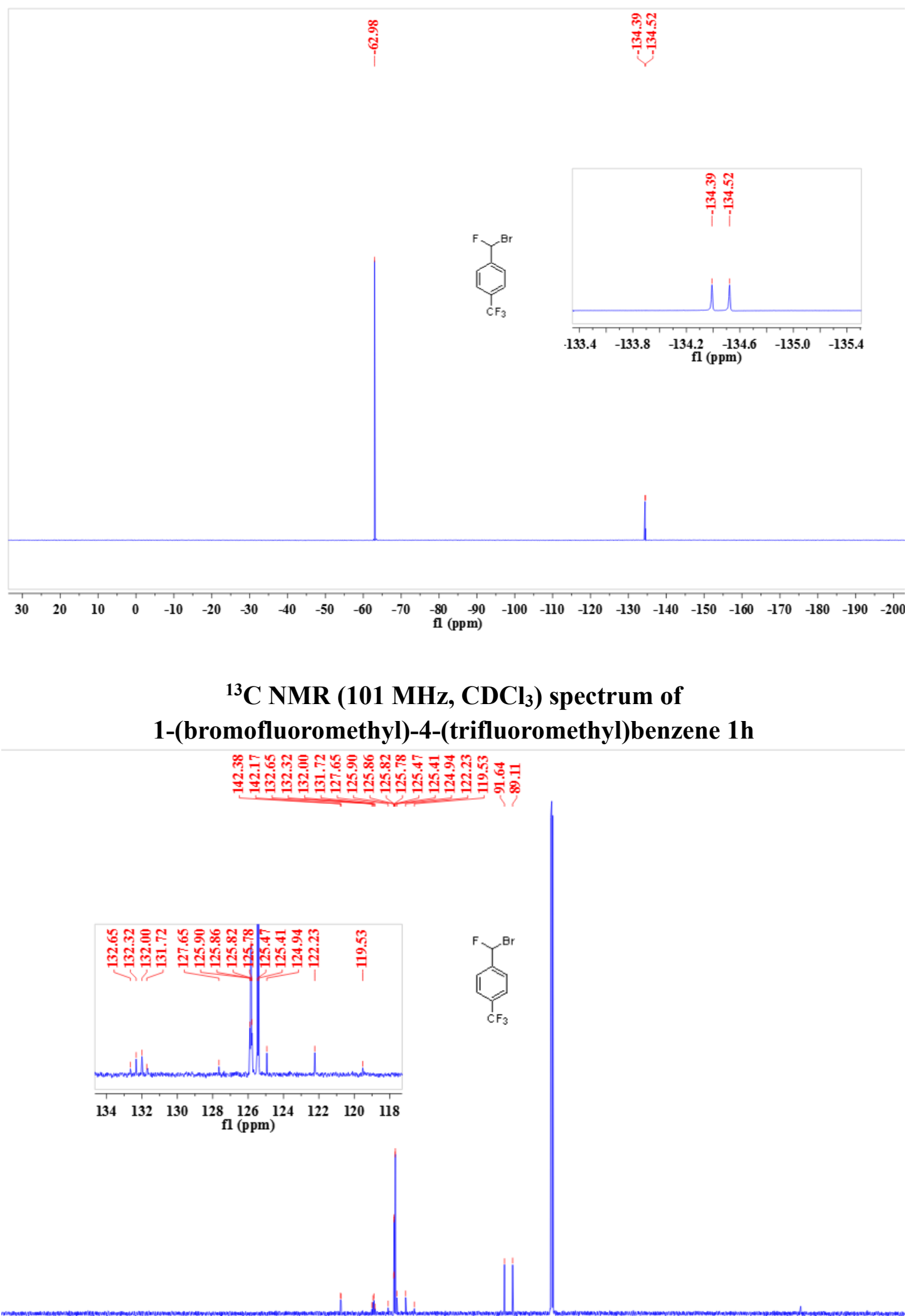

$\begin{array}{lllllllllllllllllllllllllllll}240 & 230 & 220 & 210 & 200 & 190 & 180 & 170 & 160 & 150 & 140 & 130 & 120 & 110 & 100 & 90 & 80 & 70 & 60 & 50 & 40 & 30 & 20 & 10 & 0 & -10 & -20 & -30\end{array}$ fl (ppm) 
${ }^{1} \mathrm{H}$ NMR (400 MHz, $\mathrm{CDCl}_{3}$ ) spectrum of 1-(bromofluoromethyl)-3-(trifluoromethyl)benzene 1i

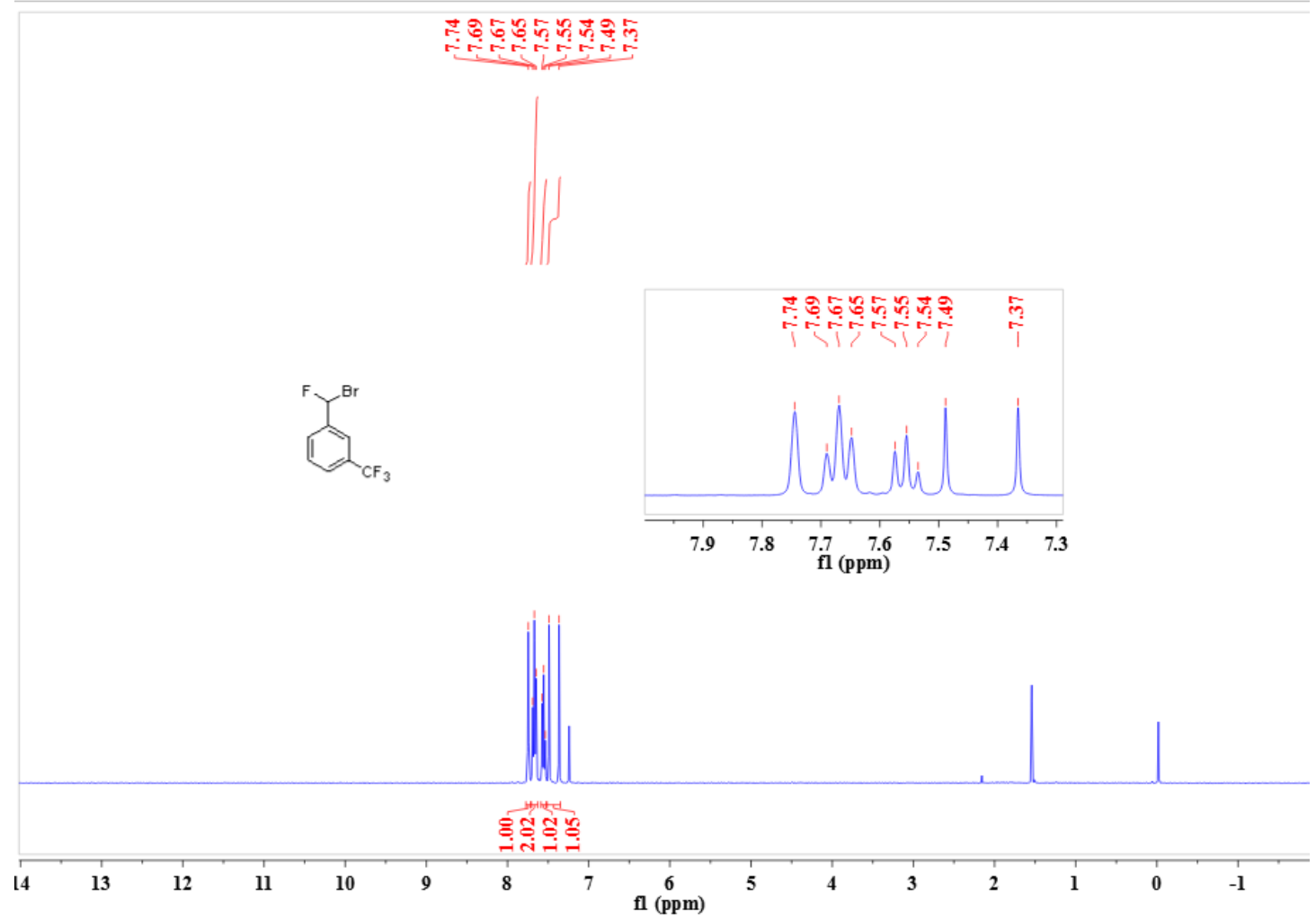

${ }^{19} \mathrm{~F}$ NMR (376 $\mathrm{MHz}, \mathrm{CDCl}_{3}$ ) spectrum of 1-(bromofluoromethyl)-3-(trifluoromethyl)benzene 1i

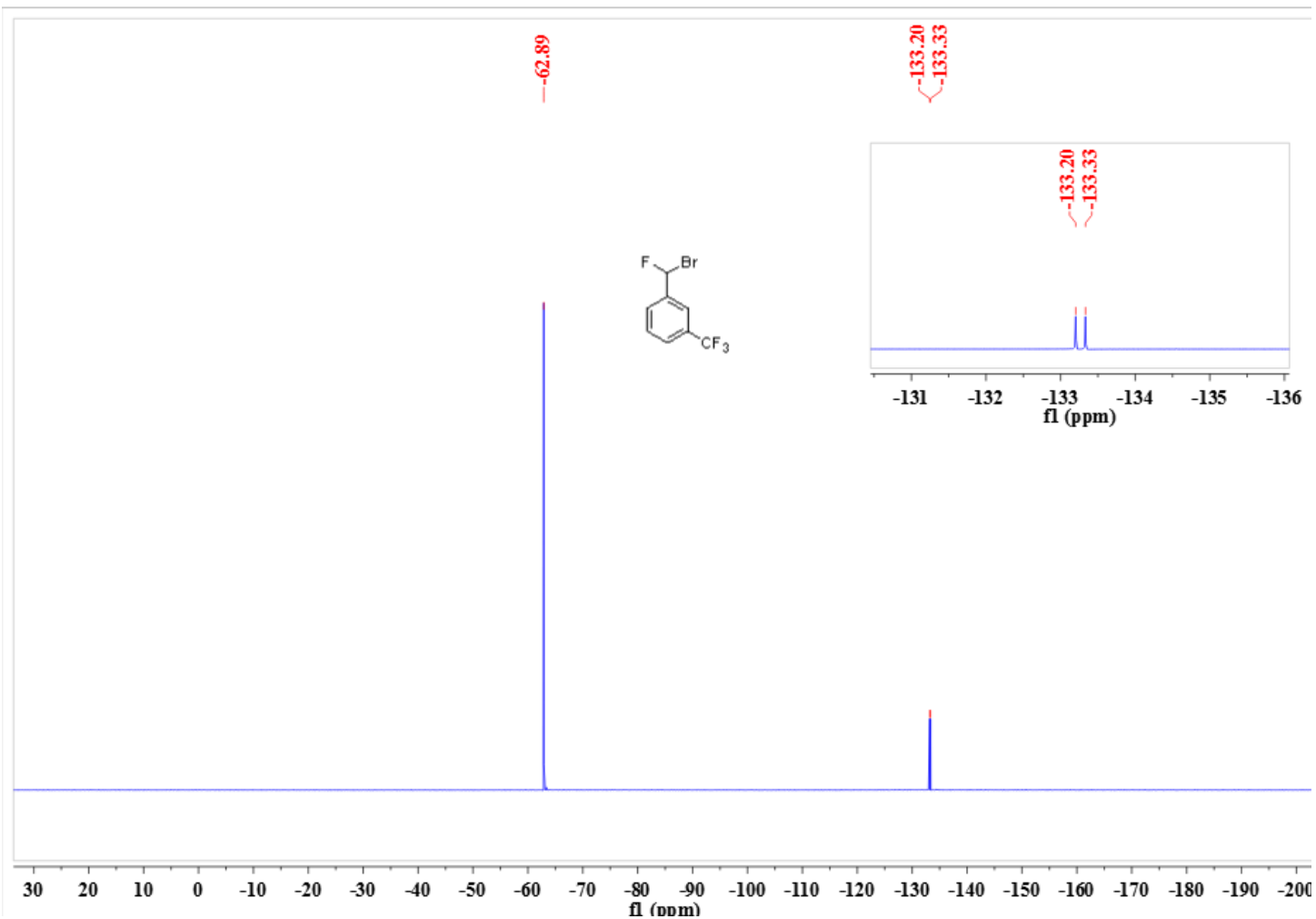


${ }^{13} \mathrm{C}$ NMR (101 MHz, $\left.\mathrm{CDCl}_{3}\right)$ spectrum of 1-(bromofluoromethyl)-3-(trifluoromethyl)benzene 1i
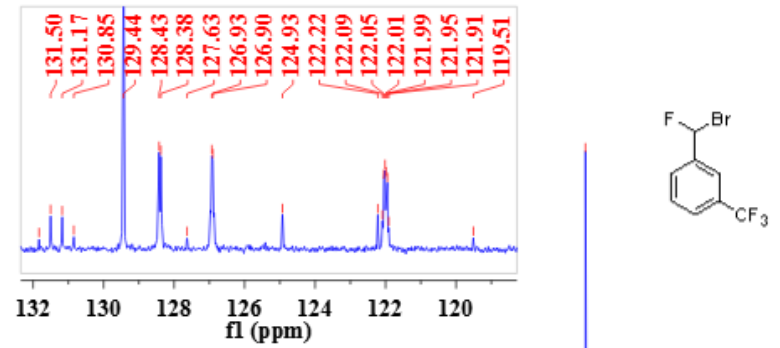

$\begin{array}{llllllllllllllllllllllllllllll}240 & 230 & 220 & 210 & 200 & 190 & 180 & 170 & 160 & 150 & 140 & 130 & 120 & \begin{array}{c}110 \\ \mathrm{fl}\end{array}(\mathrm{ppm}) & 80 & 90 & 70 & 50 & 40 & 30 & 20 & 10 & 0 & -10 & -20 & -\end{array}$

${ }^{1} \mathrm{H}$ NMR (400 $\mathrm{MHz}, \mathrm{CDCl}_{3}$ ) spectrum of 1-bromo-4-(bromofluoromethyl)benzene 1j
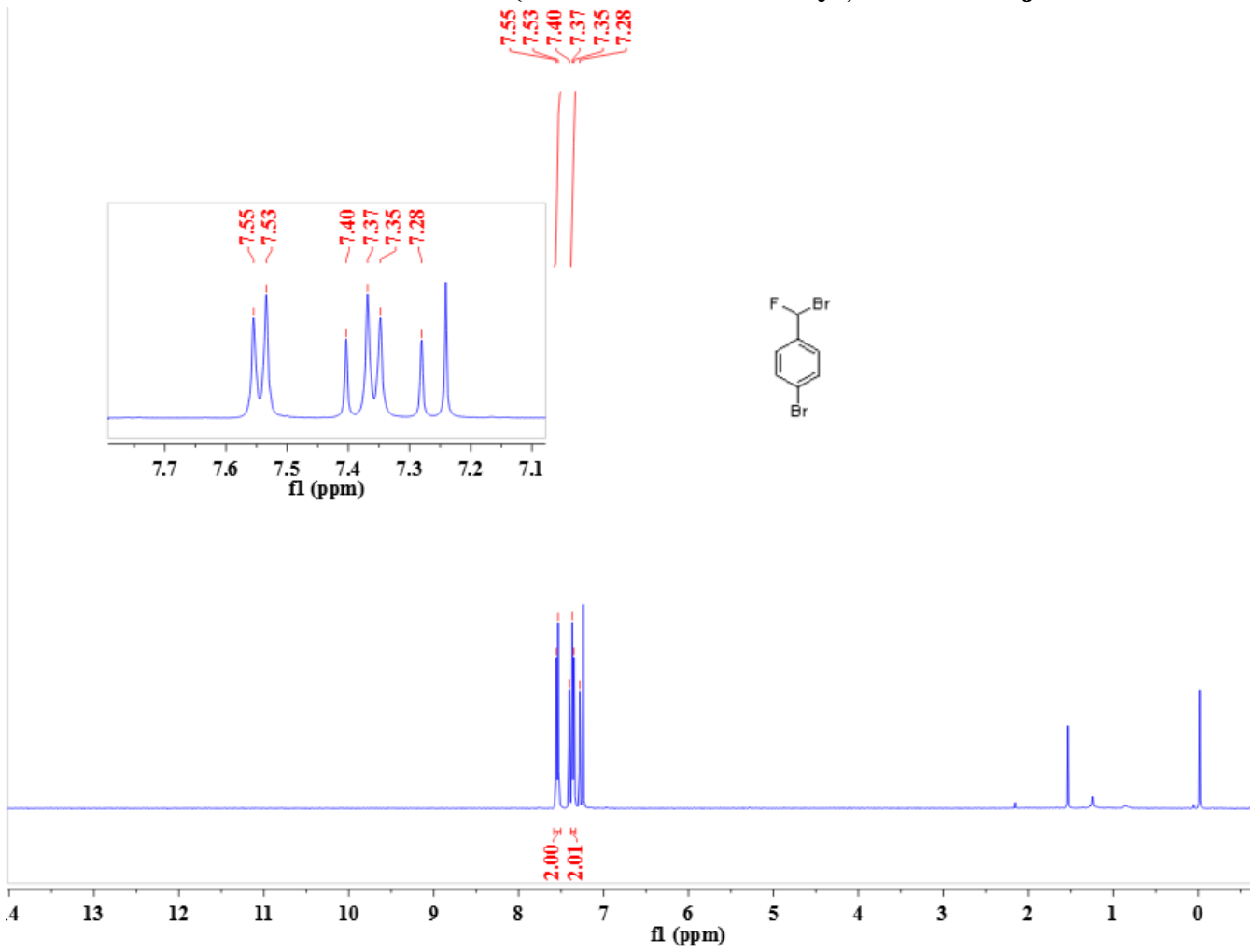
${ }^{19} \mathrm{~F}$ NMR (376 $\mathrm{MHz}, \mathrm{CDCl}_{3}$ ) spectrum of 1-bromo-4-(bromofluoromethyl)benzene $1 \mathrm{j}$

लै
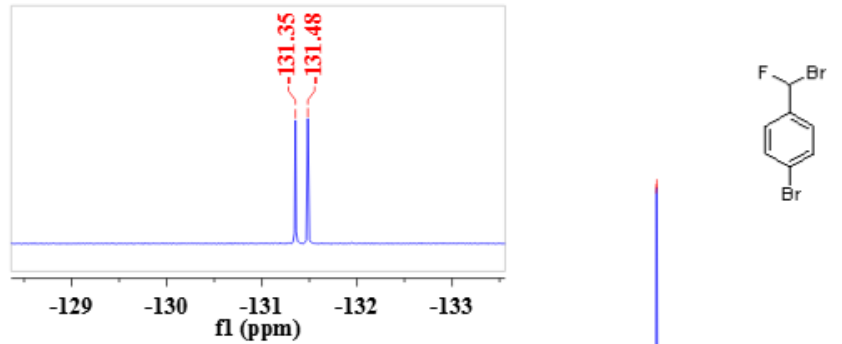

$30 \quad 10$

$10 \quad-10 \quad-30$

$\begin{array}{llll}-50 & -70 & -90 & -110\end{array}$

$-130 \quad-150$

$-170-190$

$\begin{array}{llll}-210 & -230 & -250 & -270\end{array}$

${ }^{13} \mathrm{C}$ NMR (101 MHz, $\left.\mathrm{CDCl}_{3}\right)$ spectrum of

1-bromo-4-(bromofluoromethyl)benzene $1 \mathrm{j}$ 


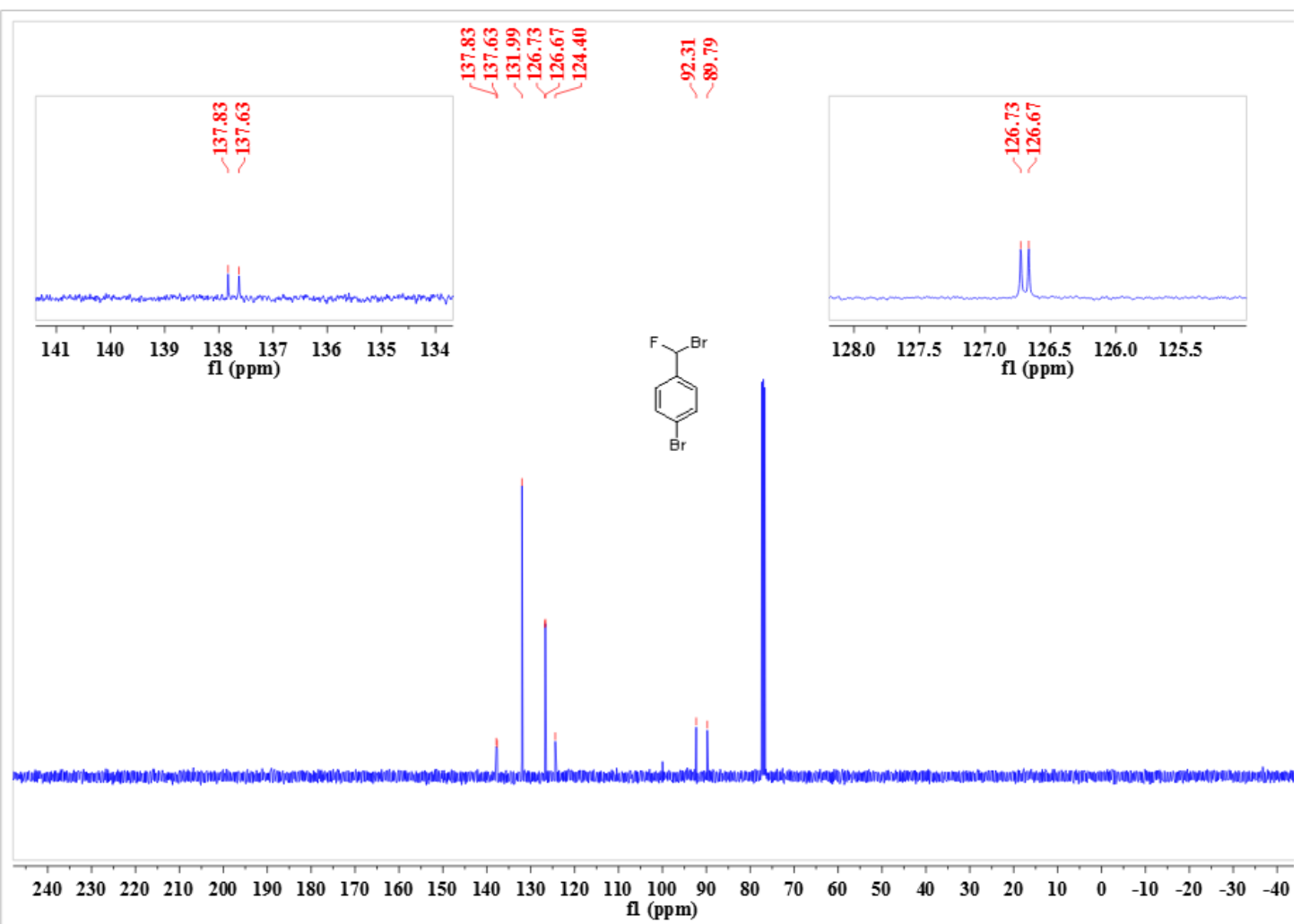

${ }^{1} \mathrm{H}$ NMR (400 $\mathrm{MHz}, \mathrm{CDCl}_{3}$ ) spectrum of 1-bromo-3-(bromofluoromethyl)-5-fluorobenzene $1 \mathrm{k}$

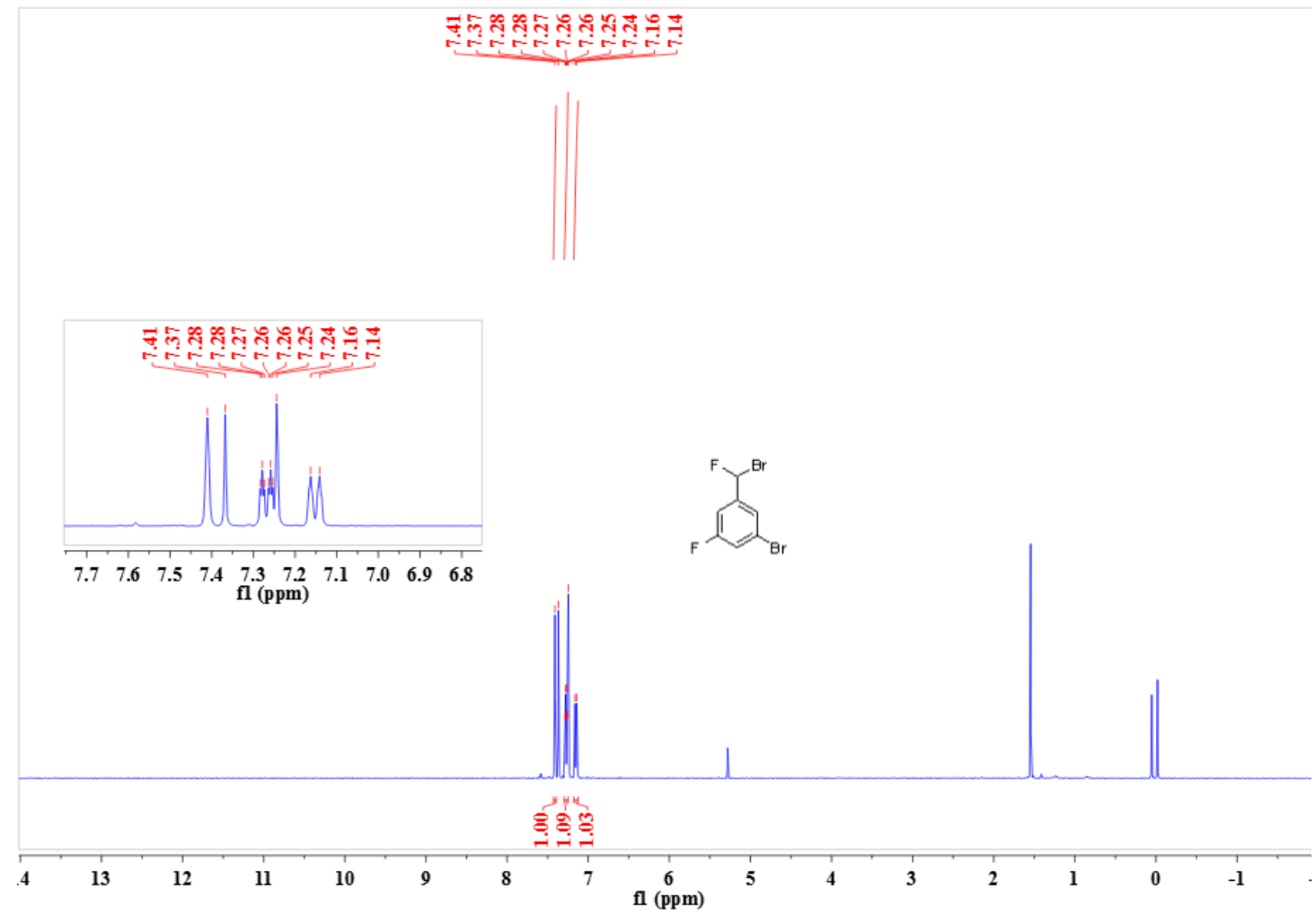

${ }^{19} \mathrm{~F}$ NMR (376 MHz, CDCl $)$ spectrum of 
1-bromo-3-(bromofluoromethyl)-5-fluorobenzene $1 \mathrm{k}$

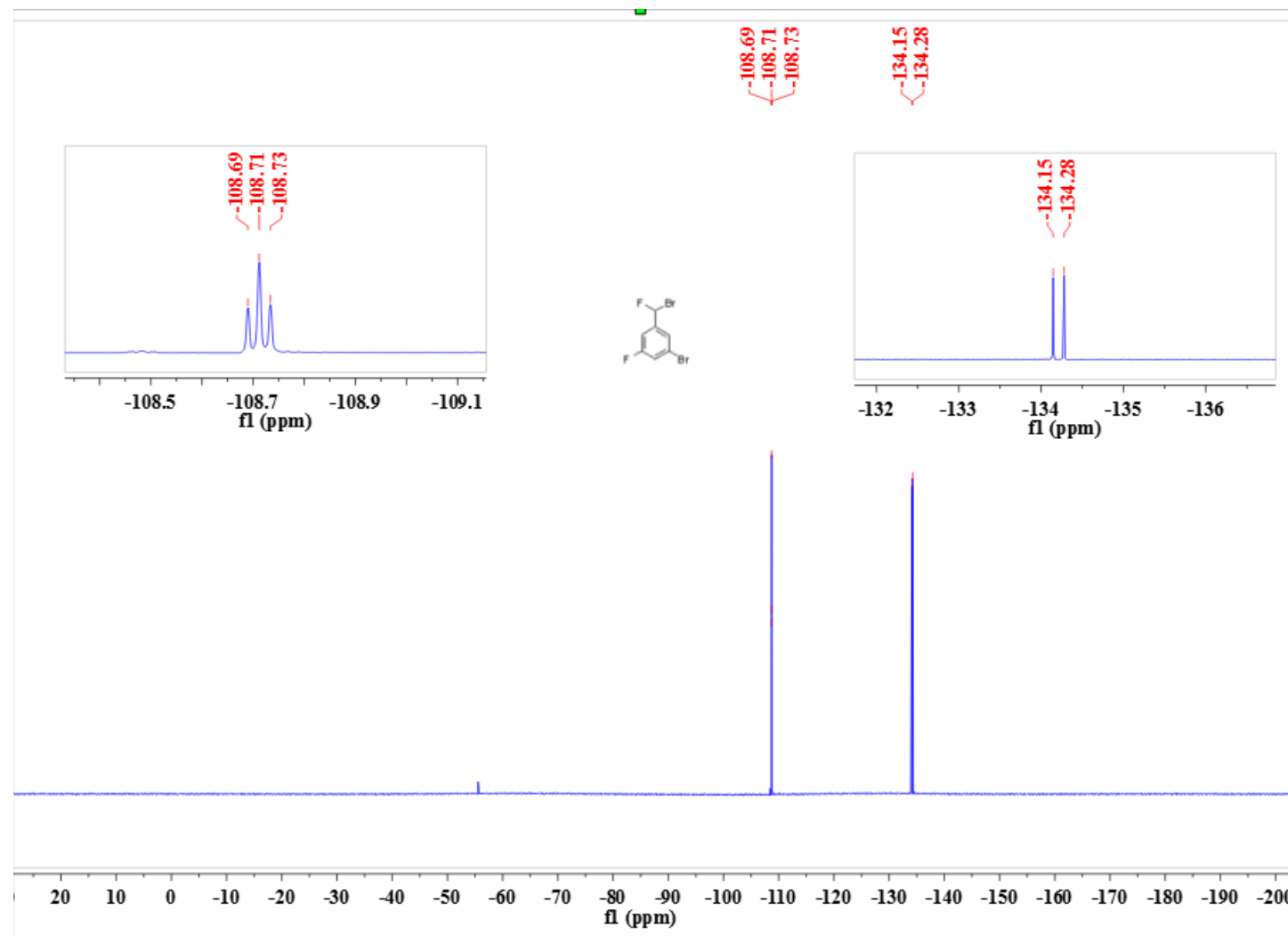

${ }^{13} \mathrm{C}$ NMR (101 MHz, $\left.\mathrm{CDCl}_{3}\right)$ spectrum of 1-bromo-3-(bromofluoromethyl)-5-fluorobenzene $1 \mathrm{k}$

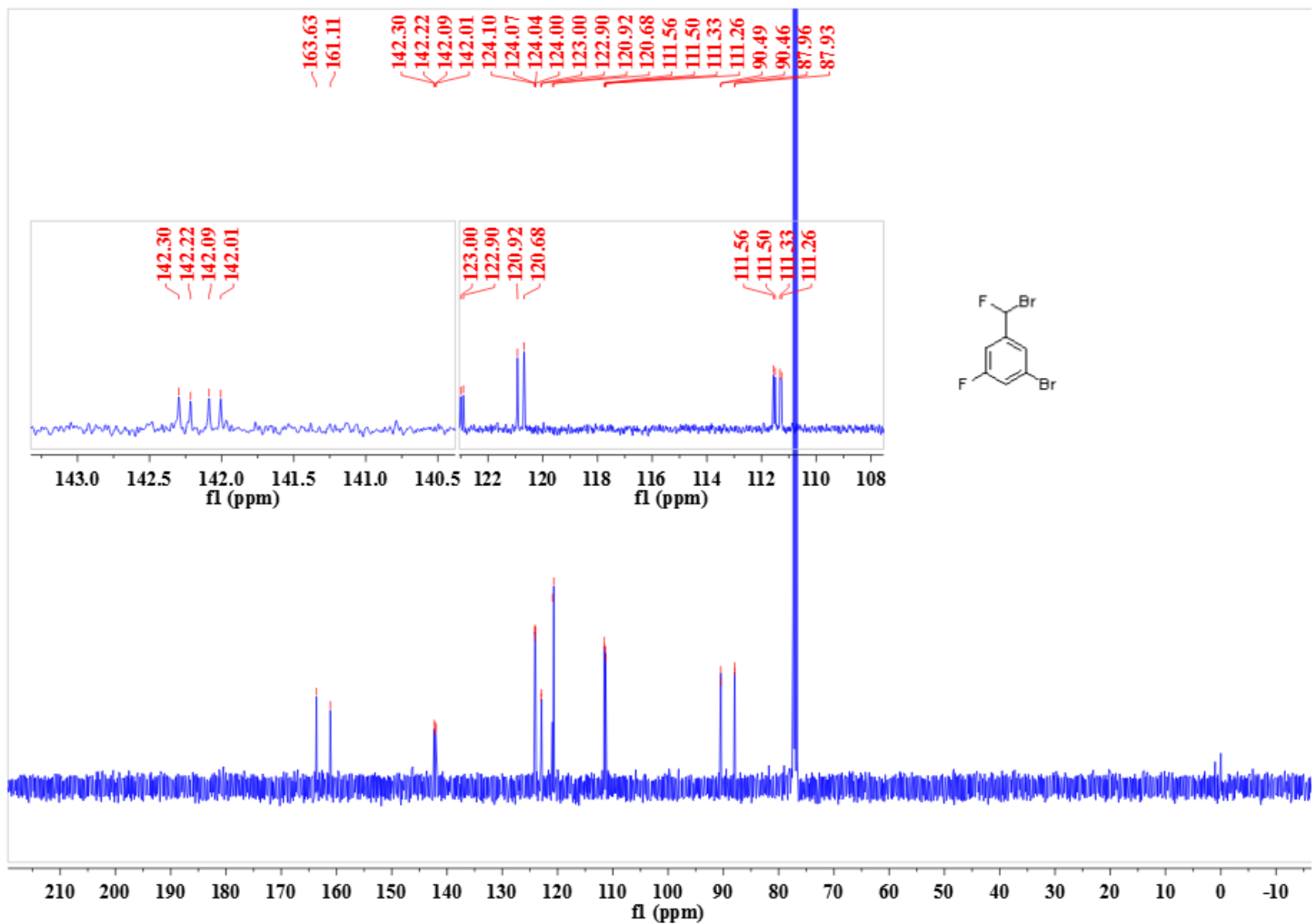


${ }^{1} \mathrm{H}$ NMR (400 $\left.\mathrm{MHz}, \mathrm{CDCl}_{3}\right)$ spectrum of

2-bromo-4-(bromofluoromethyl)-1-chlorobenzene 11

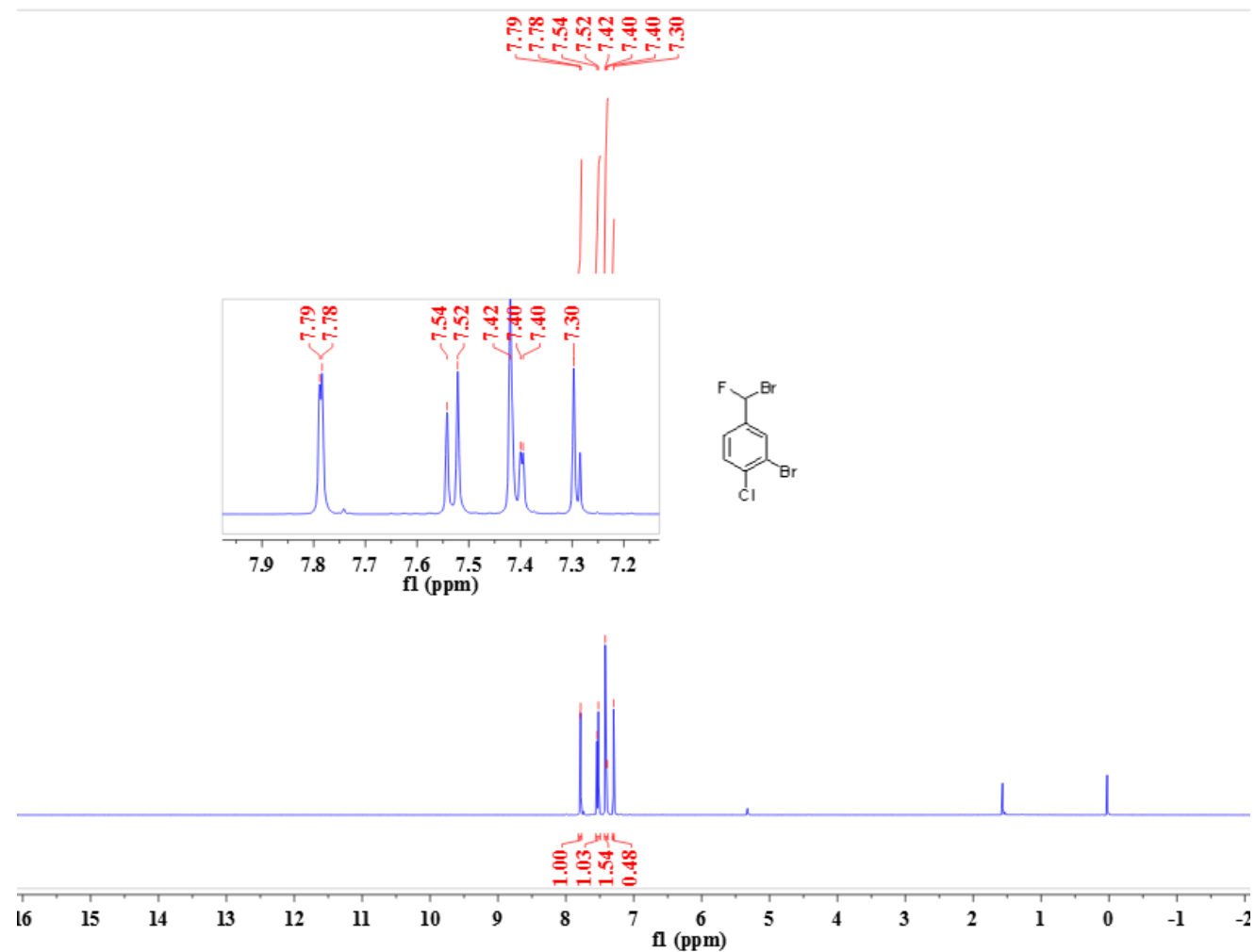

${ }^{19}$ F NMR (376 $\mathrm{MHz}, \mathrm{CDCl}_{3}$ ) spectrum of

2-bromo-4-(bromofluoromethyl)-1-chlorobenzene 11

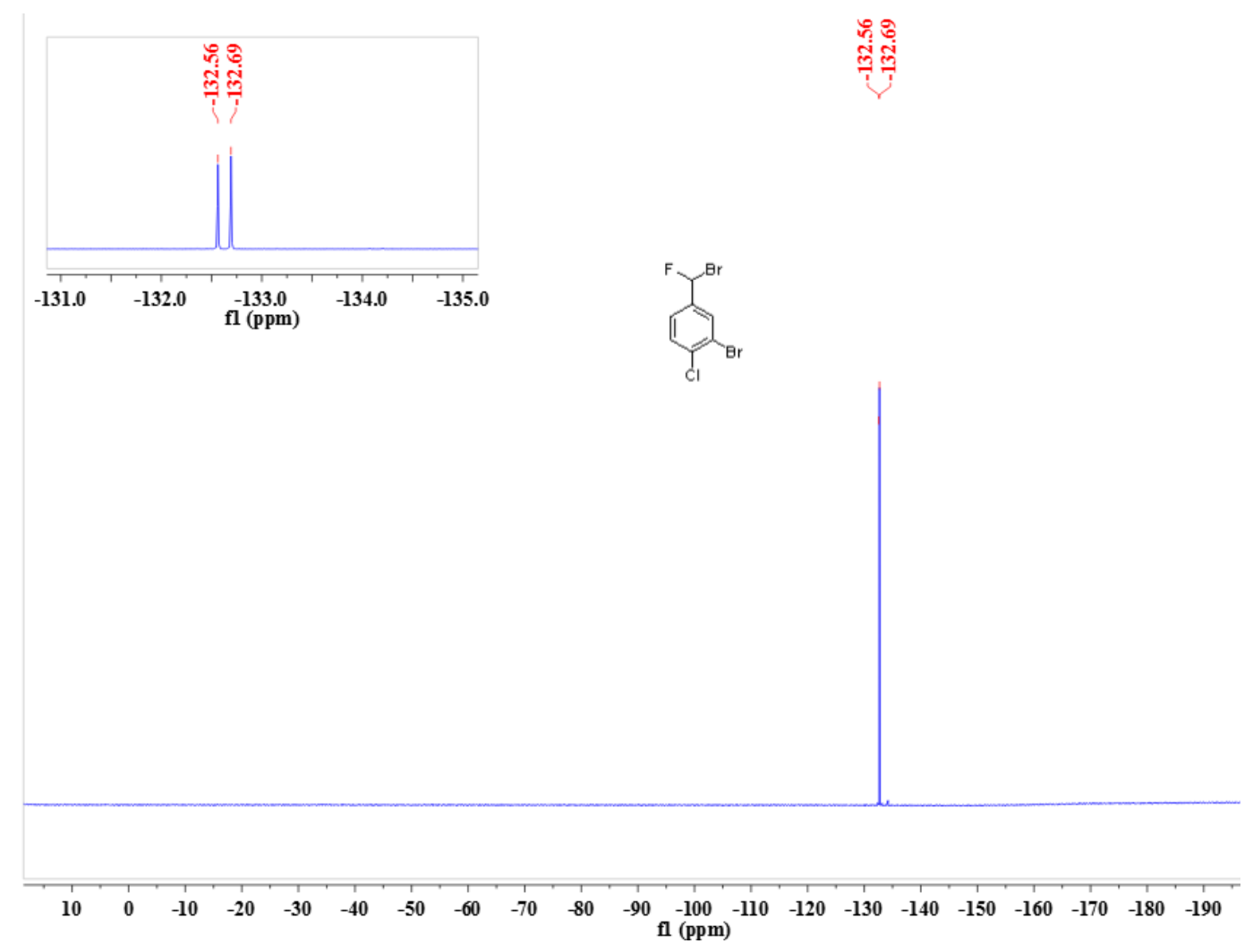


${ }^{13} \mathrm{C}$ NMR (101 MHz, $\left.\mathrm{CDCl}_{3}\right)$ spectrum of 2-bromo-4-(bromofluoromethyl)-1-chlorobenzene 11

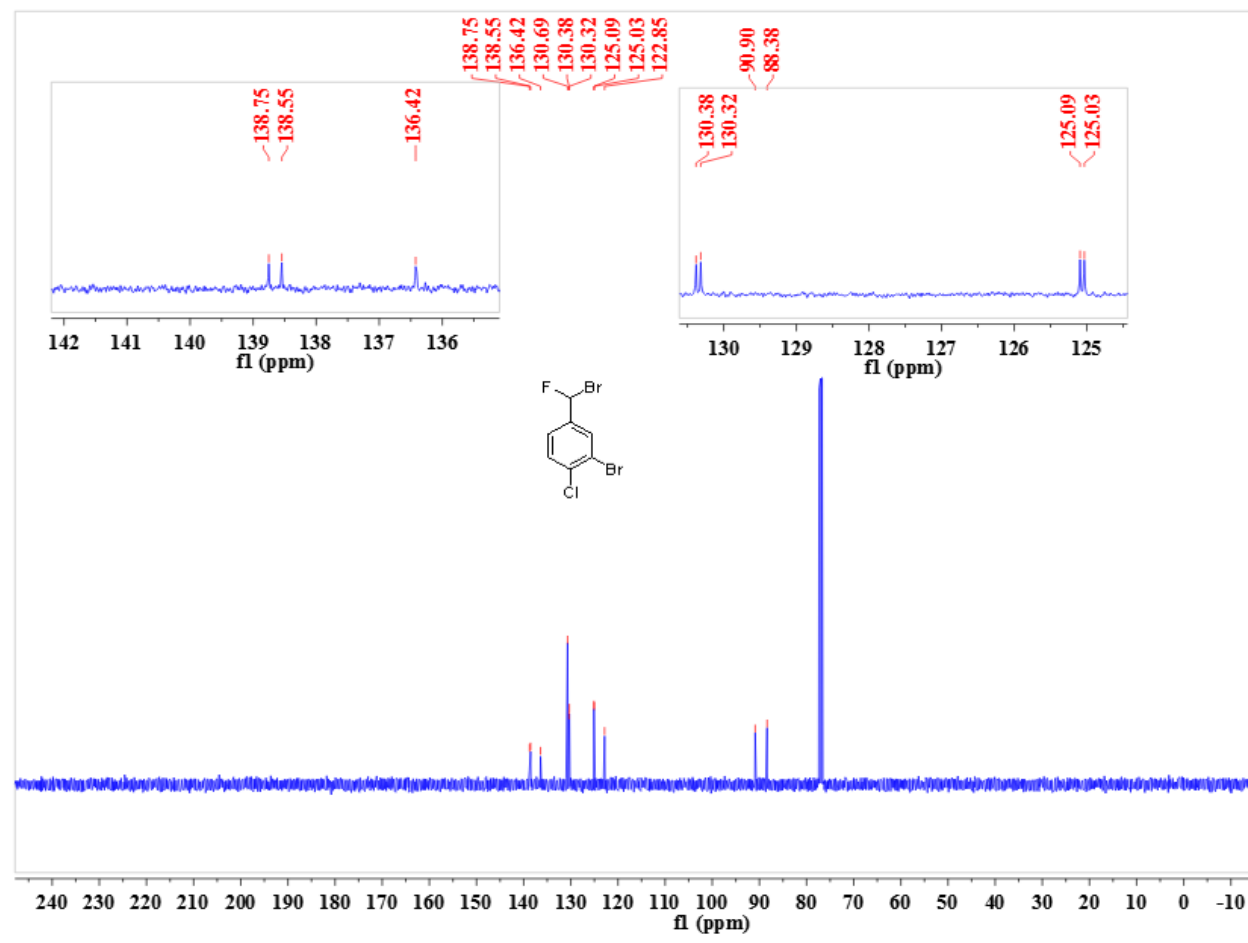

${ }^{1} \mathrm{H}$ NMR (400 MHz, $\mathrm{CDCl}_{3}$ ) spectrum of 1-(Bromofluoromethyl)-4-chlorobenzene $1 \mathrm{~m}$

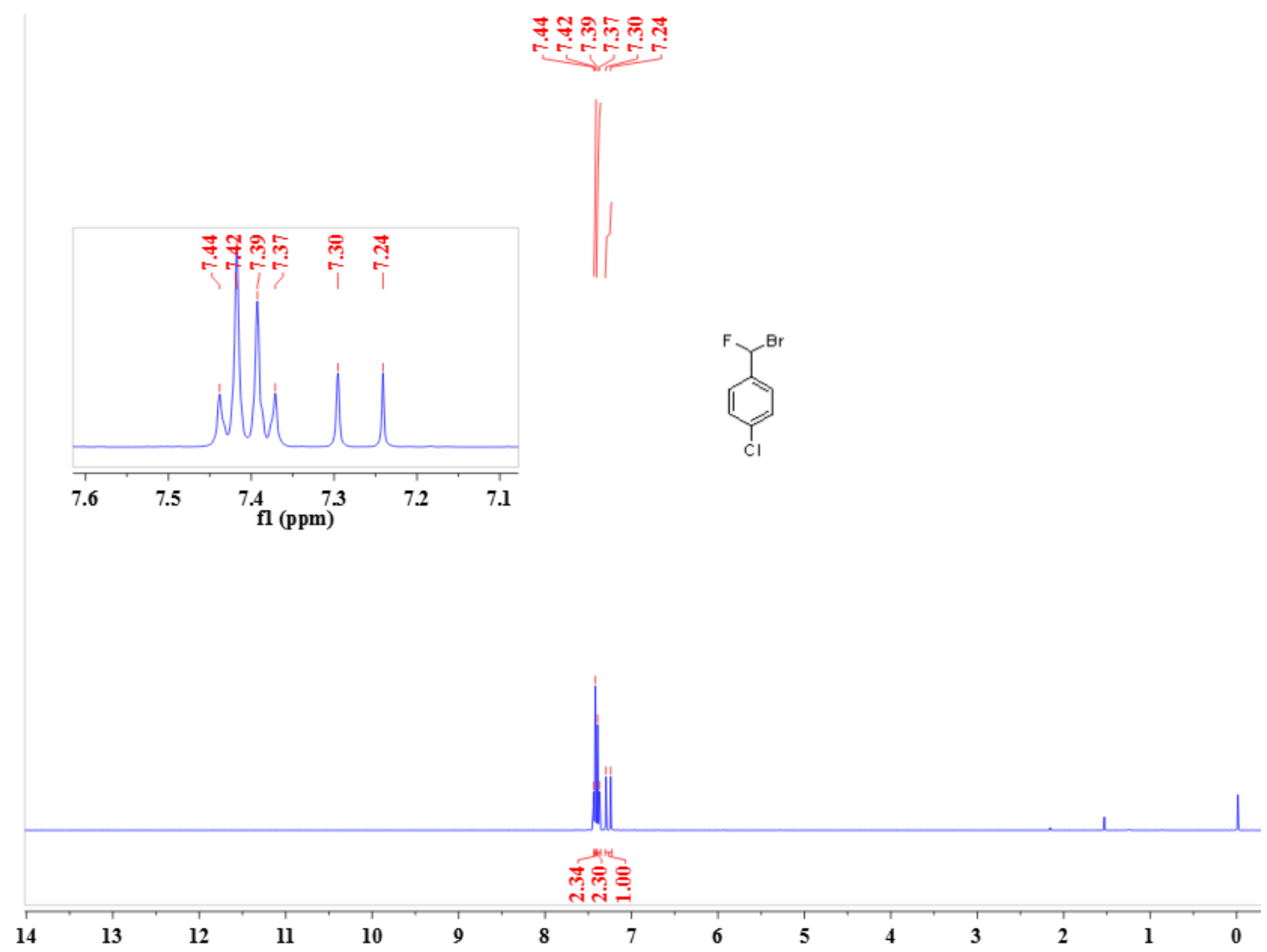


${ }^{19} \mathrm{~F}$ NMR (376 $\mathrm{MHz}, \mathrm{CDCl}_{3}$ ) spectrum of

1-(bromofluoromethyl)-4-chlorobenzene $1 \mathrm{~m}$

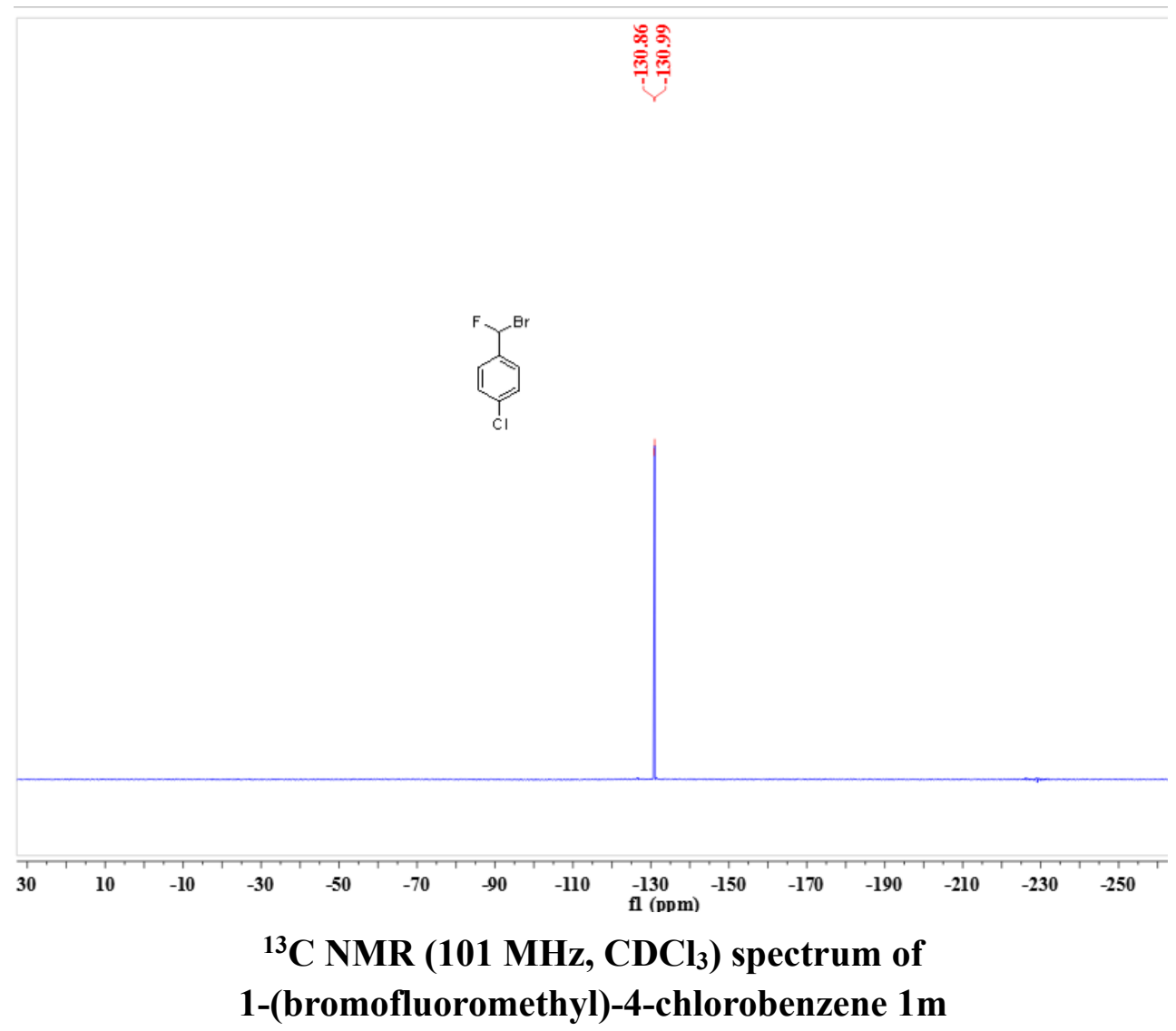




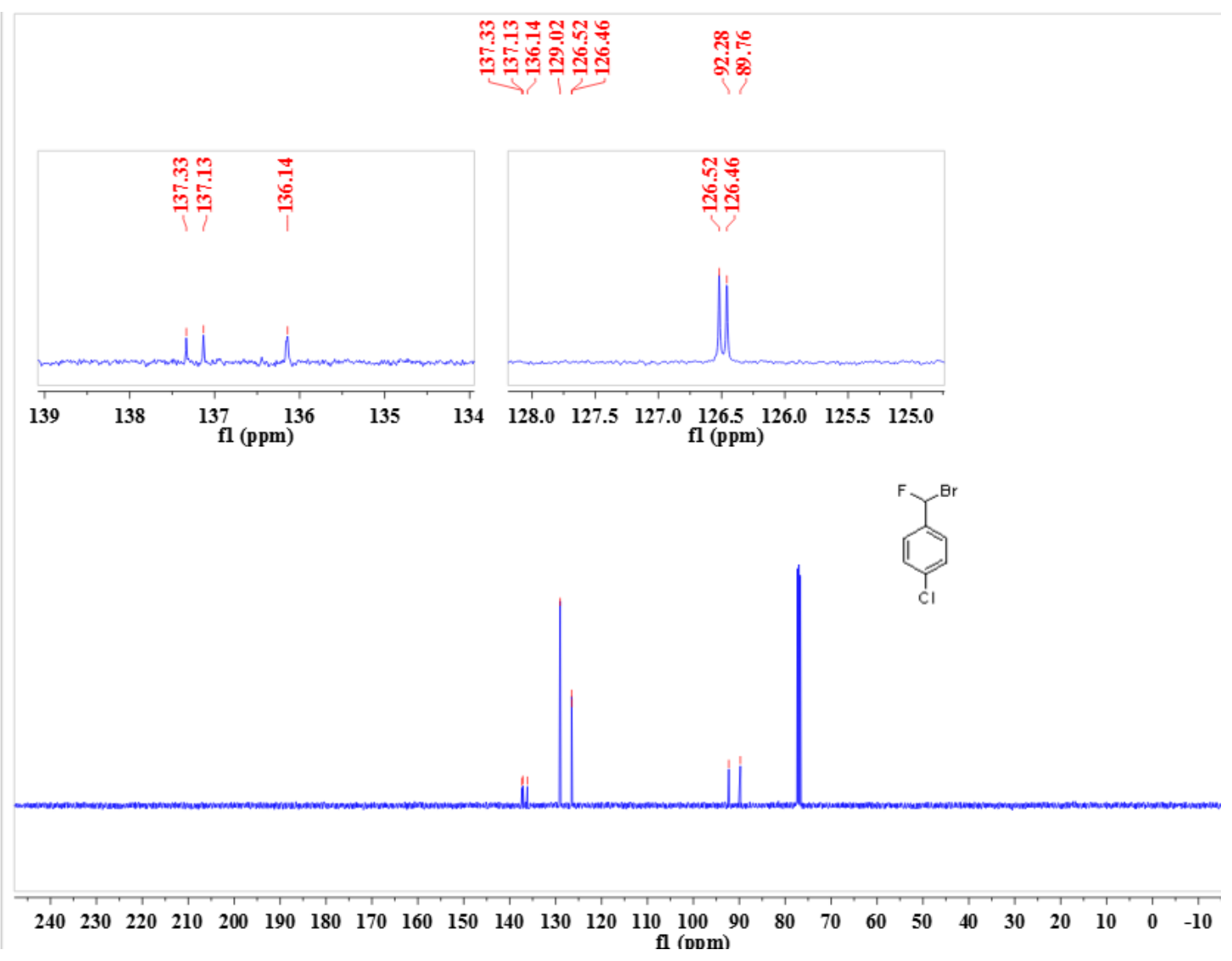

${ }^{1} \mathrm{H}$ NMR (400 $\mathrm{MHz}, \mathrm{CDCl}_{3}$ ) spectrum of 4-(bromofluoromethyl)-1,2-dichlorobenzene 1n

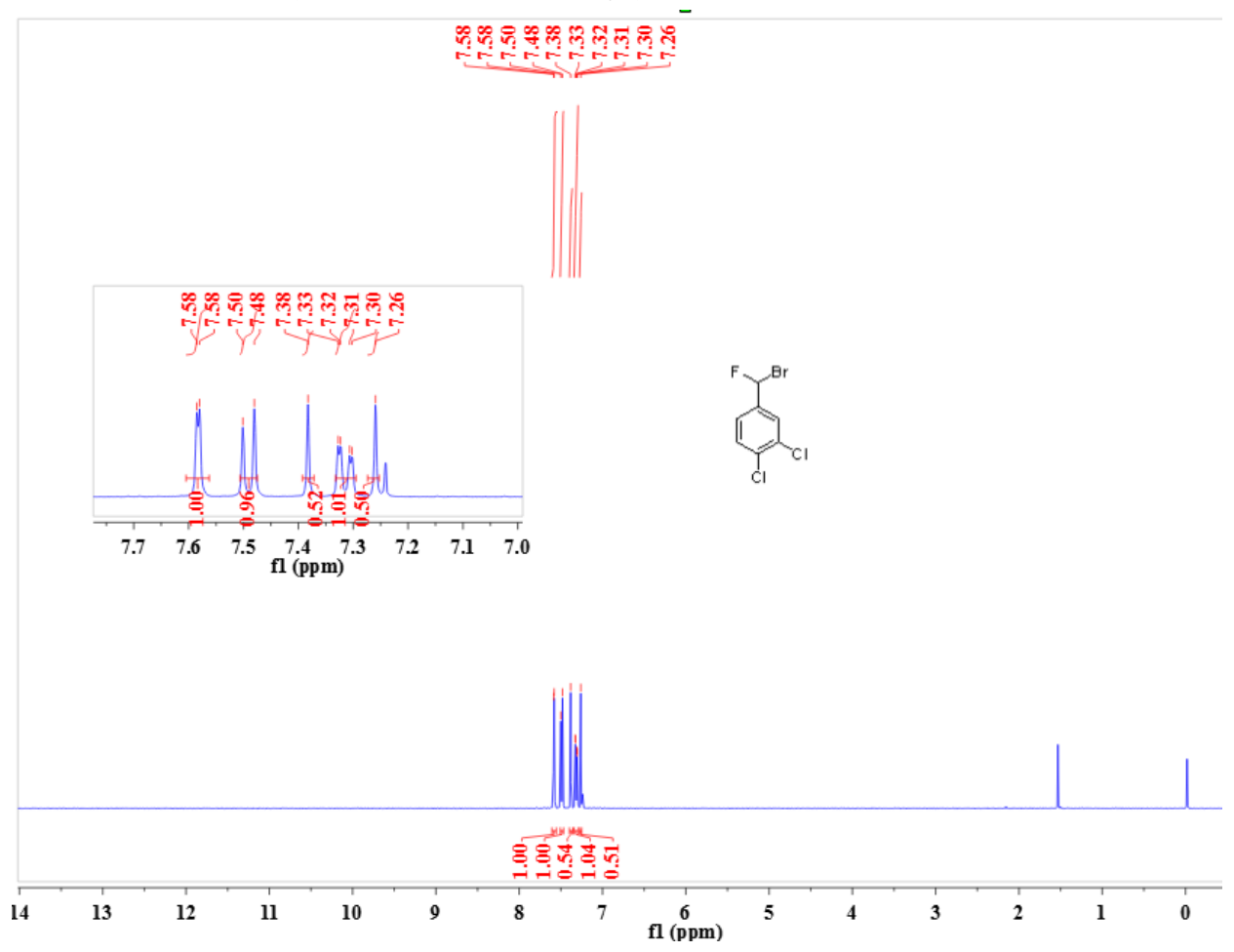

${ }^{19}$ F NMR (376 $\mathrm{MHz}, \mathrm{CDCl}_{3}$ ) spectrum of 4-(bromofluoromethyl)-1,2-dichlorobenzene 1n 


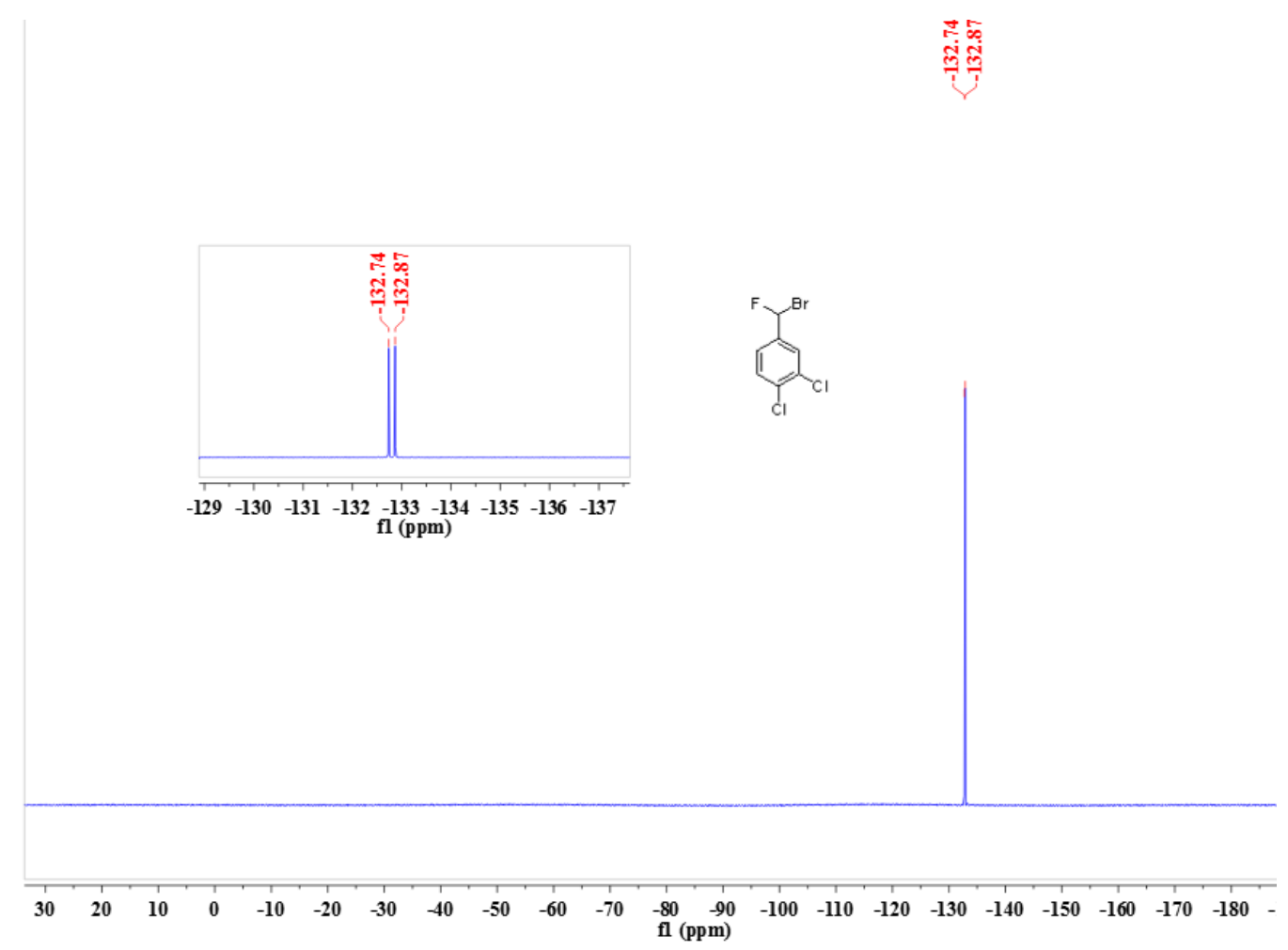

${ }^{13} \mathrm{C}$ NMR (101 $\left.\mathrm{MHz}, \mathrm{CDCl}_{3}\right)$ spectrum of

4-(bromofluoromethyl)-1,2-dichlorobenzene 1n

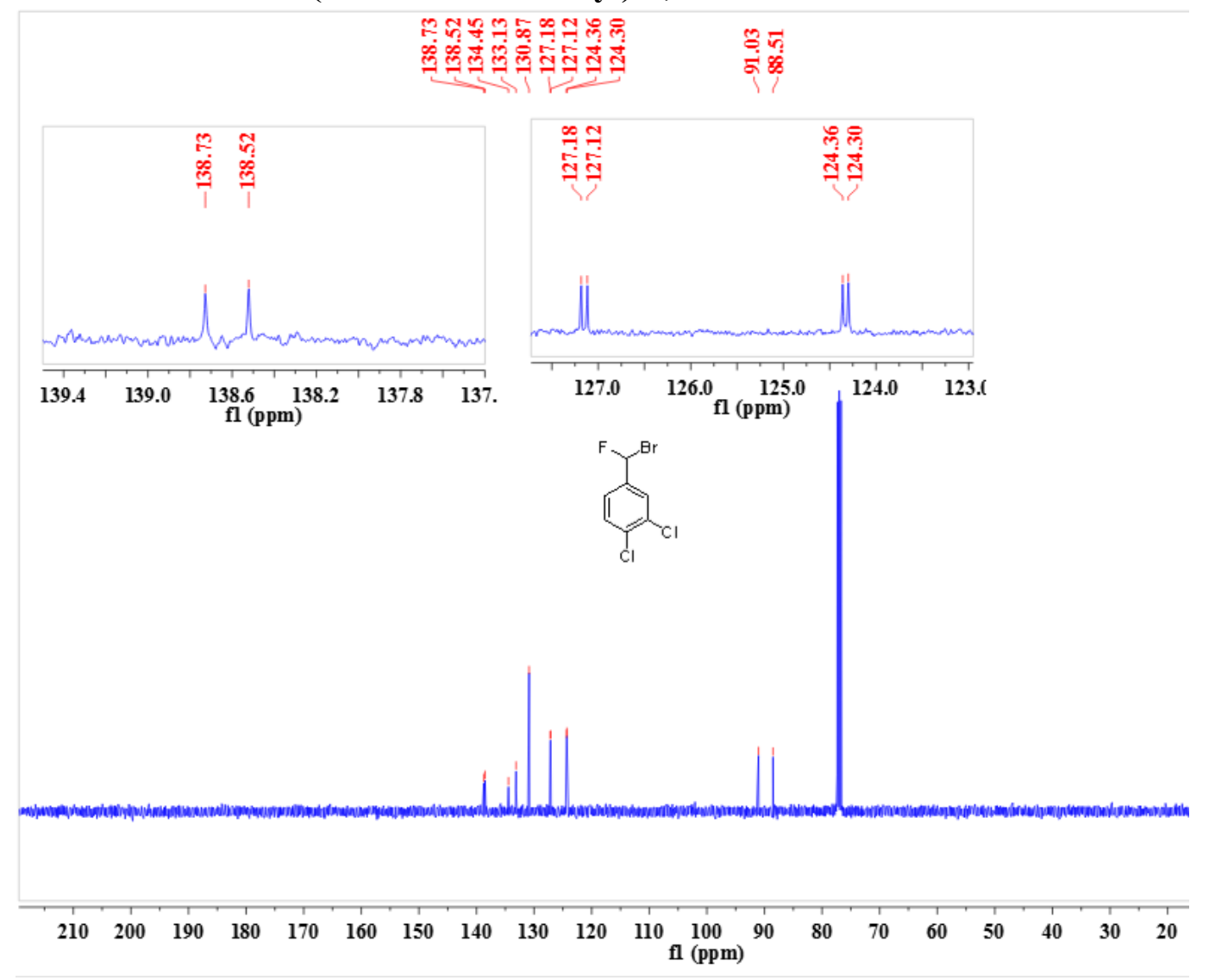

${ }^{1} \mathrm{H}$ NMR (400 MHz, $\mathrm{CDCl}_{3}$ ) spectrum of

1-(bromofluoromethyl)-3,5-dichlorobenzene 10 

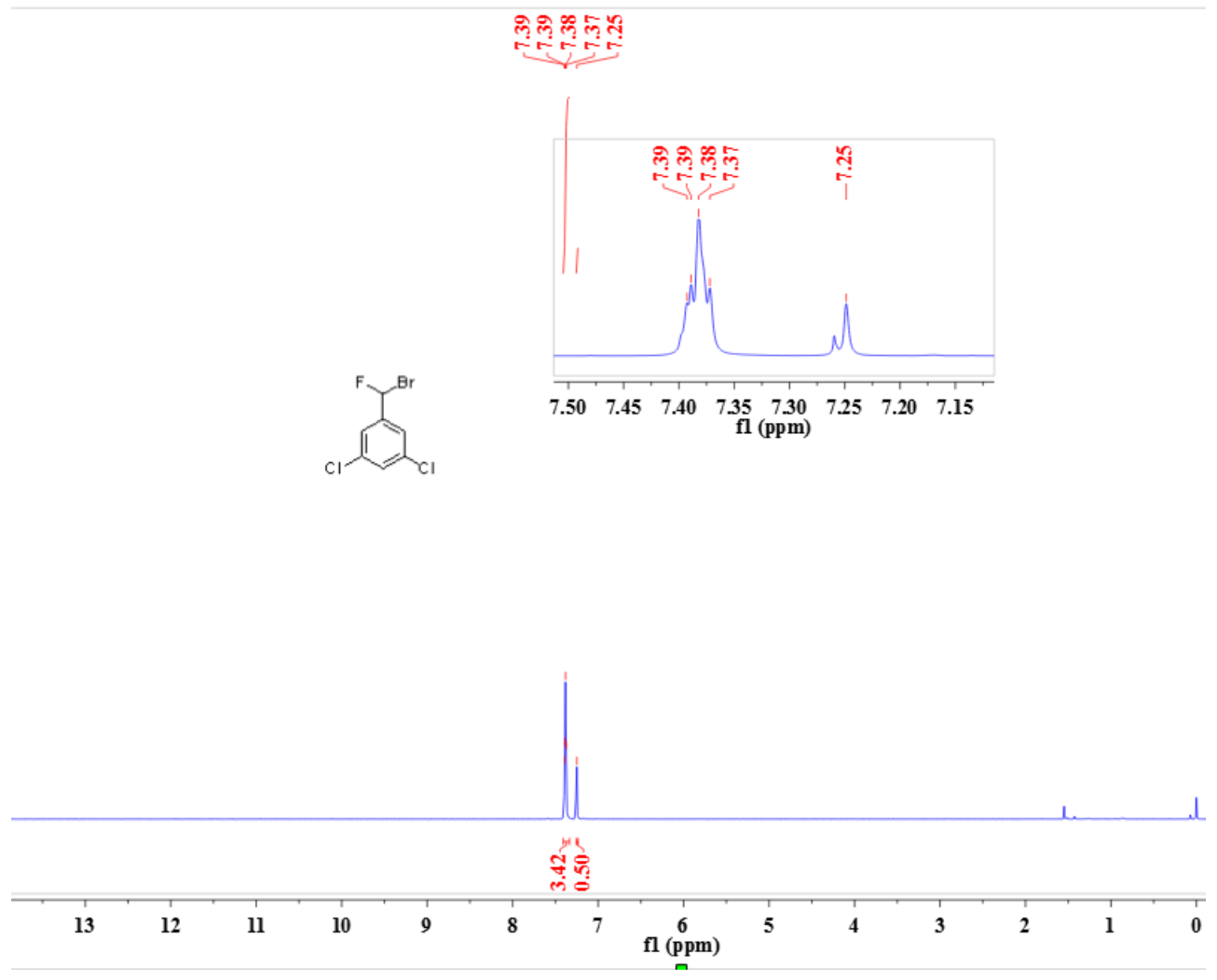

${ }^{19}$ F NMR (376 $\left.\mathrm{MHz}, \mathrm{CDCl}_{3}\right)$ spectrum of 1-(bromofluoromethyl)-3,5-dichlorobenzene 10

लैं
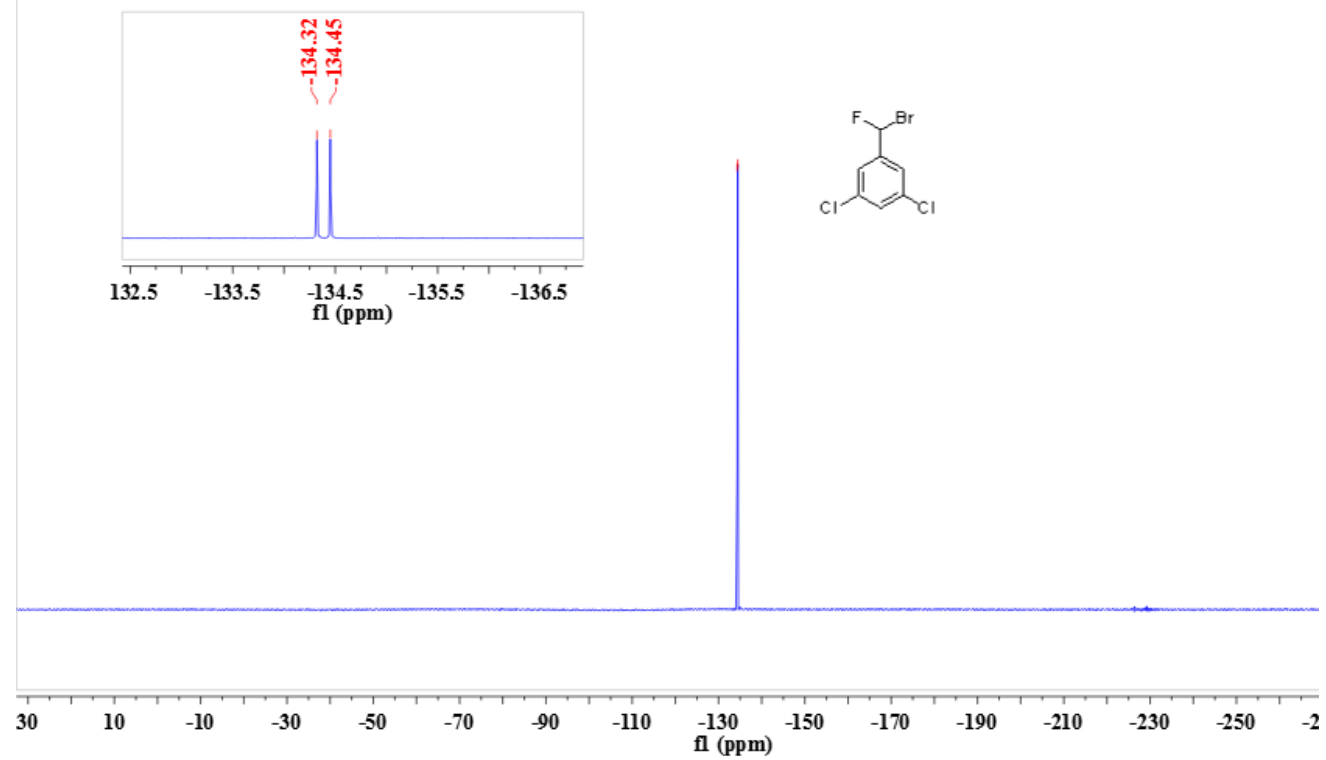

${ }^{13} \mathrm{C}$ NMR (101 MHz, $\left.\mathrm{CDCl}_{3}\right)$ spectrum of 2-(bromofluoromethyl)-3,5-dichlorobenzene 10 


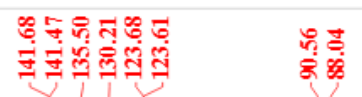

\section{象}
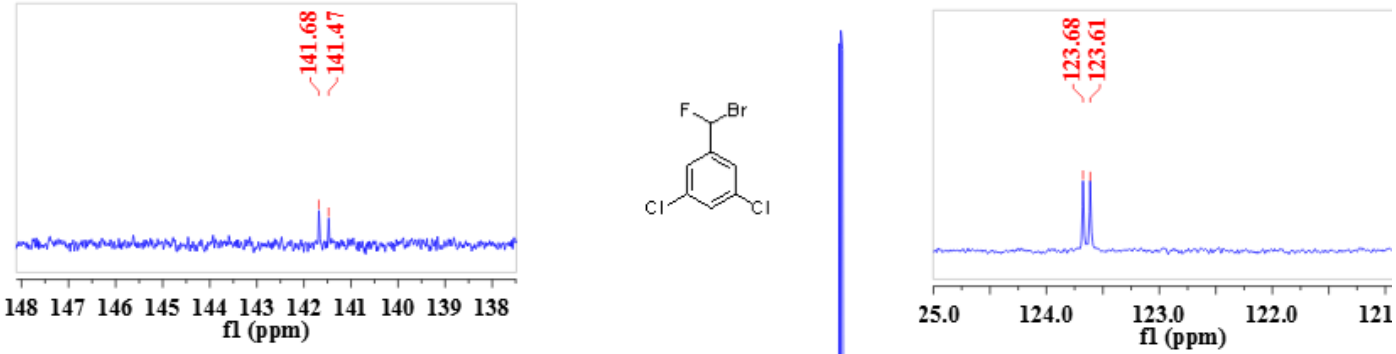
$148147146145144 \quad 143142141140139138$
$\mathrm{fl}(\mathrm{ppm})$
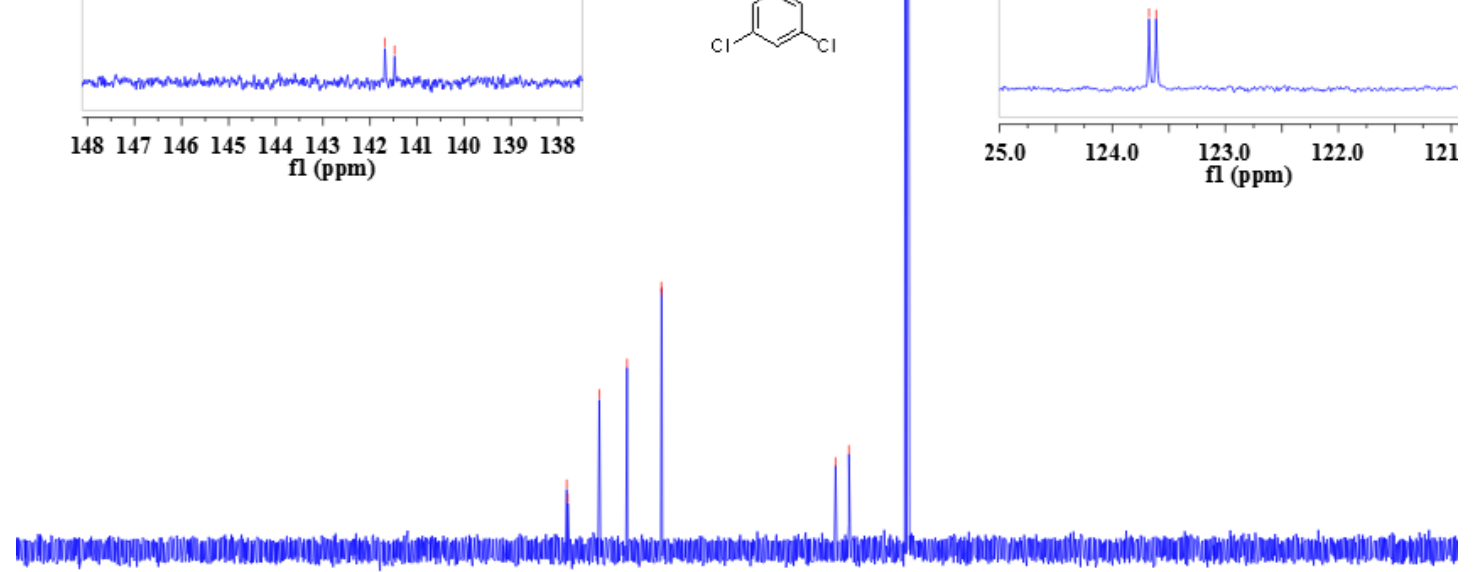

$\begin{array}{llllllllllllllllllllllllllll}240 & 230 & 220 & 210 & 200 & 190 & 180 & 170 & 160 & 150 & 140 & 130 & 120 & 110 & 100 & 90 & 80 & 70 & 60 & 50 & 40 & 30 & 20 & 10 & 0 & -10 & -20 & -\end{array}$

${ }^{1} \mathrm{H}$ NMR (400 MHz, CDCl3) spectrum of 4-(bromofluoromethyl)-1-chloro-2-fluorobenzene 1p

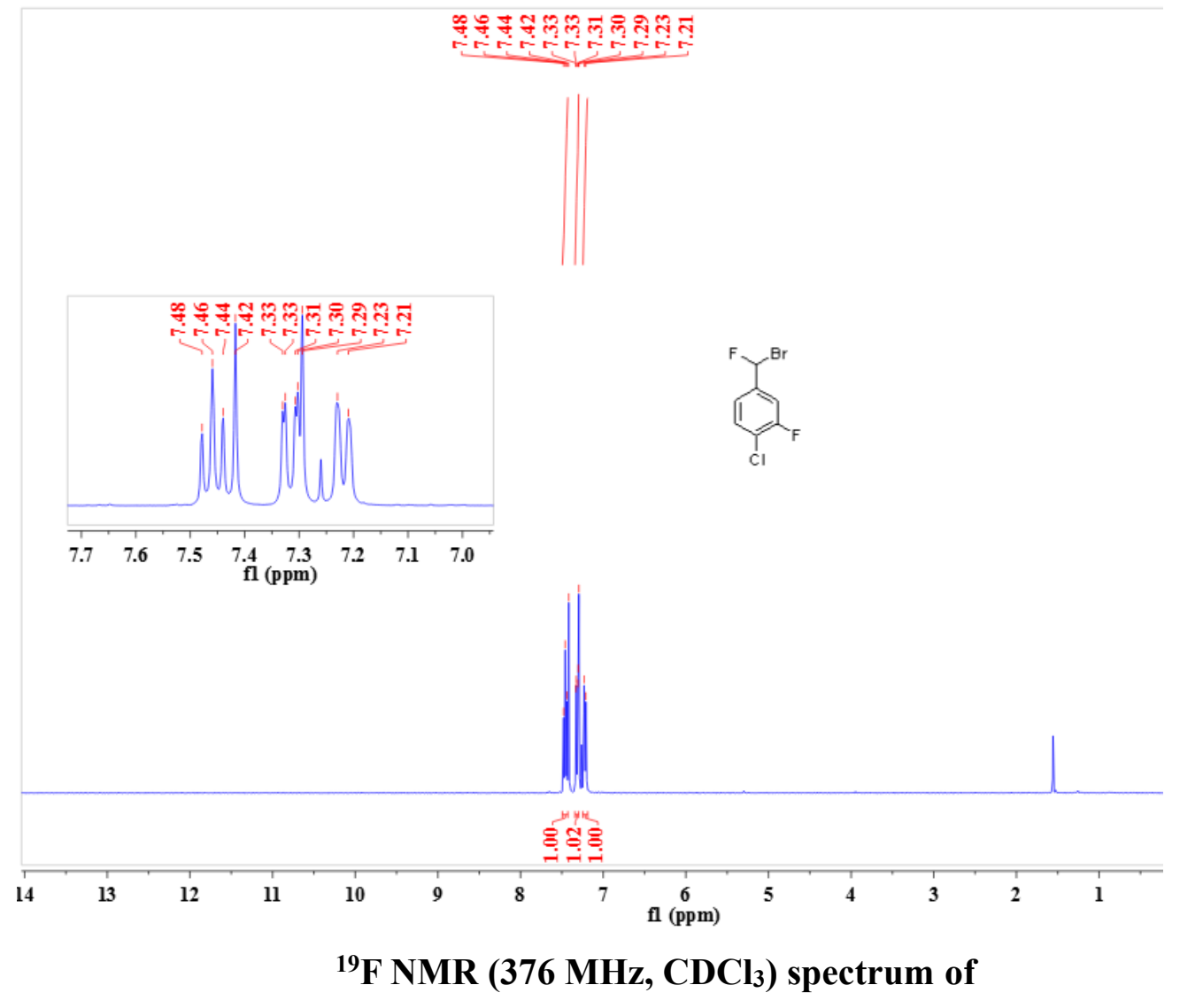


4-(bromofluoromethyl)-1-chloro-2-fluorobenzene 1p
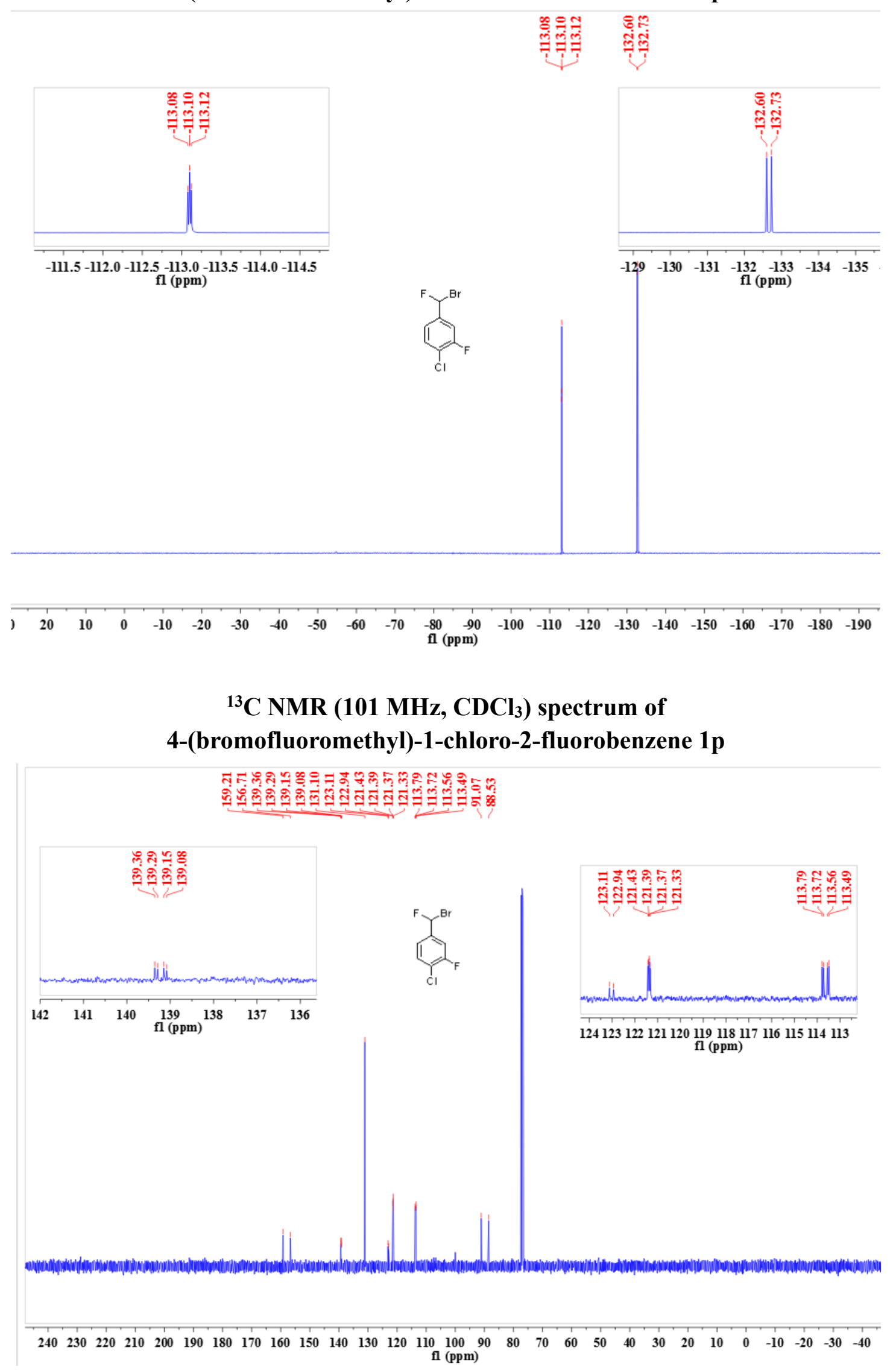

${ }^{1}$ H NMR (400 MHz, CDCl3) spectrum of 
methyl (S)-4-(fluoro(phenyl)methyl)benzoate 3a

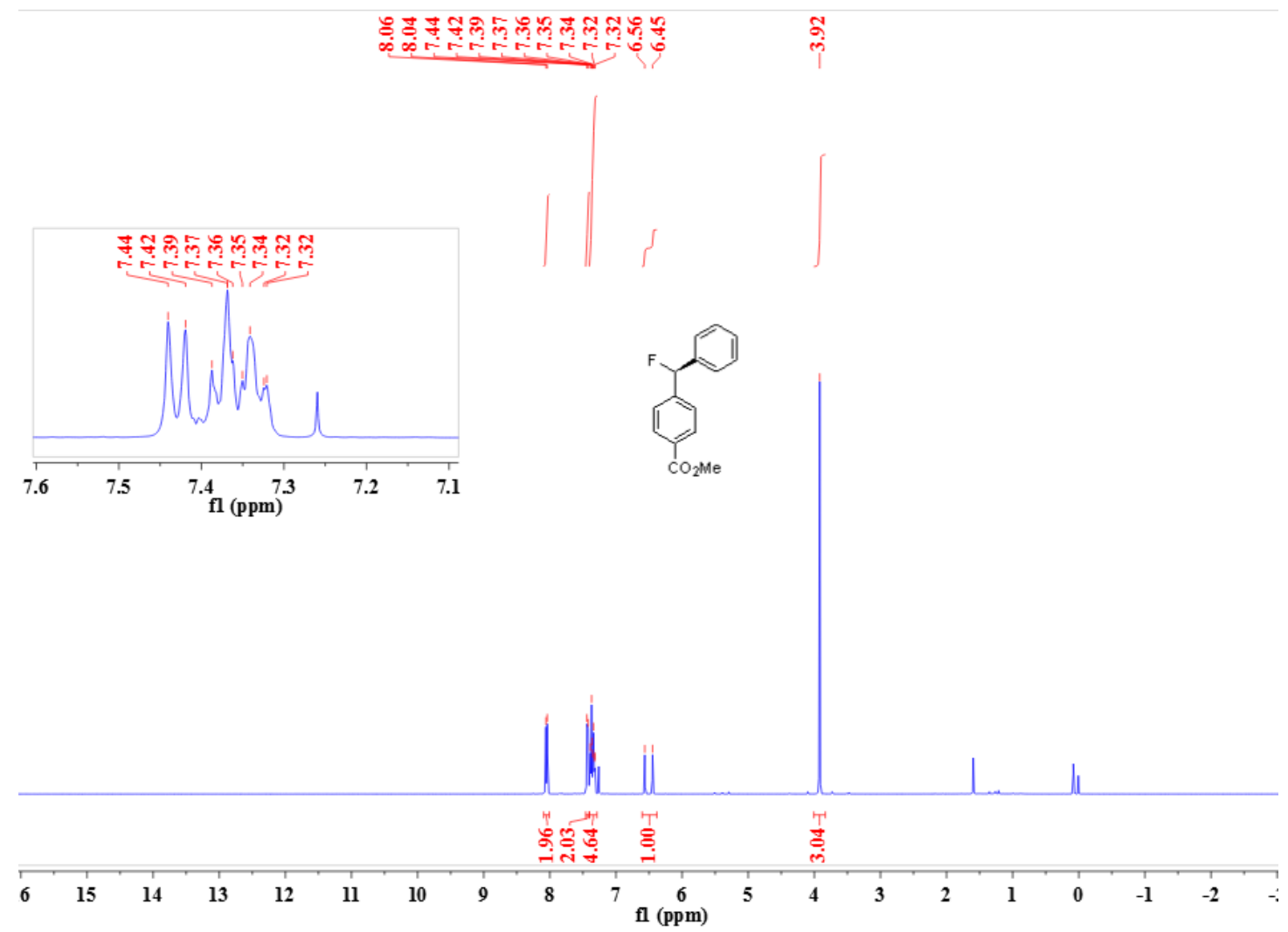

${ }^{19}$ F NMR (376 MHz, $\mathrm{CDCl}_{3}$ ) spectrum of methyl (S)-4-(fluoro(phenyl)methyl)benzoate 3a

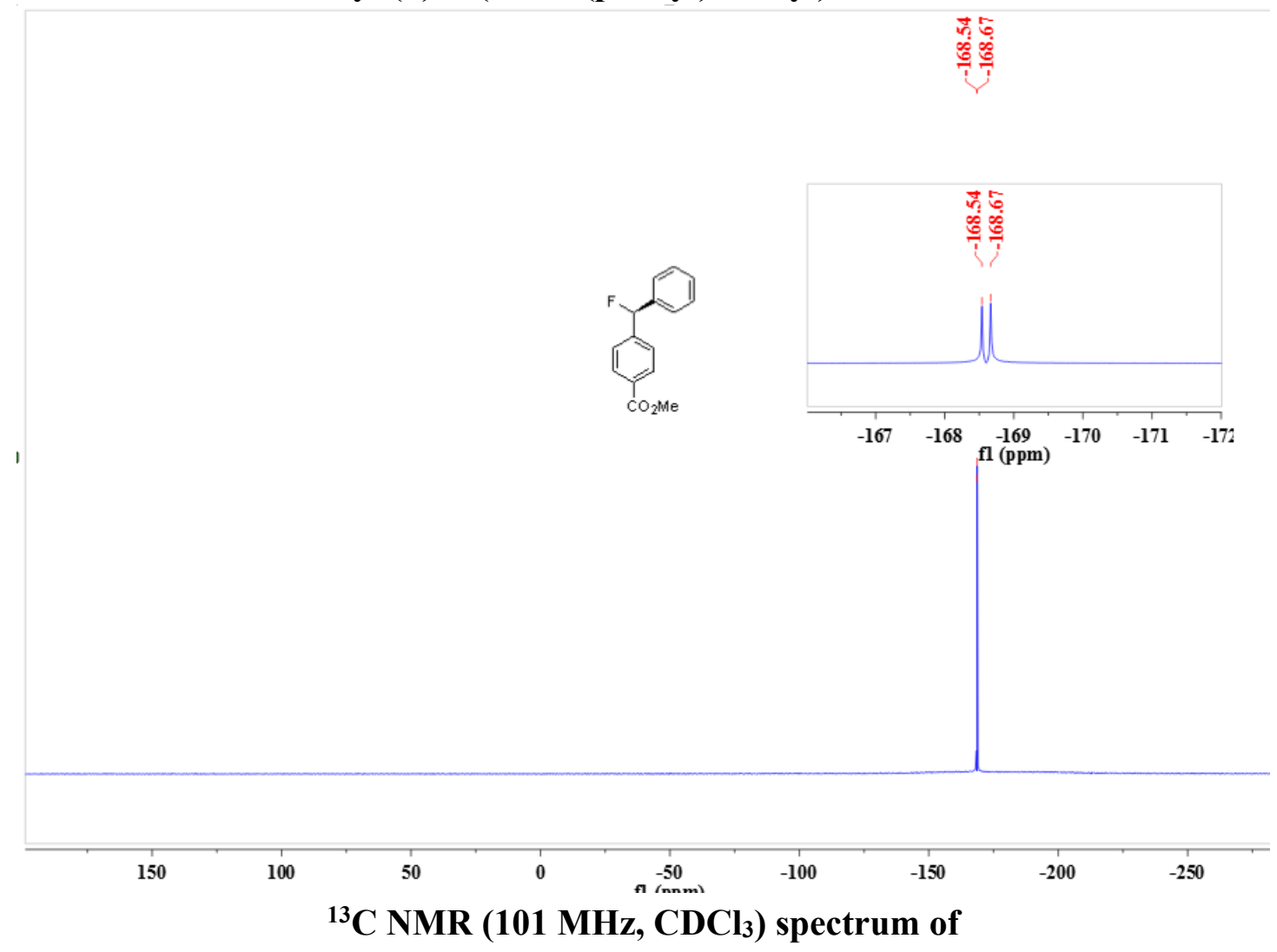


methyl (S)-4-(fluoro(phenyl)methyl)benzoate 3a

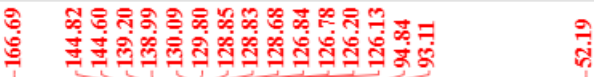

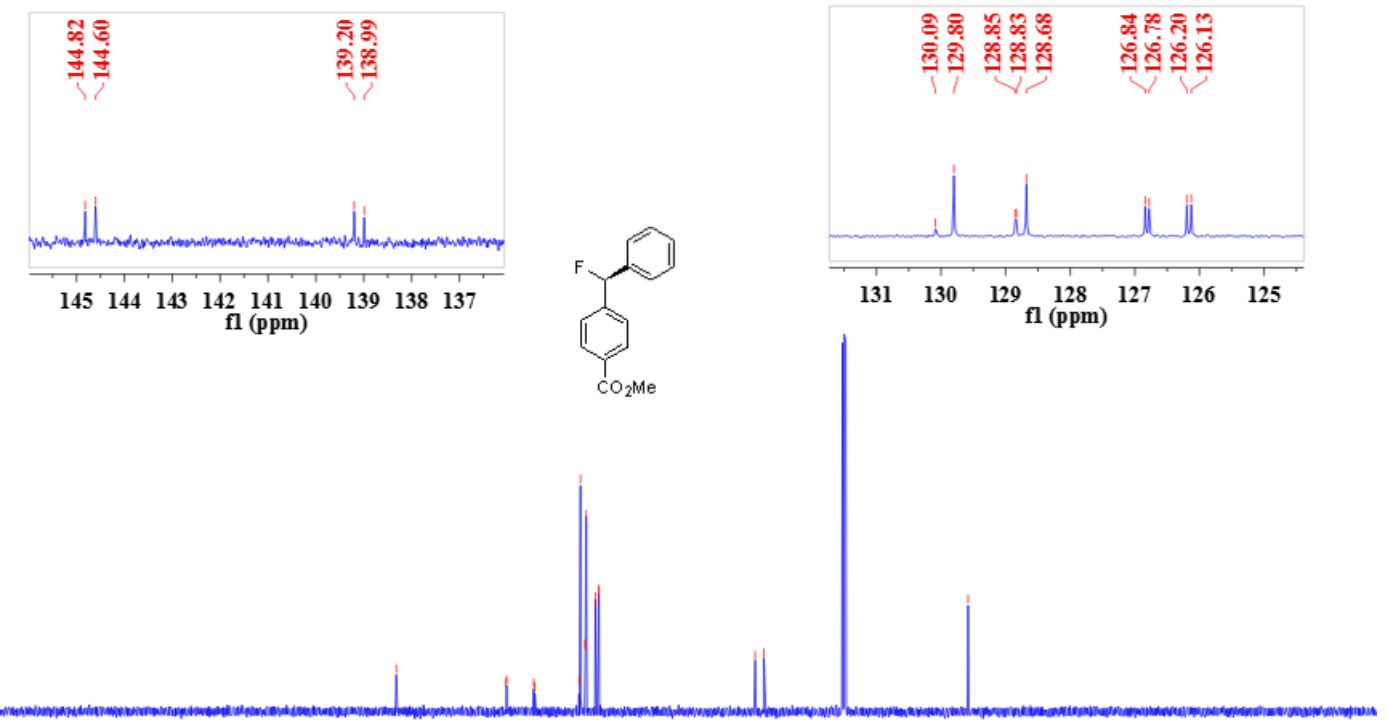

\begin{tabular}{lllllllllllllllllllllllllllllllllll}
\hline 240 & 230 & 220 & 210 & 200 & 190 & 180 & 170 & 160 & 150 & 140 & 130 & 120 & 110 & 100 & 90 & 80 & 70 & 60 & 50 & 40 & 30 & 20 & 10 & 0 & -10 & -20 & -4
\end{tabular}

${ }^{1} \mathrm{H}$ NMR (400 $\mathrm{MHz}, \mathrm{CDCl}_{3}$ ) spectrum of methyl (R)-4-(fluoro(m-tolyl)methyl)benzoate 3b

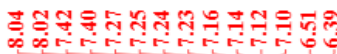
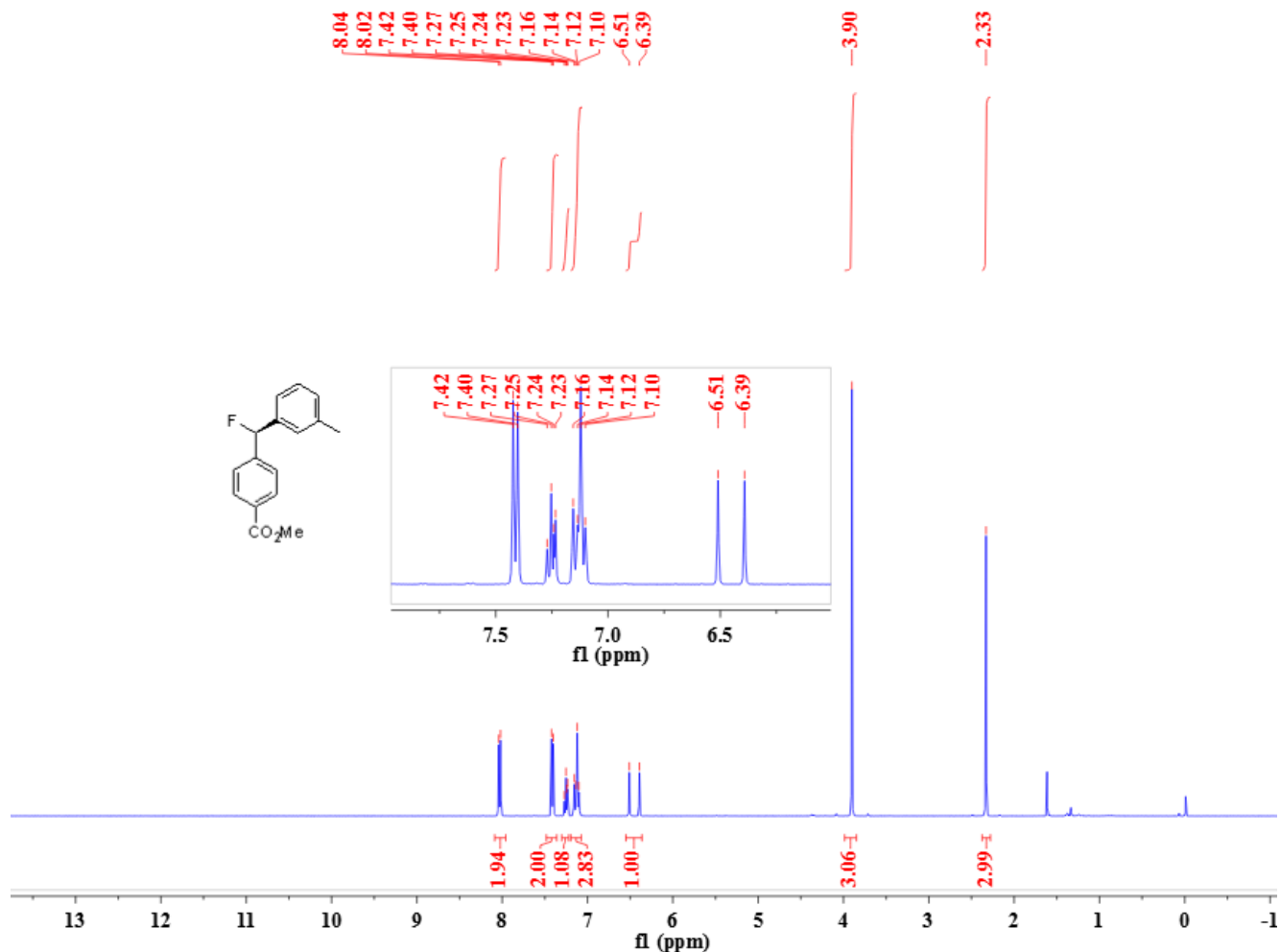
${ }^{19} \mathrm{~F}$ NMR (376 MHz, $\mathrm{CDCl}_{3}$ ) spectrum of methyl (R)-4-(fluoro(m-tolyl)methyl)benzoate $3 \mathrm{~b}$
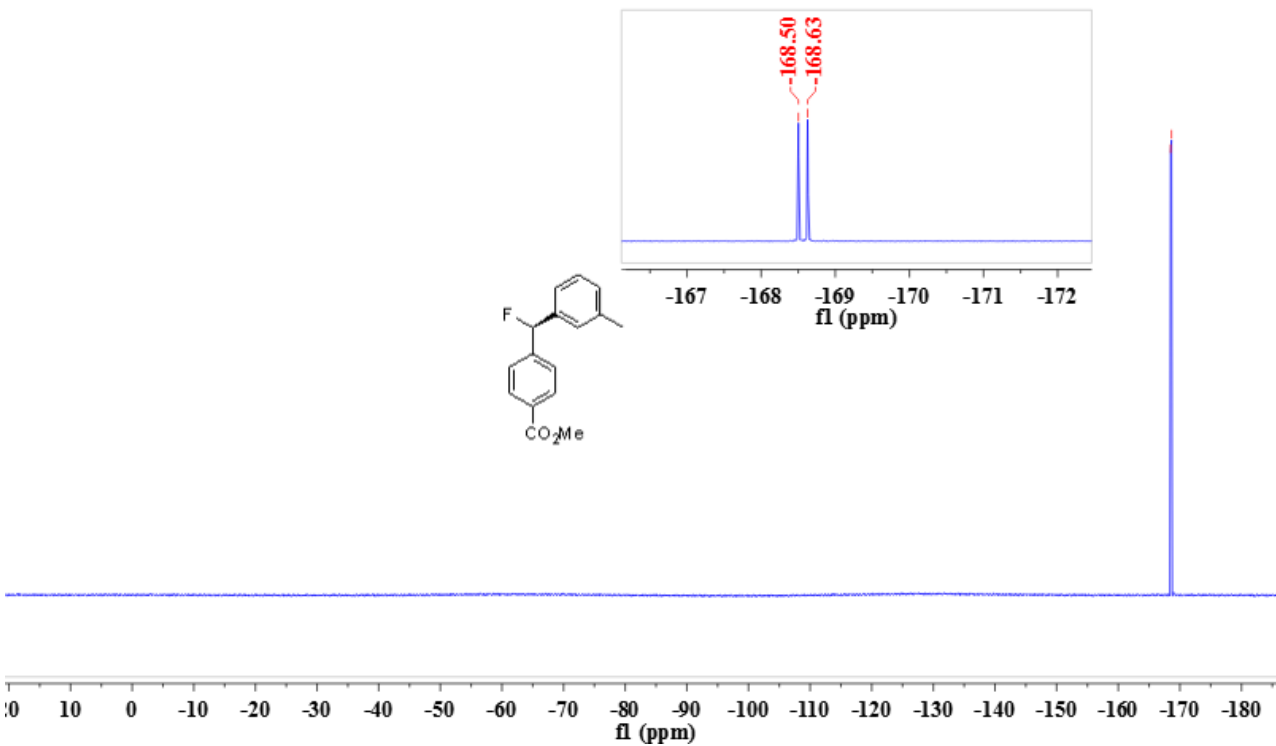

${ }^{13} \mathrm{C}$ NMR (101 MHz, $\left.\mathrm{CDCl}_{3}\right)$ spectrum of methyl (R)-4-(fluoro(m-tolyl)methyl)benzoate $3 \mathrm{~b}$

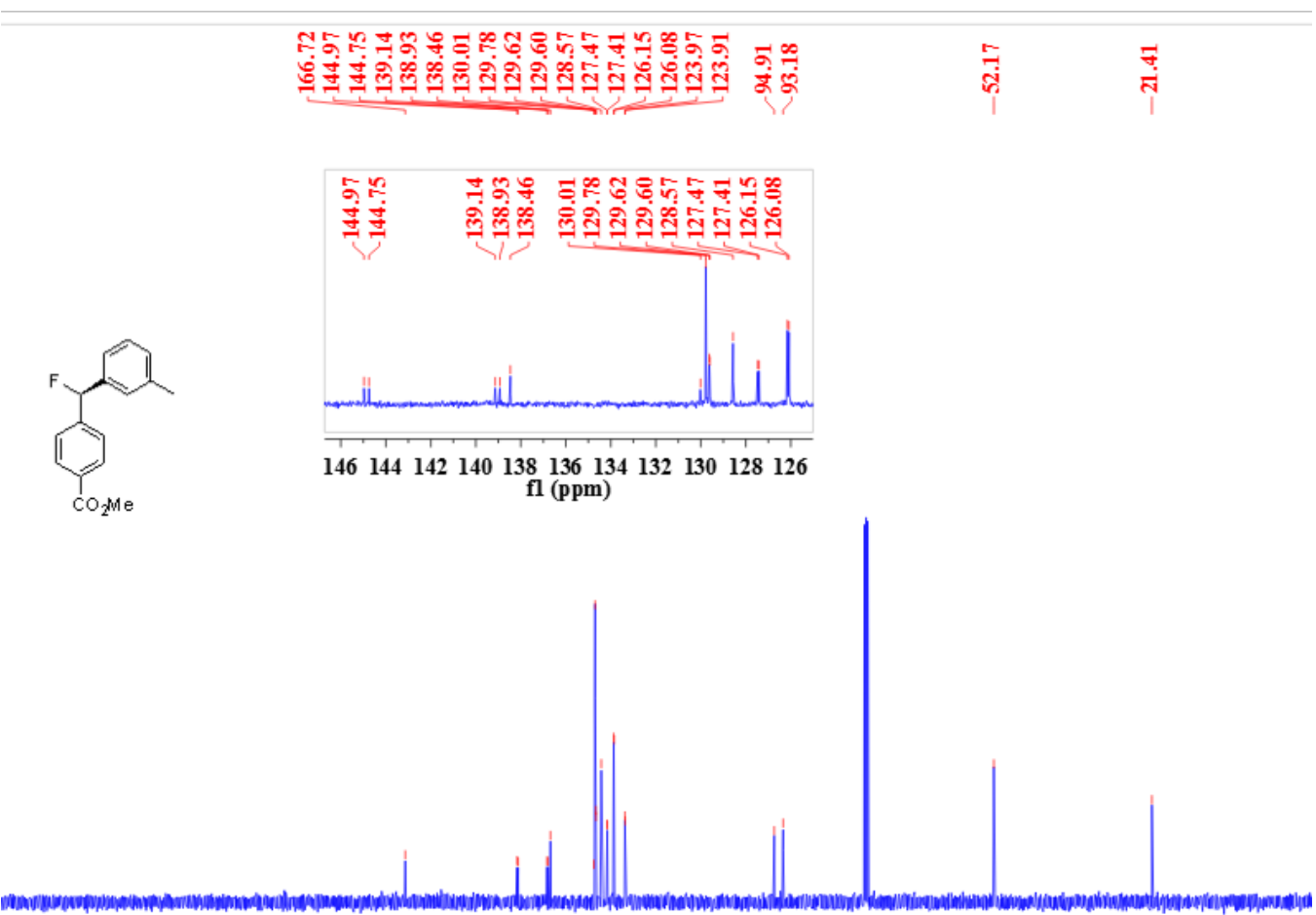

$\begin{array}{llllllllllllllllllllllllllllll}240 & 230 & 220 & 210 & 200 & 190 & 180 & 170 & 160 & 150 & 140 & 130 & 120 & 110 & 100 & 90 & 80 & 70 & 60 & 50 & 40 & 30 & 20 & 10 & 0 & -1\end{array}$ 
${ }^{1} \mathrm{H}$ NMR (400 MHz, $\mathrm{CDCl}_{3}$ ) spectrum of methyl (R)-4-(fluoro(3-morpholinophenyl)methyl)benzoate 3c
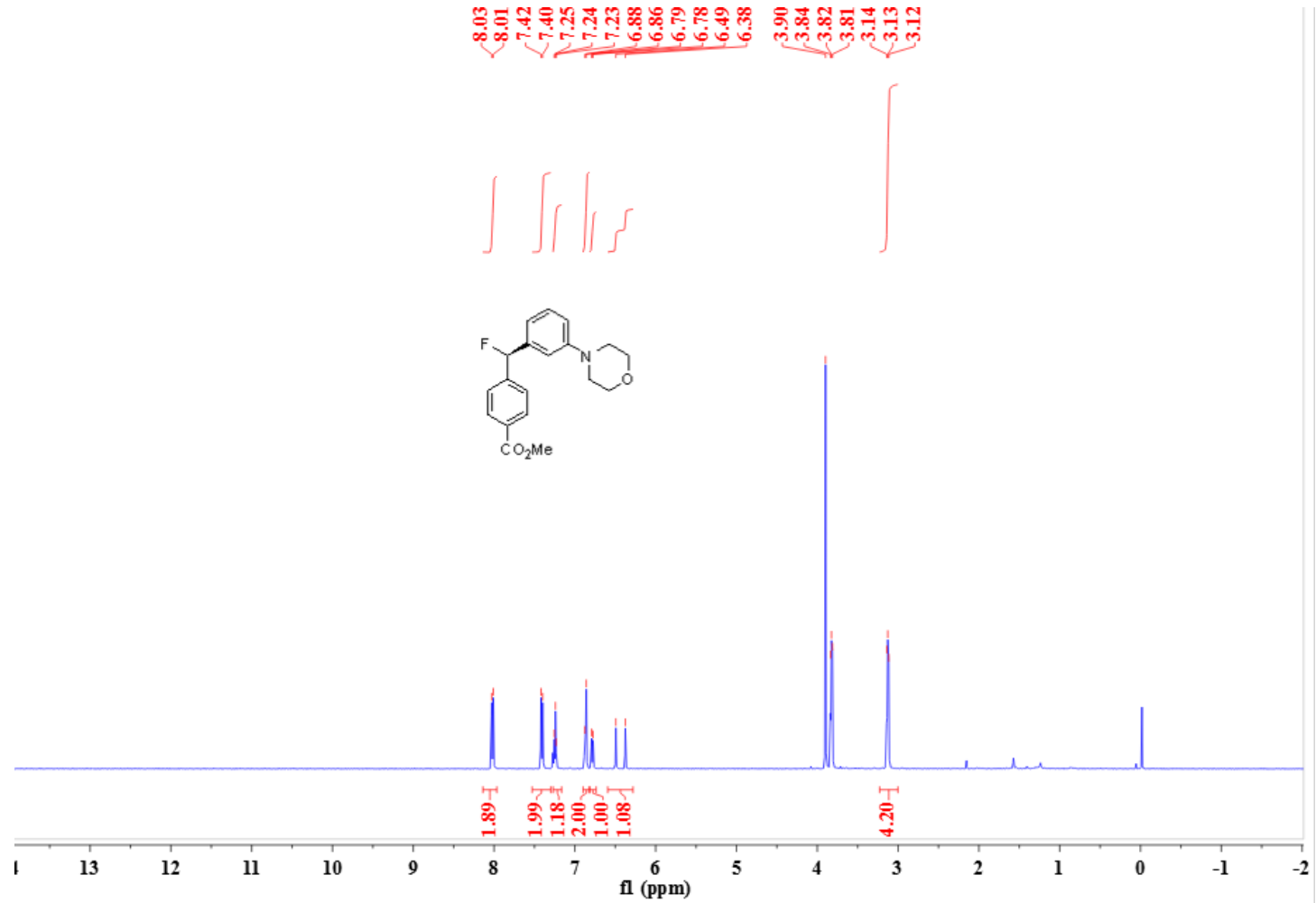

${ }^{19}$ F NMR (376 $\mathrm{MHz}, \mathrm{CDCl}_{3}$ ) spectrum of methyl (R)-4-(fluoro(3-morpholinophenyl)methyl)benzoate 3c
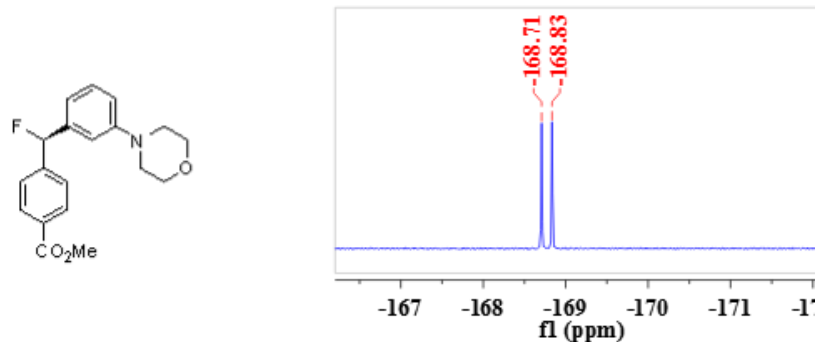
${ }^{13} \mathrm{C}$ NMR (101 MHz, $\left.\mathrm{CDCl}_{3}\right)$ spectrum of methyl (R)-4-(fluoro(3-morpholinophenyl)methyl)benzoate 3c
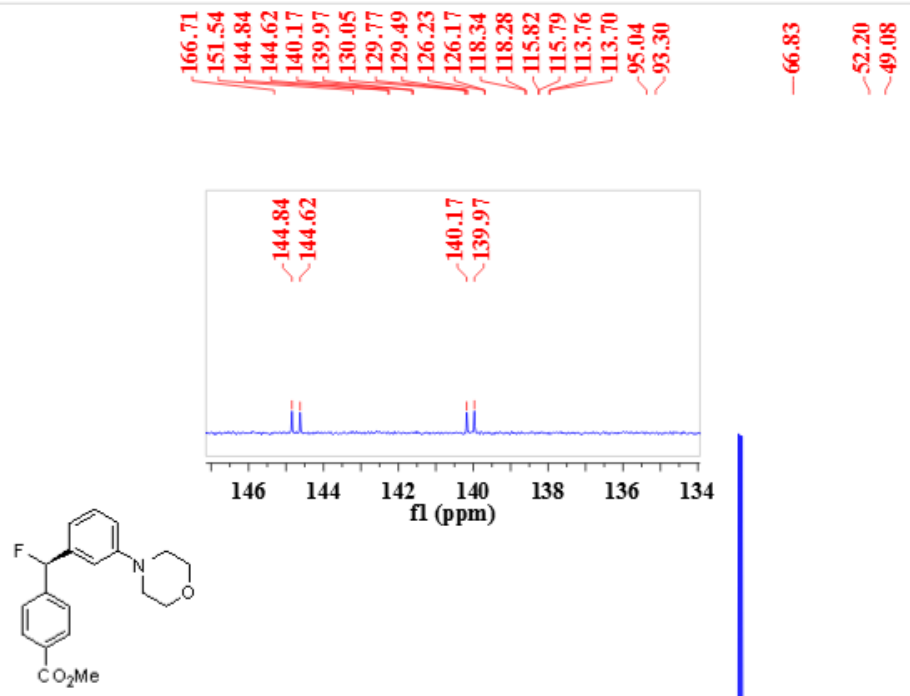

$\begin{array}{lllllllllllllllllllllllllllll}240 & 230 & 220 & 210 & 200 & 190 & 180 & 170 & 160 & 150 & 140 & 130 & 120 & 110 & 100 & 90 & 80 & 70 & 60 & 50 & 40 & 30 & 20 & 10 & 0 & -10 & -20 & -30\end{array}$

${ }^{1} \mathrm{H}$ NMR (400 $\mathrm{MHz}, \mathrm{CDCl}_{3}$ ) spectrum of methyl (R)-4-(fluoro(3-methoxyphenyl)methyl)benzoate 3d

מ.
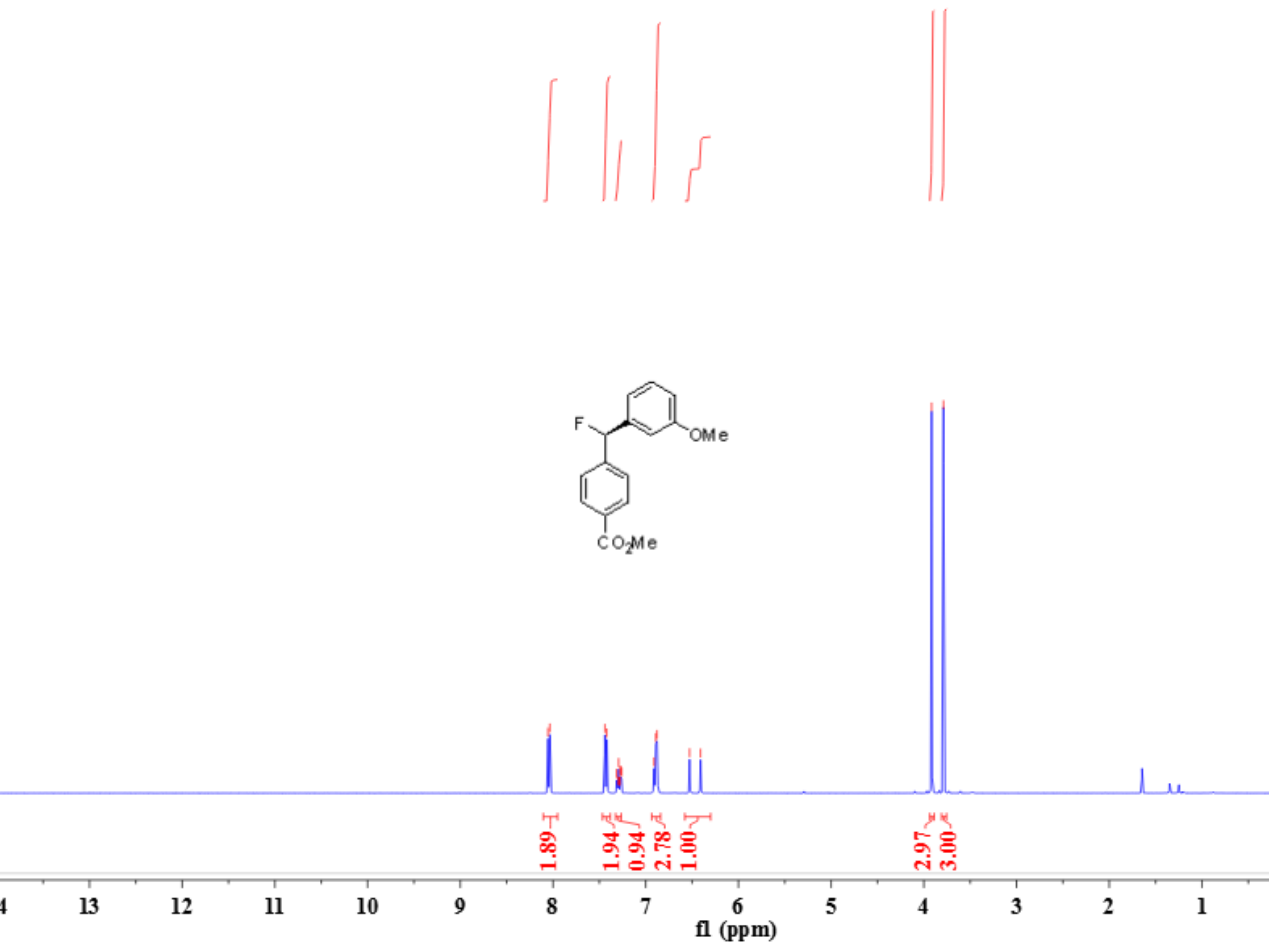
${ }^{19}$ F NMR (376 MHz, $\mathrm{CDCl}_{3}$ ) spectrum of methyl (R)-4-(fluoro(3-methoxyphenyl)methyl)benzoate 3d

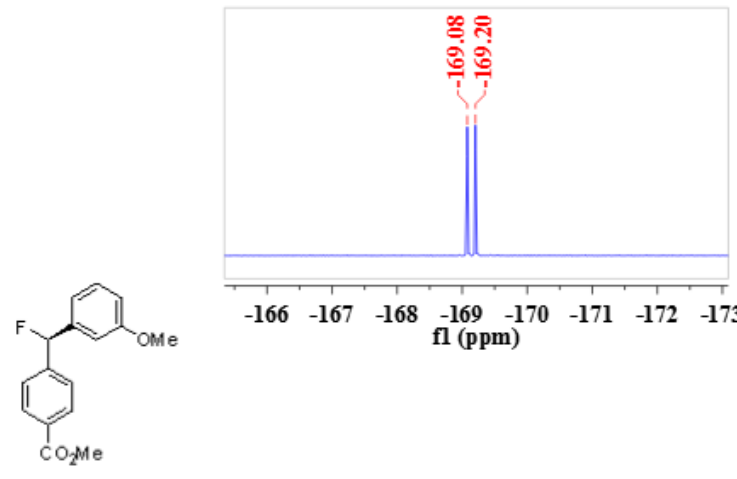

$\begin{array}{llllllllllllllllllllll}0 & 20 & 10 & 0 & -10 & -20 & -30 & -40 & -50 & -60 & -70 & -80 & -90 & -100 & -110 & -120 & -130 & -140 & -150 & -160 & -170 & -180\end{array}$ fl (ppm)

${ }^{13} \mathrm{C}$ NMR (101 MHz, $\left.\mathrm{CDCl}_{3}\right)$ spectrum of methyl (R)-4-(fluoro(3-methoxyphenyl)methyl)benzoate 3d

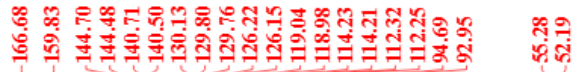
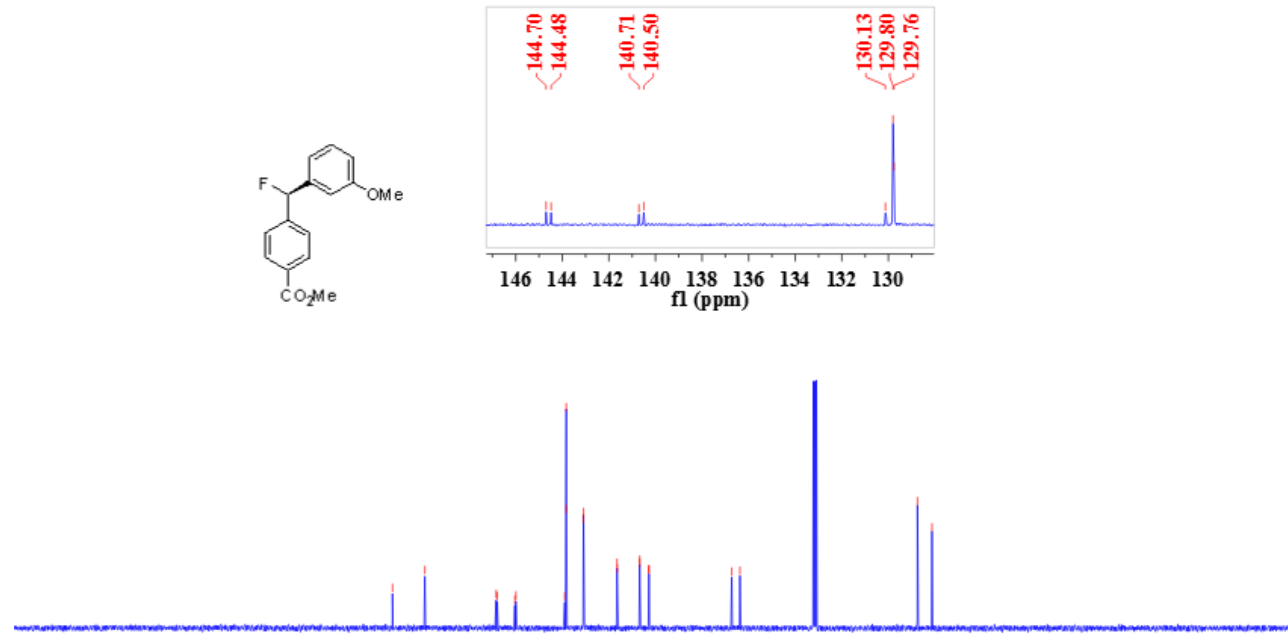

$\begin{array}{lllllllllllllllllllllllllllllll}240 & 230 & 220 & 210 & 200 & 190 & 180 & 170 & 160 & 150 & 140 & 130 & 120 & 110 & 100 & 90 & 80 & 70 & 60 & 50 & 40 & 30 & 20 & 10 & 0 & -10 & -20\end{array}$ 
${ }^{1} \mathrm{H}$ NMR (400 MHz, $\mathrm{CDCl}_{3}$ ) spectrum of methyl (R)-4-(fluoro(3-fluorophenyl)methyl)benzoate 3e

bon

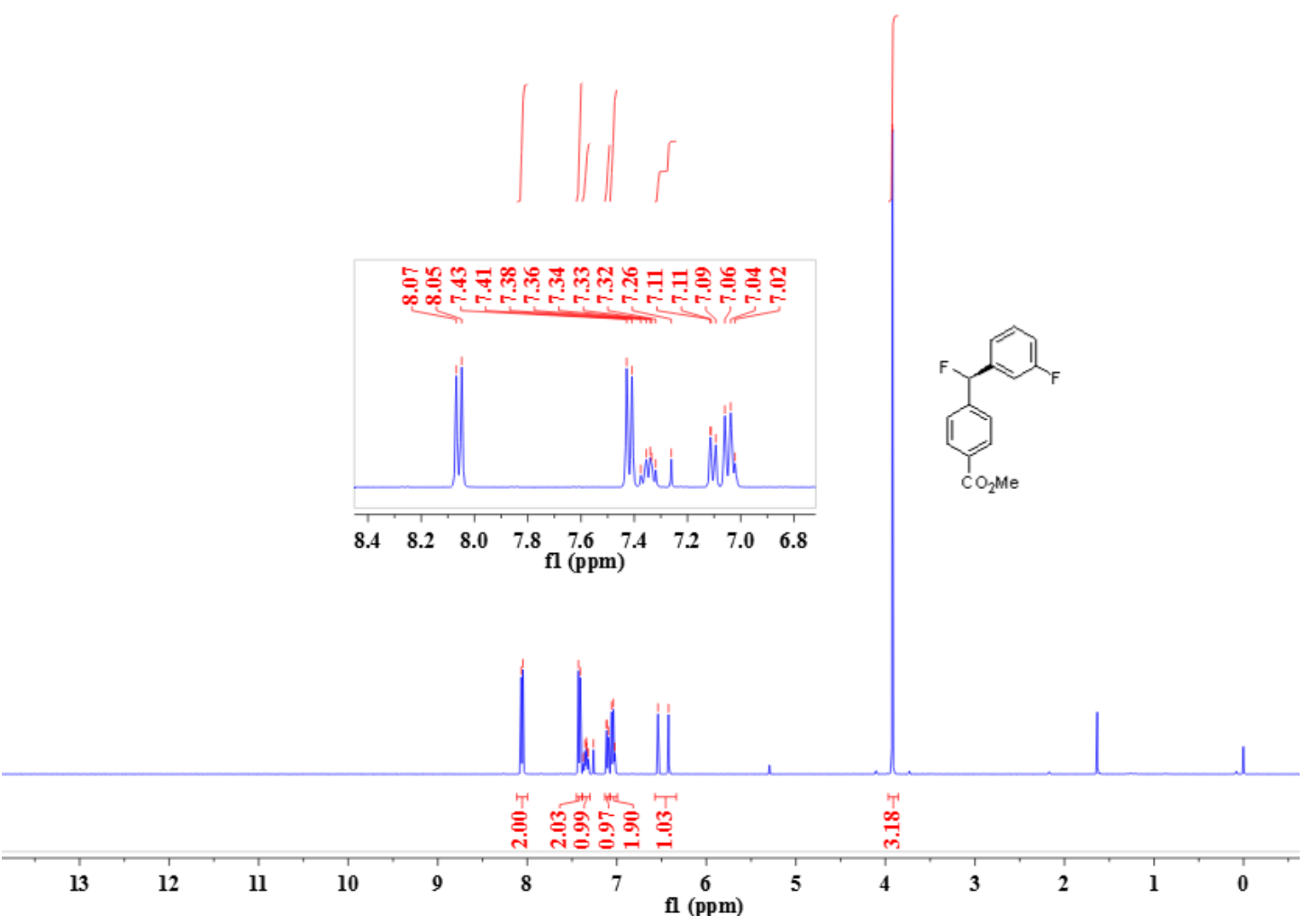

${ }^{19} \mathrm{~F}$ NMR (376 $\left.\mathrm{MHz}, \mathrm{CDCl}_{3}\right)$ spectrum of methyl (R)-4-(fluoro(3-fluorophenyl)methyl)benzoate 3e

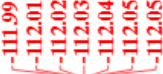
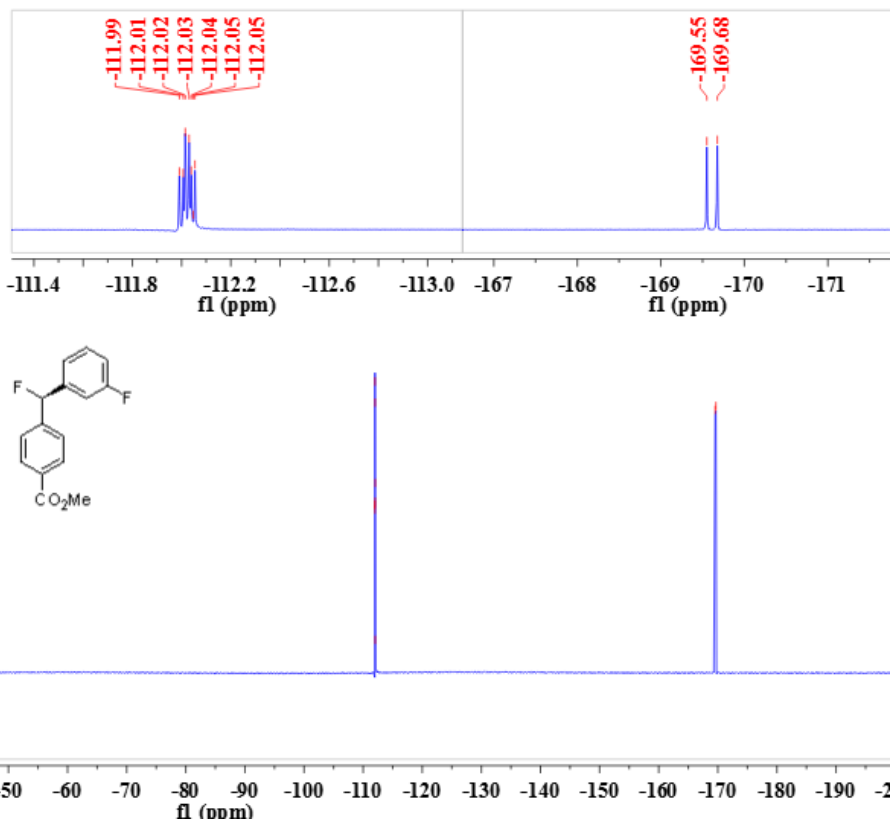
${ }^{13} \mathrm{C}$ NMR (101 MHz, $\left.\mathrm{CDCl}_{3}\right)$ spectrum of methyl (R)-4-(fluoro(3-fluorophenyl)methyl)benzoate 3e

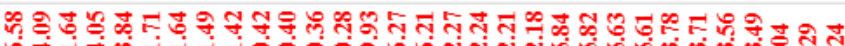

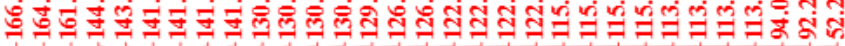
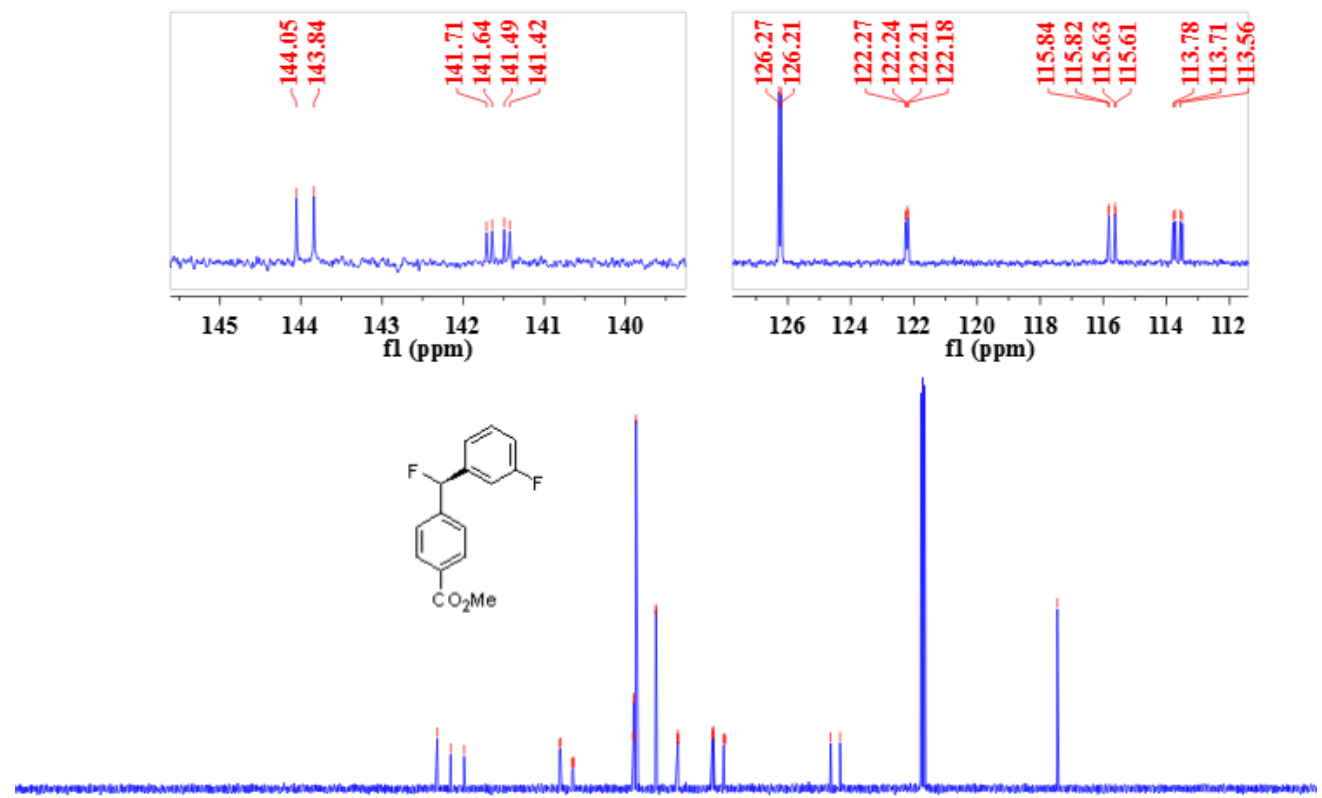

$\begin{array}{llllllllllllllllllllllll}240 & 230 & 220 & 210 & 200 & 190 & 180 & 170 & 160 & 150 & 140 & 130 & 120 & 110 & 100 & 90 & 80 & 70 & 60 & 50 & 40 & 30 & 20 & 10\end{array}$

${ }^{1} \mathrm{H}$ NMR (400 $\mathrm{MHz}, \mathrm{CDCl}_{3}$ ) spectrum of methyl (R)-4-(fluoro(4-fluorophenyl)methyl)benzoate $3 f$

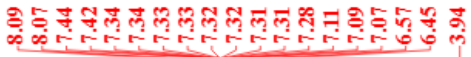

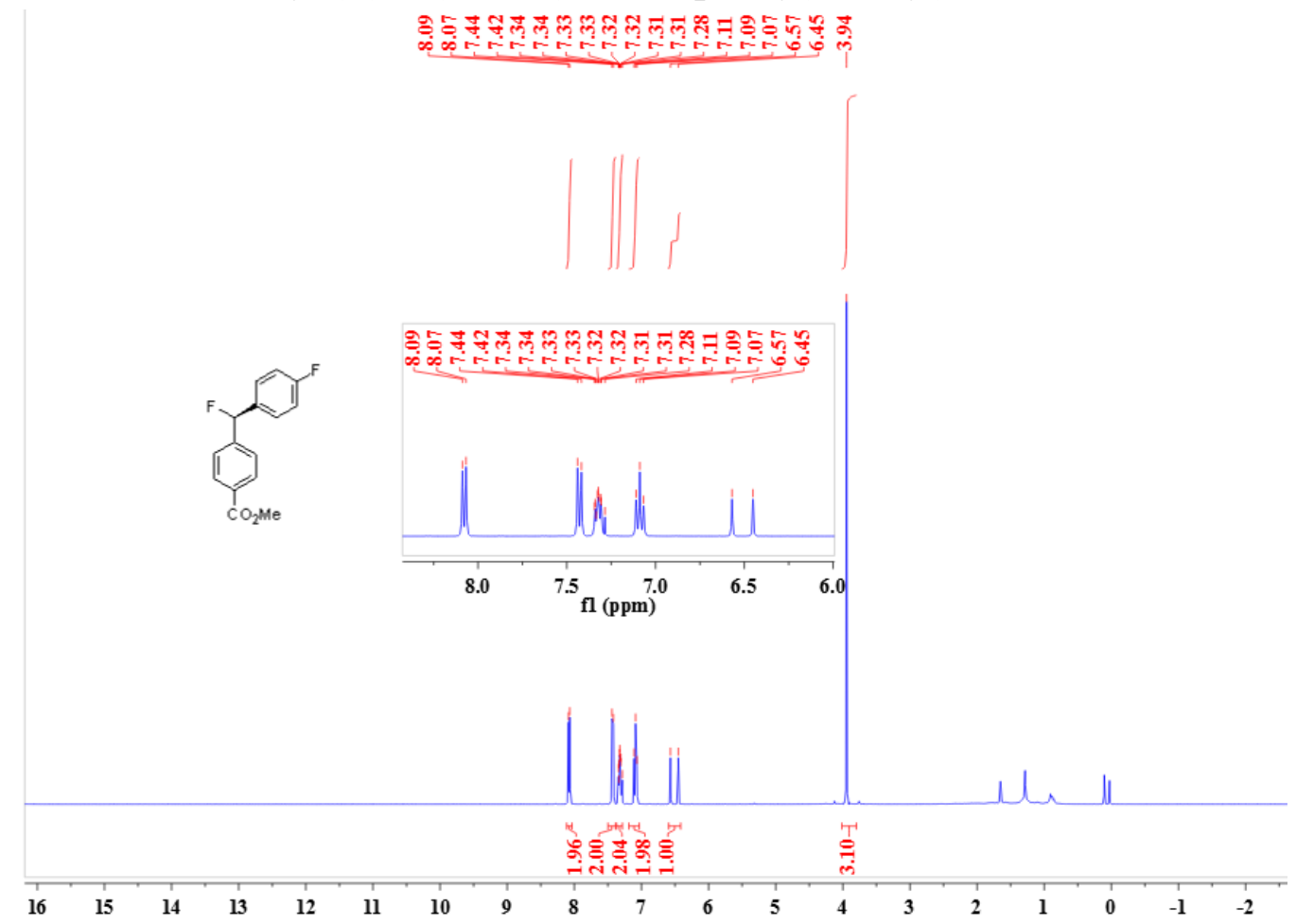


${ }^{19}$ F NMR (376 MHz, $\mathrm{CDCl}_{3}$ ) spectrum of methyl (R)-4-(fluoro(4-fluorophenyl)methyl)benzoate $3 f$
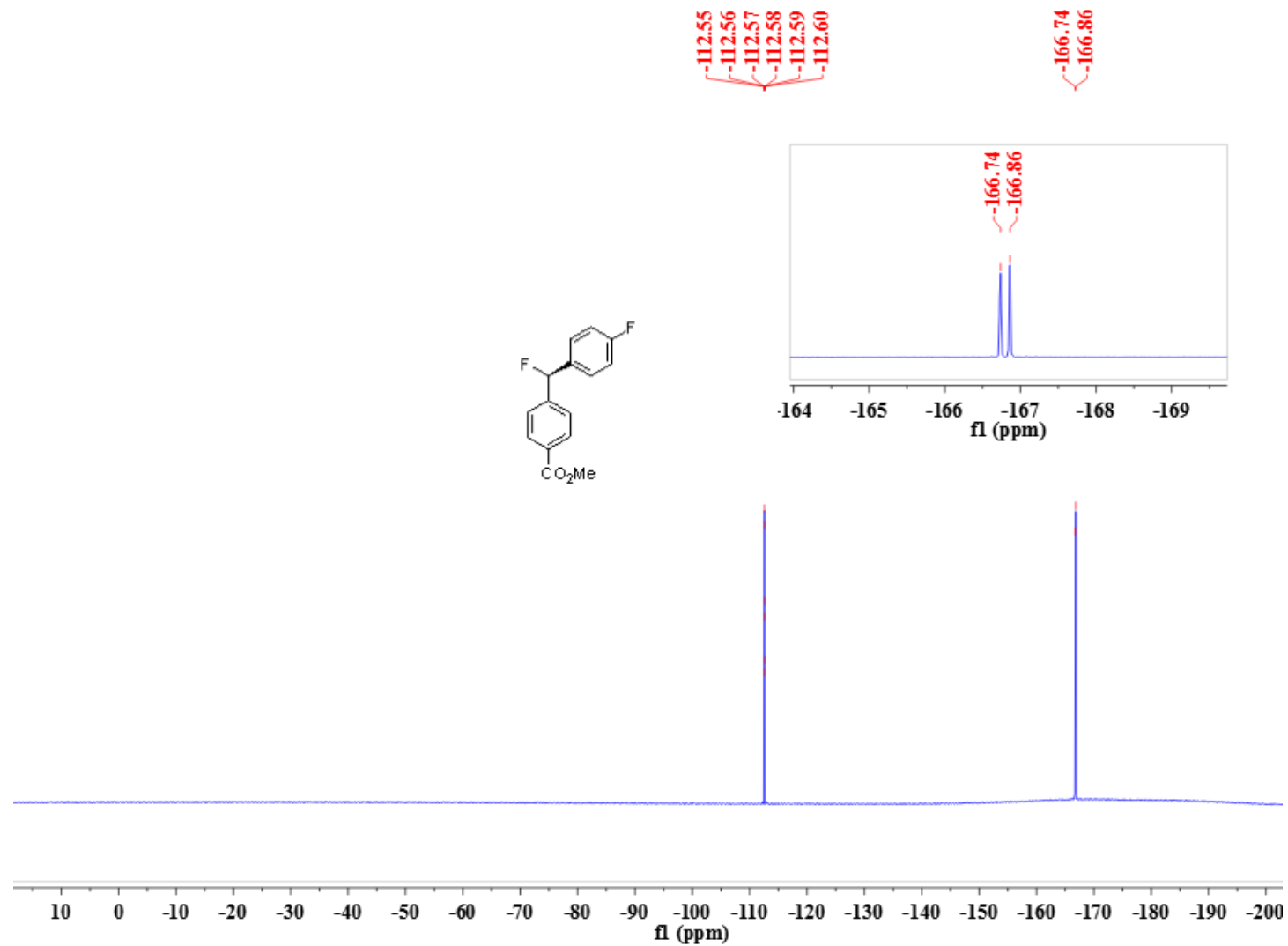

${ }^{13} \mathrm{C}$ NMR (101 MHz, $\left.\mathrm{CDCl}_{3}\right)$ spectrum of methyl (R)-4-(fluoro(4-fluorophenyl)methyl)benzoate 3f

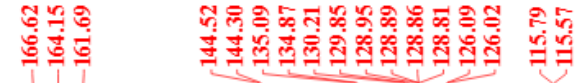

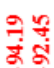

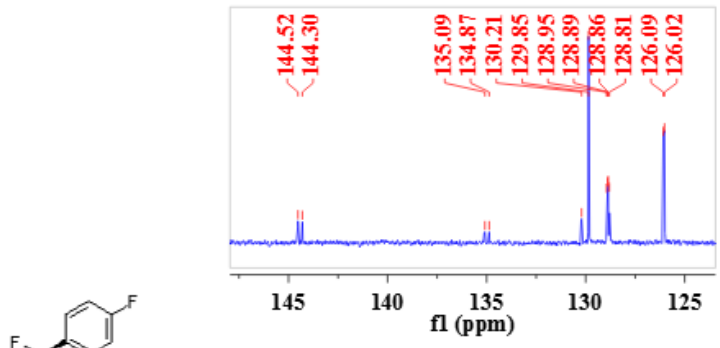

$\begin{array}{llllllllllllllllllllllllllllll}225 & 220 & 215 & 210 & 205 & 200 & 195 & 190 & 185 & 180 & 175 & 170 & 165 & 160 & 155 & 150 & 145 & 140 & 135 & 130 & 125 & 120 & 115 & 110 & 105 & 100 & 95 & 90 & \text { fl }\end{array}$ 
${ }^{1} \mathrm{H}$ NMR (400 MHz, $\mathrm{CDCl}_{3}$ ) spectrum of methyl (R)-4-(fluoro(3-fluoro-4-methylphenyl)methyl)benzoate 3g

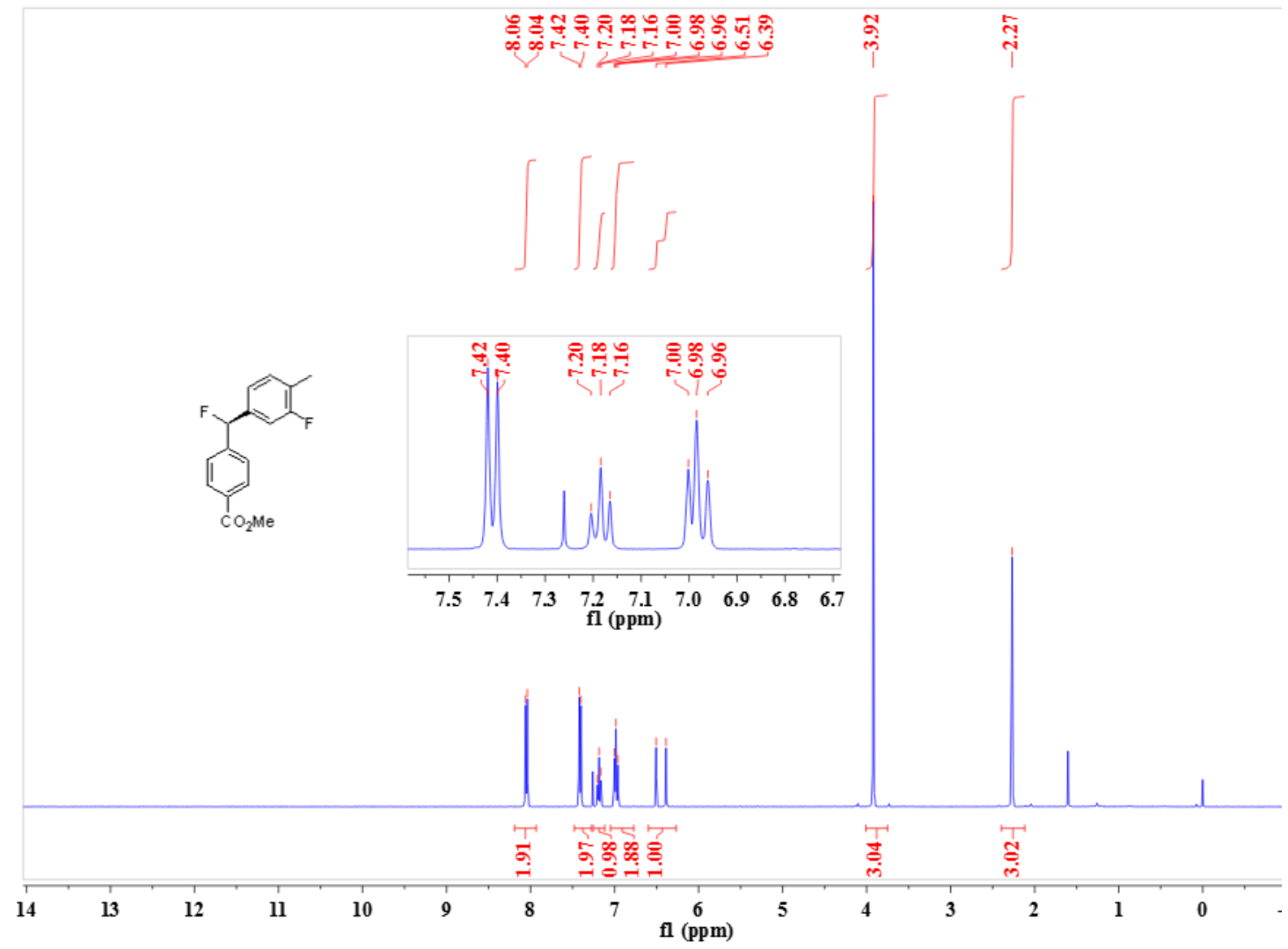

${ }^{19}$ F NMR (376 $\mathrm{MHz}, \mathrm{CDCl}_{3}$ ) spectrum of methyl (R)-4-(fluoro(3-fluoro-4-methylphenyl)methyl)benzoate 3g

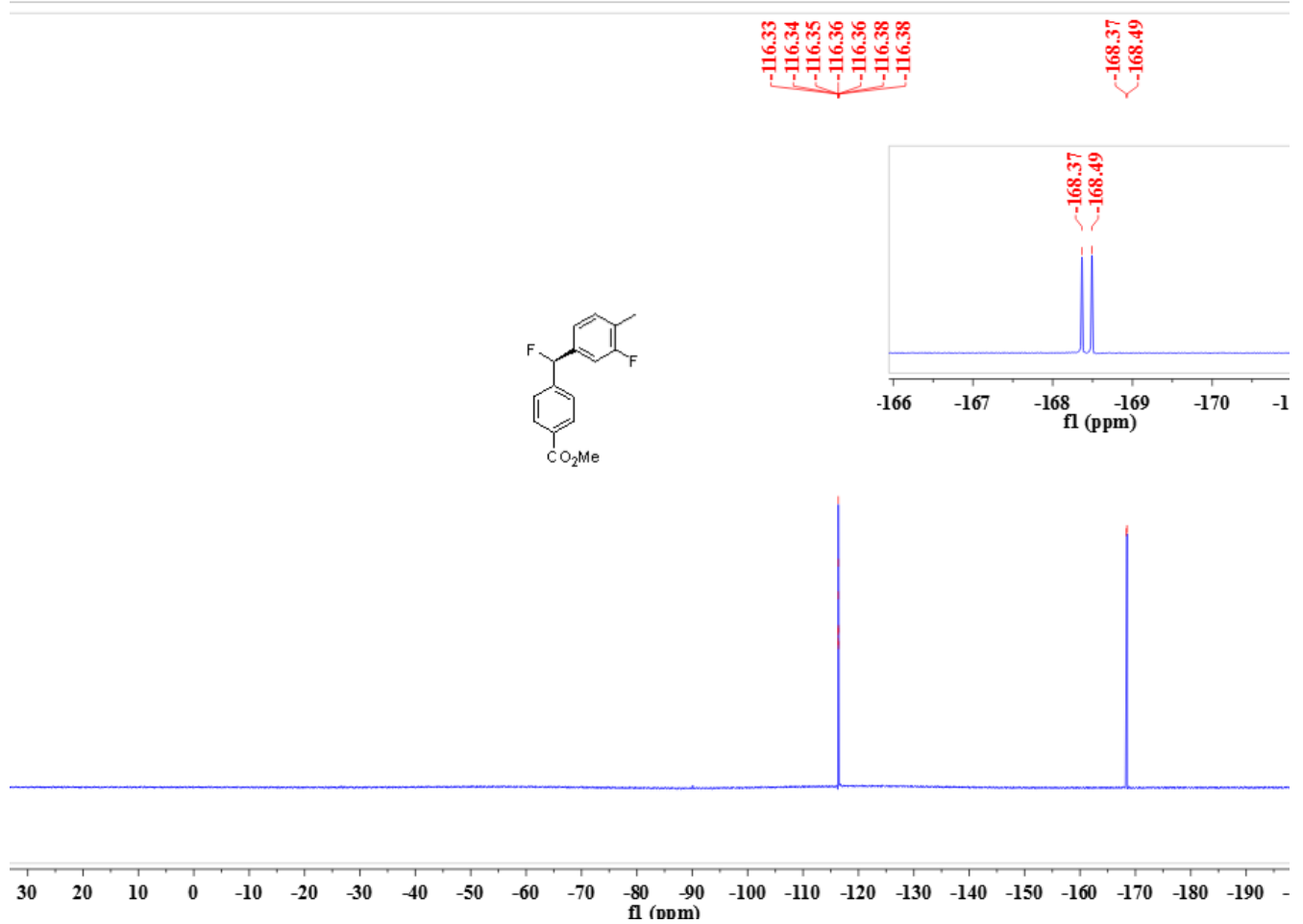


${ }^{13} \mathrm{C}$ NMR (101 MHz, $\left.\mathrm{CDCl}_{3}\right)$ spectrum of methyl (R)-4-(fluoro(3-fluoro-4-methylphenyl)methyl)benzoate 3g

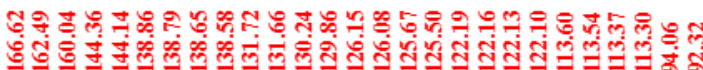
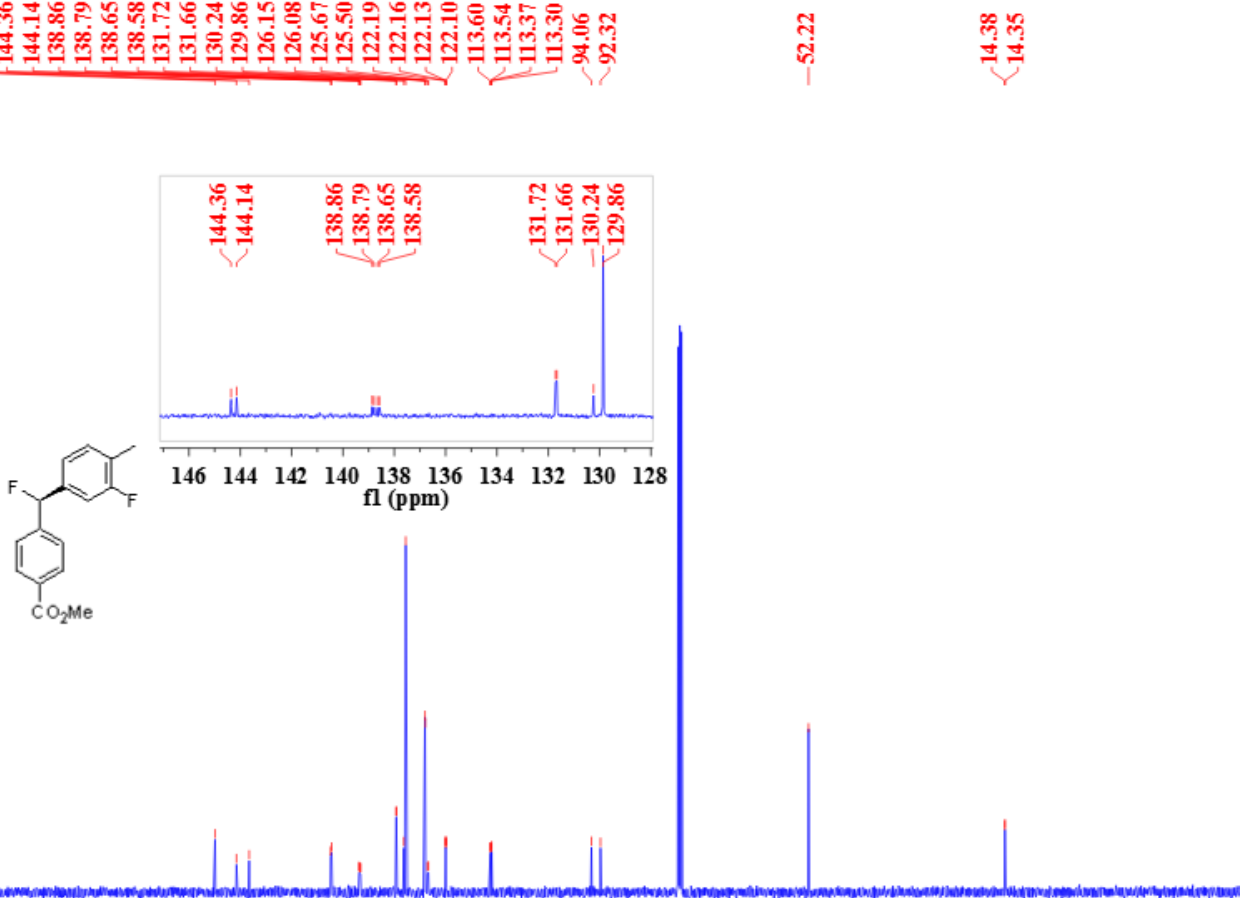

$\begin{array}{llllllllllllllllllllllllllll}240 & 230 & 220 & 210 & 200 & 190 & 180 & 170 & 160 & 150 & 140 & 130 & 120 & 110 & 100 & 90 & 80 & 70 & 60 & 50 & 40 & 30 & 20 & 10 & 0 & -10 & -20 & -3\end{array}$

${ }^{1} \mathrm{H}$ NMR (400 MHz, $\left.\mathrm{CDCl}_{3}\right)$ spectrum of methyl (S)-3-(fluoro(phenyl)methyl)benzoate $3 \mathrm{~h}$ 象 के<smiles>CC(=O)c1cccc(C(F)c2ccccc2)c1</smiles>

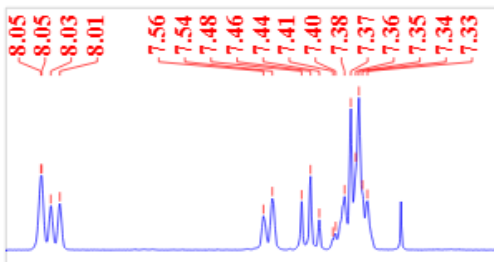

$\begin{array}{llllllllllll}8.1 & 8.0 & 7.9 & 7.8 & 7.7 & 7.6 & 7.5 & 7.4 & 7.3 & 7.2 & 7.1\end{array}$

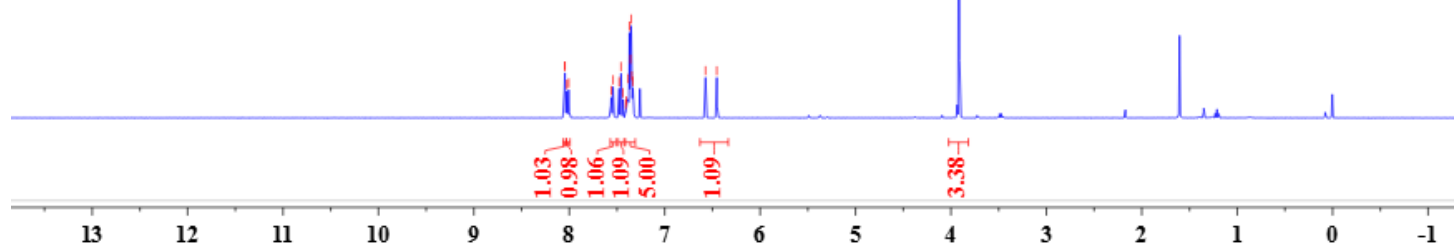


${ }^{19}$ F NMR (376 MHz, $\left.\mathrm{CDCl}_{3}\right)$ spectrum of methyl (S)-3-(fluoro(phenyl)methyl)benzoate $3 \mathrm{~h}$
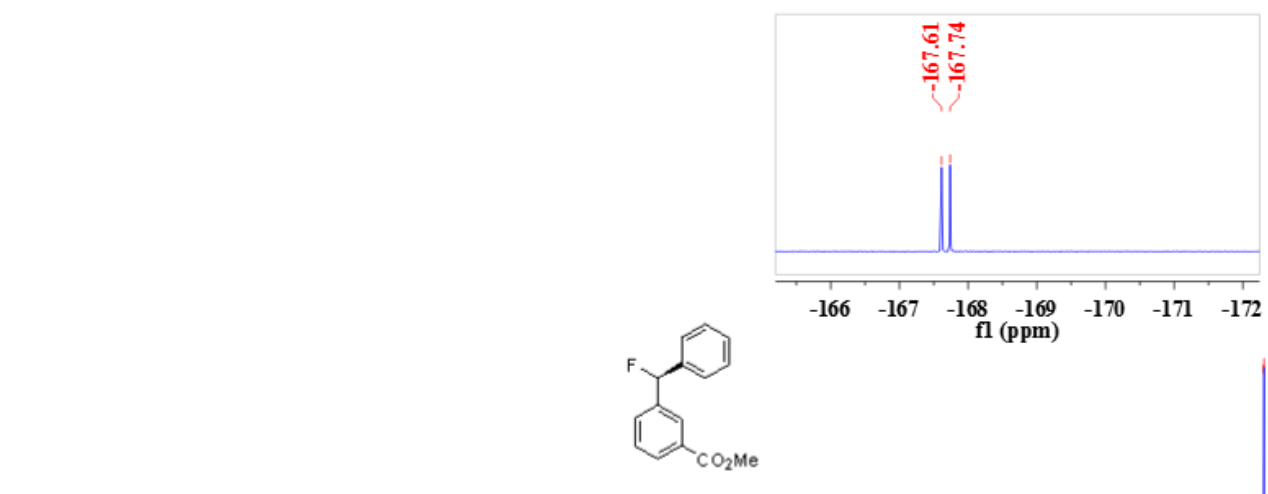

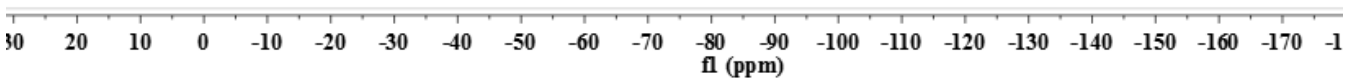

${ }^{13} \mathrm{C}$ NMR (101 MHz, $\left.\mathrm{CDCl}_{3}\right)$ spectrum of methyl (S)-3-(fluoro(phenyl)methyl)benzoate $3 \mathrm{~h}$

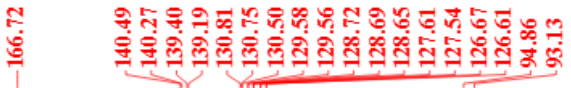
สุ่
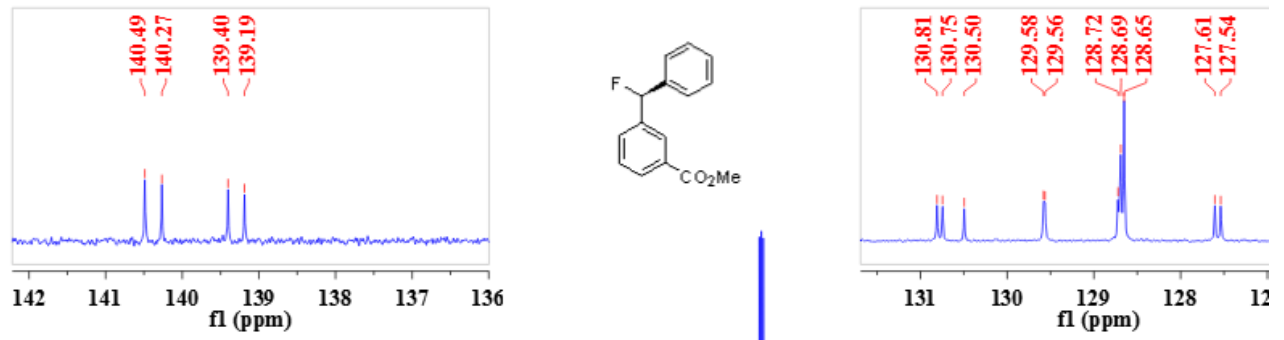

$\begin{array}{lllllllllllllllllllll}210 & 200 & 190 & 180 & 170 & 160 & 150 & 140 & 130 & 120 & 110 & 100 & 90 & 80 & 70 & 60 & 50 & 40 & 30 & 20 & 10\end{array}$ 
${ }^{1} \mathrm{H}$ NMR (400 $\mathrm{MHz}, \mathrm{CDCl}_{3}$ ) spectrum of methyl (R)-3-(fluoro(3-fluorophenyl)methyl)benzoate 3i

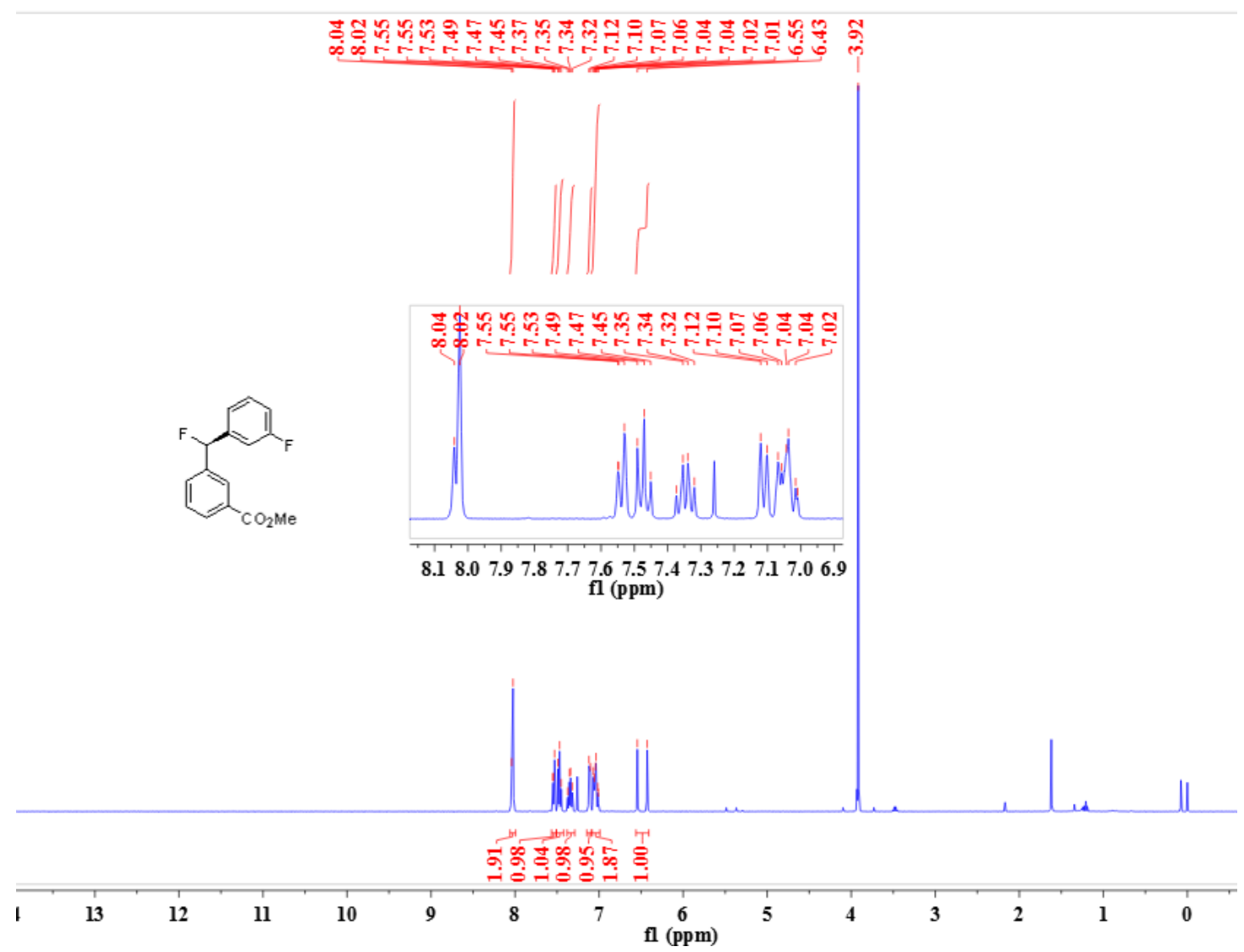

${ }^{19}$ F NMR (376 $\mathrm{MHz}, \mathrm{CDCl}_{3}$ ) spectrum of methyl (R)-3-(fluoro(3-fluorophenyl)methyl)benzoate 3i
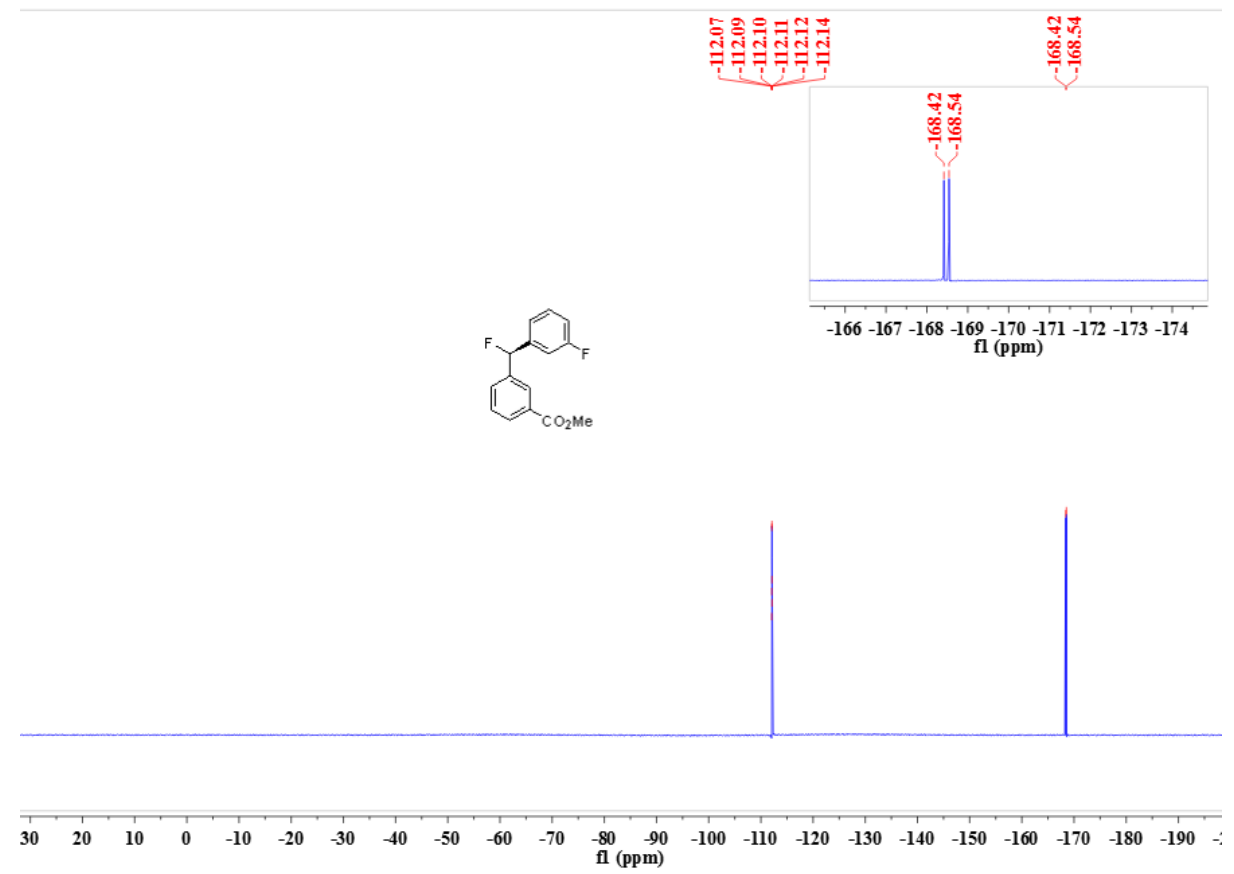
${ }^{13} \mathrm{C}$ NMR (101 $\left.\mathrm{MHz}, \mathrm{CDCl}_{3}\right)$ spectrum of methyl (R)-3-(fluoro(3-fluorophenyl)methyl)benzoate 3i की
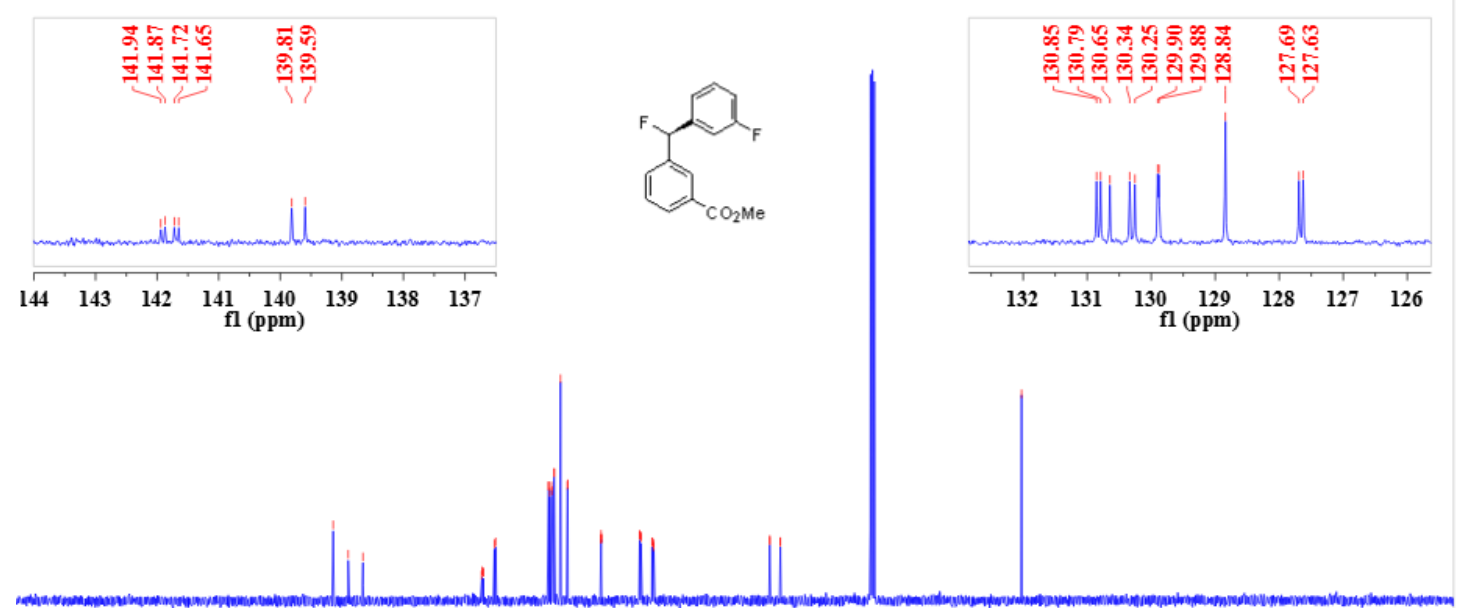

$\begin{array}{lllllllllllllllllllllll}210 & 200 & 190 & 180 & 170 & 160 & 150 & 140 & 130 & 120 & 110 & 100 & 90 & 80 & 70 & 60 & 50 & 40 & 30 & 20 & 10 & 0 & -10\end{array}$

${ }^{1} \mathrm{H}$ NMR (400 $\mathrm{MHz}, \mathrm{CDCl}_{3}$ ) spectrum of ethyl (S)-4-(fluoro(phenyl)methyl)benzoate $3 \mathbf{j}$

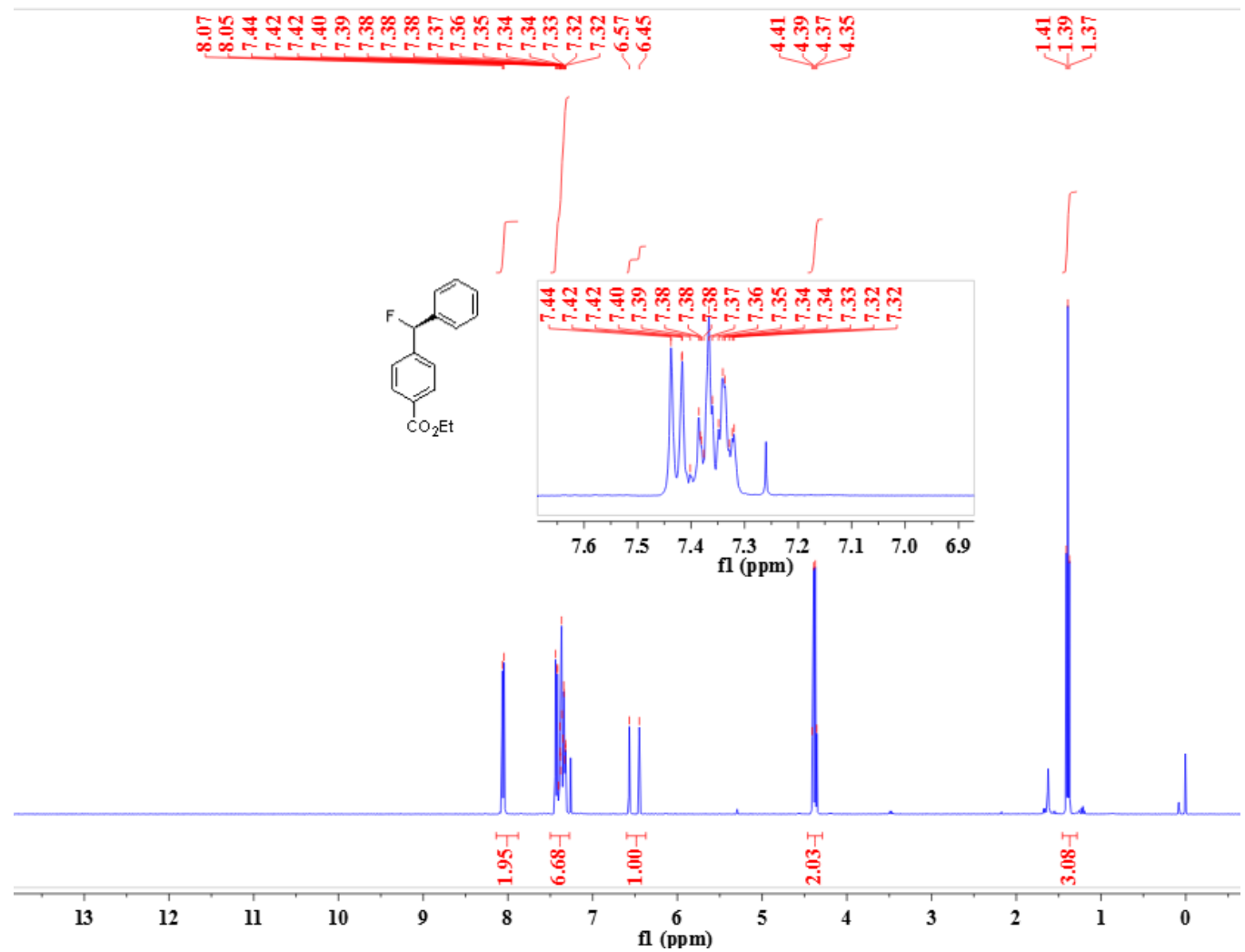


${ }^{19}$ F NMR (376 MHz, $\left.\mathrm{CDCl}_{3}\right)$ spectrum of ethyl (S)-4-(fluoro(phenyl)methyl)benzoate 3j

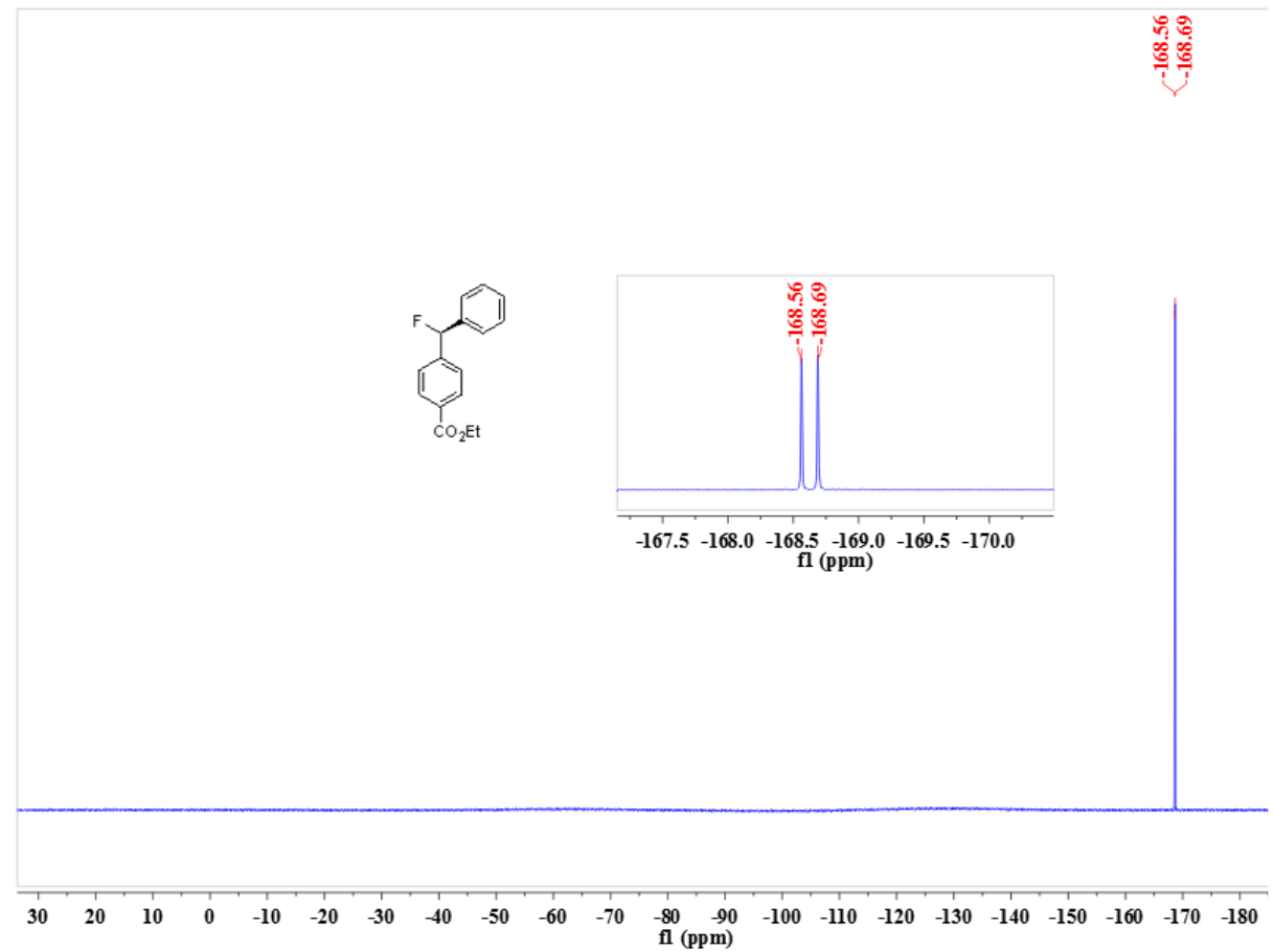

${ }^{13} \mathrm{C}$ NMR (101 MHz, $\left.\mathrm{CDCl}_{3}\right)$ spectrum of ethyl (S)-4-(fluoro(phenyl)methyl)benzoate $3 \mathbf{j}$
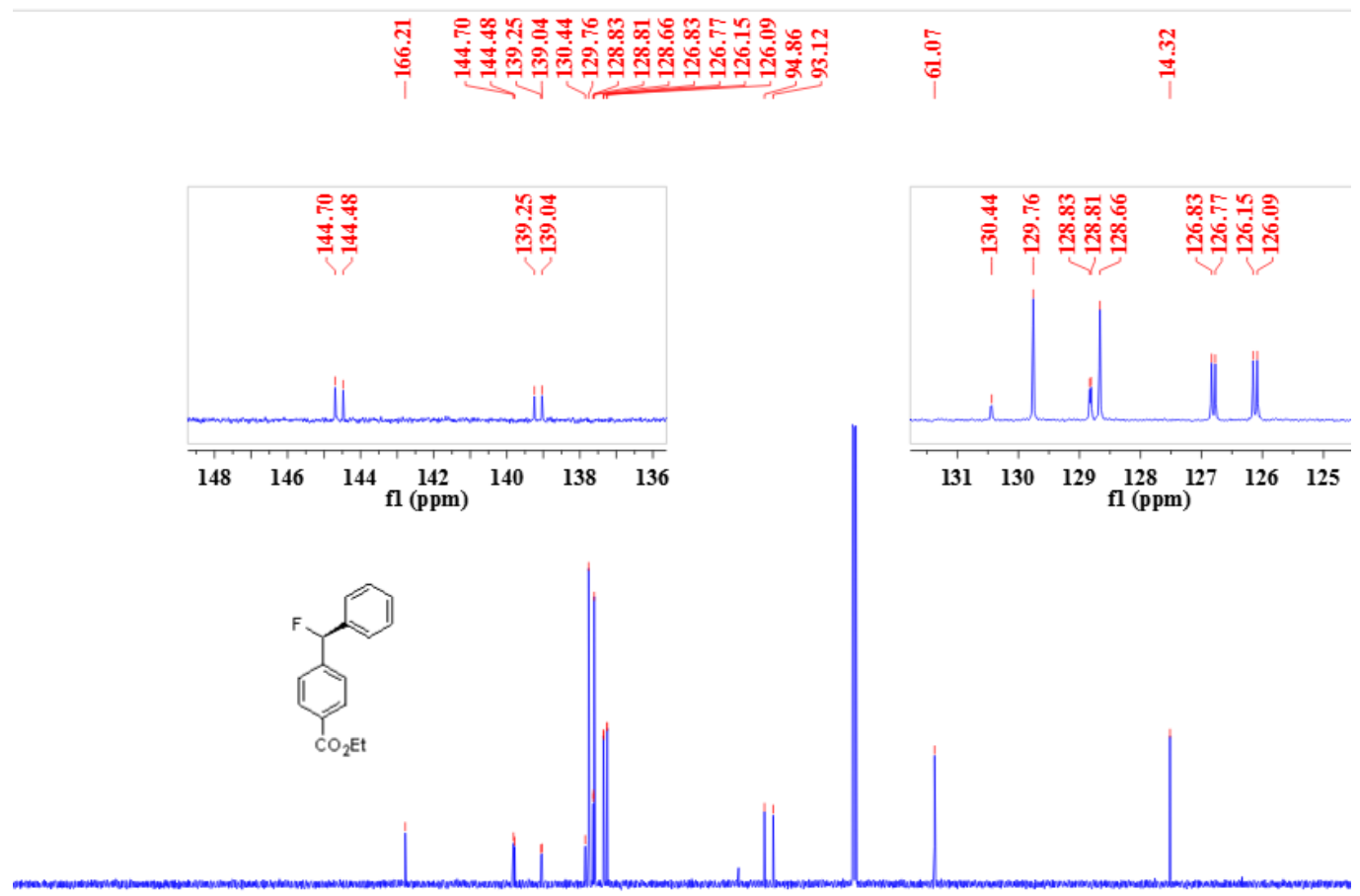

$\begin{array}{llllllllllllllllllllllllllll}240 & 230 & 220 & 210 & 200 & 190 & 180 & 170 & 160 & 150 & 140 & 130 & 120 & 110 & 100 & 90 & 80 & 70 & 60 & 50 & 40 & 30 & 20 & 10 & 0 & -10 & -21\end{array}$ 
${ }^{1} \mathrm{H}$ NMR (400 $\mathrm{MHz}, \mathrm{CDCl}_{3}$ ) spectrum of (S)-3-(fluoro(phenyl)methyl)benzonitrile 3k

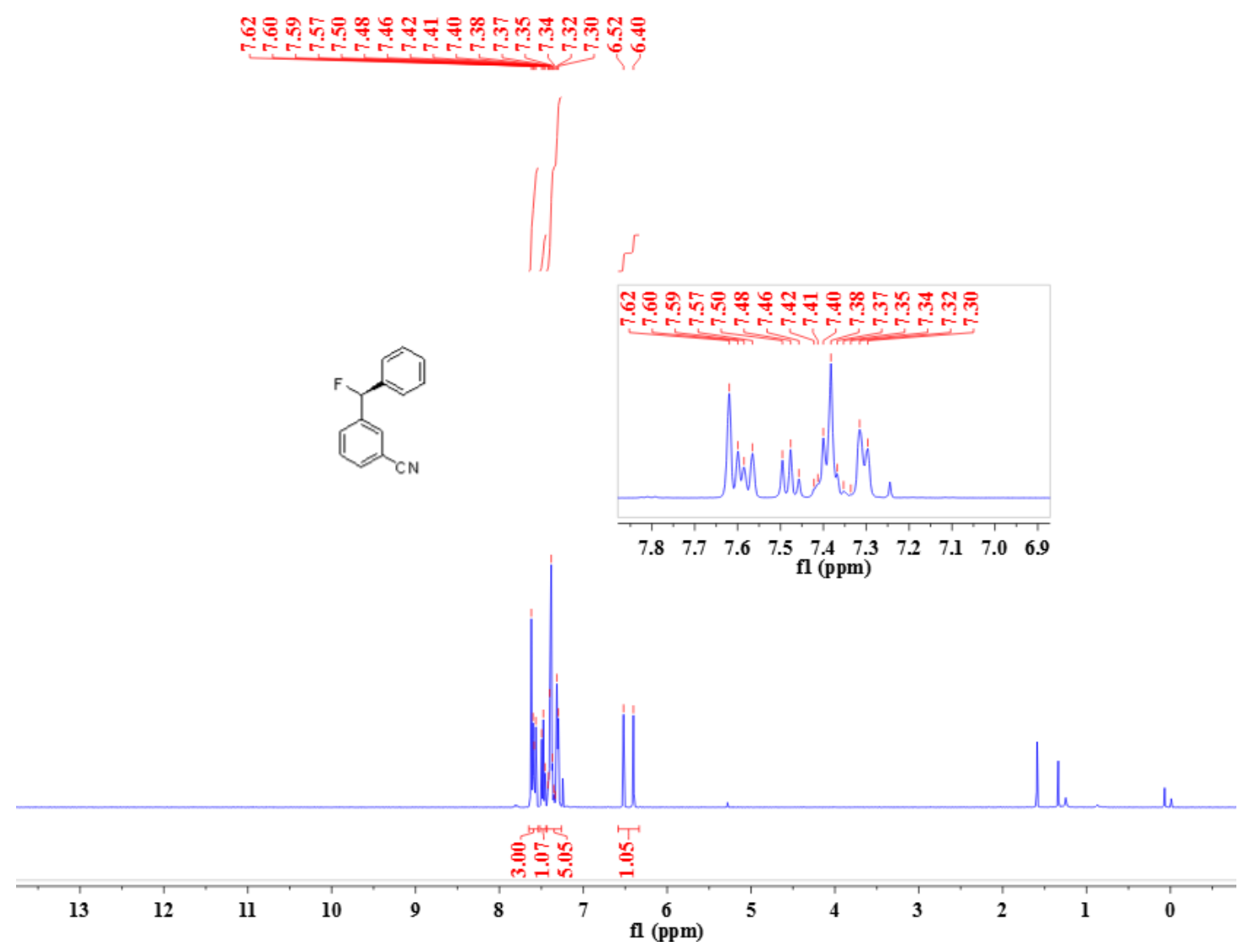

${ }^{19} \mathrm{~F}$ NMR (376 $\left.\mathrm{MHz}, \mathrm{CDCl}_{3}\right)$ spectrum of (S)-3-(fluoro(phenyl)methyl)benzonitrile 3k

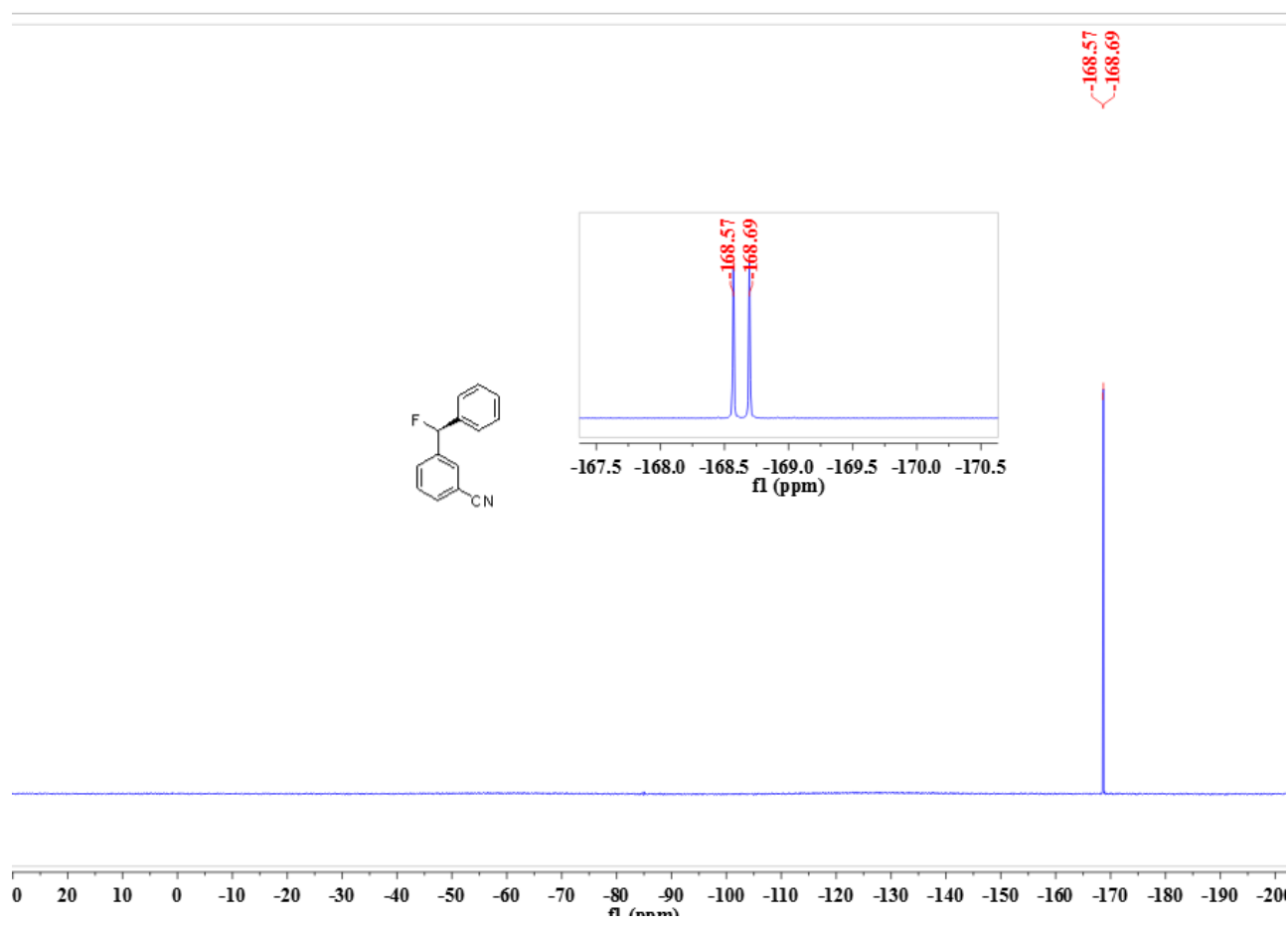


${ }^{13} \mathrm{C}$ NMR (101 MHz, $\left.\mathrm{CDCl}_{3}\right)$ spectrum of (S)-3-(fluoro(phenyl)methyl)benzonitrile 3k

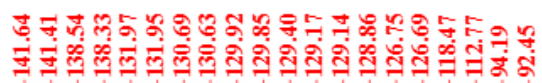

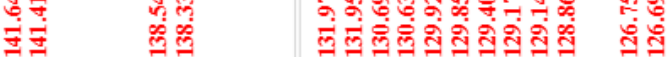

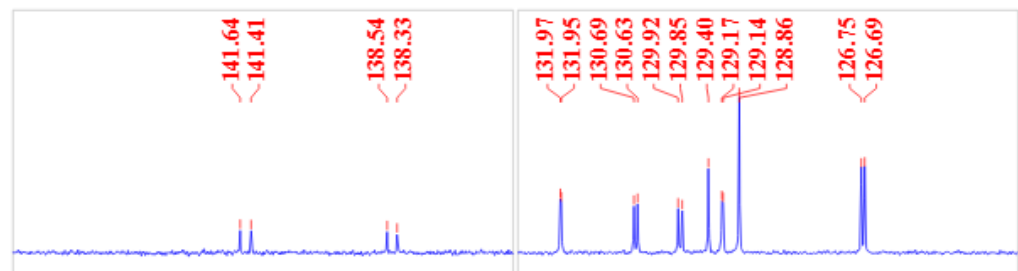

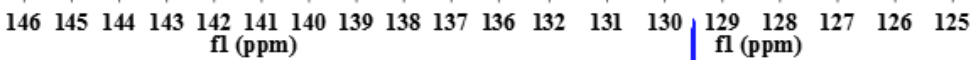
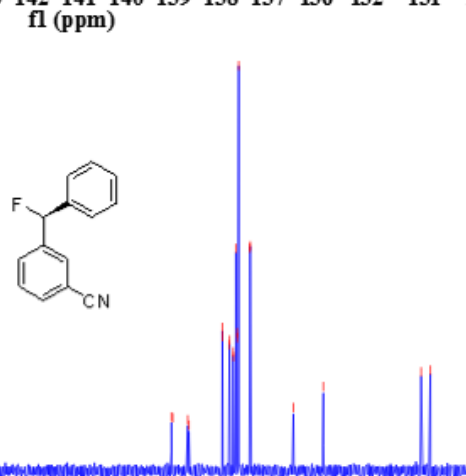

$\begin{array}{lllllllllllllllllllllllllll}240 & 230 & 220 & 210 & 200 & 190 & 180 & 170 & 160 & 150 & 140 & 130 & 120 & 110 & 100 & 90 & 80 & 70 & 60 & 50 & 40 & 30 & 20 & 10 & 0 & -10 & -20\end{array}$

${ }^{1} \mathrm{H}$ NMR (400 MHz, $\left.\mathrm{CDCl}_{3}\right)$ spectrum of

(R)-3-(Fluoro(3-fluorophenyl)methyl)benzonitrile 31

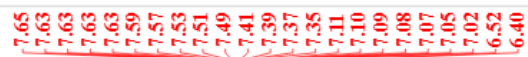
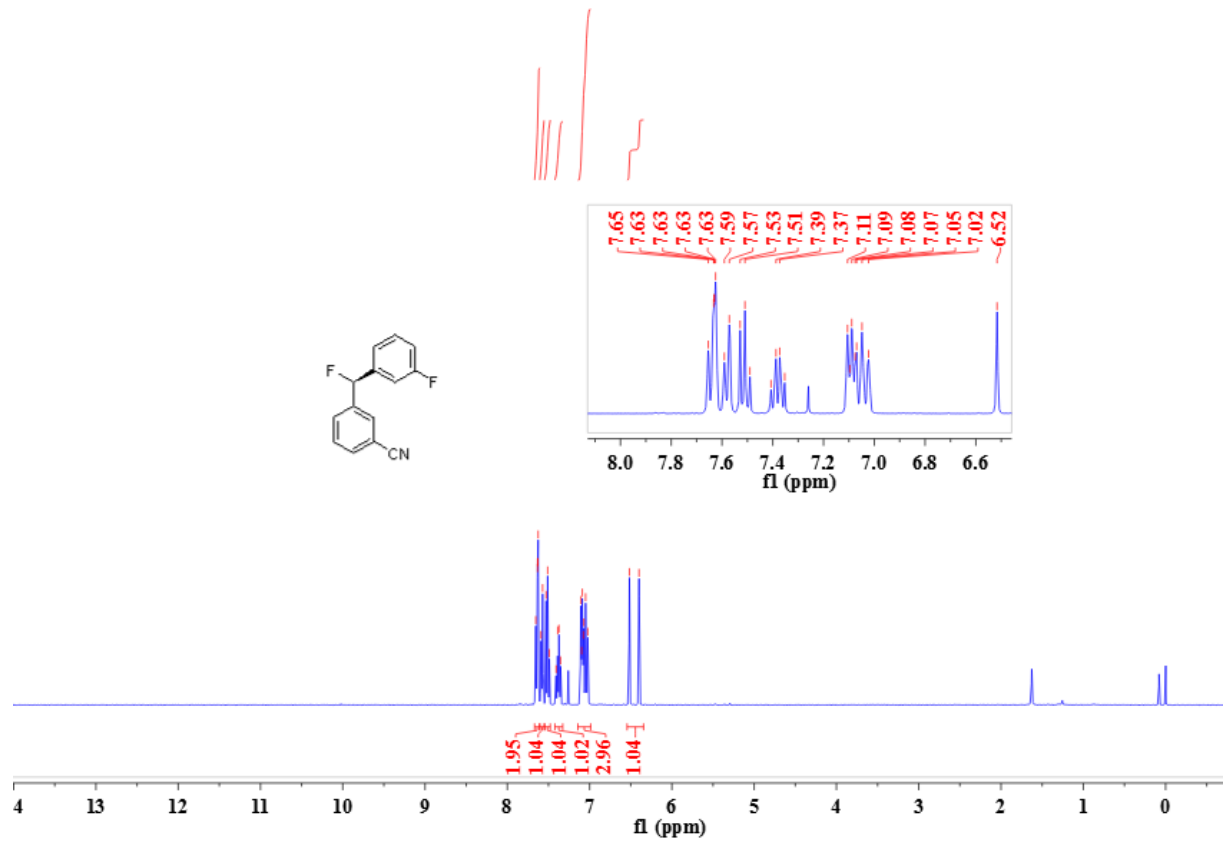
${ }^{19}$ F NMR (376 $\mathrm{MHz}, \mathrm{CDCl}_{3}$ ) spectrum of

(R)-3-(fluoro(3-fluorophenyl)methyl)benzonitrile 31

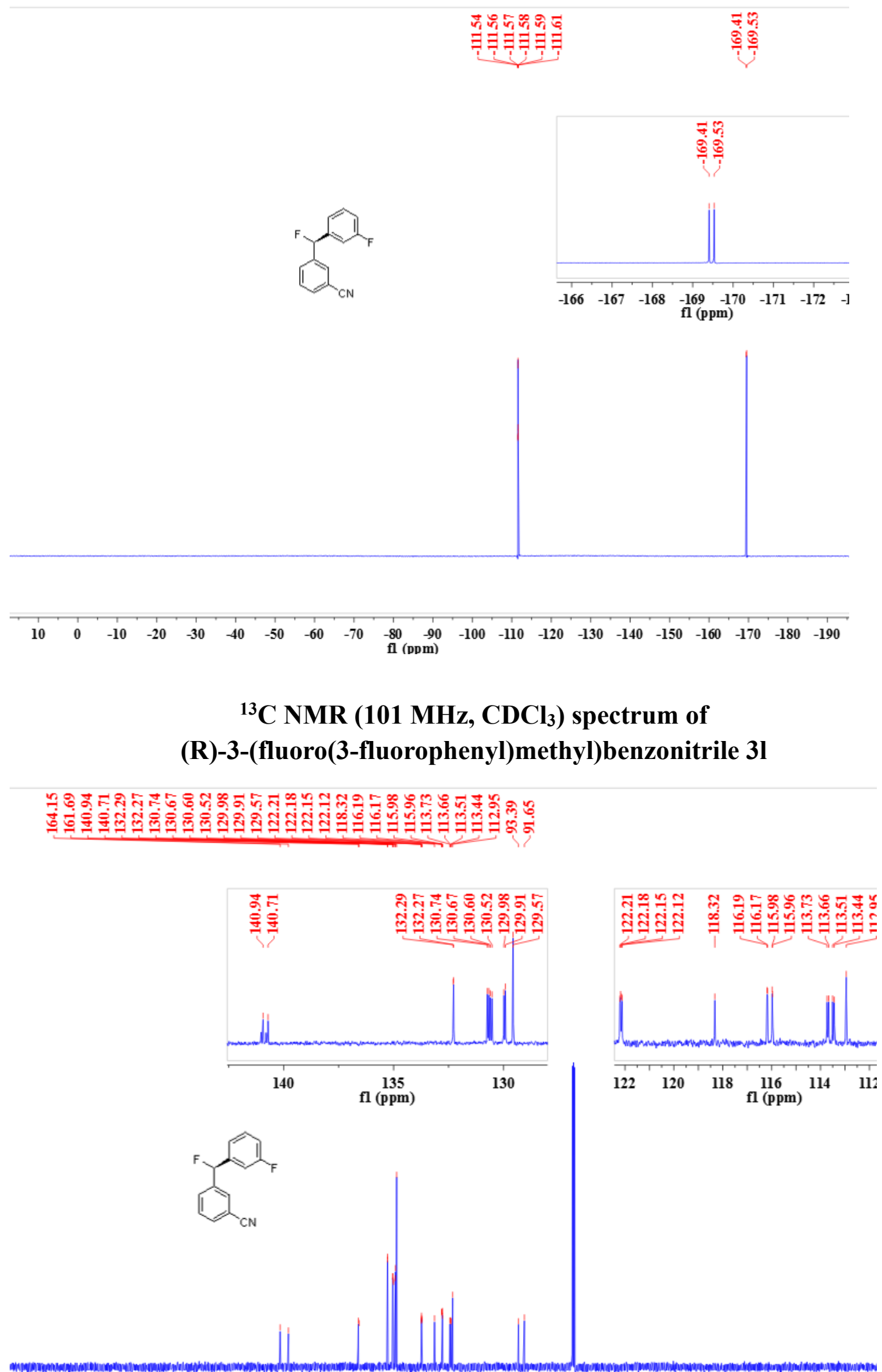

$\begin{array}{llllllllllllllllllllllllll}240 & 230 & 220 & 210 & 200 & 190 & 180 & 170 & 160 & 150 & 140 & 130 & 120 & 110 & 100 & 90 & 80 & 70 & 60 & 50 & 40 & 30 & 20 & 10 & 0 & -10\end{array}$ fl (ppm) 
${ }^{1} \mathrm{H}$ NMR (400 $\mathrm{MHz}, \mathrm{CDCl}_{3}$ ) spectrum of

(S)-3-(fluoro(4-fluorophenyl)methyl)benzonitrile 3m

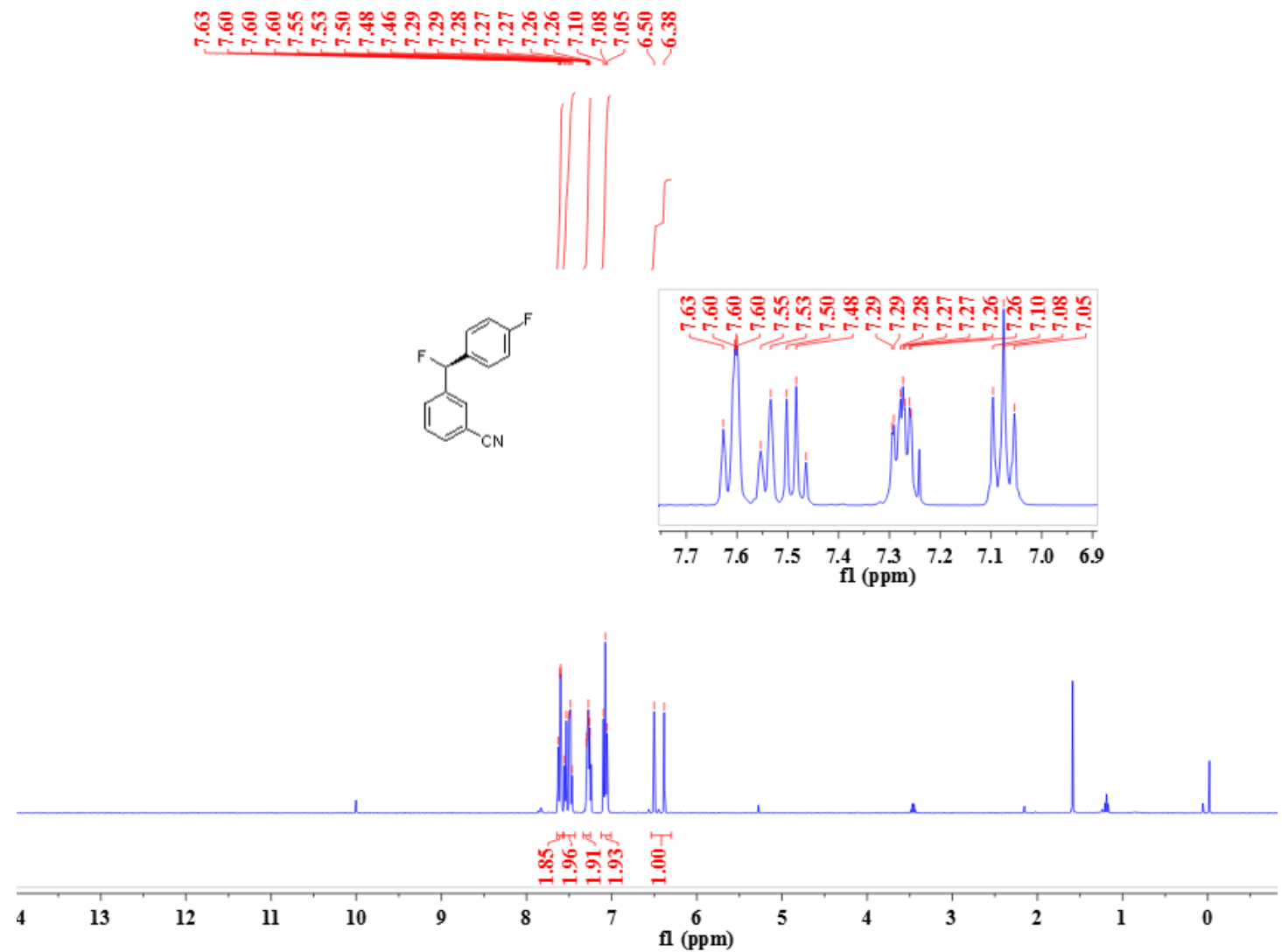

${ }^{19} \mathrm{~F}$ NMR (376 $\mathrm{MHz}, \mathrm{CDCl}_{3}$ ) spectrum of

(S)-3-(fluoro(4-fluorophenyl)methyl)benzonitrile 3m

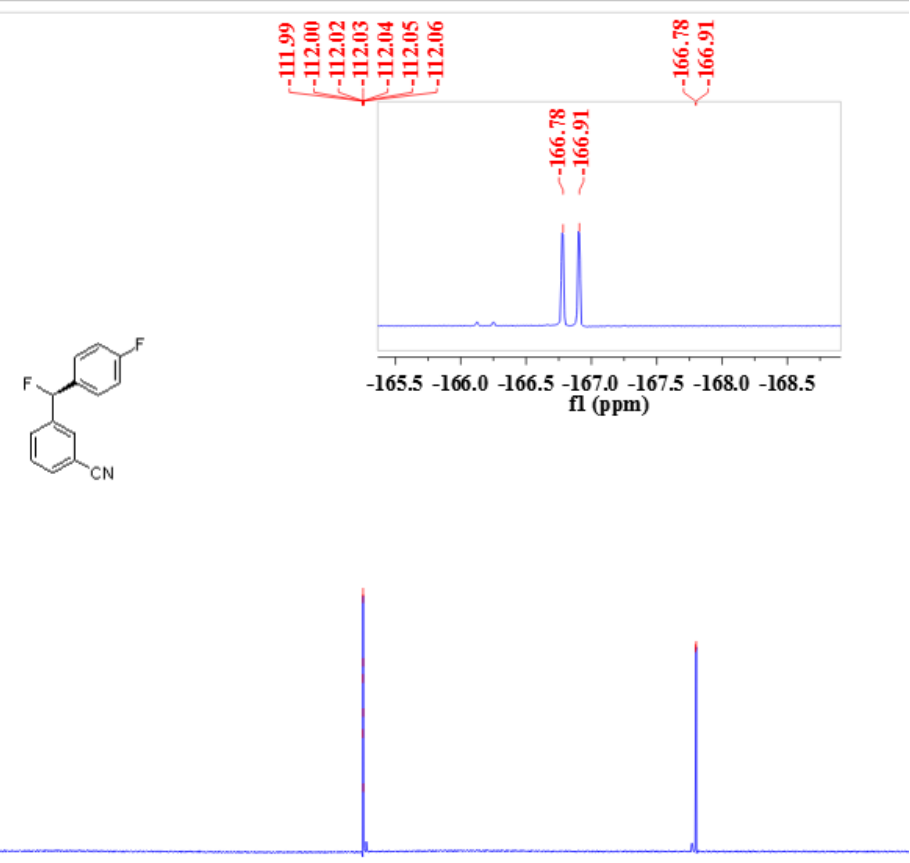

$\begin{array}{lllllllllllllllllllllllll}20 & 10 & 0 & -10 & -20 & -30 & -40 & -50 & -60 & -70 & -80 & -90 & -100 & -110 & -120 & -130 & -140 & -150 & -160 & -170 & -180 & -190 & -20\end{array}$ 
${ }^{13} \mathrm{C}$ NMR (101 MHz, $\left.\mathrm{CDCl}_{3}\right)$ spectrum of

(S)-3-(fluoro(4-fluorophenyl)methyl)benzonitrile 3m

कि

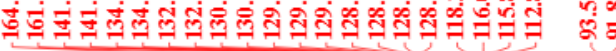

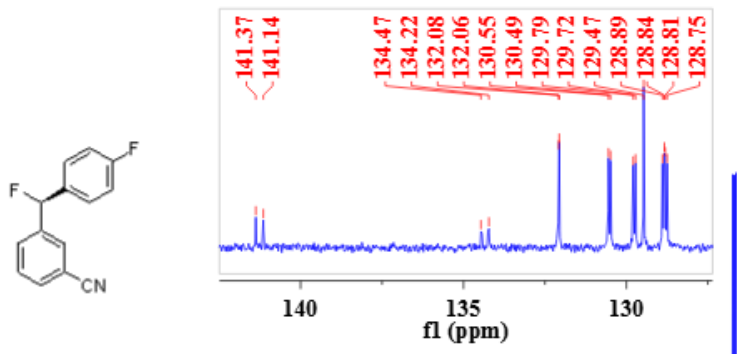

$\begin{array}{lllllllllllllllllllllllllllllllll}240 & 230 & 220 & 210 & 200 & 190 & 180 & 170 & 160 & 150 & 140 & 130 & 120 & 110 & 100 & 90 & 80 & 70 & 60 & 50 & 40 & 30 & 20 & 10 & 0 & -10 & -20 & -30\end{array}$

${ }^{1} \mathrm{H}$ NMR (400 MHz, $\left.\mathrm{CDCl}_{3}\right)$ spectrum of (R)-3-(fluoro(m-tolyl)methyl)benzonitrile 3n

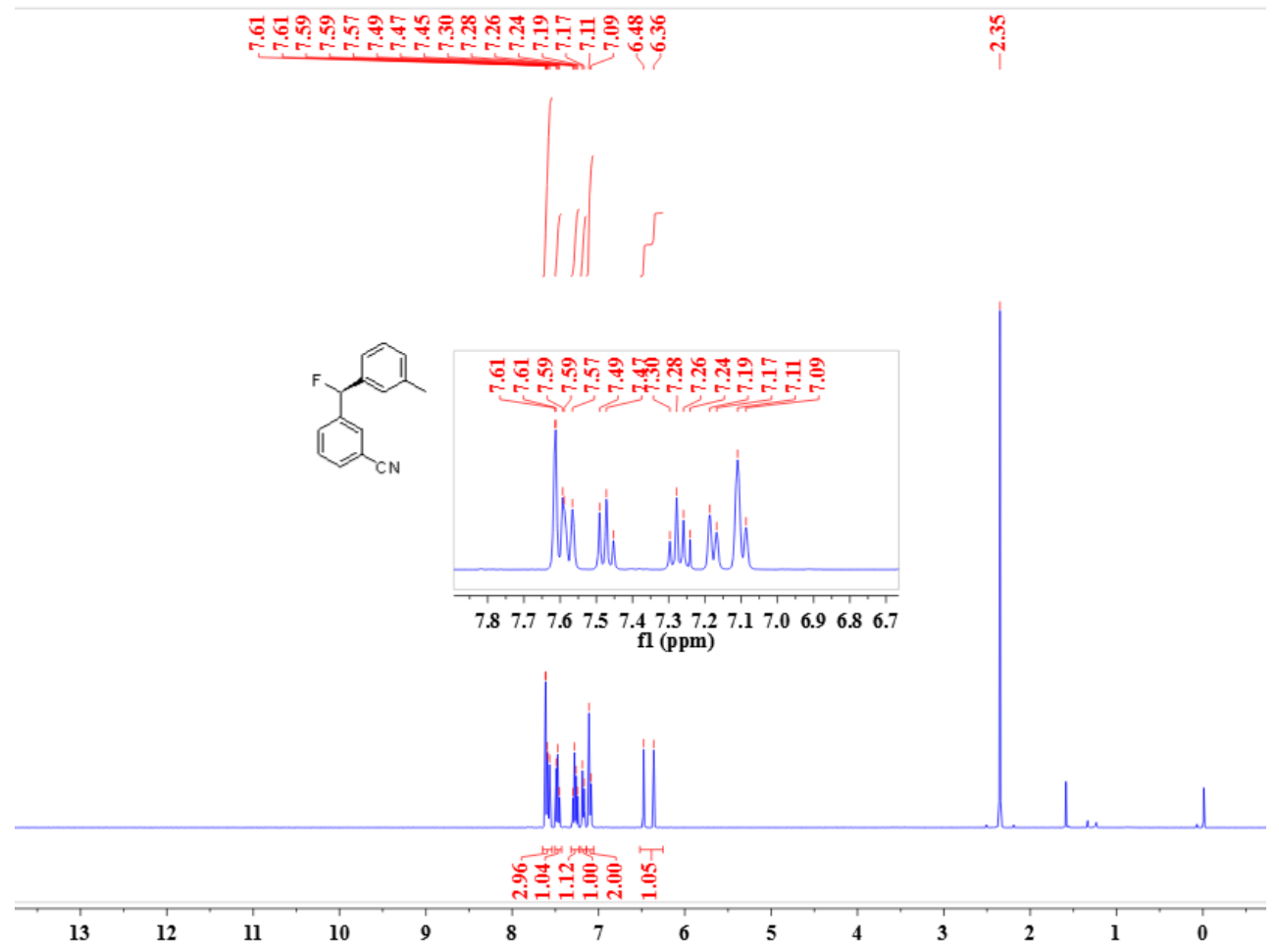


${ }^{19} \mathrm{~F}$ NMR (376 MHz, $\mathrm{CDCl}_{3}$ ) spectrum of

(S)-3-(fluoro(m-tolyl)methyl)benzonitrile 3n

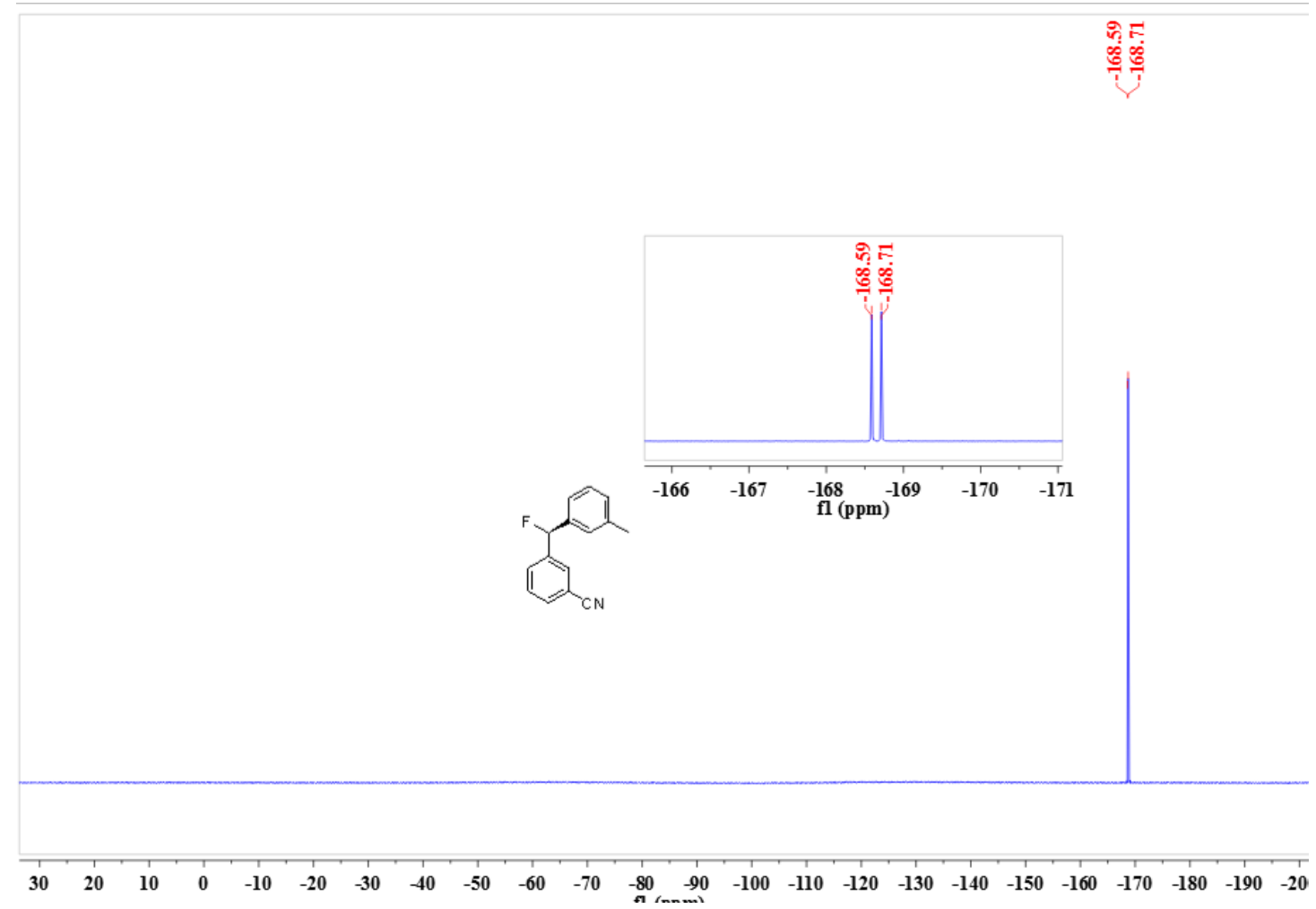

${ }^{13} \mathrm{C}$ NMR (101 MHz, $\left.\mathrm{CDCl}_{3}\right)$ spectrum of

(S)-3-(fluoro(m-tolyl)methyl)benzonitrile 3n

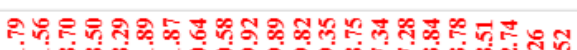

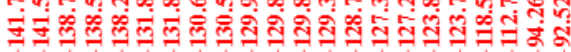

พับ

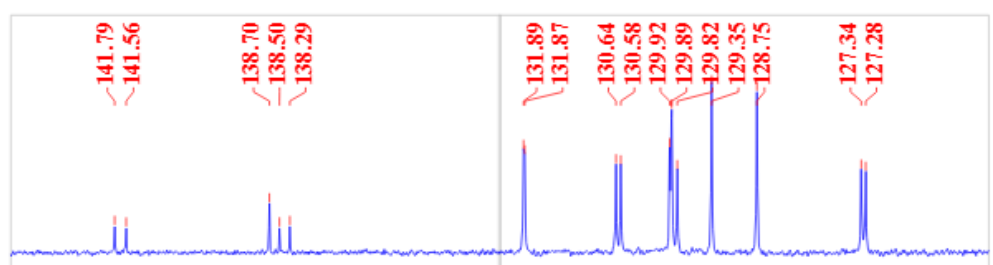

$\begin{array}{llllllllllllllllllll}143 & 142 & 141 & 140 & 139 & 138 & 137 & 136 & 135 & 132 & 131 & 130 & 129 & 128 & 127 & 126\end{array}$

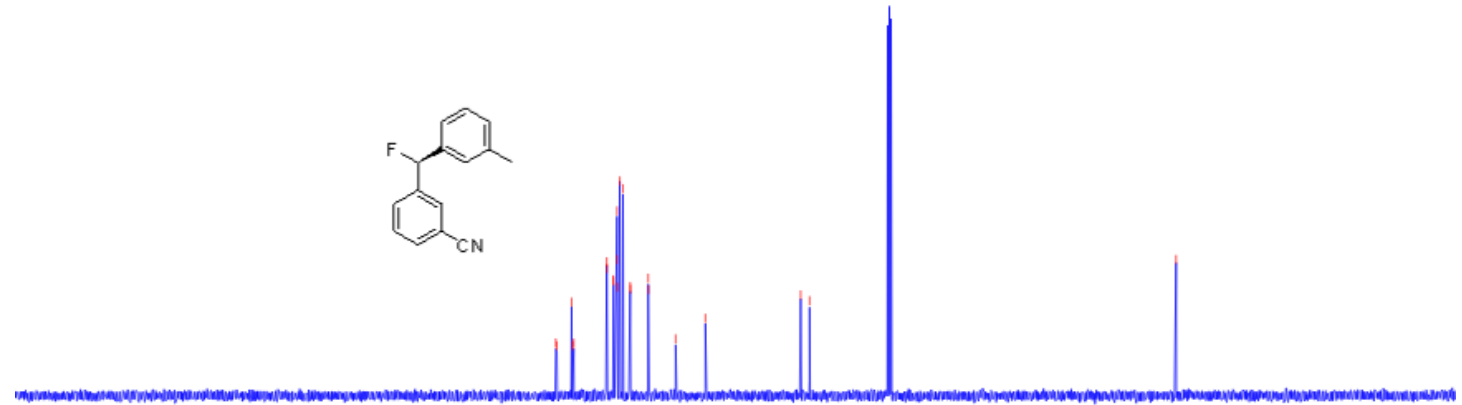

$\begin{array}{llllllllllllllllllllllllllll}240 & 230 & 220 & 210 & 200 & 190 & 180 & 170 & 160 & 150 & 140 & 130 & 120 & 110 & 100 & 90 & 80 & 70 & 60 & 50 & 40 & 30 & 20 & 10 & 0 & -10 & -20 & -30\end{array}$ 
${ }^{1} \mathrm{H}$ NMR (400 $\mathrm{MHz}, \mathrm{CDCl}_{3}$ ) spectrum of

(R)-3-(fluoro(3-methoxyphenyl)methyl)benzonitrile 30

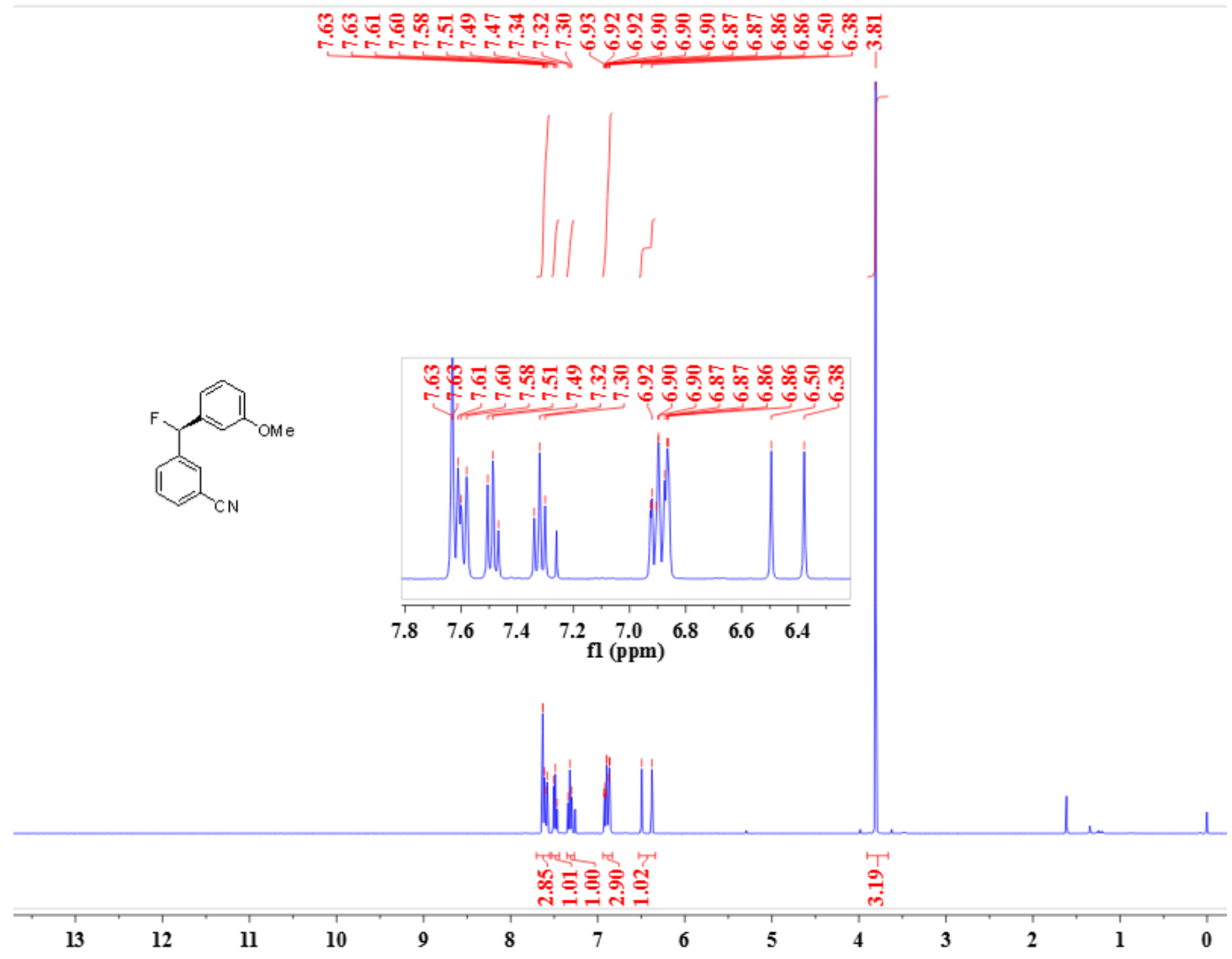

${ }^{19} \mathrm{~F}$ NMR (376 $\mathrm{MHz}, \mathrm{CDCl}_{3}$ ) spectrum of

(R)-3-(fluoro(3-methoxyphenyl)methyl)benzonitrile 30

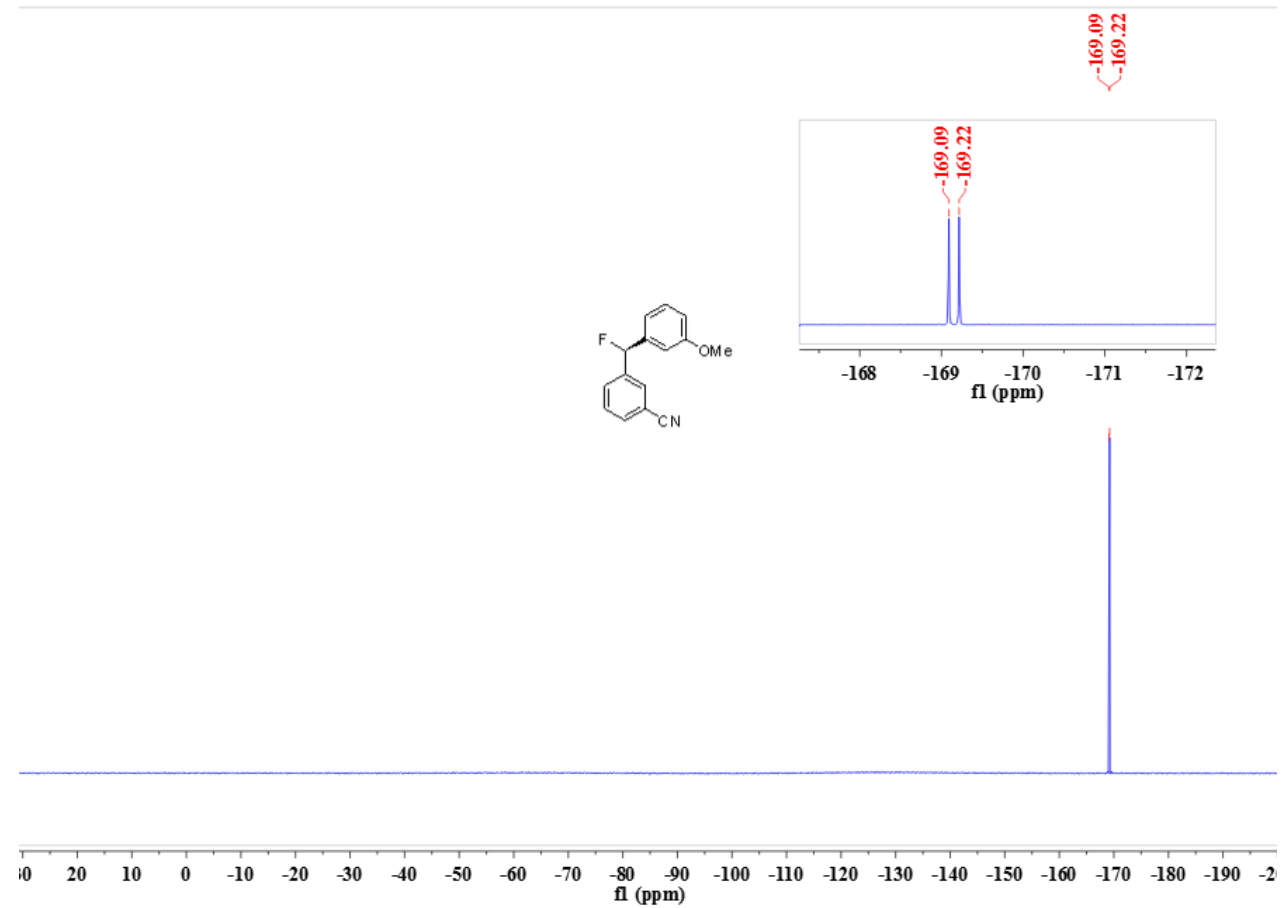


${ }^{13} \mathrm{C}$ NMR (101 MHz, $\left.\mathrm{CDCl}_{3}\right)$ spectrum of

(R)-3-(fluoro(3-methoxyphenyl)methyl)benzonitrile 30

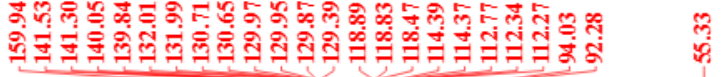
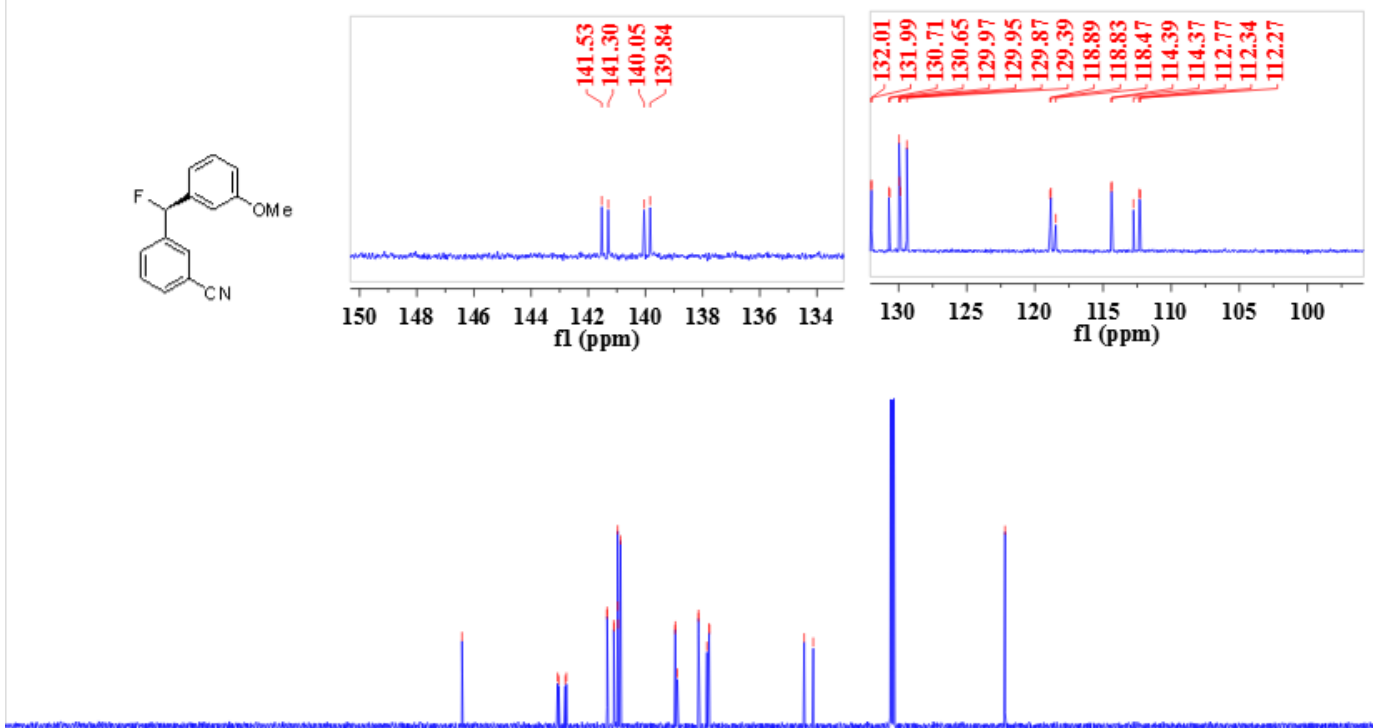

$\begin{array}{llllllllllllllllllllllllll}240 & 230 & 220 & 210 & 200 & 190 & 180 & 170 & 160 & 150 & 140 & 130 & 120 & 110 & 100 & 90 & 80 & 70 & 60 & 50 & 40 & 30 & 20 & 10 & 0 & -10\end{array}$ fl (ppm)

${ }^{1} \mathrm{H}$ NMR (400 MHz, $\left.\mathrm{CDCl}_{3}\right)$ spectrum of (R)-3-(fluoro(3-fluoro-4-methylphenyl)methyl)benzonitrile 3p

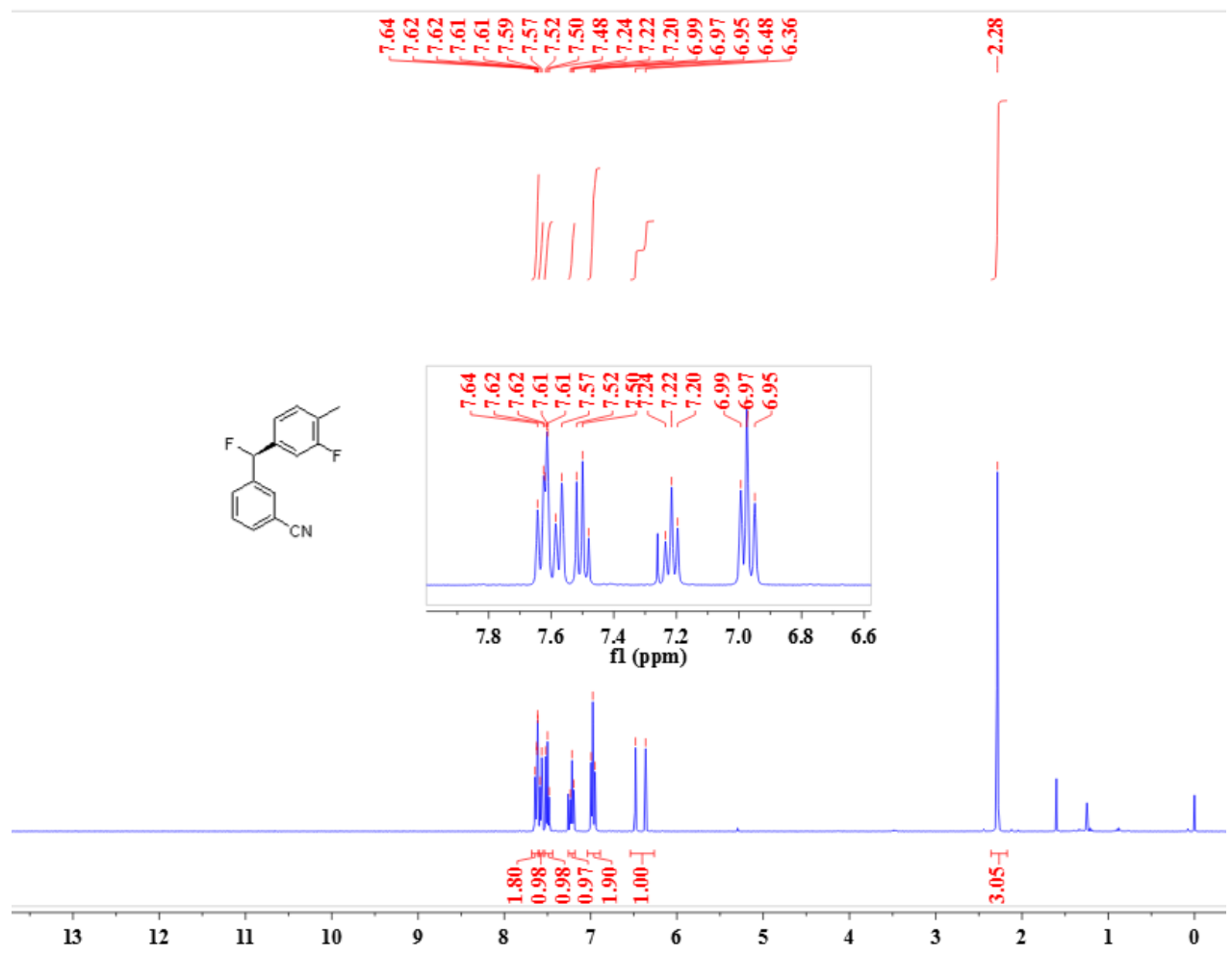


${ }^{19}$ F NMR (376 $\mathrm{MHz}, \mathrm{CDCl}_{3}$ ) spectrum of

(R)-3-(fluoro(3-fluoro-4-methylphenyl)methyl)benzonitrile 3p
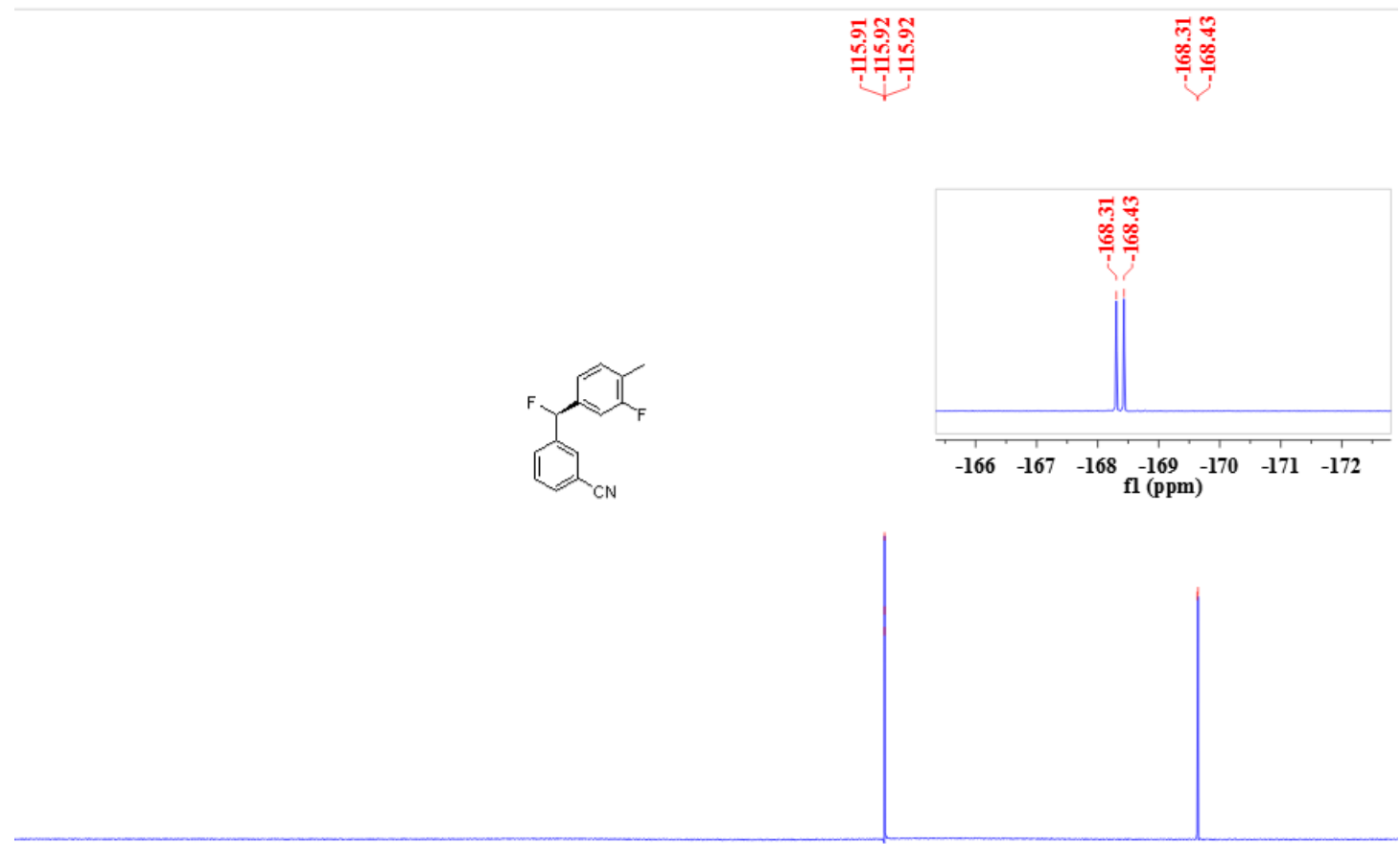

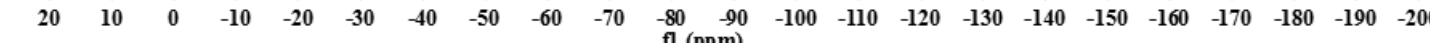

${ }^{13} \mathrm{C}$ NMR (101 MHz, $\left.\mathrm{CDCl}_{3}\right)$ spectrum of

(R)-3-(fluoro(3-fluoro-4-methylphenyl)methyl)benzonitrile 3p

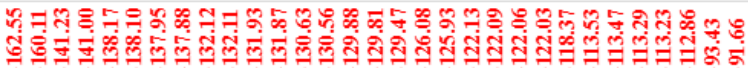
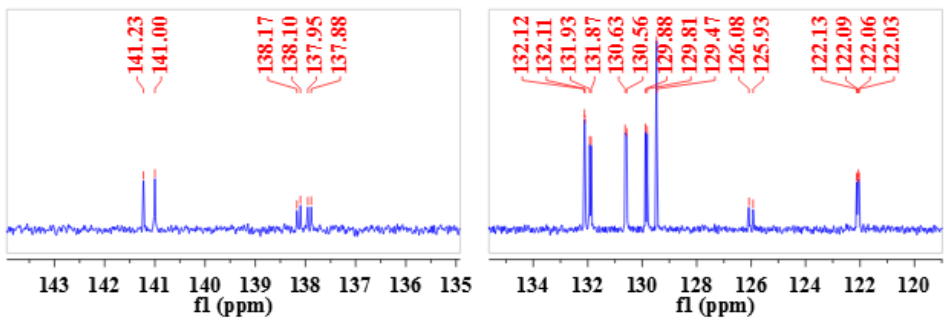

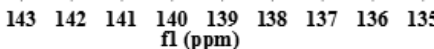

$\begin{array}{llllllll}134 & 132 & 130 & 128 & 126 & 124 & 122 & 120\end{array}$

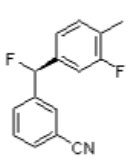

$\begin{array}{llllllllllllllll}240 & 230 & 220 & 210 & 200 & 190 & 180 & 170 & 160 & 150 & 140 & 130 & 120 & 110 & 100 & 90\end{array}$ 
${ }^{1} \mathrm{H}$ NMR (400 $\mathrm{MHz}, \mathrm{CDCl}_{3}$ ) spectrum of (S)-4-(fluoro(phenyl)methyl)benzonitrile 3q

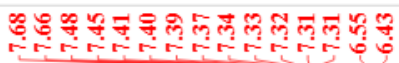
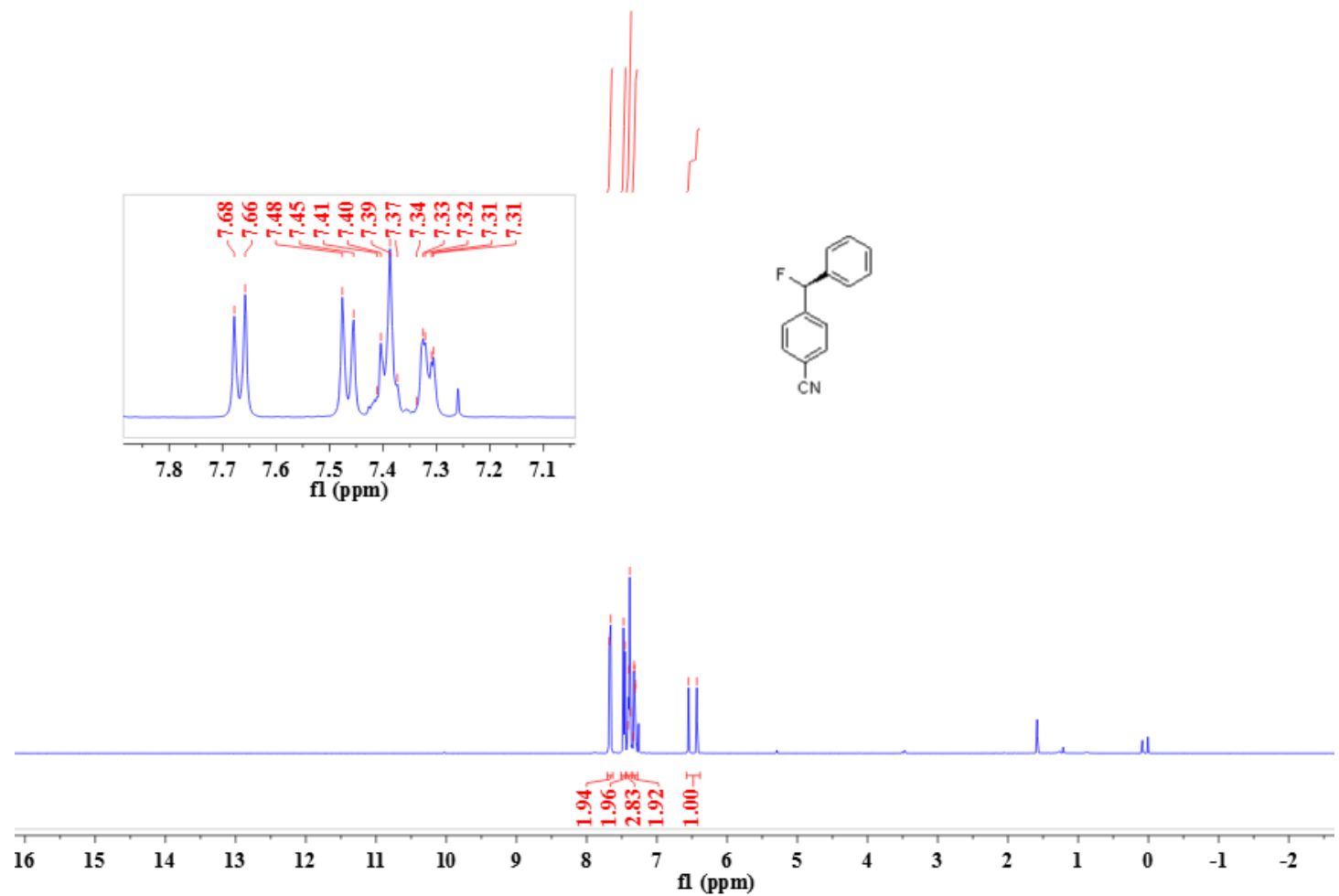

${ }^{19}$ F NMR (376 MHz, $\left.\mathrm{CDCl}_{3}\right)$ spectrum of (S)-4-(fluoro(phenyl)methyl)benzonitrile 3q

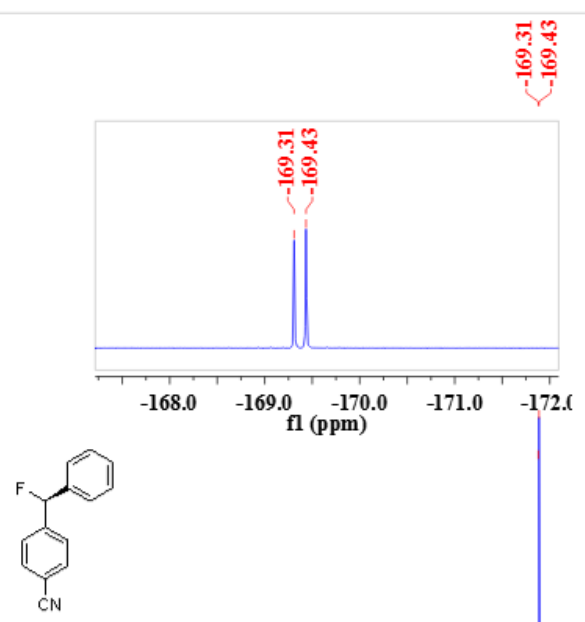

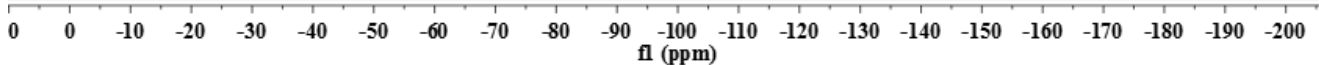


${ }^{13} \mathrm{C}$ NMR (101 MHz, $\left.\mathrm{CDCl}_{3}\right)$ spectrum of

(S)-4-(fluoro(phenyl)methyl)benzonitrile 3q

के के ले ले

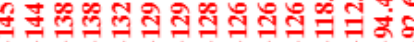
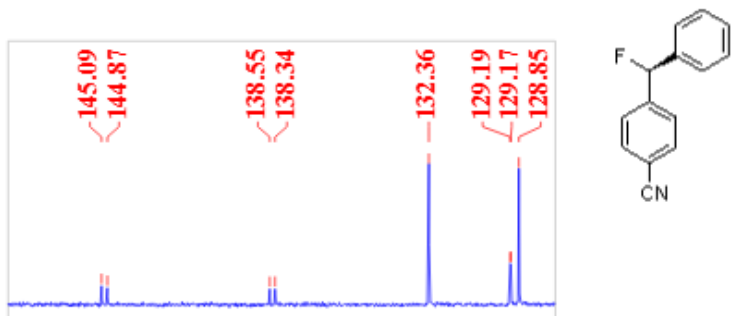

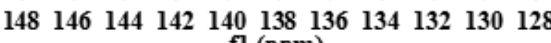
fl $(\mathrm{ppm})$

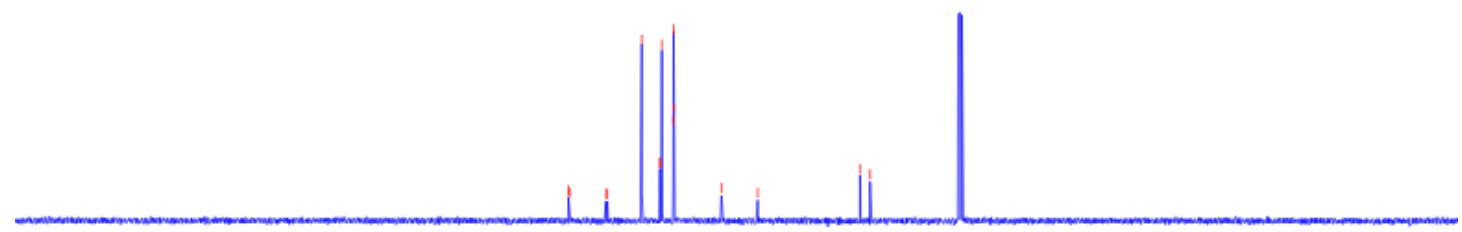

$\begin{array}{llllllllllllllllllllllllll}40 & 230 & 220 & 210 & 200 & 190 & 180 & 170 & 160 & 150 & 140 & 130 & 120 & 110 & 100 & 90 & 80 & 70 & 60 & 50 & 40 & 30 & 20 & 10 & 0 & -\end{array}$

${ }^{1} \mathrm{H}$ NMR (400 MHz, $\left.\mathrm{CDCl}_{3}\right)$ spectrum of (R)-4-(fluoro(m-tolyl)methyl)benzonitrile 3r

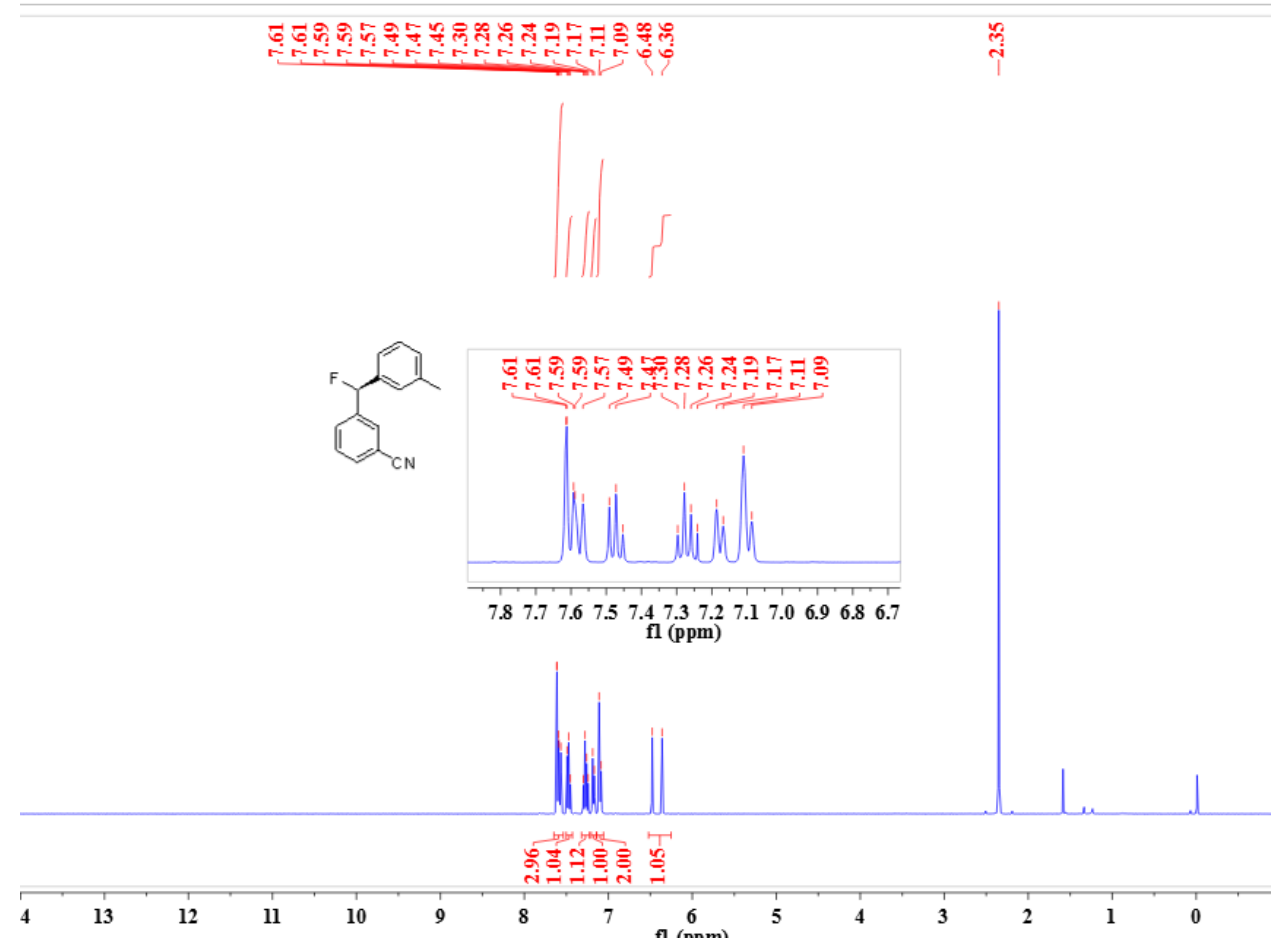


${ }^{19} \mathrm{~F}$ NMR (376 MHz, $\mathrm{CDCl}_{3}$ ) spectrum of (R)-4-(fluoro(m-tolyl)methyl)benzonitrile 3r

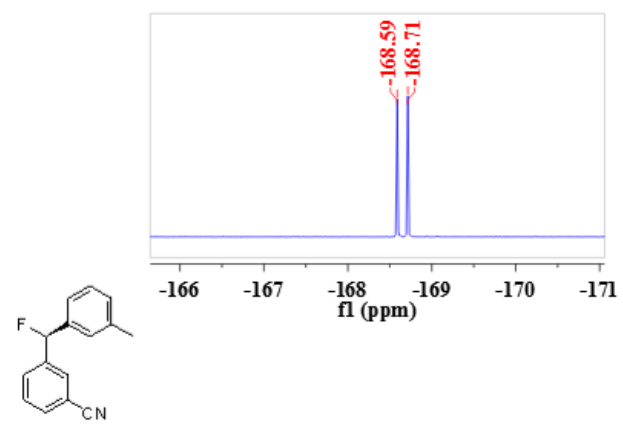

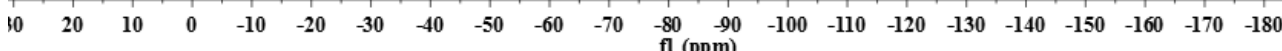

${ }^{13} \mathrm{C}$ NMR (101 MHz, $\left.\mathrm{CDCl}_{3}\right)$ spectrum of (R)-4-(fluoro(m-tolyl)methyl)benzonitrile 3r
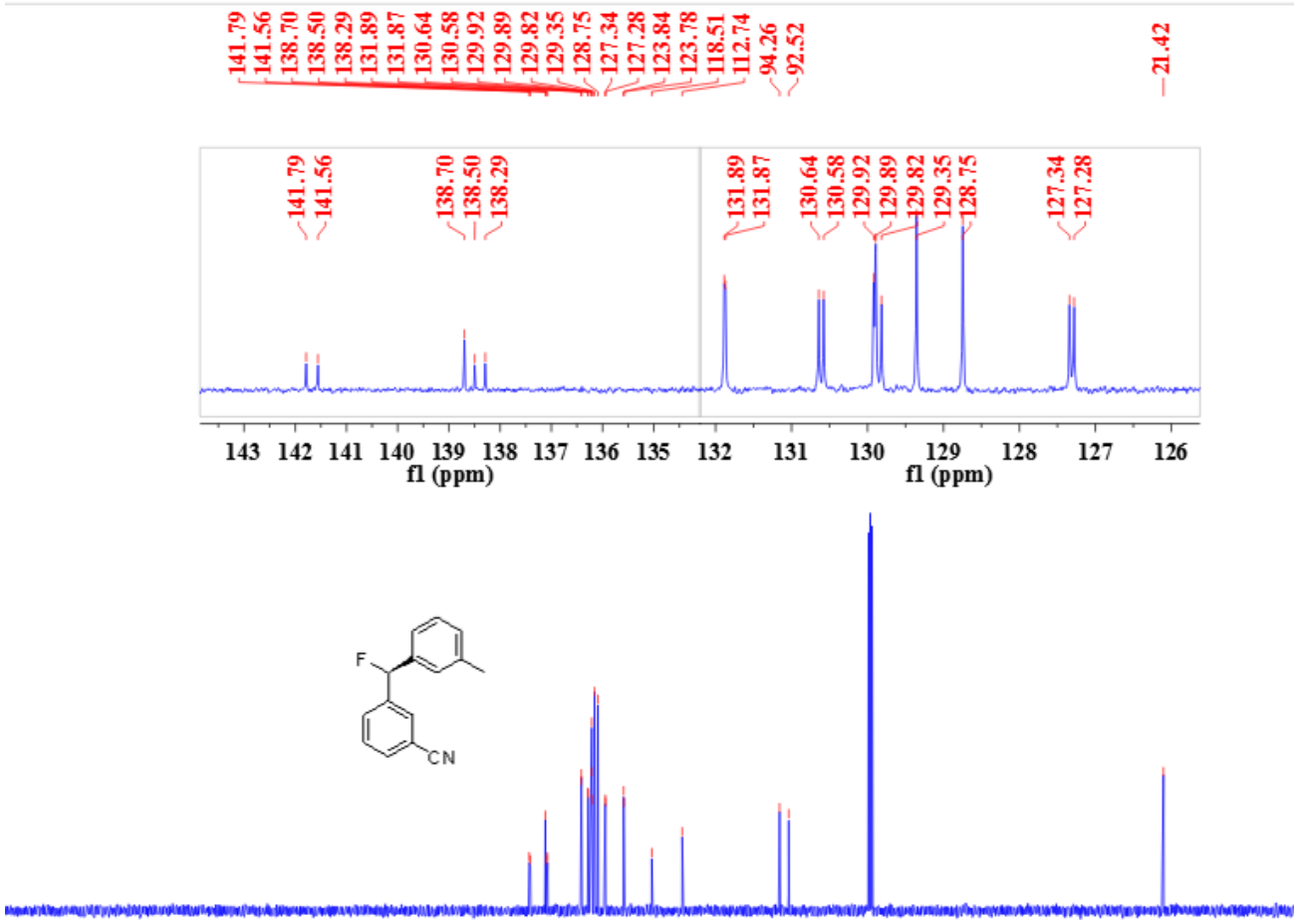

$\begin{array}{lllllllllllllllllllllllllll}40 & 230 & 220 & 210 & 200 & 190 & 180 & 170 & 160 & 150 & 140 & 130 & 120 & 110 & 100 & 90 & 80 & 70 & 60 & 50 & 40 & 30 & 20 & 10 & 0\end{array}$ 
${ }^{1} \mathrm{H}$ NMR (400 $\mathrm{MHz}, \mathrm{CDCl}_{3}$ ) spectrum of (R)-3-(Fluoro(3-fluorophenyl)methyl)benzonitrile $3 \mathrm{~s}$

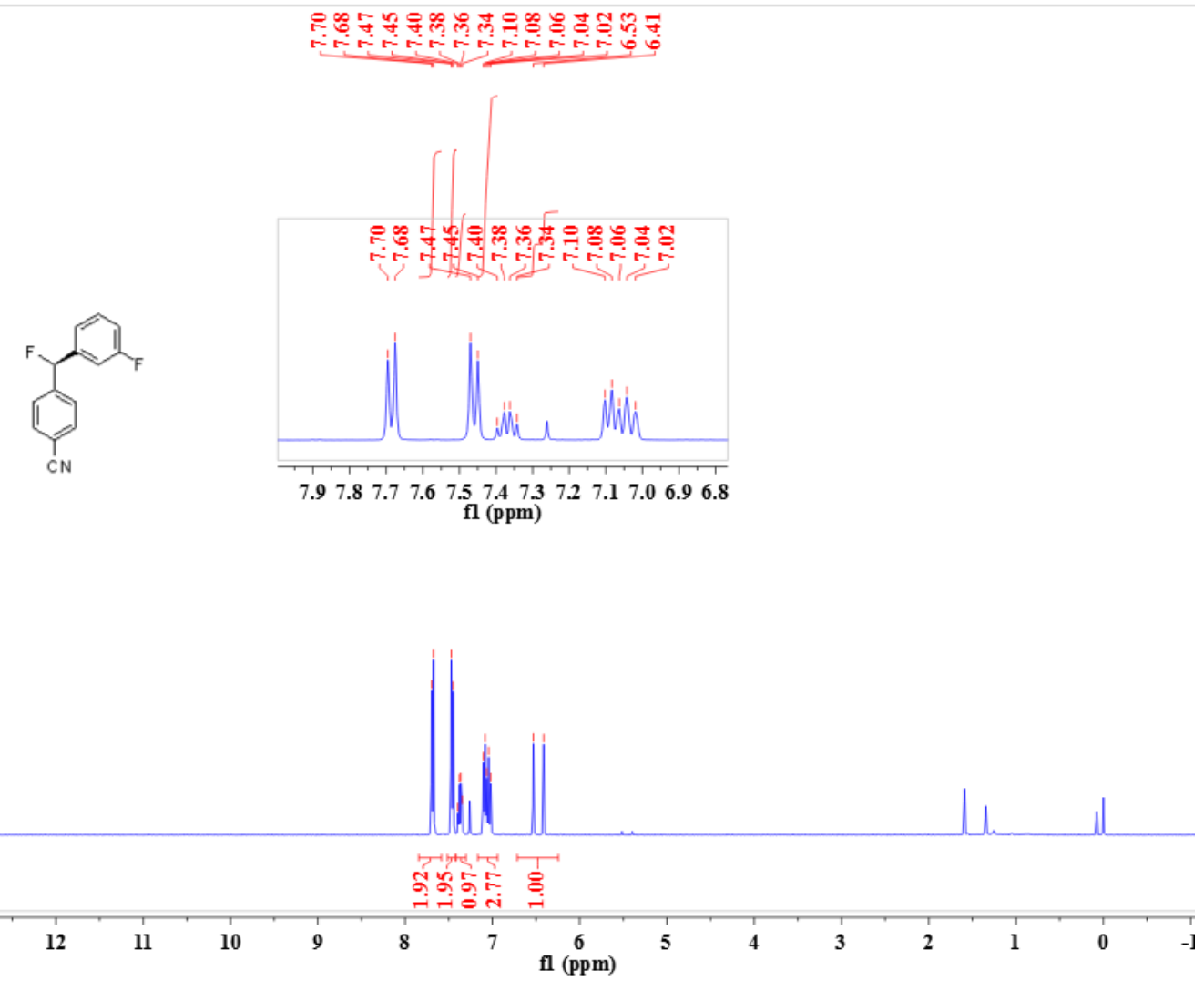

${ }^{19} \mathrm{~F}$ NMR (376 $\left.\mathrm{MHz}, \mathrm{CDCl}_{3}\right)$ spectrum of (R)-3-(fluoro(3-fluorophenyl)methyl)benzonitrile 3s

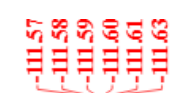
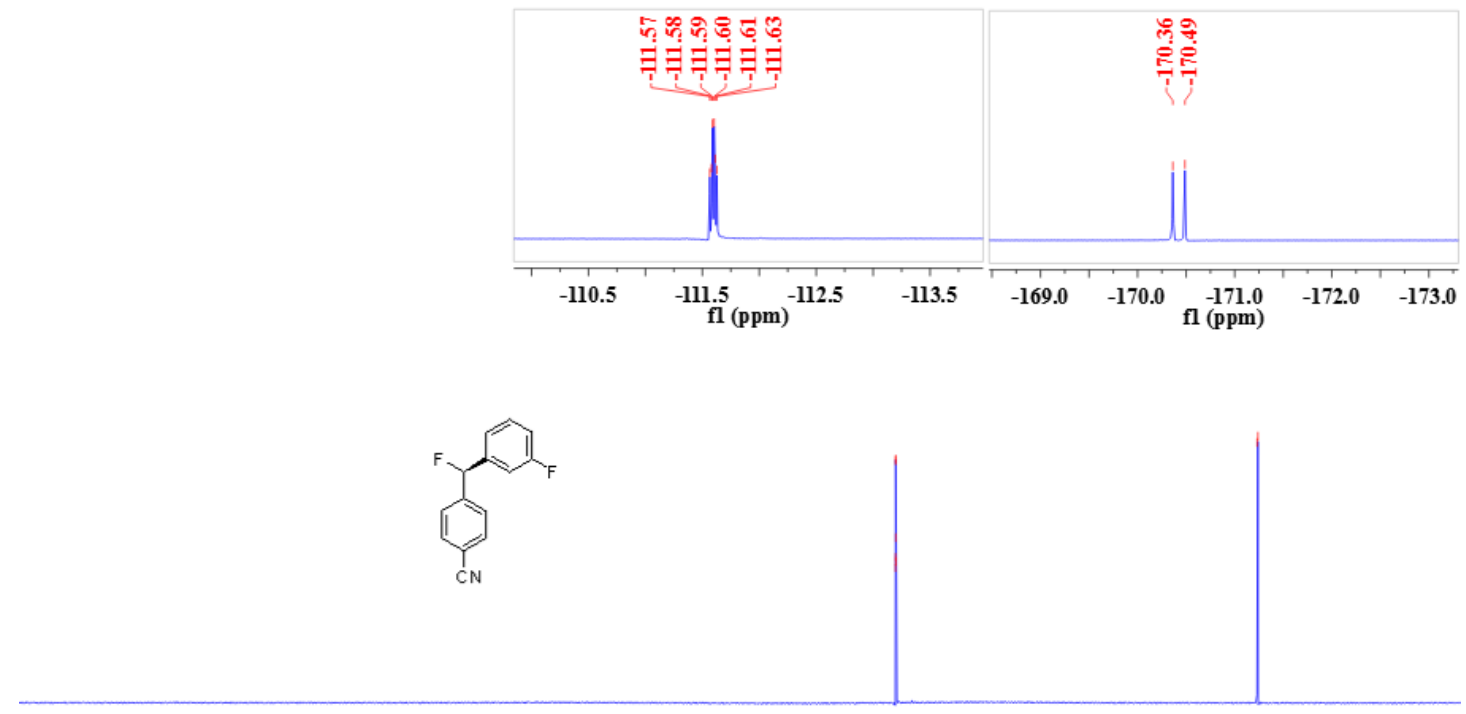

$\begin{array}{lllllllllllllllllllllll}20 & 10 & 0 & -10 & -20 & -30 & -40 & -50 & -60 & -70 & -80 & -90 & -100 & -110 & -120 & -130 & -140 & -150 & -160 & -170 & -180 & -190 & -200\end{array}$ 
${ }^{13} \mathrm{C}$ NMR (101 MHz, $\left.\mathrm{CDCl}_{3}\right)$ spectrum of

(R)-3-(fluoro(3-fluorophenyl)methyl)benzonitrile 3s

चै

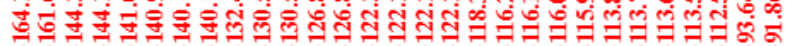
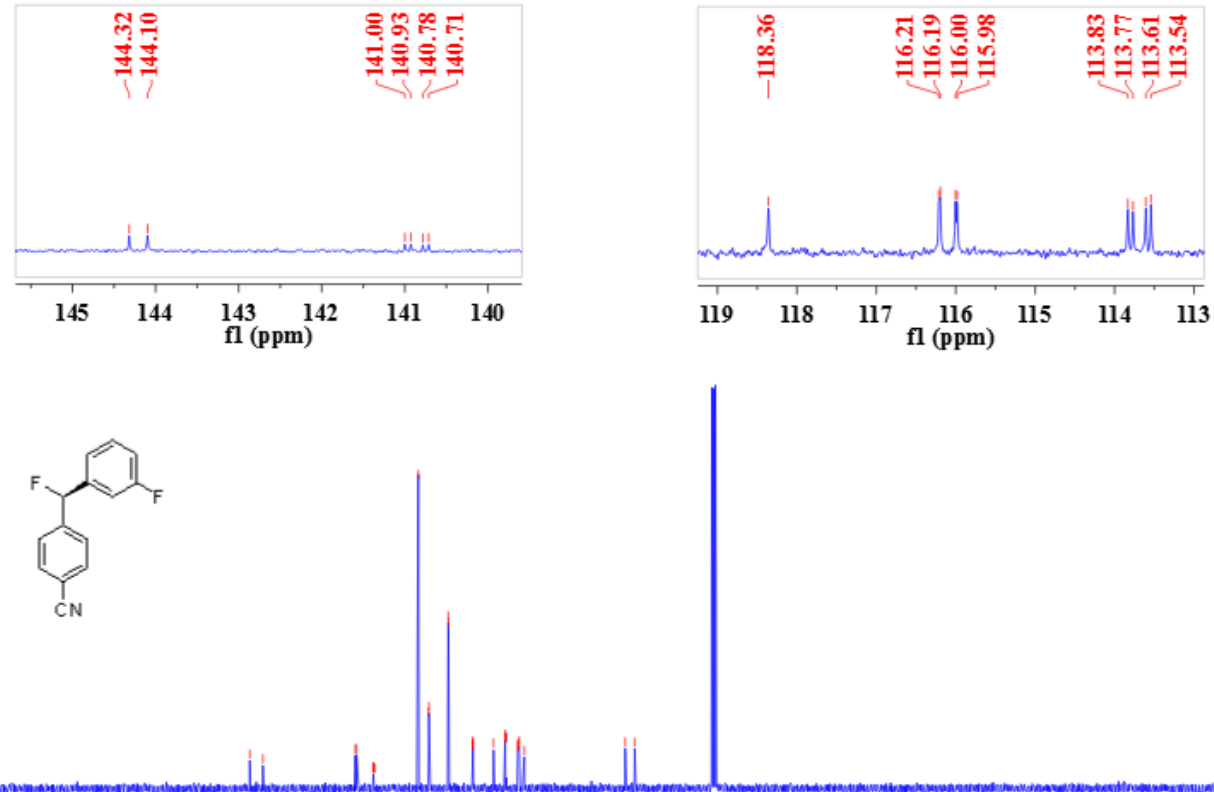

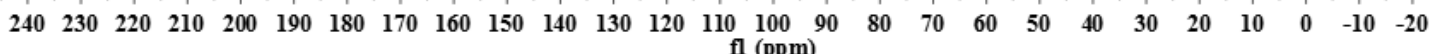

${ }^{1} \mathrm{H}$ NMR (400 MHz, $\left.\mathrm{CDCl}_{3}\right)$ spectrum of (S)-2-fluoro-4-(fluoro(3-fluorophenyl)methyl)benzonitrile 3t

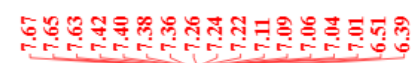
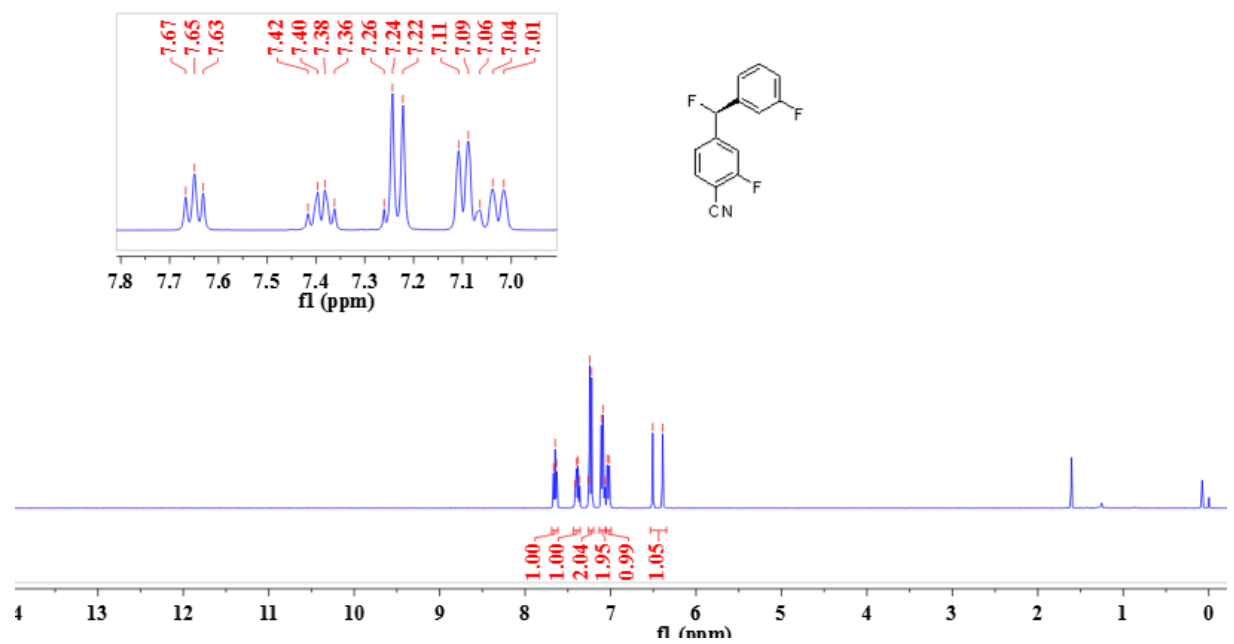
${ }^{19}$ F NMR (376 $\left.\mathrm{MHz}, \mathrm{CDCl}_{3}\right)$ spectrum of

(S)-2-Fluoro-4-(fluoro(3-fluorophenyl)methyl)benzonitrile 3t

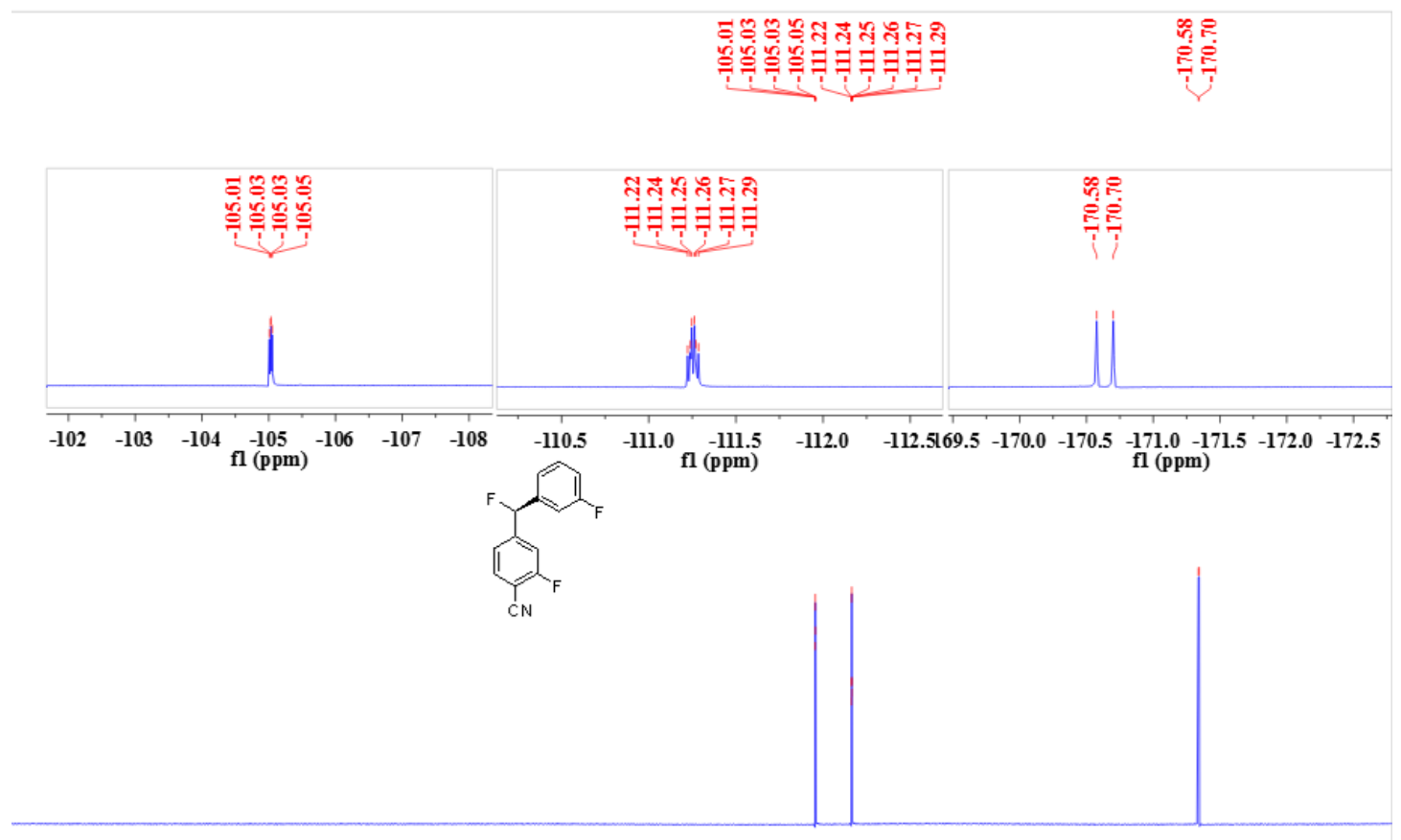

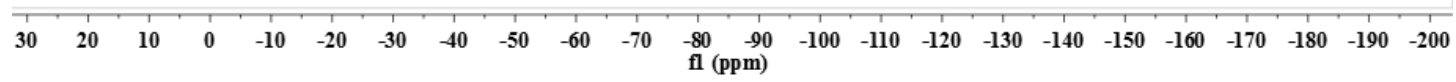

${ }^{13} \mathrm{C}$ NMR (101 MHz, $\left.\mathrm{CDCl}_{3}\right)$ spectrum of

(S)-2-fluoro-4-(fluoro(3-fluorophenyl)methyl)benzonitrile 3t

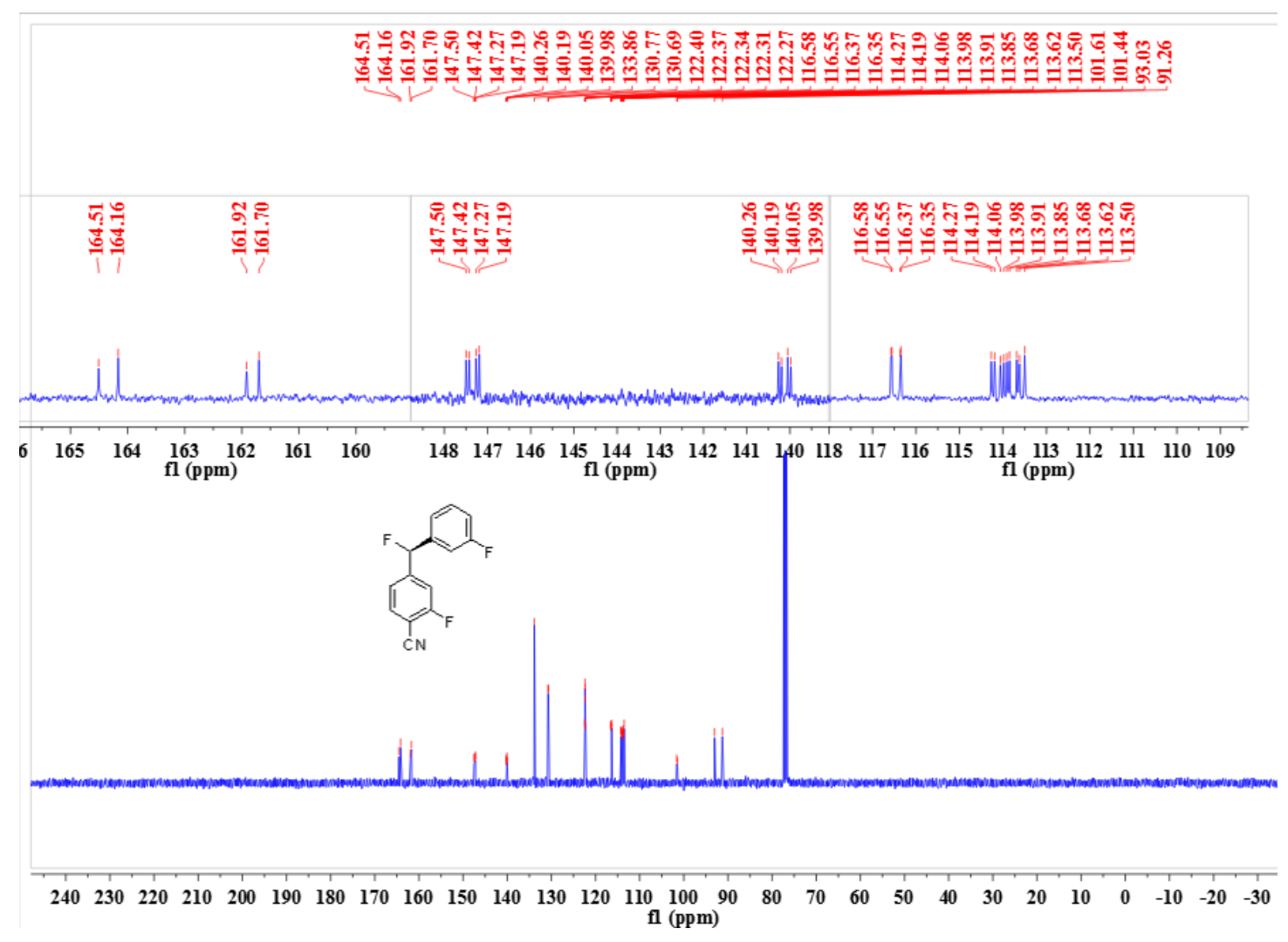


${ }^{1} \mathrm{H}$ NMR (400 $\mathrm{MHz}, \mathrm{CDCl}_{3}$ ) spectrum of

(S)-3-fluoro-4-(fluoro(3-fluorophenyl)methyl)benzonitrile 3u

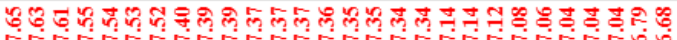
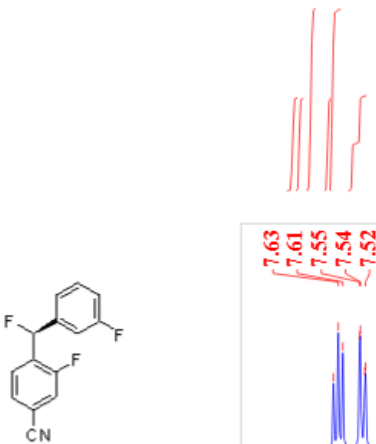

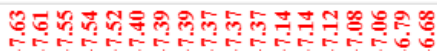
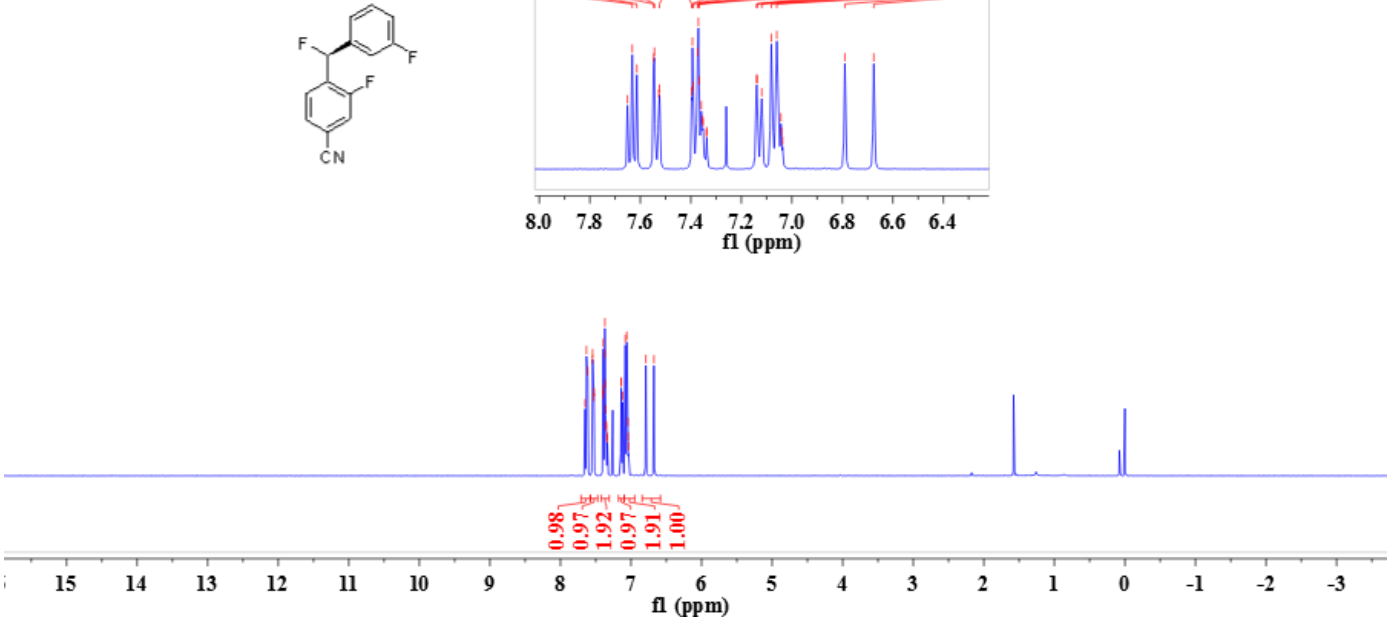

${ }^{19}$ F NMR (376 MHz, $\mathrm{CDCl}_{3}$ ) spectrum of (S)-3-fluoro-4-(fluoro(3-fluorophenyl)methyl)benzonitrile 3u
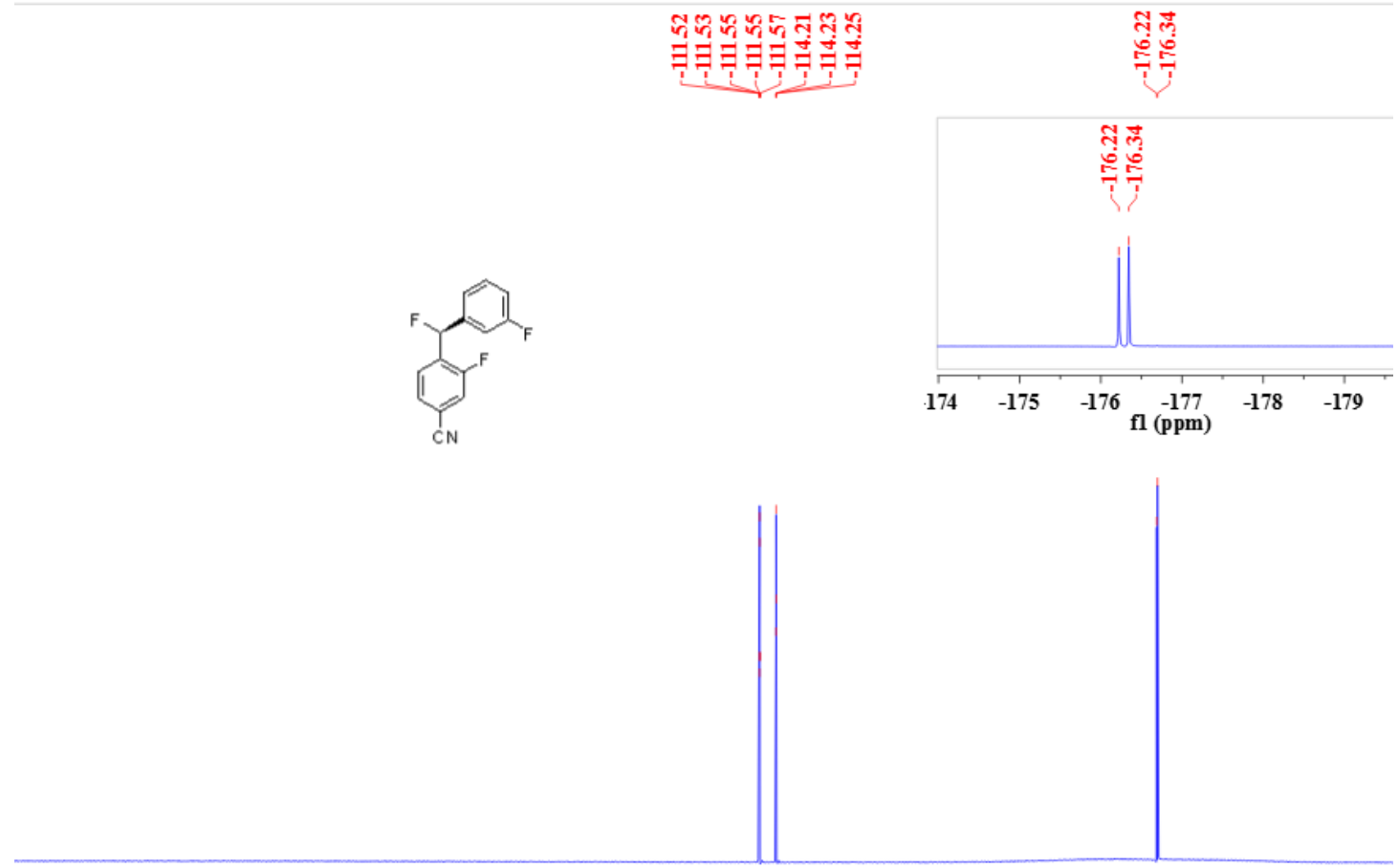

$\begin{array}{llllllllllllllllllllllll}0 & 0 & -10 & -20 & -30 & -40 & -50 & -60 & -70 & -80 & -90 & -100 & -110 & -120 & -130 & -140 & -150 & -160 & -170 & -180 & -190 & -200 & -210\end{array}$ 
${ }^{13} \mathrm{C}$ NMR (101 MHz, $\left.\mathrm{CDCl}_{3}\right)$ spectrum of (S)-3-fluoro-4-(fluoro(3-fluorophenyl)methyl)benzonitrile 3u च
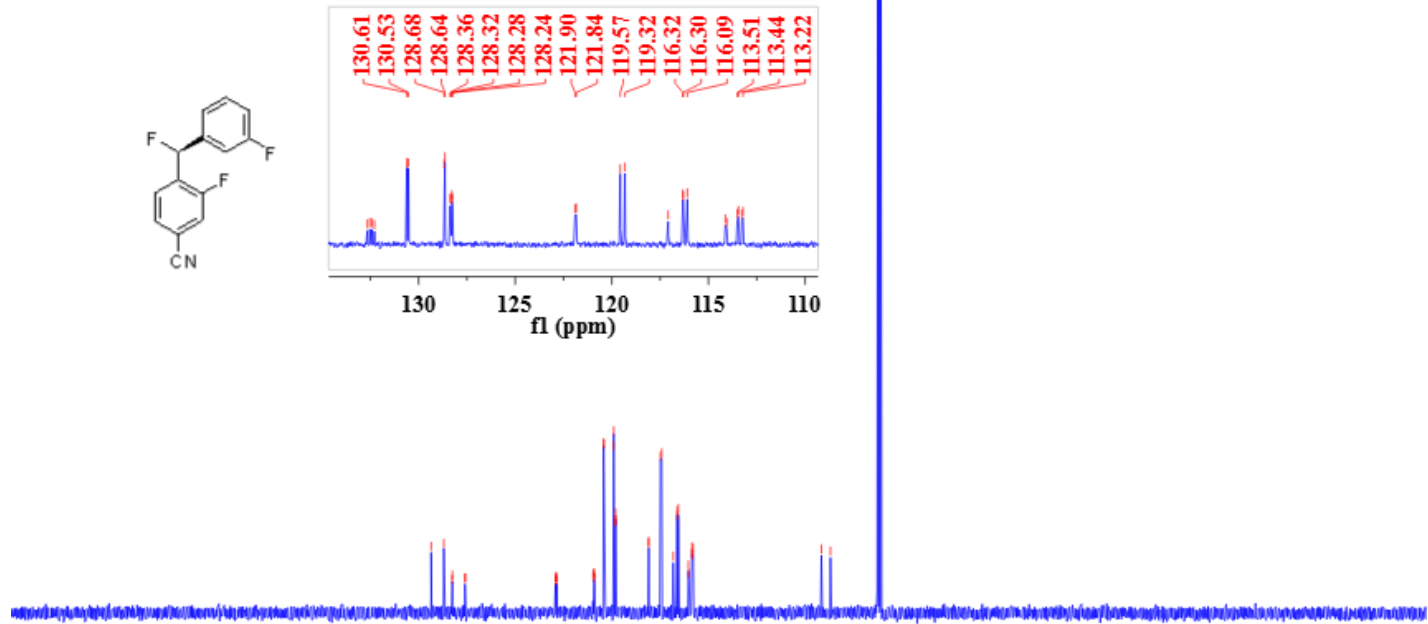

$\begin{array}{lllllllllllllllllllllllllllll}240 & 230 & 220 & 210 & 200 & 190 & 180 & 170 & 160 & 150 & 140 & 130 & 120 & 110 & 100 & 90 & 80 & 70 & 60 & 50 & 40 & 30 & 20 & 10 & 0 & -10 & -20 & -\end{array}$

${ }^{1} \mathrm{H}$ NMR (400 MHz, $\mathrm{CDCl}_{3}$ ) spectrum of (S)-1-(fluoro(phenyl)methyl)-4-(trifluoromethyl)benzene 3v

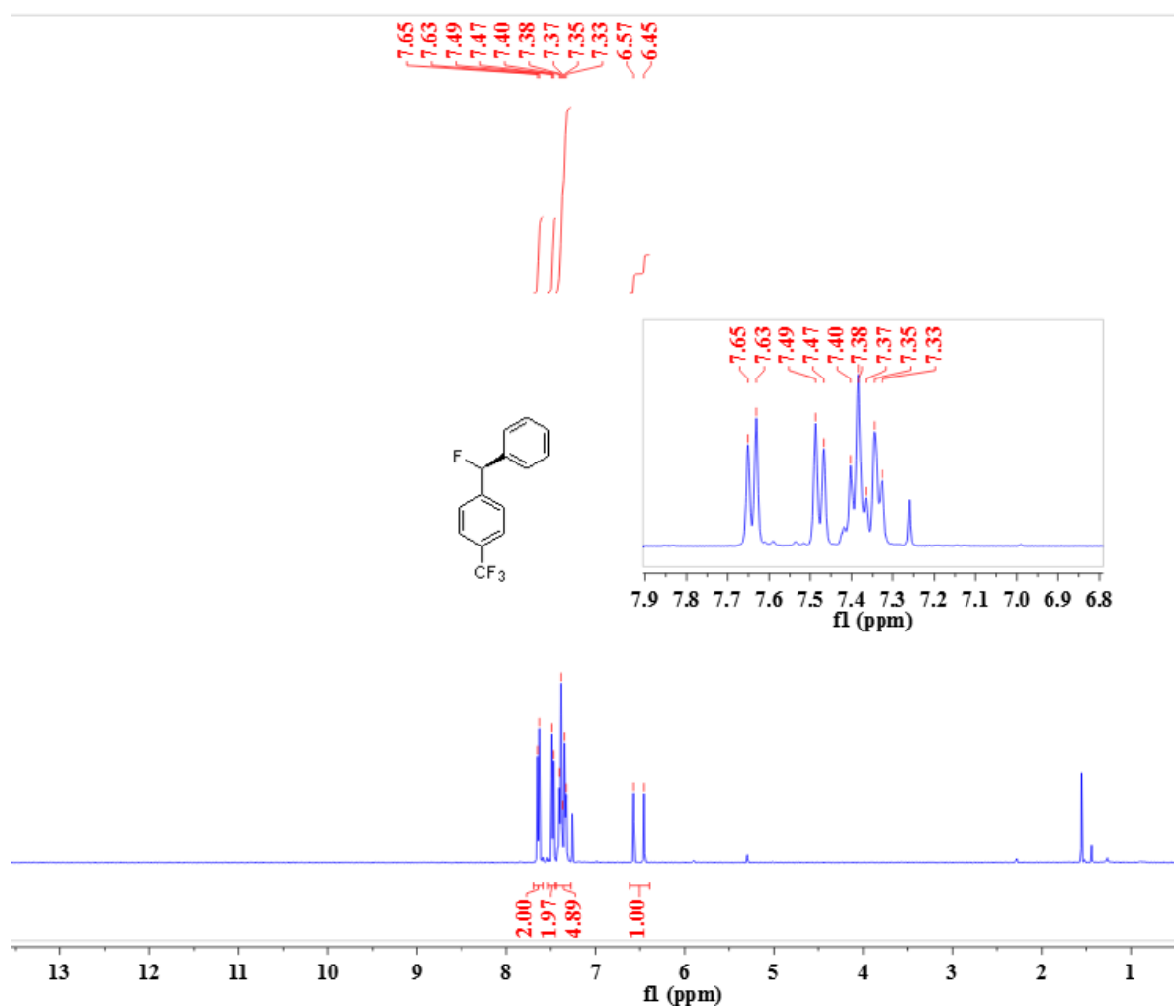


${ }^{19}$ F NMR (376 MHz, $\mathrm{CDCl}_{3}$ ) spectrum of

(S)-1-(fluoro(phenyl)methyl)-4-(trifluoromethyl)benzene 3v

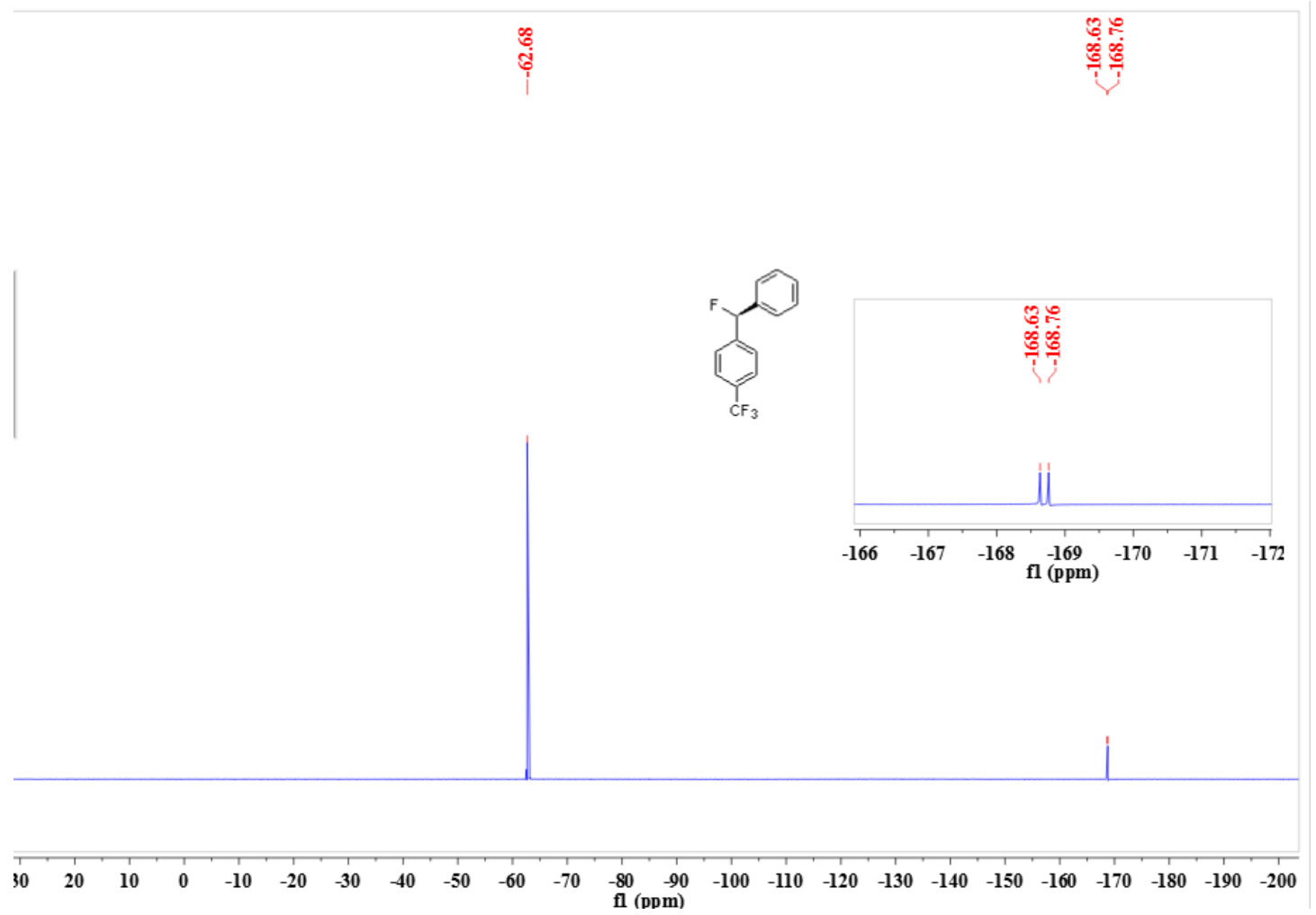

${ }^{13} \mathrm{C}$ NMR (101 MHz, $\left.\mathrm{CDCl}_{3}\right)$ spectrum of

(S)-1-(fluoro(phenyl)methyl)-4-(trifluoromethyl)benzene 3v

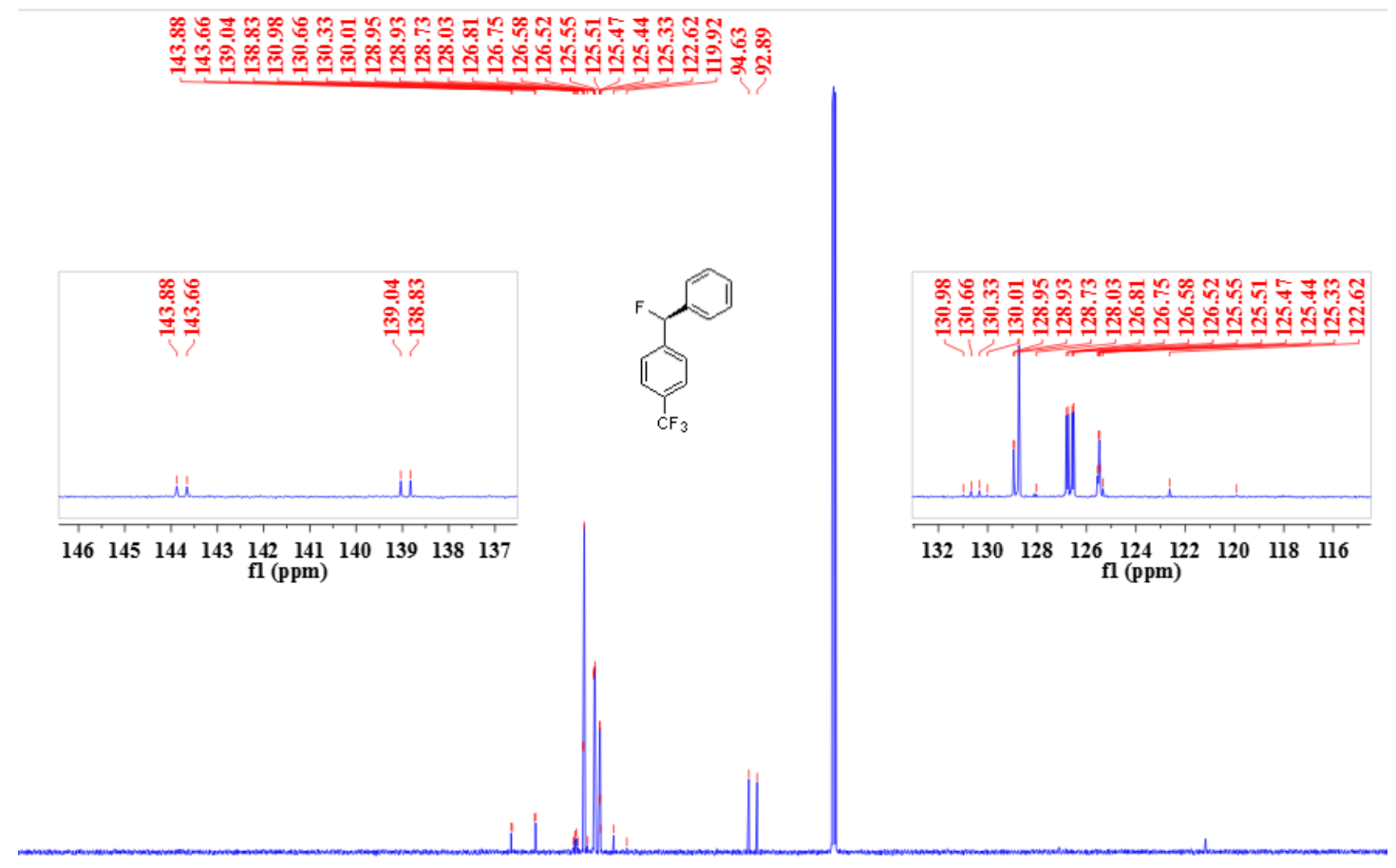

$\begin{array}{llllllllllllllllllllllllllll}240 & 230 & 220 & 210 & 200 & 190 & 180 & 170 & 160 & 150 & 140 & 130 & 120 & 110 & 100 & 90 & 80 & 70 & 60 & 50 & 40 & 30 & 20 & 10 & 0 & -10 & -20 & -30\end{array}$. 
${ }^{1} \mathrm{H}$ NMR (400 MHz, $\mathrm{CDCl}_{3}$ ) spectrum of (R)-1-fluoro-3-(fluoro(4-(trifluoromethyl)phenyl)methyl)benzene 3w

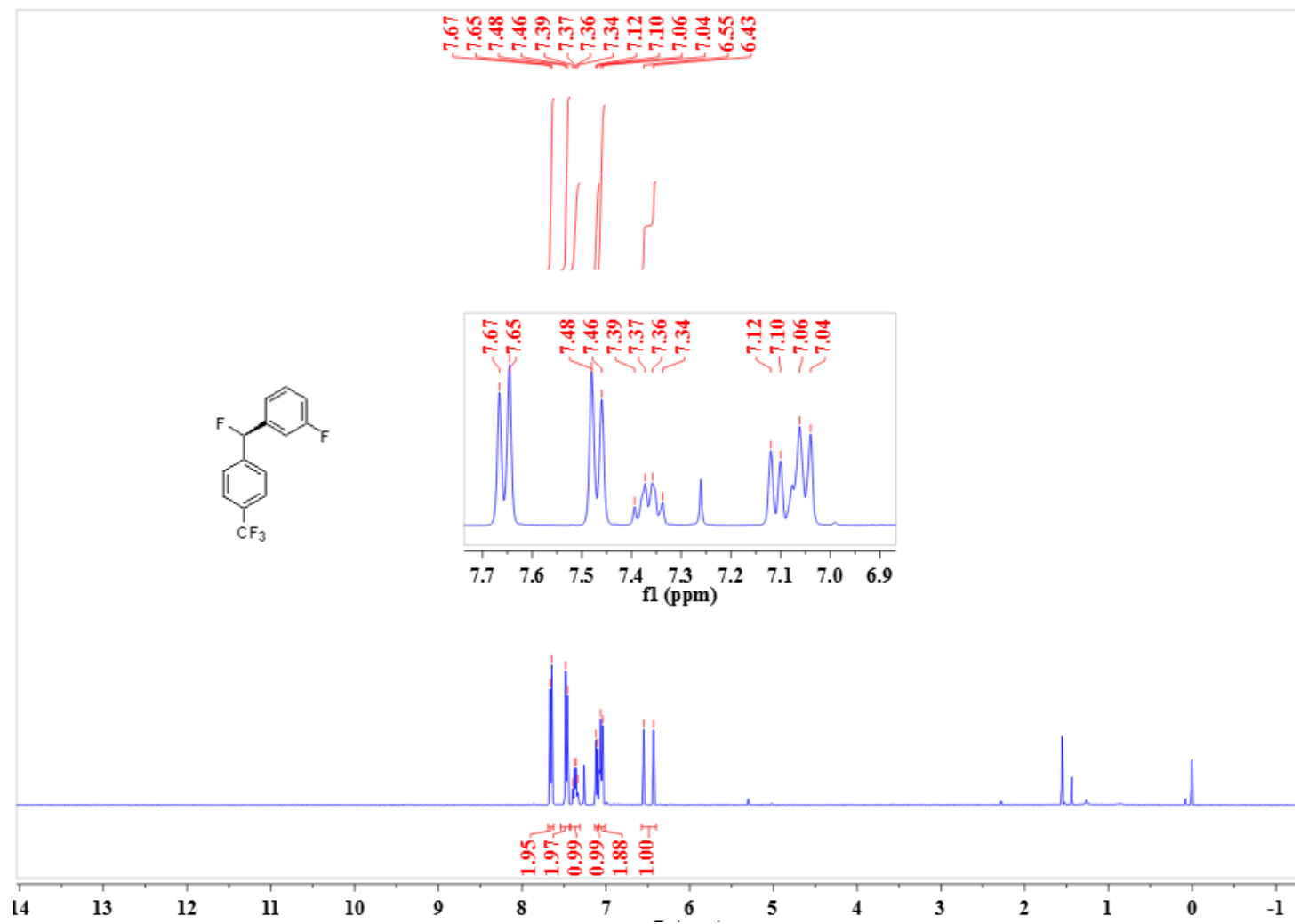

${ }^{19}$ F NMR (376 $\left.\mathrm{MHz}, \mathrm{CDCl}_{3}\right)$ spectrum of (R)-1-fluoro-3-(fluoro(4-(trifluoromethyl)phenyl)methyl)benzene 3w

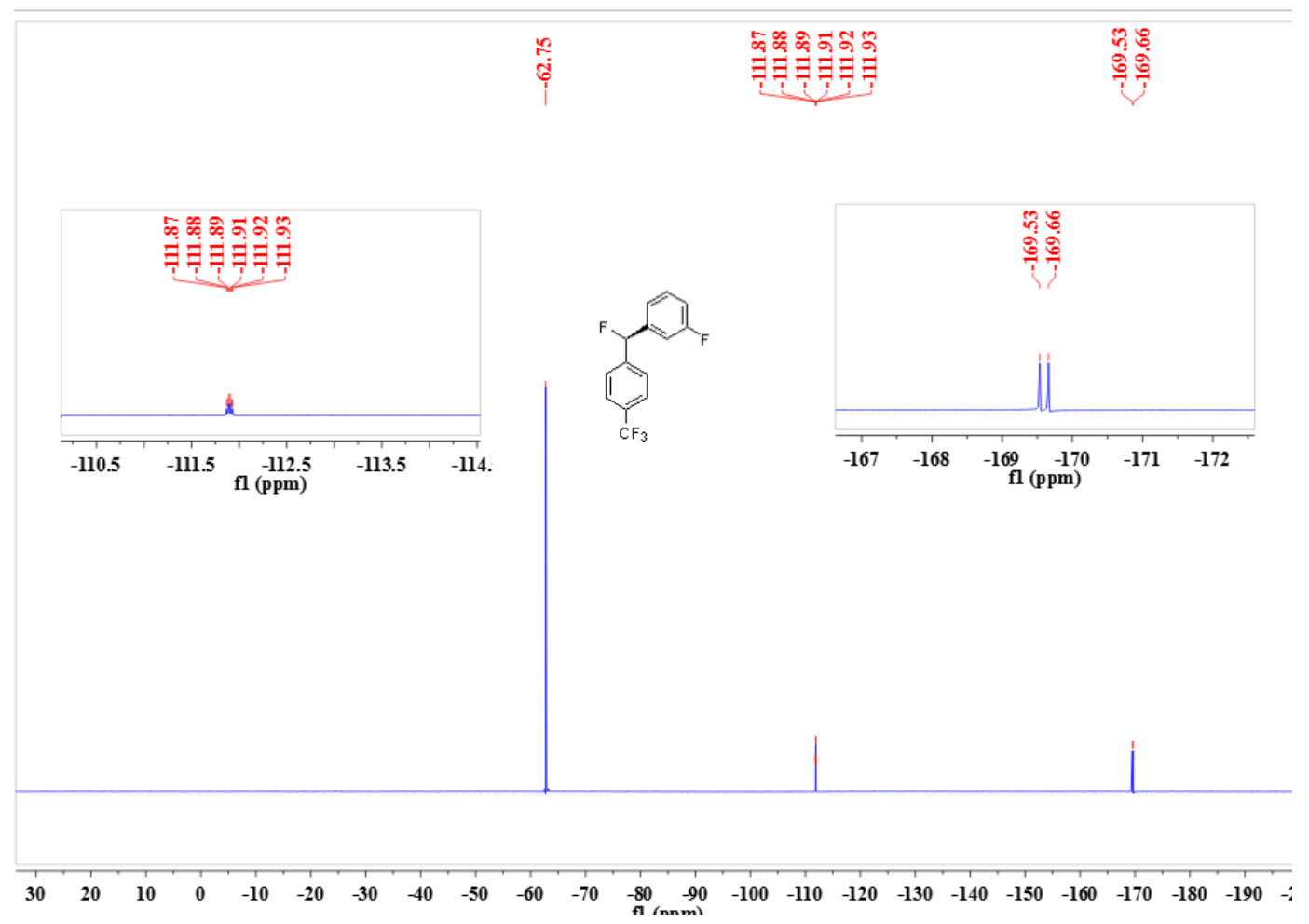


${ }^{13} \mathrm{C}$ NMR (101 MHz, $\left.\mathrm{CDCl}_{3}\right)$ spectrum of (R)-1-fluoro-3-(fluoro(4-(trifluoromethyl)phenyl)methyl)benzene 3w

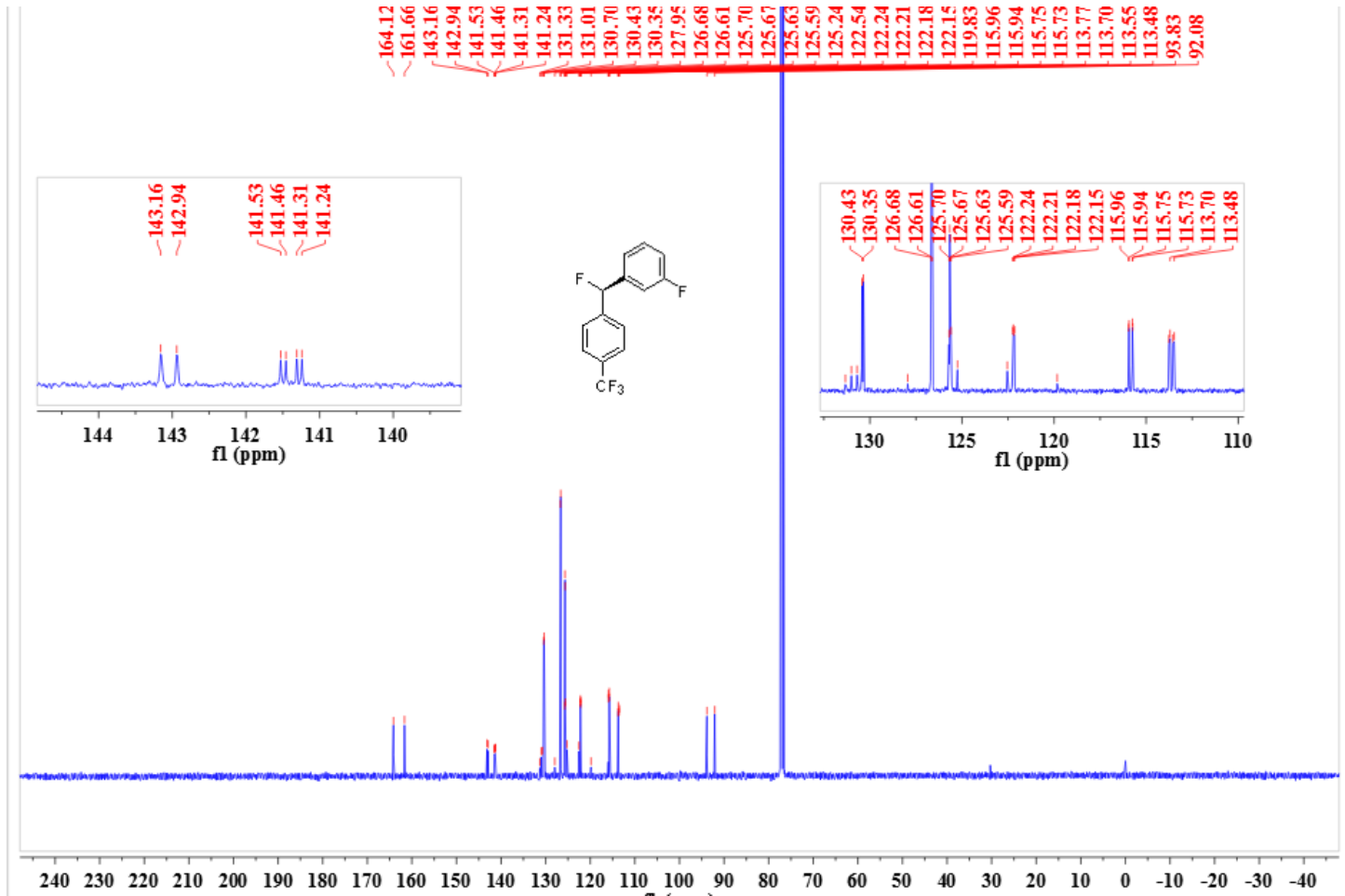

${ }^{1} \mathrm{H}$ NMR (400 MHz, $\mathrm{CDCl}_{3}$ ) spectrum of (S)-1-(fluoro(phenyl)methyl)-3-(trifluoromethyl)benzene 3x

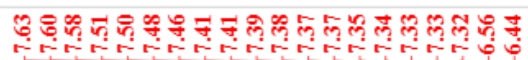
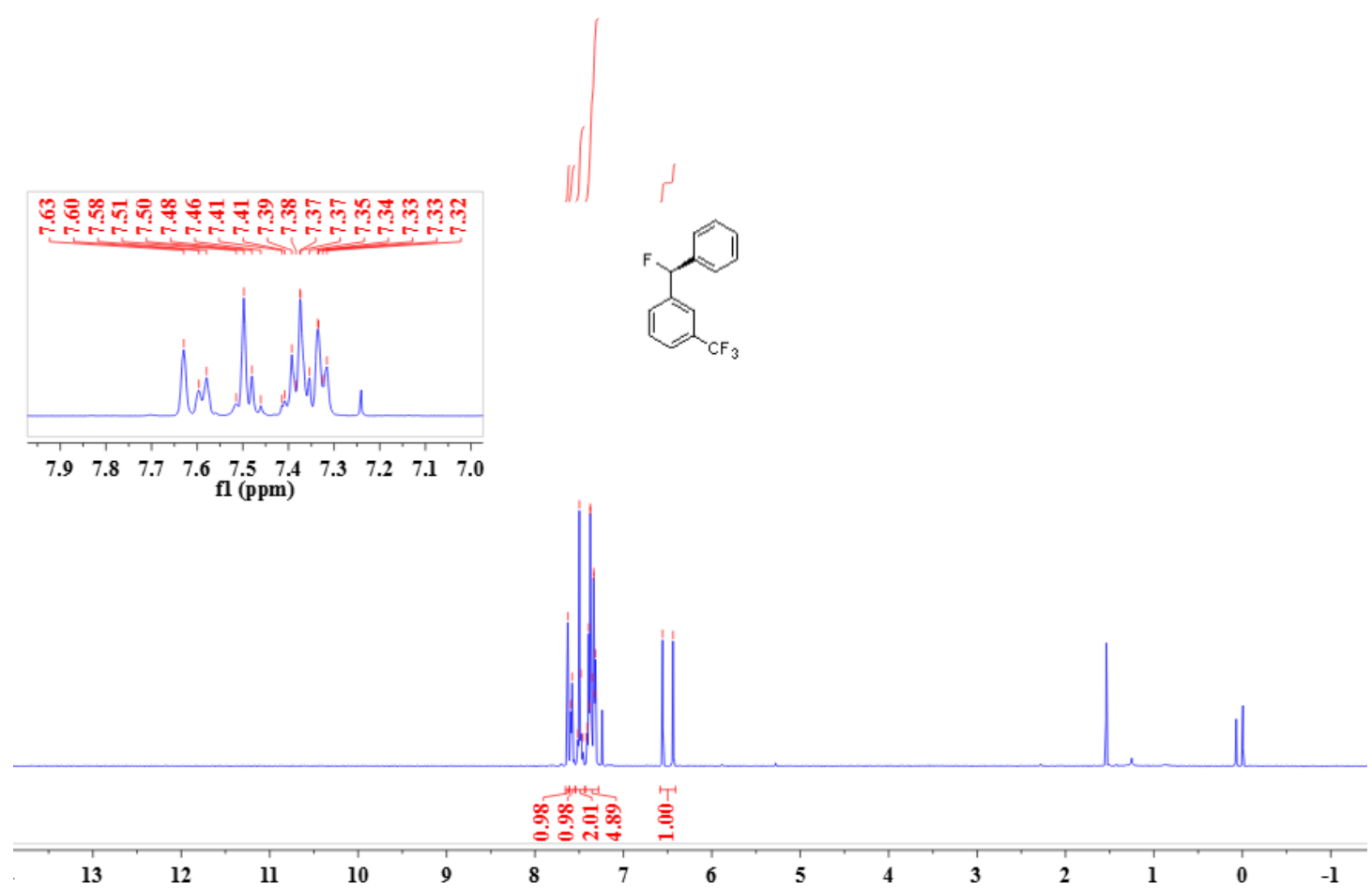
${ }^{19} \mathrm{~F}$ NMR (376 $\mathrm{MHz}, \mathrm{CDCl}_{3}$ ) spectrum of

(S)-1-(fluoro(phenyl)methyl)-3-(trifluoromethyl)benzene 3x
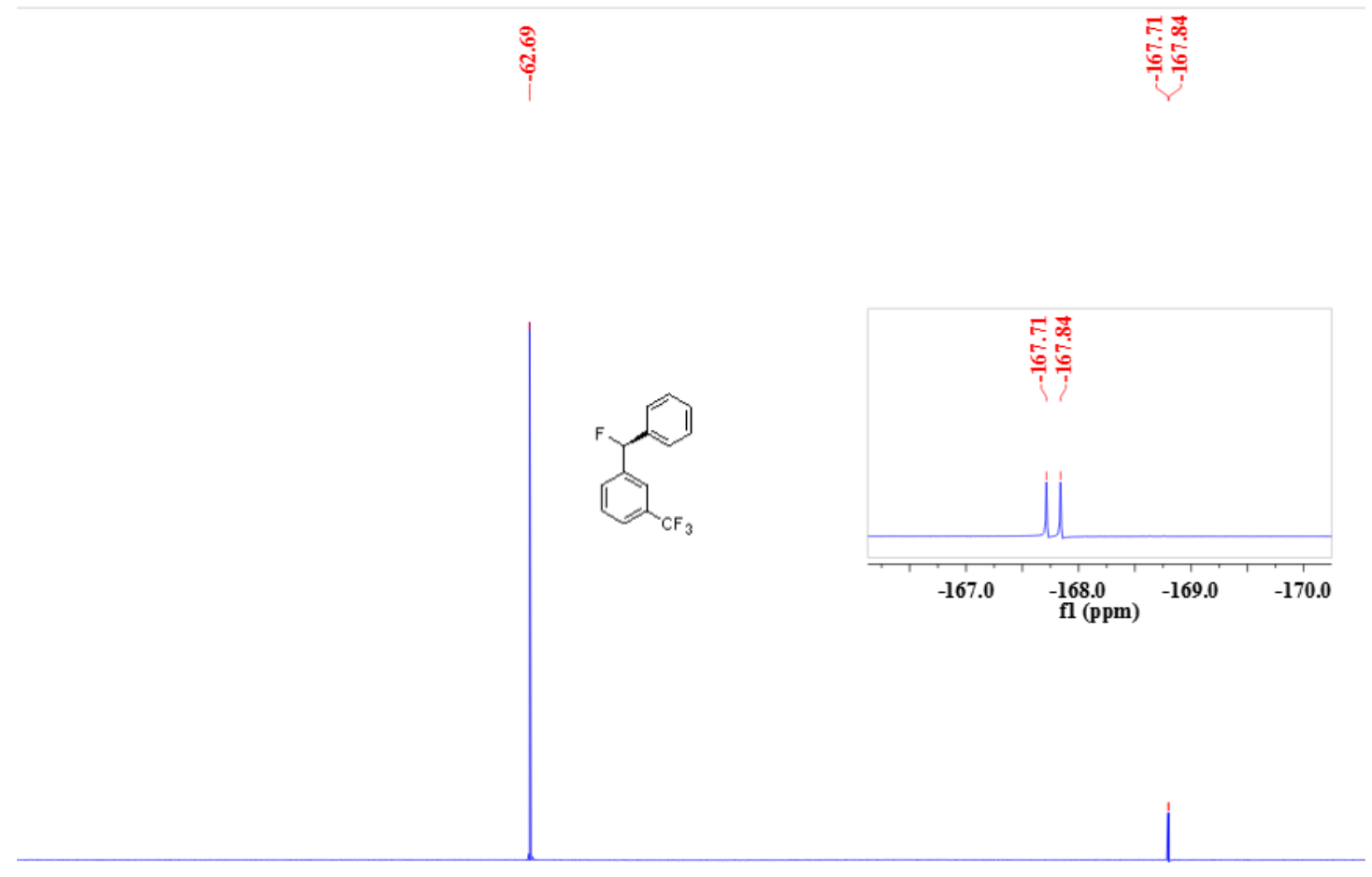

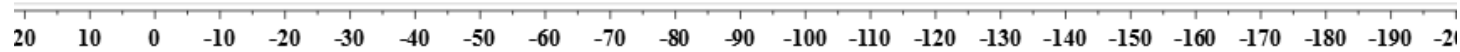

${ }^{13} \mathrm{C}$ NMR (101 MHz, $\left.\mathrm{CDCl}_{3}\right)$ spectrum of

(S)-1-(fluoro(phenyl)methyl)-3-(trifluoromethyl)benzene 3x

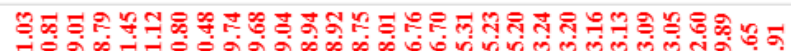

守
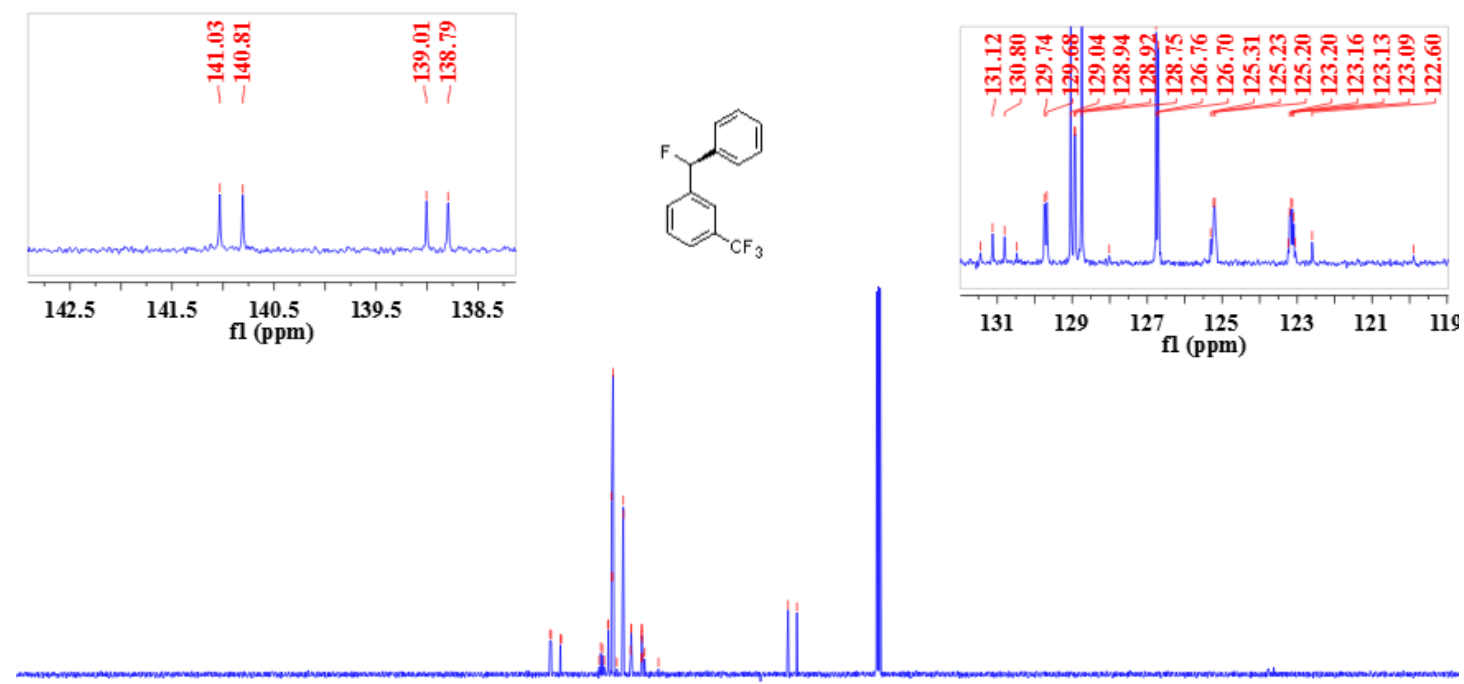

$\begin{array}{llllllllllllllllllllllllllll}240 & 230 & 220 & 210 & 200 & 190 & 180 & 170 & 160 & 150 & 140 & 130 & 120 & 110 & 100 & 90 & 80 & 70 & 60 & 50 & 40 & 30 & 20 & 10 & 0 & -10 & -20 & -30\end{array}$ 
${ }^{1} \mathrm{H}$ NMR (400 MHz, $\mathrm{CDCl}_{3}$ ) spectrum of

(R)-1-((4-bromophenyl)fluoromethyl)-3-fluorobenzene 3y

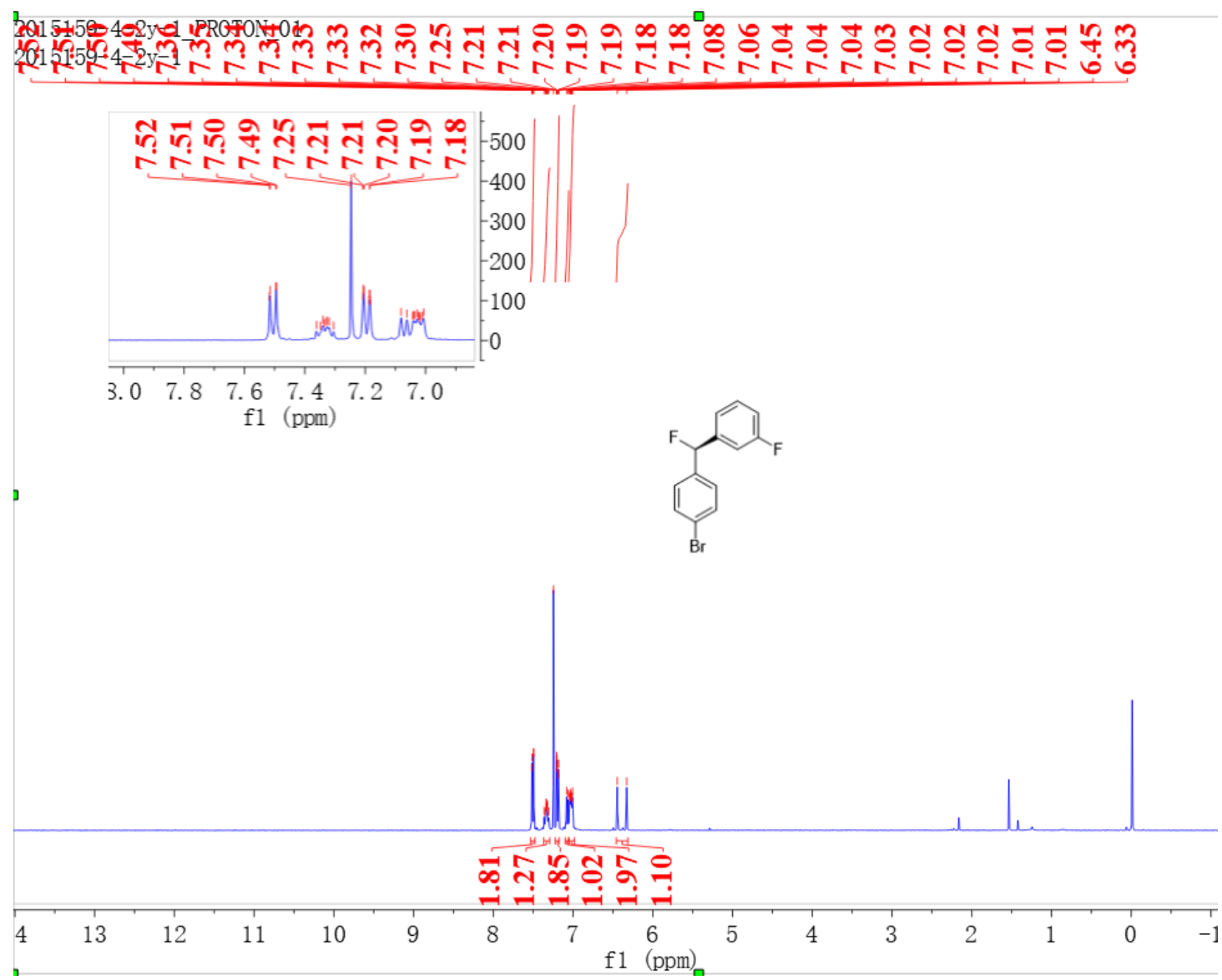

${ }^{19} \mathrm{~F}$ NMR (376 $\left.\mathrm{MHz}, \mathrm{CDCl}_{3}\right)$ spectrum of

(R)-1-((4-bromophenyl)fluoromethyl)-3-fluorobenzene 3y

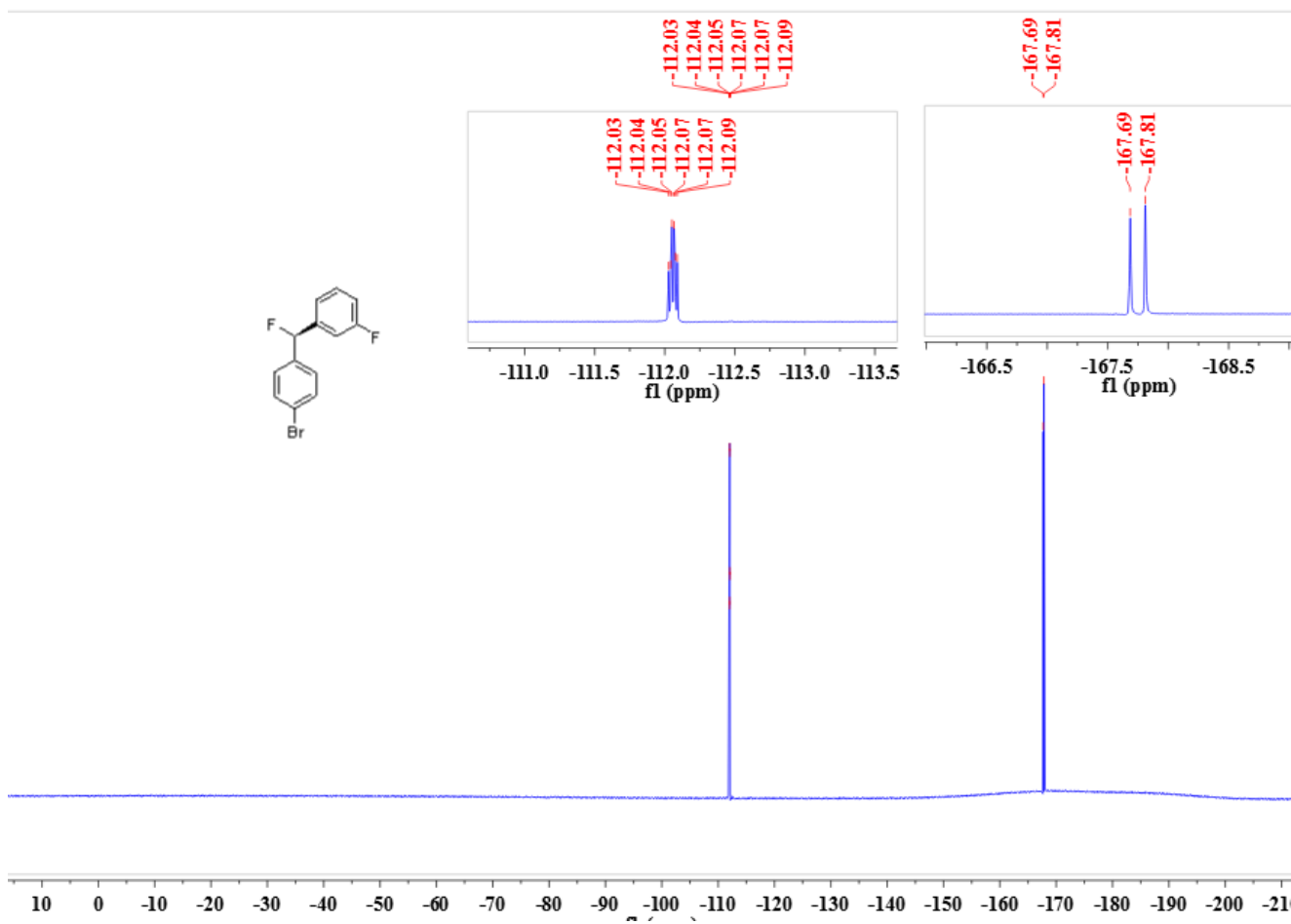


${ }^{13} \mathrm{C}$ NMR (101 MHz, $\left.\mathrm{CDCl}_{3}\right)$ spectrum of (R)-1-((4-bromophenyl)fluoromethyl)-3-fluorobenzene 3y
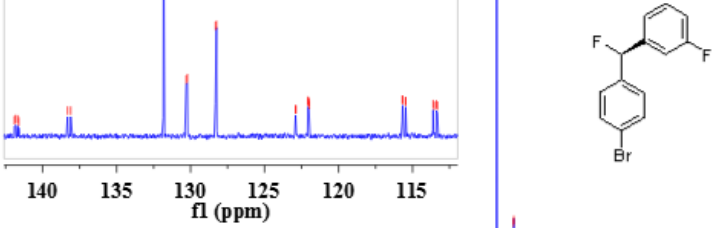

$\begin{array}{llllllllllllllllllllllllllll}240 & 230 & 220 & 210 & 200 & 190 & 180 & 170 & 160 & 150 & 140 & 130 & 120 & 110 & 100 & 90 & 80 & 70 & 60 & 50 & 40 & 30 & 20 & 10 & 0 & -10 & -20 & -3\end{array}$ fl (ppm)

${ }^{1} \mathrm{H}$ NMR (400 MHz, $\mathrm{CDCl}_{3}$ ) spectrum of (R)-1-((4-bromophenyl)fluoromethyl)-3-fluorobenzene 3z

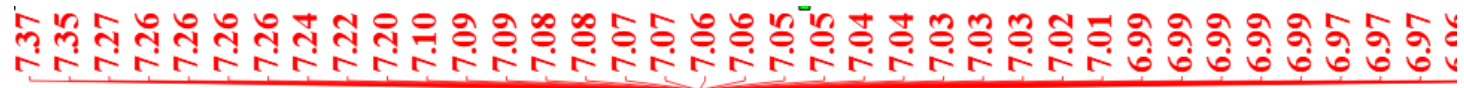

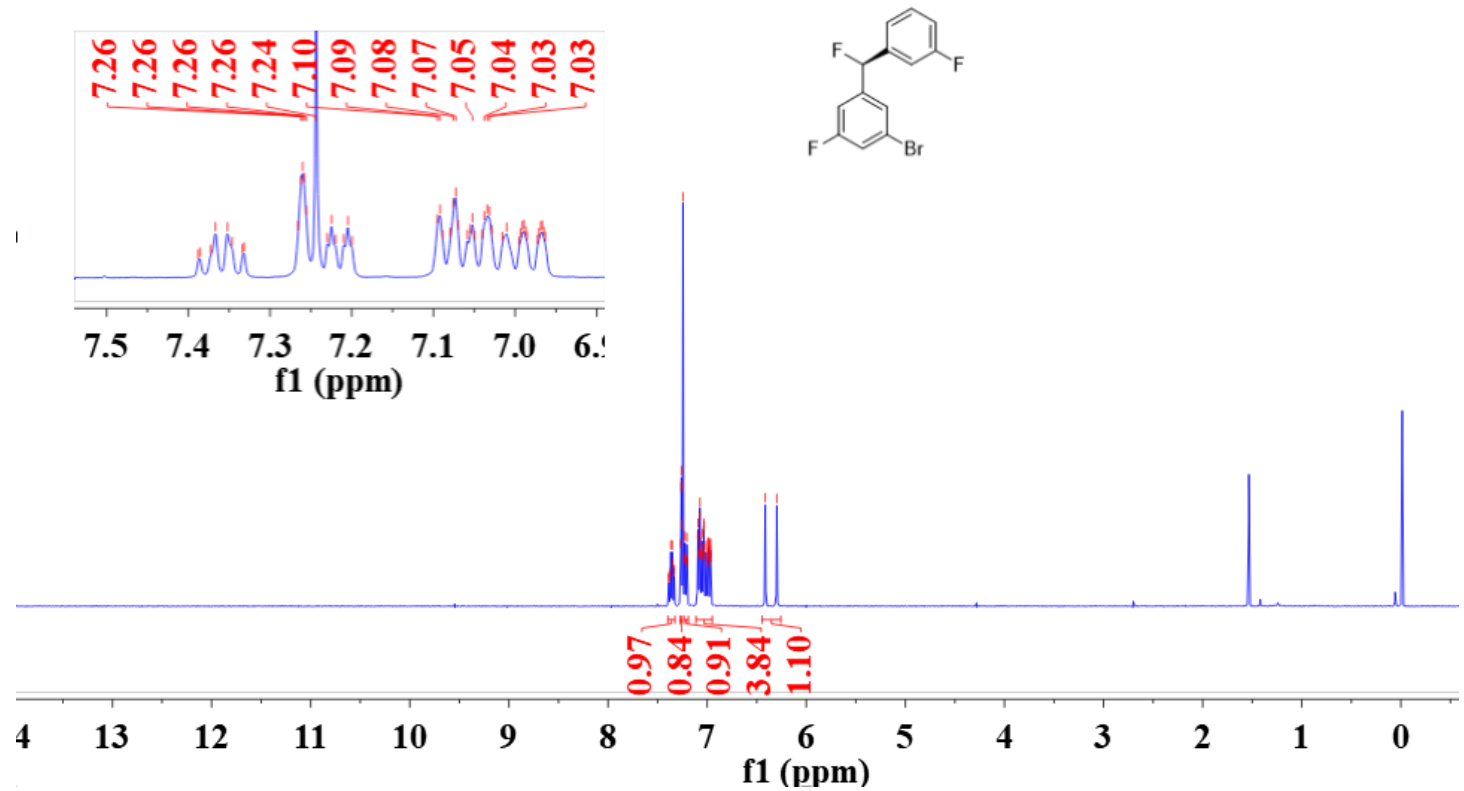


${ }^{19} \mathrm{~F}$ NMR (376 $\mathrm{MHz}, \mathrm{CDCl}_{3}$ ) spectrum of (R)-1-((4-bromophenyl)fluoromethyl)-3-fluorobenzene 3z

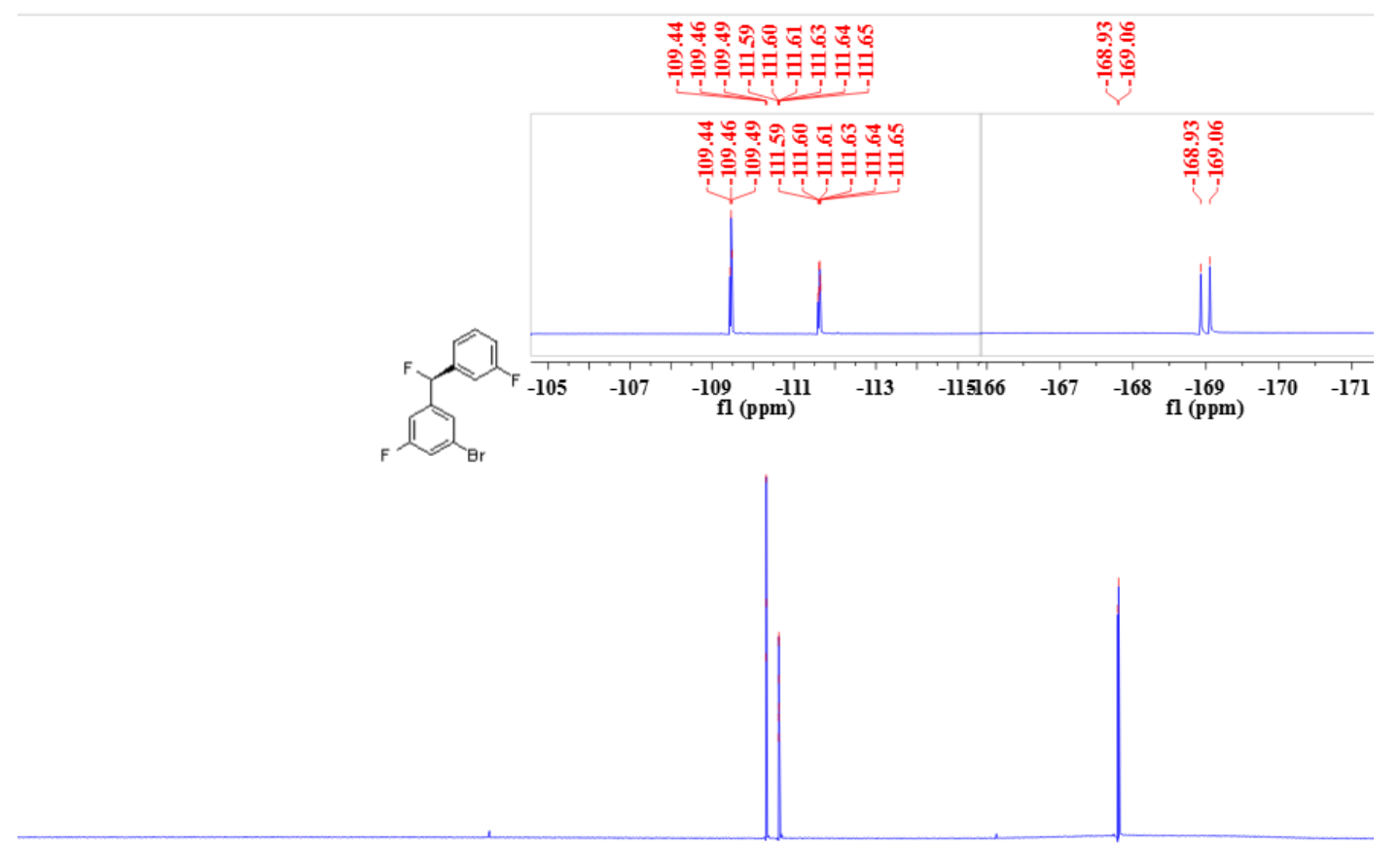

$\begin{array}{llllllllllllllllllllllllllllllll}10 & 0 & -10 & -20 & -30 & -40 & -50 & -60 & -70 & -80 & -90 & -100 & -110 & -120 & -130 & -140 & -150 & -160 & -170 & -180 & -190 & -200 & -211\end{array}$

${ }^{13} \mathrm{C}$ NMR (101 MHz, $\left.\mathrm{CDCl}_{3}\right)$ spectrum of (R)-1-((4-bromophenyl)fluoromethyl)-3-fluorobenzene $3 z$

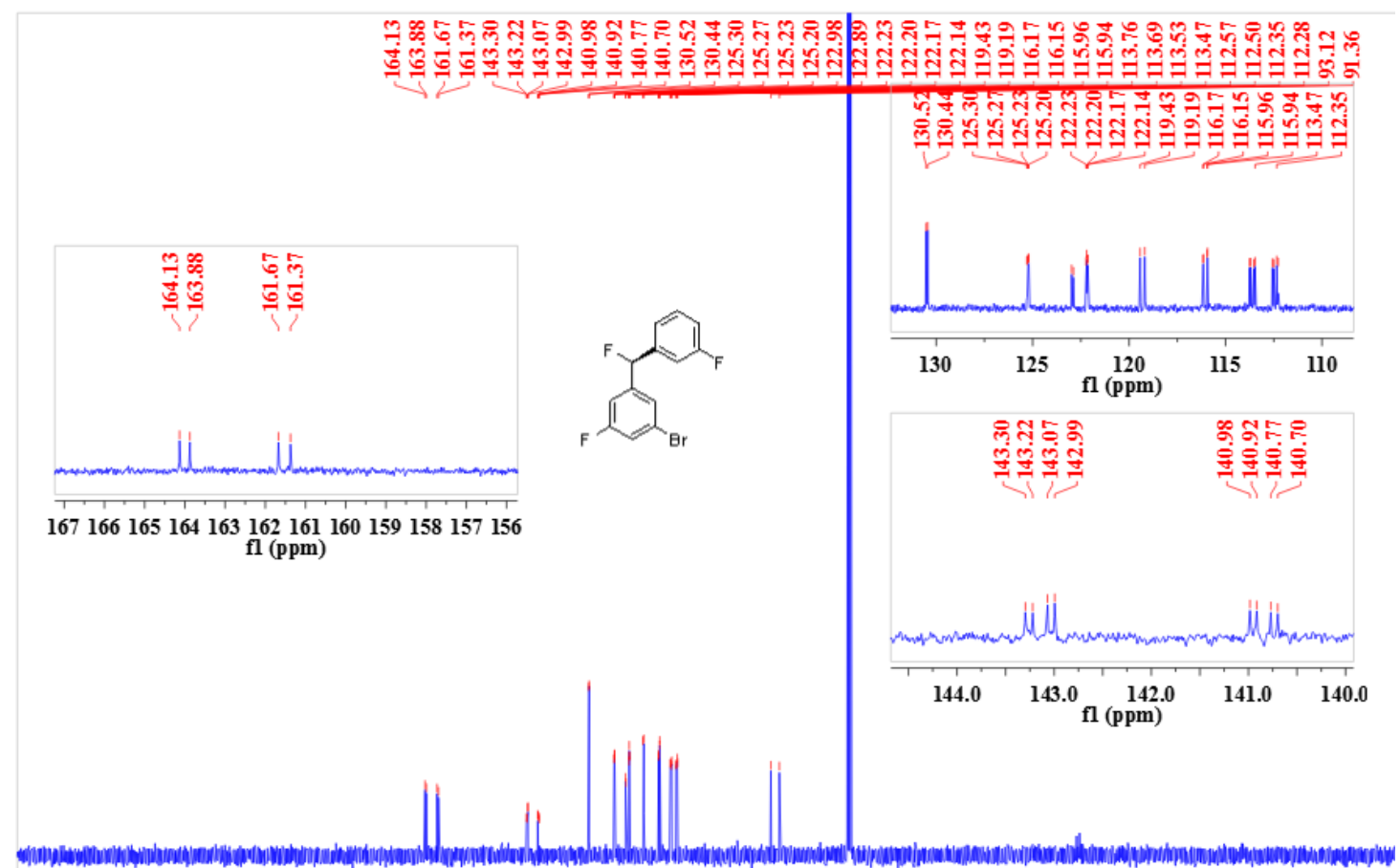

$\begin{array}{llllllllllllllllllllllllllll}240 & 230 & 220 & 210 & 200 & 190 & 180 & 170 & 160 & 150 & 140 & 130 & 120 & 110 & 100 & 90 & 80 & 70 & 60 & 50 & 40 & 30 & 20 & 10 & 0 & -10 & -20 & -30\end{array}$ 
${ }^{1} \mathrm{H}$ NMR (400 $\mathrm{MHz}, \mathrm{CDCl}_{3}$ ) spectrum of

(S)-2-bromo-1-chloro-4-(fluoro(phenyl)methyl)benzene 3aa

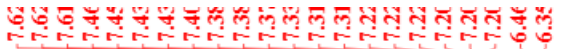
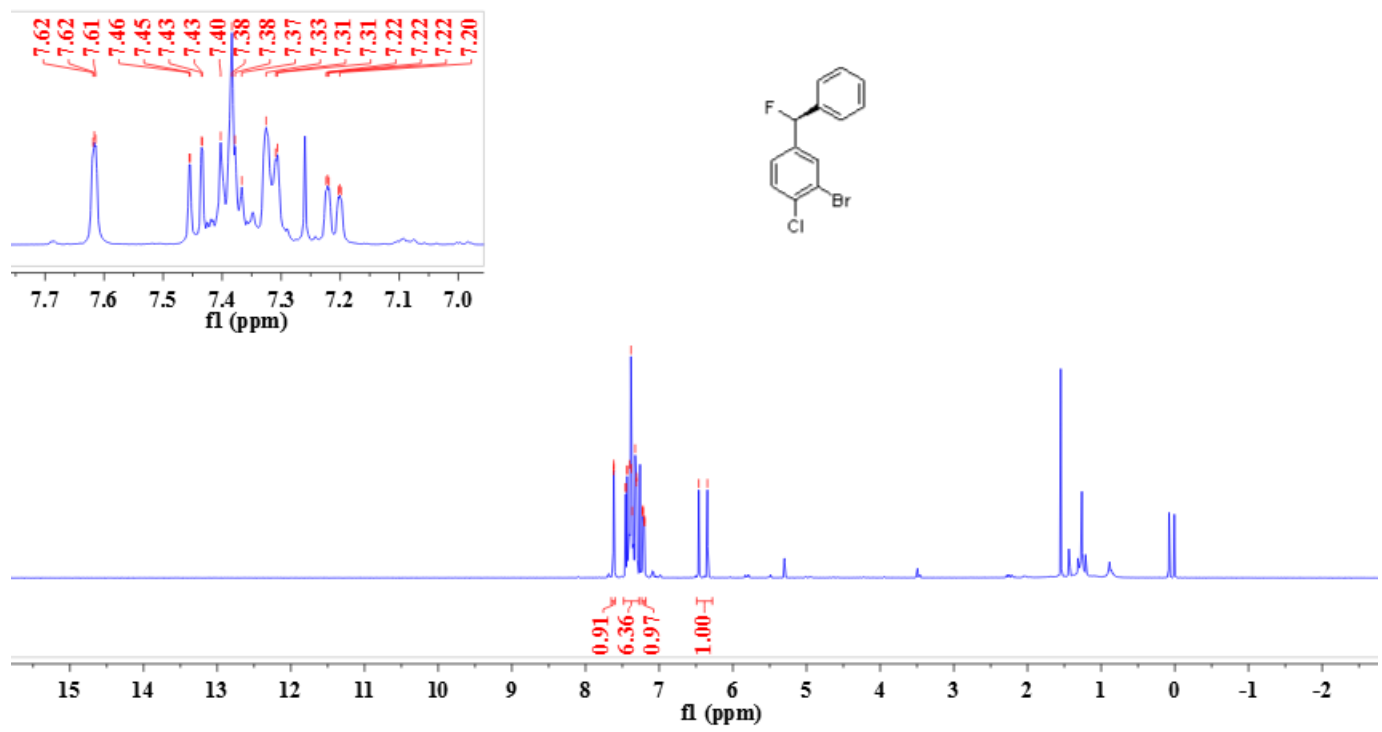

${ }^{19} \mathrm{~F}$ NMR (376 MHz, $\left.\mathrm{CDCl}_{3}\right)$ spectrum of (S)-2-bromo-1-chloro-4-(fluoro(phenyl)methyl)benzene 3aa
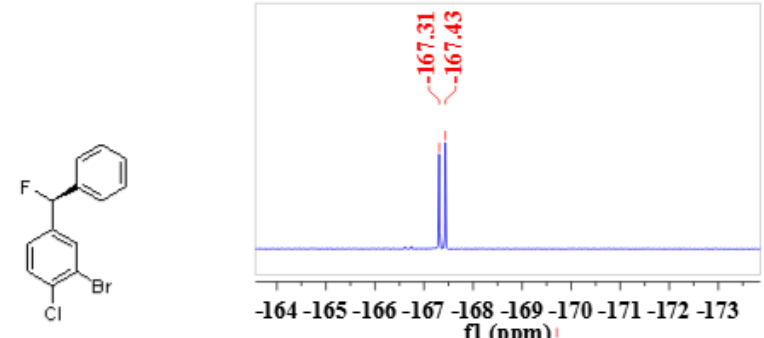

$-164-165-166-167-168-169-170-171-172-173$ 
${ }^{13} \mathrm{C}$ NMR (101 MHz, $\left.\mathrm{CDCl}_{3}\right)$ spectrum of

(S)-2-bromo-1-chloro-4-(fluoro(phenyl)methyl)benzene 3aa

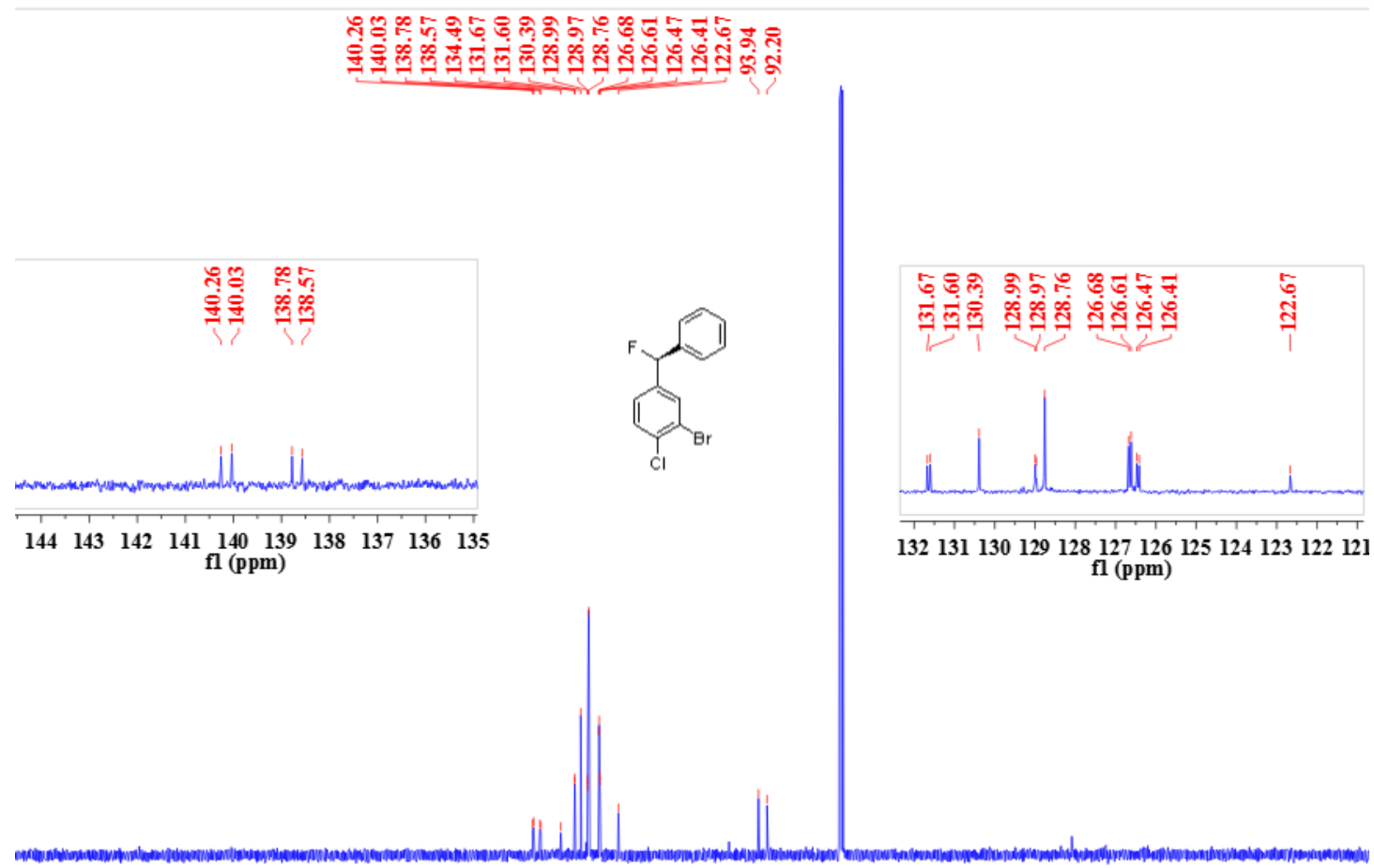

$\begin{array}{llllllllllllllllllllllllllll}240 & 230 & 220 & 210 & 200 & 190 & 180 & 170 & 160 & 150 & 140 & 130 & 120 & 110 & 100 & 90 & 80 & 70 & 60 & 50 & 40 & 30 & 20 & 10 & 0 & -10 & -20 & -3\end{array}$

${ }^{1} \mathrm{H}$ NMR (400 MHz, $\mathrm{CDCl}_{3}$ ) spectrum of (R)-1-((4-chlorophenyl)fluoromethyl)-3-fluorobenzene 3ab

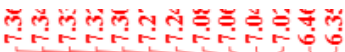
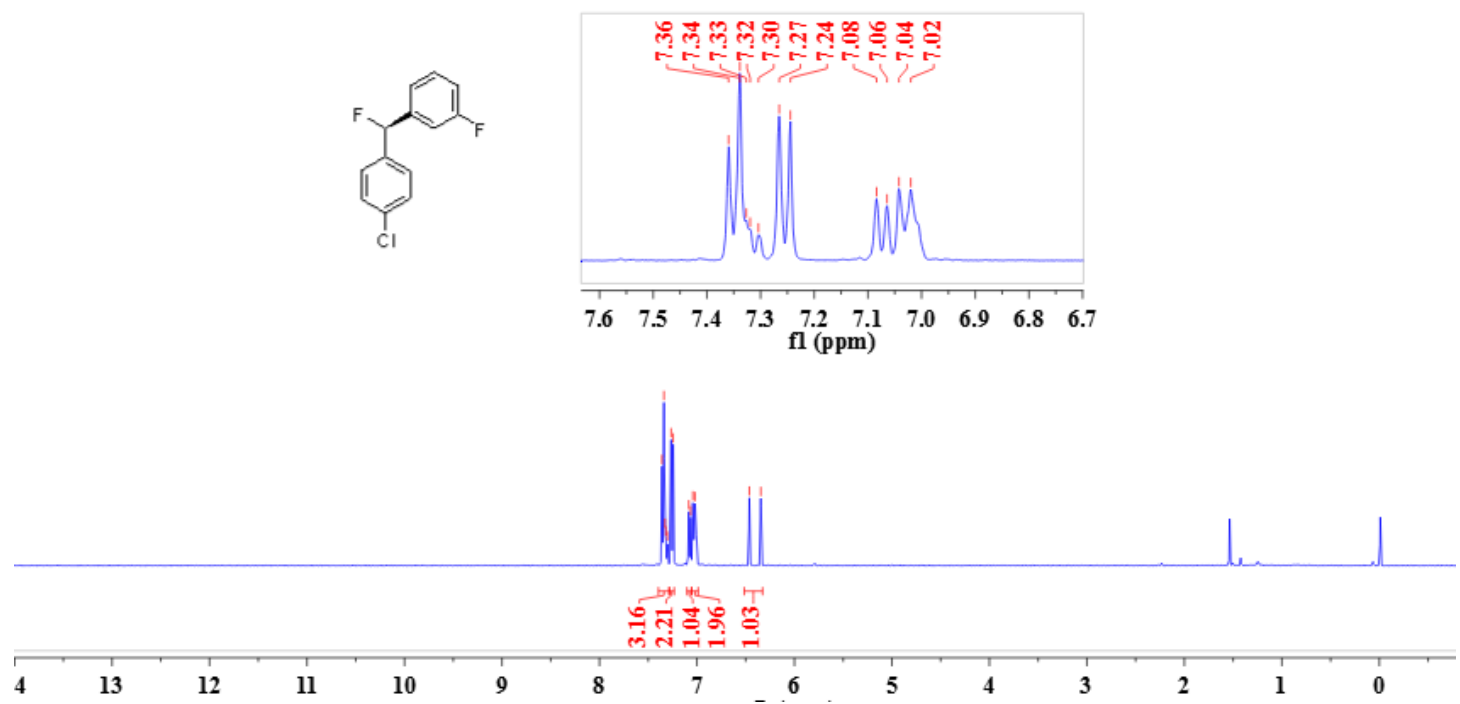
${ }^{19}$ F NMR (376 MHz, $\mathrm{CDCl}_{3}$ ) spectrum of (R)-1-((4-chlorophenyl)fluoromethyl)-3-fluorobenzene 3ab

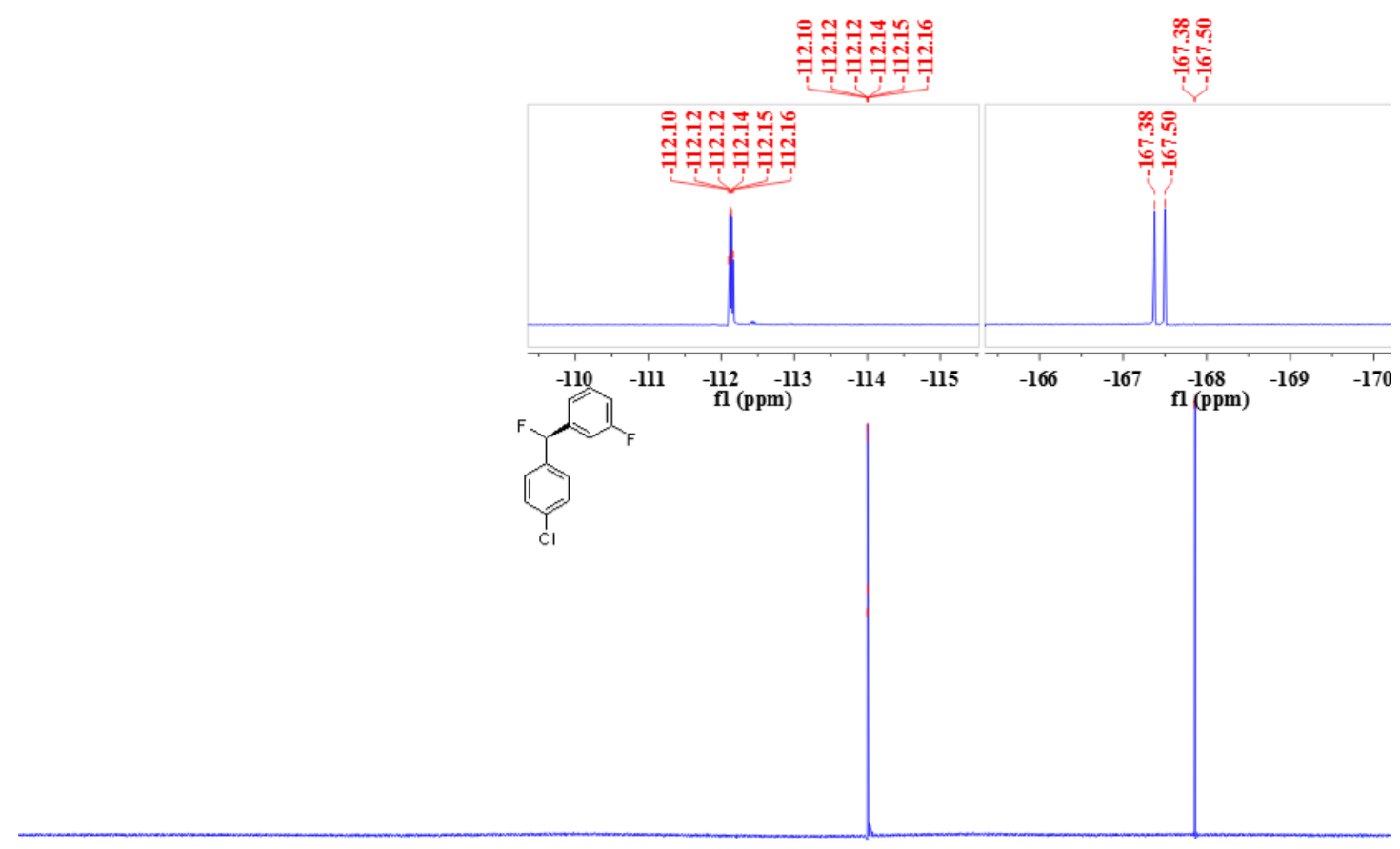

$\begin{array}{llllllllllllllllllllllllllllllll} & 30 & 20 & 10 & 0 & -10 & -20 & -30 & -40 & -50 & -60 & -70 & -80 & -90 & -100 & -110 & -120 & -130 & -140 & -150 & -160 & -170 & -180 & -190 & -2(\end{array}$

${ }^{13} \mathrm{C}$ NMR (101 MHz, $\left.\mathrm{CDCl}_{3}\right)$ spectrum of

(R)-1-((4-chlorophenyl)fluoromethyl)-3-fluorobenzene 3ab

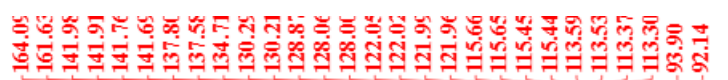

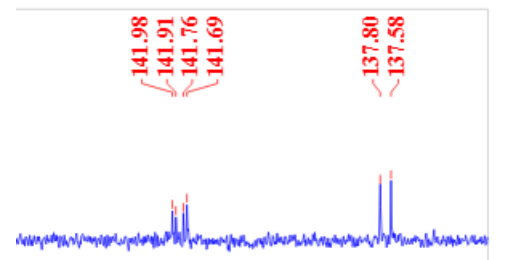

$\begin{array}{lllllllllll}45 & 144 & 143 & 142 & 141 & 140 & 139 & 138 & 137 & 136\end{array}$ fl (ppm)

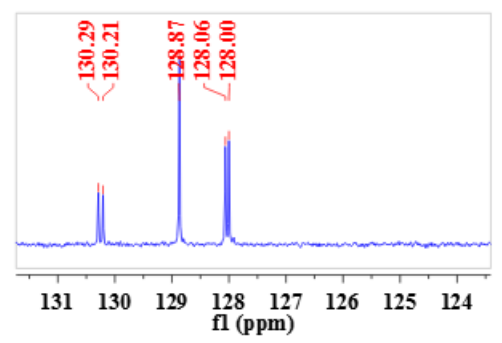

$\begin{array}{lllllll}131 & 130 & 129 & \begin{array}{l}128 \\ \text { fl (ppm) }\end{array} & 126 & 125 & 124\end{array}$<smiles>Fc1cccc(C(F)c2ccc(Cl)cc2)c1</smiles>

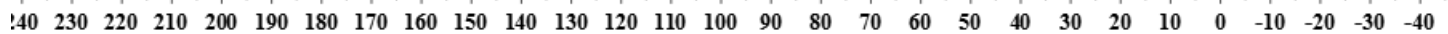


${ }^{1} \mathrm{H}$ NMR (400 MHz, $\left.\mathrm{CDCl}_{3}\right)$ spectrum of (S)-1,2-dichloro-4-(fluoro(3-fluorophenyl)methyl)benzene 3ac

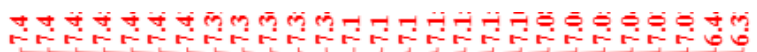
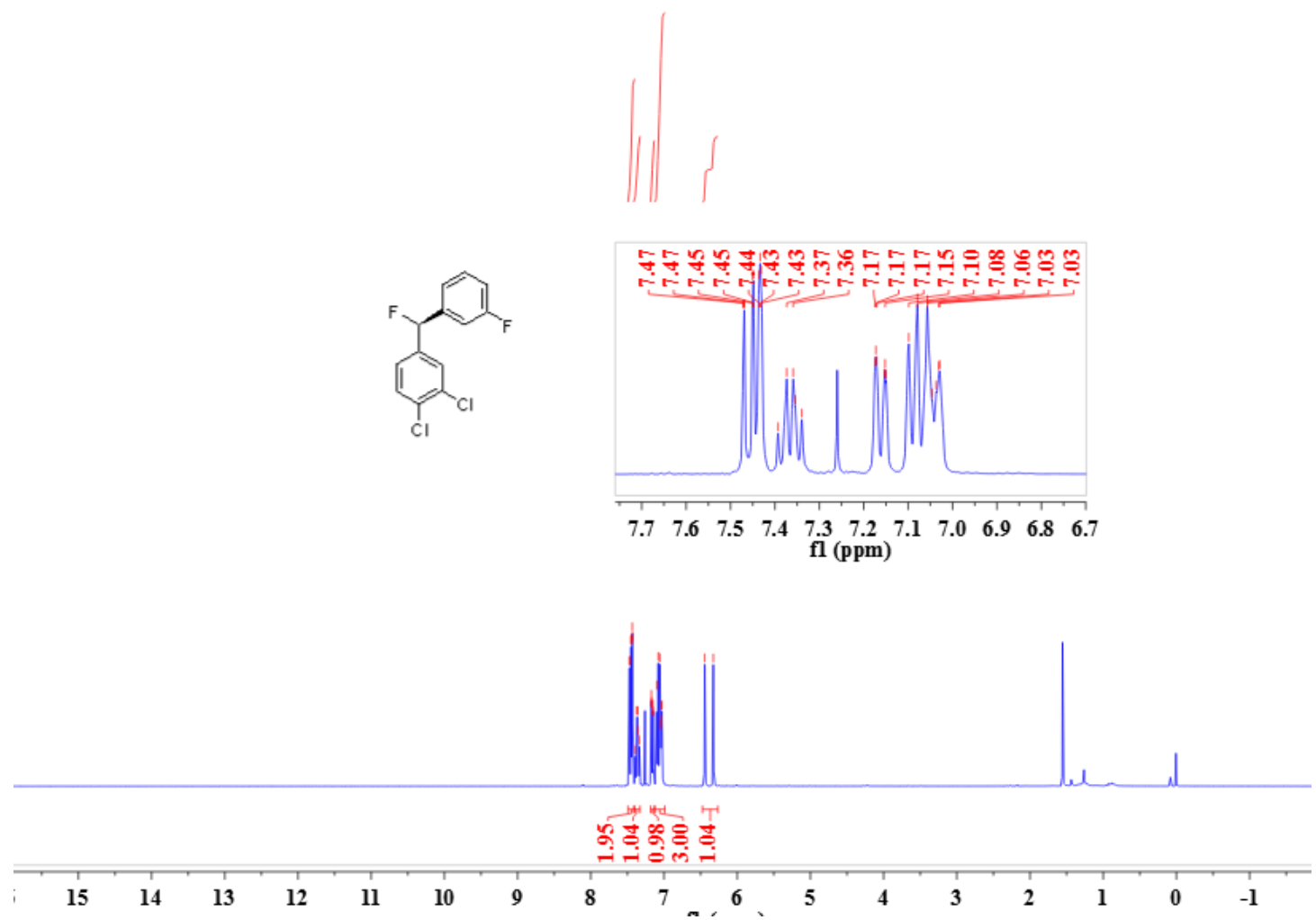

${ }^{19} \mathrm{~F}$ NMR (376 MHz, $\left.\mathrm{CDCl}_{3}\right)$ spectrum of (S)-1,2-dichloro-4-(fluoro(3-fluorophenyl)methyl)benzene 3ac

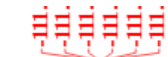
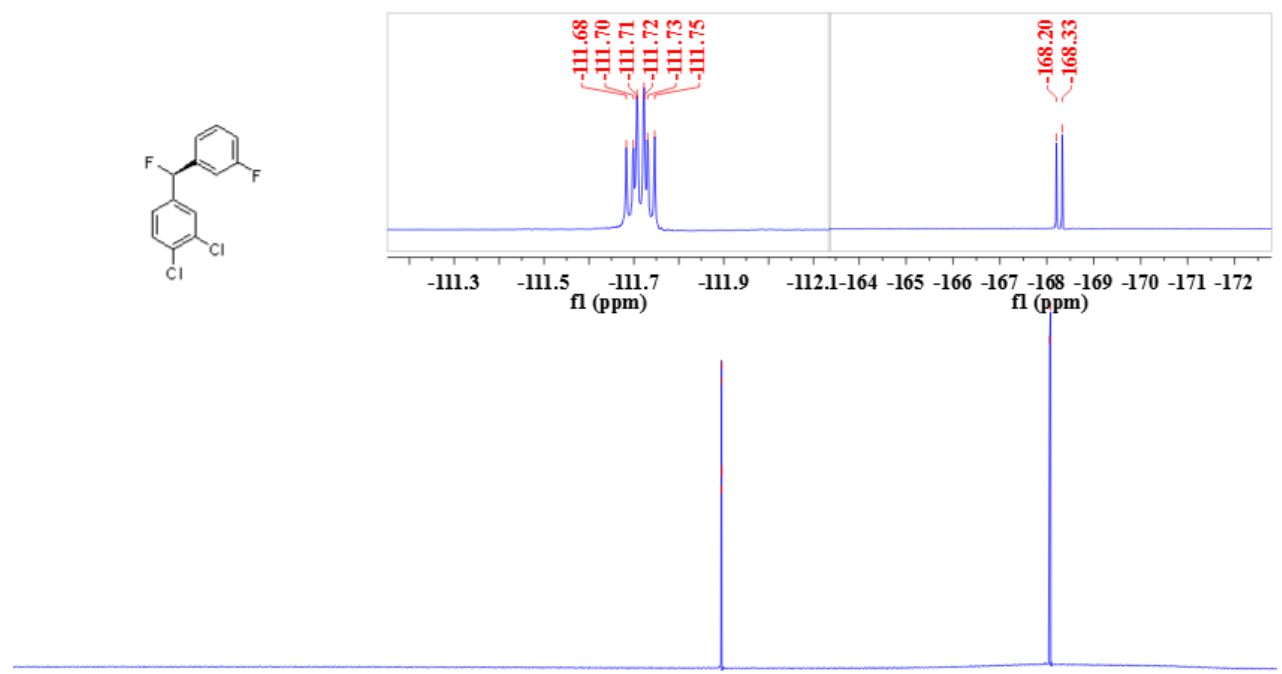

$\begin{array}{lllllllllllllllllllllll}0 & 0 & -10 & -20 & -30 & -40 & -50 & -60 & -70 & -80 & -90 & -100 & -110 & -120 & -130 & -140 & -150 & -160 & -170 & -180 & -190 & -200 & \end{array}$ 
${ }^{13} \mathrm{C}$ NMR (101 MHz, $\left.\mathrm{CDCl}_{3}\right)$ spectrum of (S)-1,2-dichloro-4-(fluoro(3-fluorophenyl)methyl)benzene 3ac

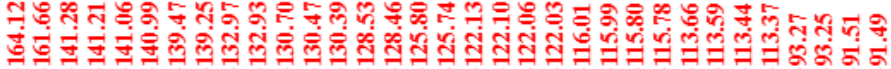
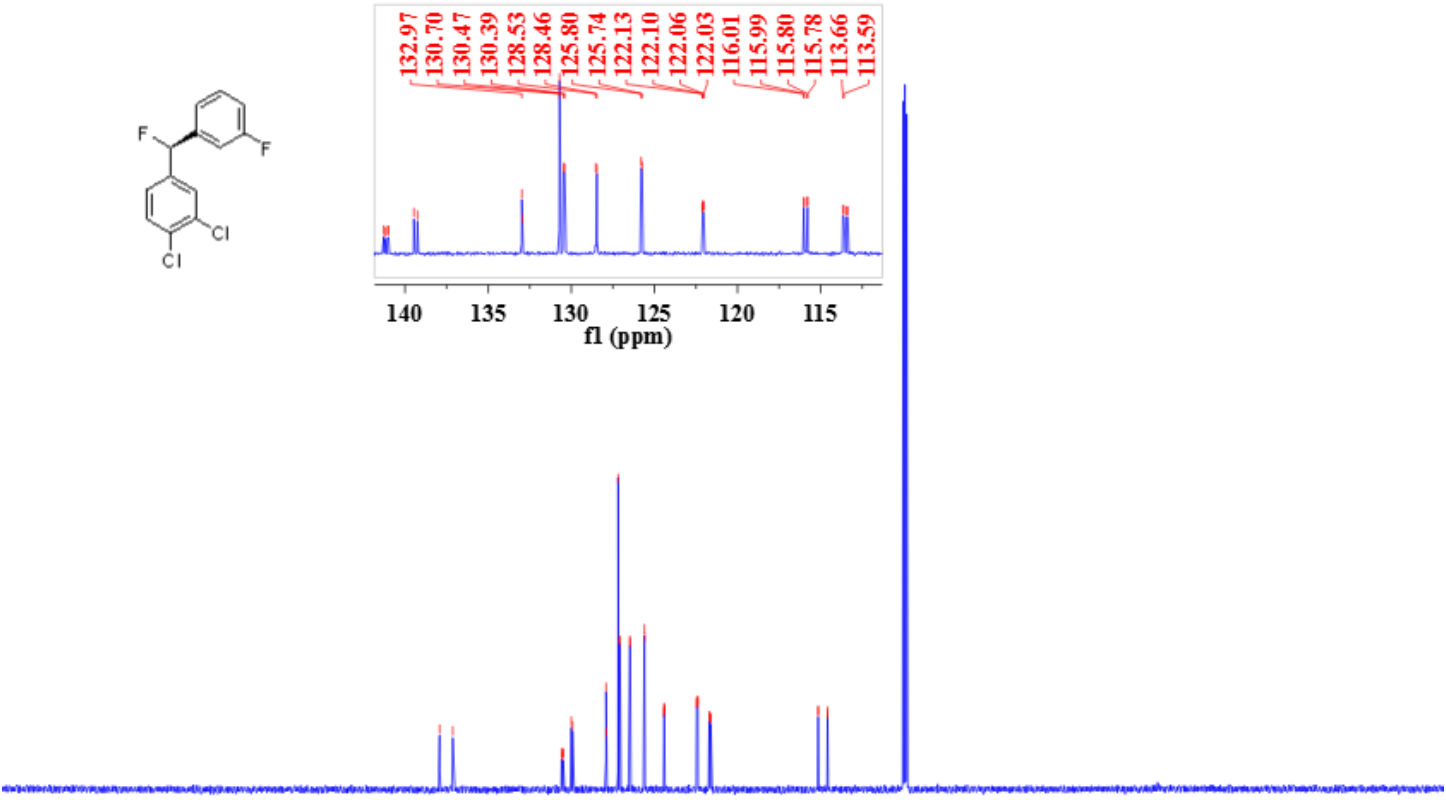

$\begin{array}{llllllllllllllllllllllllllllll}240 & 230 & 220 & 210 & 200 & 190 & 180 & 170 & 160 & 150 & 140 & 130 & 120 & 110 & 100 & 90 & 80 & 70 & 60 & 50 & 40 & 30 & 20 & 10 & 0 & -10 & -20\end{array}$

${ }^{1} \mathrm{H}$ NMR (400 MHz, $\left.\mathrm{CDCl}_{3}\right)$ spectrum of (S)-1,3-dichloro-5-(fluoro(3-fluorophenyl)methyl)benzene 3ad

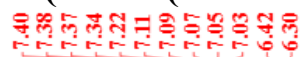

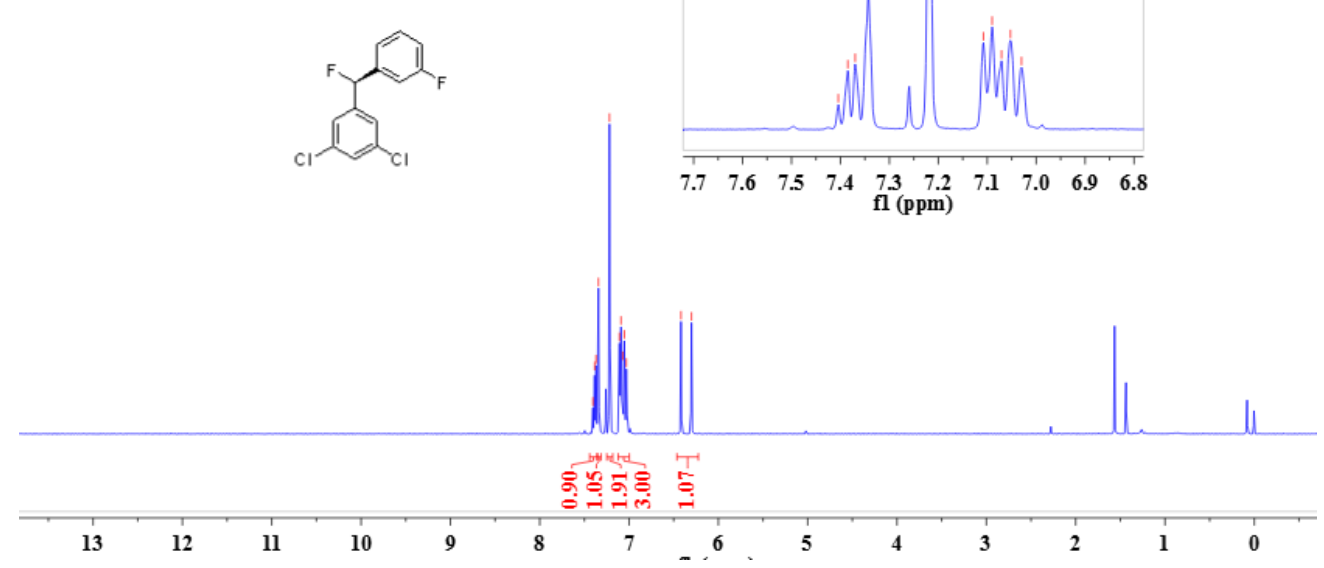


${ }^{19}$ F NMR (376 MHz, $\mathrm{CDCl}_{3}$ ) spectrum of

(S)-1,3-dichloro-5-(fluoro(3-fluorophenyl)methyl)benzene 3ad
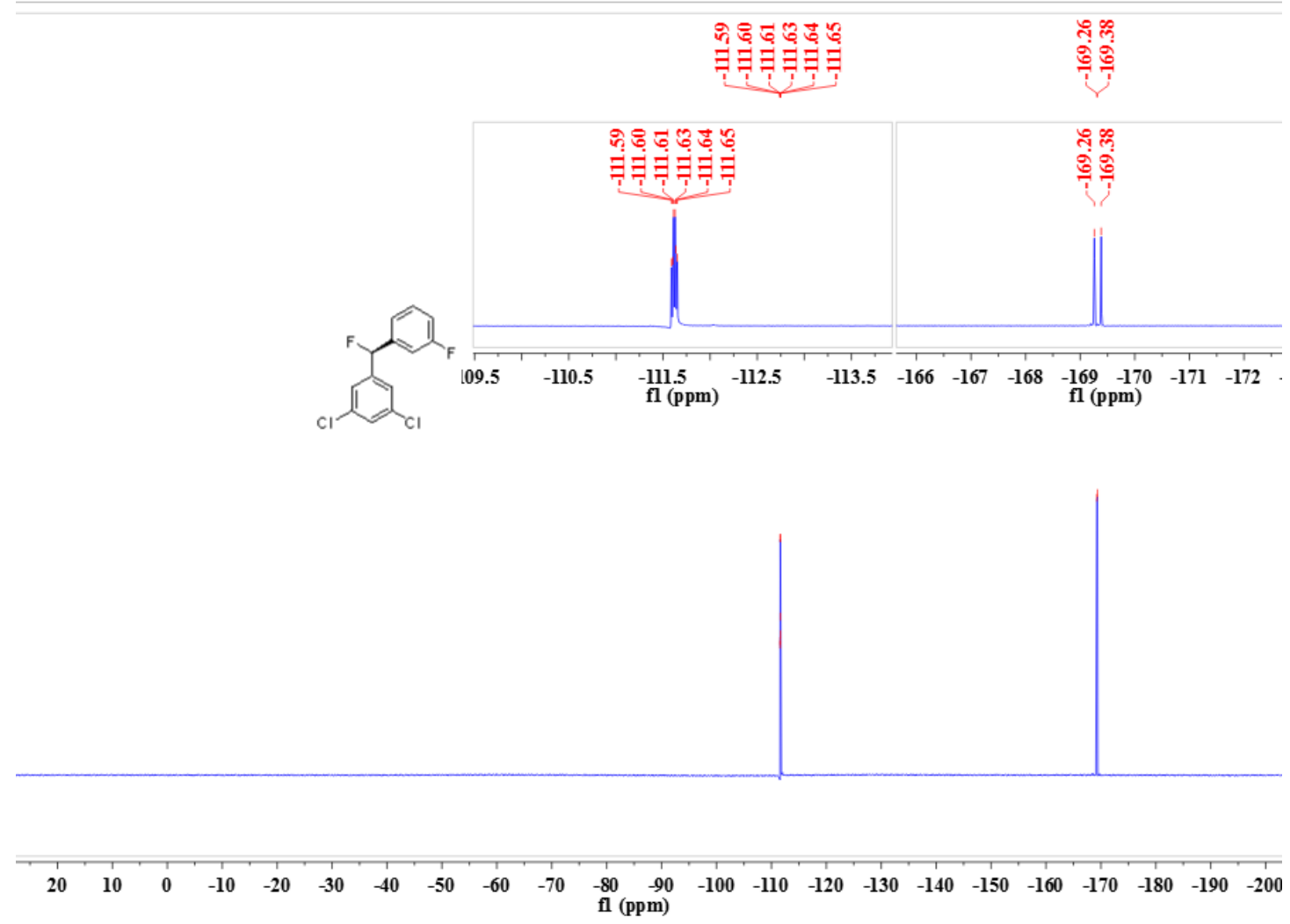

${ }^{13} \mathrm{C}$ NMR (101 MHz, $\left.\mathrm{CDCl}_{3}\right)$ spectrum of

(S)-1,3-dichloro-5-(fluoro(3-fluorophenyl)methyl)benzene 3ad

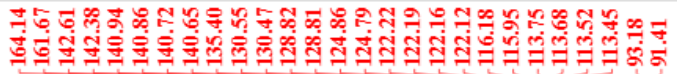
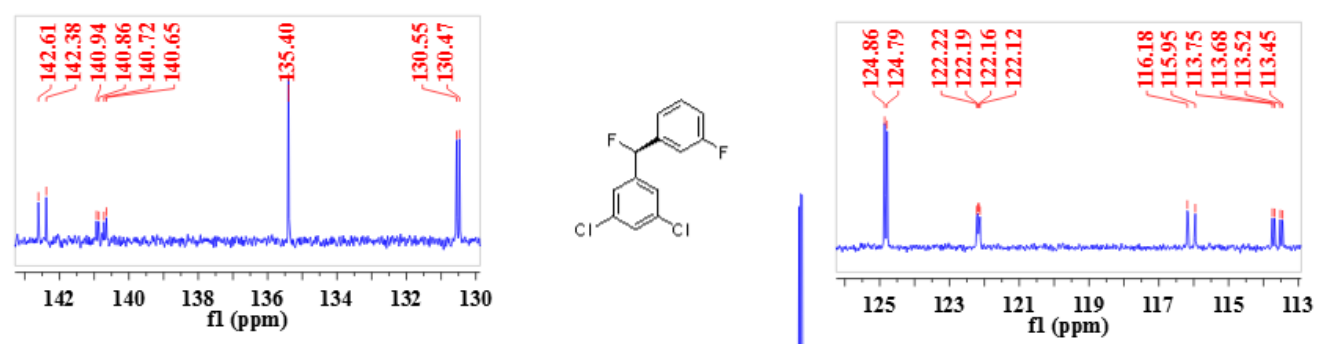

$\begin{array}{lllllllllllllllllllllllllllll}240 & 230 & 220 & 210 & 200 & 190 & 180 & 170 & 160 & 150 & 140 & 130 & 120 & 110 & 100 & 90 & 80 & 70 & 60 & 50 & 40 & 30 & 20 & 10 & 0 & -10 & -20 & -\end{array}$ 
${ }^{1} \mathrm{H}$ NMR (400 $\mathrm{MHz}, \mathrm{CDCl}_{3}$ ) spectrum of (S)-1,3-dichloro-5-(fluoro(phenyl)methyl)benzene 3ae

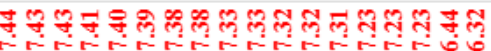
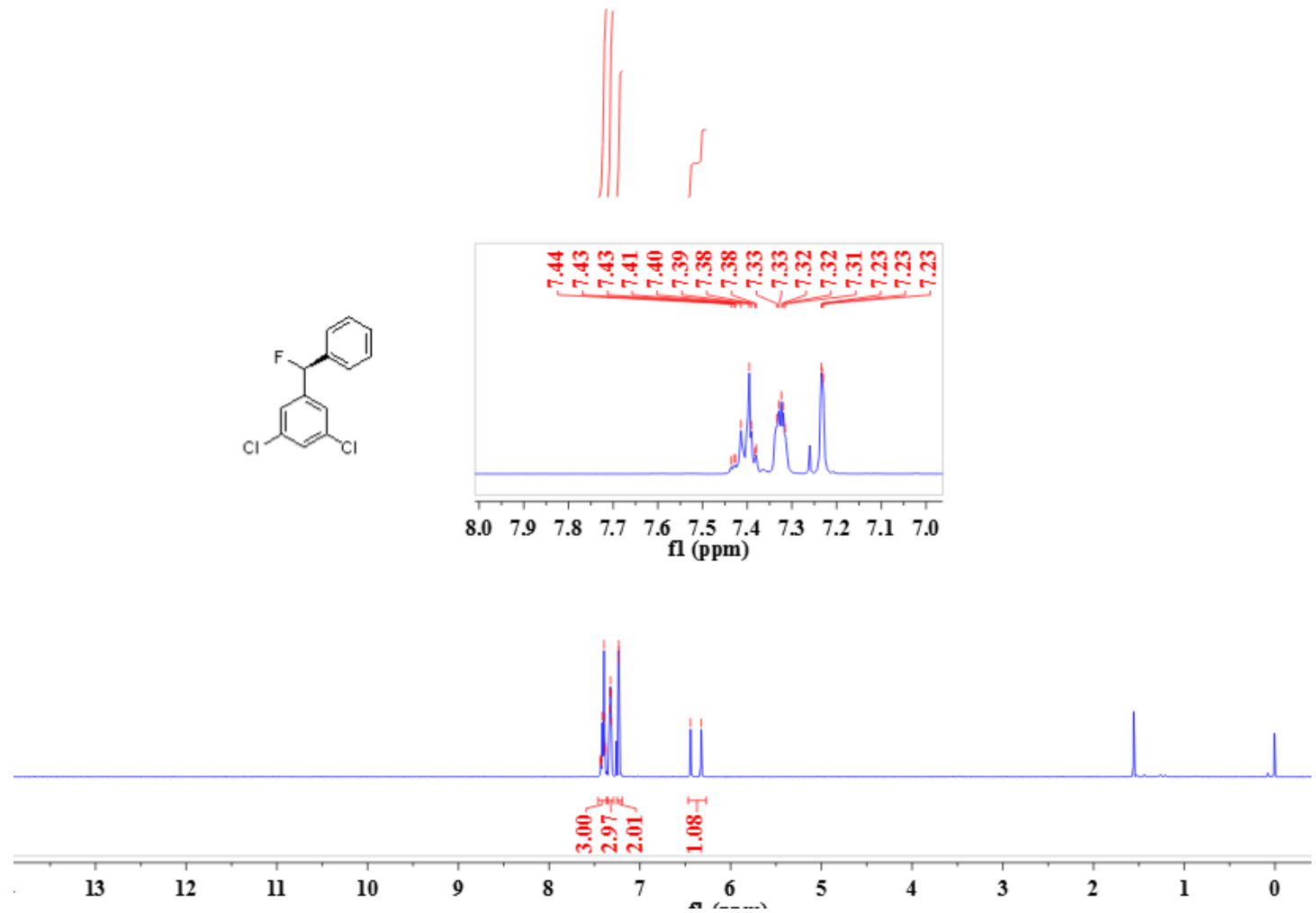

${ }^{19} \mathrm{~F}$ NMR (376 $\left.\mathrm{MHz}, \mathrm{CDCl}_{3}\right)$ spectrum of (S)-1,3-dichloro-5-(fluoro(phenyl)methyl)benzene 3ae

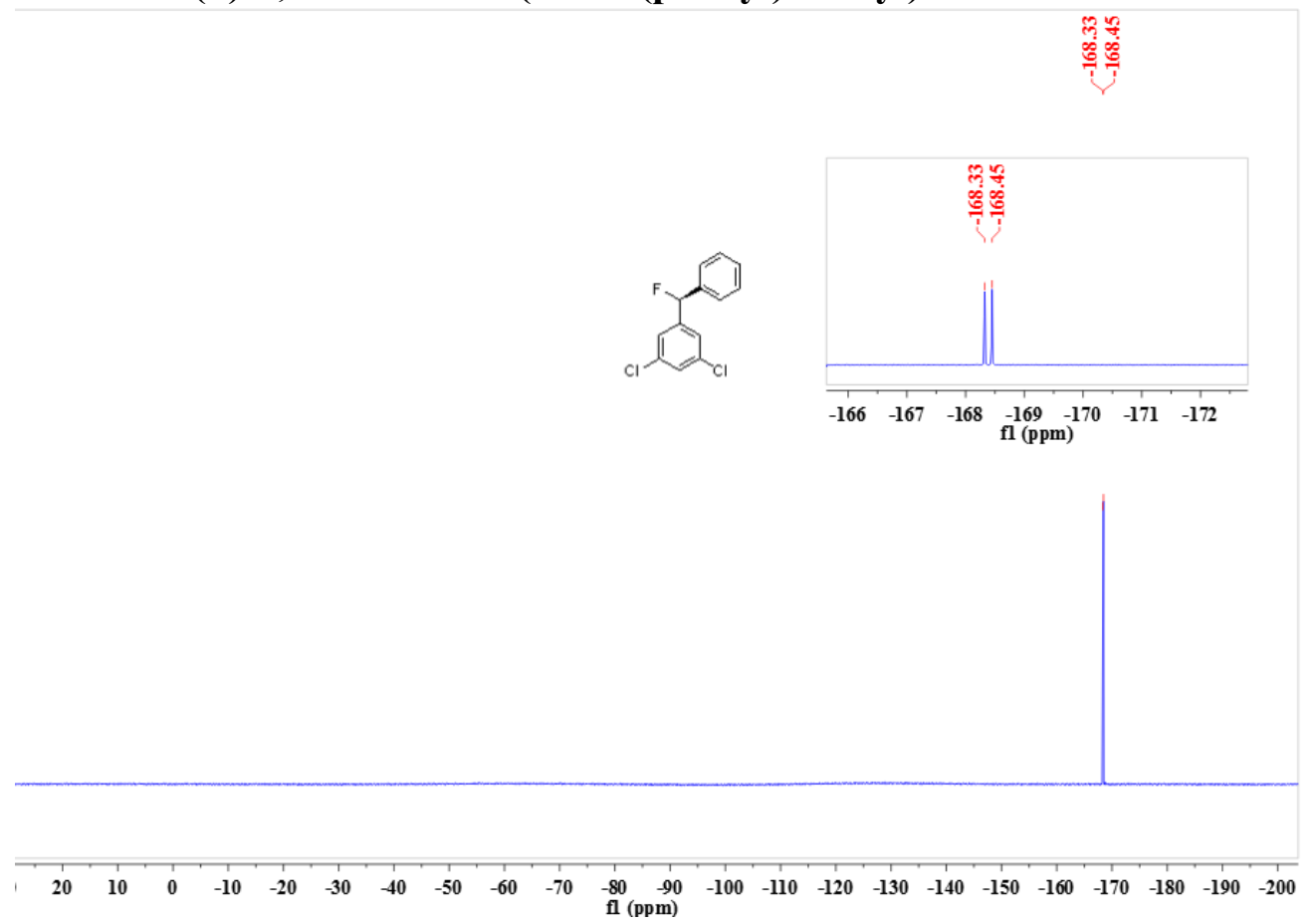


${ }^{13} \mathrm{C}$ NMR (101 MHz, $\left.\mathrm{CDCl}_{3}\right)$ spectrum of (S)-1,3-dichloro-5-(fluoro(phenyl)methyl)benzene 3ae

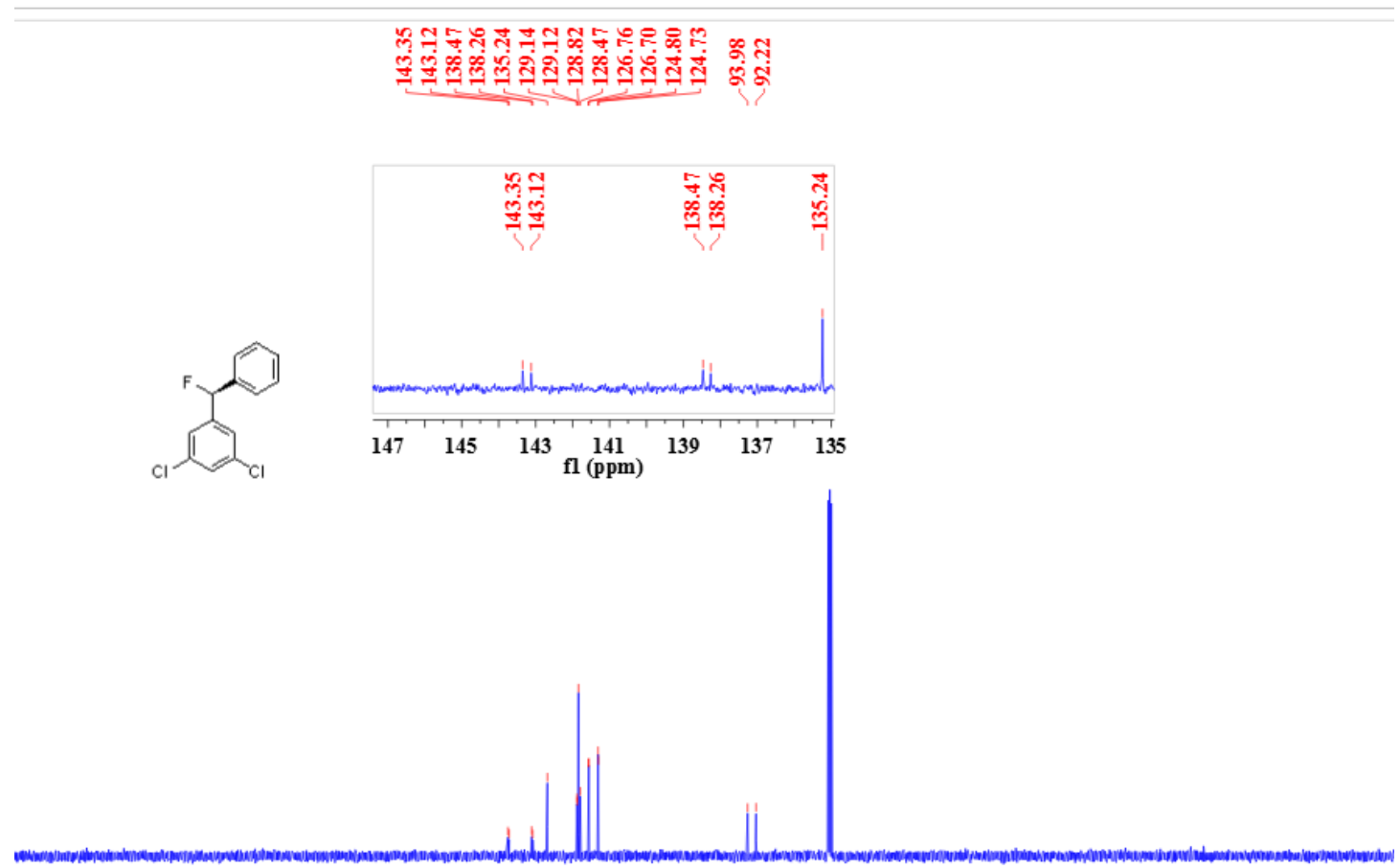

$\begin{array}{llllllllllllllllllllllllllllllllllllll}240 & 230 & 220 & 210 & 200 & 190 & 180 & 170 & 160 & 150 & 140 & 130 & 120 & 110 & 100 & 90 & 80 & 70 & 60 & 50 & 40 & 30 & 20 & 10 & 0 & -10 & -20 & -30 & -6\end{array}$

${ }^{1} \mathrm{H}$ NMR (400 $\left.\mathrm{MHz}, \mathrm{CDCl}_{3}\right)$ spectrum of

(S)-1,3-dichloro-5-((4-chlorophenyl)fluoromethyl)benzene 3af

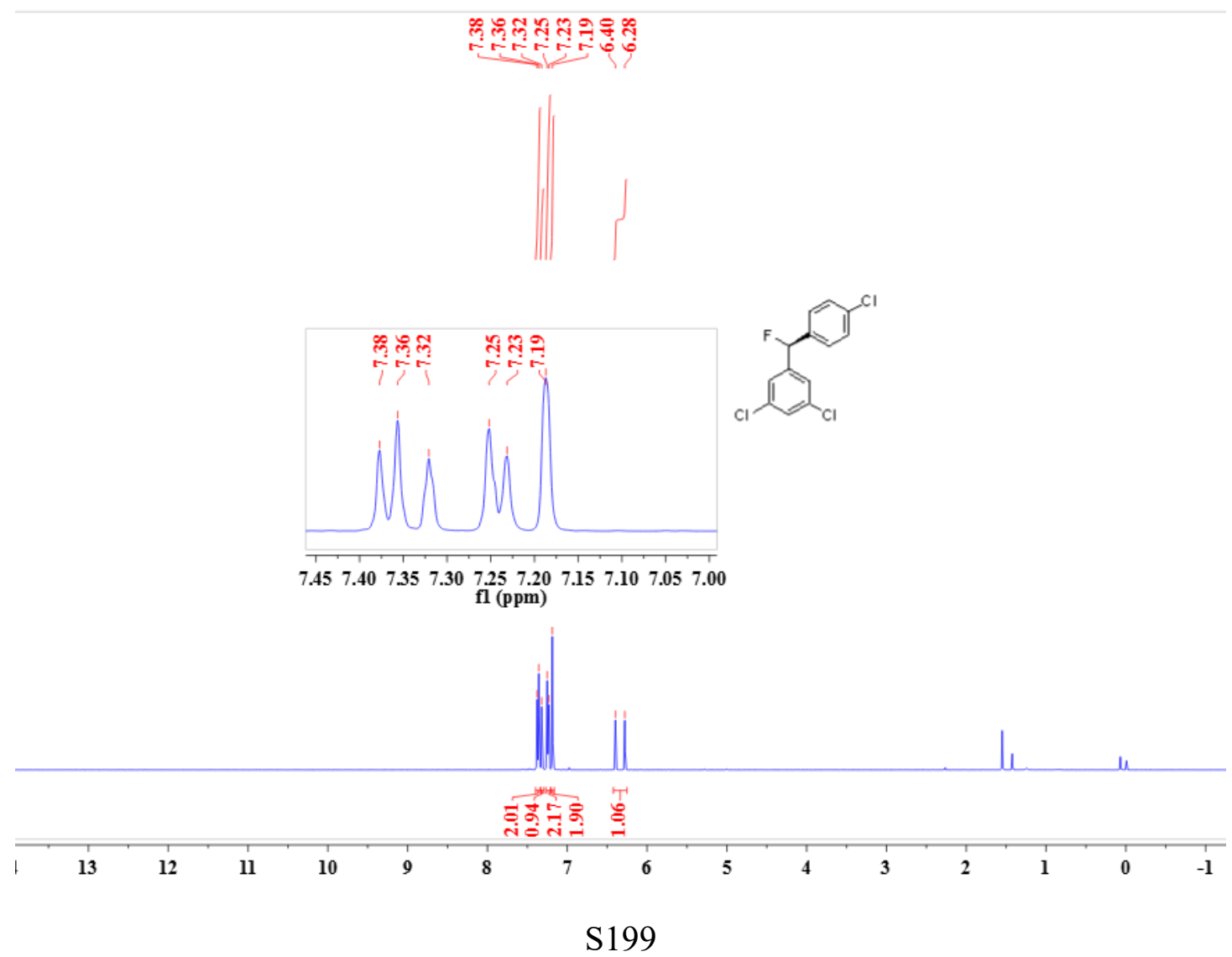


${ }^{19} \mathrm{~F}$ NMR (376 MHz, $\mathrm{CDCl}_{3}$ ) spectrum of (S)-1,3-dichloro-5-((4-chlorophenyl)fluoromethyl)benzene 3af

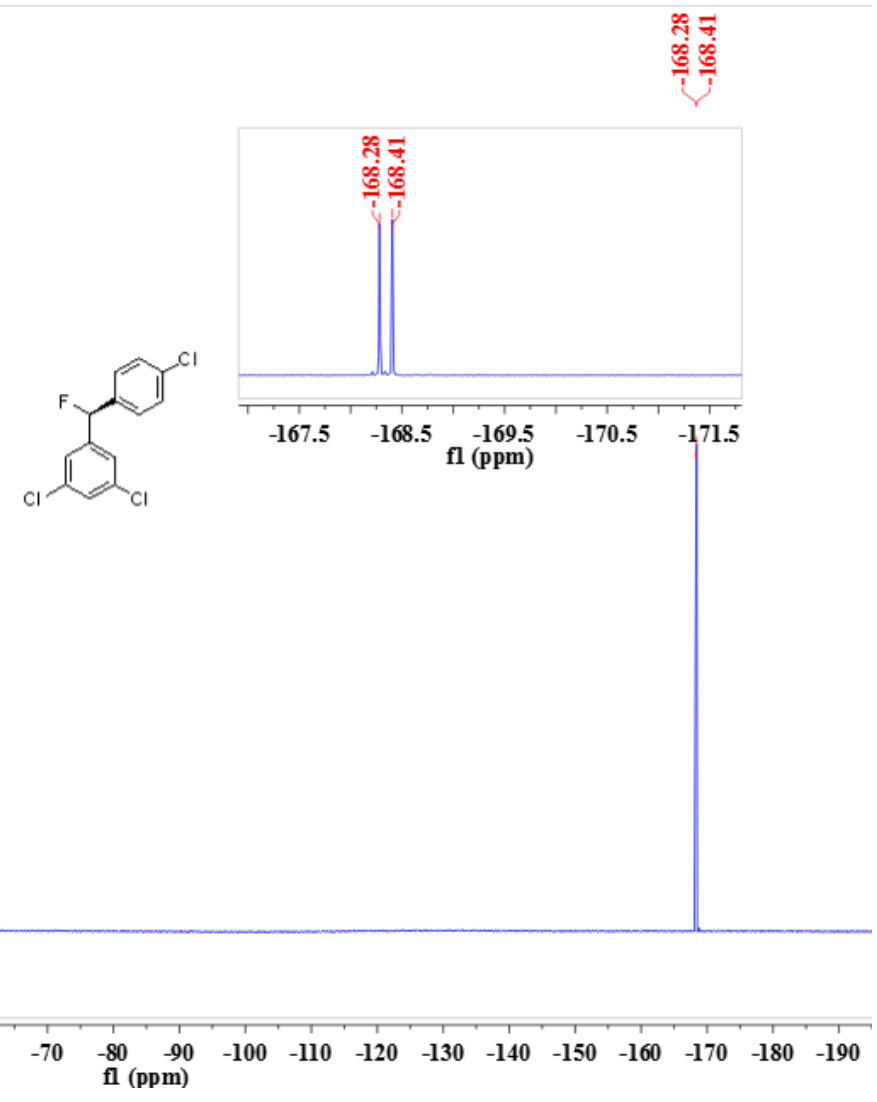

${ }^{13} \mathrm{C}$ NMR (101 MHz, $\left.\mathrm{CDCl}_{3}\right)$ spectrum of (S)-1,3-dichloro-5-((4-chlorophenyl)fluoromethyl)benzene 3af

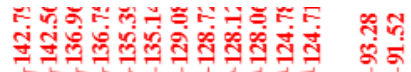

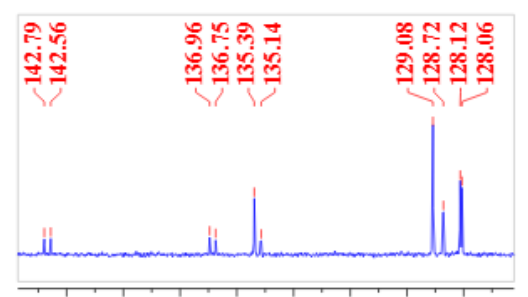
$\begin{array}{llllllll}142 & 140 & 138 & 136 & 134 & 132 & 130 & 128\end{array}$ $\begin{array}{llllllllllllllllllllllllllll}240 & 230 & 220 & 210 & 200 & 190 & 180 & 170 & 160 & 150 & 140 & 130 & 120 & 110 & 100 & 90 & 80 & 70 & 60 & 50 & 40 & 30 & 20 & 10 & 0 & -10 & -20 & -3\end{array}$ 
${ }^{1} \mathrm{H}$ NMR (400 MHz, $\left.\mathrm{CDCl}_{3}\right)$ spectrum of

(S)-1,3-dichloro-5-((3-chlorophenyl)fluoromethyl)benzene 3ag

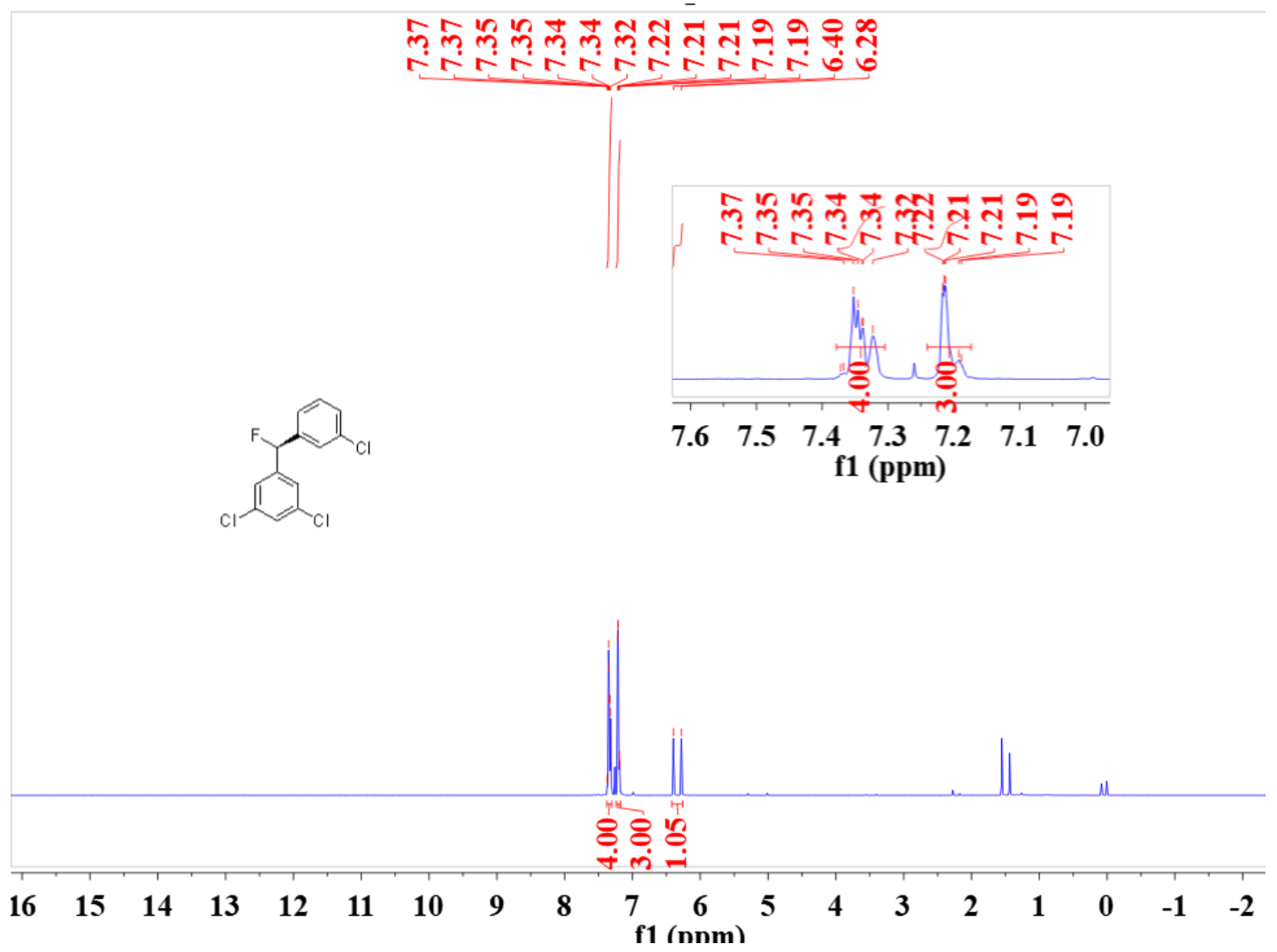

${ }^{19} \mathrm{~F}$ NMR (376 $\left.\mathrm{MHz}, \mathrm{CDCl}_{3}\right)$ spectrum of

(S)-1,3-dichloro-5-((3-chlorophenyl)fluoromethyl)benzene 3ag
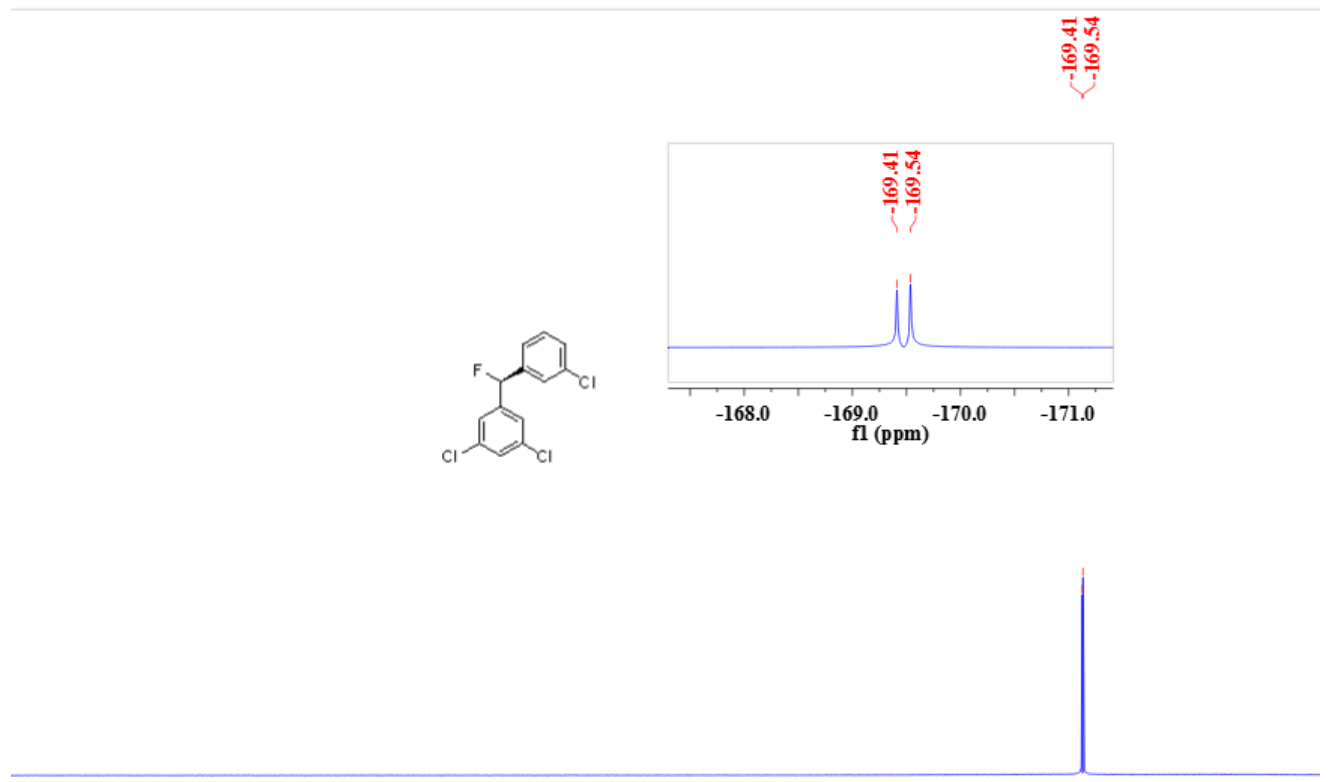

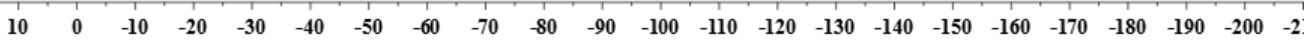


${ }^{13} \mathrm{C}$ NMR (101 MHz, $\left.\mathrm{CDCl}_{3}\right)$ spectrum of

(S)-1,3-dichloro-5-((3-chlorophenyl)fluoromethyl)benzene $3 \mathrm{ag}$

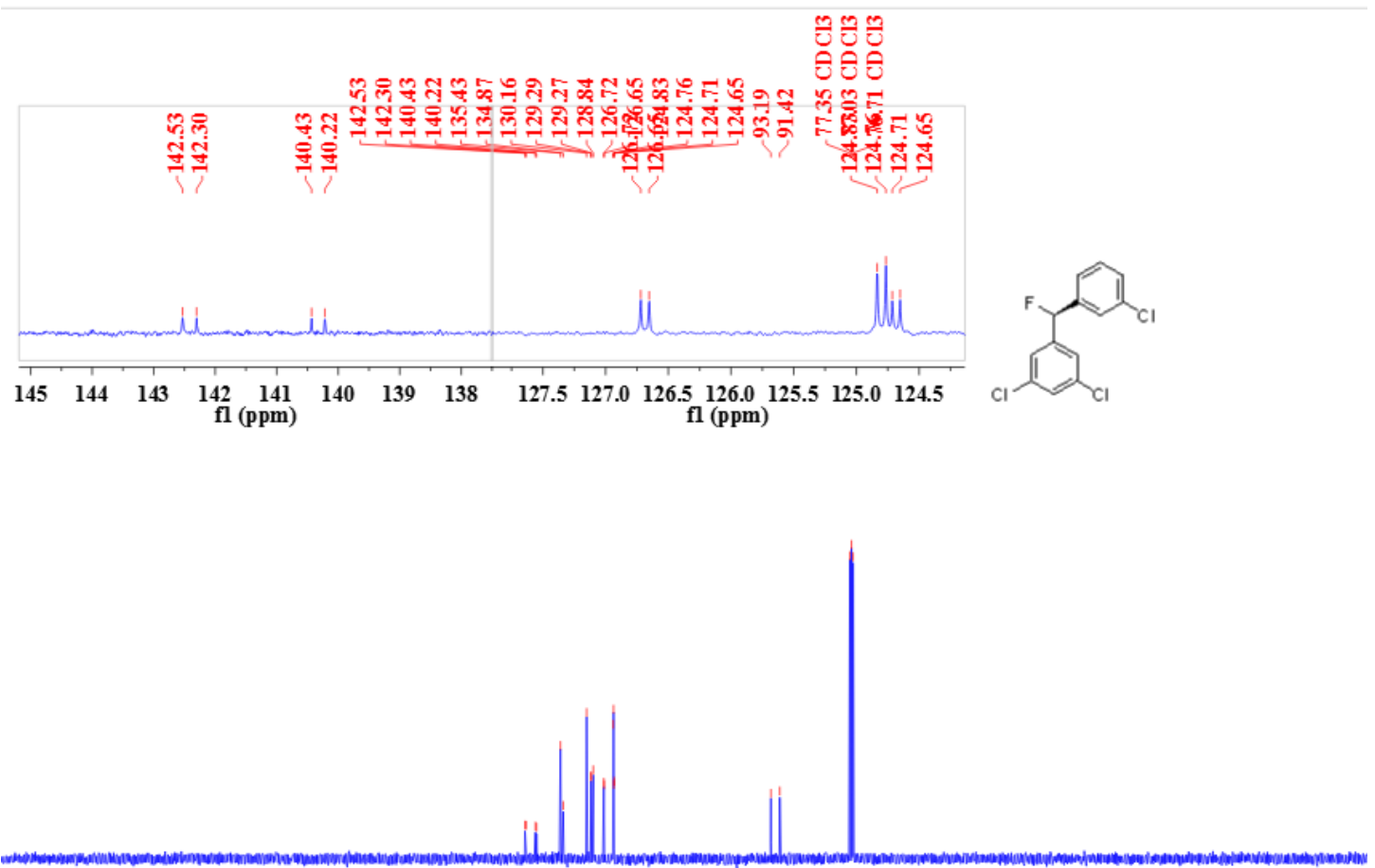

$\begin{array}{lllllllllllllllllllllllllllllllllll}240 & 230 & 220 & 210 & 200 & 190 & 180 & 170 & 160 & 150 & 140 & 130 & 120 & 110 & 100 & 90 & 80 & 70 & 60 & 50 & 40 & 30 & 20 & 10 & 0 & -10 & -20\end{array}$

${ }^{1} \mathrm{H}$ NMR (400 $\mathrm{MHz}, \mathrm{CDCl}_{3}$ ) spectrum of

(S)-1-chloro-2-fluoro-4-(fluoro(phenyl)methyl)benzene 3ah

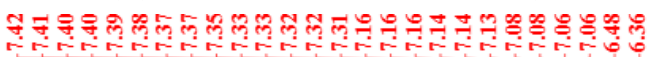
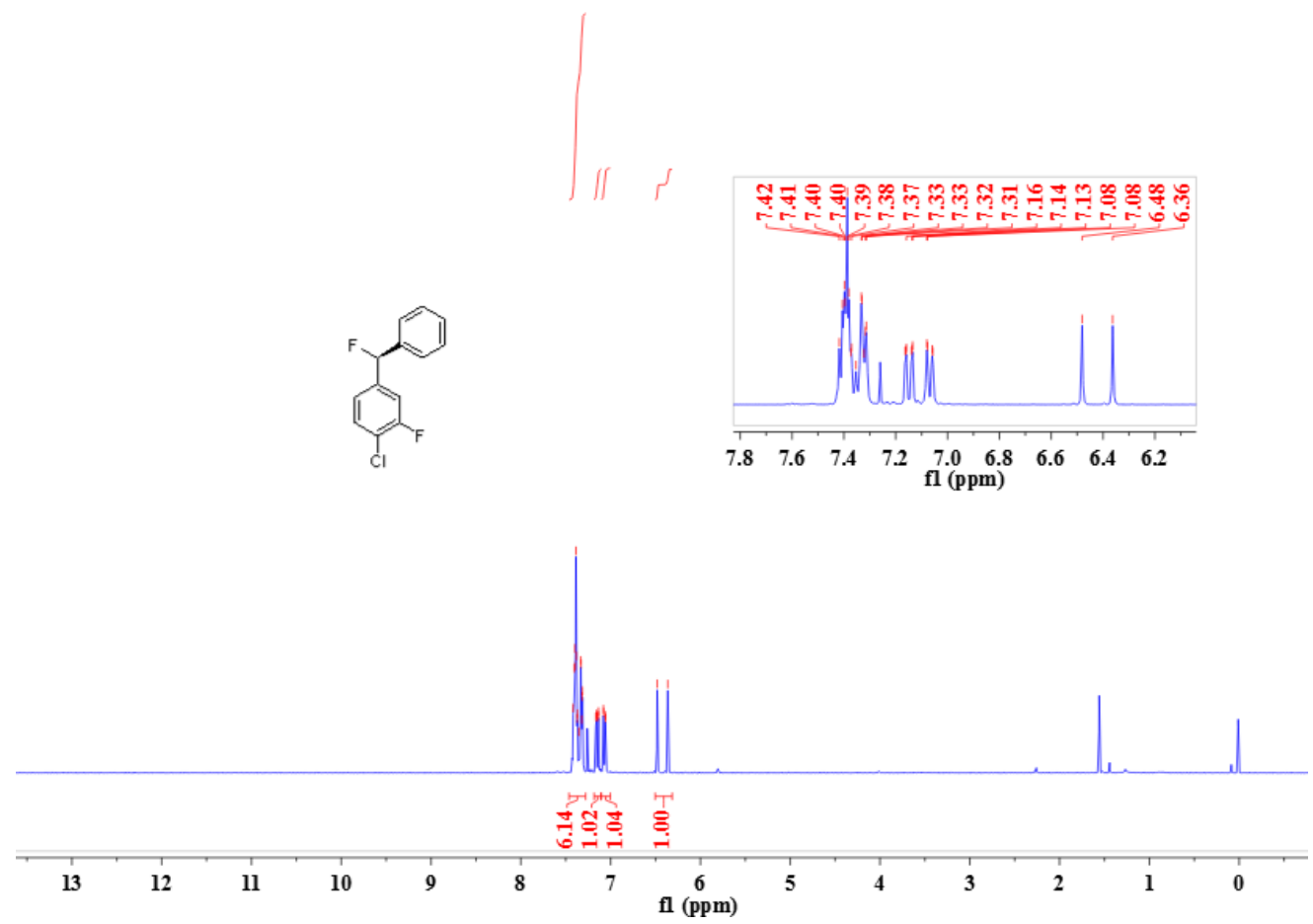
${ }^{19}$ F NMR (376 MHz, $\mathrm{CDCl}_{3}$ ) spectrum of (S)-1-chloro-2-fluoro-4-(fluoro(phenyl)methyl)benzene 3ah

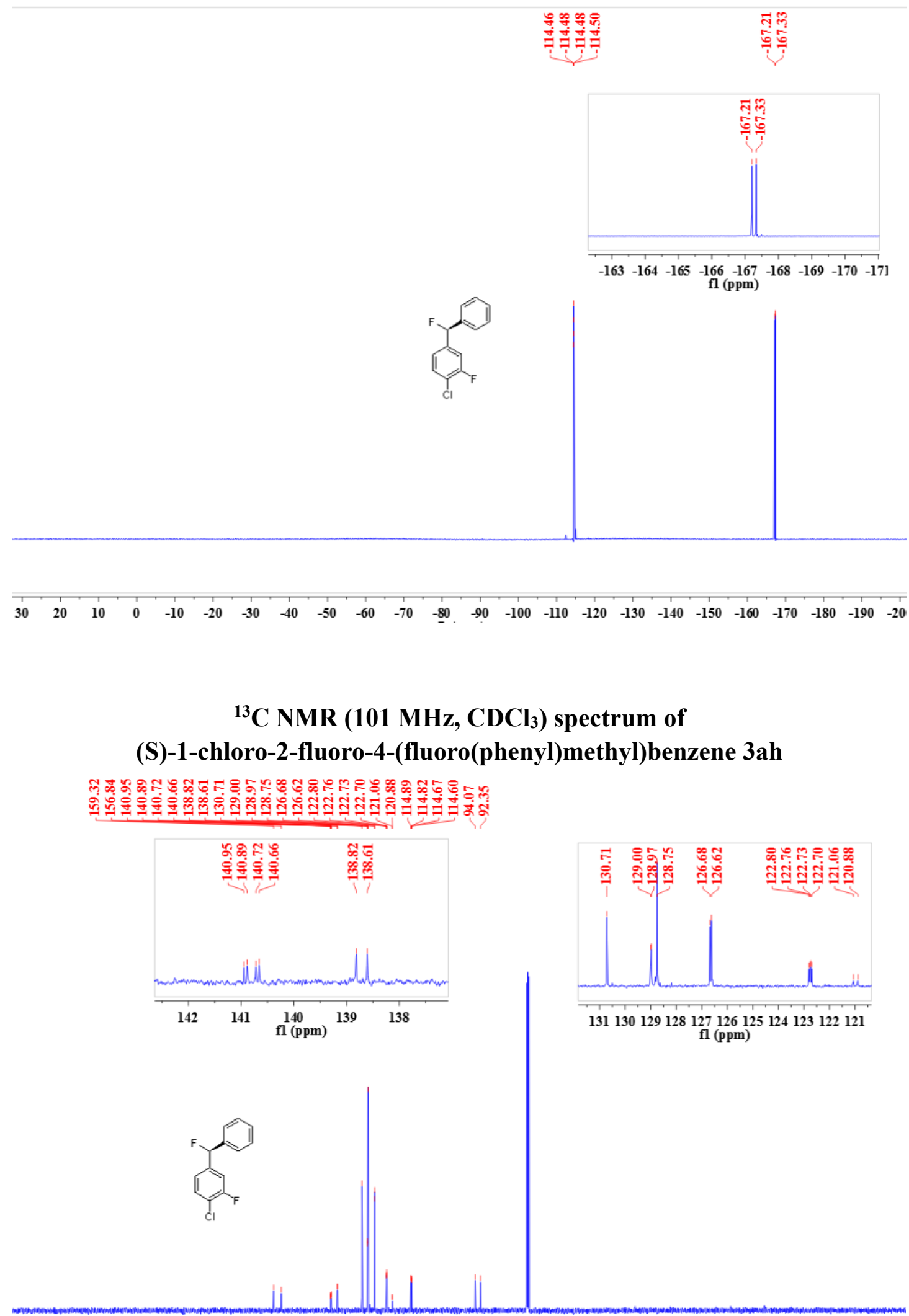

$240 \begin{array}{lllllllllllllllllllllllllllllll} & 230 & 220 & 210 & 200 & 190 & 180 & 170 & 160 & 150 & 140 & 130 & 120 & 110 & 100 & 90 & 80 & 70 & 60 & 50 & 40 & 30 & 20 & 10 & 0 & -10 & -20 & -30 & -40\end{array}$ 
${ }^{1} \mathrm{H}$ NMR (400 MHz, $\mathrm{CDCl}_{3}$ ) spectrum of (S)-1-chloro-2-fluoro-4-(fluoro(3-fluorophenyl)methyl)benzene 3ai

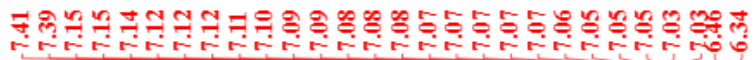

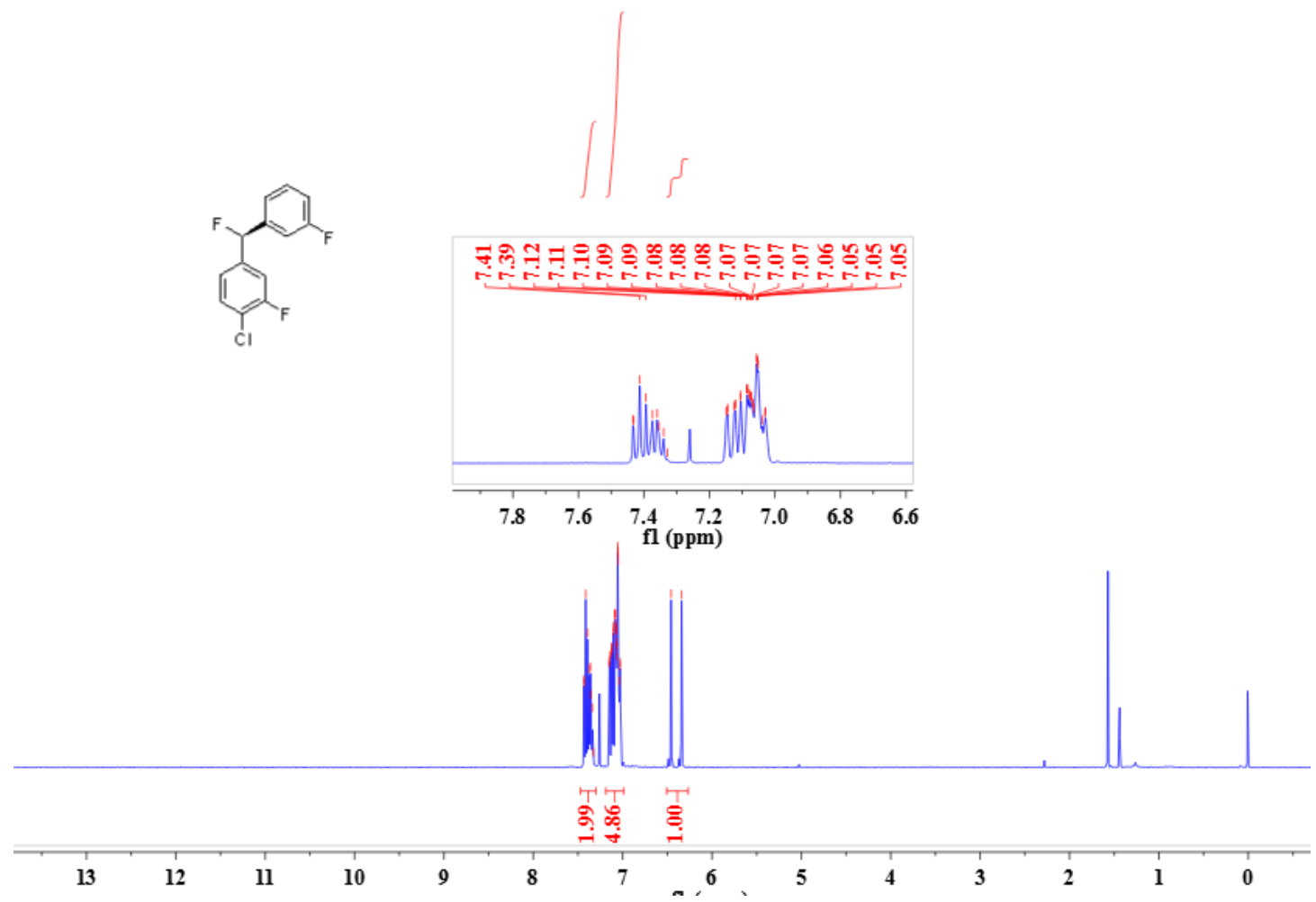

${ }^{19}$ F NMR (376 MHz, $\mathrm{CDCl}_{3}$ ) spectrum of (S)-1-chloro-2-fluoro-4-(fluoro(3-fluorophenyl)methyl)benzene 3ai

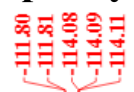
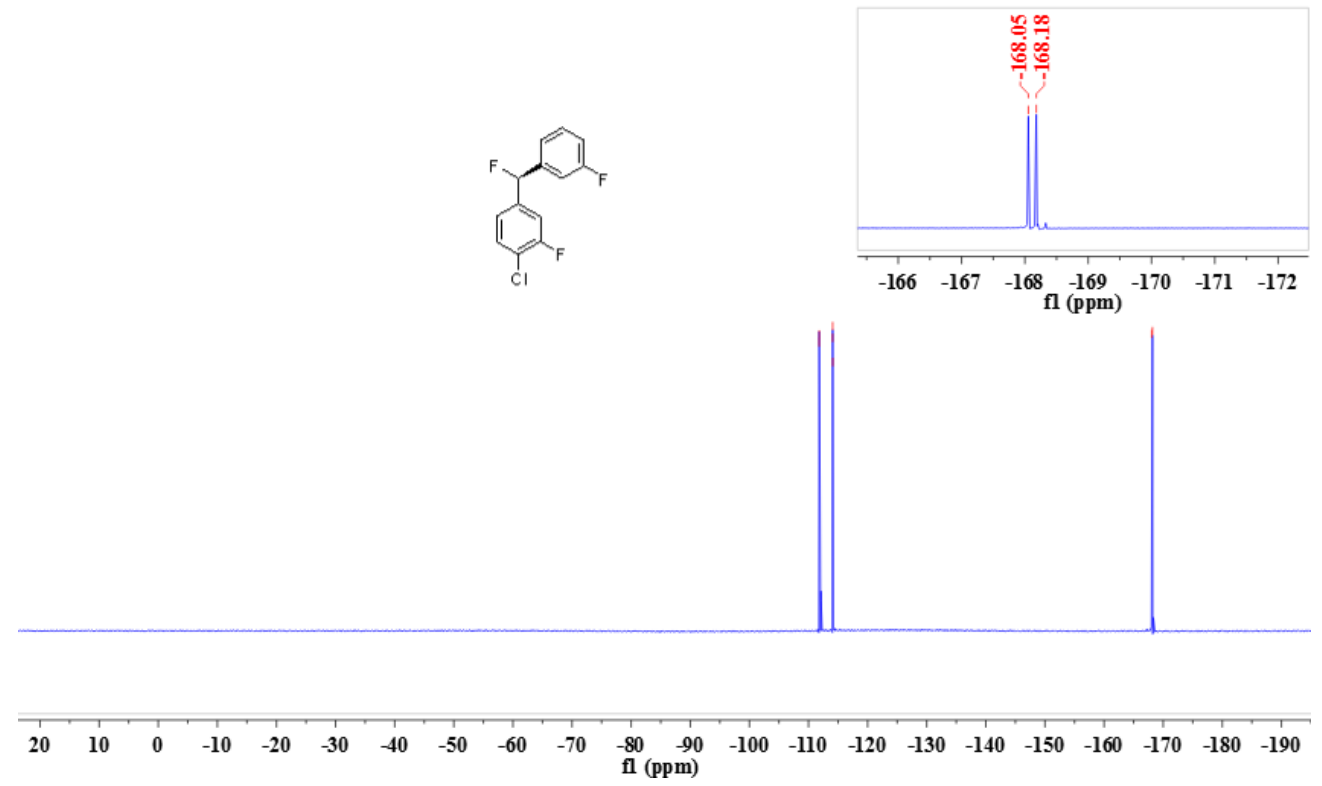
${ }^{13} \mathrm{C}$ NMR (101 MHz, $\left.\mathrm{CDCl}_{3}\right)$ spectrum of (S)-1-chloro-2-fluoro-4-(fluoro(3-fluorophenyl)methyl)benzene 3ai

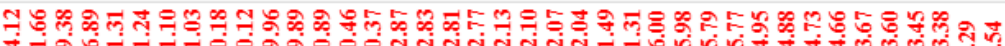

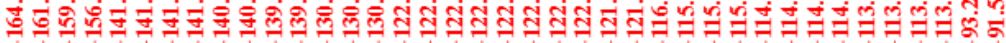

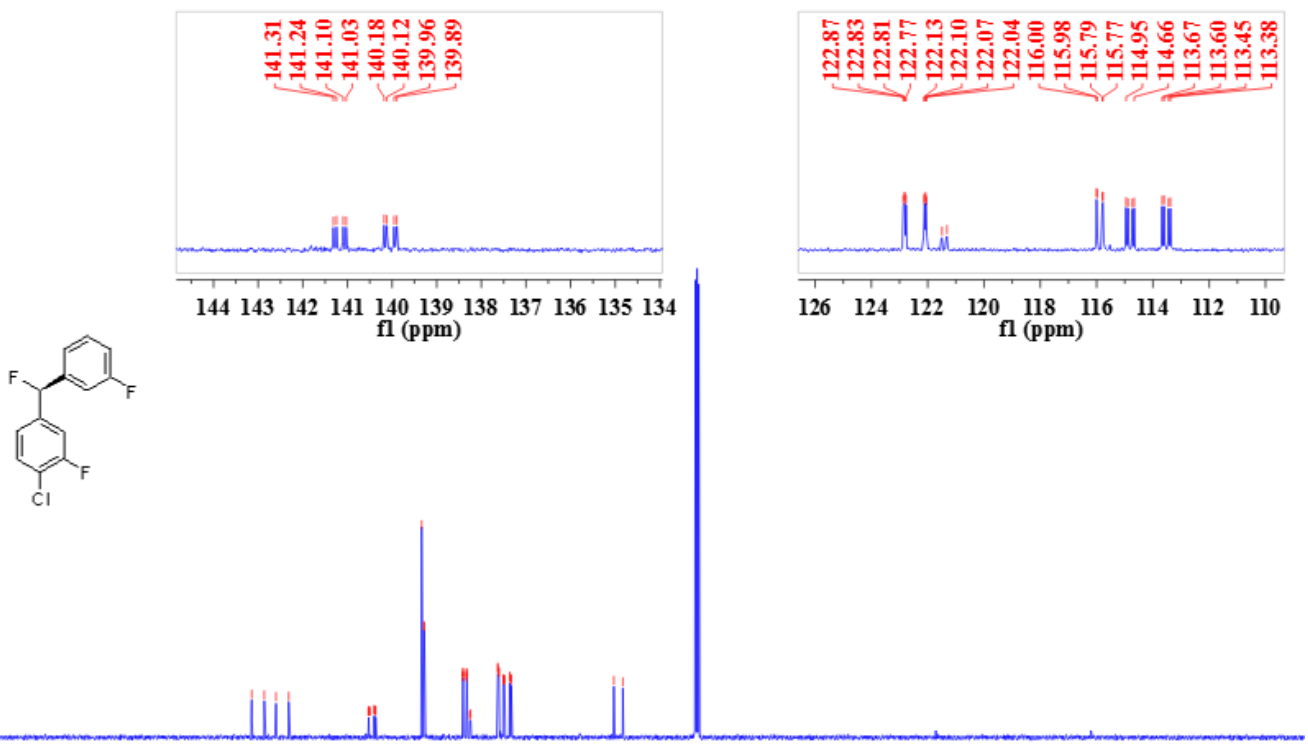

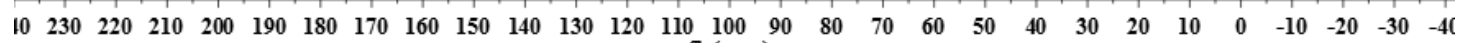

${ }^{1} \mathrm{H}$ NMR (400 MHz, CDCl 3 ) spectrum of (S)-4-(fluoro(phenyl)methyl)

- $N$-((2-hydroxy-4,6-dimethylpyridin-3-yl)methyl)benzamide 5

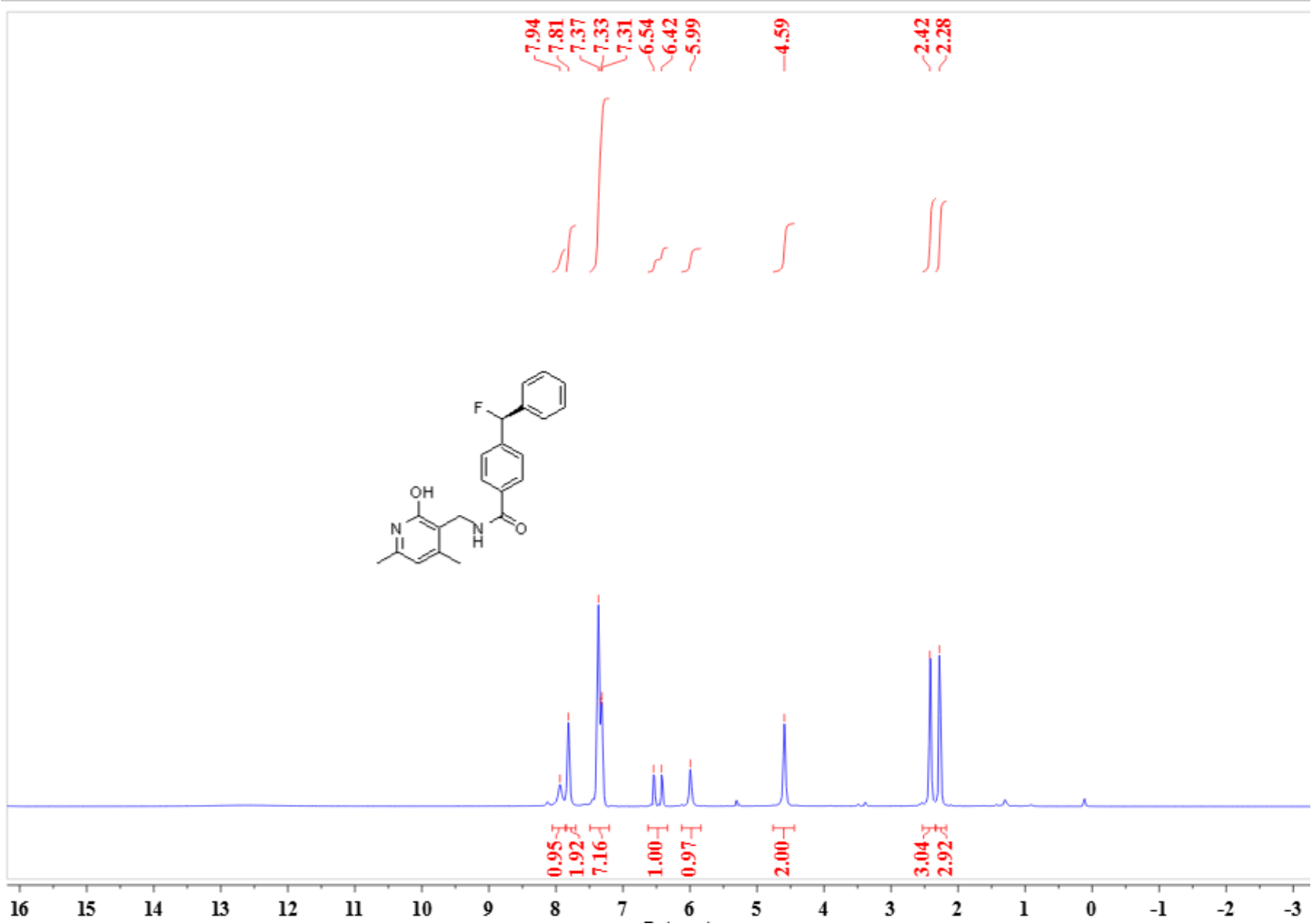


${ }^{19}$ F NMR (376 MHz, CDCl 3 ) spectrum of (S)-4-(Fluoro(phenyl)methyl) -N-((2-hydroxy-4,6-dimethylpyridin-3-yl)methyl)benzamide 5
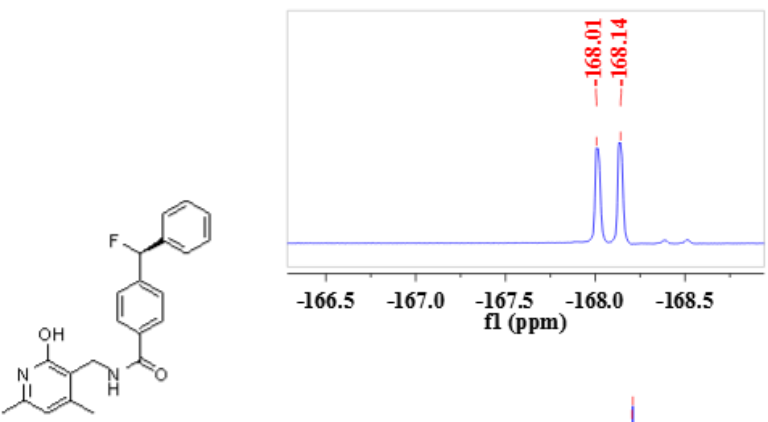

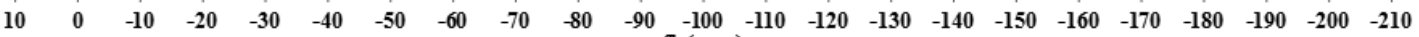

${ }^{13} \mathrm{C}$ NMR (101 MHz, CDCl 3 ) spectrum of (S)-4-(Fluoro(phenyl)methyl)

-N-((2-hydroxy-4,6-dimethylpyridin-3-yl)methyl)benzamide 5

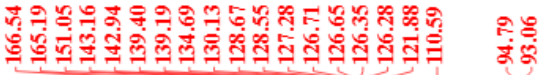
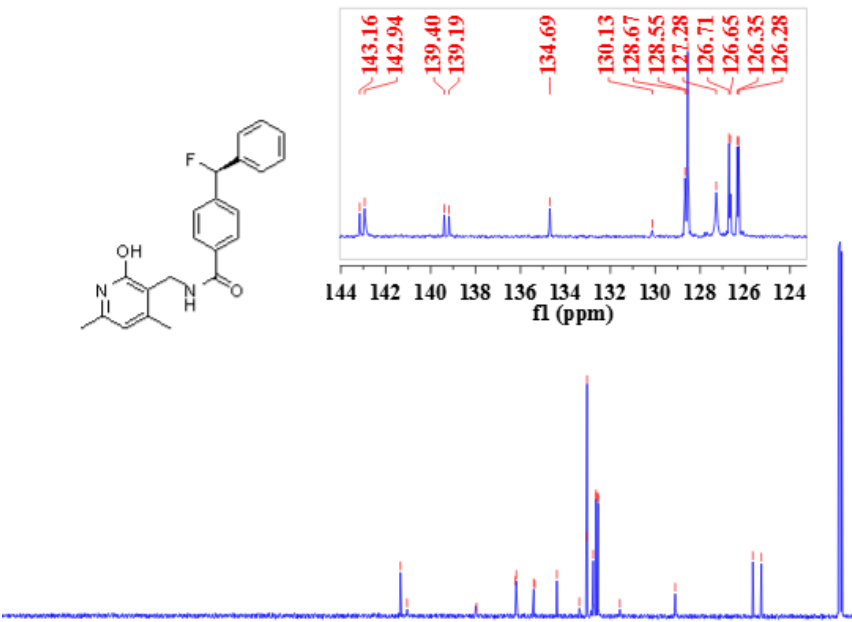

$\begin{array}{lllllllllllllllllllllllllllll}240 & 230 & 220 & 210 & 200 & 190 & 180 & 170 & 160 & 150 & 140 & 130 & 120 & 110 & 100 & 90 & 80 & 70 & 60 & 50 & 40 & 30 & 20 & 10 & 0 & -10 & -20 & -30 & -40\end{array}$ 
${ }^{1}$ H NMR (400 MHz, CDCl $)$ spectrum of (S)-(4-cyclopropyl-1,4-diazepan-1-yl) (4-(fluoro(phenyl)methyl)phenyl)methanone 6
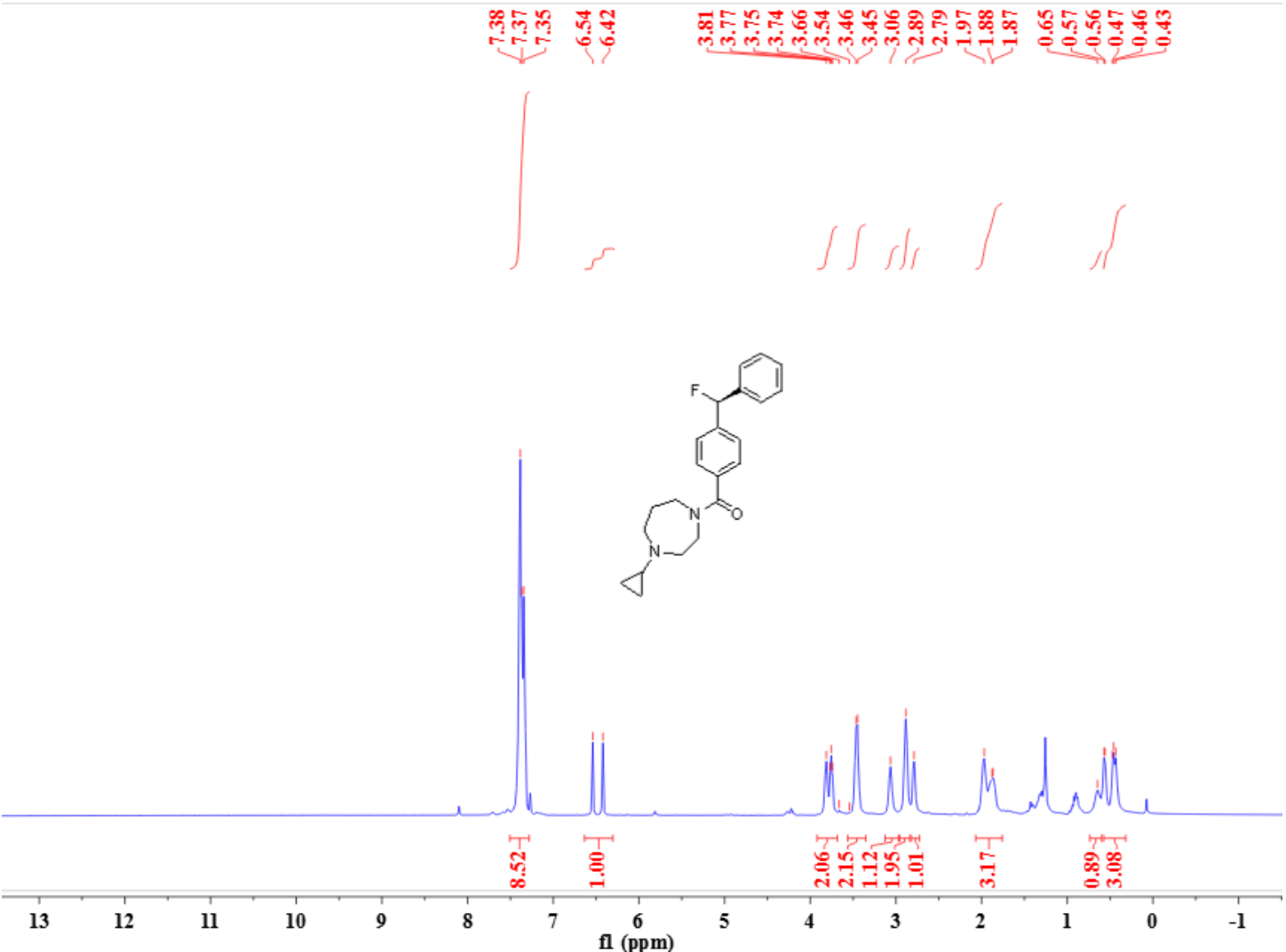

${ }^{19}$ F NMR (376 MHz, CDCl $)$ spectrum of ((S)-(4-cyclopropyl-1,4-diazepan-1-yl) (4-(fluoro(phenyl)methyl)phenyl)methanone 6

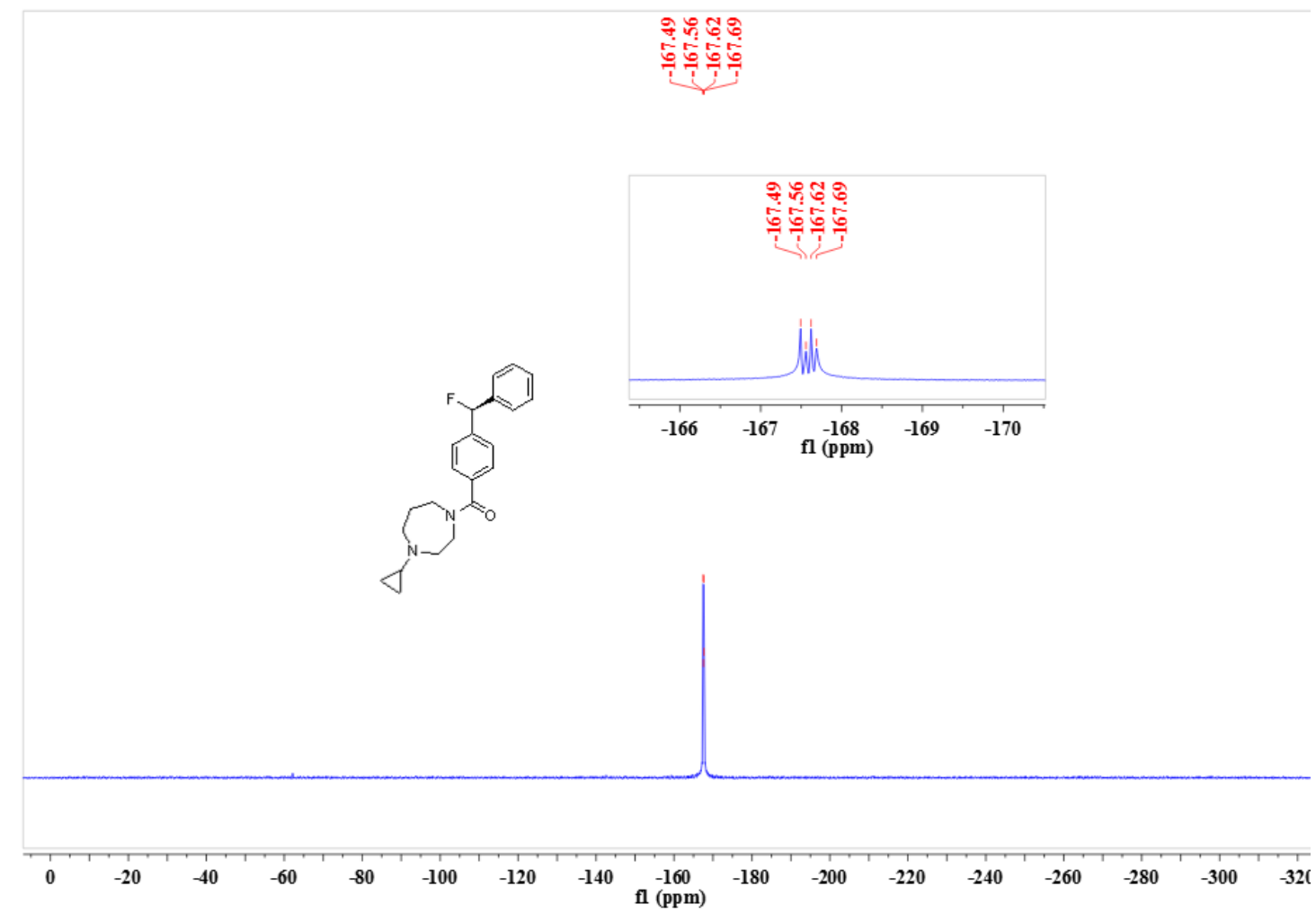


${ }^{13} \mathrm{C}$ NMR (101 MHz, $\mathrm{CDCl}_{3}$ ) spectrum of (S)-(4-cyclopropyl-1,4-diazepan-1-yl) (4-(fluoro(phenyl)methyl)phenyl)methanone 6

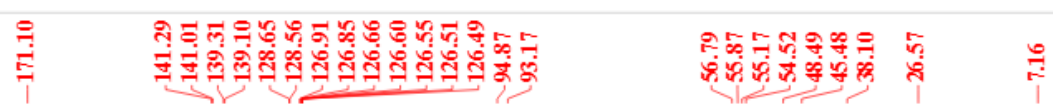
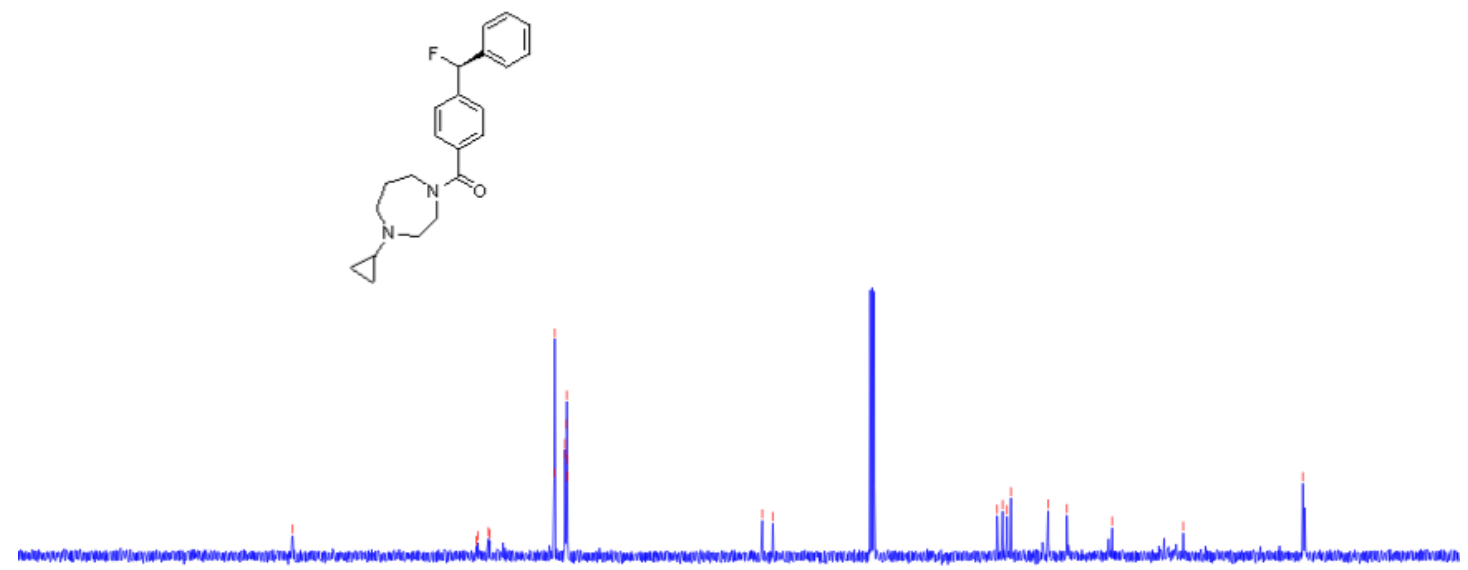

$\begin{array}{lllllllllllllllllllllll}210 & 200 & 190 & 180 & 170 & 160 & 150 & 140 & 130 & 120 & 110 & 100 & 90 & 80 & 70 & 60 & 50 & 40 & 30 & 20 & 10 & 0 & -10\end{array}$

${ }^{1} \mathrm{H}$ NMR (400 MHz, CDCl 3$)$ spectrum of (S)-3-((4-(((tert-butyldimethylsilyl) oxy)methyl)phenyl)fluoromethyl)benzonitrile 7

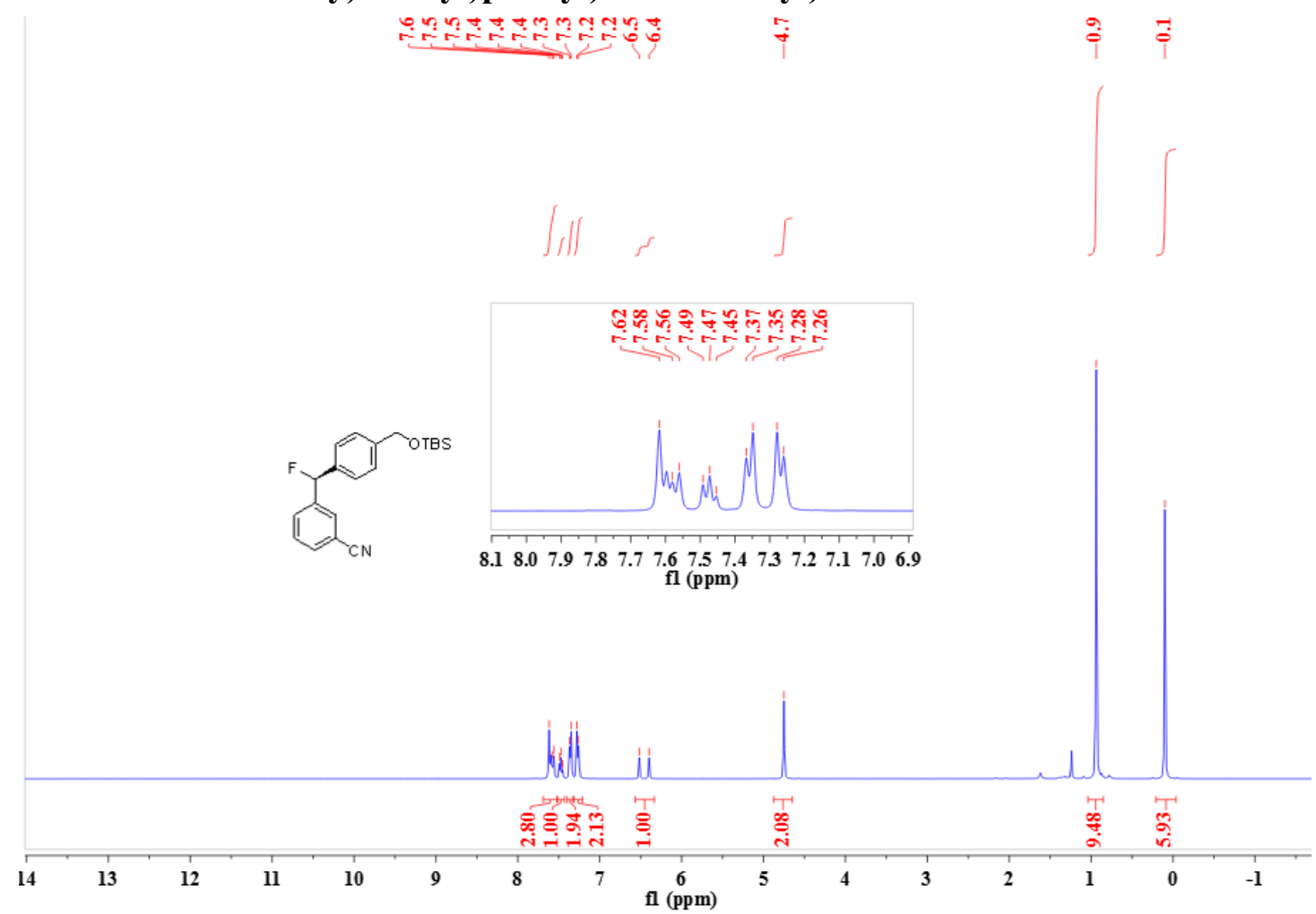

${ }^{19}$ F NMR (376 MHz, $\left.\mathrm{CDCl}_{3}\right)$ spectrum of (S)-3-((4-(((tert-butyldimethylsilyl) 
oxy)methyl)phenyl)fluoromethyl)benzonitrile 7

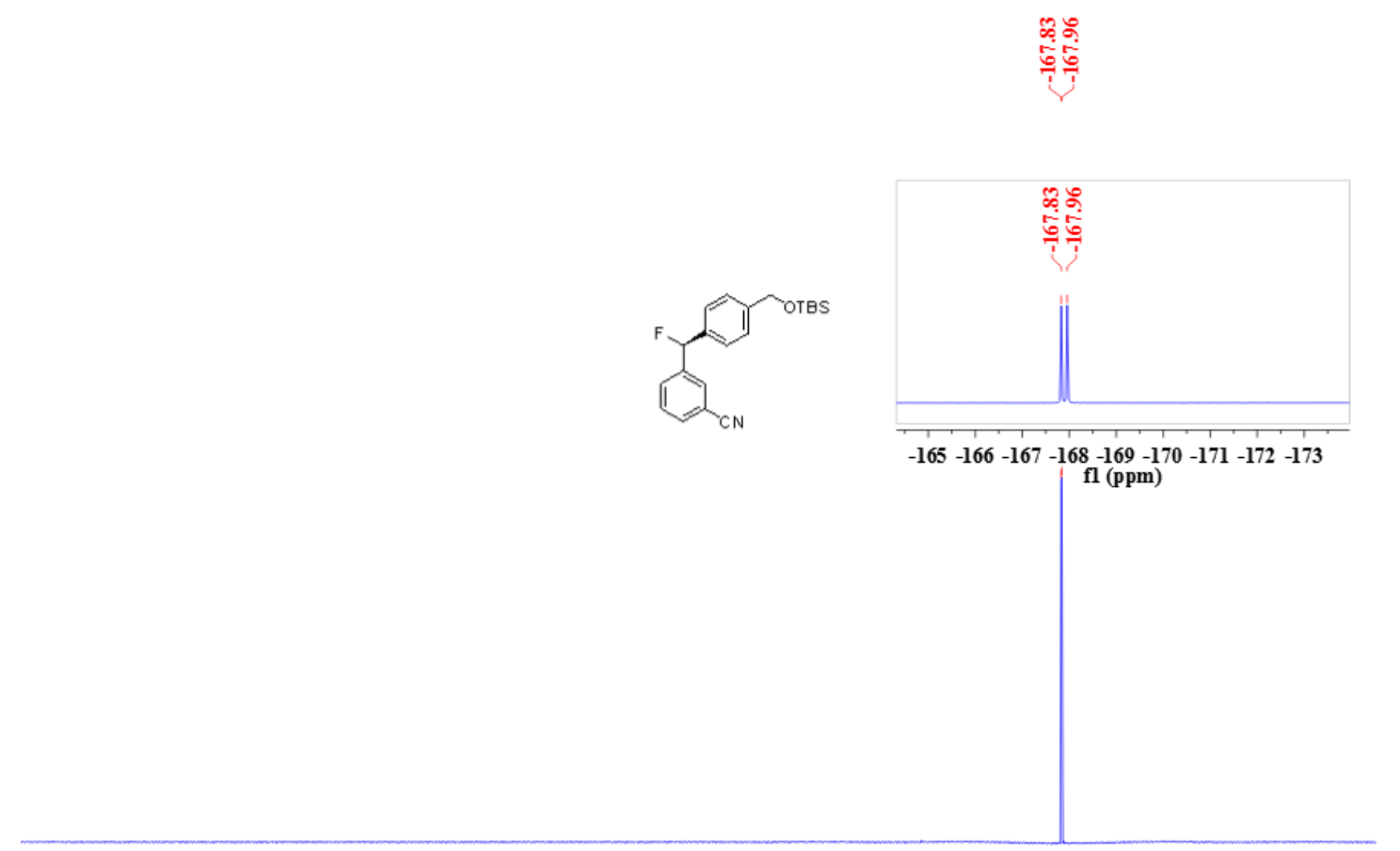

$\begin{array}{lllllllllllllllllllllllllllllllllll}0 & 10 & 0 & -10 & -20 & -30 & -40 & -50 & -60 & -70 & -80 & -90 & -100 & -110 & -120 & -130 & -140 & -150 & -160 & -170 & -180 & -190 & -200 & -210 & -220\end{array}$

${ }^{13} \mathrm{C}$ NMR (101 MHz, CDCl$)$ spectrum of (S)-3-((4-(((tert-butyldimethylsilyl) oxy)methyl)phenyl)fluoromethyl)benzonitrile 7

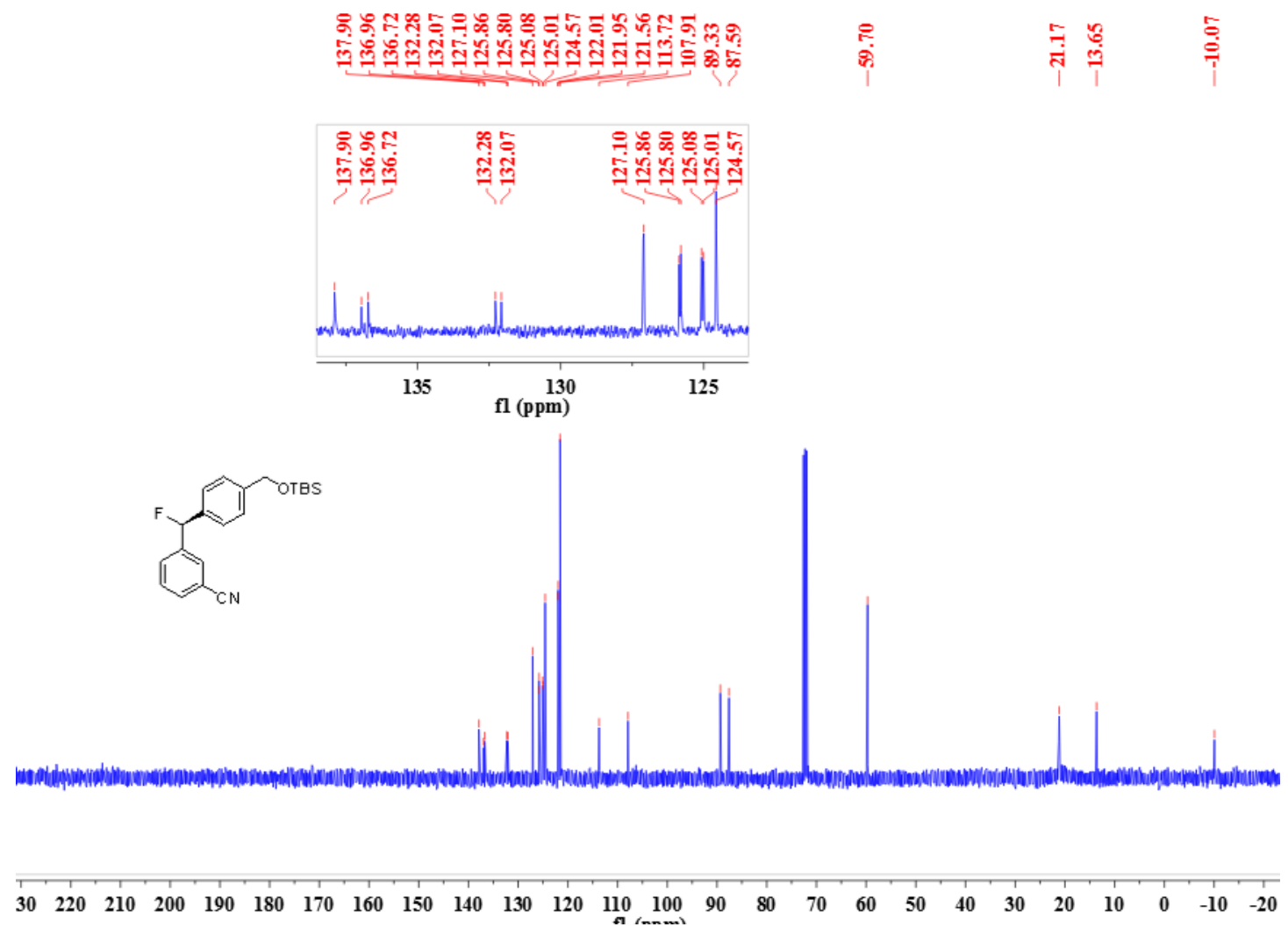


${ }^{19}$ F NMR (376 $\mathrm{MHz}, \mathrm{CDCl}_{3}$ ) spectrum of dimethyl 4,4'-(1,2-difluoroethane-1,2-diyl)dibenzoate 3a'

5159-17-14-3_FLUORTNE_01 $.5159-17-14-3$
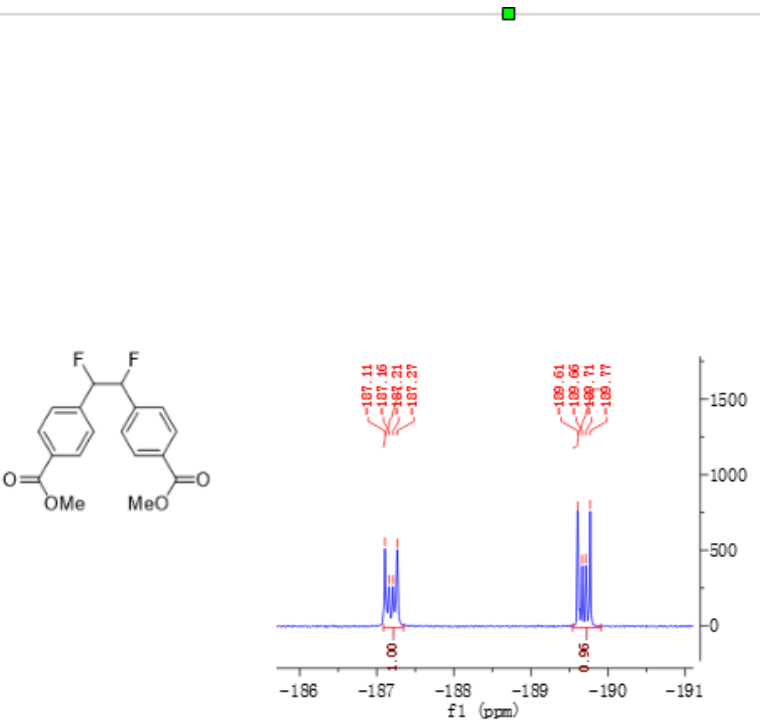

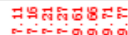

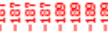

||

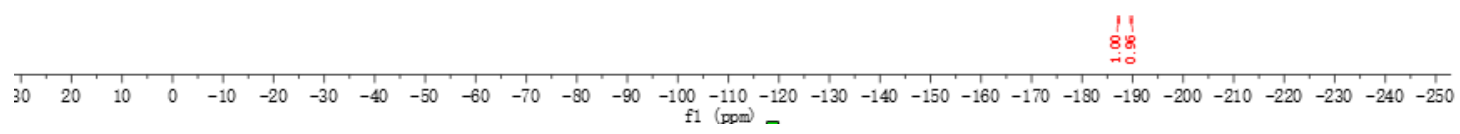

${ }^{1} \mathrm{H}$ NMR (400 $\mathrm{MHz}, \mathrm{CDCl}_{3}$ ) spectrum of

dimethyl 4,4'-(1,2-difluoroethane-1,2-diyl)dibenzoate 3a'

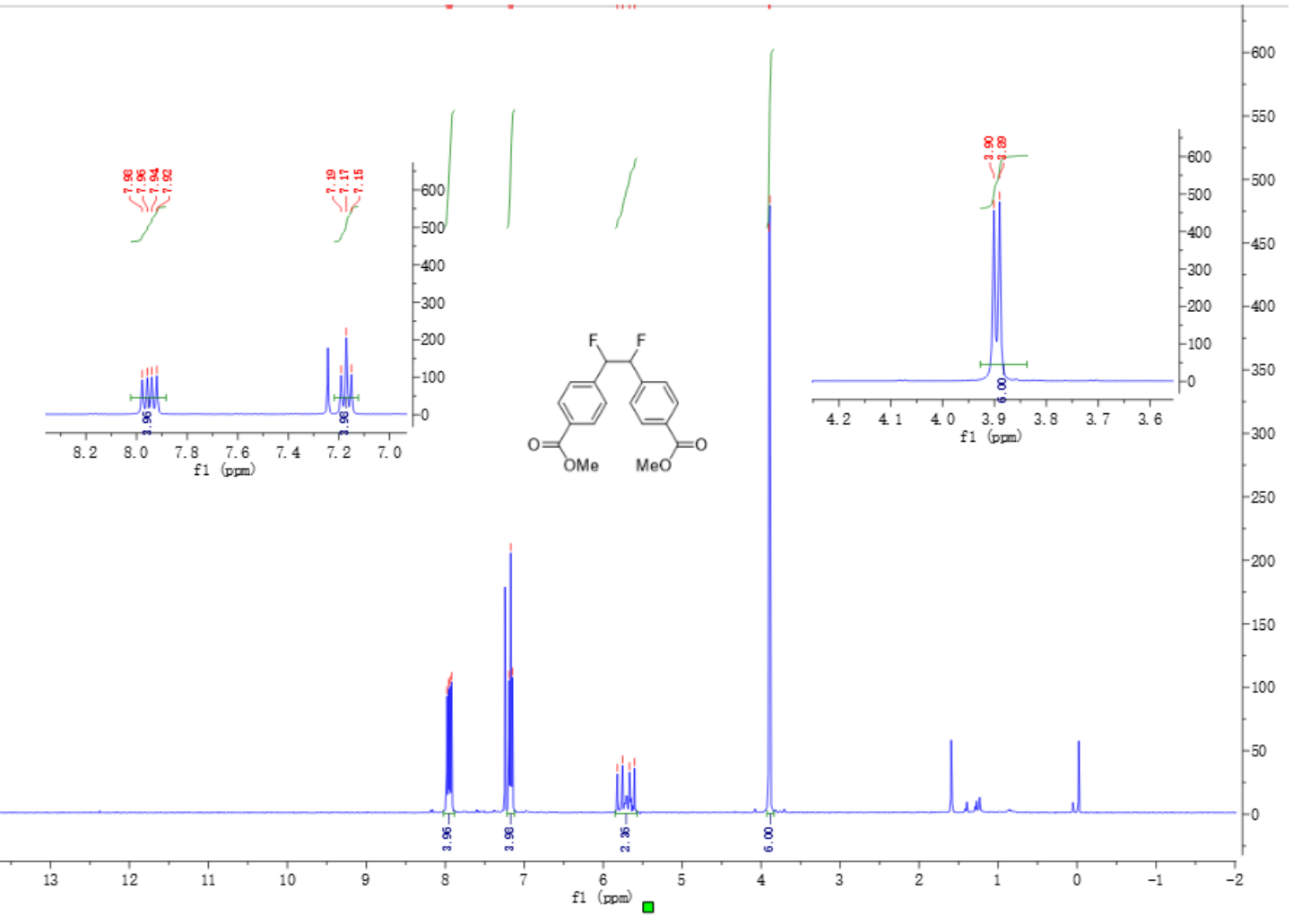


${ }^{13} \mathrm{C}$ NMR (101 MHz, $\left.\mathrm{CDCl}_{3}\right)$ spectrum of dimethyl 4,4'-(1,2-difluoroethane-1,2-diyl)dibenzoate 3a'

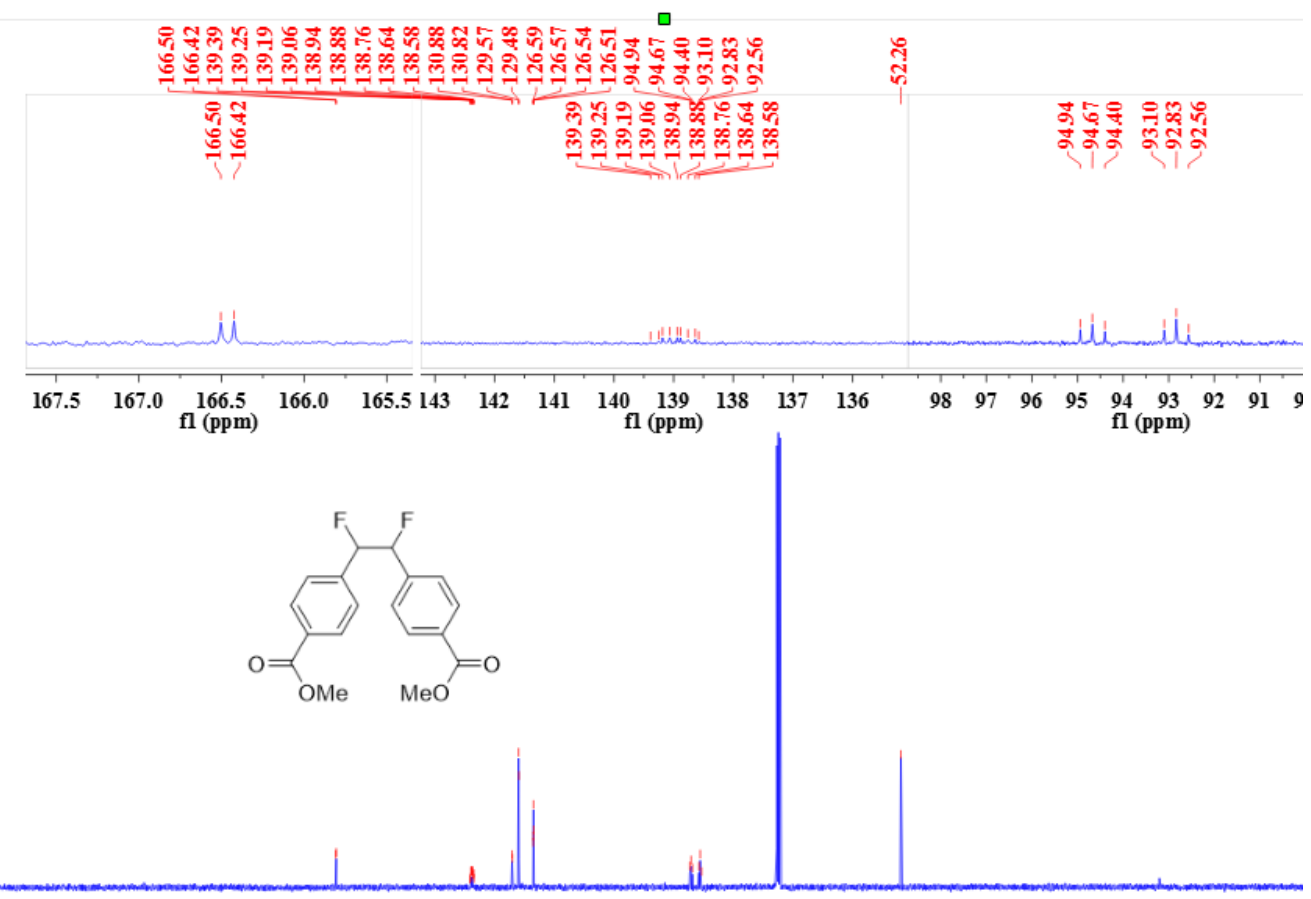

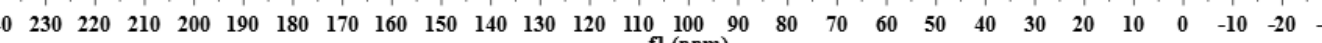




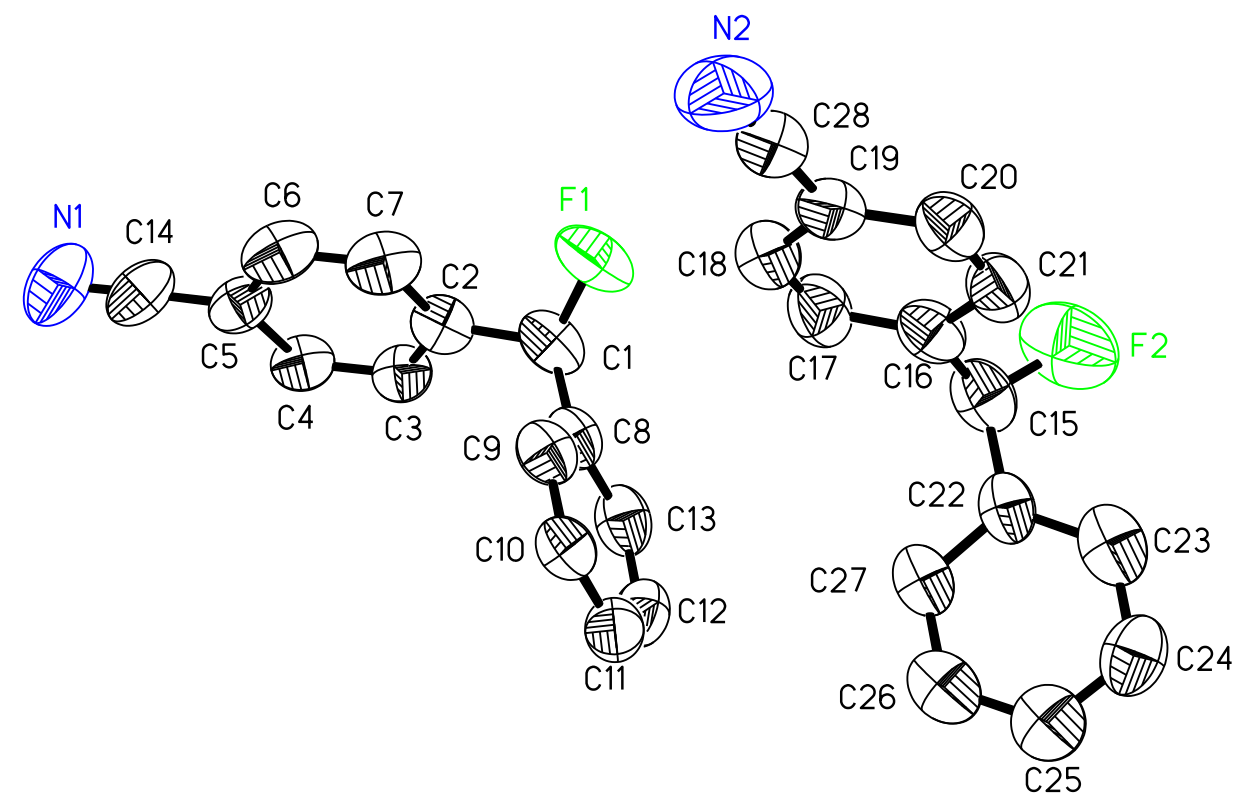

Figure S3. ORTEP diagram of compound 3q. Ellipsoids are shown at the 50\% probability level. 
Table S1. Crystal data and structure refinement for 623 .

Identification code

Empirical formula

Formula weight

Temperature

Wavelength

Crystal system, space group

Unit cell dimensions

Volume

Z, Calculated density

Absorption coefficient

$\mathrm{F}(000)$

Crystal size

Theta range for data collection

Limiting indices

Reflections collected / unique

Completeness to theta $=65.074^{\circ}$

Absorption correction

Max. and min. transmission

Refinement method

Data / restraints / parameters

Goodness-of-fit on $\mathrm{F}^{2}$

Final $\mathrm{R}$ indices [I $>2 \operatorname{sigma}(\mathrm{I})]$

$\mathrm{R}$ indices (all data)

Absolute structure parameter

Extinction coefficient

Largest diff. peak and hole
623

$\mathrm{C} 14 \mathrm{H} 10 \mathrm{FN}$

211.23

297(2) K

$1.54178 \AA$

Triclinic, $\mathrm{P} 1$

$a=7.3593(13) \AA \quad \alpha=105.097(9)^{\circ}$.

$\mathrm{b}=9.5269(15) \AA \quad \beta=110.708(10)^{\circ}$.

$\mathrm{c}=9.6955(17) \AA \quad \gamma=103.559(10)^{\circ}$.

572.37(18) $\AA^{3}$

2, $\quad 1.226 \mathrm{Mg} / \mathrm{m}^{3}$

$0.677 \mathrm{~mm}^{-1}$

220

$0.160 \times 0.120 \times 0.080 \mathrm{~mm}$

5.267 to $65.074^{\circ}$

$-8<=\mathrm{h}<=8,-11<=\mathrm{k}<=11,-11<=\mathrm{l}<=11$

$5230 / 3417[\mathrm{R}(\mathrm{int})=0.0615]$

$98.6 \%$

Semi-empirical from equivalents

0.7531 and 0.5746

Full-matrix least-squares on $\mathrm{F}^{2}$

3417 / 3 / 289

1.026

$\mathrm{R} 1=0.0863, \mathrm{wR} 2=0.2340$

$\mathrm{R} 1=0.1424, \mathrm{wR} 2=0.2875$

$0.5(2)$

$\mathrm{n} / \mathrm{a}$

0.582 and -0.265 e. $\AA^{-3}$ 